\title{
Residual Radioactivity Guidelines for the Heavy Water Components Test Reactor at the Savannah River Site
}

by

M. B. Owen

Westinghouse Savannah River Company

Savannah River Site

Aiken, South Carolina 29808

U.S. Enerov Corporation

\section{MASTER}

A document prepared for ATTACHMENT TO SPEC FOR DEMOLITION HWCTR at, , from - .

DOE Contract No. DE-AC09-96SR18500

This paper was prepared in connection with work done under the above contract number with the $U$. $S$.

Department of Energy. By acceptance of this paper, the publisher and/or recipient acknowledges the U.S.

Government's right to retain a nonexclusive, royalty-free license in and to any copyright covering this paper, along with the right to reproduce and to authorize others to reproduce all or part of the copyrighted paper. 



\section{DISCLAIMER}

This report was prepared as an account of work sponsored by an agency of the United States Government. Neither the United States Government nor any agency thereof, nor any of their employees, makes any warranty, express or implied, or assumes any legal liability or responsibility for the accuracy, completeness, or usefulness of any information, apparatus, product, or process disclosed, or represents that its use would not infringe privately owned rights. Reference herein to any specific commercial product, process, or service by trade name, trademark, manufacturer, or otherwise does not necessarily constitute or imply its endorsement, recommendation, or favoring by the United States Government or any agency thereof. The views and opinions of authors expressed herein do not necessarily state or reflect those of the United States Government or any agency thereof.

This report has been reproduced directly from the best available copy.

Available to DOE and DOE contractors from the Office of Scientific and Technical Information, P.O. Box 62, Oak Ridge, TN 37831; prices available from (615) 576-8401.

Available to the public from the National Technical Information Service, U.S. Department of Commerce, 5285 Port Royal Road, Springfield, VA 22161. 


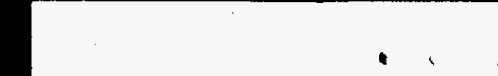




\title{
WSRC-TR-97-0135
}

\section{Residual Radioactivity Guidelines for the}

Heavy Water Components Test Reactor at the Savannah River Site

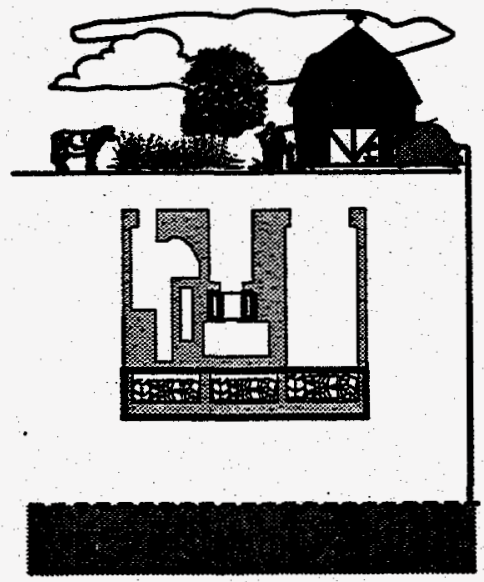

\author{
April 1997 \\ Prepared by \\ DOES NOT CONTAN \\ UACLASSIFED CONTROLED \\ $\operatorname{spc} 8$

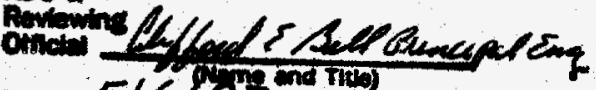

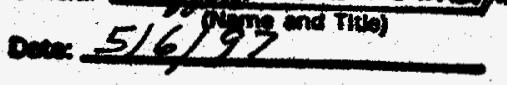 \\ U S ENERGY Corporation of Aiken, S.C.

\section{In Association With} \\ Life Cycle Environmental of Charleston, S.C. \\ - For the \\ Westinghouse Savannah River Company \\ Under Subcontract No. AB72522N
}

UNCLASSIFIED 


\section{Table of Contents}

Residual Radioactivity Guidelines for the

Heavy Water Components

Test Reactor at the

Savannah River Site
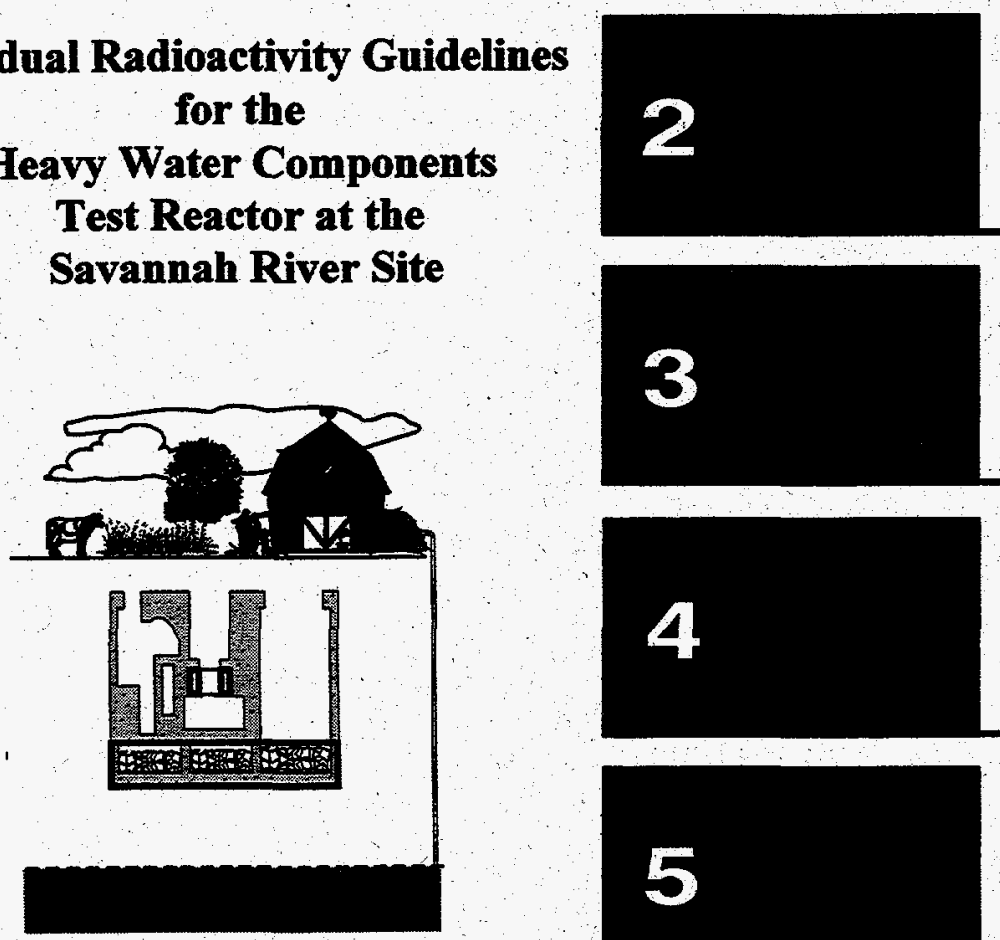

EXECUTIVE SUMMARY

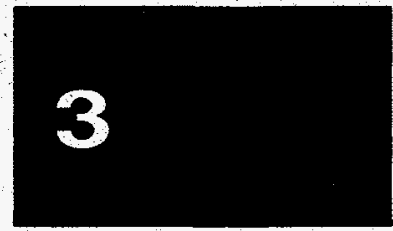

\section{REPORT}

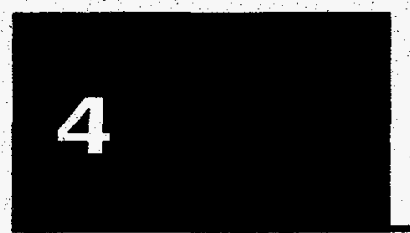

\section{APPENDIX A}

PARAMETERS USED IN ANALYSIS - SCENARIO A

April 1997

Prepared by

Ron Smith

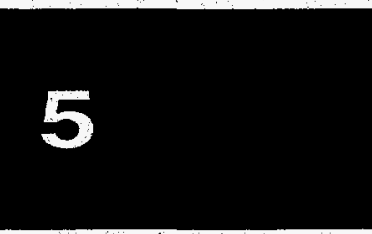

\section{APPENDIX B}

PARAmeters USEd in ANALysis - Scenario B

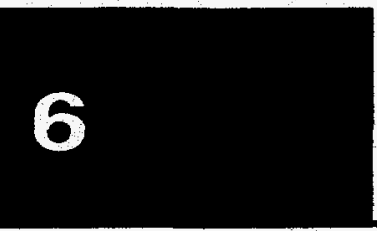

\section{APPENDIX C}

PARAMETERS Used In ANALYSIS - ScenARIO A (WITH ADDTIOONAL SOURCE TERM)

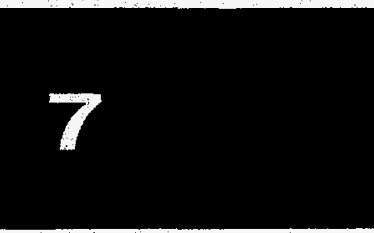

\section{APPENDIX D} and

Jim McNeil

Life Cycle Environmental
RESRAD SUMMARY REPORT - BIOLOGICAL SHIELD

\section{APPENDIX E}

8

RESRAD SUMMARY REFOFT - CONTAMINATED CONCRETE AND EMBEDDED PIPING

\section{APPENDIX F}

RESRAD SUMMARY REPORT - RADIOACTIVE EQUIPMENT, CONTAMINATED CONCRETE AND EMBEDDED PIPING

APPENDIX G

10 


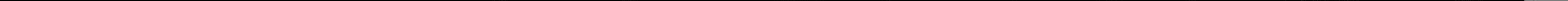




\section{DISCLAMMER}

Portions of this document may be illegible in electronic image products. Images are produced from the best available original document. 



\section{Contents}

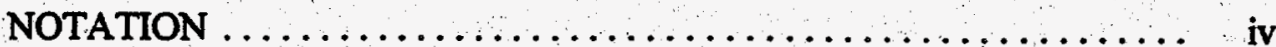

EXECUTIVE SUMMARY $\ldots \ldots \ldots \ldots \ldots \ldots \ldots \ldots \ldots \ldots \ldots \ldots$

1.0 INTRODUCTION $\ldots \ldots \ldots \ldots \ldots \ldots \ldots \ldots \ldots \ldots \ldots \ldots \ldots \ldots$

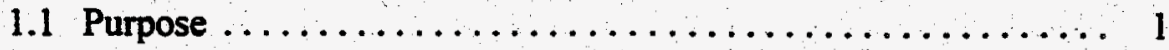

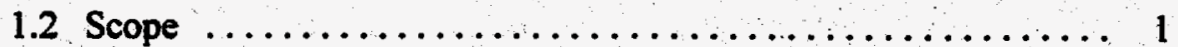

1.3 Background $\ldots \ldots \ldots \ldots \ldots \ldots \ldots \ldots \ldots \ldots \ldots \ldots \ldots \ldots \ldots \ldots \ldots \ldots$

1.4 About RESRAD $\ldots \ldots \ldots \ldots \ldots \ldots \ldots \ldots \ldots \ldots \ldots, 3$

1.5 About RESRAD-BUILD $\ldots \ldots \ldots \ldots \ldots \ldots \ldots \ldots \ldots \ldots$

1.6 Derived Concentration Guideline Levels ............ 5

1.7 Assumptions and Inherent Conservatism ............ 5

2.0 FACILITY DESCRIPTION $\ldots \ldots \ldots \ldots \ldots \ldots \ldots \ldots \ldots \ldots \ldots$

3.0 FACILITY HISTORY $\ldots \ldots \ldots \ldots \ldots \ldots \ldots \ldots \ldots \ldots \ldots \ldots \ldots \ldots \ldots$

4.0 HWCTR SURVEY UNITS $\ldots \ldots \ldots \ldots \ldots \ldots \ldots \ldots \ldots \ldots \ldots \ldots$

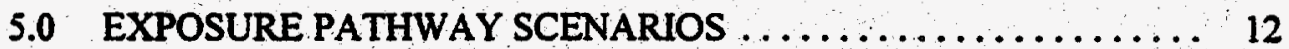

5.1 Scenario A-Residential Use $\ldots \ldots \ldots \ldots \ldots \ldots \ldots \ldots \ldots \ldots$

5.2 Scenario B - Administrative Building $\ldots \ldots \ldots \ldots \ldots \ldots \ldots \ldots$

5.3 Considerations in Scenario Selection $\ldots \ldots \ldots \ldots \ldots \ldots \ldots \ldots 14$

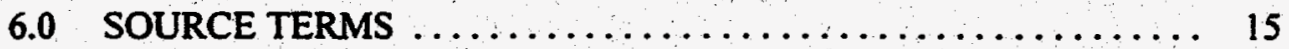

6.1 The Calculated Source Terms $\ldots \ldots \ldots \ldots \ldots \ldots \ldots \ldots \ldots .16$

6.2 The 1996 Characterization Study Data ............. 18

6.3 Induced Radioactivity in the Reactor Biological Shield ....... 19

6.4 Radioactivity Embedded in Concrete Floors . . . . . . . . . 21

6.5 Radioactivity Inside Floor Drains and Other Embedded Piping . . . 22

6.6 Radioactivity Inside Equipment $\ldots \ldots \ldots \ldots \ldots \ldots \ldots \ldots \ldots 24$

6.7 Removable Contamination $\ldots \ldots \ldots \ldots \ldots \ldots \ldots \ldots \ldots \ldots .24$

6.8 Methodology for Calculating the Source Terms . . . . . . . 25

6.8.1 Biological Shield Activity ................. 25

6.8.2 Contaminated Concrete and Rubble Activity ........ 26

6.8.3 RESRAD-BUILD Wall Activity $\ldots \ldots \ldots \ldots \ldots \ldots \ldots 26$ 
6.9 Assumptions Used in Computing Source Terms $\ldots \ldots \ldots \ldots 26$

7.0 OTHER PARAMETERS FOR RESRAD AND RESRAD-BUILD . . . 28

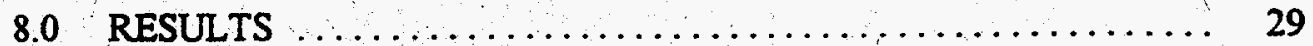

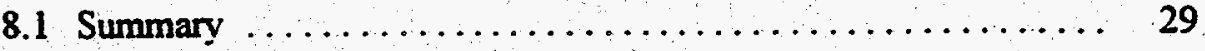

8.2 Detailed RESRAD Results $\ldots \ldots \ldots \ldots \ldots \ldots \ldots \ldots \ldots \ldots .30$

8.3 Detailed RESRAD Results - With Radioactive Equipment in Place

8.4 Water-Dependent Pathways $\ldots \ldots \ldots \ldots \ldots \ldots \ldots \ldots \ldots \ldots, 37$

8.5 Radionuclides of Most Significance $\ldots \ldots \ldots \ldots \ldots \ldots \ldots \ldots, 38$

8.6 Detailed RESRAD-BUILD Results $\ldots \ldots \ldots \ldots \ldots \ldots \ldots \ldots \ldots$

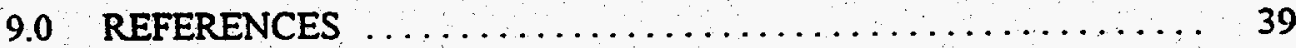

APPENDIX A Parameters Used in Analysis - Scenario A

B Parameters Used in Analysis - Scenario B

C Parameters Used in Analysis - Scenario A (With Additional Source Term)

D RESRAD Summary Report, Biological Shield

E RESRAD Summary Report, Contaminated Concrete and Embedded Piping

F RESRAD Summary Report, Radioactive Equipment. Contaminated Concrete and Embedded Piping

G RESRAD-BUILD Summary Report

\section{Figures}

1 Location of the HWCTR Facility on the Savannah River Site ....... 2

2 RESRAD Exposure Pathways . ..................... 4

3 The HWCTR Property $\ldots \ldots \ldots \ldots \ldots \ldots \ldots \ldots \ldots \ldots \ldots \ldots \ldots$

4 Section View of Reactor Building ................ 8

5 Section View of Reactor Building Showing Steam Generators ....... 8

6 Reactor and Biological Shield ..................... 10

$7 \quad$ Plan View of Underground Structure $\ldots \ldots \ldots \ldots \ldots \ldots \ldots \ldots \ldots$

8 Biological Shield Showing Core Samples ................ 21 
10 RESRAD Results With Present Source Terms For Biological Shield .. 29

11. RESRAD Results With Present Source Terms For Contaminated Concrete $\ldots \ldots \ldots \ldots \ldots \ldots \ldots \ldots \ldots \ldots \ldots \ldots \ldots \ldots \ldots \ldots . \ldots \ldots$

12 RESRAD Results With Present Source Term for Radioactive Equipment and Contaminated Concrete $\ldots \ldots \ldots \ldots \ldots \ldots \ldots \ldots, 36$

13 RESRAD Doses from Water-Dependent Pathways $\ldots \ldots \ldots \ldots \ldots \ldots$

\section{Tables}

1 Typical HWCTR Survey Units $\ldots \ldots \ldots \ldots \ldots \ldots \ldots \ldots \ldots \ldots \ldots \ldots$

2 Exposure Pathways for Scenario A $\ldots \ldots \ldots \ldots \ldots \ldots \ldots \ldots \ldots$

3 Exposure Pathways for Scenario B ................... 14

$4 \quad$ Calculated Source Term - Reactor Biological Shield . . . . . . . 16

5 Calculated Source Term - Rubble $\ldots \ldots \ldots \ldots \ldots \ldots \ldots \ldots \ldots$

6 Calculated Source Term - Rubble, Installed Equipment and Biological

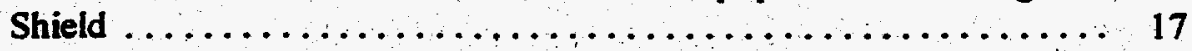

7 Biological Shield Activation Analysis Results $\ldots \ldots \ldots \ldots \ldots \ldots \ldots$

8 Concrete Sample Results $\ldots \ldots \ldots \ldots \ldots \ldots \ldots \ldots \ldots \ldots \ldots \ldots \ldots \ldots \ldots \ldots \ldots$

$9 \quad$ Embedded Radioactive Piping $\ldots \ldots \ldots \ldots \ldots \ldots \ldots \ldots \ldots \ldots \ldots \ldots \ldots \ldots$

10 Smears Showing Removable Activity Above Limits $\ldots \ldots \ldots \ldots \ldots .24$

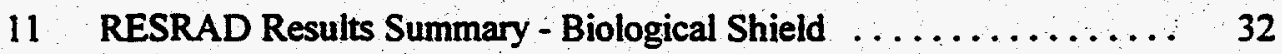

12. RESRAD Results Summary - Concrete and Rubble $\ldots \ldots \ldots \ldots \ldots . \ldots 33$

13 Calculated DCGLs For Concrete Floors, Based on $15 \mathrm{mrem} / \mathrm{yr}$

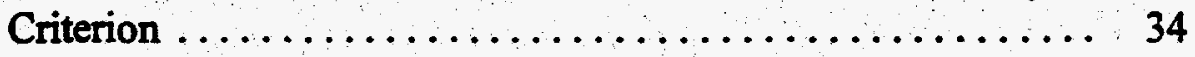

14 Calculated DCGLs For Concrete Floors, Based on $100 \mathrm{mrem} / \mathrm{yr}$

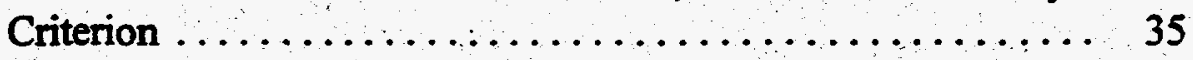

15 RESRAD Results Summary - With Radioactive Equipment in Place 


\section{NOTATION}

The following acronyms, initialisms and abbreviations (including units of measure) are used in this document.

\section{ACRONYMS, INITLALISMS AND ABBREVIATIONS}

$\begin{array}{ll}\text { ALARA } & \text { as low as reasonably achievable } \\ \text { DCGL } & \text { derived concentration guideline level } \\ \text { DOE } & \text { U.S. Department of Energy } \\ \text { HPLS } & \text { Horizontal power level sleeve } \\ \text { HWCTR } & \text { Heavy Water Components Test Reactor } \\ \text { RESRAD } & \text { residual radioactive material guideline computer code } \\ \text { RESRAD-BULLD } & \text { residual radioactive computer code for buildings } \\ \text { SRS } & \text { Savannah River Site }\end{array}$

\section{UNITS OF MEASURE}

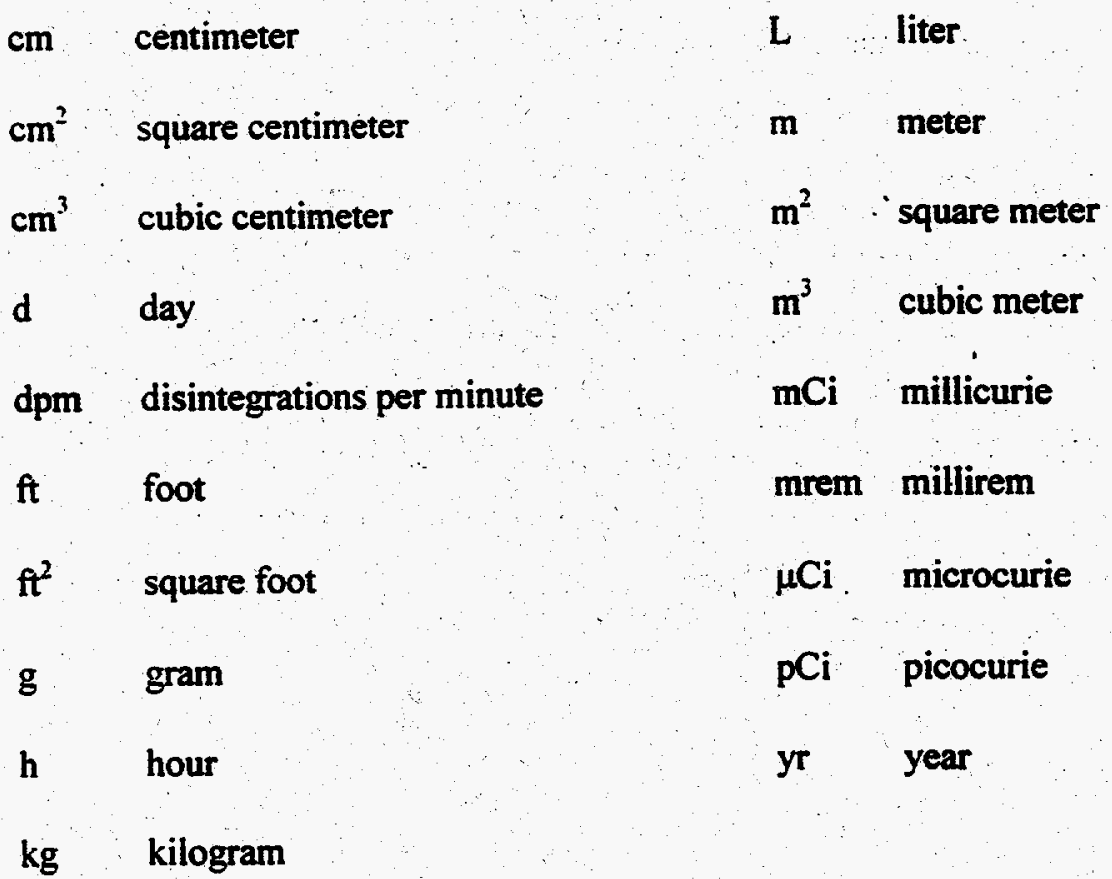




\section{EXECUTIVE SUMMARY}

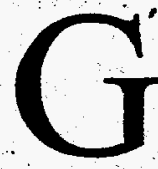
uidelines were developed for acceptable levels of residual radioactivity in the Heavy Water Components Test Reactor (HWCTR) facility at the conclusion of its decommissioning, which will entail an unconditional release of the property. These guidelines are expressed as derived concentration guideline levels (DCGLs) for the radionculides that will remain in the facility.

The calculated DCGLs, when compared to existing concentrations of radionuclides measured during a 1996 characterization program, indicate that no decontamination of concrete surfaces will be necessary. Also, based on the results of the calculations, activated concrete in the reactor biological shield does not have to be removed, and imbedded radioactive piping in the facility can remain in place.

Viewed in another way, the results of the calculations showed that the present inventory of residual radioactivity in the facility (not including that associated with the reactor vessel and steam generators) would produce less than one millirem per year above background to a hypothetical individual on the property. The residual radioactivity is estimated to be approximately 0.04 percent of the total inventory in the facility as of March, 1997.

According to the results, the only radionuclides that would produce greater than 0.01 millirem per year are Am-241 $(0.013 \mathrm{mrem} / \mathrm{yr}$ at 300 years $), C-14$ (0.022 mrem/yr at 1000 years) and U-238 (0.034 mrem/yr at 6000 years). Human exposure would occur only through the ground-water pathways, that is, from water drawn from a well on the property. The maximum exposure would be approximately one percent of the 4 millirem per year ground water exposure limit established by the U.S. Environmental Protection Agency.

The HWCTR is a pressurized heavy water cooled and moderated test reactor located at the Savannah River Site (SRS). Housed in a steeldomed containment structure, the HWCTR was operated in the 1960s to test experimental fuel assemblies for heavy water power reactors. It is being dismantled and the two-acre site released for unrestricted use.

For this project, the U. S. Department of Energy (DOE) plans to use the release criteria of DOE Order 5400.5, Radiation Protection of the 
Public and the Environment. These criteria require that exposure from residual radioactivity be as low as reasonably achievable (ALARA), but no more than 100 millirem per year.

Using source terms developed from data generated in a detailed 1996 characterization study, RESRAD and RASRAD-BUILD residual radioactivity computer codes were utilized to calculate DCGLs for radionuclides in the facility. These radionuclide limits also inctuded embedded radioactive piping. A timeframe of 10,000 years for potential releases of residual radioactivity to the environment was used. Two sets of DCGLs were calculated, corresponding to exposure rates of 100 millirem per year and 15 millirem per year to a hypothetical individual at the site.

Two exposure pathway scenarios were studied. Scenario A was the basic RESRAD scenario, which entails having a family farm on the property. In Scenario $A$ it was assumed that groundwater from a well on the property would be used for drinking and other purposes. One standard RESRAD exposure pathway - ingestion of fish from an onsite pond - was not considered plausible for the HWCTR site, and was not taken into account. In consideration of current SRS futureuse plans; it was assumed that Scenario $\mathrm{A}$ would begin in 30 years.

Scenario B entailed use of the property for an administrative building where one existing wall in the upper part of the underground structure would form part of a building basement occupied by workers.

The assumptions used in the study were realistic but generally on the conservative side. The RESRAD results using Scenario A were considered to be more meaningful than the RESRAD-BUIID results using scenario $B$ : 


\subsection{Purpose}

The purpose of this report is to describe the results of using the RESRAD and RESRAD-BUILD residual radioactivity computer codes to calculate guidelines for residual radioactivity in the Heavy Water Components Test Reactor (HWCTR) facility at the Savannah River Site (SRS) at the conclusion of its decommissioning. The HWCTR decommissioning entails an unconditional release of the site property.

\subsection{Scope}

The study focused on the reactor containment building. It considered radiation exposure pathway scenarios appropriate for a 10,000-year timeframe. It produced residual radioactivity guidelines based on two different criteria: 100 mrem per year above background and 15 mrem per year above background to a hypothetical individual exposed to radioactivity on the site property. The 100 mrem per year value is the maximum limit of the current Department of Energy (DOE) unrestricted release criteria. The $15 \mathrm{mrem}$ per year value corresponds to the criterion of the Nuclear Regulatory Commission's proposed radiation cleanup rule. It is considered appropriate for a reference value corresponding to as low as reasonably achievable (ALARA), given the limited amounts of radioactive contaminants in the HWCTR facility.

The study also produced two estimates of the maximum annual radiation exposure to a hypothetical individual on the property. One estimate assumed that the radioactivity associated with the biological shield, contaminated concrete and embedded radioactive piping would remain in place. The other assumed that this radioactivity would be left in place along with all radioactivity in installed equipment, with the exception of the reactor vessel assembly and the steam generators.

\subsection{Background}

The DOE is undertaking the decommissioning of the HWCTR. This process will involve dismantlement of the facility.

Equipment contaminated with radioactivity will be removed from the facility, along with hazardous materials. The steel containment dome will be removed. The 
The underground concrete structure of the reactor building will be removed to five feet below grade. A final radiation staus survey will be accomplished. After it has been confirmed that residual radioactivity levels meet cleanup guidelines. concrete rubble produced during the decommissioning will be placed at the bottom of the building cavity. Clean earth will be used to fill the cavity. Then the property will be graded and seeded with grass. and made available for any suitable use.

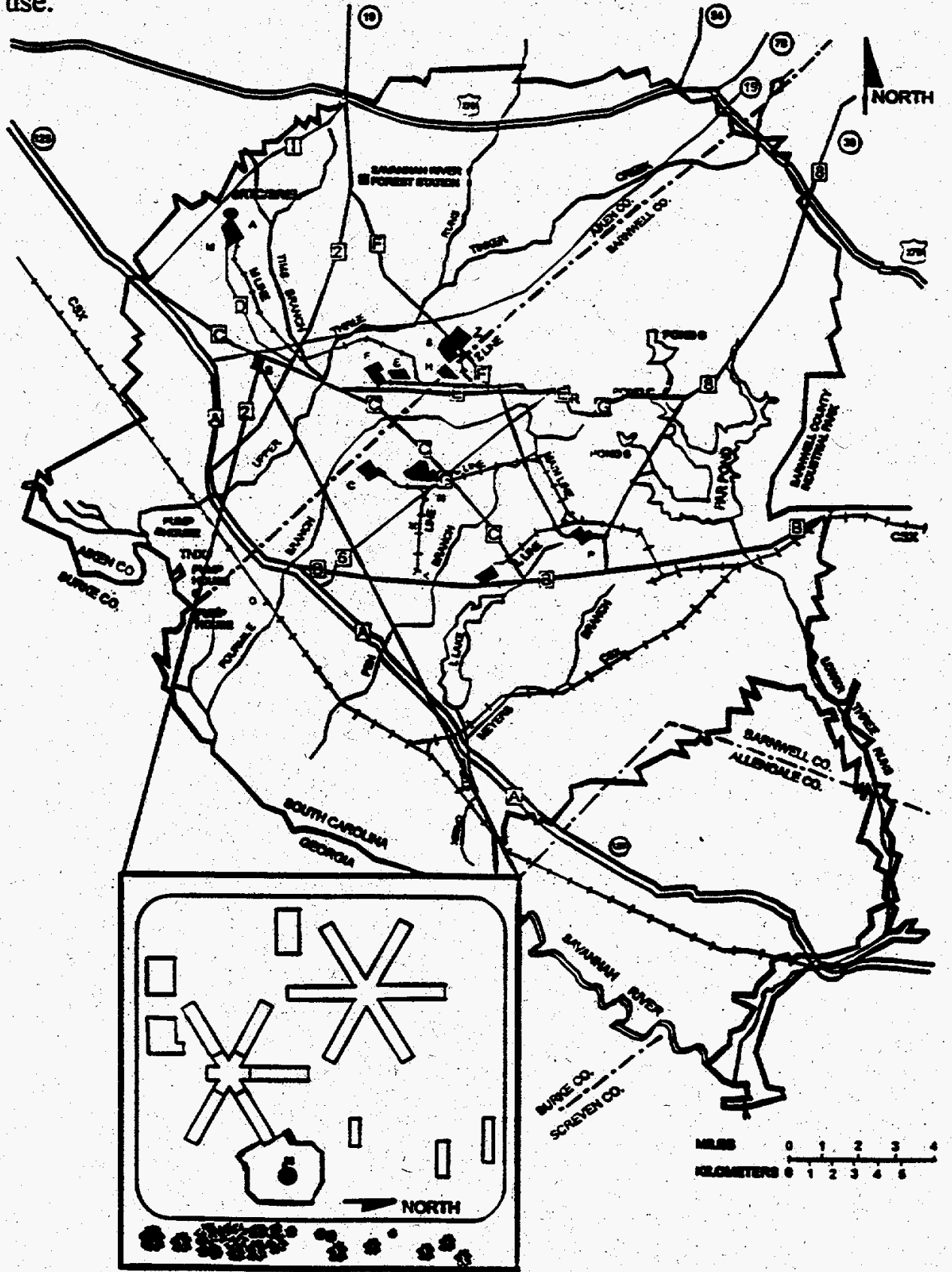

Figure 1. Location of the HWCTR Facility on the Savannah River Site. The HWCTR property, shown in the lower part of the inset. comprises U-Area. U-Area lies within B-Area which is designated an administrative area in long-term site property plans. 
For this project, the DOE plans to use the release criteria of DOE Order 5400.5, Radiation Protection of the Public and the Environment (reference 1). These criteria require that exposure to members of the public from residual radioactivity be less than 100 millirem per year for all exposure pathways, and as low as reasonably achievable below this value. The DOE order also specifies that the exposure rate inside occupied structures shall be less than $20 \mu \mathrm{rem}$ per hour above background, although it states that this criterion does not necessarily apply to buried structures or buildings planned for demolition.

To implement the release criteria, guidelines are required that relate the amounts of different radionuclides remaining in the facility to the annual exposure limit. These guidelines are expressed in terms of $\mathrm{dpm} / 100 \mathrm{~cm}^{2}$ and $\mathrm{pCi} / \mathrm{g}$. They are known as derived concentration guideline levels, or DCGLs.

DOE Order 5400.5 specifies that authorized limits for each property shall be set equal to generic or derived guidelines. According to the order, the authorized limits (i.e., the DCGLs) are to be approved by the DOE headquarters program office.

The DOE Decommissioning Resource Mamual (reference 2) states that sitespecific guidelines for radioactivity in soils and remaining structures are to be developed using the DOE material code RESRAD, employing a realistic pathway analysis. Procedures for using RESRAD appear in the Mamual for Implementing Residual Radioactive Material Guidelines Using RESRAD (reference 3).

When a building remaining on a decommissioned site may be occupied in a realistic future scenario, a related computer code may be used to calculate site-specific guidelines. This code is RESRAD-BUILD. It is described in RESRAD-BUILD, $A$ Computer Model for Analyzing the Radiological Doses Resulting from the Remediation and Occupancy of Buildings Contaminated with Radioactive Material (reference 4). RESRAD-BUILD also may be used to establish guidelines associated with possible occupancy of a portion of a buried contaminated structure.

\subsection{About RESRAD}

As explained in reference 3, RESRAD is a computer code developed by Argonne National Laboratory that can be used to model the transport of radionuclides in 
soil and determine the resulting radiation exposure to humans. Outputs include tables and graphs which show annual radiation exposure for periods as long as 10,000 years. Also, radionuclide guideline levels that equate to particular annual exposures can be produced by the code.

The basic RESRAD exposure pathway scenario, which reference 3 indicates is usually the most conservative, entails a family farm. The family living on the property could receive radiation exposure from residual radioactivity through eight different pathways, such as direct exposure to contaminated soil, eating plant foods grown on the property and drinking milk from on-site livestock. The model includes provisions for contamination from soil (or for the HWCTR case, buried structures) leaching into the ground water and then reaching the environment of those living on the property. Figure 2 illustrates the RESRAD pathways.

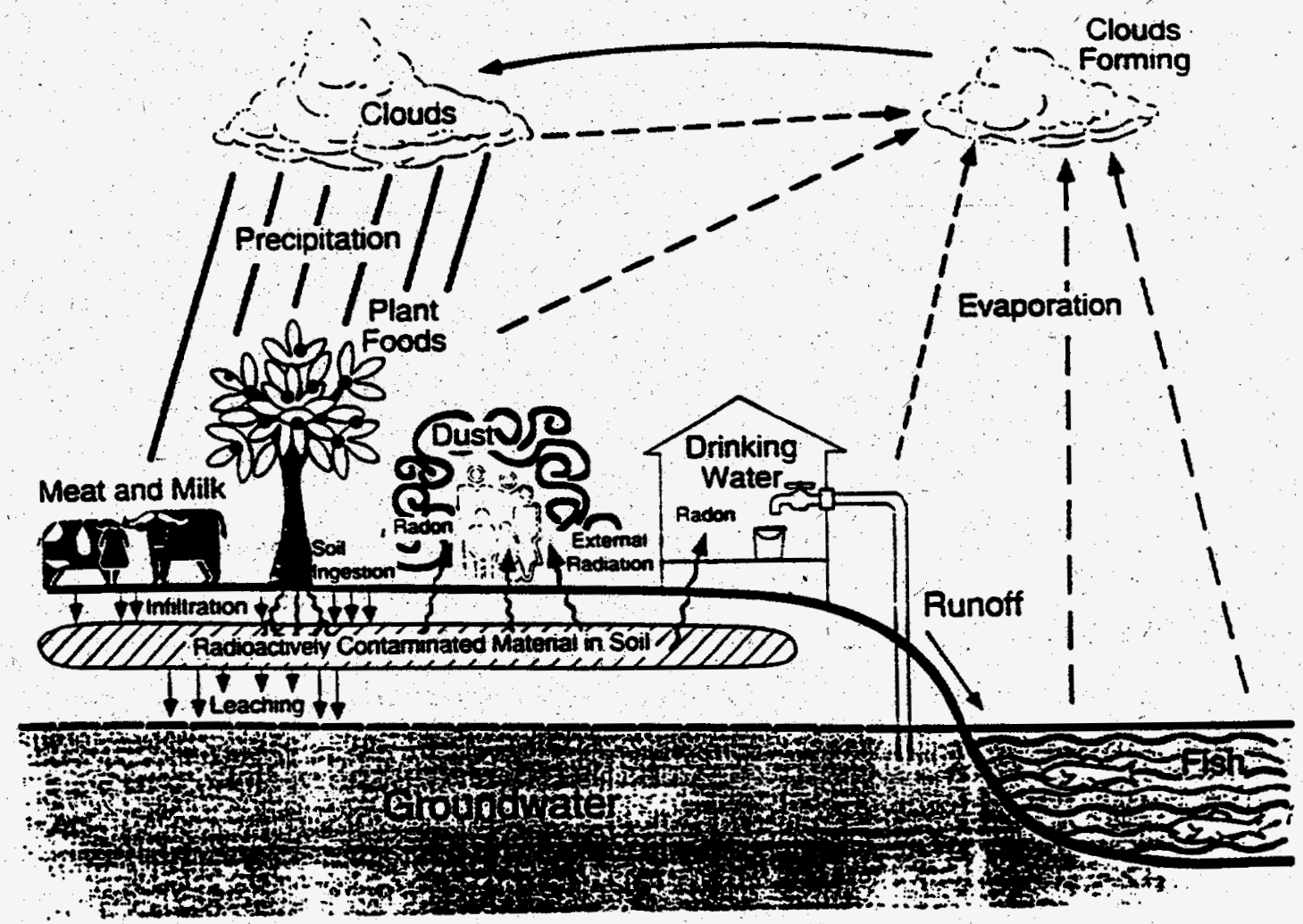

Figure 2. RESRAD Exposure Pathways.

RESRAD requires a variety of inputs. A source term that describes the concentrations of different radionuclides present is one key input. The geometry of the source, especially with respect to ground water, is another. Then there are numerous other inputs such as characteristics of the soil and the rates of intakes of foodstuffs. 


\subsection{About RESRAD-BUILD}

As described in reference 4, RESRAD-BUILD is a pathway analysis model developed by Argonne National Laboratory to evaluate the potential radiological dose to an individual who lives or works in a building contaminated with radioactive material. Outputs include tables of exposures associated with building occupancy. Radionuclide guideline levels associated with a particular annual exposure can also be calculated. Unlike RESRAD, the program does not produce graphs.

The program makes use of an air quality model that considers the transport of radioactive material from one compartment in a building to another. Six exposure pathways are considered, including external exposure from the source and inhalation of airborne radioactive particles.

Like RESRAD, the program requires a variety of inputs, such as the radionuclide concentrations, the source geometry and the building description.

\subsection{Derived Concentration Guideline Levels}

The primary products of the RESRAD and RESRAD BUILD calculations are the DCGLs.

A final radiation status survey will be performed to ensure that cleanup criteria have been achieved. This survey will be performed in accordance with a detailed Final Survey Plan, which will follow guidelines in the HWCTR Decommissioning Plan (reference 5). The level of detail for this survey should be commensurate with need, based on the results of this study.

The final status survey will entail the use of DCGLs. These are radionuclidespecific activity concentrations within a survey unit that correspond to the release criteria. The HWCTR survey units are discussed in Section 4. A survey unit is basically an area for which a separate decision is made on whether the unit meets the cleanup standard.

\subsection{Assumptions and Inherent Conservatism}

Assumptions made in performing this study are described in Sections 5 and 6. Assumptions were generally realistic but on the conservative side. For example, in source term calculations the highest measured contamination levels were 
considered to be average contamination levels. One inherent conservatism in the RESRAD process is modeling buried concrete structures as soil, which was done for the HWCTR project. Radionuclide migration from and through concrete would, of course, occur at a slower rate than through soil, allowing for more radioactive decay to take place before contaminants reached the environment. Another conservative assumption involves the lower axial shield below the reactor vessel. This shield, which contains most of the Co-60 in the facility, was assumed to remain in place. But it more likely will be removed during the dismantlement work.

\subsection{FACILTYY DESCRIPTION}

The HWCTR facility is located on approximately two acres in the northwest quadrant of the SRS, on property known as U-Area. U-Area lies three miles from the nearest SRS property boundary and about two and one-half miles from any major nuclear materials production facilities on the site. See Figure 1.

The federally-owned SRS reservation is located near Aiken, South Carolina. The DOE Savannah River Operations Office manages the SRS. The Westinghouse Savannah River Company operates the site under contract to DOE.

The SRS occupies an area of approximately 310 square miles in western South Carolina in a mostly rural area of Aiken, Barnwell and Allendale counties, about 25 miles southeast of Augusta, Georgia.

The area east of the HWCTR facility is wooded. Adjoining the HWCTR property to the west is the site security force headquarters: 'Other administrative buildings of B-Area are nearby.

The elevation of the HWCTR site is approximately 288 feet above sea level. No stream runs near the property. The water table at the site is some 50 feet below the lowest point in the reactor containment building.

Figure 3 on the next page shows the HWCTR property. Figures 4 and 5 on page 9 show the reactor containment building.

The HWCTR was a pressurized heavy water test reactor used to test candidate fuel designs for heavy water power reactors. The nominal reactor power of 50 megawatts (thermal) was dissipated to the atmosphere through a muffler, which lies approximately 110 yards east of the reactor building. 


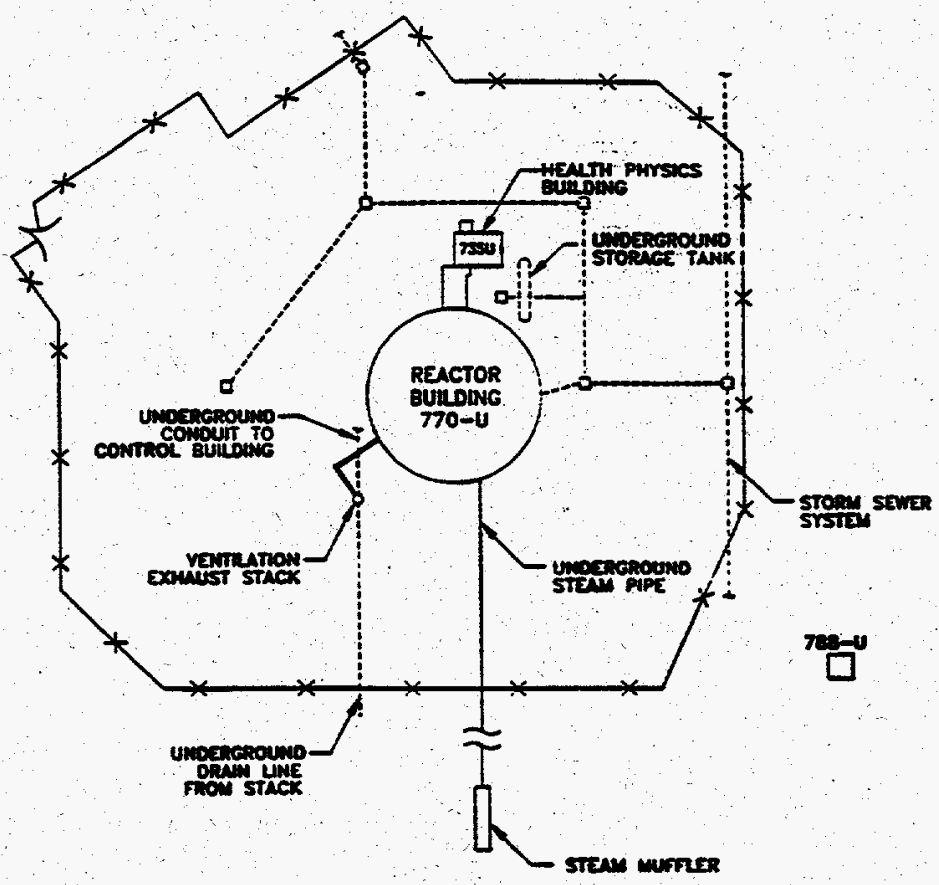

Figure 3. The HWCTR Property

The containment building is 70 feet in diameter. The steel dome structure rises approximately 65 feet above the ground; the floor of the lowest level is approximately 52 feet below grade. The below-grade part was constructed of prestressed concrete.

The containment building houses the reactor and coolant systems, the refueling machine, the spent fuel basin and the reactor instrumentation. Arrangement of the reactor within the containment building is shown in Figure 4.

The reactor vessel has an overall height of 36.75 feet (including the monitor pin nozzles), a diameter of about eight feet and weighs approximately 100 tons. The vessel is made of carbon steel, three to five inches thick, clad internally with $\mathbf{0 . 2 5}$ inch stainless steel. The reactor vessel and its associated biological shielding are shown in Figure 6 on page 10.

The reactor main cooling system operated at 1200 pounds per square inch (gage) and 250 degrees Centigrade. Two test loops, isolated from the primary cooling system, provided special test conditions. These loops are comprised of four-inch diameter stainless steel piping. 


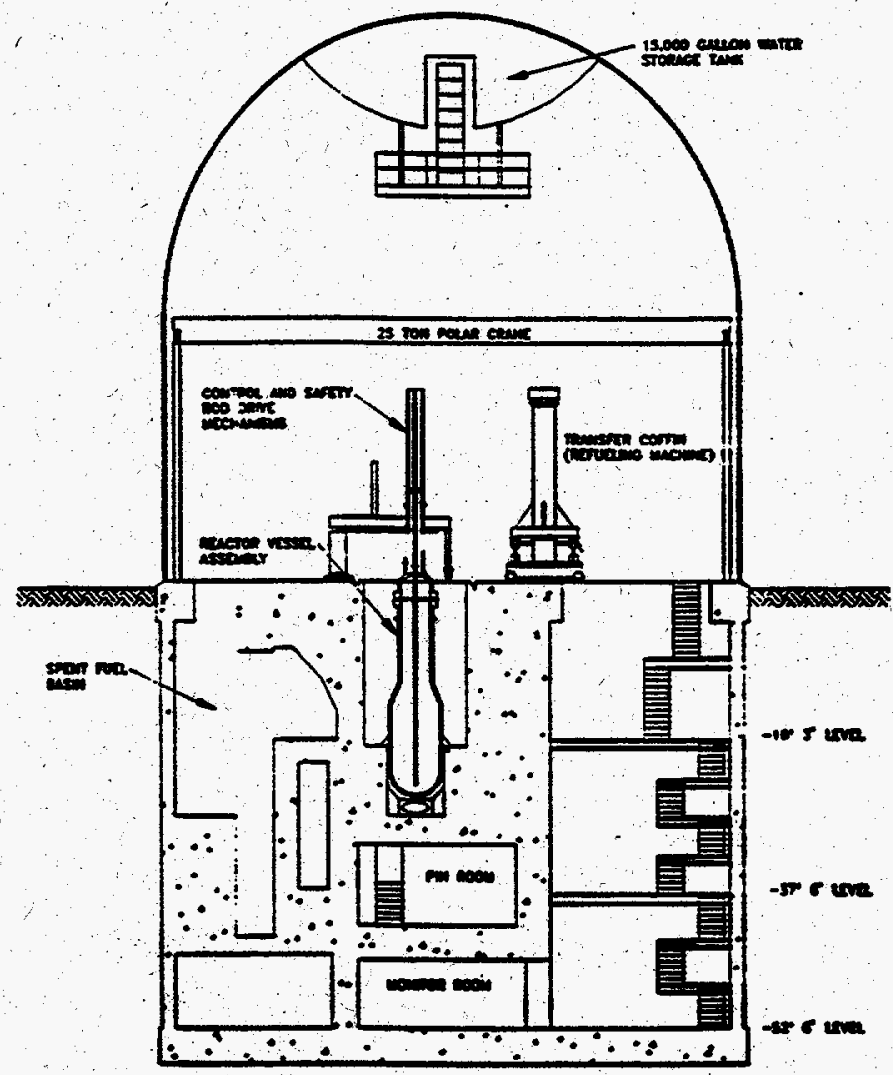

Figure 4. Section View of Reactor Building

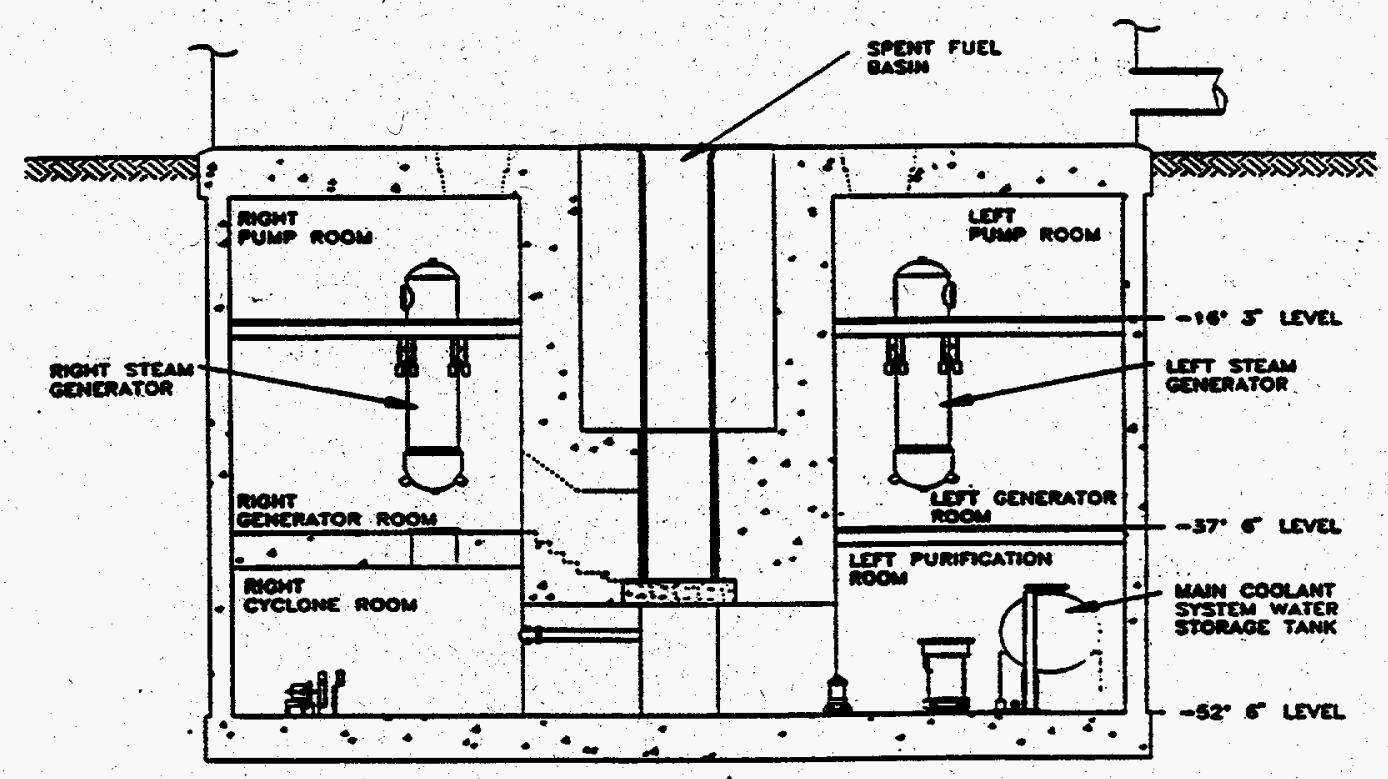

Figure 5. Section View of Reactor Building Showing Steam Generators. (This view is rotated 90 degrees from the view of Figure 4.) 
Also in the reactor building is a small spent fuel basin made of reinforced concrete lined with stainless steel. This basin, 9 feet by 13 feet wide, extends from the ground floor to the minus 27 -foot level. It contains a 54-inch by 54inch shipping cask pit that extends 15 feet below the basin floor.

A system of floor drains leads to a 350 gallon sump tank located in the monitor room at the minus 52 -foot level.

\subsection{FACILITY HISTORY}

The HWCTR was operated from March 1962 to December 1964 to test fuel elements and other reactor components of potential use in heavy water moderated and cooled power reactors. The total power history of the HWCTR was 13.882 megawatt-days.

During operation. 36 different fuel assemblies were tested. Ten of these experienced cladding failures as operational capabilities of the different designs were being established, releasing fission products, uranium and transuranics into the primary cooling system. Spills of radioactive water from the primary cooling system and other systems occurred frequently in most areas of the reactor building during the operating period.

In December of 1964, operations were terminated and the facility was placed in a standby condition. as a result of the decision by the U. S. Atomic Energy Commission to redirect research and development work on heavy water power reactors to reactors cooled with organic materials. For about one year. site personnel maintained the facility in a standby status. then retired the reactor in place.

In the 1970s, the U.S. Energy Research and Development Administration made preparations to decommission the HWCTR. To support planning for the decommissioning, a characterization study was accomplished in 1975 to determine the location and amounts of radiological contaminants in the facility. The study showed that radiation and radioactive contamination levels were low, and that most of the radioactivity was contained within the reactor vessel.

A decommissioning plan was developed in 1976 , but this plan was never carried out. The facility condition for the past twenty years has approximated safe storage or protective confinement. 


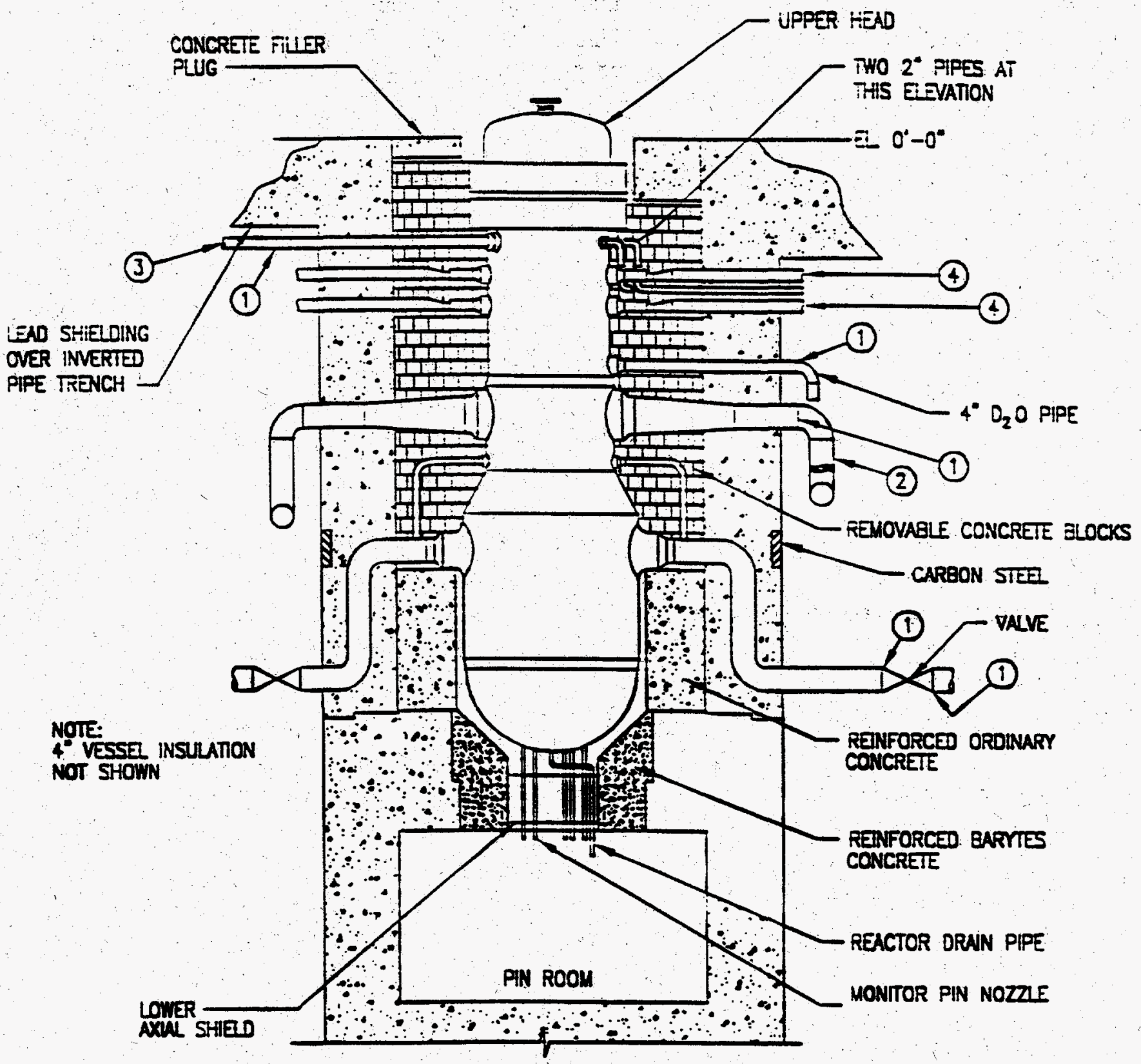

(1) Carbon steel to stainless steel transition

(2) Ten-inch carbon steel pipe

(4) Two 4-inch instrument pipes at this location

(4) Six 4-inch isolated coolant loop stainless steel pipes at this location

Figure 6. Reactor and Biological Shield. The reactor vessel and piping which penetrates the biological shield will be removed, as will the removable concrete shielding blocks. The lower part of the biological shield was found during the 1996 characterization to have detectable radioactivity from neutron activation of the concrete at a maximum depth of 41 inches from the shielding inner surface. 
Additional work on the HWCTR decommissioning project began several years ago. In Fiscal Year 94, four auxiliary buildings on the HWCTR site were demolished.

In early Fiscal Year 95 detailed radiological contamination surveys of the reactor building were performed. These showed that removable contamination levels in the facility were low.

In early 1996, the control building was demolished. During the summer of 1996 a detailed characterization study of the facility was performed. Details of the results appear in the HWCTR Facility Characterization Report (reference 6).

The characterization study showed that most areas of the facility had radiation levels below one millirem per hour and no detectable transferable radioactive contamination. Based on laboratory analysis of 50 samples from concrete surfaces, only low concentrations of radioactivity were found to be embedded in concrete floors. Dominant radionuclides were Cs-137, Sr-90 and Co-60. The reactor biological shield was determined to be activated a maximum of 41 inches from its inner surface, with Eu-152 the most abundant radionuclide. The data from the 1996 characterization study forms the basis for source terms used in this study. These source terms are discussed in Section 6.

As of April 1997, only the reactor containment building and two other small buildings remained on the property. One building is the health physics building, which is connected to the reactor building by a short passageway. The other is the smail deluge control bunker, Building 788-U, which actually stands about 150 yards north of the site property. Also still in place are the ventilation exhaust stack (which is designated Building 791-U) and the steam muffler, which lies outside the security fence, 110 yards east of the reactor containment building. See Figure 3.

\subsection{HWCTR SURVEY UNITS}

At the time this study was accomplished, the draft HWCTR Decommissioning Plan (reference 5) specified that the underground part of the facility which will remain in place would be divided into survey units. A survey unit is a part of the facility for which a separate decision will be made about whether the unit meets the cleanup standard. 
Typical survey units that could be used for the HWCTR are described in Table 1 . These were considered during accomplishment of the RESRAD study. Note that the plans for using such survey units may be changed based on the results of this study.

Table 1. Typical HWCTR Survey Units (Refer to Figures 4 and 5)

\begin{tabular}{|c|l|}
\hline Unit & Location \\
\hline 1 & Floors and lower walls (up to 6') of monitor room at minus $52^{\prime}$ level \\
\hline 2 & Floors and lower walls of right instrument room at minus $52^{\prime}$ level \\
\hline 3 & Floors and lower walls of right cyclone room at minus $52^{\prime}$ level \\
\hline 4 & Floors and lower walls of left purification room at minus $52^{\prime}$ level \\
\hline 5 & Floors, walls and ceiling of pin room at minus $41^{\prime}$ ' level \\
\hline 6 & Floors and lower walls of right generator room at minus $37^{\prime}$ level \\
\hline 7 & Inside surfaces of reactor biological shield \\
\hline 8 & Inside surfaces of spent fuel basin \\
\hline 9 & Upper walls and ceiling of minus 52 ' level \\
\hline 10 & Remaining upper concrete walls of underground structure \\
\hline 11 & Outer walls of reactor biological shield and spent fuel basin shieid wall \\
\hline 12 & Building stairwell, minus $52^{\prime}$ level to 0 level \\
\hline
\end{tabular}

These survey units cover only the parts of the underground reactor building structure that will remain in place. It is these areas for which DCGLs would be necessary, uniess the final survey is simplified based on the results of this study.

As discussed in Section 8, the DCGLs calculated for the HWCTR turned out to be substantially higher than the levels of radioactivity determined to be present in the facility during the 1996 characterization study. In view of this situation, fewer survey units would be warranted. Also, one set of DCGLs could suffice for all of the survey units as explained in Section 8.

\subsection{EXPOSURE PATHWAY SCENARIOS}

Two potential exposure scenarios were considered in this study. The potential radiation exposures that could be associated with these scenarios and DCGLs for the HWCTR facility were calculated over a 10,000-year period using RESRAD and RESRAD-BUILD. Parameters used in the calculations are listed in Appendix $A$ and Appendix B, respectively. 


\subsection{Scenario A - Residential Use}

Scenario A assumed residential use of the site as a family farm. Reference 3 notes that this is typically the worst case scenario and that in cases where it is not realistic but still plausible, it can generally be assumed to be the most restrictive use.

It was assumed that the farm would not be built on the property for at least $\mathbf{3 0}$ years. This assumption is consistent with current future-use plans for the SRS, which do not entail residential use (reference 7).

A hypothetical resident was assumed to spend 50 percent of his time indoors in a house built on the remediated HWCTR property. Twenty-five percent of his time would be spent outdoors on the property, and the remaining 25 percent of time away from the site. The resident was assumed to eat homegrown produce and meat and drink milk from livestock fed with forage grown onsite. Groundwater drawn from a well located on the property would be the only source of water for drinking, household use and irrigation.

Note that RESRAD analyses also can model radiation exposures associated with eating fish from an onsite pond. This pathway was not considered plausible for the HWCTR site, given the small size of the property. The seven exposure pathways for Scenario A are described in Table 2.

Table 2. Exposure Pathways for Scenario A

\begin{tabular}{|c|l|}
\hline No. & Pathway \\
\hline 1 & Direct gamma exposure to contaminated soil \\
\hline 2 & Internal dose from inhalation of dust \\
\hline 3 & Internal dose from inhalation of radon \\
\hline 4 & Internal dose from ingestion of plant foods \\
\hline 5 & Internal dose from ingestion of meat and milk from onsite livestock \\
\hline 6 & Internal dose from drinking water \\
\hline 7 & Internal dose from ingestion of soil \\
\hline
\end{tabular}

\subsection{Scenario B - Administrative Building}

Another plausible use for the HWCTR property would involve constructing a building on the site. Scenario $B$ assumes that an administrative building with a 
basement four meters deep is built on the property. An administrative building would be more likely to be constructed than an industrial facility, since future-use plans for this part of the SRS call for it to remain an administrative area. Such a building may or may not have a basement. Any basement would be unlikely to be more than four meters deep.

It is assumed that the building basement incorporates as one of its walls an existing wall of the HWCTR underground structure. The wall shown in Figure 7 on page 19 was selected for the analysis since it could plausibly be used for this purpose. It would not be plausible for the curved outer walls of the containment building, the sides of the small spent fuel basin or the opening in the reactor biological shield to be utilized in such an application.

Under this scenario, a hypothetical individual was assumed to work eight hours per day inside the building basement, five days per week for 50 weeks per year. The exposure pathways associated with the scenario are shown in Table 3.

Table 3. Exposure Pathways for Scenario B

\begin{tabular}{|l|l|}
\hline 1 & Direct gamma exposure to contaminated wall \\
\hline 2 & External exposure due to air submersion \\
\hline 3 & Internal dose from inhalation of airborne radioactive particulates \\
\hline 4 & Internal dose for inadvertent ingestion of radioactive material \\
\hline
\end{tabular}

Note that two pathways considered by RESRAD-BUILD were not included because they were not applicable to the HWCTR project. These are inhalation of indoor radon progeny and external exposure from radioactive materials deposited on a floor. Radon was included in the RESRAD calculation as indicated in Section 5.2. But because radon is not generated by the radioactive materials which will remain in the HWCTR facility (apart from naturally-occurring radionuclides) it was not a significant factor in that calculation.

\subsection{Considerations in Scenario Selection}

DOE Order 5400.5 (reference 1) and the Decommissioning Resource Manual (reference 2) both indicate that a realistic pathway analysis should be employed in establishing the site-specific cleanup guidelines. The two scenarios selected for analysis may not be considered entirely realistic, given present expectations for future uses of the SRS property, but they are plausible. 
The family farm scenario is a basic RESRAD exposure pathway scenario. It has been used in other RESRAD studies at the SRS. An example is the study of the Solid Waste Disposal Facility in E-Area, which is described in reference 8. Even though the present SRS future-use plan of reference 7 does not accommodate residential use, such use is plausible in the long term. Because the HWCTR property is not located in the central region of the site near highly-contaminated nuclear facilities, it would be more likely to eventually become a residential area than would property located in the central part of the SRS. The administrative building scenario also would be a plausible use for the HWCTR property. The assumption of a basement is plausible as well. This assumption opens up exposure pathways beyond those in the RESRAD analysis, as one can see by comparing Table 3 to Table 2.

Note that one could have assumed a basement in the family farmhouse of Scenario A. But the Scenario B building basement assumption was chosen because it could be more conservative. This is because Scenario A includes a 30-year period during which no structure is expected to be built, which allows for radioactive decay. (Note, however, that the source term was not corrected for 30 years decay since transport of the radionuclides into the soil column would begin immediately.)

The administrative building basement assumption is consistent with exposure pathway scenarios used in connection with decommissioning of the Shippingport Atomic Power Station in Pennsylvania (reference 9). The Shippingport project entailed dismantlement of that facility and unrestricted release of the site property. Some underground concrete structures with low levels of residual radioactivity remained in place. These were filled with building rubble and clean earth, as will be the case for the HWCTR project.

Please note that the scenarios selected for the HWCTR are not necessarily applicable to other similar SRS facilities to be decommissioned, such as the larger production reactors.

\subsection{SOURCE TERMS}

Section 6.1 identifies the three HWCTR source terms. Sections 6.2 through 6.7 describe the 1996 characterization data used in the calculations. In Section 6.8, the methodology for the calculations is described. Section 6.9 identifies assumptions made. 


\subsection{The Calculated Source Terms}

As one can see in Table 4 below, the predominant radionuclide in the biological shield source term is Co- 60 at approximately $620 \mathrm{mCi}$. The majority of this activity is found in the lower axial shield, which contains a concrete mixture with 90 percent steel shot. In the other concrete, the dominant radionuclides are Ba133 at $103 \mathrm{mCi}, \mathrm{Eu}-152$ at $57 \mathrm{mCi}$ and Eu-154 at $3.58 \mathrm{mCi}$. The total amount of radioactivity in the shielding was calculated to be approximately $785 \mathrm{mCi}$. This amount includes radioactivity in reinforcing steel (rebar) and in piping embedded in the biological shield wall.

Table 4. Calculated Source Term - Reactor Biological Shield

Biodedd mase (8) : $\quad 393 E+07$

\begin{tabular}{|c|c|c|c|c|c|c|c|c|}
\hline Redienechele & $\begin{array}{l}\text { primery pope } \\
\text { DCi }\end{array}$ & $\begin{array}{c}\text { copper } \\
\mathrm{pCi}\end{array}$ & $\begin{array}{l}\text { HPLS } \\
\text { pCi }\end{array}$ & $\begin{array}{l}\text { Reber } \\
\text { PCi }\end{array}$ & $\begin{array}{l}\text { Lowner exvel } \\
\text { shiedd (pCi) }\end{array}$ & 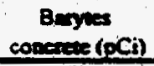 & $\begin{array}{c}\text { Ordinaty } \\
\text { conerese (pCi) }\end{array}$ & seoming \\
\hline $82-133$ & & & & & & $1.03 \mathrm{E}+11$ & & TISE+03 \\
\hline En-152 & 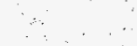 & & & & & $2.395+10$ & $3.29 E+10$ & $636 E+02$ \\
\hline Evise & & & & & & $1.41 E+09$ & & 3.98E*01 \\
\hline seseo & $3.00 E+07$ & & & & & & & 3.368 .01 \\
\hline $\mathrm{Ni} 63$ & $3.23 E+\infty 8$ & & & & & & & $3.62 E+60$ \\
\hline C-14 & $5.89 E+05$ & & & & & & & $6.592-03$ \\
\hline Ni-s9 & $4.04 E+06$ & & & & & & & 4.538 .02 \\
\hline $\begin{array}{l}\text { Coso } \\
\text { th-3 }\end{array}$ & $\begin{array}{l}1.62 E+08 \\
2.42 E+07\end{array}$ & $1.778+06$ & 273E+07 & $9.67 E+07$ & $6.005+11$ & $\begin{array}{l}2.01 E+10 \\
1.36 E+09\end{array}$ & $\begin{array}{l}3.658+08 \\
2.665+08\end{array}$ & $\begin{array}{l}6.958+03 \\
1.27 \pi+101\end{array}$ \\
\hline $\operatorname{col} 137$ & 1:12E=06. & & & v & $\because$ & & & $1.2 \operatorname{sen}, 02$ \\
\hline Po-238 & $1.73 E+05$ & & & & & & & 1.84803 \\
\hline Pu-239 & $1.396+06$ & & & $\ldots$ & & & & $1.55 E-02$ \\
\hline 1238 & $6.58 \mathrm{E}+02$ & 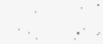 & & & & $\because$ & & 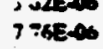 \\
\hline$\therefore-7.201$ & $2.0 x=+06$ & & & . & & & & $123 E-02$ \\
\hline
\end{tabular}

HPLS = horizontal power level sleeve

Note the biological shield mass is specified in Tabie 4 as $8.93 E+07$ grams. This figure was based on the densities of the concrete in the shielding. An assumption was made that the radioactivity in the shield was uniformly distributed over the shield volume shown in the conceptual model of Figure 9. The concentrations of radionuclides (in $\mathrm{pCi} / \mathrm{g}$ ) in Table 4 are used as input to the RESRAD computer code for the biological shield model.

In the concrete floors of the facility, a total of approximately $6.6 \mathrm{mCi}$ was caiculated to be present. Cesium 137 was the predominant radionuclide at 4.5 $\mathrm{mCi}$, followed by $\mathrm{H}-3$ at $1.3 \mathrm{mCi}, \mathrm{C}-14$ at $0.34 \mathrm{mCi}, \mathrm{Sr}-90$ at $0.30 \mathrm{mCi}$ and $\mathrm{Co}-$ 60 at $0.05 \mathrm{mCi}$. These quantities include radioactivity inside embedded piping and the spent fuel basin. Table 5 shows the details of the rubble source term. 
Table 5. Calculated Source Term - Rubble

\begin{tabular}{|c|c|c|c|c|c|c|c|}
\hline Radiomuctide & $\begin{array}{c}\text { conerete tloor } \\
\quad{ }_{2 \mathrm{Ci}}\end{array}$ & $\begin{array}{c}\text { Zero elevición } \\
\text { Drain lines } \\
\text { pCi }\end{array}$ & $\begin{array}{c}-37 \text { elevation } \\
\text { Drain lines } \\
\text { oCi }\end{array}$ & $\begin{array}{c}\text {-52 devanoa } \\
\text { Drin lines } \\
\text { pCi }\end{array}$ & $\begin{array}{c}-52 \sin \theta \\
\operatorname{sectin}=0 x \\
p C i\end{array}$ & $\begin{array}{c}\text { Spent fucl basin } \\
\text { PCI }\end{array}$ & $\begin{array}{l}\text { Aervingy } \\
=\mathrm{Ci}_{2}\end{array}$ \\
\hline $\mathrm{Sr}-90$ & $3.00 \mathrm{E}+07$ & $1.61 E+08$ & $3.26 E+07$ & $6.96 E+07$ & $9.6 \mathrm{IE}+06$ & 6.00E+05 & $1.09 E-01$ \\
\hline$C \cdot 14$ & $3.35 E+08$ & & & & & $1.18 E+04$ & $: .20 \mathrm{E}-0 !$ \\
\hline $\cos 60$ & $3.88 E+06$ & $1.83 E+07$ & $3.70 E+06$ & $7.89 E+06$ & $5.31 E+05$ & $3.23 E+06$ & $1 Z A E-02$ \\
\hline $\mathrm{H}-3$ & $5.64 \varepsilon+08$ & & & & $1.43 E+06$ & $4.85 E+05$ & $2.03 \varepsilon-01$ \\
\hline$C_{5-137}$ & $3.88 E+09$ & $3.85 E+08$ & $7.79 E+07$ & $1.66 \mathrm{E}+08$ & $1.36 E+07$ & 5.95E+06 & $1.62 E+60$ \\
\hline$P v-238$ & & $1.87 E+02$ & $3.77 E+01$ & $8.05 E+01$ & $1.72 E+03$ & $3.46 E+03$ & 1.97E-O6 \\
\hline Pu-239 & $1.53 \mathrm{E}+05$ & 2.95E+04 & $5.98 E+03$ & $1.27 \mathrm{E}+04$ & $2.728+05$ & $2.77 E+04$ & 1.soe-o4 \\
\hline U-234. & $=29 E+07$ & & & & & $6.47 E+\infty 0$ & $3.20 E_{-03}$ \\
\hline U.235 & TEE+OS & & & & $\therefore$ & , & S..SE-O4 \\
\hline U-238 & $1.73 \varepsilon+07$ & & & & : & $1.39 x+01$ & $5.18 E-03$ \\
\hline$A m-241$ & & & & & & $5.773+04$ & :.OTEAS \\
\hline Ni-59 & & & & & & s.onetan & $2896-0 S$ \\
\hline $\mathrm{Ni}-63$ & & & & $\therefore$ & ... & $6.475+06$ & $231 E-03$ \\
\hline
\end{tabular}

In calculating the mass of the contaminated rubble. a density of $1.6 \mathrm{~g} / \mathrm{cm}^{3}$ was assumed. This value. approximately two-thirds of the density of reinforced concrete. was selected to account for voids in the rubble volume. Radioactivity in embedded piping and in the spent fuel basin was included. The activity in $\mathrm{pCi} / \mathrm{g}$ in the last column of Table 5 is the total for each radionuclide divided by the total mass of the contaminated concrete and rubble.

To determine the effect of leaving installed radioactive equipment in the facility, a modified source term was calculated. It is shown in Table 6 . In this source term the radioactivity associated with all installed equipment except the reactor vessel assembly and steam generators is included. Also included is induced activity in the reactor biological shield.

Table 6. Calculated Source Term - Rubble. Installed Equipment and Biological Shield

\begin{tabular}{|c|c|c|c|c|c|}
\hline Radioniclide & $\begin{array}{l}\text { Bioshield } \\
\text { activity (pCi) }\end{array}$ & $\begin{array}{c}\text { Rubble } \\
\text { accivity (pCi) }\end{array}$ & $\begin{array}{c}\text { Systems } \\
\text { activity (pCi) }\end{array}$ & $\begin{array}{l}\text { Tori } \\
\text { activity (pCi) }\end{array}$ & $\begin{array}{l}\text { Concencranon } \\
\mathrm{pCl} / \mathrm{g} \text { - rubble }\end{array}$ \\
\hline$B a-133$ & $1.03 E+11$ & & & $1.03 \mathrm{E}+11$ & $3.69 E+01$ \\
\hline En-152 & $5.68 E+10$ & & & $5.682+10$ & $2.03 E+01$ \\
\hline Ev-154 & $3.55 E+09$ & & & $3.55 E+09$ & $1.27 \mathrm{E}+00$ \\
\hline Sr-90 & $3.00 E+07$ & $3.04 E+08$ & +.21E+09. & $455 E+09$ & $1.63 E+00$ \\
\hline $\mathrm{Ni}-63$ & $3.23 E+08$ & $6.47 E+06$ & $4.54 E+10$ & $+.57 E+10$ & $1.64 E+01$ \\
\hline C.14 & $5.89 E+05$ & $3.35 E+08$ & $8.26 E+07$ & $4.18 E+08$ & $1.50 E-01$ \\
\hline Ni-s9 & $+.04 E+06$ & $8.08 E+04$ & $5.67 E+08$ & $5.71 E+08$ & 2.04E-01 \\
\hline $\operatorname{Co} 60$ & $6.21 E+11$ & $4.955+07$ & $2.27 E+10$ & $6.44 E+11$ & $230 E+02$ \\
\hline H-3 & $1.67 E+09$ & $1.25 E+09$ & $3.40 E+09$ & $6.32 E+09$ & $2.26 \mathrm{E}+00$ \\
\hline$C_{s-137}$ & $1.12 E+06$ & $4.53 E+09$ & $1.57 E+08$ & $+68 E+09$ & $1.68 E+\infty 0$ \\
\hline Pu-238 & $1.73 E+05$ & $5.49 E+03$ & $2.43 E+07$ & $2.45 E+07$ & $8.76 E-03$ \\
\hline Pu-239 & $1.39 E+06$ & $5.02 E+05$ & $1.94 E+08$ & $1.96 E+08$ & $7.03 E-02$ \\
\hline U-234 & $3.23 E+02$ & $2.29 E+07$ & $+.54 E+04$ & $2.30 E+07$ & $8.22 \mathrm{E}-03$ \\
\hline U-235 & & 7.76E+OS & & $7.76 E+05$ & $2.78 E-04$ \\
\hline U.238 & $6.93 \mathrm{E}+02$ & $7.58 E+07$ & 9.72E+04 & $7.59 E+07$ & $2.72 E-02$ \\
\hline \multirow[t]{2}{*}{$A m-241$} & $2.89 E+06$ & 5.T7E +04 & $+.05 E+08$ & $4.08 E+08$ & $1.46 \mathrm{E}-01$ \\
\hline & $7.86 \mathrm{E}+11$ & $6.57 \mathrm{E}+09$ & $7.71 E+10$ & $8.70 E+11$ & \\
\hline
\end{tabular}


One can see from this table that the part of the source term associated with radioactive systems is 0.0771 curies. This value is somewhat larger than the source term for contaminated concrete (rubble) and smaller than the one for the biological shield.

For the RESRAD-BUILD calculation, a source term was not calculated based in the 1996 characterization data. This approach would not have been meaningful because, based on that data, and surveys taken previously in the facility, the contamination levels on the walls are essentially background.

\subsection{The 1996 Characterization Study Data}

The 1996 characterization study (reference 6) served as the basis for the source term calculations. This study provides detailed information on the radioactive contaminants in the facility. All radioactive equipment will be removed from the HWCTR facility before the underground structure is filled with building rubble and earth. Given this situation, the following data from the 1996 characterization is most pertinent to this study:

- Induced radioactivity in the reactor biological shield, based on laboratory analysis of 35 samples from five cores,

- Radioactivity embedded in concrete floor surfaces, based on 49 concrete samples analyzed in a laboratory,

- Radioactivity inside floor drains and other embedded piping, and

- Transferable surface contamination based on the results of 970 smears taken on external surfaces in the facility.

These data are discussed in detail in the following sections. Figure 7 shows the areas of the facility involved. 


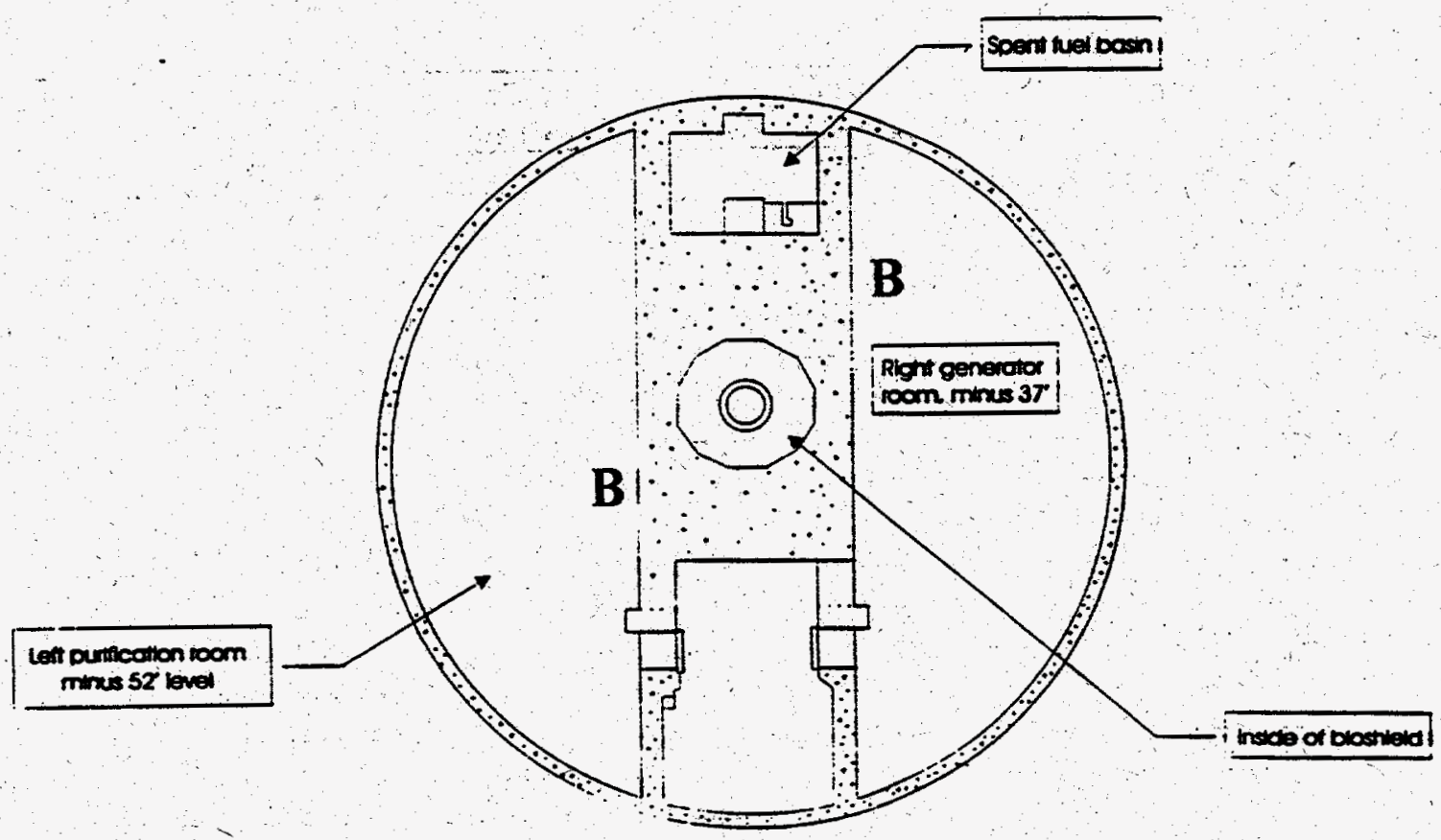

Figure 7. Plan View of Underground Structure. The different areas of the underground structure can be seen here. The top surfaces of the concrete will be five feet below grade. In Scenario B, one of the walls identified in the figure by a " $B$ " was assumed to form one wall of a basement in a hypothetical building.

\subsection{Induced Radioactivity in the Reactor Biological Shield}

To measure induced radioactivity in the biological shield, five cores were taken through the shield from the right generator room. Figure 8 on page 21 shows the location of the cores and, with a dashed line, the approximate limits of detectable activation in the materials. The results of the sample analyses are summarized in Table 7.

Table 7. Biological Shield Activation Analysis Results ("<" signifies that the radionuclide was not identified by gamuna spectroscopy.)

\begin{tabular}{|c|c|c|c|c|c|c|}
\hline Sanplé ID Number & $\begin{array}{l}\text { Elevation } \\
\text { * (fét) }\end{array}$ & $\begin{array}{c}\text { Sample } \\
\text { Depth (in) }\end{array}$ & $\begin{array}{l}{ }^{\infty} \mathrm{Co} \\
\mathrm{pCi} / \mathrm{gm}\end{array}$ & 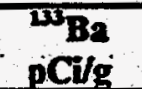 & $\begin{array}{l}{ }^{152} \mathrm{Eu} \\
\mathrm{pCO} / \mathrm{g}\end{array}$ & pcis \\
\hline USE-H-15-J9-08-001-6-1 & $-285^{\prime \prime}$ & 15 & $<6.0 \mathrm{E}-02$ & $<$ & $<$ & $<$ \\
\hline USE-H-15-J9-08-001-5-1 & $-28^{\prime} 5^{\prime \prime}$ & 27 & $<1.0 \mathrm{E}-01$ & $<$ & $<$ & $<$ \\
\hline USE-HL-15-J9-08-001-5-2 & $-285^{\prime \prime}$ & 39 & $7.3 \mathrm{E}-01$ & $<$ & $<$ & $<$ \\
\hline USE-H-15-J9-08-001-1 & $-28^{\prime} 5^{n}$ & 45 & $<1.0 \mathrm{E}-0 \mid$ & $<$ & $<$ & $<$ \\
\hline USE-H-15-J9-08-001-3-1 & $-28^{\prime} 5^{n}$ & 51 & $<1.0 \mathrm{E}-01$ & $<$ & $2.3 E+00$ & $<$ \\
\hline USE-H-15-J9-08-001-2-1 & $-28^{\prime} 5^{n}$ & 67 & $2.1 E+00$ & $<$ & $6.0 \mathrm{E}+01$ & $5.7 E+00$ \\
\hline USE-H-15-J9-08-001-1-1 & $-28^{\prime} 5^{n}$ & 86 & $8.0 \mathrm{E}+02$ & $4.1 E+03$ & $9.5 \mathrm{E}+02$ & $5.6 \mathrm{E}+01$ \\
\hline$-59-08-005-6-1$ & $-26^{\circ} 8^{\prime \prime}$ & 15 & $<9.0 \mathrm{E}-02$ & $<$ & $<$ & $<$ \\
\hline$-59-08-005-5-1$ & $-26^{\prime} 8^{\prime \prime}$ & 27 & $<8.0 E-02$ & $<$ & $<$ & $<$ \\
\hline USE-H-15-J9-08-005-4-1 & $-26^{\prime} 8^{\prime \prime}$ & 39 & $<1.0 \mathrm{E}-01$ & $<$ & $2.2 E+00$ & $<$ \\
\hline USE-H-15-J9-08-005-3-1 & $-26^{\circ} 8^{\prime \prime}$ & 45 & $<1.0 \mathrm{E}-01$ & $<$ & $7.6 E+00$ & $<$ \\
\hline
\end{tabular}


RESIDUAL RADIOACTIVIT GUIDELINES FOR THE HWCTR

Table 7 (continued)

\begin{tabular}{|c|c|c|c|c|c|c|}
\hline Sample ID Number & $\begin{array}{c}\begin{array}{c}\text { Elevation } \\
\text { (feet) }\end{array} \\
\end{array}$ & \begin{tabular}{|c|} 
Sample \\
Depth (in)
\end{tabular} & $\begin{array}{c}{ }^{60} \mathrm{Co} \\
\text { pCijgm }\end{array}$ & $\begin{array}{l}{ }^{133} \mathbf{B a} \\
\mathrm{pCi} / \mathrm{g}\end{array}$ & $\begin{array}{l}{ }^{135} \mathbf{E u} \\
\mathrm{pCV} / g\end{array}$ & $\begin{array}{l}{ }^{134} \mathbf{E u} \\
\mathrm{pCi} / \mathrm{g}\end{array}$ \\
\hline USE-H-15-J9-08-005-3-2 & $-26^{\prime} 8^{\prime \prime}$ & 51 & $3.0 \mathrm{E}+00$ & $<$ & $3.9 \mathrm{E}+01$ & $1.6 \mathrm{E}+00$ \\
\hline USE-H-15-J9-08-005-2-1 & $-26^{\prime} 8^{\prime \prime}$ & 67 & $2.5 E+02$ & $<$ & $5.1 E+03$ & $2.0 \mathrm{E}+02$ \\
\hline USE-H-1 5-J9-08-004-6-1 & $-25^{\prime} 2^{\prime \prime}$ & 15 & $<9.0 \mathrm{E}-02$ & $\overline{<}$ & $<$ & $<$ \\
\hline USE-H-15-J9-08-004-5-1 & $-25^{\prime} 2^{\prime \prime}$ & 27 & $<4.0 \mathrm{E}-02$ & $<$ & $<$ & $<$ \\
\hline USE-H-15-J9-08-004-4-1 & $-25^{\prime} 2^{\prime \prime}$ & 39 & $1.4 \mathrm{E}-01$ & $<$ & $<$ & $<$ \\
\hline USE-H-15-J9-08-004-3-1 & $-25^{\prime} 2^{\prime \prime}$ & 45 & $3.4 \mathrm{E}-01$ & $<$ & $2.0 E+0.1$ & $1.1 E+00$ \\
\hline USE-H-15-J9-08-004-2-1 & $-25^{\prime} \cdot 2^{\prime \prime}$ & 51 & $9.2 \mathrm{E}-01$ & $<$ & $5.8 \mathrm{E}+01$ & $4.6 \mathrm{E}+00$ \\
\hline USE-H-15-19-08-004-1-1 & $-25^{\prime} 2^{\prime \prime}$ & 67 & $1.5 \mathrm{E}+01$ & $<$ & $1.1 \mathrm{E}+03$ & $4.7 E+01$ \\
\hline USE-H-15-j9-08-004-8-1 & $-25^{\prime} 2^{\prime \prime}$ & 79 & $1.8 \mathrm{E}+02$ & $<$ & $1.5 E+04$ & $7.7 \mathrm{E}+02$ \\
\hline USE-H-15-J9-08-003-6-1 & $-23^{\prime} 2^{\prime \prime}$ & 15 & $<1.0 \mathrm{E}-01$ & $<$ & $<$ & $<$ \\
\hline USE-H & $-23^{\prime} 2^{\prime \prime}$ & 27 & $<1.0 \mathrm{E}-01$ & $<$ & $<$ & $<$ \\
\hline USE-H-15-J9-08-003-5-2 & $-23: 2^{\prime \prime}$ & 39 & $<1.0 \mathrm{E}-01$ & $<$ & $<$ & $<$ \\
\hline USE-H-15-J9-08-003-4-1 & $-23^{\prime} 2^{\prime \prime}$ & 45 & $5.7 E-01$ & $<$ & $1.2 \mathrm{E}+01$ & $7.0 \mathrm{E}-01$ \\
\hline USE-H-15-J9-08-003-4-2 & $-23^{\prime} 2^{\prime \prime}$ & 51 & $9.4 \mathrm{E}-01$ & $<$ & $<$ & $<$ \\
\hline USE $=H-15-59-08-003-2-1$ & $-23^{\prime} 2^{\prime \prime}$ & 67 & $2.8 \mathrm{E}+01$ & $<$ & $2.3 E+03$ & $9.9 E+01$ \\
\hline USE-H-15-J9-08-003-1-1 & $-23^{\prime} 2^{\prime \prime}$ & 83 & $2.7 E+02$ & $<$ & $2.3 \mathrm{E}+04$ & $1.5 E+03$ \\
\hline USE-H-15-J9-08-002-6-1 & $-2110^{\prime \prime}$ & 15 & $<1.0 \mathrm{E}-01$ & $<$ & $<$ & $<$ \\
\hline USE-H-15-39-08-002-6-2 & $-21 \cdot 10^{\prime \prime}$ & 27 & $<6.0 \mathrm{E}-02$ & $<$ & $<$ & $<$ \\
\hline USE-H-15-J9-08-002-5-1 & $-2 l^{\prime} 10^{\prime \prime}$ & 39 & $<8.0 \mathrm{E}-02$ & $<$ & $<$ & $<$ \\
\hline USE-H-15-J9-08-002-4-1 & $-21^{\prime} 10^{\prime \prime}$ & 51 & $1.1 \mathrm{E}+00$ & $<$ & $4.5 \mathrm{E}+01$ & $2.0 \mathrm{E}+00$ \\
\hline USE-H-15-39-08-002-3-1 & $-21^{\prime} 10^{\prime \prime}$ & 67 & $3.0 \mathrm{E}+01$ & $\leq$ & $1.6 \mathrm{E}+03$ & $8.4 \mathrm{E}+01$ \\
\hline USE-H-15-j9-08-002-2-1 & $-21^{\prime} 10^{\prime \prime}$ & 83 & $3.9 E+02$ & $<$ & $1.4 E+04$ & $8.8 E+02$ \\
\hline
\end{tabular}

Due to physical interferences, the cores were not taken along a radial from the reactor vessel center. A six-inch correction is required to correct core depth to radial depth (e.g., 39 inch core depth is equivalent to 33 inch radial depth). The detectable concentration ranged from a radial depth of 33 inches to 74 inches (inner biological shield wall adjacent to the reactor vessel).

The radial depth of activation in the region of the core with the highest thermal neutron flux is 41 inches. This region basically extends from minus 26 feet 8 inches elevation to minus 21 feet 10 inches, approximately five feet. Figure 8 displays the activation depth in the active core region. As can be seen from this figure. the activation depth varies from 25 inches to 41 inches over the entire height of the active core region. approximately 10 feet.

Based on the radial activity profile and axial neutron flux distribution, the activity inventory of Eu-152 was calculated. Europium 152 is the predominant radionuclide with an inventory of $3.29 \times 10^{10} \mathrm{pCi}$ in the activated concrete opposite the active core region.

The lower shield is a high density concrete. The aggregate in this concrete is barite which contains barium. The entire lower shield was conservatively calculated to have an inventory of $1.03 \times 10^{11} \mathrm{pCi} \mathrm{Ba}-133$. This estimate was based on a single data point. 
The lower axial shield is removable. It consists of high density concrete containing 90 percent steel shot, encased in cadmium-plated carbon steel. Although this shield may be removed during the decommissioning, the activity associated with it was included in the source term in case it is left in place.

\subsection{Radioactivity Embedded in Concrete Floors}

During the 1996 characterization. a total of 50 concrete surface samples were taken in 47 different locations (three were duplicates for quality assurance purposes). All came from floor surfaces. except one. which was obtained from the wall of the spent fuel basin in the left generator room. Table 8 on page 22 summarizes the results.

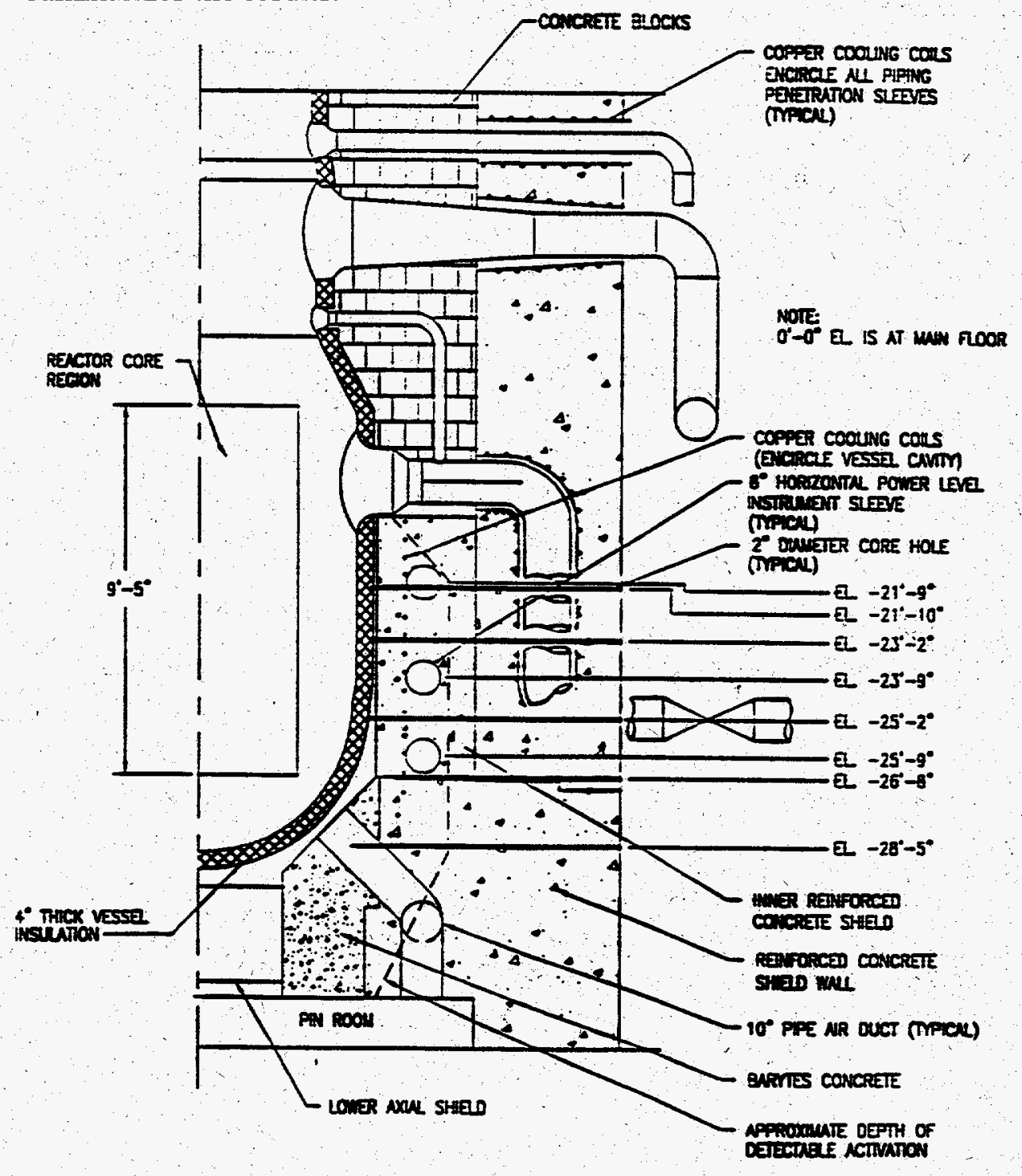

Figure 8. Biological Shield Showing Core Samples. The locations of the five core samples obtained during the 1996 characterization program. 
Table 8. Concrete Sample Results ${ }^{1,2}$

(Selected radionuclide concentrations in $\mathrm{pCi} / \mathrm{g}$ )

\begin{tabular}{|c|c|c|c|c|c|c|c|}
\hline Loeation & Na. & $\mathbf{H} \leq \mathbf{3}$ & $\mathrm{Co}-60$ : & Sr-90 & Cs-137: & $\mathrm{U}=238$ & Pu-239 \\
\hline $\begin{array}{l}\text { Concrete slab outside } \\
\text { equipment hatch }\end{array}$ & 5 & - & $<.03$ & $\overline{-}$ & $<.06-14$ & - & .057 \\
\hline Health physics building & 3 & $\overline{-}$ & $<.05$ & - & $.26-.97$ & $\overline{-}$ & - \\
\hline R. V. head area & $\overline{3}$ & $23-71$ & $<.005-1$ & 1.7 & $.84-7.7$ & - & $<-.01$ \\
\hline Main floor & 11 & $14-33$ & $<.05-.9$ & .74 & $.36-220$ & $.054-4.3$ & $<.008-.06$ \\
\hline Left pump room & 1 & - & 0.04 & - & .4 & - & 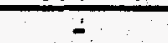 \\
\hline Right generator room & 5 & $<12$ & $.15-.57$ & $=$ & $.028-2.2$ & $\overline{-}$ & - \\
\hline Pin room & $\overline{2}$ & - & $.06-.12$ & - & $.24-.56$ & - & - \\
\hline Left purification room & $\overline{6}$ & - & $<.04-18$ & $=$ & $1.1-27$ & - & $\overline{-}$ \\
\hline Right cyclone room & $\overline{4}$ & $z$ & $<.06-.21$ & - & $.73-7.6$ & $\overline{-}$ & - \\
\hline Right instrument room & 3 & - & $.11-.26$ & - & $.86-27$ & - & $\overline{-}$ \\
\hline Monitor room & $\overline{5}$ & - & $<.03-.49$ & - & $4.5-84$ & - & - \\
\hline Stairwell & 2 & - & $.09, .13$ & - & $.5-2.2$ & - & - \\
\hline
\end{tabular}

${ }^{1}$ Dashes indicate not measured. $>$ indicates less than minimum detectable value.

As seen from this table:

- H-3 concentrations ranged up to approximately seven times the detection limit,

- Co-60 concentrations are relatively low, with $0.9 \mathrm{pCi} / \mathrm{g}$ being the highest value,

- Cs-137 levels ranged up to $220 \mathrm{pCi} / \mathrm{g}$, with the highest value in the expected location - between the reactor vessel head and the spent fuel basin,

- U-238 levels ranged from 0.54 to $4.3 \mathrm{pCi} / \mathrm{g}$. with the highest near the spent fuel basin, and

- Pu-239 concentrations ranged from 0.01 to $0.06 \mathrm{pCi} / \mathrm{gm}$, with the highest value on the concrete slab outside the equipment hatch.

The results of analyses of concrete samples from floor surfaces therefore show low levels of Co-60 and somewhat higher leveis of Cs-137 contamination. Analysis of two core samples from the 0 -level floor showed no detectable Co-60 or Cs-137 three inches deep in the concrete. It is likely that the contamination penetrates less than one inch deep, based on experience.

\subsection{Radioactivity Inside Floor Drains and Other Embedded Piping}

Beta-gamma scans of the openings to floor drains in the 0-level floor showed up to $380,000 \mathrm{dpm} / 100 \mathrm{~cm}^{2}$; alpha scans showed $20 \mathrm{dpm} / 100 \mathrm{~cm}^{2}$ in two of these drains. 
The 4-inch diameter lines from these drains run through the shield wall of the spent fuel basin down to the sump tank at the minus 52-foot level.

At the time of the characterization work, most floor drains at the minus 52-foot level contained water. Water samples showed radioactivity concentrations in the following ranges:

- H-3 $1.1 \times 10^{5}$ to $6 \times 10^{5} \mathrm{pCiL}$

- Co-60<20 to $150 \mathrm{pCi} / \mathrm{L}$

- $\mathrm{Cs}-137100$ to $2.9 \times 10^{5} \mathrm{pCi} / \mathrm{L}$

Table 9 lists embedded radioactive piping in the facility. It does not show small diameter piping - mostly instrument sample lines - that penetrates the walls of the monitor room at the minus 52 -foot level.

Table 9. Embedded Radioactive Piping

\begin{tabular}{|c|c|c|c|}
\hline Location $\quad$ ou & $\begin{array}{l}\text { Diameter } \\
\text { (inches) }\end{array}$ & $\begin{array}{l}\text { Length } \\
\text { (feet) }\end{array}$ & Drawings \\
\hline Biological shield: & - & $\dot{-}$ & \\
\hline Reactor piping & $2-16$ & 97 & $\begin{array}{l}\text { W231080, W231081, } \\
\text { W230740 }\end{array}$ \\
\hline Power level sleeves ${ }^{2}$ & 10 & 180 & W231486. W231756 \\
\hline $\begin{array}{l}\text { Copper cooling coils (except for coils } \\
\text { around embedded pipes) }\end{array}$ & $7 / 8$ & 365 & W231438 \\
\hline Spent fuel basin shield wall & $1-4$ & 228 & $\begin{array}{l}\text { W231443, W231252, } \\
\text { W230856 }\end{array}$ \\
\hline 0 -level floor ${ }^{3}$ & $1 / 2-6$ & 477 & W231537. W231477 \\
\hline \multicolumn{4}{|l|}{ Floor drains } \\
\hline 0-level floor & 4 & 108 & W231085. W231227 \\
\hline -37-foot level floor & 4 & 68 & W231086 \\
\hline -52-foot level floor & 4 & 145 & W238873 \\
\hline
\end{tabular}

This table includes representative embedded piping that has been verified. or is expected to be activated or contaminated. It does not include piping that penetrates the monitor room wall.

${ }^{2}$ Not all portions of the power level sleeves and cooling coils will be radioactive.

${ }^{3}$ Not all piping embedded in the floor is radioactive. Much of this piping is under the floor.

In calculating the RESRAD source term, it was assumed that all of this embedded piping would remain in place. Average contamination levels in the system piping were assumed to be the maximum levels measured during system sampling in the 1996 characterization program. Average contamination levels in the floor drain system were likewise assumed to be the maximum levels measured during the 1996 characterization. 


\subsection{Radioactivity Inside Equipment}

During the 1996 characterization, radioactivity inside installed equipment was measured in three ways. Smears were taken inside various systems. Water samples were taken from the spent fuel basin purification and cooling water systems. Metal coupons were cut from the main coolant and steam piping of each loop. All of the samples were analyzed in a laboratory.

The smears showed the average activity in the main coolant system to be approximately $2,500 \mathrm{pCi} / 100 \mathrm{~cm}^{2}$ gross alpha and $65,000 \mathrm{pCi} / 100 \mathrm{~cm}^{2}$ gross betagamma. Contamination levels measured in the two isolated coolant loops were slightly lower than these values.

The most significant of the system data was considered to be from analysis of the metal coupons taken from the main coolant system. The average of the two samples showed $18 \mathrm{pCi} / \mathrm{g} \mathrm{H}-3,135 \mathrm{pCi} / \mathrm{g} \mathrm{Co}-60$ and $0.78 \mathrm{pCi} / \mathrm{g} \mathrm{Pu}-239$.

\subsection{Removable Contamination}

During the 1996 characterization program, approximately 970 smears were taken to measure removable contamination on various external surfaces of the facility, with emphasis on cracks and crevices. Each one was analyzed for gross $\alpha$ and gross $\beta-\gamma$. Representative ones showing significant activity were analyzed by $\gamma$ spectroscopy for Co-60, Cs-137 and other $\gamma$ emitters. Eighteen smears showed significant activity, all but two of these were taken on equipment which will be removed. Table 10 shows the activity on the two smears located on facility surfaces. The surfaces invoived have a combined area of less than three square feet.

Table 10. Smears Showing Significant Removable Activity'

\begin{tabular}{|c|c|c|}
\hline 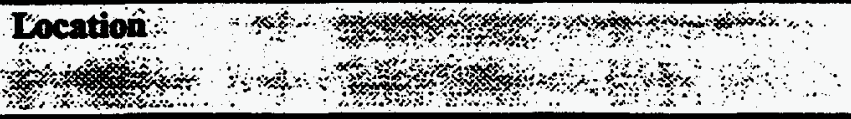 & $\begin{array}{r}\alpha \text { activity }>20 \\
\mathrm{dpm} / 100 \mathrm{~cm}^{2}\end{array}$ & $\begin{array}{l}\text { Pryactivity } \\
>1000 \\
\text { dpmil100 } \mathrm{cm}^{2}\end{array}$ \\
\hline $\begin{array}{l}\text { CRDM and reactor vessel head area, under plastic near head } \\
\text { (less than } 1 \mathrm{f}^{2} \text { ) }\end{array}$ & 33 & 134,200 \\
\hline Right cyclone room at $-52^{\prime}$, in radiation area & * & 1432 \\
\hline
\end{tabular}

${ }^{1}$ Note that a value of $20 \mathrm{dpm} / 100 \mathrm{~cm}^{2}$ was used for significant $\alpha$ activity. A value of $1000 \mathrm{dpm} /$ $100 \mathrm{~cm}^{2}$ was used for $\beta-\gamma$ radioactivity. *Amount below significant value.

Overall, the results of the smear surveys were consistent with those made in 1994 (approximately 1300 smears were taken in 1994 to support downgrading the facility status to a radiological buffer area) and show that removable contamination levels are low in the facility, even in cracks and crevices. 
Because extensive surveys showed only very low levels of removable contamination on facility surfaces, it was assumed that there is no significant activity associated with removable contamination in the facility, apart from inside the spent fuel basin. Consequently, only the spent fuel basin activity was included in the source terms.

In the spent fuel basin, eight atomic wipes were used to measure surface contamination during the 1996 characterization program. These smears showed a maximum of $174 \mathrm{pCi} / 100 \mathrm{~cm}^{2} \mathrm{Co}-60$ and $370 \mathrm{pCi} / 100 \mathrm{~cm}^{2} \mathrm{Cs}-137$. These values were used as average contamination levels for these radionuclides in calculating the source term from the basin. Levels for other radionuclides were estimated using scaling factors developed from the results of laboratory analyses of contamination from other areas.

\subsection{Methodology for Calculating the Source Terms}

Because of their different locations and characteristics, the biological shield activity and the activity associated with contaminated concrete and rubble were treated as two different source terms.

\subsubsection{Biological Shield Activity}

Activity in the lower 10-inch piping in the reactor biological shield (which may remain in place) was calculated using the laboratory analysis resuits of a primary system metal sample activity averaged over the piping inner surface area.

The induced activity associated with activated copper cooling coils inside the biological shield was calculated using the laboratory analysis results of a piece of this material obtained from coring. Induced activity in the power level sleeves and steel reinforcing bar in the biological shield was calculated in the same fashion. Induced activity in the lower axial shield was calculated using information in the 1976 Decommissioning Plan (reference 11). In all three cases, Co-60 was the predominant radionuclide.

The induced activity in the barytes concrete lower biological shielding was calculated based on the laboratory analysis results of the one core sample segment which detected Ba-133 (sample USE-H-15-J9-08-001-1-1 of Table 7). The induced activity in the ordinary concrete biological shielding also was calculated using laboratory analysis results of core samples.

To support the RESRAD calculations, a conceptual model of the biological shield was developed. This model, which shows the location of the radioactivity in the underground structure, is illustrated in Figure 9. Note that the $\mathrm{Ba}-133$ activity was assumed to be uniformly distributed over the 
modeled volume. This assumption is equivalent in the RESRAD calculation to considering the activity to reside only in the lower portion of the modeled volume.

\subsubsection{Contaminated Concrete and Rubble Activity}

The activity in the embedded piping of the floor drain system was calculated based on the maximum measured surface contamination from $\beta$ $\gamma$ and $\alpha$ scans averaged over the inner surface area of the piping, using the resuits of laboratory analysis of sediment in the drains for the fractional abundance of the radionuclides present. Activity inside the sump tank was calculated based on the analysis results of a sediment sample from the tank, assuming an average sediment thickness in the bottom of the tank of two centimeters.

The activity associated with contaminated concrete floors of the facility was calculated using the highest concentration of each radionuclide measured in laboratory analysis of the 49 samples from concrete floors. These values appear in Table 5. The average depth of contamination in the concrete floors was assumed to be one centimeter.

The activity inside installed equipment was calculated using estimates of system surface area derived from drawings and assuming average contamination levels from laboratory analysis of metal coupons taken from the main coolant system.

Figure 9 shows the conceptual model of the radioactivity associated with contaminated concrete and embedded piping.

\subsubsection{RESRAD-BUILD Wall Activity :}

As noted in Section 6.1 , it would not have been meaningful to calculate a RESRAD-BUILD source term because contamination levels on the walls of the facility are essentially background.

\subsection{Assumptions Used in Computing Source Terms}

In using the 1996 characterization data to calculate the source terms, certain assumptions were necessary. These were made in a conservative fashion. Some assumptions were mentioned above. Others include:

- The average length of the activated section of the power level sleeves is six feet. 
RESIDUAL RADIOACTIVITY GUIDELINES FOR THE HWCTR

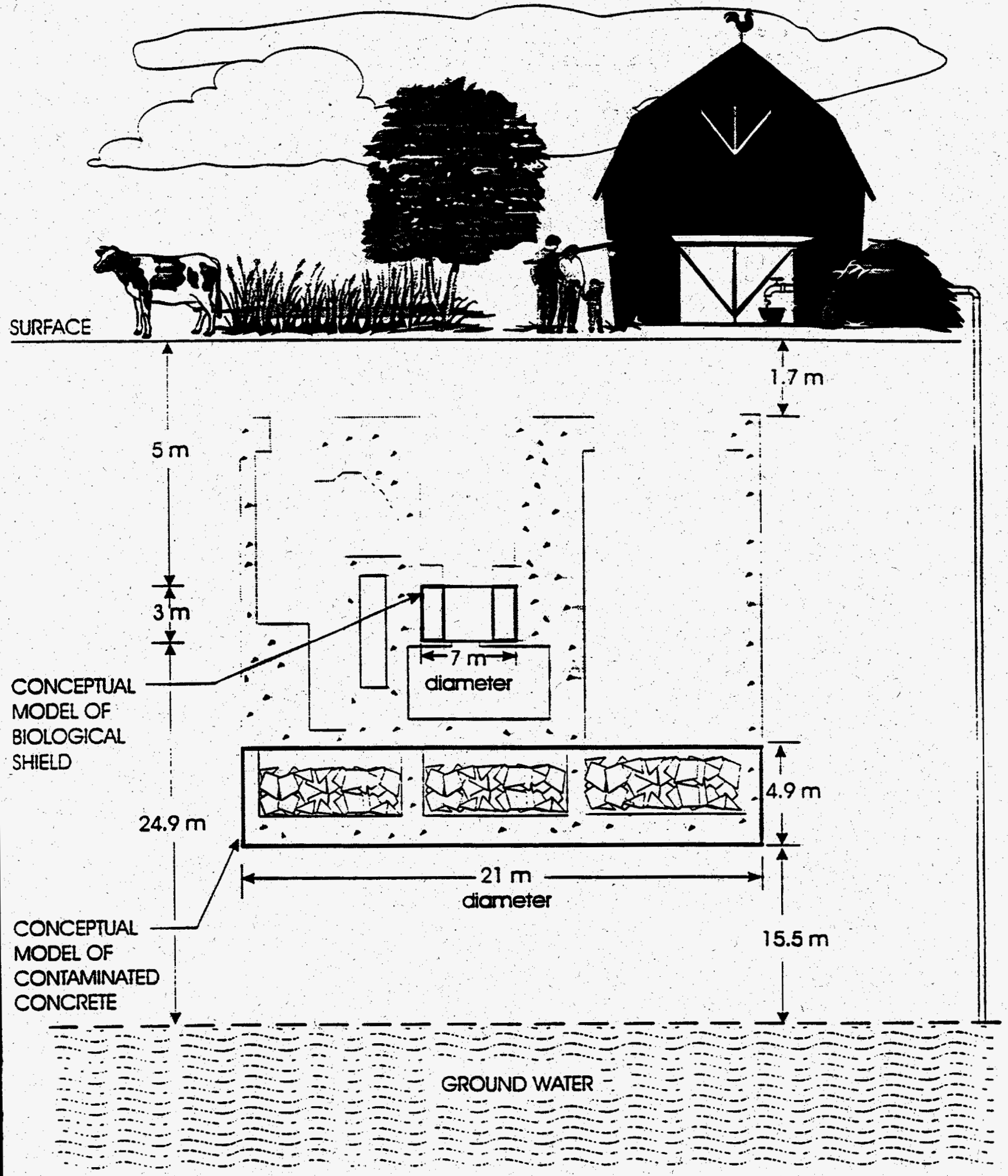

Figure 9. Conceptual Models of Radioactivity in the Facility 
- The amount of radioactivity associated with removable contamination in the facility is insignificant compared to contamination in the embedded piping and concrete floors, and induced activity in the biological shield. Consequently, the removable contamination was not included in the model.

- For the calculation of the source term which inciuded the installed radioactive equipment, the conceptual model of Figure 9 was used. This concept assumed that the radioactivity associated with contaminated concrete, embedded piping, the biological shield and the installed equipment was contained within the contaminated rubble zone shown in the illustration. Use of this model is more conservative than the biological shield model in this instance because the contaminated zone is nearer to the ground water.

- Standard radiological control practices will be followed during dismantlement of radioactive systems so that the low existing removable contamination levels in the facility will not be substantially increased during the course of work.

\subsection{OTHER PARAMETERS FOR RESRAD AND RESRAD-BUILD}

In addition to the source terms discussed in Section 6, there are a variety of other input parameters to RESRAD and RESRAD-BUILD. These are identified in Appendix A and Appendix B, respectively. Appendix C identifies the input parameters for RESRAD with the additional source term associated with leaving radioactive equipment, except for the reactor vessel and steam generators, in the facility. In each case, a brief statement of the source of the value used is included. References are specified, as appropriate.

In most cases, the HWCTR values for environmental parameters are the same as those used in other RESRAD studies at the SRS, such as the one documented in reference 8. 


\subsection{RESULTS}

\subsection{Summary}

The RESRAD calculations showed that radioactivity which would remain in the facility with no decontamination would produce under Scenario A a maximum annual exposure to a hypothetical individual of $0.037 \mathrm{mrem}$ per year. This peak would occur in about the year 6962 as shown in Figure 10 and Figure 11 on the following pages. Calculated DCGLs for residual radioactivity which would produce 15 mrem per year were well above levels measured during the 1996 characterization program.

The second RESRAD calculations, which assumed that all installed radioactive equipment except the reactor vessel and steam generators would remain in place, also showed a maximum annual exposure of approximately $0.037 \mathrm{mrem}$ per year. The slight increase in the source term of approximately nine percent from the installed equipment did not result in a significant increase in exposure.

The DCGLs from the two RESRAD runs were identical. The values are expressed in Table 13 for $15 \mathrm{mrem}$ per year and Table 14 for $100 \mathrm{mrem}$ per year. The calculated values substantially exceed the existing average radioactivity levels used in the calculations. The DCGLs for $15 \mathrm{mrem}$ per year range from $13 \mathrm{pCi} / \mathrm{g}$ and $1.09 \mathrm{E}+05 \mathrm{dpm} / 100 \mathrm{~cm}^{2}$ for U-234 to $1.13 \mathrm{E}+15 \mathrm{pCi} / \mathrm{g}$ and $3.03 \mathrm{E}+18 \mathrm{dpm} / 100 \mathrm{~cm}^{2}$ for Co-60. Cobalt-60 and other radionuciides marked with asterisks in the tables appeared at the individual nuclide limits as calculated by RESRAD. That is, no concentration of $\mathrm{Co}-60$ in the facility as modeled could produce the exposure limit because it would decay to minuscule levels before reaching the ground water.

Because the calculated DCGLs are far above the radioactivity levels in the facility, one set of DCGLs may suffice for all surfaces in all survey units in the containment building.

The RESRAD-BUILD calculation indicated that the DCGLs for this scenario were $15,000 \mathrm{dpm} / 100 \mathrm{~cm}^{2} \beta-\gamma$ or $150 \mathrm{dpm} / 100 \mathrm{~cm}^{2} \alpha$, based on the most restrictive radionuclides. These DCGLs were calculated to equate to $15 \mathrm{mrem}$ per year. The actual existing contamination levels for Scenario B are so low - effectively background - that calculating the exposure from these levels using RESRADBUIID was not meaningful. The results also show that the highest exposure is associated only with the first year of occupancy; each subsequent year the annual exposure decreases.

Comparison of the RESRAD-BUILD values of $15,000 \mathrm{dpm} / 100 \mathrm{~cm}^{2} \beta-\gamma$ and 150 $\mathrm{dpm} / 100 \mathrm{~cm}^{2} \alpha$ with the DCGLs of Table 13 shows that the former levels are much lower than the latter. Note that the calculated DCGLs would not apply to 
activation in the biological shield which should be treated differently from contamination in the facility in appiying cleanup guideline values.

TOTAL DOSE: All Isotopes and Pathways Summed

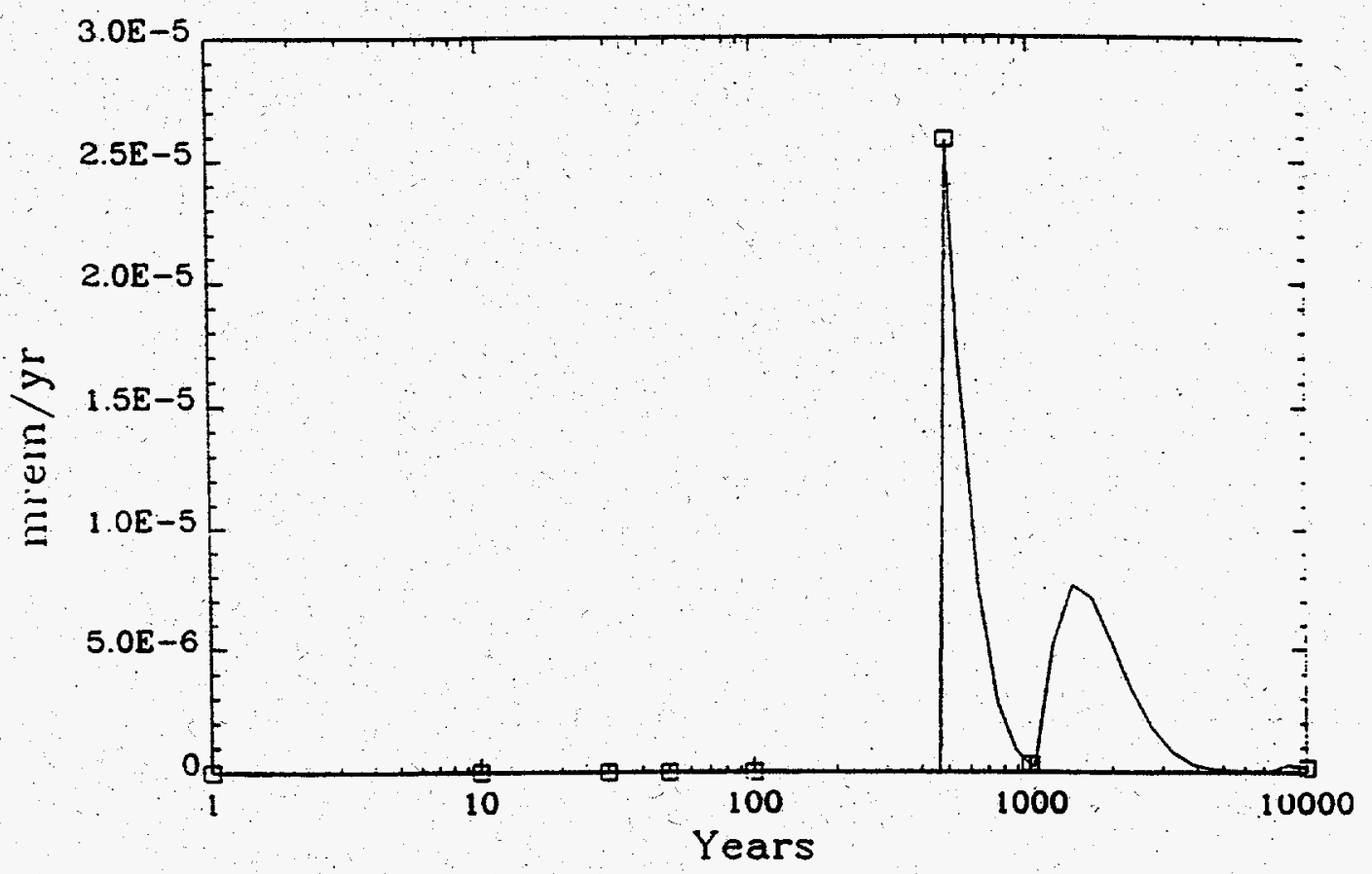

Figure 10. RESRAD Results With Present Source Terms For Biological Shieid. This exposure projection is based primarily on activated concrete.

\subsection{Detailed RESRAD Results}

The RESRAD summary report for the biological shield activity assessment appears in Appendix D. The summary report for the radioactivity associated with contaminated concrete and embedded piping appears in Appendix E.

Figures 10 and 11 show the annual radiation exposure to a hypothetical individual on the property as calculated by RESRAD. Comparison of the two figures shows the contaminated concrete (which also includes radioactive embedded piping and contamination in the spent fuel basin) to be a much more significant source of radiation exposure than the biological shield activation. The peak annual exposure from the contaminated concrete is approximately $0.037 \mathrm{mrem}$. This value for the biological shield is less than 0.00003 mrem, a factor of about 1200 smaller. 
RESIDUAL RADIOACTIVITY GUIDELINES FOR THE HWCTR

TOTAL DOSE: All Isotopes and Pathways Summed

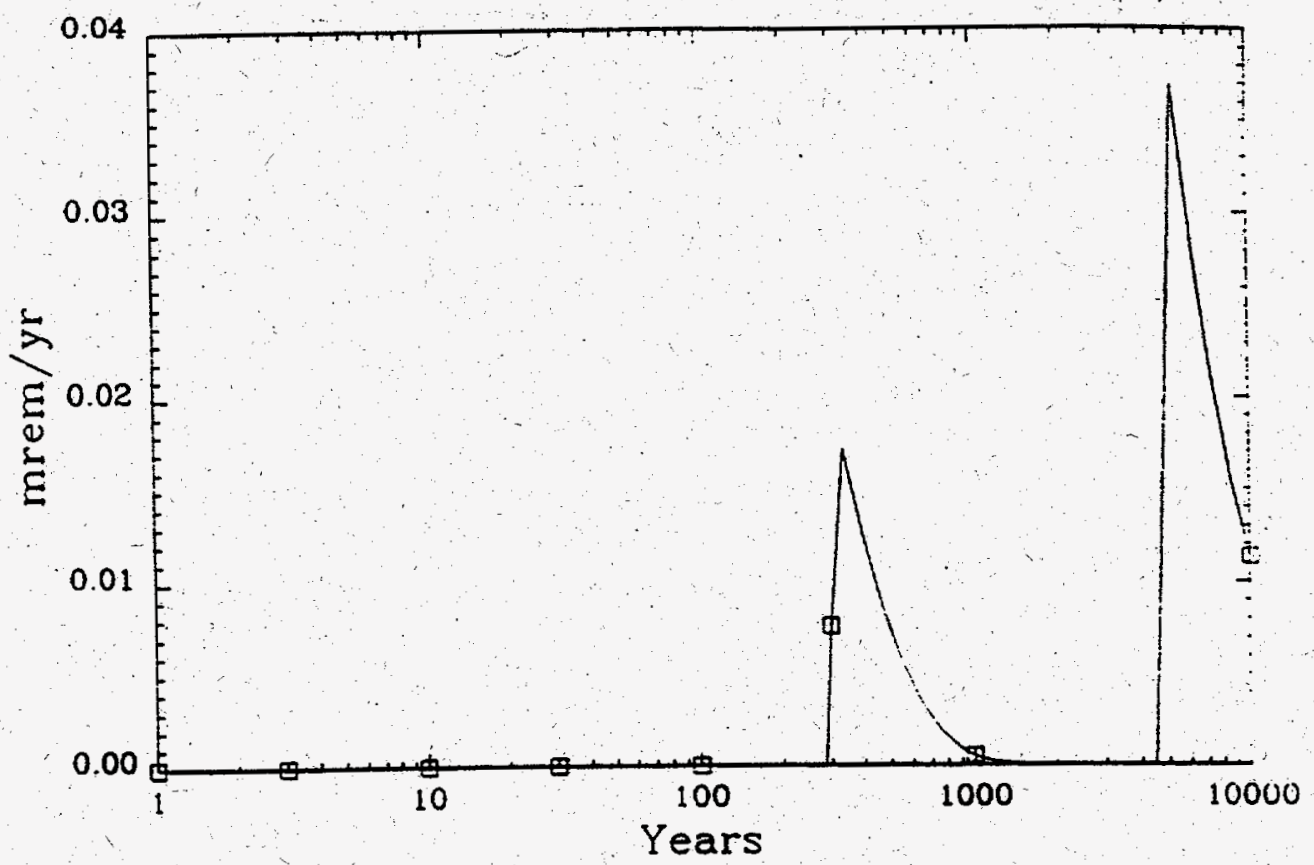

Figure 11. RLSRAD Resuits With Present Source Terms For Contaminated Concrete. This exposure projection is based on removal of radioactive equipment but no decontamination of the facility structure which will remain in place.

The key results of the biological shield assessment, which appear on page 28 of Appendix D, are given in Table 11. The primary values in this table are the $\mathrm{G}(\mathrm{i} / \mathrm{tmin})$ values. which are the calculated DCGLs for an arinual exposure of 15 mrem per year to a hypothetical person on the property. 
RESIDUAL RADIOACTIVITY GUIDELINES FOR THE HWCTR

\section{Table 11. RESRAD Resuits Summary - Biological Shieid}

RESRAD, Version $5.621 \quad T^{-}$Limit $=0.5$ yeax

Sumary : Bio-Shield Activity Asgessmenc HACIR
$03 / 06 / 97$ 12:11 Page 28

File: A IAIOSETD.DAT

Summed Dose/Source Ratios DSR(i,t) in (mrem/yr)/(pCi/g)

and single Radionuclide Soil Guidelises $G(i . t)$ in $\mathrm{pli} / \mathrm{g}$

at tmin = time of minimum single radionucide soil guideline

and at tmax $=$ time of maximum total dose $=464.9 \mathrm{p} 0.5$ years

\begin{tabular}{|c|c|c|c|c|c|c|}
\hline $\begin{array}{c}\text { Nuclide } \\
\text { (i) }\end{array}$ & $\begin{array}{c}\text { Inirial } \\
\text { pCi/g }\end{array}$ & $\begin{array}{l}\text { tmin } \\
\text { (years) }\end{array}$ & $\operatorname{DSR}(i, \operatorname{tmin})$ & $\begin{array}{l}G(i, t m i n) \\
(p c i / g)\end{array}$ & $\operatorname{DSR}(i, \operatorname{cmax})$ & $\begin{array}{c}G(i, t \text { max }) \\
\text { (pci/g) }\end{array}$ \\
\hline Iááá & aáasaasá & abaakabaáaAaka & åaลaลaลa & aลaลaลaลa & áâabąaa & isasa \\
\hline-241 & $230 E-02$ & $189 \mathrm{~b} \mathrm{I}$ & $2.389 E-04$ & $6.2792+04$ & $0.000 \mathrm{z}+00$ & $=3.4305+12$ \\
\hline$c-24$ & $6.590 \mathrm{E}-03$ & $465.3 \mathrm{~b} 0.5$ & $5.305 E-03$ & $2.828 E+03$ & $5.324 E-03$ & $2.817 E+03$ \\
\hline$C 0-60$ & $6.950 E+03$ & $0.000 E+00$ & $2.839 E-24$ & $=1.131 E+15$ & $0.000 E+00$ & $-1.131 E+15$ \\
\hline Cs -137 & 1.250 & $0.000 \mathrm{E}+00$ & $79 E-30$ & $-8.701 E+13$ & $0.000 \mathrm{E}+00$ & $=8.701 E+13$ \\
\hline $24-152$ & 1.79 & $0.000 E+00$ & $2.018 E-26$ & $-1.765 E+14$ & $0.000 E+00$ & $-2.765 E+14$ \\
\hline Eu-154 & $3.980 E+02$ & $0.000 E+00$ & $9.072 E-26$ & $2.639 E+14$ & $0.0005+00$ & $=2.639 E+14$ \\
\hline $\mathrm{H}-3$ & $1.870 E+01$ & 258.2 b 0.3 & $1.5412-10$ & $9.737 E+10$ & $5.0708-17$ & $\cdot 9.594 E+15$ \\
\hline 9 & 4.5 & 0000 p 0. & $E+00$ & $185 E+10$ & $0.000 E+00$ & $\cdot 8.085 E+10$ \\
\hline $\mathrm{N} i-63$ & 3.62 & 0.0000 p 0.0000 & $0.0008+00$ & $-5.9168+13$ & $0.0008+00$ & $-5.926 E+13$ \\
\hline$P u-238$ & 1.9401 & $7558 \mathrm{p} 8$ & $1.6308-05$ & $9.2028+05$ & $1.383 E-08$ & $1.0855+09$ \\
\hline Pu-239. & $1.550 z-02$ & $1.0008+04$ & $1.193 E-07$ & $1.258 E+08$ & $0.000 E+00$ & $-6.2128+10$ \\
\hline 90 & 3. & 2 & $.230 E-23$ & $1.365 E+14$ & $0.000 \mathrm{E}+00$ & $=1.365 E+14$ \\
\hline$v$ & 3.6 & $7282 \mathrm{P} 7$ & $5.1258-02$ & $2.927 E+02$ & $6.3578-05$ & $2.360 E+05$ \\
\hline $0-238$ & $7.760 \mathrm{E}-06$ & $7282 \mathrm{~b} 7$ & $4.6548-02$ & $3.223 E+02$ & $2.7318-08$ & $-3.360 E+05$ \\
\hline $\begin{array}{l}\text { ceses } \\
\text { tAe }\end{array}$ & $\cdots$ & 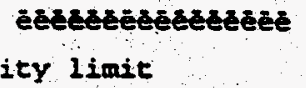 & $\varepsilon$ & sé & See & ĖEZXEEAEE \\
\hline
\end{tabular}

The key resuits of the concrete and rubble assessment, which appear on page 27 of Appendix $E$, are given in Table 12 . 


\section{Table 12. RESRAD Results Summary - Concrete and Rubble}

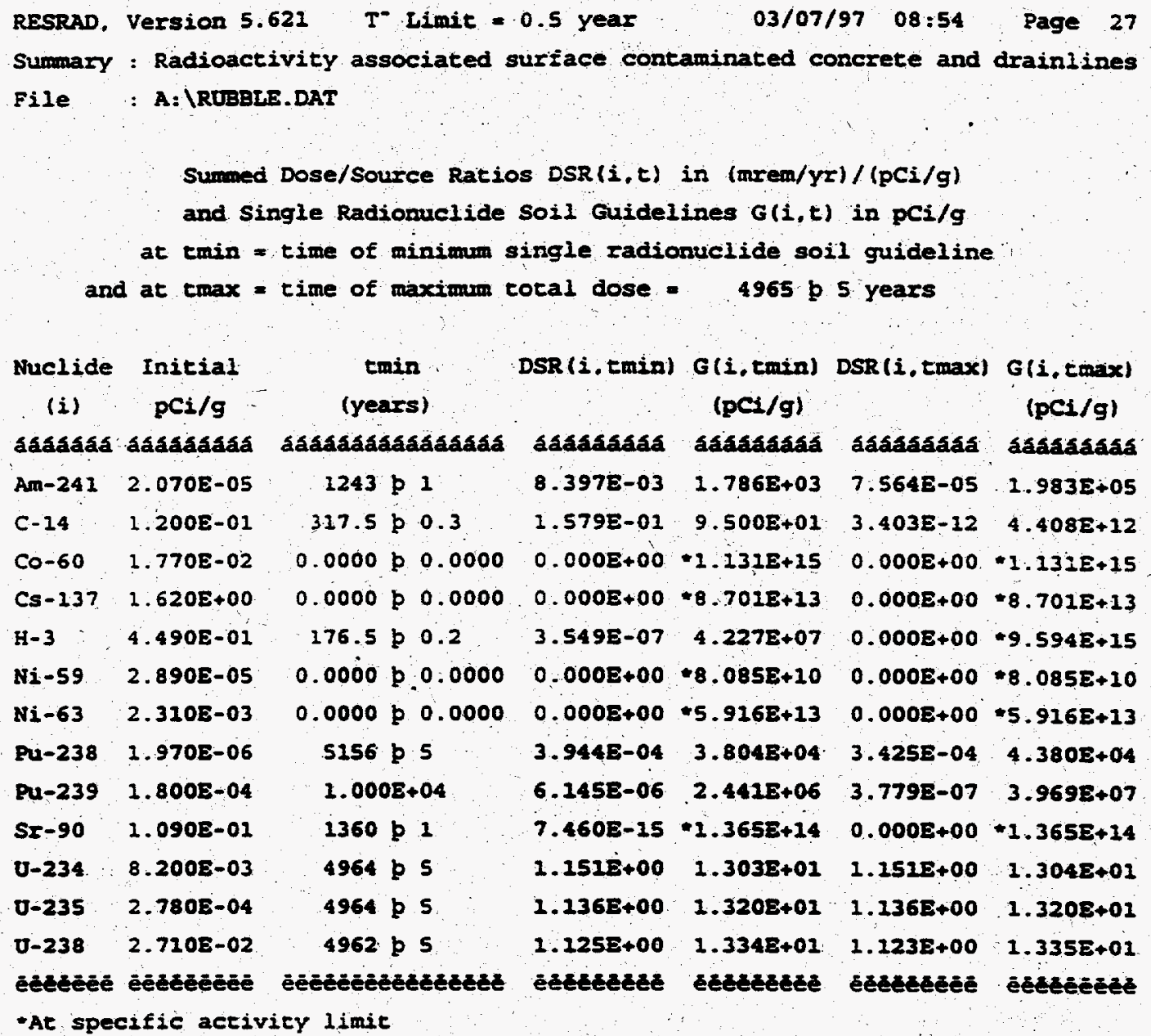

\begin{tabular}{|c|c|c|c|c|c|c|}
\hline $\begin{array}{l}\text { Nuclide } \\
\text { (i) }\end{array}$ & $\begin{array}{l}\text { Initial } \\
\mathrm{pCi} / \mathrm{g}\end{array}$ & $\begin{array}{l}\text { tmin } \\
\text { (years) }\end{array}$ & $D S R$ (i.tmin) & $\begin{array}{c}G(i, t m i n) \\
(p c / g)\end{array}$ & $\operatorname{DSR}\left(i, t_{\max }\right)$ & $\begin{array}{c}G(i, \text { cmaix) } \\
\text { (pCi/g) }\end{array}$ \\
\hline aaasąa & áááááá & 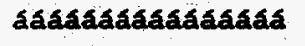 & aáaáaaá & aáaaaaáa & 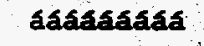 & asaakaaka \\
\hline$A m-241$ & $2.070 E-05$ & 1243 p 1 & $8.3978-03$ & $1.786 \mathrm{E}+03$ & $7.564 \mathrm{E}-05$ & $1.983 E+05$ \\
\hline$:-14$ & $1.200 E-01$ & $3.17 .5 \mathrm{p} 0.3$ & $1.579 E-01$ & $9.500 E+01$ & $3.403 E-12$ & $4.408 E+12$ \\
\hline $\mathrm{CO}-60$ & $1.770 \mathrm{E}-02$ & 0.0000 p 0.0000 & $0.000 E+00$ & $1.131 E+15$ & $0.000 E+00$ & $-1.132 E+15$ \\
\hline $\mathrm{Cs}-137$ & $1.620 E+00$ & $0.0000 \mathrm{p} 0.0000$ & $0.000 E+00$ & $-8.701 E+13$ & $0.000 E+00$ & $-8.701 E+13$ \\
\hline$H-3$ & $4.490 E-01$ & 176.5 p 0.2 & $3.549 E-07$ & $4.2278+07$ & $0.000 E+00$ & $-9.594 E+15$ \\
\hline $\mathrm{Ni}-59$ & $2.890 \mathrm{E}-05$ & $0.0000 \mathrm{~b} .0 .0000$ & $.000 E+00$ & $+8.085 E+10$ & $0.0005+00$ & $-8.085 E+10$ \\
\hline $\mathrm{Ni}-63$ & $2.3105-03$ & 0.0000 b 0.0000 & $.000 \mathrm{E}+00$ & $-5.916 E+13$ & $0.000 E+00$ & $-5.916 E+13$ \\
\hline Pu-238 & $1.9705-06$ & 5156 p 5 & 3.944 E-04 & $3.8048+04$ & $3.425 E-04$ & $4.3805+04$ \\
\hline$P u-239$ & $1.8005-04$ & $1.0008+04$ & $6.145 E-06$ & $2.442 E+06$ & $3.7795-07$ & $3.9698+07$ \\
\hline SI-90 & $1.090 \mathrm{E}-01$ & $1360 \mathrm{bl}$ & $7.460 \mathrm{E}-15$ & $1.3652+14$ & $0.000 E+00$ & $-1.3658+14$ \\
\hline $\mathbf{U}-234$. & $8.200 E-03$ & 4964 b 5 & $1.1513+00$ & $1.303 E+01$ & $1.1528+00$ & $1.304 E+01$ \\
\hline $0-235$ & $2.7808-04$ & 4964 & $1.1362+00$ & $1.3208+02$ & $1.136 E+00$ & $1.3208+01$ \\
\hline $0-238$ & 2.7108 & 4962 & $1.1258+00$ & $1.3348+03$ & $1.123 E+00$ & $1.335 E+01$ \\
\hline èezeēé & èezẺëēēè & Eèèè & zescezes & ezetezede & 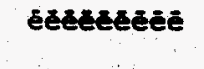 & écLsêEes \\
\hline
\end{tabular}

These tables show that the contaminated concrete and rubble have more limiting concentrations than the biological shield activation.

Table 13 compares the calculated DCGLs for the concrete floors, based on an annual exposure of 15 mrem per year to a hypothetical person on the property, to the average values used in the modeling. The nuclides listed are those which were identified in laboratory analyses of the concrete samples. 
Table 13. Calculated DCGLs For Concrete Floors, Based on the $15 \mathrm{mrem} / \mathrm{yr}$ Criterion

\begin{tabular}{|l|c|c|c|c|}
\hline Nuclide & $\begin{array}{c}\text { DCGL- } \\
\text { unit mass } \\
\text { pCi/g }\end{array}$ & $\begin{array}{c}\text { Modeled } \\
\text { average } \\
\text { pCi/g }\end{array}$ & $\begin{array}{c}\text { Ratio- } \\
\text { modeled } \\
\text { average to } \\
\text { DCGL }\end{array}$ & $\begin{array}{c}\text { DCGL-surface } \\
\text { contamination }^{{ }^{2}} \\
\text { dpm/100 } \text { cm }^{2}\end{array}$ \\
\hline $\mathrm{H}-3$ & $4.23 \mathrm{E}+07$ & $4.490 \mathrm{E}-01$ & $1.06 \mathrm{E}-08$ & $3.53 \mathrm{E}+11$ \\
\hline $\mathrm{C}-14$ & $9.50 \mathrm{E}+01$ & $1.200 \mathrm{E}-01$ & $1.07 \mathrm{E}-03$ & $7.95 \mathrm{E}+05$ \\
\hline $\mathrm{Co}-60$ & $* 1.13 \mathrm{E}+15$ & $1.770 \mathrm{E}-02$ & $1.57 \mathrm{E}-17$ & $3.03 \mathrm{E}+18$ \\
\hline $\mathrm{Sr}-90$ & $* 1.37 \mathrm{E}+14$ & $1.090 \mathrm{E}-01$ & $7.96 \mathrm{E}-16$ & $1.13 \mathrm{E}+17$ \\
\hline $\mathrm{Cs}-137$ & $* 8.70 \mathrm{E}+13$ & $1.620 \mathrm{E}+00$ & $1.86 \mathrm{E}-14$ & $6.24 \mathrm{E}+17$ \\
\hline $\mathrm{U}-234$ & $1.30 \mathrm{E}+01$ & $8.200 \mathrm{E}-03$ & $6.90 \mathrm{E}-04$ & $1.09 \mathrm{E}+05$ \\
\hline $\mathrm{U}-235$ & $1.32 \mathrm{E}+01$ & $2.780 \mathrm{E}-04$ & $1.46 \mathrm{E}-05$ & $1.10 \mathrm{E}+05$ \\
\hline $\mathrm{U}-238$ & $1.33 \mathrm{E}+01$ & $2.710 \mathrm{E}-02$ & $2.04 \mathrm{E}-03$ & $1.10 \mathrm{E}+05$ \\
\hline $\mathrm{Pu}-239$ & $2.44 \mathrm{E}+06$ & $1.800 \mathrm{E}-04$ & $7.38 \mathrm{E}-11$ & $6.34 \mathrm{E}+09$ \\
\hline
\end{tabular}

Notes: a- Assumes all contamination concentrated in top $1 \mathrm{~cm}$ of floors. homogeneously distributed within that volume. The maximum values measured in concrete samples were used in calculating the total radionuclide inventory.

b- Average used in modeling.

c- Assumes all surface contamination contained in top $1 \mathrm{~mm}$ of surface.

- These nuclides were at the individual nuclide limit as calculated by RESRAD.

The ratios of the concentrations modeled to the DCGLs provide a measure of the difference between the concentrations required to produce 15 mrem per year and the average contamination in the contaminated floors of the structure. Note that the highest ratio is 0.00204 for U-238. The ratios for most radionuclides fall far below this value. The three radionuclides marked with as asterisk are shown to be insignificant in producing exposure under the RESRAD pathway scenario.

Table 14 shows the calculated DCGLs for an annual exposure of 100 mrem per year to a hypothetical person on the property. 
Table 14. Calculated DCGLs For Concrete Floors, Based on $100 \mathrm{mrem} / \mathrm{yr}$ Criterion

\begin{tabular}{|l|c|c|c|c|}
\hline Nuclide & $\begin{array}{c}\text { DCGL- } \\
\text { unit mass } \\
\text { pCi/g }\end{array}$ & $\begin{array}{c}\text { Modeled } \\
\text { average } \\
\text { pCi/g }\end{array}$ & $\begin{array}{c}\text { Ratio- } \\
\text { modeled } \\
\text { average to } \\
\text { DCGL }\end{array}$ & $\begin{array}{c}\text { DCGL- surface } \\
\text { contamination }^{\mathbf{d}} \\
\text { dpm/100 cm }\end{array}$ \\
\hline $\mathrm{H}-3$ & $2.81 \mathrm{E}+08$ & $4.490 \mathrm{E}-01$ & $1.60 \mathrm{E}-09$ & $2.35 \mathrm{E}+12$ \\
\hline $\mathrm{C}-14$ & $6.33 \mathrm{E}+02$ & $1.200 \mathrm{E}-01$ & $1.89 \mathrm{E}-04$ & $5.30 \mathrm{E}+06$ \\
\hline $\mathrm{C} 0-60$ & $2.41 \mathrm{E}+15$ & $1.770 \mathrm{E}-02$ & $7.33 \mathrm{E}-18$ & $2.02 \mathrm{E}+19$ \\
\hline $\mathrm{Sr}-90$ & $9.01 \mathrm{E}+13$ & $1.090 \mathrm{E}-01$ & $1.21 \mathrm{E}-15$ & $7.54 \mathrm{E}+17$ \\
\hline $\mathrm{Cs}-137$ & $4.97 \mathrm{E}+14$ & $1.620 \mathrm{E}+00$ & $3.26 \mathrm{E}-15$ & $4.16 \mathrm{E}+18$ \\
\hline $\mathrm{U}-234$ & $8.67 \mathrm{E}+01$ & $8.200 \mathrm{E}-03$ & $9.46 \mathrm{E}-05$ & $7.25 \mathrm{E}+05$ \\
\hline $\mathrm{U}-235$ & $8.80 \mathrm{E}+01$ & $2.780 \mathrm{E}-04$ & $3.16 \mathrm{E}-06$ & $7.36 \mathrm{E}+05$ \\
\hline $\mathrm{U}-238$ & $9.87 \mathrm{E}+01$ & $2.710 \mathrm{E}-02$ & $3.06 \mathrm{E}-04$ & $7.42 \mathrm{E}+05$ \\
\hline $\mathrm{Pu}-239$ & $4.97 \mathrm{E}+06$ & $1.800 \mathrm{E}-04$ & $3.62 \mathrm{E}-011$ & $4.16 \mathrm{E}+10$ \\
\hline
\end{tabular}

Notes: a- Assumes all contamination concentrated in top $1 \mathrm{~cm}$ of floors. homogeneously distributed within that volume. The maximum values measured in concrete samples were used in calculating the total radionuclide inventory.

b- Average used in modeling.

c- Assumes all surface contamination contained in top $1 \mathrm{~mm}$ of surface.

\subsection{Detailed RESRAD Results - With Radioactive Equipment in Place}

The RESRAD summary report for the radioactivity associated with radioactive equipment, contaminated concrete and embedded piping appears in Appendix F.

Figure 12 shows the annual radiation exposure to a hypothetical individual on the property as calculated by RESRAD. Comparison of this figure with Figure 11 shows that there is not a significance difference associated with the installed equipment. Table 15 shows the RESRAD summary from page 28 of Appendix $F$. One can see that the DCGLs which appear in the $G(i, t m i n)$ column are identical to those of Table 12. This situation shows that neither the additional contamination inside of installed equipment nor the induced radioactivity in the biological shield affect the outcome of the RESRAD calculation. 
RESIDUAL RADIOACTIVTY GUIDELINES FOR THE HWCTR.

TOTAL DOSE: All Isotopes and Pathways Summed

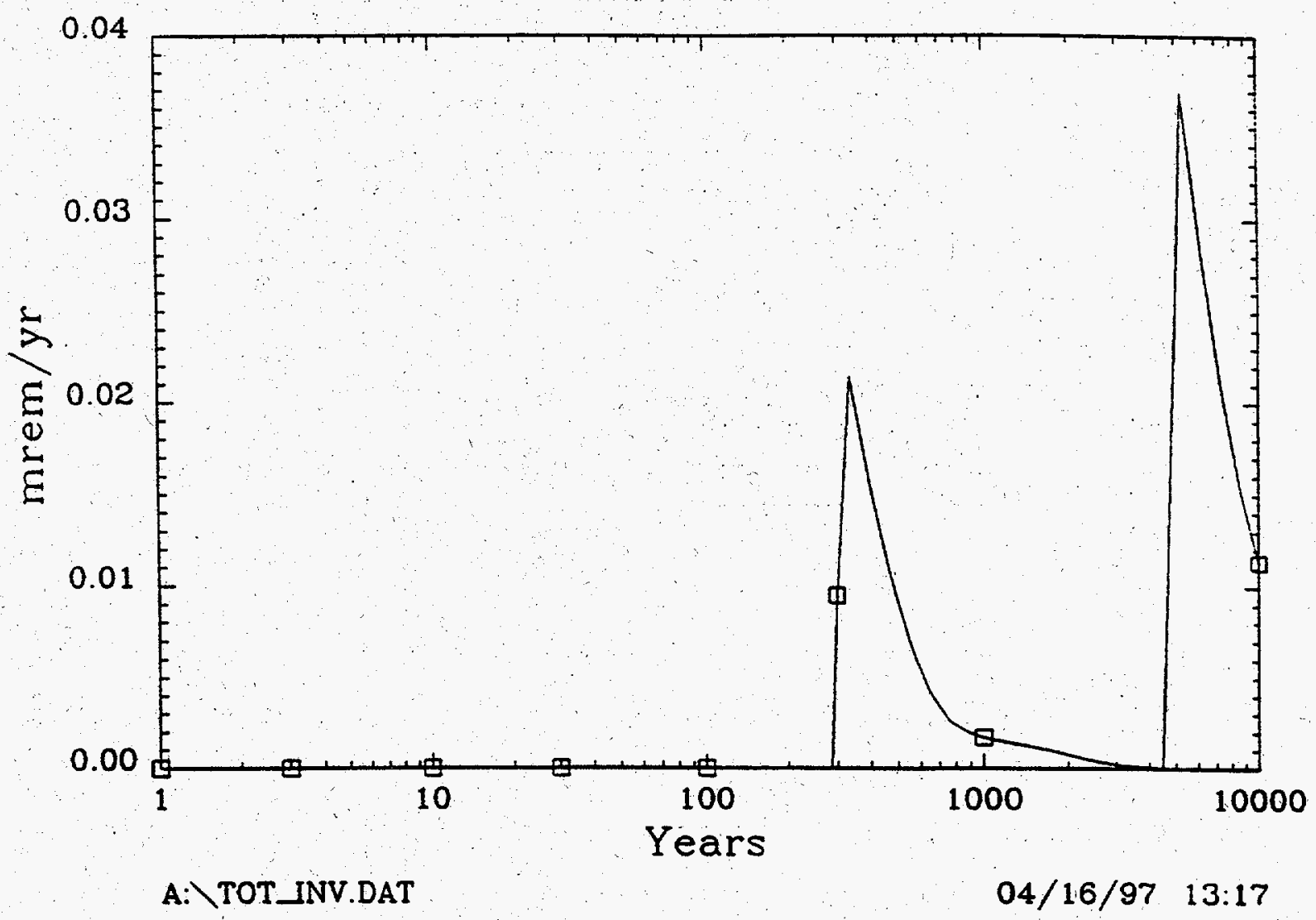

Figure 12. RESRAD Results With Present Source Term for Radioactive Equipment and Contaminated Concrete. 
RESIDUAL RADIOACTIVITY GUIDEUINES FOR THE HWCTR

Table 15. RESRAD Results Summary - Installed Equipment, Biological Shield and Concrete and Rubble.

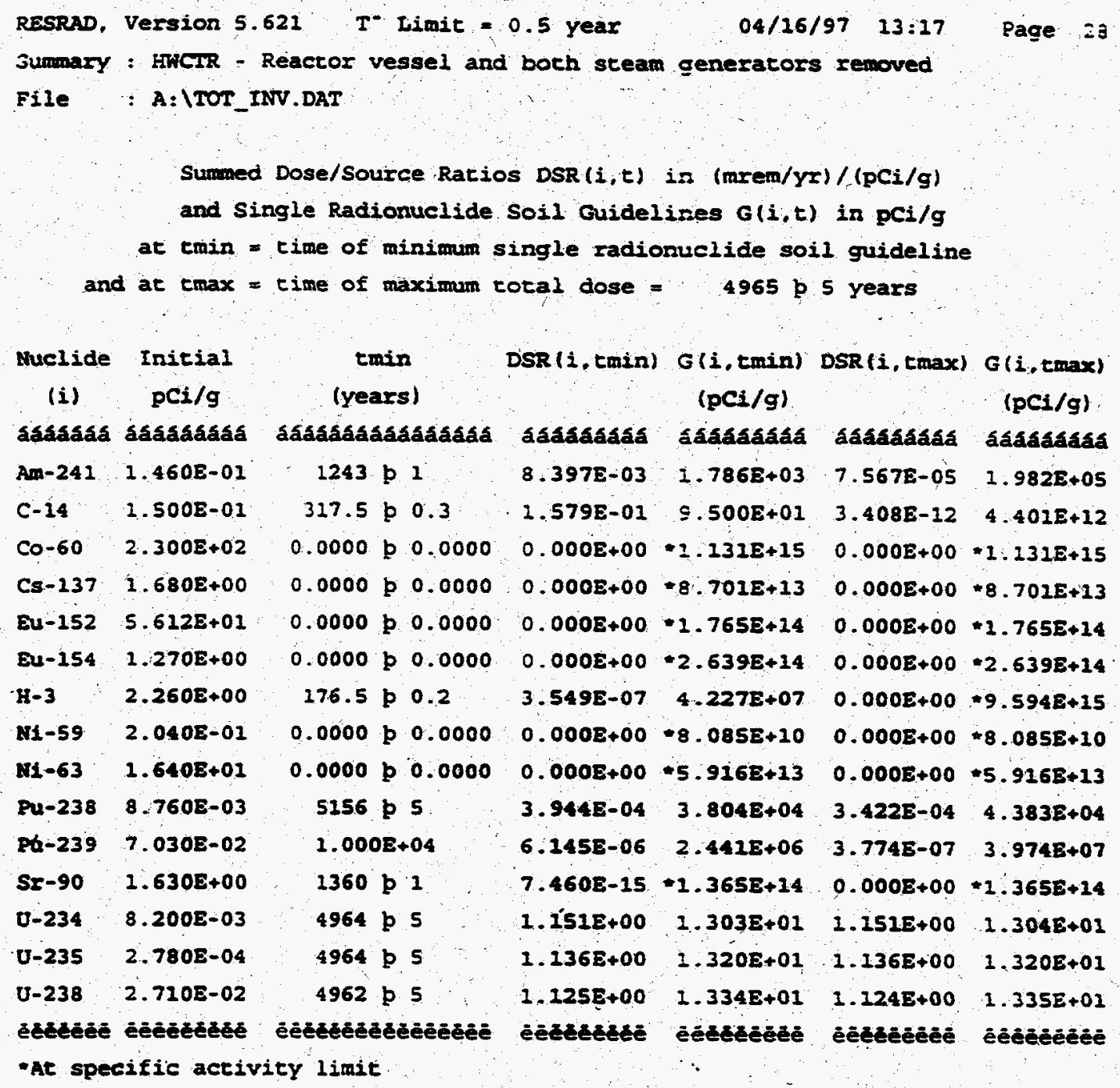

\subsection{Water-Dependent Pathways}

The RESRAD calculations showed that radiation exposure to humans would come through water-dependent pathways. That is, radioactivity from the facility enters the ground water and is drawn from the ground water by a well on the property. Humans living on the property drink water from the well. They eat foodstuffs grown in a garden irrigated with water from the well. They drink milk from cows who drink water from the well and eat forage grown on the property. They also eat meat from these livestock.

The effects of these pathways are displayed in Figure 13. 
DOSE: All Isotopes Summed

Water Dependent Pathways

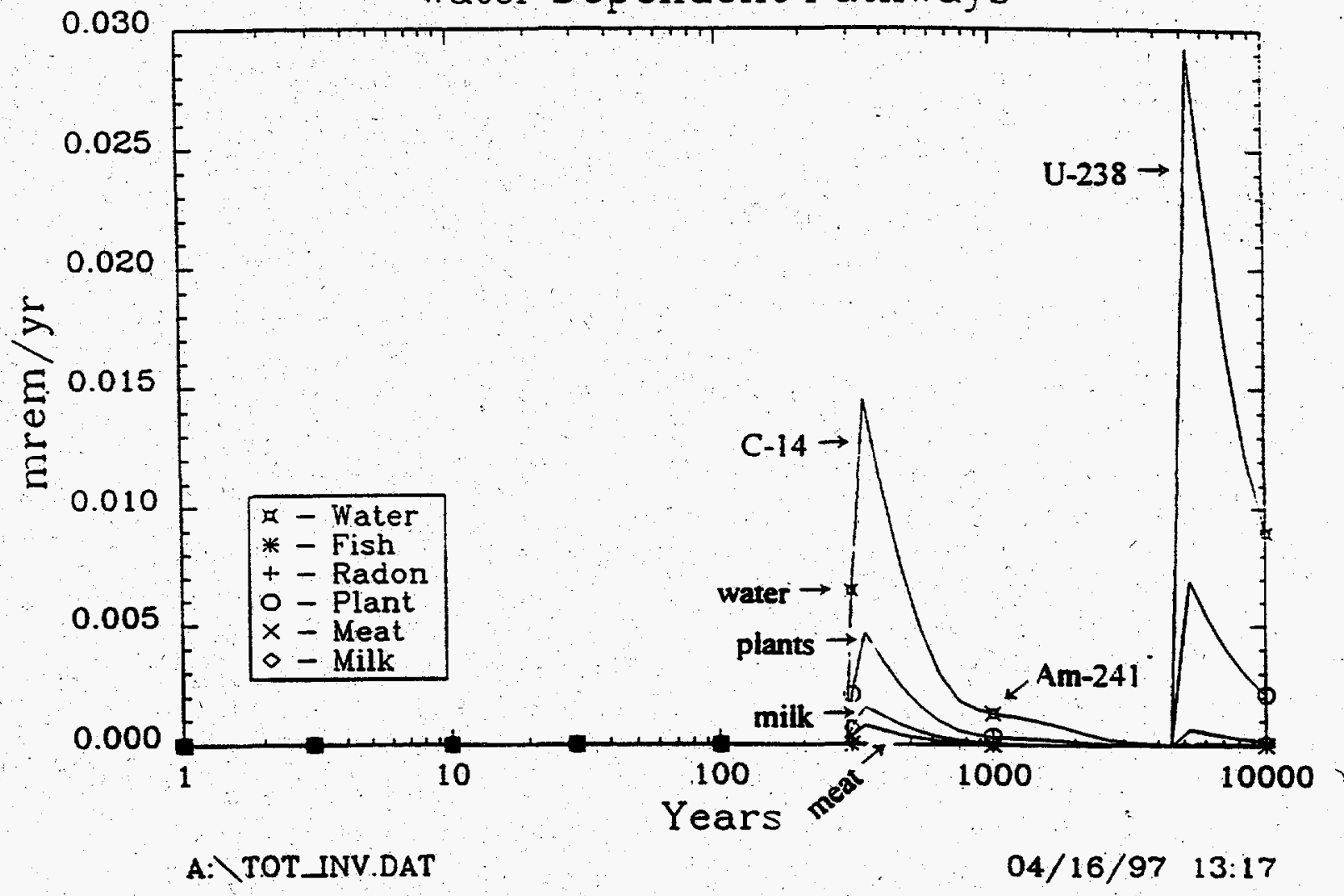

Figure 13. RESRAD Doses from Water-Dependent Pathways

\subsection{Radionuclides of Most Significance}

One can see from Figure 13 that the most-significant radionuclides in the RESRAD calculation results are C-14, Am-241 and U-238. The C-14 peak occurs at approximately 300 years. The Am-241 peak occurs at approximately 100 years. At approximately 6000 years the peak associated with U-238 appears.

From this figure one can also see the relative contributions from water, plants, milk and meat. The dose fractions associated with each of these pathways are 0.76 , $0.18,0.04$ and 0.02 , respectively. Note that the contributions from the different pathways are additive. For example, the $C-14$ peaks together amount to 0.02 mrem per year. The total for Am-241 is 0.013 mrem per year and for U-238, 0.034 mrem per year. 


\subsection{Detailed RESRAD-BUILD Results}

The RESRAD-BUILD summary report appears in Appendix G. The principal results appear on page 5 of the appendix. It is from the table on this page that the DCGLs of $15,000 \mathrm{dpm} / 100 \mathrm{~cm}^{2} \beta-\gamma$ and $150 \mathrm{dpm} / 100 \mathrm{~cm}^{2} \alpha$ described in Section 8.1 were drawn.

The RESRAD-BUILD computer code does not produce graphs as does RESRAD.

\subsection{REFERENCES}

ok1. Department of Energy Order 5400.5, Radiation Protection of the Public and the Environment. U.S. Department of Energy, Washington, D.C.

ok2. Decommissioning Resource Manual. U.S. Department of Energy, Office of Environmental Management Manual DOE /EM-0246, August 1995.

¿k3. Yu. C., et. al., Manual for Implementing Residual Radioactivity Material Guidelines Using RESRAD, Version 5.0. Argonne National Laboratory, ANL/EAD/LD-2 (September 1993).

6K4. Yu, C.. et. al., RESRAD-BUILD, A Computer Model for Analyzing the Radiological Doses Resulting from the Remedial and Occupancy of Buildings Contaminated With Radioactive Material, Argonne National Laboratory, ANL/EAD/LD-3 (November 1994).

o K 5. Decommissioning Plan for the Heavy Water Components Test Reactor. U. S. Department of Energy, Savannah River Site. Aiken. SC 29808. D PST.75.417

1K 6. Heavy Water Components Test Reactor Facility Characterization Report. U. S. Department of Energy, Savannah River Site. Aiken, SC 29808 (September 1996). WSR C-ms- $96-0636$

6K7. Savannah River Site Future Use Report, U. S. Department of Energy, Savannah River Site. Aiken, SC 29808 (January 1996).

OK8. Hsu, R. H. and Cook, J. R., Soil Release Guidelines - Expanded RESRAD Study, WSRC-RP-95-287, Westinghouse Savannah River Company, Savannah River Technology Center, Aiken, SC 29808 (March 1, 1995).

OK9. Eger, K.J., Final Consolidated Implementation Plan for the Shippingport Station Decommissioning Project Site Release Criteria, KJE87-144a, Revision 1 (July 13, 1988). 
RESIDUAL RADIOACTIVITY GUIDELINES FOR THE HWCTR

oK 10. Heavy Water Components Test Reactor Screening Characterization Study, U S ENERGY Corporation, Aiken, SC 29803 (April 1996).

OK 11. Field, F. R., A Decommissioning Plan for the Heavy Water Components Test Reactor, U.S. Atomic Energy Commission Report DPST-75-417, duPont Savannah River Plant, Aiken, SC 29808 (January 1976). 


\section{APPENDIX A}

\section{PARAMETERS USED IN ANALYSIS - SCENARIO A}

This appendix identifies in tables the parameters used for the Scenario A calculation. Scenario A assumes that a family farm would be built on the HWCTR site, 30 or more years in the future. All RESRAD pathways were considered, except for the ingestion of fish from a pond on the property. References for this appendix can be found following the tables. Each of the parameters is defined in the RESRAD manual (reference 1).

\section{Table 1. RESRAD Parameters For Biological Shield Calculation}

\begin{tabular}{|c|c|c|c|}
\hline Parameter & Wnit & VIalue & Basis. \\
\hline Area of contaminated zone & $\mathbf{m}^{2}$ & $1.240 \mathrm{E}+01$ & Area of activated region. \\
\hline Zane & $m$ & 3 & Height of activated region \\
\hline Length parallel & $\mathrm{m}$ & 7 & Size of activated zone. \\
\hline Base & renty & 100,15 & DOE limit and $A L A R A$ \\
\hline Times for & $\mathbf{y r}$ & $0-10^{4} \mathrm{yr}$ & RESRAD standard values, ref. 1 \\
\hline Concentr & -3 & 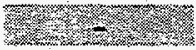 & Radronnclide values were \\
\hline $\mathrm{H}-3$ & $\mathrm{pCi} / \mathrm{g}$ & $1.870 \mathrm{E}+01$ & the 1996 characterization data in \\
\hline C 14 & pelg & $6590 \mathrm{E}-03$ & Ief 2 averaged over the masso \\
\hline $\mathrm{Ni}-59$ & $\mathrm{pCi} / \mathrm{g}$ & $4.530 \mathrm{E}-02$ & different components in the \\
\hline $\mathrm{Co}-60$ & pets & $6950 \mathrm{E}+03$ & activated region such as \\
\hline $\mathrm{Ni}-63$ & $\mathrm{pCi} / \mathrm{g}$ & $3.620 E+00$ & and concrete. \\
\hline $\mathrm{S}=90$ & pCig & $3360 \mathrm{E} 01$ & 3at \\
\hline $\mathrm{Ba}-133$ & $\mathrm{pCi} / \mathrm{g}$ & - & $\begin{array}{l}\text { Eu-152 was used as a surrogate for } \\
\mathrm{Ba}-133 \text { (Ba-133 not in RESRAD) }\end{array}$ \\
\hline 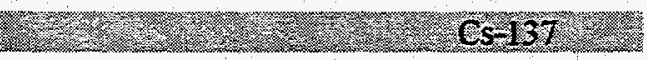 & peif & $1250 \mathrm{E} 02$ & 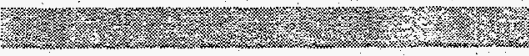 \\
\hline Eu-152 & $\mathrm{pCi} / \mathrm{g}$ & $1.790 \mathrm{E}+03$ & \\
\hline Eu-154 & pCrg & $3980 \mathrm{E}+01$ & -2 \\
\hline$U-234$ & $\mathrm{pCi} / \mathrm{g}$ & $3.620 \mathrm{E}-06$ & \\
\hline $\mathrm{y}-238$ & peig & $7260 \mathrm{E} 06$ & 2 \\
\hline $\mathrm{Pu}-238$ & $\mathrm{pCi} / \mathrm{g}$ & $1.940 \mathrm{E}-03$ & \\
\hline (1) Pu-239 & pefg & $1550 \mathrm{E}-02$ & \pm \\
\hline Am-241 & $\mathrm{pCi} / \mathrm{g}$ & $3.230 \mathrm{E}-02$ & \\
\hline Initial principal radionucide concentration & pCll & not used & $\begin{array}{l}\text { Groundwater contanination not } \\
\text { applicable to HWCTR }\end{array}$ \\
\hline Cover depth & m & 5 & Depth to activated region. \\
\hline Bensity of cover material & $\mathrm{g} / \mathrm{cm}$ & 1.44 & Based on soil borings, ref $1 \& 3$. \\
\hline Cover depth erosion rate & $\mathrm{m} / \mathrm{yr}$ & $9.000 \mathrm{E}-05$ & Ref. 4. \\
\hline Contaninated zone density & $g / \mathrm{cm}^{3}$ & 2.400 & Ref 1 density of concrete. \\
\hline Contaminated zone erosion rate & $\mathrm{m} / \mathrm{yr}$ & 0.000 & Ref. 1 default: \\
\hline Contaminated zone total porosity & (2)- & 043 & Ref 185, data from well $\mathrm{P}-29$ \\
\hline Contaminated zone effective porosity & - & 0.33 & Ref. $1 \& 5$, data from well P-29. \\
\hline Contaninated zone hy dranlic conduct & $\mathrm{m} / \mathrm{x}$ & 1.520 & Ref 6, fom laboratory tests. \\
\hline Contaminated zone b parameter & 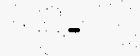 & 5.300 & ef. $1 \& 3$, data from soil \\
\hline
\end{tabular}


Table 1 (continued)

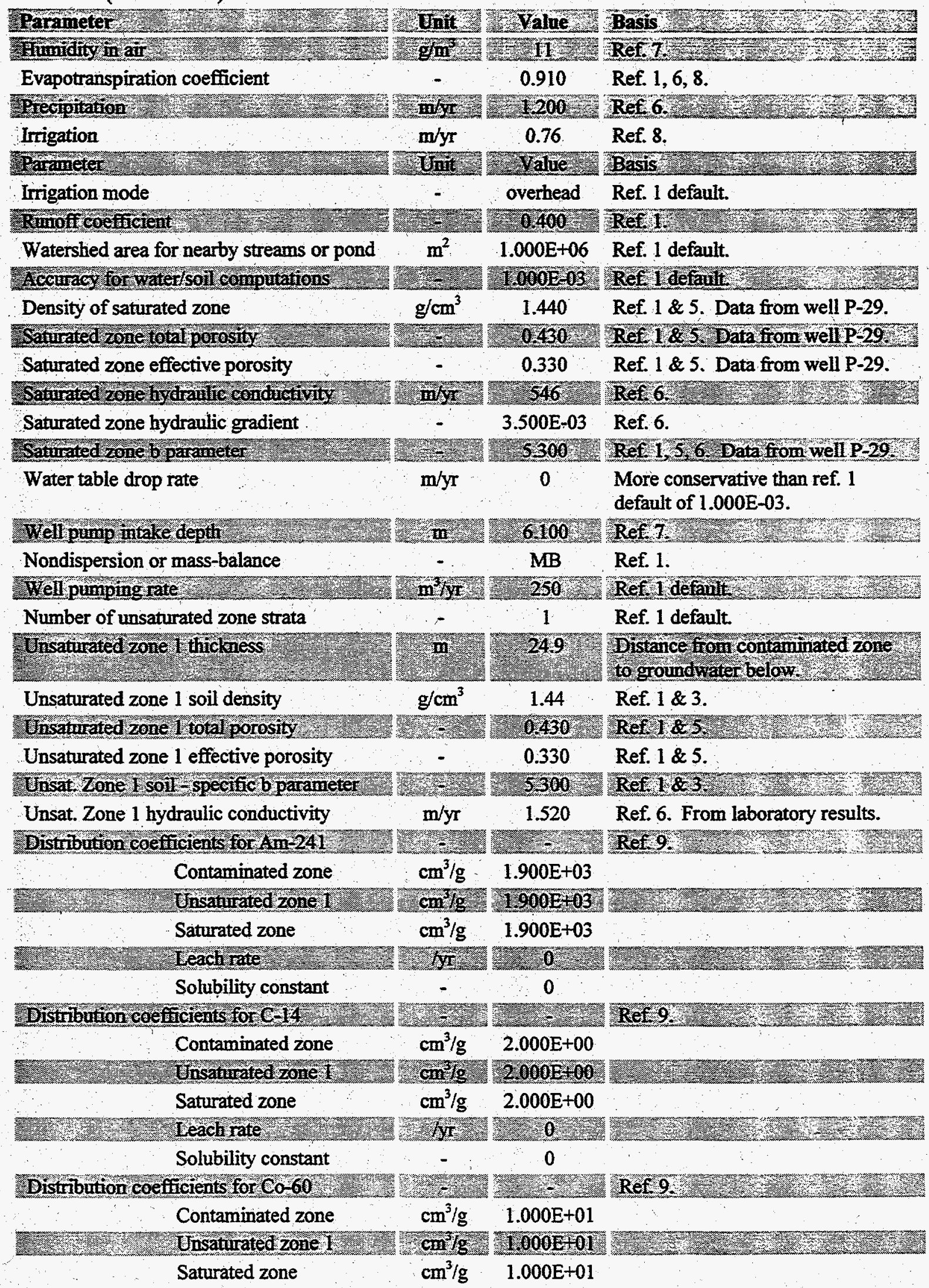


Table 1 (continued)




Table 1 (continued)

Parameter

Distribution coefficients for Pu-239

Unit

Unsaturated zone 1

2. Saturated zone

Leach rate

27.

Distribution coefficients for $\mathrm{Sr}-90$

20.15aminated zone

Unsaturated zone 1

20.2 Saturated zone

Leach rate

201- Solubility constant

Distribution coefficients for U-234

Congrominated zone

Unsaturated zone 1

Saturated zone

Leach rate

Solibility constant
Distribution coefficients for U -238

Contaminated zone

Unsaturated zone 1

Saturated zone

Leach rate

Solubility constant

Distribution coefficients - daughter Ac-227

Contaminated zone

2.0 Unsaturated zone

Saturated zone

Leach rate

Solubility constant

Distribution coefficients - daughter Gd-152

Contaminated zone

E. 20.0 Unsaturated zonel

Saturated zone

Leach rate

Solubility constant

Distribution coeffcients - daughter Np 237

Contaminated zone

Uns Unsaturated zone

Saturated zone

Leach rate

Solubility constant

Distribution coefficients - daughter $\mathrm{P}-231$

Contaminated zone

carig $1000 \mathrm{E}+01$ $\mathrm{cm}^{3} / \mathrm{g} \quad 3.500 \mathrm{E}+01$ c $\mathrm{cm}^{3} / \mathrm{g}$ 3.500E+01 $\mathrm{cm}^{3} / \mathrm{g} \quad 3.500 \mathrm{E}+01$

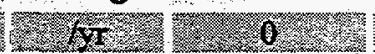

$-$

$\mathrm{cm}^{3} / \mathrm{g} / 1.000 \mathrm{E}+02$

$\mathrm{cm}^{3} / \mathrm{g} \quad 1.000 \mathrm{E}+02$

$\mathrm{cm} / \mathrm{g} / \mathrm{0} / 00 \mathrm{E}+02$

/yr

0

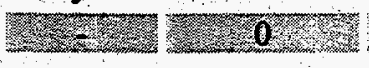

Ref. 9.

cming T000ET0I

$\mathrm{cm}^{3} / \mathrm{g} \quad 1000 \mathrm{E}+01$

tyr

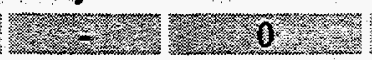

-

$\therefore \quad$ Ref. 9.

$\mathrm{cm}^{3} \mathrm{~g} 3 \mathrm{~s}, 300 \mathrm{E}+01$

$\mathrm{cm}^{3} / \mathrm{g} \quad 3.500 \mathrm{E}+01$

$\mathrm{cm}^{3} \mathrm{tg} / 3.500 \mathrm{E}+01$

/yr

0

$=2.00$

Ref. 9.

$20-30$ -

1.2. 310 -

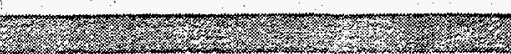

2.

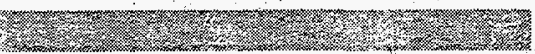

78

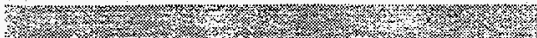

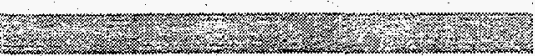

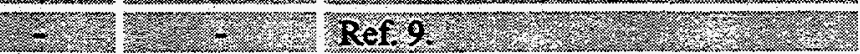

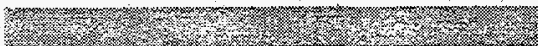

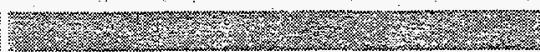

- 0

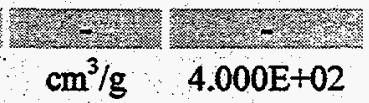

Ref 9.20

cmlg $4.000 \mathrm{E}+02$

$\mathrm{cm}^{3} / \mathrm{g} \quad 4.000 \mathrm{E}+02$

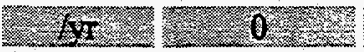

$-\quad 0$

\begin{tabular}{cc}
\hline & \\
\hline $\mathrm{cm}^{3} / \mathrm{g}$ & $-1.00 \mathrm{E}+00$ \\
$\mathrm{~cm}^{2} / \mathrm{g}$ & $-1.00 \mathrm{E}+00$ \\
$\mathrm{~cm}^{3} / \mathrm{g}$ & $-1.00 \mathrm{E}+00$
\end{tabular}

17. 0

$-\quad \because 0$

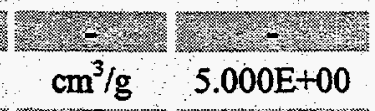

cnitg $5000 \mathrm{E}+00$.

$\mathrm{cm}^{3} / \mathrm{g} \quad 5.000 \mathrm{E}+00$

- $/ \mathrm{y}$

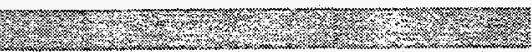

0

$3-2$

Ref. 9

$\mathrm{cm}^{3} / \mathrm{g} \quad 5.500 \mathrm{E}+02$
Ref I default

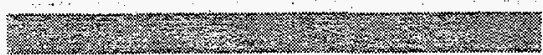

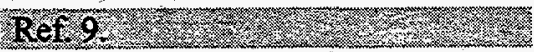

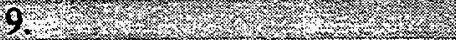


Table 1 (continued)

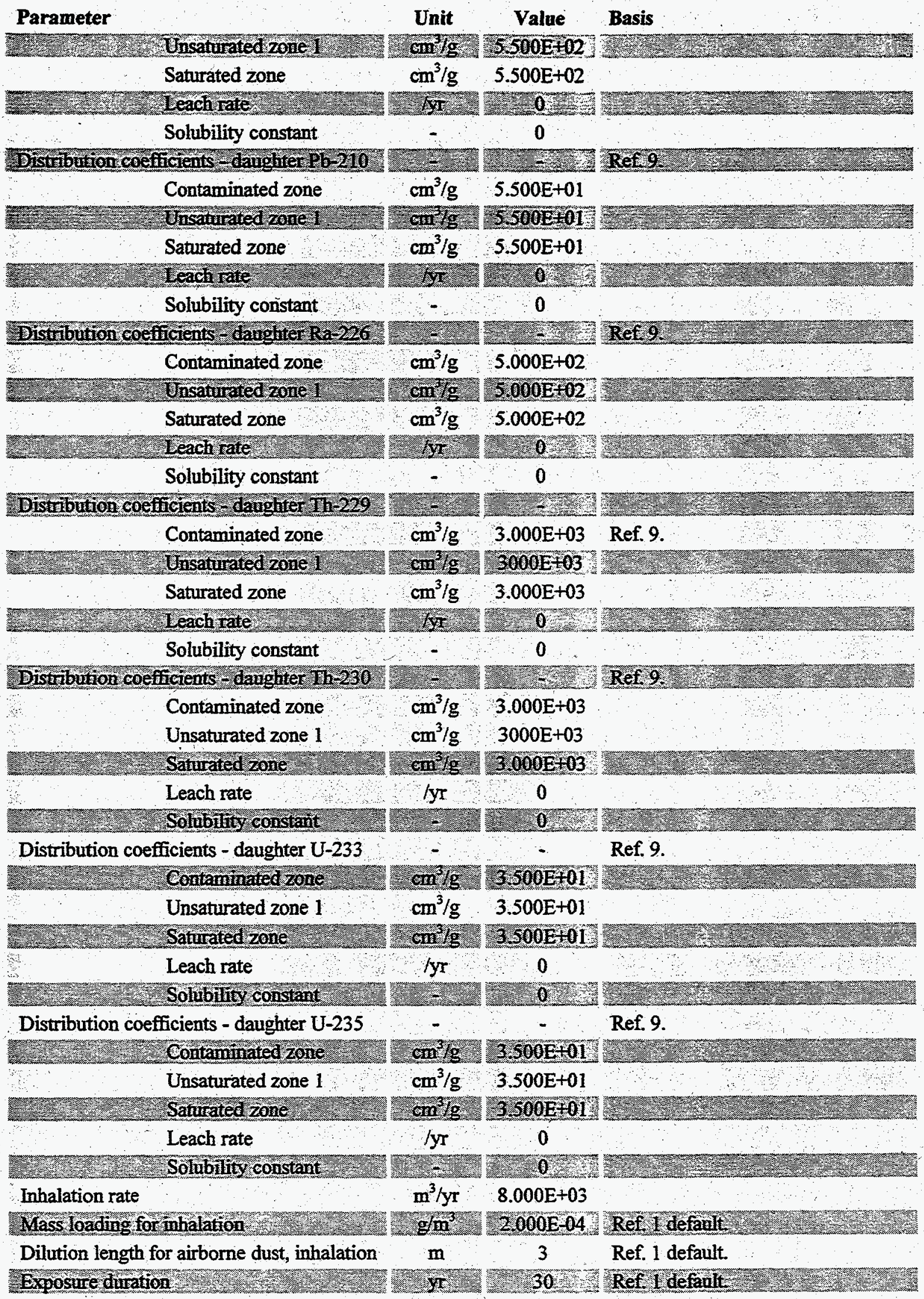


Table 1 (continued)

Parameter

Unit Value Basis

Sinelding factor, infialation

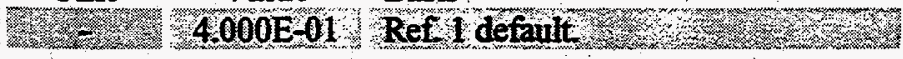

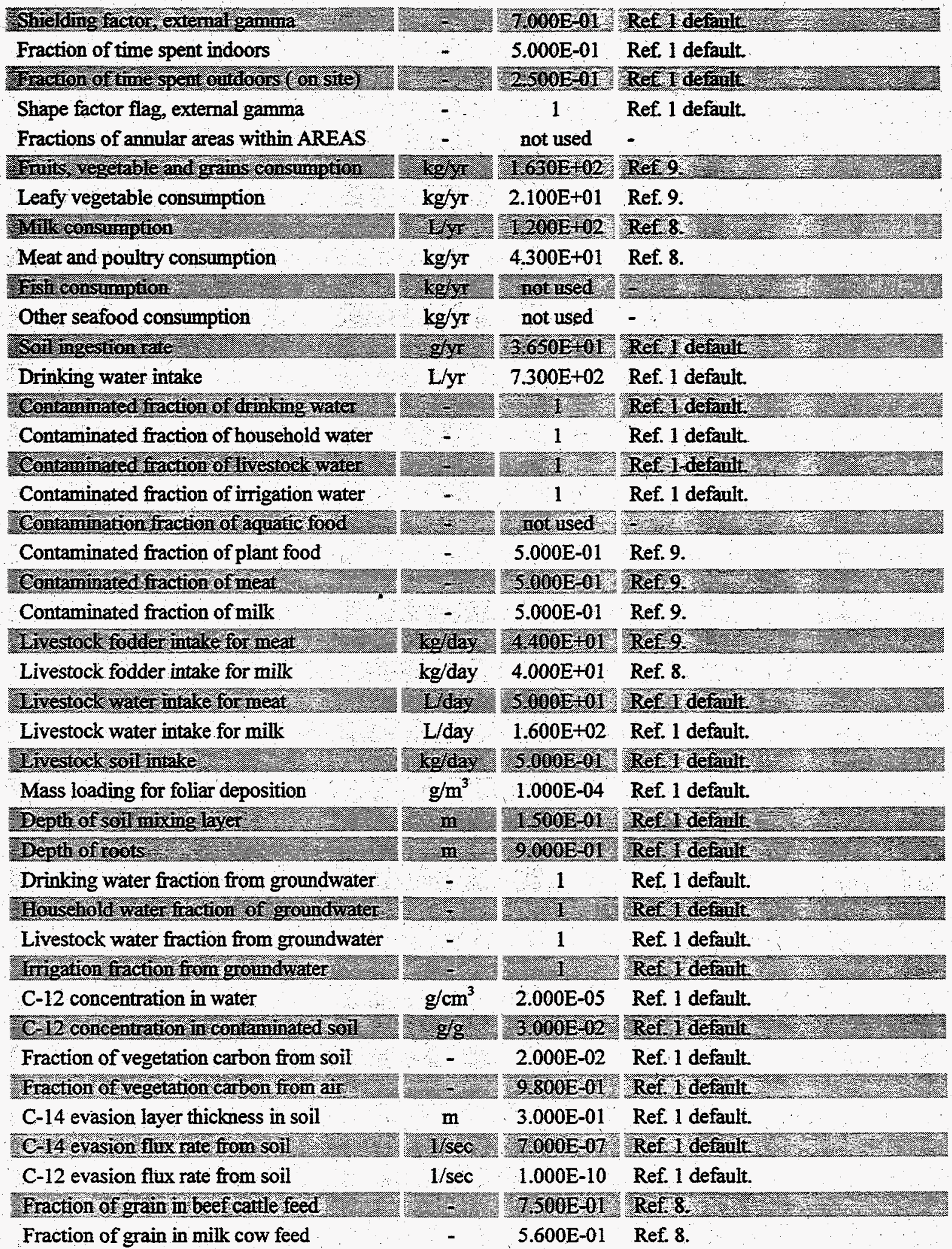


Table 1 (continued)

\begin{tabular}{|c|c|c|c|}
\hline Parameter & Unit & Value & Basis \\
\hline Storage times for contaninated food suifs & 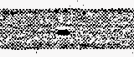 & 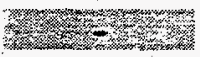 & 8 \\
\hline Fruits, non-leafy vegetables and grains & days & $1.400 \mathrm{E}+01$ & Ref. 1 default. \\
\hline I Eafy regetables & days & $2+1.125$ & Refil defaulie \\
\hline Milk & days & 3 & Ref. 1 default \\
\hline Weat and poulus & dass: & 6 & Ref I defant \\
\hline Fish & days & not used & - \\
\hline Grustaces and nollusks & days & Dotised & -2 \\
\hline Well water & days & 1. & Ref. 1 default \\
\hline Surface water & days & 1 & Ref. I default \\
\hline Livestock fodder & days & 45 & Ref. 1 default. \\
\hline Thickness of builaing foundation & in & OSOOOE.018 & Ref 1 defant: \\
\hline Bulk density of building foundation & $\mathrm{g} / \mathrm{cm}^{3}$ & 2.4 & Ref. 1 default. \\
\hline Total porosity of cover materal & $2=0$ & 804 & Ref I default: \\
\hline Total porosity of building foundation & - & 0.1 & Ref. 1 default. \\
\hline Wolumetric yater content of cover material & 20 & $5000 \mathrm{E}=02$ & Ref, 1 defailt \\
\hline Volumetric water content of the foundation & - & $3.000 \mathrm{E}-02$ & Ref. 1 default. \\
\hline Difiusion coefficient for radon gas & $2=$ & $=$ & 40 \\
\hline In cover material & $\mathrm{m} / \mathrm{sec}$ & $2.000 \mathrm{E}-06$ & Ref. 1 default \\
\hline In foundatron matenal. & misec & $3000 \mathrm{E} 07$ & Ref I defaull \\
\hline In contaminated zone soil & $\mathrm{m} / \mathrm{sec}$ & $2.500 \mathrm{E}-08$ & Ref. 9. \\
\hline Radon vertical dimension of mixing & $\mathrm{m}$ & 2 & Ref I defailt \\
\hline Average annual wind speed & $\mathrm{m} / \mathrm{sec}$ & 2 & Ref. I default. \\
\hline Average building air exchange rate & Whir & S000E-01 & Ref. 1 defanlt \\
\hline Height of the building (room) & $\mathbf{m}$ & 2.5 & Ref. 1 default. \\
\hline Building inter bor area factor & 2 & 0 & Ref I default: \\
\hline Building depth below ground surface & $\mathbf{m}$ & -2.5 & Ref. 1 default. \\
\hline Emanating power of Rn-222 gas & -8 & $2500 \mathrm{E} 01$ & Ref 1 defauit \\
\hline Emanating pover of Rn-220 & 8 & not used & 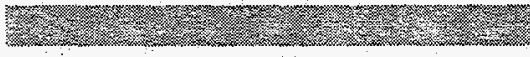 \\
\hline
\end{tabular}

Parameters for the second RESRAD run for contaminated concrete and imbedded radioactive piping appear in Table 2 .

\section{Table 2. RESRAD Parameters For Contaminated Concrete Calculation}

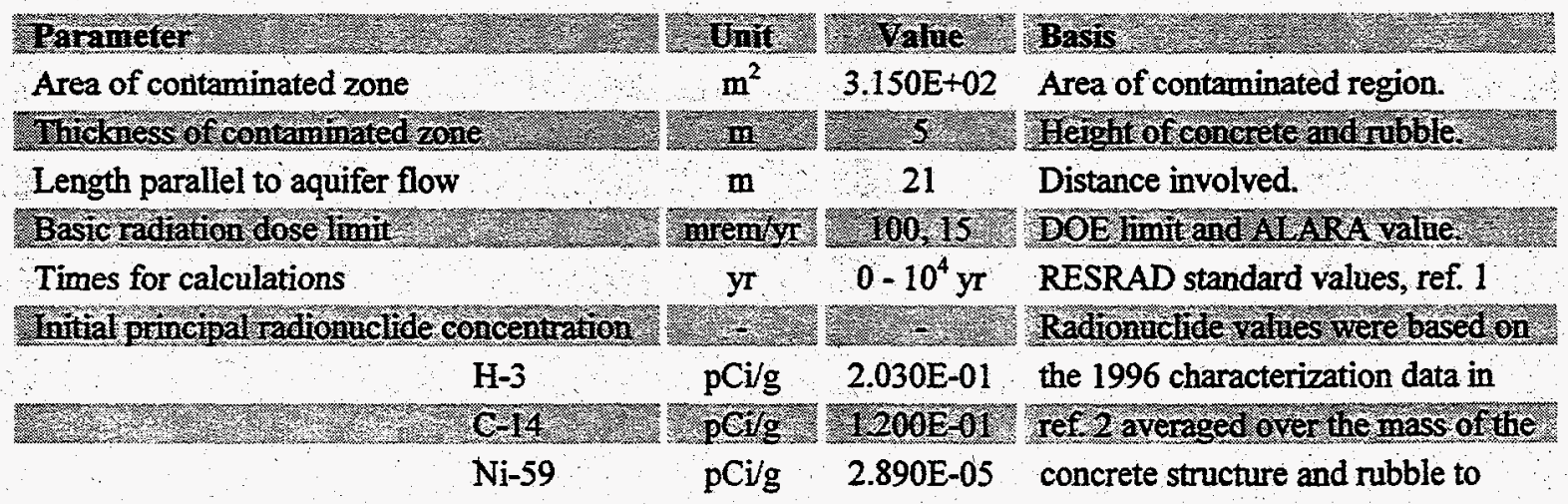


Table 2 (continued)

Parameter

Unit Value Basis

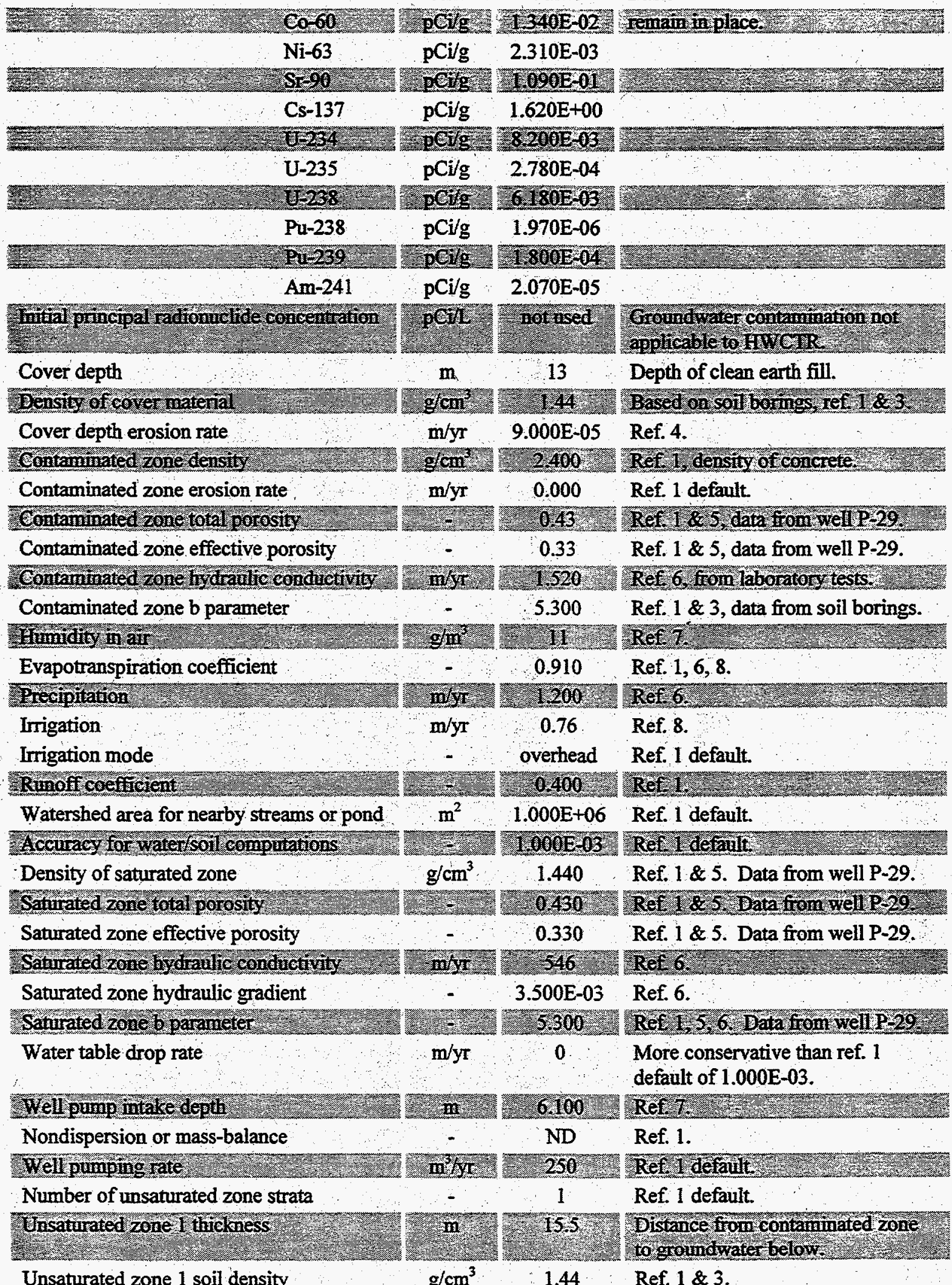

Unsaturated zone 1 soil density

$\mathrm{g} / \mathrm{cm}^{3}$

1.44

Ref. 1 \& 3 


\section{Table 2 (continued)}

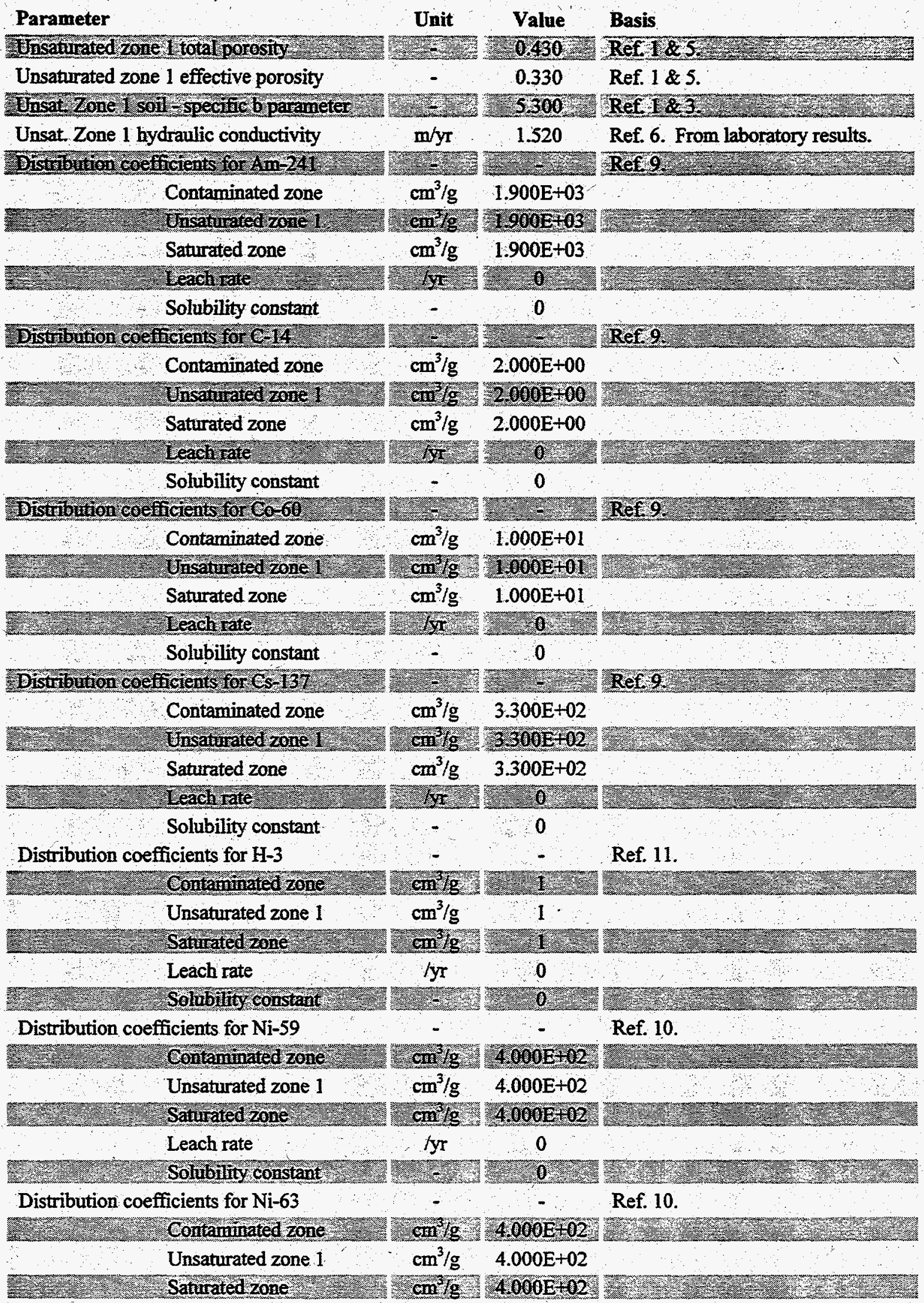


Table 1 (continued)

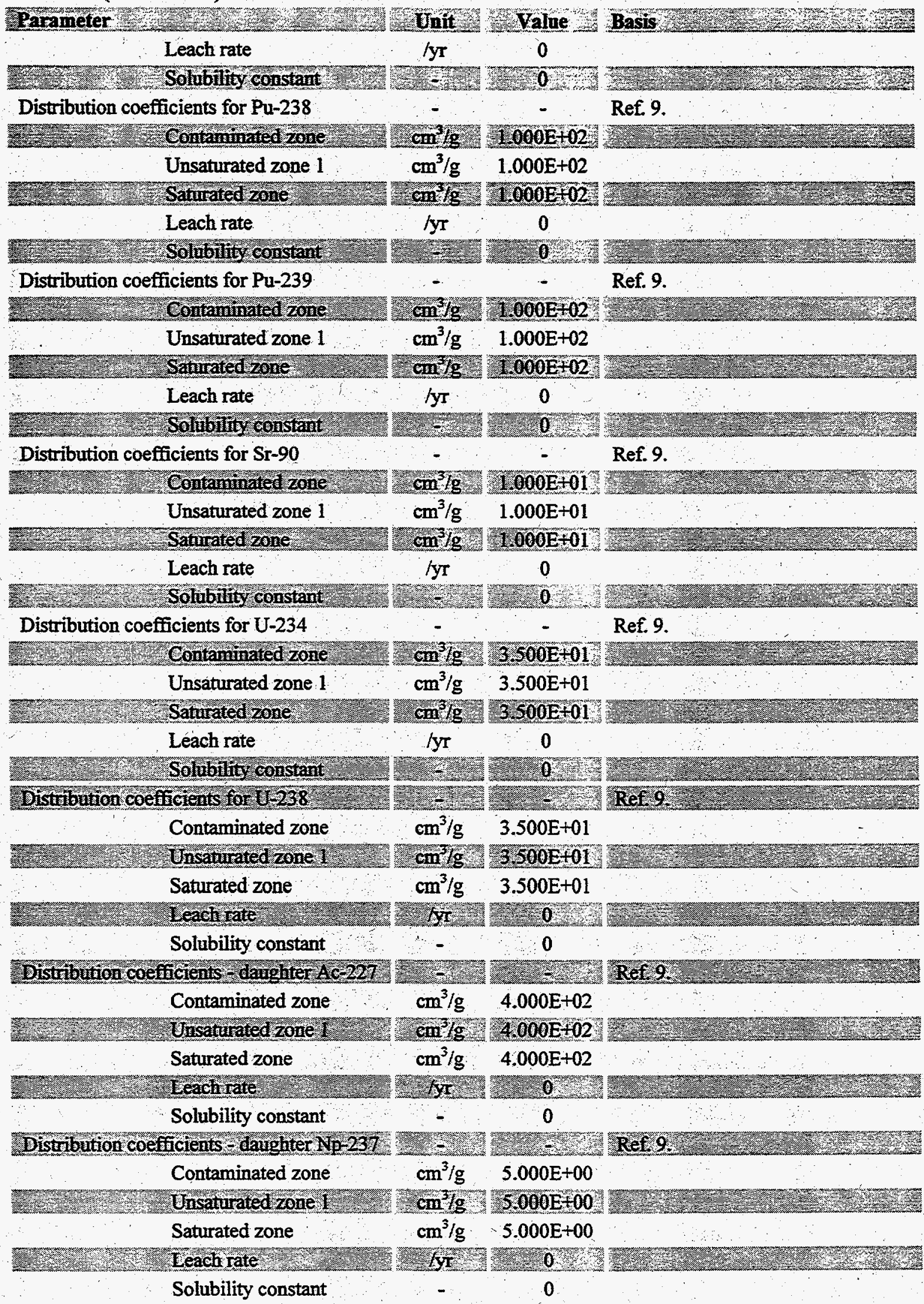


Table 2 (continued)

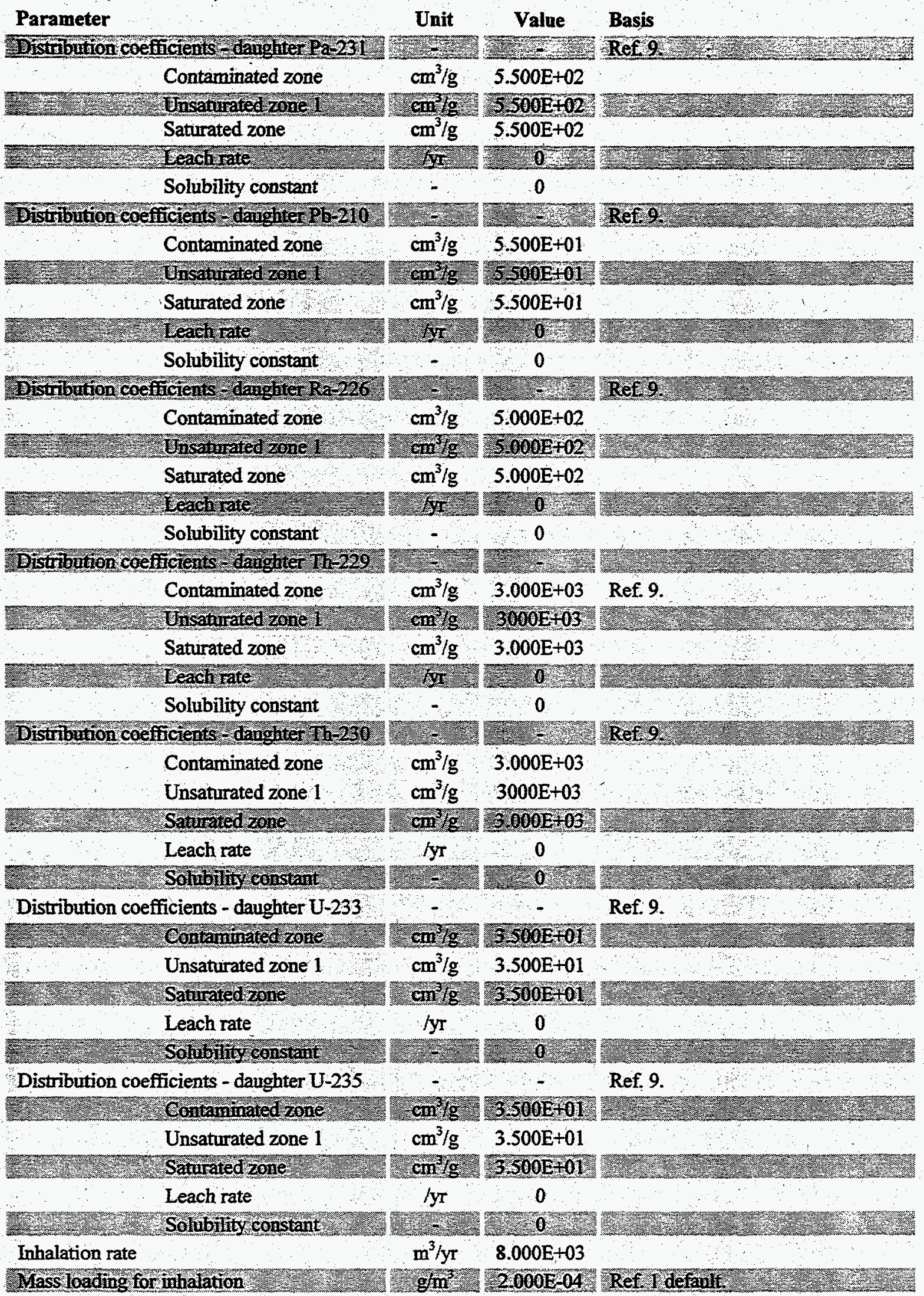


Table 2 (continued)




Table 2 (continued)

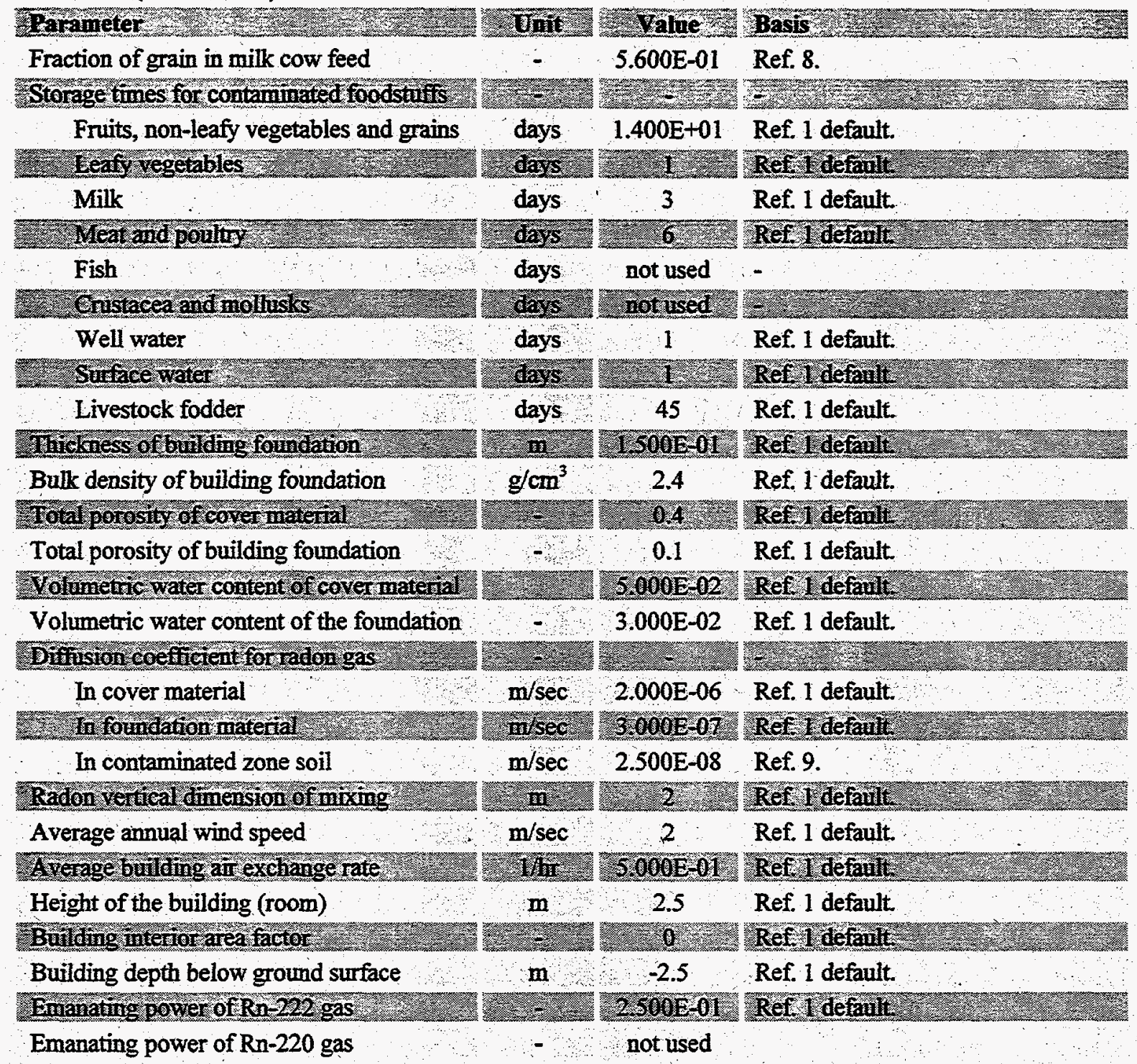

\section{References}

1. Yu, C. et. al., Manual for Implementing Residual Radioactive Material Guidelines Using RESRAD, Version 5.0, Argonne National Laboratory, ANL/EAD/LD-2 (September 1993).

2. Heavy Water Components Test Reactor Facility Characterization Report, U.S. Department of Energy, Savannah River Site, Aiken, SC 29808 (September 1996).

3. Looney, B. B., et. al., Geochemical and Physical Properties of Soils and Shallow Sediments at the Savannah River Site (U), Report WSRC-RP-901031, Westinghouse Savannah River Company, Aiken, SC 29808 (1990). 
4. Rodgers, V. A., Erosion Factor For Waste Site Cap, Report SRT-ESS-94113, Westinghouse Savannah River Company, Aiken, SC 29808 (1994).

V 5. Bledsoe, H. W., SRP Baseline Hydrogeologic Investigation - Phase III, Report DPST-88-627, E. I. du Pont de Nemours \& Co., Savannah River Laboratory, Aiken, SC 29808 (August 1988).

16. Aadland, R. K., et. al., Hydrogeologic Framework of West-Central South Carolina, State of South Carolina Department of Natural Resources, Water Resources Division Report 5, Columbia, SC 29202 (1995).

$\checkmark$ 7. Hamby, D. M. Site-Specific Parameters for the NRC Food pathway Dose Model $(U)$, Report WSRC-MS-91-210, Westinghouse Savannah River Company, Aiken, SC 29808 (1991).

V 8. Hamby, D. M. Guidelines for Acceptable Soil Concentrations in the Old Fand H-Area Retention Basins, Report WSRC-TR-94-0194, Westinghouse Savannah River Company, Aiken, SC 29808 (April 18, 1994).

$\checkmark$ 9. Hse, R. H., Soil Release Guidelines - RESRAD Calculations (U), Report WSRC-RP-94-1342-Westinghouse Savannah River Company, Aiken, SC 29808 (December 19, 1994).

10. Radiological Performance Assessment for the E-Area Vaults Disposal Facility (U), Report WSRC-RP-94-218, Westinghouse Savannah River Company, Aiken, SC 29808 (April 15, 1994).

$\checkmark$ 11. Hse, R. H. and J. R. Cook, Soil Release Guidelines - Expanded RESRAD Study (U), Report WSRC-RP-95-287, Westinghouse Savannah River Company, Savannah River Research Laboratory, Aiken, SC 29808 (March 1, 1995). 


\section{APPENDIX B}

\section{PARAMETERS USED IN ANALYSIS - SCENARIO B}

This appendix identifies in tables the parameters used for the Scenario B calculation. Scenario B entails constructing an administrative building on the property. The building is assumed to have a 4-meter-deep basement. References for this appendix can be found following the tables. Each of the parameters is defined in the RESRAD-BUILD manual (reference 1).

\section{Table 1. RESRAD-BUILD Parameters for Scenario B}

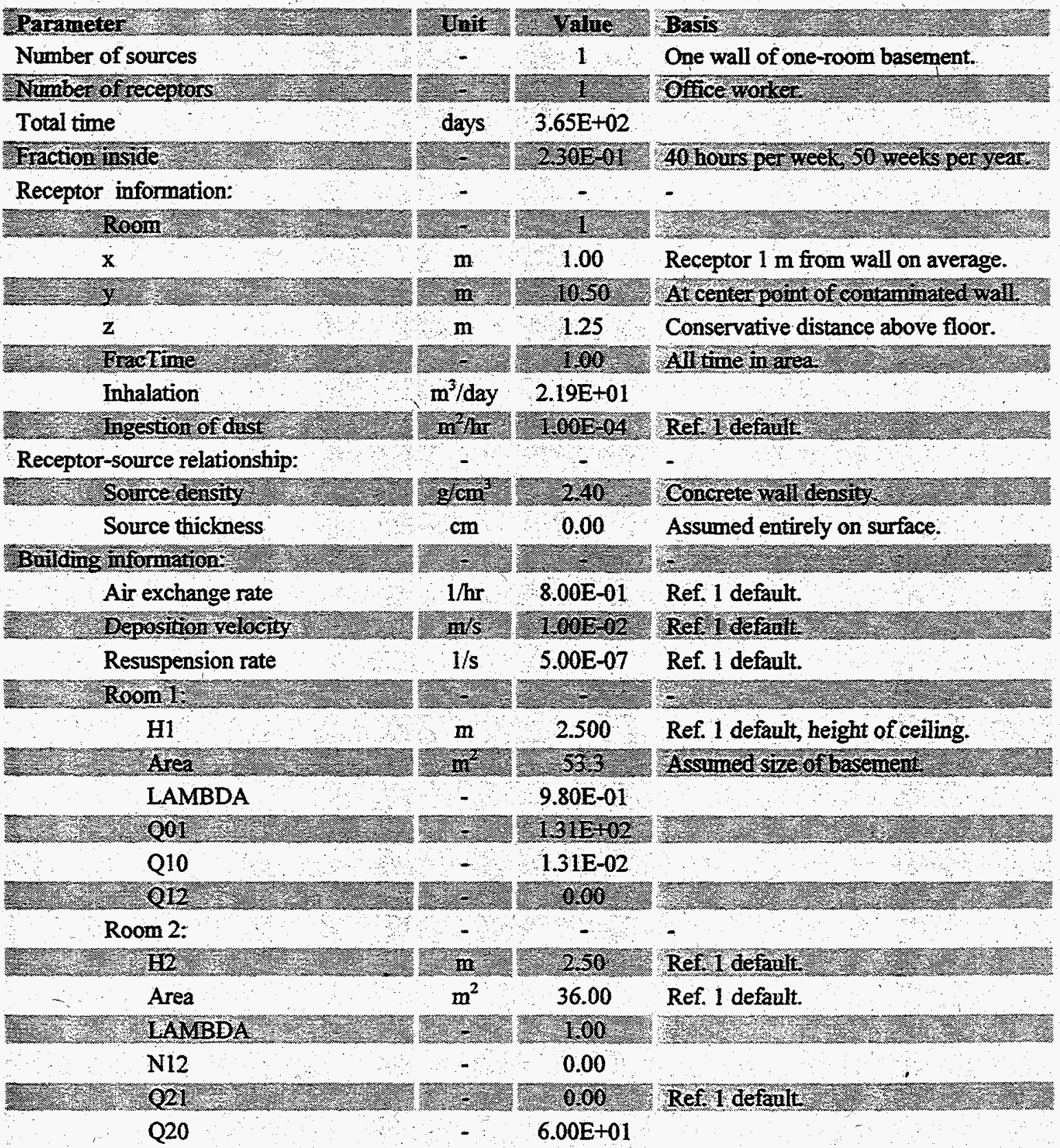




\section{References}

1. Yu, C. et. al., RESRAD-BUILD, A Computer Model for Analyzing the Radiological Doses Resulting from the Remediation and Occupancy of Buildings Contaminated with Radioactive Material, Argonne National Laboratory, ANL/EAD/LD-3 (November 1994).

V. Heavy Water Components Test Reactor Facility Characterization Report, U.S. Department of Energy, Savannah River Site, Aiken, SC 29808 (September 1996). 



\section{APPENDIX C}

\section{PARAMETERS USED IN ANALYSIS - SCENARIO A (WITH ADDITIONAL SOURCE TERM)}

This appendix identifies the parameters used for the Scenario A calculation, with the added assumption that all of the radioactive hardware but the reactor vessel and steam generators would remain in place. Scenario A assumes that a family farm would be built on the HWCTR site, 30 or more years in the future. All RESRAD pathways were considered, except for the ingestion of fish from a pond on the property. The values in this table are similar to those of Table 2 of Appendix A except for the radionuclide concentrations which were increased to account for leaving the radioactive hardware in place, and Ba-133, Eu-152 and Eu-154 added because the biological shield activity was included in the calculation.

References for this appendix can be found following the tables. Each of the parameters is defined in the RESRAD manual (reference 1).

\section{Table 1. RESRAD Parameters With Radioactive Hardware in Place}

\begin{tabular}{|c|c|c|c|}
\hline Cis: & 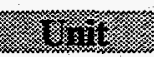 & & sastas \\
\hline Contaminated zone & 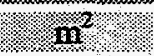 & & Area of contaminated region. \\
\hline 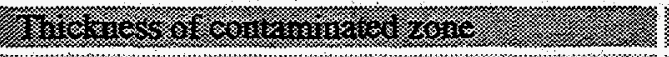 & aix & & 20 \\
\hline & in & 21 & $x \rightarrow$ \\
\hline 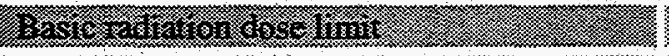 & $\operatorname{res} 20$ & 20 & 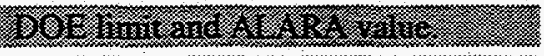 \\
\hline Emes for calculations & yr & & \\
\hline a & -1 & & Rerenomitide \\
\hline 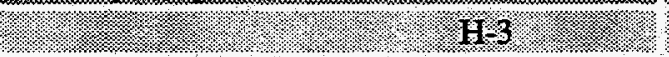 & pelg. & $2.260 \mathrm{E}+00$ & the 190 \\
\hline m & (2) & & $\operatorname{set} x=2 x$ \\
\hline (18.7: & pelg & $2040 \mathrm{E}-01$ & concretest \\
\hline 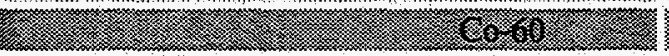 & rels & 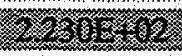 & 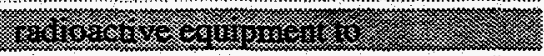 \\
\hline Ni-63 & tevg. & $1.640 \mathrm{E}+01$ & acriviny \\
\hline (2) & EC. & 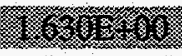 & 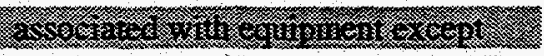 \\
\hline 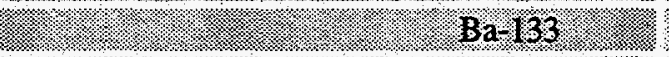 & PCirg. & & The reactor vessel and \\
\hline 10 & (2) & 610 & 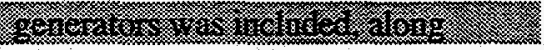 \\
\hline च च & pelg & (1) & gical \\
\hline ry & (2) & 12701 & 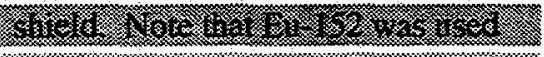 \\
\hline " & pCig & 8.2 & tise \\
\hline$\sqrt{20}$ & mell: & 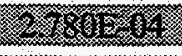 & Tatculaker \\
\hline 1. & pêf: & 272 & \\
\hline$\sqrt{20}$ & refe & 3.164: & \\
\hline 110.0 Pu- 239 & ievg & & (2) \\
\hline s. & 1. & & \\
\hline & & & 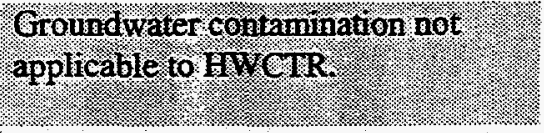 \\
\hline
\end{tabular}


Table 1 (continued)

\begin{tabular}{|c|c|c|c|}
\hline 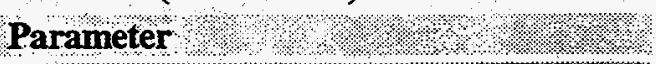 & Oumt & & \\
\hline Covereapth & inten & 18 & Ementing \\
\hline Density of cover material & $\mathrm{g} / \mathrm{cm}^{3}$ & 1.44 & Based on soll borings, ref $1 \& 3$. \\
\hline 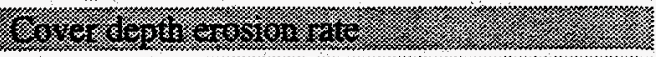 & 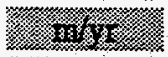 & Wren & (x) \\
\hline Conkaminated zone densily & $\mathrm{g} / \mathrm{cm}^{3}$ & 2400 & Ref 1 , densi \\
\hline 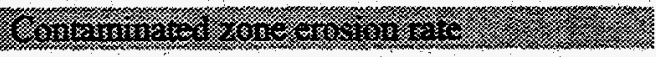 & notr & Oa) & 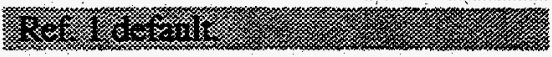 \\
\hline tatated zone total:p & & 0.43 & Ref. $1.8,5$, data fir \\
\hline 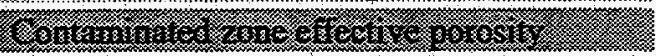 & 2 & 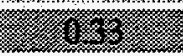 & 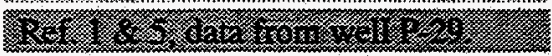 \\
\hline dated zone bydre & $\mathrm{m} / \mathrm{y}$ & 52 & Ref 6 from laboratory \\
\hline 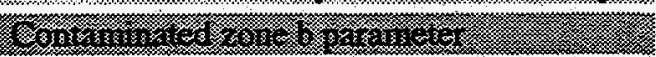 & & 36 & 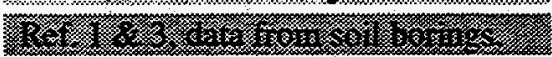 \\
\hline Humbily in air & $9 / m^{3}$ & 11 & Ref 7 \\
\hline 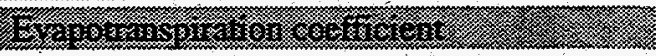 & 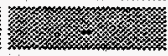 & (1961) & Rer. $16.8=0$ \\
\hline Ion & $\mathrm{m} / \mathrm{yz}$ & 1,200 & Ref 6 . \\
\hline 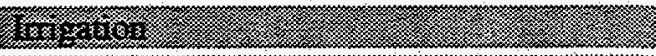 & 2 & ]. & ref. \\
\hline A node & 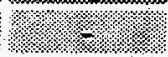 & orertiead: & Ref l defanlt \\
\hline Wherinan & 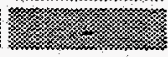 & 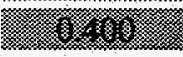 & Rer: I \\
\hline da area for nearby stre & $\mathbf{m}^{2}$ & 10001.66 & Ref 1 Lefault: \\
\hline 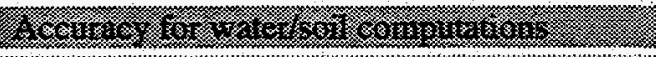 & & WOSOE & 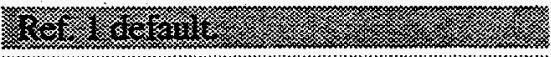 \\
\hline of sakurated zone. & g/cm³ & 1440 & Ref 1 \& 5 . Data from Well P 29 . \\
\hline 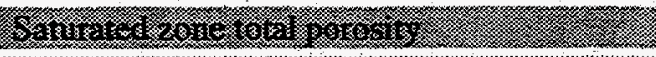 & & (1) & 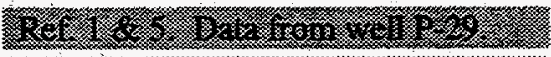 \\
\hline ad zone effective porosity & 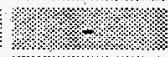 & 0330 & Ref 1 \& 5 Data from urell P 29 \\
\hline Congainervity. & 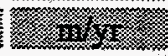 & 3.46 & Vir \\
\hline zone hy draulic gradient & 2 & $3.500 \mathrm{E}-03$ & Ref 6 . \\
\hline d rond briananeter? & 2 & 35 & i \\
\hline Water table arop rate & mi & 0 & $\begin{array}{l}\text { Wore conservative thaia ref } 1 \\
\text { default of } 1000 \mathrm{E}-03\end{array}$ \\
\hline 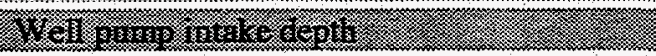 & . & 6.108 & 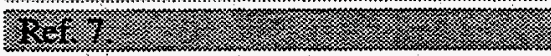 \\
\hline Ecion or mass balance & & $=$ & Ref. 1. \\
\hline (m) & $\sqrt{13} 32$ & 25 & 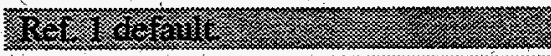 \\
\hline Of unsalurated zone stata & 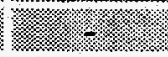 & 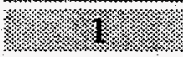 & Ref I defanll: \\
\hline 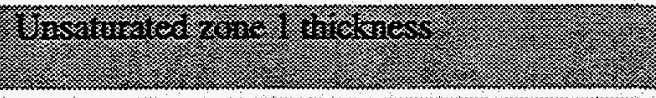 & 裙 & & 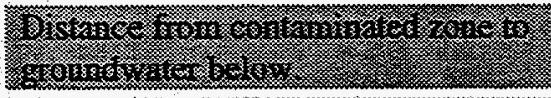 \\
\hline arated zone I soil density & $\mathrm{g} / \mathrm{cm}^{3}$ & 1.44 & Ref. 1 \&.3: \\
\hline 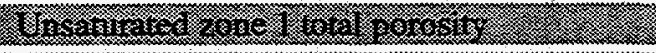 & 20 & 68 & W \\
\hline ated zone 1 effective porosity & & 0.330 & Ref 1 \& 5 \\
\hline 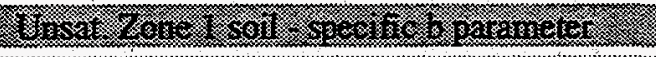 & 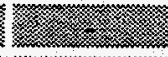 & - 300 & rer \\
\hline Unsat Zone I hydraulic conductivity. & $\mathrm{m} / \mathrm{m}$ & 1528 & Ref 6. From laboratowy resulls: \\
\hline 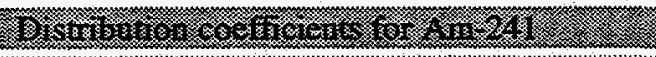 & 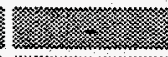 & 2 & Thes: \\
\hline Contaminated zone & $\mathrm{cm}^{3} \mathrm{~g}$ & $1000 \mathrm{E}+03$ & 4 \\
\hline 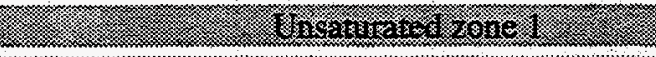 & $\cos 3: 28$ & 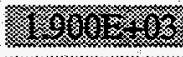 & $x_{2 x}$ \\
\hline Saturated zone & $\mathrm{cm} / \mathrm{g}$ & $1900 \mathrm{E}+03$ & 2 \\
\hline 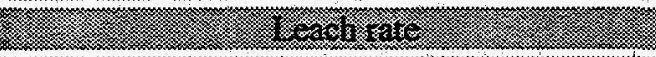 & $\sqrt{2} 2$ & $\sqrt{20}$ & 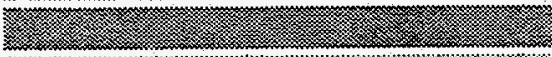 \\
\hline Solubility constant & 3.1 .8 & & : \\
\hline & & & \\
\hline
\end{tabular}


Table 1 (continued)

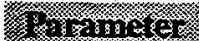

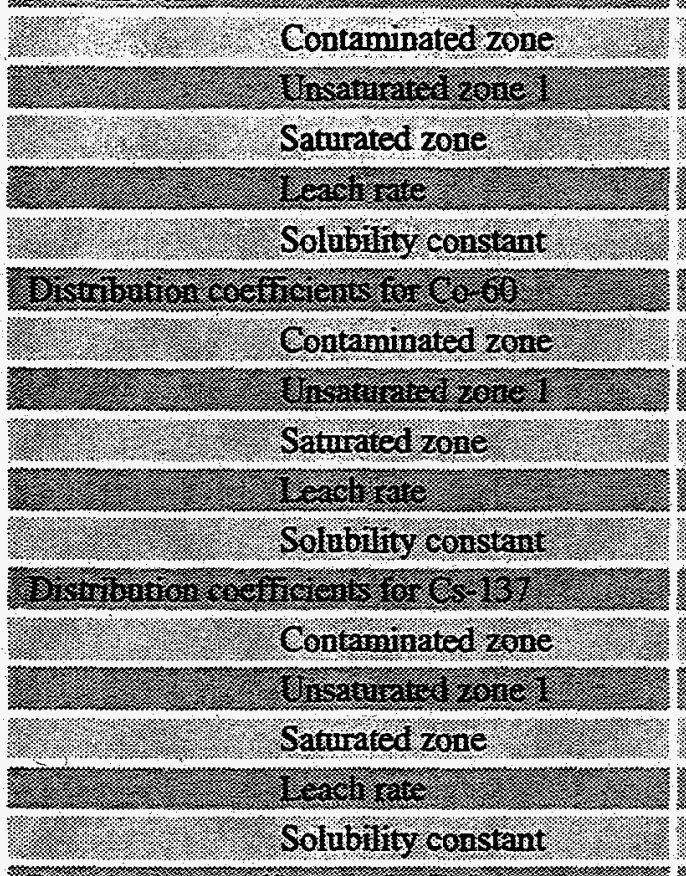

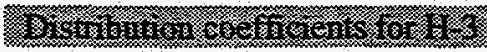

Contaminated zone

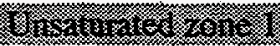

Saturated zone:

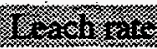

Solubility Constar:

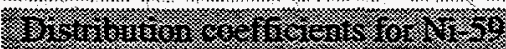

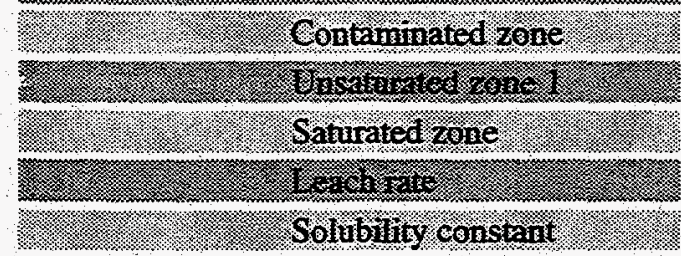

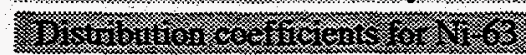

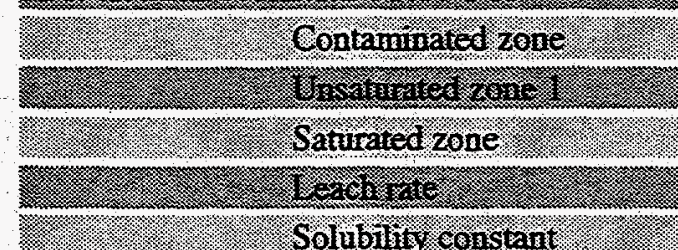

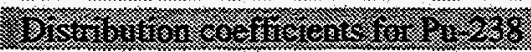

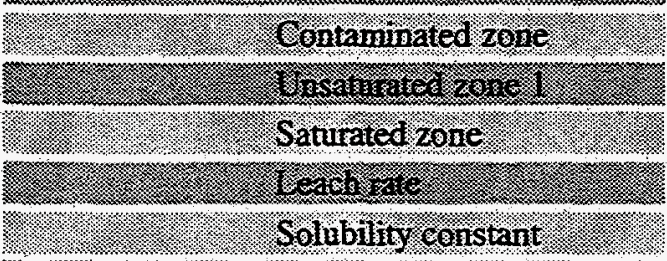

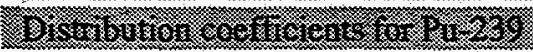

Contaminated zone

\begin{tabular}{|c|c|c|}
\hline Sint & Yhation & 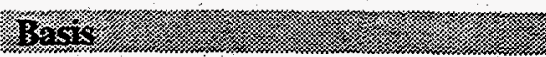 \\
\hline $\mathrm{cm}^{3} / \mathrm{g}$ & $2.000 \mathrm{E}+00$ & \\
\hline (3) & 2 20018-96 & 2 \\
\hline $\mathrm{cm} / \mathrm{g}$ & $2.000 \mathrm{E}+00$ & 14\% \\
\hline 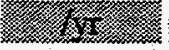 & 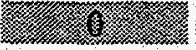 & (2) \\
\hline-2 & (3) 9 & 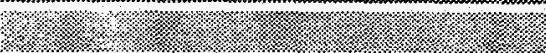 \\
\hline s & & 3) \\
\hline cmils: & $1.000 \mathrm{E}+01$ & 2 \\
\hline $\sin$ & 2. & $x^{2}+x_{x}$ \\
\hline $\mathrm{cm} / \mathrm{g}$ & $1.000 \mathrm{E}+01$ & 1:1: \\
\hline 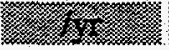 & (2) & 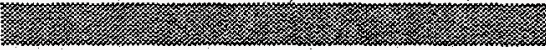 \\
\hline (3) & 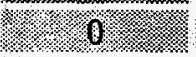 & 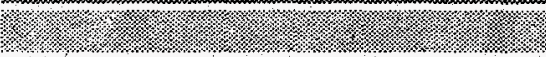 \\
\hline 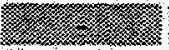 & (n) & 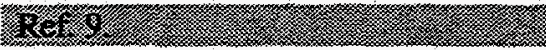 \\
\hline $\mathrm{cm} / \mathrm{g}$ & $3300 \mathrm{E}+02$ & 1.:. \\
\hline sting & 3. & s. \\
\hline $\mathrm{cm}^{3} \mathrm{~g}$ & $3,300 \mathrm{E}+02$ & . \\
\hline (2) & 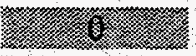 & (2) \\
\hline 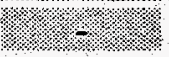 & 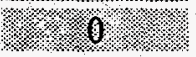 & 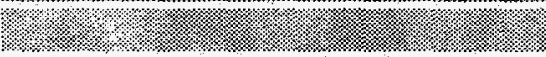 \\
\hline 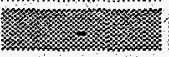 & (2) & R(I. III \\
\hline $\mathrm{cm}^{2} / \mathrm{g}$ & $1=1=$ & 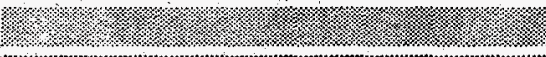 \\
\hline sents: & $1=1$ & 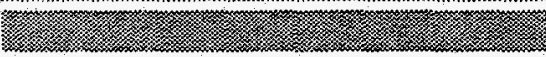 \\
\hline $\mathrm{cm}^{3} / \mathrm{g}$ & 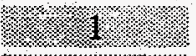 & 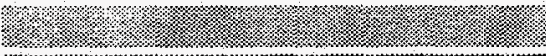 \\
\hline 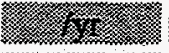 & 28 & !) \\
\hline & 0.0 & 10.7 \\
\hline 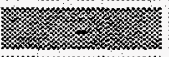 & 2 & 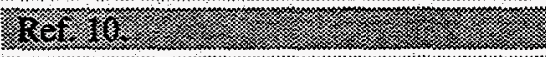 \\
\hline $\mathrm{cm}^{3} / \mathrm{g}$ & $4000 \mathrm{E}+02$ & 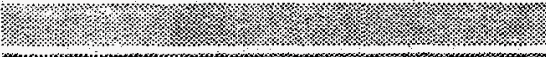 \\
\hline aristis: & 200009102 & st \\
\hline cino & $4.000 \mathrm{E}+02$ & 8 \\
\hline WXI & 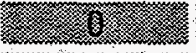 & 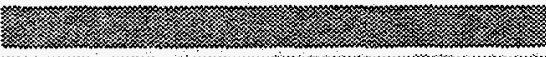 \\
\hline 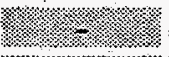 & $1=0$ & 197. \\
\hline & 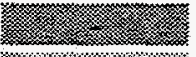 & $18=10$ \\
\hline $\mathrm{cm} / \mathrm{g}$ & $4.000 \mathrm{E} * 02$ & 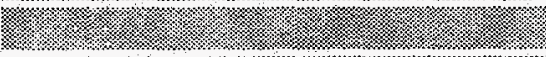 \\
\hline s.18) & 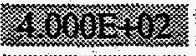 & (2) \\
\hline $\mathrm{cm}^{3} / \mathrm{g}$ & $4000 \mathrm{E}+02$ & 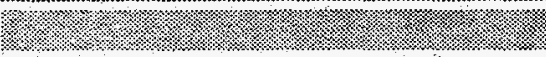 \\
\hline rax & 20 & 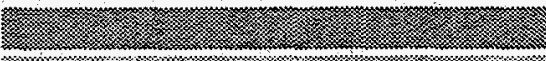 \\
\hline$\sqrt{2}$ & (12. 0 & 4 \\
\hline 2 & 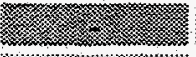 & leEg 9 \\
\hline $\mathrm{cm}^{3} / \mathrm{g}$ & $1.000 \mathrm{E}+12$ & 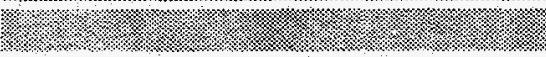 \\
\hline 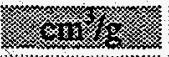 & $20064=62$ & $y_{3}$ \\
\hline cming. & $1.0000 \mathrm{E}+02$ & 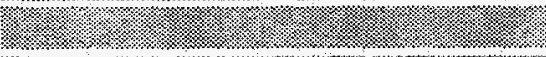 \\
\hline 18 & 2 & 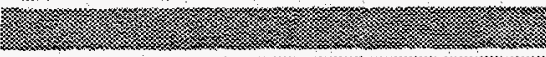 \\
\hline$=$ & 0 & 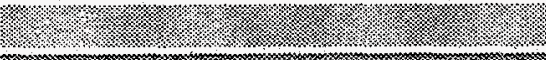 \\
\hline 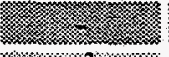 & 20 & Re 9 \\
\hline & & \\
\hline
\end{tabular}


Table 1 (continued)

Parameter

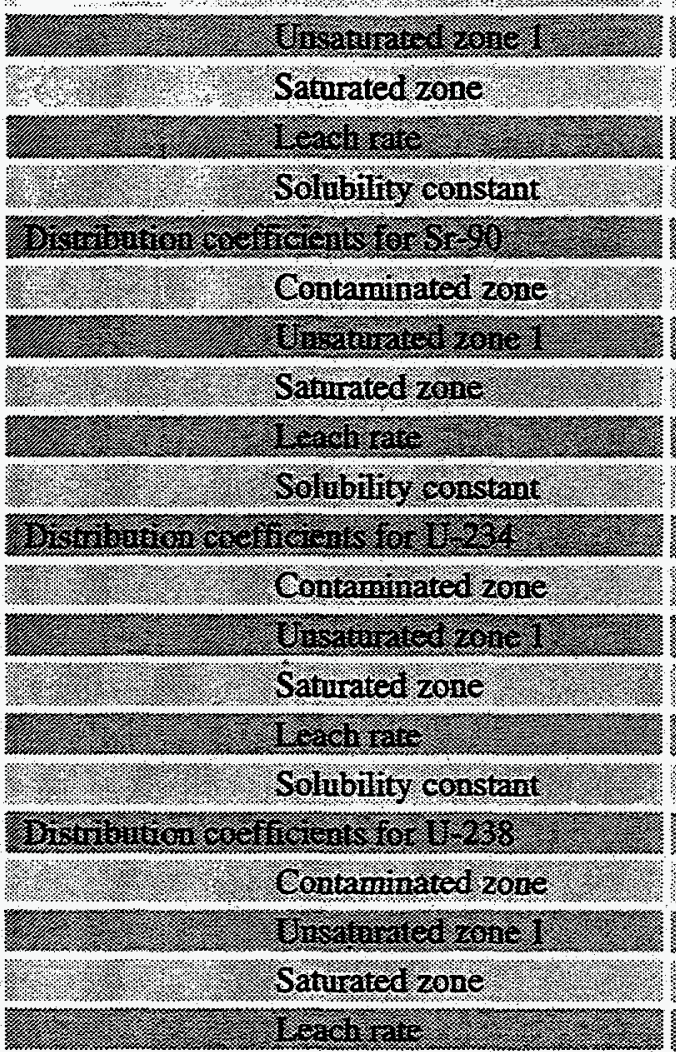

Solubility constant.

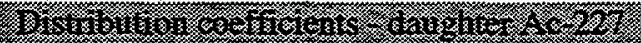

\begin{tabular}{|c|c|}
\hline & in \\
\hline 2 & 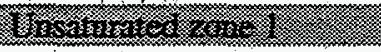 \\
\hline (2) & Sat \\
\hline 2 & Lerch rate \\
\hline & Solub \\
\hline
\end{tabular}

Distribution coefficterts d dughiter Me:23.

Contaminated zane

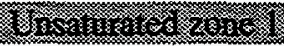

Saturated zone

Tezhi mate

Solubility constant

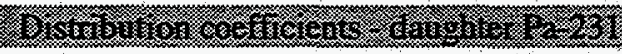

Contaminated zone

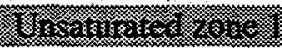

Saburated zone

Leati arte

Solvbility constant

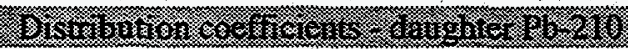

Contamirated zoge

Unsanratef zoneI
Unit

chils: 1000E+12

$\mathrm{cm} / \mathrm{g} / 1.000 \mathrm{E}+02$

s.t.

1.

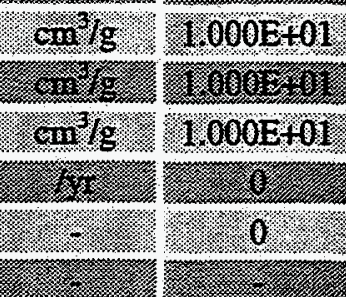

$\mathrm{cm}^{3} / \mathrm{g}: / 3500 \mathrm{E}+01$

$2 \mathrm{~cm}^{3} \mathrm{~m} / \mathrm{3} / 3500 \mathrm{~s}+61$

$\mathrm{cm}^{3} / \mathrm{g} / 3.500 \mathrm{E}+01$

$6 \mathrm{x}=0$

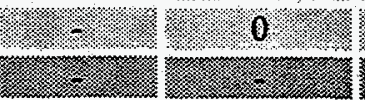

$\mathrm{cm}^{3} / \mathrm{g} / 3.500 \mathrm{E}+01$

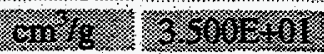

$\mathrm{cm}^{3} / \mathrm{g}: 3.500 \mathrm{E}+01$

TI. 0

2.0

$\mathrm{cm}^{3} / \mathrm{g}, 4.000 \mathrm{E}+02$

$\mathrm{cm}^{\mathrm{m}} \mathrm{gg} / 4.000 \mathrm{E}+\mathrm{62}$

$\mathrm{cm}^{3} / \mathrm{g}: 4.000 \mathrm{E}+02$

19.

$12-0.0$

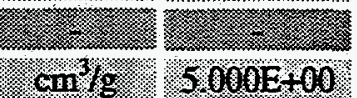

13et:2.

cantg $15000 \mathrm{E}+60$

$\mathrm{cm}^{3} / \mathrm{g} / 5000 \mathrm{E}+00$

7.

0

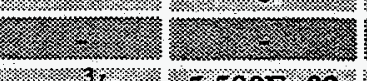

$\mathrm{cm}^{3} / \mathrm{g} / 5500 \mathrm{E}+02$

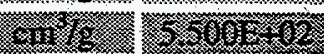

$\mathrm{cm}^{3} / \mathrm{g} / 5.500 \mathrm{E}+02$

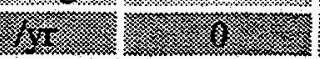

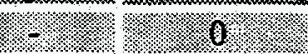

$\mathrm{cm}^{3} / \mathrm{g} / \frac{2500 \mathrm{E}+01}{2.10}$

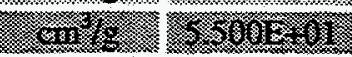

Basis

\section{Ter 2}

\section{रे: $=9$} Ref 8 .
.

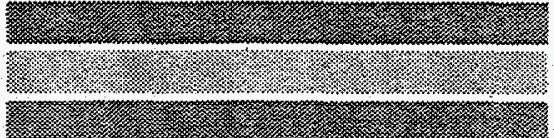

Tet:9:

\section{स w}


Table 1 (continued)

\begin{tabular}{|c|c|c|c|}
\hline & Unit & & \\
\hline 2. Saturated zone & $\mathrm{cm}^{3} / \mathrm{s}$ & $5.500 \mathrm{E}+01$ & 0.2 \\
\hline Tereli ate & bix: & 0 & x \\
\hline fol sol & $=2$ & 1.0 & 묘. \\
\hline 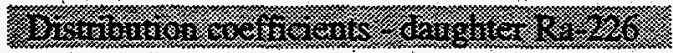 & 2 & & (ncerts. \\
\hline Cont & $\mathrm{cm}^{3} / \mathrm{g}$ & $5.000 E+02$ & 10 \\
\hline 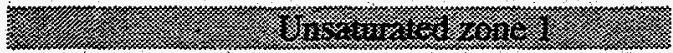 & 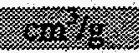 & $6062+02$ & $s^{2}$ \\
\hline San & $\mathrm{cm}^{3} \mathrm{ig}$ & $5.0000+02$ & 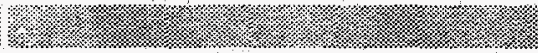 \\
\hline 2 & 2.: & a) & 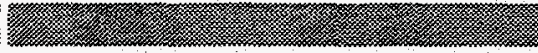 \\
\hline SoI & $=$ & 0 & \\
\hline 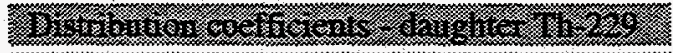 & $=0$ & & 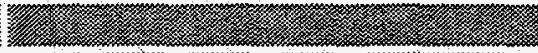 \\
\hline 2.20 & $\mathrm{~cm} / \mathrm{s}$ & $3.000 \mathrm{E}+03$ & Ref 9 \\
\hline 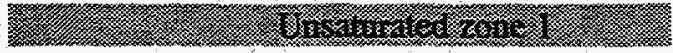 & cinis. & 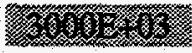 & 2 \\
\hline Sat & $\mathrm{cm}^{3} / \mathrm{g}$ & 3.0001E+03 & 2 \\
\hline $2=2.1 .2 .2$ & (121: & 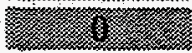 & 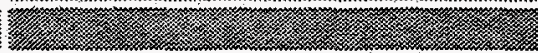 \\
\hline Sol & 0. & 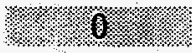 & $\sqrt{2}-\sqrt{2}-2$ \\
\hline 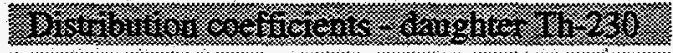 & 2 & & Ifer: 9 \\
\hline 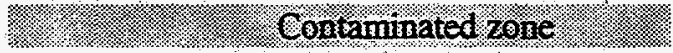 & $\mathrm{cm}^{3} \mathrm{fg}$ & $3.000 \mathrm{E}+03$ & 2. \\
\hline 20.12 & 2 & 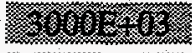 & \\
\hline 2.0 San & $\mathrm{en}^{3} / \mathrm{g}$ & $3000 E+03$ & $10.2=$ \\
\hline 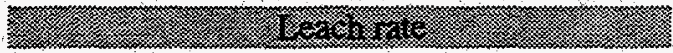 & 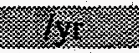 & $=21$ & 2 \\
\hline 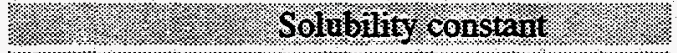 & -2 & 0 & 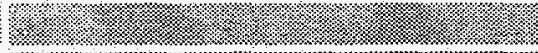 \\
\hline encoefitic & 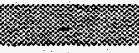 & 2 & 20 \\
\hline$=\mathrm{Co}_{0}$ & $\mathrm{~cm}^{3} / \mathrm{g}$ & 401 . & $\sqrt{10}=1$ \\
\hline 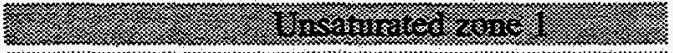 & $\mathrm{cm}$ ts: & 850 & 12 \\
\hline $2.2 .5 a t$ & $\mathrm{~cm}^{3} / \mathrm{g}$ & 30.50 & 10 \\
\hline E & 25 & d. & $=0$ \\
\hline$=\mathrm{S}$ & 10 & 0 & 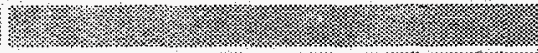 \\
\hline ismbulion coefficic & $2=$ & $=2$ & Ref.9. \\
\hline$C_{e}$ & $\mathrm{~cm}^{3} / \mathrm{g}$ & $3500 E+01$ & $\sqrt{2}$ \\
\hline 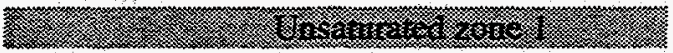 & $\operatorname{con} / 8$ & 3550 & 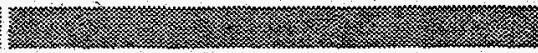 \\
\hline 2IIIn & $\mathrm{cm}^{3} \mathrm{~g}$ & 3.500 & 20 \\
\hline 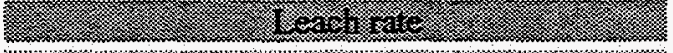 & 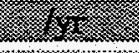 & -2 & 2 \\
\hline 20 & 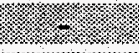 & e. & 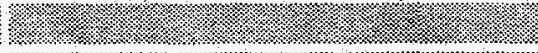 \\
\hline 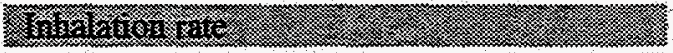 & $\mathrm{m}$ & 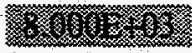 & 20 \\
\hline & $g / m^{3}$ & 20 & t. \\
\hline Minhatoo & in & & it: \\
\hline 20 & 2 & 30 & L: \\
\hline$x$ & & 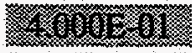 & Rer \\
\hline 28 & $=2$ & 7.00 & iti : \\
\hline & & 20 & Ref: \\
\hline 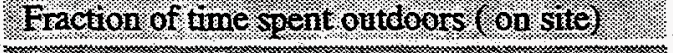 & $=2$ & $2500 \mathrm{E}$ & 2 \\
\hline & & & \\
\hline & & & \\
\hline
\end{tabular}




\section{Table 1 (continued)}

\section{Parameter}

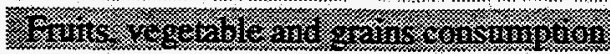

Leafy vegetable consumption

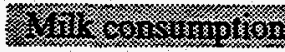

Meat and poultry consumption

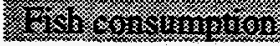

Other seafood consumption

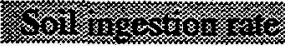

Drinking water intake

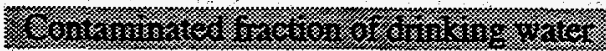

Contaminated fraction of household water

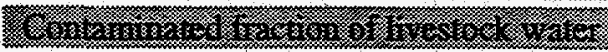

Contarimated fraction of imgation water

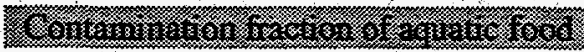

Contaminated fraction of plant food

Comb.r.

Contanisated fraction of milk

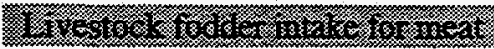

Livestodk fodder bake for milk

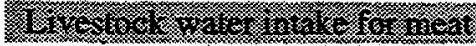

Livestodk water intake for milk

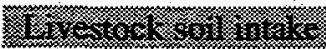

Mass loading for follar deposition

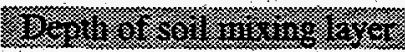

Depti of roots:

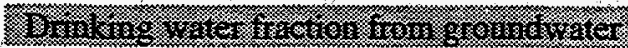

Housebold water fraction of ground water

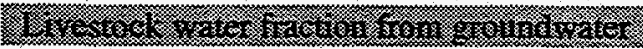

Irrigation fraction from groundwater

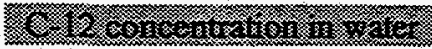

C 12 concentration in contaminated soil

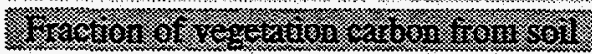

Fraction of vegetation carbon from ait

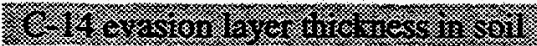

C- 14 evasion flux rate from sol

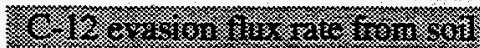

Fraction of grain in beef cattle feed

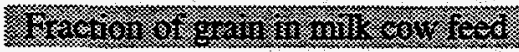

Storage times for contaminated foodstuffs

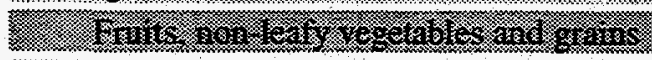

Leafy regetables

14:

Meat and poultry

Tris:

\begin{tabular}{|c|c|c|}
\hline Enit & & 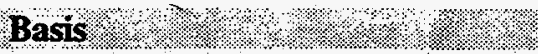 \\
\hline I. & 690 & 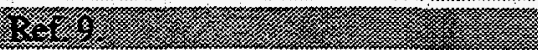 \\
\hline $\mathrm{kg} / \mathrm{yr}$ & 11. & ef. 9 . \\
\hline 18 & 管 & res \\
\hline $\log / \mathrm{m}$ & 330 & Ref: 8 \\
\hline 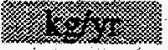 & 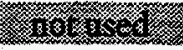 & $x_{2}$ \\
\hline $\operatorname{lgg} / x$ & Bot used & \\
\hline ex & 6515 & 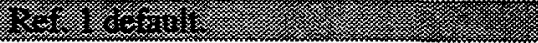 \\
\hline L/y & 300 & Ref. I \\
\hline$\sqrt{x+2}$ & 2 & 2) \\
\hline 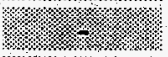 & $1=1$ & Ref I defanil \\
\hline 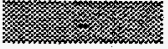 & (2) & 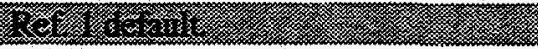 \\
\hline - & 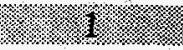 & Ref I a franl \\
\hline & $108=8$ & (s) \\
\hline & 5.0001001 & Ref 9 \\
\hline & 80 & 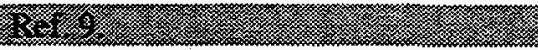 \\
\hline & $50000-01$ & Ret 9 \\
\hline 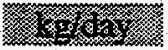 & 1.1006 & 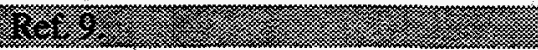 \\
\hline ggday. & $4400 E+01$ & Ref.9. \\
\hline (magy. & 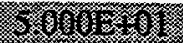 & Rer I A \\
\hline Way & $1600 \mathrm{E}+02$ & Ref I default (-2) \\
\hline (x) & 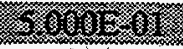 & 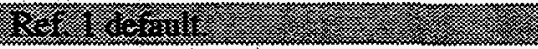 \\
\hline$g m^{3}$ & 1100 & Ref \\
\hline$\sqrt{\mathrm{an}}$ & 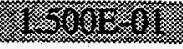 & 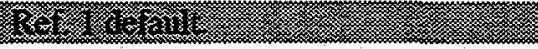 \\
\hline $\mathrm{an}$ & $9000 \mathrm{E}-01$ & Ref i d default: \\
\hline & (2) & 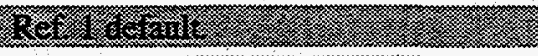 \\
\hline & 1 & Ref I defanl: \\
\hline & the & 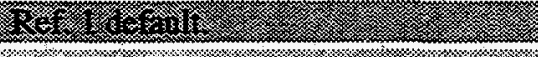 \\
\hline 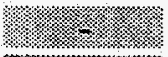 & 14 & Ref I default \\
\hline 8 (6)=12 & 200109 & 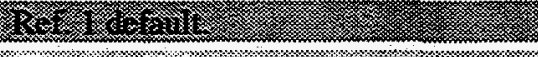 \\
\hline$g / g$ & $3000 \mathrm{E} 92$ & 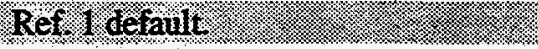 \\
\hline 2 & 2.200061869 & 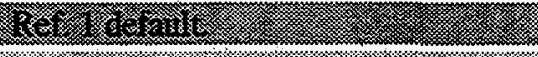 \\
\hline 2 & $9800 \mathrm{E}$ OI & Ref I default \\
\hline tis: & 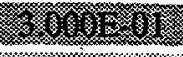 & 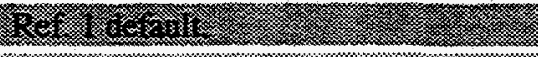 \\
\hline Usec & 700 & Ref: \\
\hline 1meres & I. & r \\
\hline .8 & 7500 & $\operatorname{Ref} 8$ \\
\hline 党 & 3.6091691 & R R \\
\hline & (2.) & 4 \\
\hline Sh & 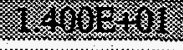 & Ret f defarilis \\
\hline days: & 1.1. & Ref I default: \\
\hline Gas: & 3 & 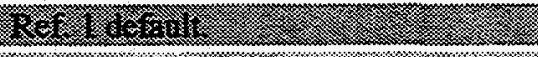 \\
\hline days & (:) & Ref I default: \\
\hline & & \\
\hline
\end{tabular}


Table 1 (continued)

\begin{tabular}{|c|c|c|c|}
\hline Parameter & Une & Valies: & Bastris \\
\hline Crustacea and mollusks & days & not used & 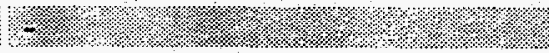 \\
\hline mel rater: & 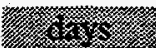 & 1 & Ret I defanter. \\
\hline Surface water & days & 1. & Ref 1 default \\
\hline 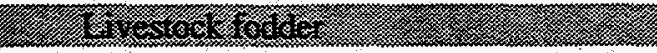 & (d) & 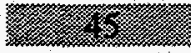 & 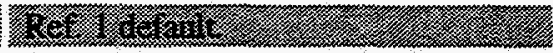 \\
\hline Thickness of building Toundation & III & 1 S00E-01 & Ref 1 default: \\
\hline 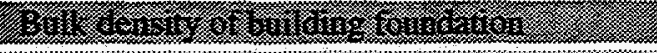 & genit & 224 & Ref I I G taril \\
\hline Total poresity of cover material & $2=$ & 0.04 & Ref 1 deffalt: \\
\hline 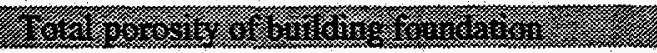 & 2 & $1=1.10$ & 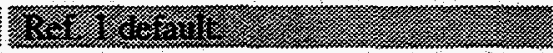 \\
\hline Volumeric water content of cover naterial & & $5.000 \mathrm{E}-02$ & Ref I defanil: \\
\hline 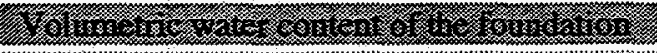 & 2 & 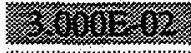 & 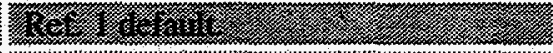 \\
\hline Diffision coefficient for radon gas & 2.2 & $2-2-2$ & $14-2.020$ \\
\hline 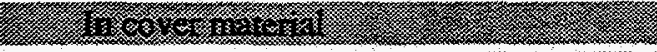 & mbet & $20001=18$ & 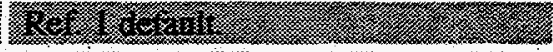 \\
\hline In fourdation material & insec & $3000 \mathrm{E}-07$ & Ref 1 default \\
\hline 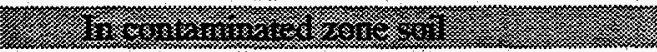 & nseec & 2.500 E 08 & Ref. 2) \\
\hline Radon verical dimension of minking & $m$ & 2 & Ref. 1 default \\
\hline 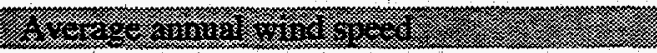 & finser & 2 & 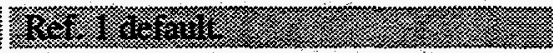 \\
\hline Average bullaing air exchange rate & This & $5.000 \mathrm{E}-01$ & Ref 1 defanil: \\
\hline 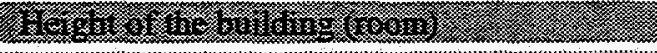 & 211 & $2=23$ & IRef I deramil \\
\hline Burlding interior area factor & 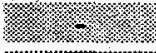 & $=0$ & Ref 1 defaur: \\
\hline 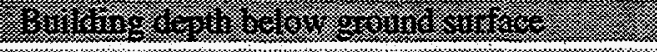 & (3) & -25 & Ref.1 defarli: \\
\hline Enranating power of Rn-2222 gas & $=$ & $2.500 \mathrm{E}-01$ & Ref. I default \\
\hline FofR & & & \\
\hline
\end{tabular}

\section{References}

1. Yu, C. et. al., Manual for Implementing Residual Radioactive Material Guidelines Using RESRAD, Version 5.0, Argonne National Laboratory, ANL/EAD/LD-2 (September 1993).

2. Heavy Water Components Test Reactor Facility Characterization Report, U.S. Department of Energy, Savannah River Site, Aiken, SC 29808 (September 1996).

V3. Looney, B. B., et. al., Geochemical and Physical Properties of Soils and Shallow Sediments at the Savannah River Site (U), Report WSRC-RP-901031, Westinghouse Savannah River Company, Aiken, SC 29808 (1990).

/4. Rodgers, V. A., Erosion Factor For Waste Site Cap, Report SRT-ESS-94113, Westinghouse Savannah River Company, Aiken, SC 29808 (1994).

V5. Bledsoe, H. W., SRP Baseline Hydrogeologic Investigation - Phase III, Report DPST-88-627, E. I. du Pont de Nemours \& Co., Savannah River Laboratory, Aiken, SC 29808 (August 1988). 
V6. Aadland, R. K., et. al., Hydrogeologic Framework of West-Central South Carolina, State of South Carolina Department of Natural Resources, Water Resources Division Report 5, Columbia, SC 29202 (1995).

7. Hamby, D. M. Site-Specific Parameters for the NRC Food pathway Dose Model $(U)$, Report WSRC-MS-91-210, Westinghouse Savannah River Company, Aiken, SC 29808 (1991).

8. Hamby, D. M. Guidelines for Acceptable Soil Concentrations in the Old Fand H-Area Retention Basins, Report WSRC-TR-94-0194, Westinghouse Savannah River Company, Aiken, SC 29808 (April 18, 1994).

9. Hse, R. H., Soil Release Guidelines - RESRAD Calculations (U), Report WSRC-RP-94-134? Westinghouse Savannah River Company, Aiken, SC 29808 (December 19, 1994).

10. Radiological Performance Assessment for the E-Area Vaults Disposal Facility (U), Report WSRC-RP-94-218, Westinghouse Savannah River Company, Aiken, SC 29808 (April 15, 1994).

11. Hse, R. H. and J. R. Cook, Soil Release Guidelines - Expanded RESRAD Study $(U)$, Report WSRC-RP-95-287, Westinghouse Savannah River Company, Savannah River Research Laboratory, Aiken, SC 29808 (March 1, 1995). 
RESIDUAL RADIOACTIVITY GUIDELINES FOR THE HWCTR

\section{Appendix D}

RESRAD Summary Report Biological Shield 

RESRAD, Vergion $5.621 T^{*}$ Limit $=0.5$ year Suntary : Bio-Shield Activity Assessment HWCTR
$03 / 06 / 9712: 11$ Page 1 File: A: IBIOSHDD.DAT

Tabie of concents aasasakasasasasas

Part I: Mixcure Sums and single Radionuclide Guidelines

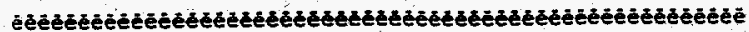

Dose Conversion Factor (and Reiated) Paramecer Sumary ... 2

Sice-Specific paramecer sumary $\ldots \ldots \ldots \ldots \ldots \ldots \ldots \ldots \ldots 7$

Sumnary of Pachway selections $\ldots \ldots \ldots \ldots \ldots \ldots \ldots \ldots \ldots ; 14$

Contaminaced zone and Total Dose Summary $\ldots \ldots \ldots \ldots \ldots \ldots, 15$

Total Dose Components

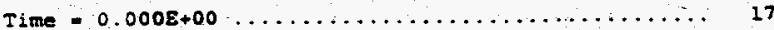

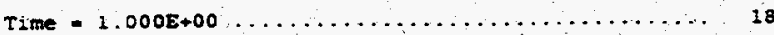

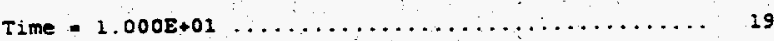

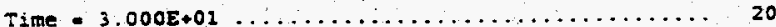

Time $=5.000 E+01 \ldots \ldots \ldots \ldots \ldots \ldots \ldots \ldots \ldots \ldots \ldots, 21$

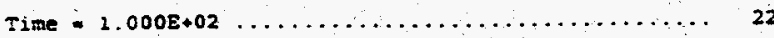

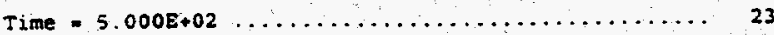

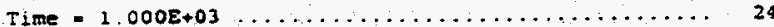

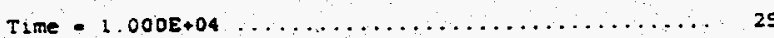

Dose/Source Racros Summed Over All Pachways .......... 26

Single Radionuclide Soil Guidelines ................ 27

sose per Nuclede Surmed over All Pachways . . . . . . . . . 29

Sol 1 Conceneracion Per Nuclide $\ldots \ldots \ldots \ldots \ldots \ldots \ldots \ldots \ldots \ldots$ 
RESRAD. Version 5.621 T" Limit $=0.5$ year Sumary : Bio-Shield ACtivity Assesgment HWCIR
03/06/97 12:11、 Page 2 File: A: UBIOSTRD.DAT

Dose Conversion Factor (and Relaced) Parameter Summary

File: DOSFAC.BIN

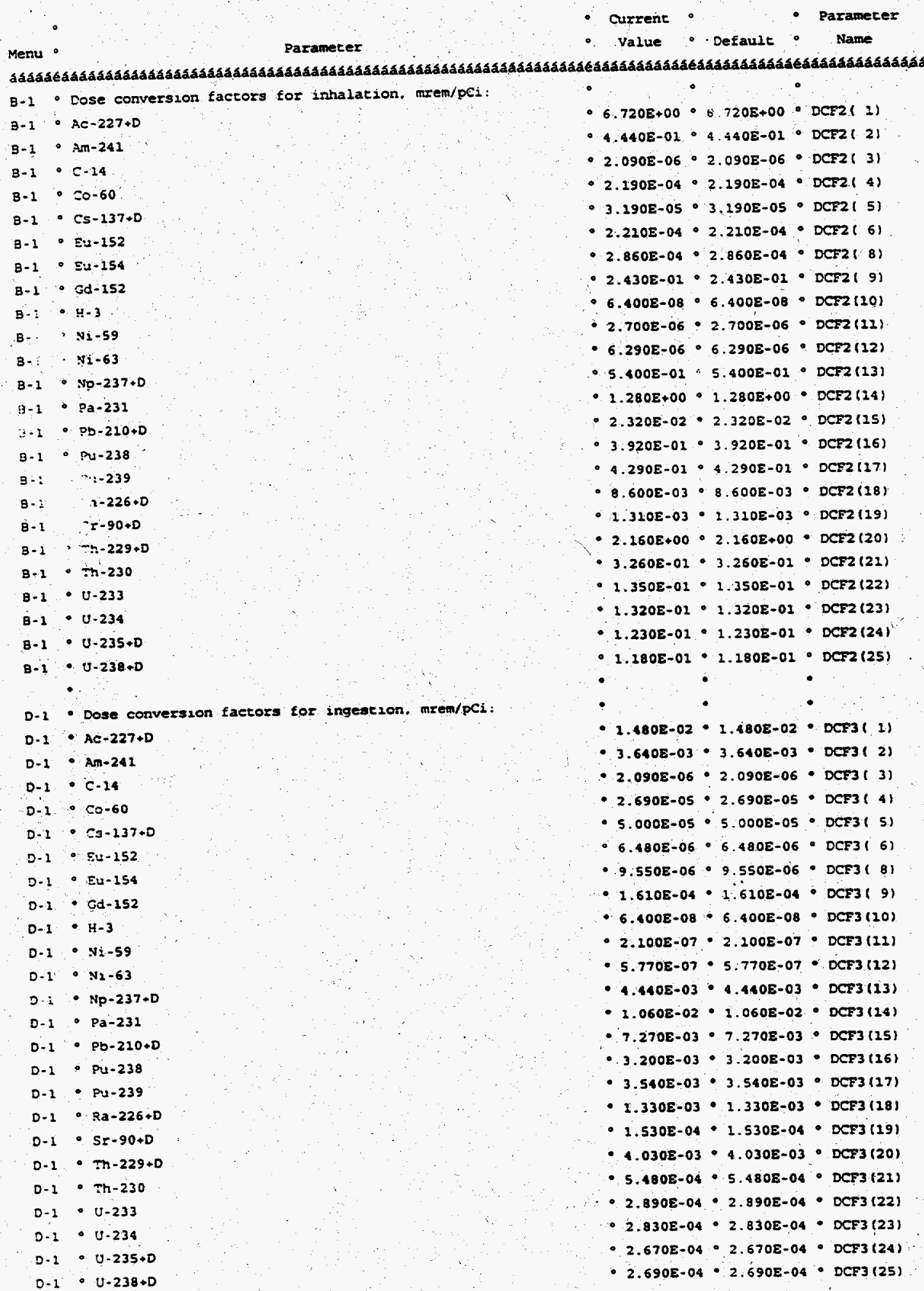


RESRAD, Version 5.623 T Timit $=0.5$ year

Sumary : Bio-Shield Activity Assessment kNCTR
03/06/97 12:11 Page 3

File: A: \BIOSHID.DAT

Dose Conversion Factor (and Related) Parameter Summary. (concinued)

File: DOSFAC. BIN

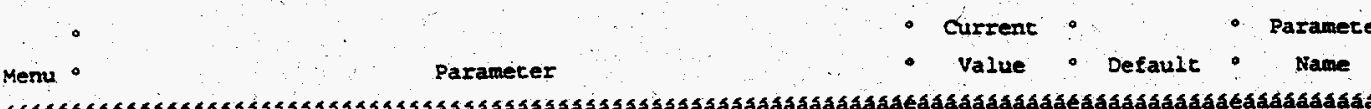

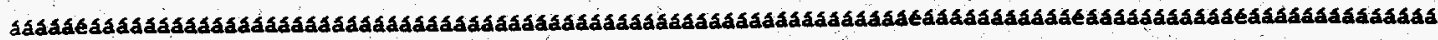

0.34 - Food eransfer factors

D-34 Ac-227+D, plant/soil concentration ratio. dimensionless

$2.500 E-03 \cdot 2.5008-03 \cdot \operatorname{RTE}(1.1)$

D-34 AC-227+D, beef/livestock-intake ratio, $(\mathrm{pCi} / \mathrm{kg}) /(\mathrm{pCi} / \mathrm{d})$

- 2.000E-05 $2.000 E-05 \cdot \operatorname{RTF}(1.2)$

D-34. AC-227+D. milk/liveatock-intake ratio, (pCi/L)/(pCi/d)

$0-34$.

D-34 Am-241 planc/soil concentratson ratio, dimensionless

$\mathrm{D}=34$ Am-241. beef/livescock-intake ratio. $(\mathrm{pCi} / \mathrm{kg}) /(\mathrm{pCi} / \mathrm{d})$

D.34 Am-241, milk/liveseock-ineake ratio, $(\mathrm{pCi} / \mathrm{L}) /(\mathrm{pCl} / \mathrm{d})$

D-34:

$0-34 \cdot C-14$

$0-34 \cdot c-14$

plant/soil corcentration ratio, dimengionless beef/livescock-intake ratio. $(\mathrm{pci} / \mathrm{kg}) /(\mathrm{pci} / \mathrm{d})$

$0.34 \cdot C-14$

milk/livestock-intake rac 2o. $(p C i / L) /(p C i / d)$

$0-34 \%$

$0.34 \cdot C 0.60$

plant/soil conceneracion rac 20 . dimensionless

$5.34 \cdot \operatorname{co} .60$

beef/livestock-2ncake rat $10,(p \mathrm{pl} / \mathrm{kg}) /(\mathrm{pCi} / \mathrm{d})$

$0.34 \cdot 60.60$

$m 11 k / 1$ ivescock-intake ratio, $(p C i / L) /(p c i / d)$

$D-34^{\circ}$

2.000E-05 2.000E-05 RTF( 1.3$)$

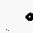

- $1.0008-03 \cdot 1.000 E-03 \cdot \operatorname{RTF}(2,1)$

- 5.000E-05 - 5.000E-05 RTF( 2.2)

2.000E-06.2.000E-06 RTF(2.3)

-.

- $5.500 E+00 \cdot 5.500 E+00 \cdot \operatorname{RTE}(3.1)$

- 3.200E-02 $3.100 \mathrm{E}-02 \cdot \operatorname{RTF}(3.2)$

1.200E-02 $: 1.200$ E-02 RTF( 3.3)

- $\quad \because \quad$ a

-9.000E-02 8.000E-02 RTF( 4.1)

- 2.000E-02 2.000E-02 - RTF(4.2)

- 2.000E-03 2.000E-03 $\bullet \operatorname{RTF}(4.3)$

- $\quad$ - $\quad \therefore$

- 4.000E-02 4.000E-02 - RTF( 5.3)

- 3.000E-02 $3.000 E-02 \cdot \operatorname{RTE}(5.2)$

$0-34 \cdot \operatorname{cs}-137+0$, beef $/ 1$ ivestock-2ntake rat $10 .(\mathrm{pCi} / \mathrm{kg}) / \mathrm{pCi} / \mathrm{d})$

$0-34$ - Cs-137.0. mlik/livescock-incake ratso. (pCi/L)/(pCi/d)

$0.34 \cdot$

D-34 Eu-152 planc/soil concentracion racio. dimensionless

D-34 su-152, beef/livestock-intake rat2o. (pci/kg)/(pCidd)

D-34 Eu-152, milk/livestock-intake ratio, (pCi/L)/(pCi/d)

D-34?

D-34 Eu-154 plane/soil concencration ratio. dimensionleas

D-34 Eu-154 , beet/livescock-incake racio, (pCi/kg)/(pCl/d)

D-34 Eu-154 milk/1ivescock-intake racio. $(p C 1 / L) /(p C L / d)$

$D-34 \cdot$

D-34 - Gd-152, plant/soil concentration ratio, dimensionlesa

$0-34$ - cd-152 beet/1 ivescock-intake ratso. $(\mathrm{pct} / \mathrm{kg}) /(\mathrm{pci} / \mathrm{d})$

$0-34$ - Gd-152 . milk/livescock-incake rac10, (pci/L)/(pCi/d)

$0-34$.

D-34 - H-3 plant/sol 1 concentracion rat10. dimensionless

D-34 H-3 Heef/1ivestock-2ntake ratio. $(\mathrm{pCl} / \mathrm{kg}) /(\mathrm{pCl} / \mathrm{d})$

0-34 H-3 $\quad$ milk/1ivescoek-intake ratio. $(\mathrm{pCi} / \mathrm{L}) /(\mathrm{pCi} / \mathrm{d})$

D-34.

D-34 - Ni-59 plant/soll concentration racio, dimensionless

D-34 - Ni-59 beef/1iveatock-incake ratio. $(\mathrm{pcl} / \mathrm{kg}) /(\mathrm{pCl} / \mathrm{d})$

D-34 Ni-59 milk/1ivescock-intake racio. $(\mathrm{pCl} / \mathrm{L}) /(\mathrm{pCl} / \mathrm{d})$

D-34.

D-34 Ni-63 plant/so21 concencration ratio, dimensionless

- $.000 E-03 \cdot 8.000 E-03 \cdot R T F(5.3)$

-

2.5008-03 2.500E-03・RTF( 6.1$)$

2.000E-03 $\cdot 2.0008-03 \cdot \operatorname{RTF}(6.2)$

2.0008-05 2.000E-05 $\cdot \operatorname{RTF}(6.3)$

2.500E-03 $2.500 E-03 \cdot \operatorname{RTF}(8,1\}$

- 2.000E-03 2.000E-03 - RTF( 8,2$)$

2.0008-05 2.000E-05 - RTF(8.3)

- $\quad$. . .

2.500E-03 2.5008-03 - RTF( 9.1$)$

2.000E-03 2.000E-03 - RTF( 9.2$)$

2.000E-05:2.000E-05 $\because \operatorname{RTF}(9.3)$

- C C

- 4.800E+00 4.800E+00 RTF $(20.1)$

- 1.200E-02 $1.200 E-02 \cdot \operatorname{RTF}(20.2)$

1.000E-02 $\cdot 1.000 E-02 \cdot \operatorname{RTF}(10.3)$

5.000E-02 $-5.000 E-02 \cdot \operatorname{RTF}(11.1)$

- 5.000E-03 - 5.000E-03 RTF(13.2)

- 2.000E-02 -2.000E-02 RTF(21.3)

-

a $5.000 E-02 \cdot 5.000 E-02 \cdot \operatorname{RTF}(12,1)$

5.000E-03 $\cdot 5.000 E-03 \cdot$ RTF (22.2)

- $2.0008-02 \cdot 2.000 \mathrm{E}-02 \cdot \operatorname{RTE}(12.3)$

2.000E-02 $2.0008-02 \cdot \operatorname{RTF}(13,2)$

- $1.000 E-03 \cdot 1.000 E-03 \cdot \operatorname{RTF}(13,2)$

- 5.000E-06 5.000E-06 - RTE (13,3)

D-34 Np-237+D : mik/livestock-intake racio. (pci/L)/(pCi/d) D-34. 
RESRAD, Version 5.622 T LImit $=0.5$ year Summary : Bio-shield Activity Assessment HWCTR
$03 / 06 / 97$ 12:12 Page 4

File: A: \BIOSHL.DAT

Dose Conversion Factor (and Related) Parameter Sumnary (continued)

File: DOSFAC. BIN

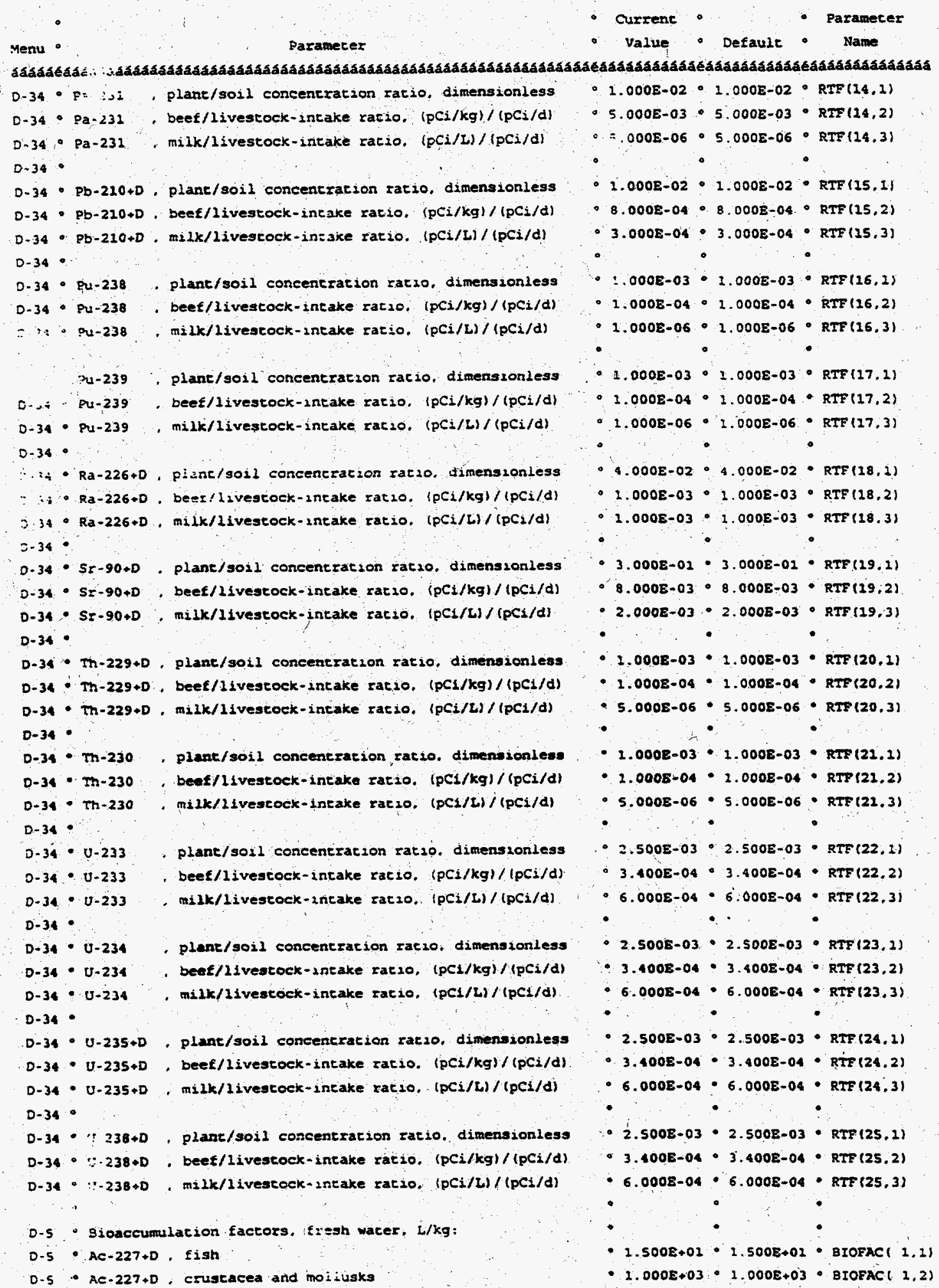


RESRAD. Version $5.622 \quad T^{*}$ Limit $* 0.5$ year Sumfary : Bio-Shield Activicy Assegsment HWCTR
$03 / 06 / 9712: 11$ Page 5

File: A: \aIOSTLD.DAT

Dose Comversion Eaccor fand Related) Paramecer Summary (continued)

File: DOSEAC.a:

Menu - Y Paramerer Parameter

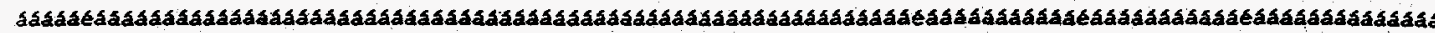

$0.5 \cdot A m-241$, Eish

0.5 An-242, crustacea and mollusks

$0-5$.

$0-5 \cdot C-14 \quad \therefore$ Eish

$0-5$ - -14 , erustacea and mollusks

$0.5:$

D-5 $\operatorname{Co-60}$. Eish

$0-5$ Co-60 : crustacea and mollusks

$0.5 \circ$

$0.5 \cdot \operatorname{Cs}-137 \cdot 0$, Eish

$0-5$ - $C s-137+D$. Crustacea and mollusks

$0-5$ :

$0.5 \cdot E u-152$, fish

0-5 Eu-152. cruscacea and mollusks

0.5 :

$0-5 \cdot E u-154$, Eish

$0.5 \cdot 5 u-154$. cruscacea and mollusks

$0-5.0$

$0.5 \cdot G d-152$, Eish

0.5 - Gd-152 cruscacea and mollusks

D.5.

$0-5 \cdot \mathrm{H}-3$ Eish

D-5 H-3 . Cruscacea and mollusks

0.5 .

$0.5 \cdot \mathrm{N}_{2} .59$..Eish

D-5 Ni-59 . srietacea and mollusks

D.5 -

D-5 Ni-63 fish

$0-5$ Ni-63. erustacea and mollusika

0.5.

$0-5 \cdot N p-237+0$, fish

D-5 No-237+D. Cruseacea and mollusks

$2.5:$

-5. Pa-231 . Eish

D.5 Pa-231 , crustaces and mollusks

D-5 -

D-5 Pb-220+D, fish

$0-5$ - $P b-210+D$. Gruscaces and mollusks

0.5 .

D-5 Pu-238: Eish

$0-5$ Pu-238 . erustacea and mollusks

$0-5$.

$0-5 \cdot P u-239$ Ejsh

0.5 - pu-239 C crustacea and mollusks

$0-5$.

D-S Ra-226+D, fish

$0.5 \cdot R a-226 \cdot 0$. crustaced and mollusks

$0.5:$

$0.5 \cdot 5 r-90+D$, Eish

$0.5 \cdot 5 x-90+0$ cruseacea and mollusks

0.5 .
- 3,000E+01 3.000E+01 : BrofAC (2,1).

$0.0008+03 \cdot 1.0008+03 \cdot \operatorname{BrOFAC}(2.2)$

- 5.000E+04 5.000E+04: BIOFAC(3.1)

$0.100 E+03 \cdot 9.100 E+03 \cdot \operatorname{BIOFAC}(3.2)$

$\therefore$

- 3.000E+02 $3.000 E+02 \cdot$ BIOFAC( 4,1 )

-2.000E+02 $2.000 E+02 \cdot \operatorname{BIOEAC}(4.2)$

$\circ$

- 2.0008+03*2.0008+03 elogac( 5.1$)$

- 1.000E+02 $\cdot 1.000 E+02 \cdot \operatorname{BrOFAC}(5,2)$

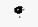

- 5.000E+01 $\cdot 5.000 E+01$ - BIOFAC $(6.1)$

- 2.000E+03-1.000E+03 BIOEAC $(6,2)$

-5.0008+01 -5.000E+01 - eloraci 8.1)

- 1.000E+03:1.000E+03 aIOFAC $(8.2)$

- 2.500E+01 $\cdot 2.500 E+01 \cdot$ Brofaci 9.3$)$

- $1.000 E+03 \cdot 1.000 E+03 \cdot \operatorname{BrOFAC}(9.2)$

-

- $1.0008+00 \cdot 1.0008+00 \cdot \operatorname{BrOFAC}(10,1)$

- 2.000E+00 1.000E+00 B eloeac $(10.2)$

:

$\because 1.0008+02 \cdot 1.0008+02 \cdot \operatorname{BIOFAC}(11.1)$

$-1.000 E+02 \cdot 1.0008+02-\operatorname{BIOFAC}(12,2)$

$\because$

-1.0005+02 $\because 1.000 E+02 \cdot \operatorname{BIOEAC}(12.1)$

- $1.0008+02 \cdot 1.0008+02 \cdot \operatorname{BIOFAC}(22.2)$

$-$

- 3.000E+02 $3.000 E+01 \cdot \operatorname{BrosaC}(23.2)$

- 4.000E+02-4.000E+02 $\cdot \operatorname{BIOFAC}(23.2)$

- $\quad \cdots \quad \therefore$.

$-2.0008+01,2.0008+01 \cdot \operatorname{BIOFAC}(24,1)$

- $1.100 E+02 \cdot 1200+02 \cdot$ BIOFAC $(14,2)$

$-$

- 3.000E+02-3.000E+02-8208AC (15.1)

- 1.0008+02 - 1.000E+02 - BTOFac(15.2)

-

-3.000E+01 - 3.000E+01 - BIOEAC $(16,1)$

$-1.000 E+02 \cdot 1.0008+02 \cdot$ BIOEAC $(16.2)$

-

- 3.000E+01-3.000E+02 BIOFAC (17. I)

- $1.0008+02 \cdot 1.0008+02 \cdot$ BIOFAC $(17.21$

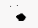

- 5.000E+01 -5.000E+01 BIOFAC $(18.1$

- $2.500 E+02-2.500 E+02 \cdot \operatorname{BrOFAC}(18,2)$

$\because$

- 6.000E+02 $6.000 E+01 \cdot$ BIOFAC $(19.1)$

- 2.000E+02-1.000E+02-3I0FAC $(19,2)$ 
RESRAD. Version $5.62 I$ L Limit $=0.5$ year Summary : Bio-Shield Activity Assessment HWCTR
$03 / 06 / 9712: 21$ Page 6

File: A: IBIOSHLD.DAT

Dose Conversion factor (and Relaced) Parameter Summary (continuéd)

File: DOSFAC.BIN

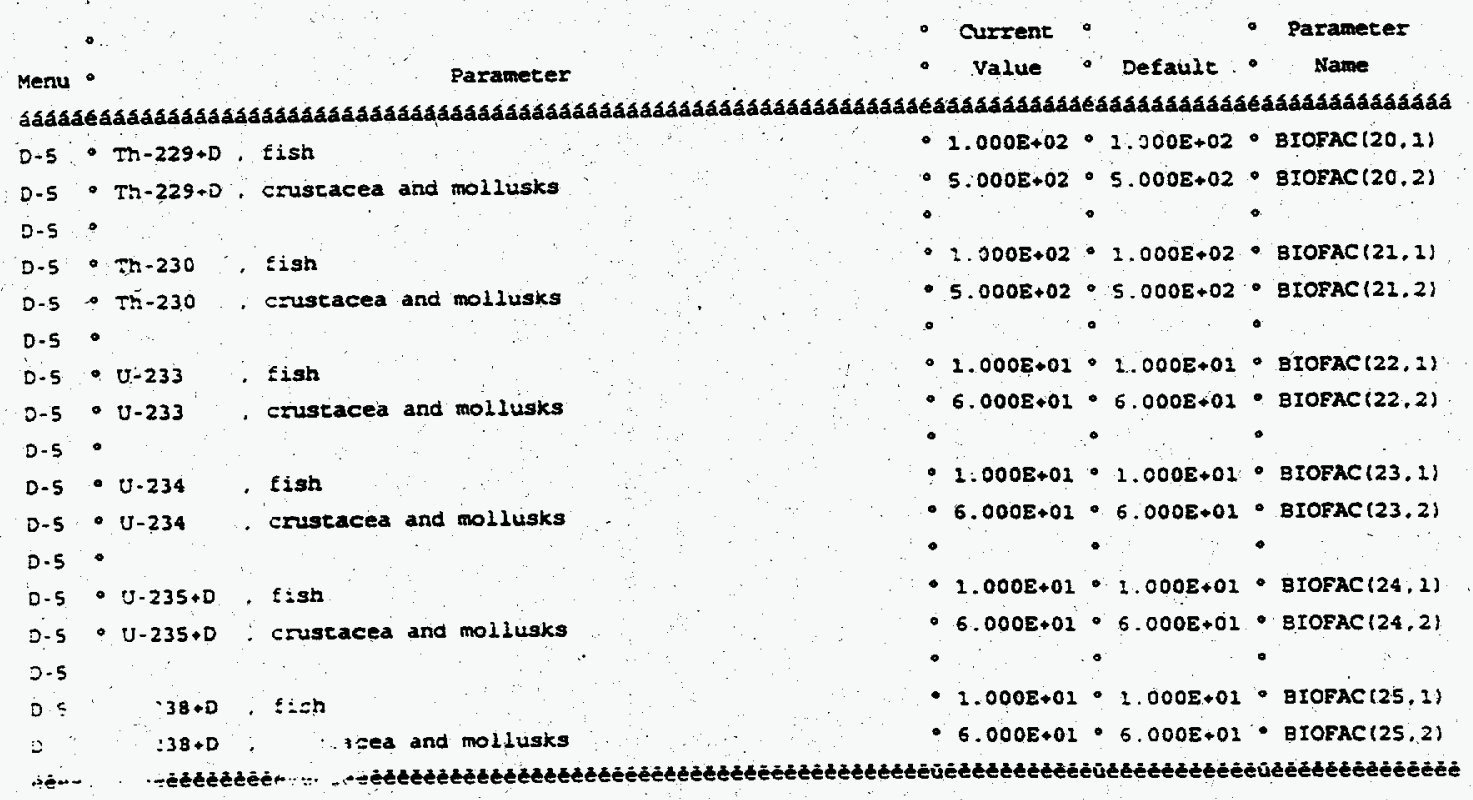




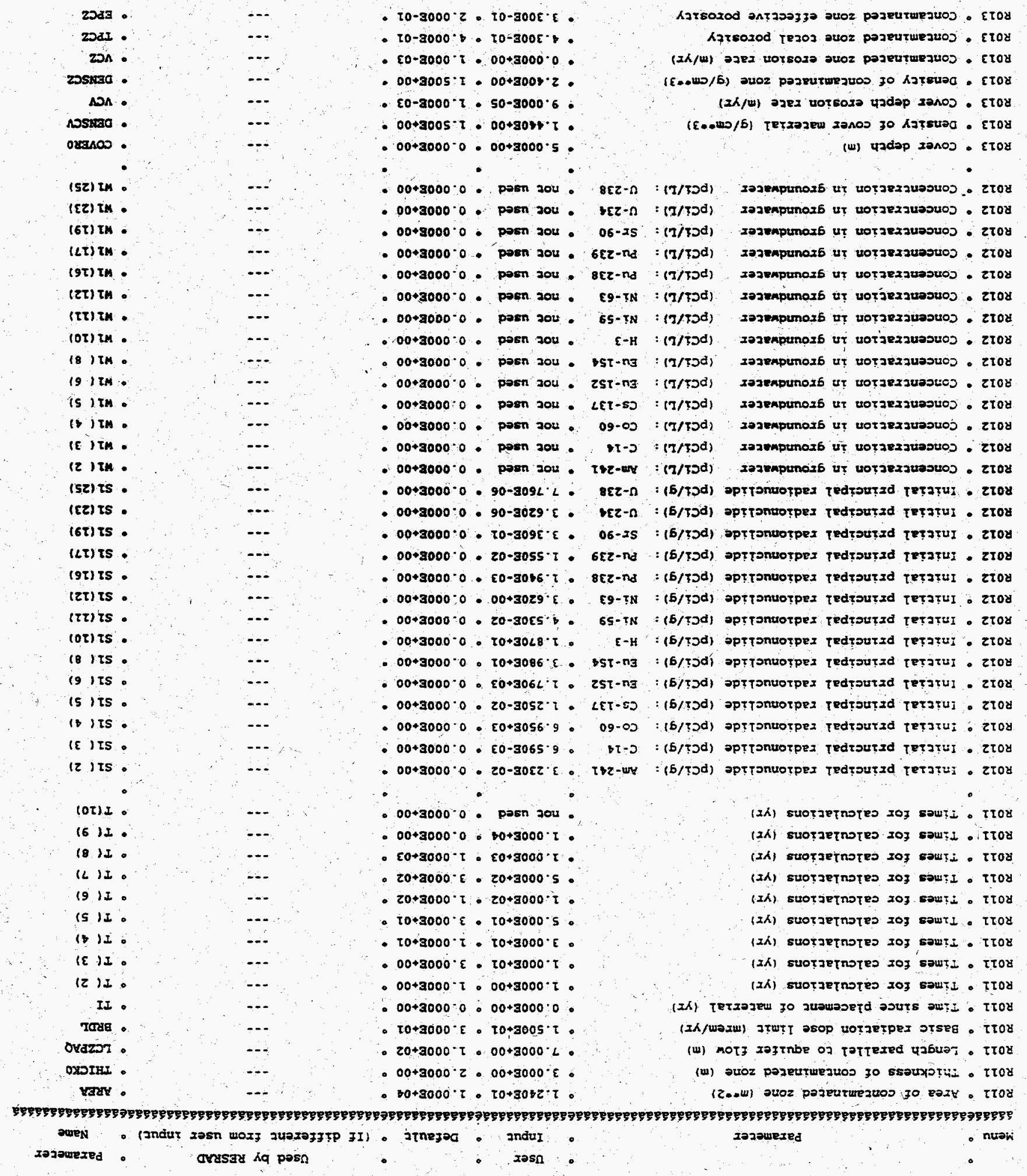

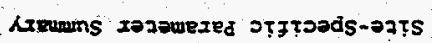

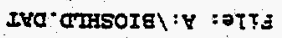

$L$ OSe II:ZI $\angle 6 / 90 / E 0$

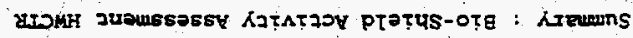

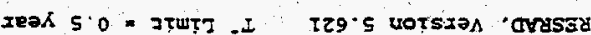


RESRAD, Version 5.62I T Timit $=0.5$ year Surmary : Bio-Snield Activity Asgessment HACIR
03/06/97, 22:21. Page 8 File: A: \BIOSHD.DAT

Site-Specific Parameter Summary (continued)

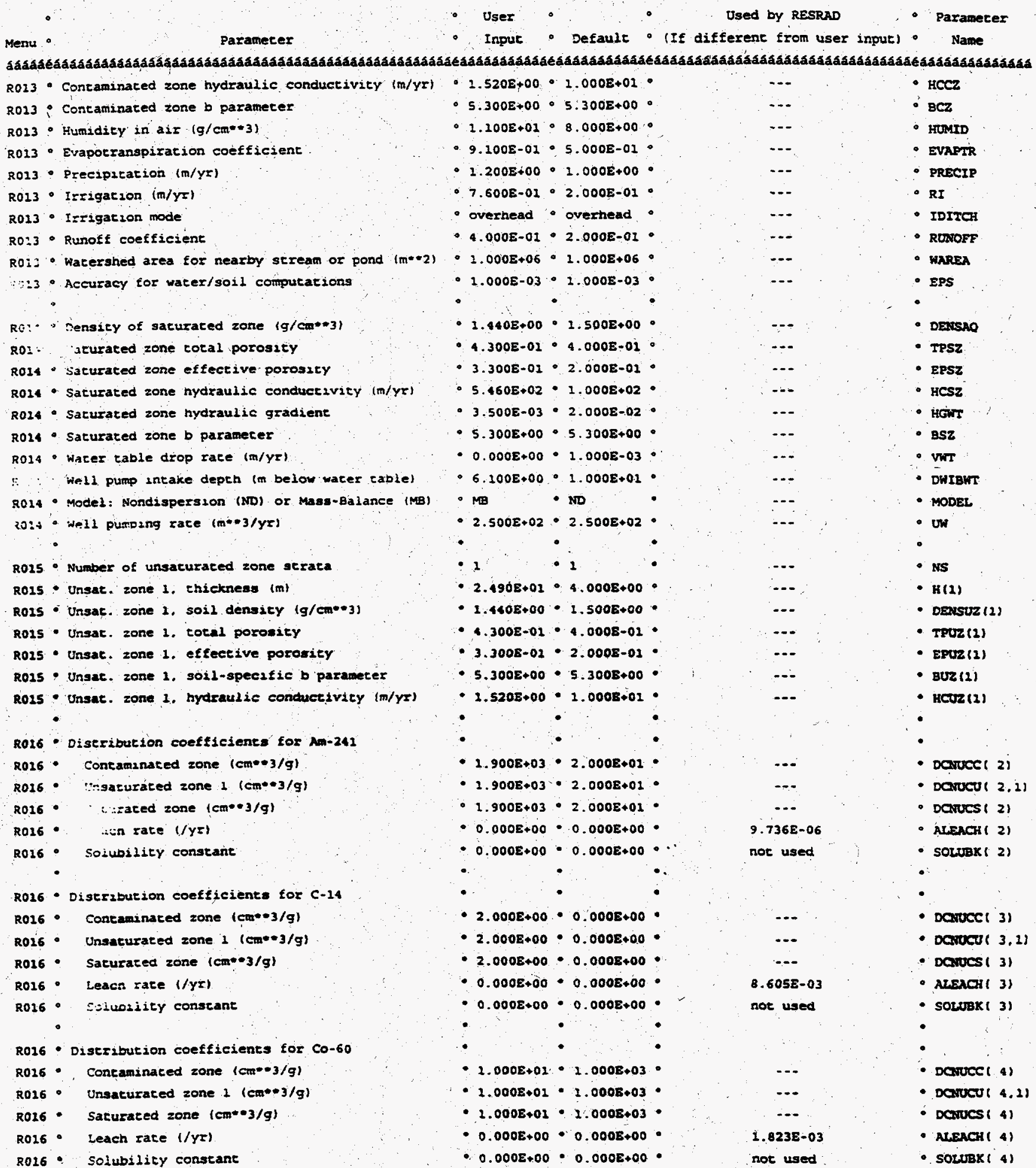


RESRAD, Version $5.621 \quad T^{*}$ Limit $=0.5$ year

Sunmary : Bio-Shield Activity Assessment HWCTR
03/06/97 $12: 11$ Page 9

File: A: VBIOSHO.DAT

Site-Specific Parameter Summary (continued)

Menu:

User - Used by RESRAD

- Paramecer

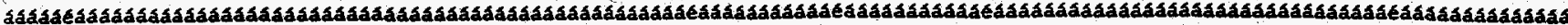

R016 Distribution coefficients for Cs-137

R016 Contaminated zone $(\mathrm{cm}+3 / \mathrm{g})$

R016 - Unsaturated zone 1 (cm=-3/g)

$R 016$ - Saturated zone $(\mathrm{cm} * \bullet 3 / \mathrm{g})$

R016- Leach rate (/Yr)

R016 - Solubility constant

R016 Distribucion coefficients for Eu-1S2

R016 - Contaninated zore $(\mathrm{cm} * * 3 / \mathrm{g})$.

R016 - Unsacuraced zone $1(\mathrm{~cm} * 3 / \mathrm{g})$

Ro26 Saturated zone $\left(\mathrm{cm}^{*} 3 / \mathrm{g}\right)$

Ro16. Leach race $|/ \mathrm{yr}|$

R016 Solubility conscant

R016 - Discribueion coefficients tor Eu-154

R026 Coneaminaced zone $(\mathrm{cm} * * 3 / \mathrm{g})$

R016 Unsaturated zone $1(\mathrm{~cm}=3 / \mathrm{g})$

R016 - Sacurated zone $(\mathrm{cm} \cdots 3 / \mathrm{g})$

R016 - Leach race (Jyr)

R016 - Solubility constant

R016 - Distribution coefficients for $\mathrm{H}-3$

R016 - Contaminated zone (cm-03/g)

R016 - Unsacuraced zone $1(\mathrm{~cm} * 3 / \mathrm{g})$

R016 Saturaced zone $(\mathrm{cm} * 3 / \mathrm{g})$

R016 Leach race $(/ Y y)$

R026 - Solubility conscant

R016 Distribution coefficients for Ni-59

R016 - Concaninated zone $(\mathrm{cm} \bullet \cdot 3 / 9)$

R016 Unsacurated zone $1(\mathrm{~cm}=43 / \mathrm{g})$

R016 - Saturated zone $(\mathrm{cm} * 3 / \mathrm{g})$

R016 - Leach race (/yr)

R016 Solubility constant

2016 Dustribucion coefficients for $\mathrm{Ni}-63$

Ro16 - Contaminated zone (cm*-3/g)

R016 Unancuraced zone $1(\mathrm{~cm} * 3 / \mathrm{g})$

R016 - Saturated zone $(\mathrm{cm}=-3 / \mathrm{g})$

R016 Leach race $\left(/ / y^{2}\right)$

R016 - Solubility conseant

R016 - Distriburion coefficients for Pu-238

R016 - Concaminaced zone $(\mathrm{cm} * 03 / \mathrm{g})$

Ro16 - Unsaturated zone 1 (cm**3/g)

R016 - Sacurated zone $(\mathrm{cm}-3 / \mathrm{g})$

Ro16 - Leach rate $(/ y)$

R016 - Solubility constant

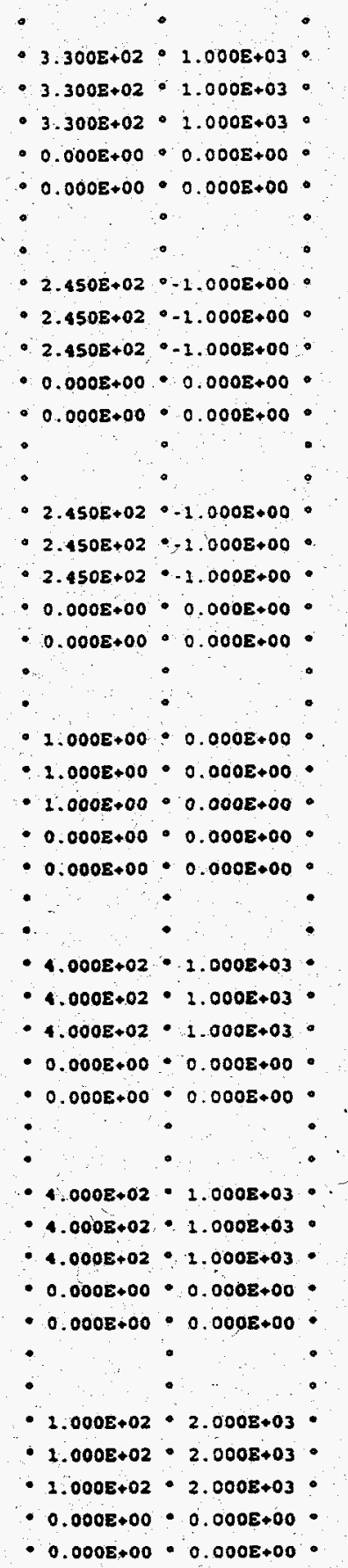

-.

- Denucci 5$)$

- Denucu ( 5.1)

- Dasues ( 5 )

- alsach (5)

- soturker 5$)$

not used

-

- Danuce (6)

- Darucar 6:3)

- Davues ( 6)

- Atraci $(6)$

- Soluak (6)

not used

-

- Danucer gi

- DanuCu( 8.1)

- Darucs (8)

- areachi 81

- sorvaki bi

not used

-

- Danucc(10)

- DaruCu(10.1)

- Darues (10)

- Arprate (10)

- soluak(10)

not used

- Darucc(11)

- Darucu (11.i)

- Dovues (11)

- ALEACH (12)

- SOLUBX (II)

not used

-

- Darucc (12)

- Dcrucu (22. 2)

- bavícs (12)

- Nesrat(32)

- solumak (22)

$4.623 E-05$
not used

-

- Deruce (26)

- DCruCu(16.2)

- Donucs (16)

- Aleaci (26)

- SOLUBK (16) 
RESRAD, Version $5.621 \quad T^{-}$Limit $=0.5$ year Sumary : Bio-Shield Activity Assessmene HWCIR
03/06/97 12:12 Page 10

File: A: \BIOSHD.DAT

Site-Specific Paramecer Sumary (concinued)

\begin{tabular}{|c|c|c|c|c|c|c|}
\hline & 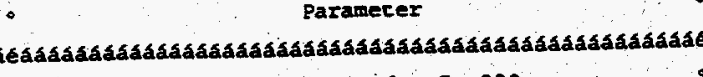 & aesaáasabááá & aéááasaáasáa & 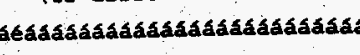 & saaáabą & 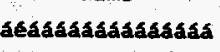 \\
\hline 026 & - Distribution coefficientes for Pu-239 & $\circ$ & - & - & & $\circ$ \\
\hline 316 & - Contaminated zone $\left(\mathrm{cm}^{* * 3 / \mathrm{g})}\right.$ & - $1.000 E+02$ & - $2.000 E+03$ & $\cdots$ & & - Danucc (17) \\
\hline 016 & - Unsacurated zone $1(\mathrm{~cm}=* 3 / \mathrm{g})$ & - $1.000 E+02$ & - $2.000 \mathrm{E}+03$ & $\cdots$ & & - Dorucu $(17 ; 1)$ \\
\hline 516 & - Sacurated zone $(\mathrm{cm} * * 3 / \mathrm{g})$ & $\therefore 1.000 E+02$ & - $2.0008+03$ & $\cdots$ & & - Darucs (17) \\
\hline 016 & - Leach rate $(/ y r)$ & $=0.000 \mathrm{E}+00$ & $0.000 E+00$ & $2.8478-04$ & & - ALEACH (17) \\
\hline 316 & - Solubility constant & $0.000 \mathrm{E}+00$ & $0.000 E+00$ & not used & & - soLuex $\{17\}$ \\
\hline 1016 & - Distribution coefficients for SY-90 & : & - & . & & - \\
\hline 016 & - Contaminated zone $\left(\mathrm{cm}^{* * 3 / g)}\right.$ & $=1.000 E+01$ & $03.0008+01$ & $\cdots$ & & - Darucc $( \pm 9)$ \\
\hline 16 & - Gacuraced zone $(c \mathrm{~cm}-3 / \mathrm{g})$ & $1.000 \mathrm{E}+01$ & $=3: 0008+02$ & $\cdots$ & & - DCNuCU(19.1) \\
\hline 16 & 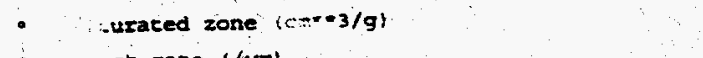 & $1.000 E+01$ & $3.000 E+01$ & $\cdots$ & & - DCrues (19) \\
\hline 016 & - den race $(/ y r)$ & $=0.000 E+00^{\circ}$ & $=0.000 E+00^{\circ}$ & $1.823 \mathrm{E}-03$ & & - arenct (19) \\
\hline R016 & Solubility constant & $0.000 \mathrm{E}+00$ & $\begin{array}{r}-0.000 E+00 \\
.\end{array}$ & not used & & SOLUBX (19) \\
\hline 016 & - Distribution coefficients for U-234 & $\cdots$ & • & $\therefore$ & & $\therefore$ \\
\hline 16 & - Contaminaced zone $(\mathrm{cm} * 3 / \mathrm{g})$ & $-3.500 E+01$ & $5.000 E+01$ & $\cdots$ & & - Daruce (23) \\
\hline 16. & $\therefore$ Unsaturated zone $1(\mathrm{~cm} * 3 / \mathrm{g})$ & - $3.500 E+01$ & $5.000 E+02$ & $\cdots$ & & - Darucu(23.1) \\
\hline 0.16 & 5. Sacuraced zone $(\mathrm{cm} * 3 / 9)$ & - $3.500 E+01$ & - $5.000 E+01^{\circ}$ & $\cdots$ & & - Davues (23) \\
\hline 16 & 6. Leach race $(/ y r)$ & $-0.000 E+00$ & $=0.000 E+00^{\circ}$ & $5.263 E-04$ & & - ALEACH $\{23\}$ \\
\hline 8016 & 6 Solubility constant & $0.000 E+0.0$ & $\because 0.000 E+00$ & not used & & : SOLURK(23) \\
\hline 116 & 6. Distribution coefficients for U-238 & $\bullet$ & $\bullet$ & - & & \\
\hline 026 & 6 Contaminated zone $(\mathrm{cm}-* 3 / \mathrm{g})$ & $\cdot 3.500 E+01$ & $1 \cdot 5.000 E+02^{\circ}$ & $\cdots$ & & - Darucc (25) \\
\hline 2016 & zone $1(\mathrm{~cm} * * 3 / \mathrm{g})$ & $3.500 E+01$ & $5.000 \mathrm{E}+01$ & $\cdots$ & & - Davucu(25.1) \\
\hline 1016 & 6 - Sacuraced zone $\left(\mathrm{cm}^{*} \cdot 3 / \mathrm{g}\right)$ & $3.500 E+02$ & - $5.0008+01$ & $\cdots$ & & - darues (25) \\
\hline 16. & 6. Leach race $|/ y r|$ & $-0.0008+00$ & $\cdot 0.000 E+00$ & $5.263 E-04$ & & - ALEACH (25) \\
\hline 136 & 6. Solubility conseane & $0.0008+00$ & $\because 0.0008+00$ & not used & & sownBk125) \\
\hline 116 & 16 - Distribution coefficients for daugher Ac-22? & $\bullet$ & $\bullet$ & $\bullet$ & & \\
\hline 2016 & 6. Concaminaced zone $(\mathrm{cm} * \cdot 3 / \mathrm{g})$ & $-4.000 \mathrm{E}+02$ & $2 \cdot 2.0008+01$ & $\cdots$ & & - Darocel 11 \\
\hline R016 & 6. Unsacurated zone $1(\mathrm{~cm} * \cdot 3 / \mathrm{g})$ & $-4.000 E+02$ & $2 \cdot 2,000 E+01$ & $\cdots$ & & - Davucu (3.1) \\
\hline 16 & 6 - Saturated zone $(\mathrm{cm}-* 3 / \mathrm{g})$ & $-4.000 E+02$ & $2 \cdot 2.0008+01$ & $\because \cdot$ & & - darues (1) \\
\hline 26 & 6 - Leach sace $(/ y z)$ & $\therefore 0.000 \mathrm{E}+00$ & $0 \cdot 0.000 E+00$ & $4.623 E-05$ & & - ALEACH (1) \\
\hline$\because 016$ & 6. Solubility constant & $0.0008+00$ & $0 \cdot 0.000 E+00$ & not used & & - SOLUBKI 11 \\
\hline 126 & 16 - Distribution coefficients for daughter Gd-152 & - & - & & & 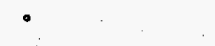 \\
\hline 016 & 16 Contammated zone $(\mathrm{cm} \cdot * 3 / \mathrm{g})$ & $-1.000 E+00$ & $0 \cdot-1.000 E+00$ & $5.758 E+02$ & & $-\operatorname{DarucC}(9)$ \\
\hline 1016 & 16 Unsaturaced zone $1(\mathrm{~cm} \bullet \cdot 3 / \mathrm{g})$ & $-1.000 E+00$ & $0 \cdot-1.000 E+00$ & $5.758 E+02$ & & - Danucu! 9.11 \\
\hline 16 & 16 - Saturated zone $(\mathrm{cm} * 3 / \mathrm{g})$ & $-1.000 E+00$ & $0 \cdot-1=000 E+00$ & $5.758 E+02$ & & - DCrucs( 91 \\
\hline 116 & 16 - Leach rate $(/ \mathrm{yr})$ & 0.00 & $0 \cdot 0.0008+00$ & $3.2328-05$ & & - MLEACH ( 9) \\
\hline 2016 & 16 Solubility constant & $0.000 E+00$ & $0 \cdot 0.0008+00$ & not used & & - SOLuak ( 9) \\
\hline 16 . & 16 Distribution coefficients for daugheer Np-237 & & & $\because$ & & \\
\hline Ro16 & 16 Contaminated zone la & -5.00 & $10 \cdot-1.000 E+00$ & $\cdots$ & & - Dorucc (13) \\
\hline Ro16 & 16 Unsacurated zone $2(\mathrm{~cm} * \cdot 3 / 9)$ & $5.0008+00$ & $0-1.0008+00$ & $\cdots$ & & - davucu(13.2) \\
\hline 026 & 16 - Saturated zone $\left(\mathrm{cm}^{* * 3} / \mathrm{g}\right)$ & $5.000 E+00$ & $10 \cdot-1.000 \mathrm{E}+00$ & 0 . & & - DCVUUCS (13) \\
\hline 6 & Leach rate $1 / y$ & 0.00 & $10 \cdot 0.000 \mathrm{0}+00$ & $3.592 \mathrm{E}-03$ & & - ALEACH (13) \\
\hline & WDility const & $-0.000 E+00$ & $10 \cdot 0.000 E+00$ & not used & & SOLUBK (13) \\
\hline
\end{tabular}


RESRAD, Version $5.621 \quad T$ Limit $=0.5$ year Summary : Bio-shield Activity Assessment HWCIR
03/06/97 $12: 11$ Page 11 File: A: NBIOSFID.DAT

Site-Specific paramecer Sumary (continued)

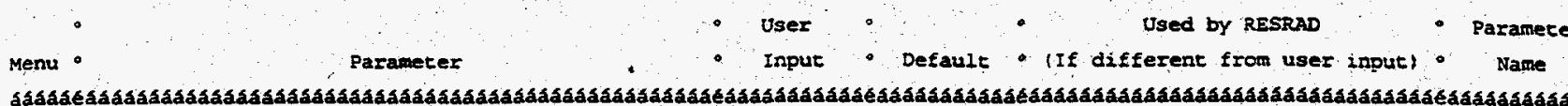

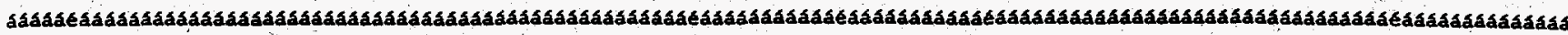

R016 - Distribution coefficients for daughter $8 a-231$

R016 Concaminaced zone $(\mathrm{cm}+* 3 / g)$

R016 Unsacurated zone $1(\mathrm{~cm} *-3 / \mathrm{g})$

Ro16 Sacuraced zone $(\mathrm{cm}=\mathbf{3} / \mathrm{g})$

R016 - Leach tate (/YI)

R016: Solubility constant

R016 Distribution coefficients for daugher $\mathrm{Pb}-210$

R016 - Contaminated zone $(\mathrm{cm} * 3 / \mathrm{g})$

RO16 - Unsaturated zone $1(\mathrm{~cm} * 3 / \mathrm{g})$

Ro16 - Sacuraced zone (cm*-*3/g)

R016 Leach race $(/ \mathrm{yr})$

Ro16 Solubility constant

Ro16 Distribution coefficiencs for daughter Ra-226

RO16 Concaminaced zone $(\mathrm{cm} * 3 / \mathrm{g})$

RoL6 Unsacurated zone $1(\mathrm{~cm}-3 / \mathrm{s} / \mathrm{g})$

R016 - Sacuraced zone $(\mathrm{cm} * 3 / \mathrm{g})$

Ro16 Leach race $(/ \mathrm{yr})$

Ro16 - Solubility constane

R016 Distribution coefElesents for daughter $\mathrm{Th}-229$

R016 - Contaminated zone $(\mathrm{cm} *-3 / \mathrm{g})$

R026 - Unsacurated zone 1 (cm*es/g)

R016 - Saturated zone $(\mathrm{cm}+03 / \mathrm{g})$

R016 - Seach race (/yr)

R016 - Solubility conseane

R016 - Distribution-coefficients for daughter Th-230

R026 - Contaminated zone $(\mathrm{cm} * 43 / \mathrm{g})$

R016 - Unsaturaced zone $1(\mathrm{~cm} * 3 / \mathrm{g})$

R026 - Saturated zone $(\mathrm{cm}-3 / \mathrm{g})$

R016 - Leach race (/yr)

R016 - Solubility constane

R026 - D1strabution coefficients for daughet U-233

R016 - Concaminaced zone $(\mathrm{cm} \cdot \bullet-3 / \mathrm{g})$

R016 - Unsacuraced zone $1(\mathrm{~cm}+* 3 / \mathrm{g})$

R016 Saturated zone $\left(\mathrm{cm}^{-0} \cdot 3 / \mathrm{g}\right)$

R016 - Leach race $(/ \mathrm{Yr})$

R016 - Solubility constant

R016 Diserzbucion coefficiente for daughter U-235

R016 - Concaminaced zone (cm+3/g)

R016: Unsaturaced zone $1(\mathrm{~cm}+\cdot 3 / \mathrm{g})$

Ro16 - Sacuraced zone $(\mathrm{cm} *-3 / \mathrm{g})$

R016 - Leach rate (/yr)

R016 - Solubility constane

R017 - Inhalacion race $(m * * 3 / y r)$

R017 Mass loading for inhalation $(\mathrm{g} / \mathrm{m} \cdots \cdot 3)$ $\therefore 5.5008+02 \div 5.0008+01$

- $5.500 E+02 \cdot 5.0008+01$.

- $5.5008+02 \cdot 5.000 E+01$.

$0.000 E+00 \div 0.000 E+00$.

$-0.0008+00 \cdot 0.0008+00$.

-

.0

- $5.500 E+01 \cdot 1.000 E+02 \cdot$

- 5.500E+01 $\cdot 1.0008+02$.

- 5.5.00E+02 $1.000 E+02$.

- $0.000 E+00 \circ 0.000 E+00$

- $0.000 E+00 \cdot 0.000 E+00$.

-

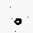

- 5.000E+02 - 7.000E+01

- 5.000E+02 - T.000E+01.

$-5.000 E+02 \div 7.000 E+01$.

- $0.000 E+00 \cdot 0.000 E+00$

$0.000 E+00 \cdot 0.000 E+00$.

-

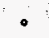

$-3.000 E+03 \div 6.000 E+04$

- 3.000E+03 - 6:000E+04

- 3.000E+03 - 6.000E+04.

- $0.000 \mathrm{E}+00 \cdot 0.000 \mathrm{E}+00$

- $0.000 E+00 \cdot 0.000 E+00$.

$-$

- 3.000E+03 $6.0008+04$.

- $3.000 E+03 \cdot 6.0008+04$

- $3.0008+03 \cdot 6.0008+04$.

- $0.000 \mathrm{E}+00 \cdot 0.0008+00$

$0.000 E+00 \cdot 0.000 E+00$

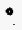

$\because$

- $3.5008+01-5.000 E+01$

- 3.5008+02 5.0008+01

- 3.5008+01 - 5.0008+01.

- $0.0005+00 \cdot 0.0008+00$

- $0.000 E+00 \cdot 0.000 E+00$.

$-$

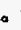

- 3.500E+01 $5.000 \mathrm{E}+01$.

- $3.5008+01 \cdot 5.0008+02$.

- $3.500 E+01 \cdot 5.000 E+01$.

$0.0008+00 \cdot 0.000 E+00$.

- $0.000 E+00 \div 0.000 E+00$.

$\therefore$

- 8.000E+03 $6.400 \mathrm{E}+03$.

-2.000E-04 2.000E-04.

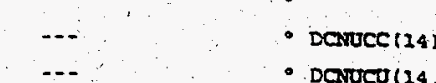

- DCNuCJ (24.1)

-.- $\quad$ DCNuCS (24)

3.363E-05 ALEACH (14)

not used SOLUBK(14)

- Danuce (25)

- Dentrou (25. 1 )

- DCNucs (25)

3.354E-04 ALEACH (25)

not used

- DCNuCC (18)

- Danucu (18.1)

- DCaves (18)

- aleach (18)

- SOLuak(18)

not used

- Darucc (20)

$\cdots$

-..

6.166E-06

- Darucu(20.1)

- davucs (20)

- Areaci(20)

not used

- sordusx(20)

- Davucc(22)

- DCrvico (22, 1$)$

- dervesizis

- Aleach (21)

6.266E-06

- solusk 21 )

not used

- Denuce (22j

- DCruco $(22,2)$

- DCrucs (22)

- alsaca (22)

5.263E-04

- sotursx (22)

。

- Darucc (24)

- DCarucu (24, I)

- Derucs (24)

- aleach (24)

- solduBK (24)

-

- ininara

- mina 
RESRAD, Version $5.621 \quad T^{-}$Limit $=0.5$ year Sumary : Bio-shield Activity Assessment tiwCTR
03/06/97 12:12 : Page 12

File: A: IBIOSTR.DAT

Site-Specific Parameter Sumary (continued)

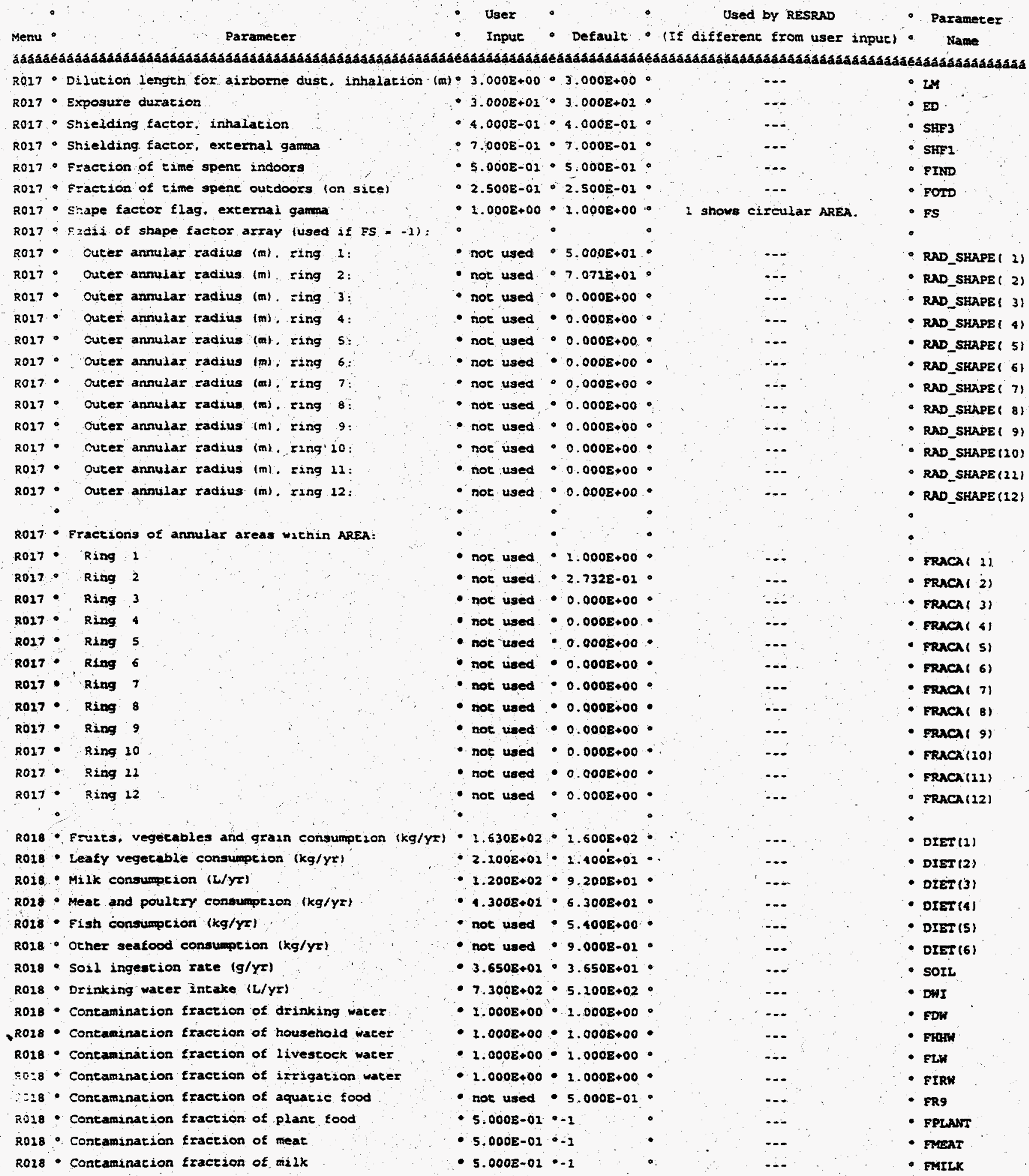




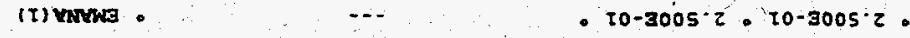

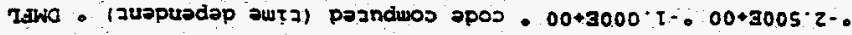
I甘s - (zuspupdop awtz) pezndwos spos - 00+3000\% 0 00+3000\%. war : $\quad .00+3005 \% \cdot 00+30052$ oxar : ... $\quad 0.30-3000.5 \cdot 70-3000.5$ ans - ... $00+3000 \cdot z \cdot 00+3000 \cdot z$.

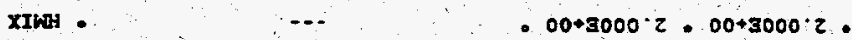
20sI0 : I3IIC . asira. Taozha. noozha Tuda -

ADd. zasnac o yoond .

(6)I yots. (8) I yars. (L)I yars . (9)I rais . (5) I yazs . (b)I zars . (E)I yors. (z) 1 zars . (T) I yors . soint osiny. nsiaza NSnz

5*o -

Erts -

3roso :

202T5 .

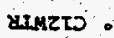

aInas.

aruas.

hungs.

maxos.

I0089 .

WI.

बSTN .

IST.

9 IMT *

SIHI 。

9IAT:

STaT :

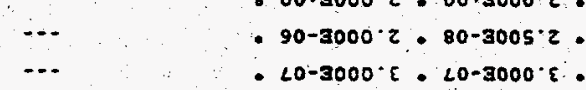

$\ldots \quad 90-3000 \%$ z $90-3000^{\circ} \mathrm{z}$.

$\therefore \quad-$

-

$-+$

$\cdots$

$\cdots$

$-$

$\cdots$

$+-$

$\ldots$

$+-$

$\because$

$\ldots$

$\cdots$

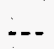

$\cdots$

$\therefore$

-..

$\cdots$

$\therefore$

$\cdots$

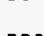

$\because$

$\ldots$

$\cdots$
$\cdots$
$\cdots$

$\therefore$

$--$

$-\ldots$

$\cdots$
$\cdots$

$-=$

--

-..

--
- 20-a000 s . $20-3000 \mathrm{~s}$.

- I0-9000. I . $10-3000$ T.

- 00+300rz o 00+300si。

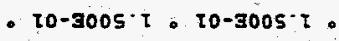

$$
\text { . }
$$

- $20+3005.20+3005 \%$

- $00+3000 \mathrm{~T} .00+3000 \mathrm{~T}$.

- 00+3000.T.00+3000\%t.

- 00+3000 L porn zol .

- 00+3000 L poen jor

- $00+3000 \cdot \tau .00+3000 \cdot \varepsilon$

- $00+3000$. 00+3000: .

- $\tau 0+300 \mathrm{\tau} \cdot \tau 0+300 \mathrm{r} \tau$.

- $20-3000.2$. $10-3009.5$.

- $20-3000$ B o to-3005:L.

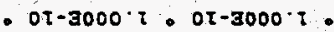

- $\angle 0-3000^{\circ} \mathrm{L} \cdot 10-3000^{\circ} \mathrm{L}$.

- $10-3000^{\circ} \varepsilon$. $10-3000^{\circ} \varepsilon$.

- I0-3008.6 : T0-3008\%。

- z0-3000. $\mathrm{z}: 20-3000 \% \mathrm{z}$

- $20-3000 \varepsilon$ o $20-3000 \varepsilon$.

- 50-3000. o 50-3000.z.

-

- $00+3000$. I $00+3000$ I .

- $00+3000 \mathrm{\tau} \cdot 00+3000 \cdot \tau$.

- $00+3000 \%$. $00+3000 \%$.

- $00+3000$ T $000+3000$.

- $20-3000: 6$ a $20-3000-6$.

- 50-3005. 2 . To-3005. 1 .

- $\$ 0-3000 \tau, 0-3000^{\circ} \tau$.

- 20-3000.5. 10-3000.5.

- $20+3009^{\circ} \mathrm{T} 20+3009^{\circ} \mathrm{\tau}$,

- $20+3000 \mathrm{~s}$. $70+3000 \mathrm{~s}$.

- $20+3005 \mathrm{~s}$ : $20+3005$.

- $\tau 0+3008.9$ o $[0+3000.6$
. $20-3000 . \varepsilon .20-3000 . \varepsilon$

- T0-3000 \& 10-3000\%.

- $10+3000 \%$ z $00+3000.9$.

se6 zzz-v8 jo zomod butaneuews - IzOd

(w) esezins punoss motaq qJdap butptịng - IZO४

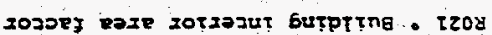

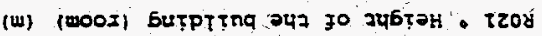

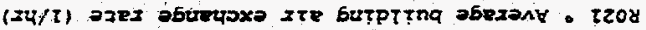

(20s/w) posas putn tenure asexany . tzoy

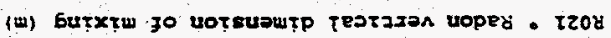
tros ouroz poseutwejuos UT - IzOz

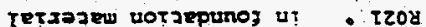
TETIOJER danos ut . T208

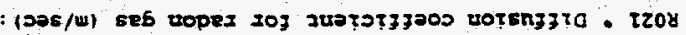

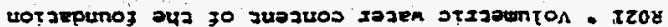

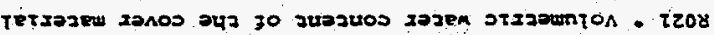
uorzepunol butpting syz 30 kJTsosod tezol . TzOU

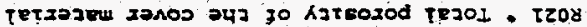

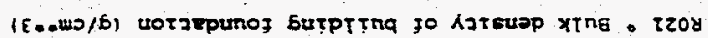
(w) uotjeptrioz butptrna go sestrotul tzos

$$
\begin{aligned}
& \text { xoppoz yooreont7. yols } \\
& \text { sejen osezins - yors } \\
& \text { zopen tTon - vars } \\
& \text { exent tow pue eoseasina - yors } \\
& \text { 4071 . yols } \\
& \text { Mrztiod pur jrow . yas } \\
& \text { XTTW . XaIs } \\
& \text { eotaejabon kjeaj - yors }
\end{aligned}
$$

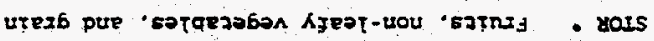

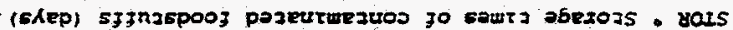

peof mos xtw ut utrezs go votzoess. ots

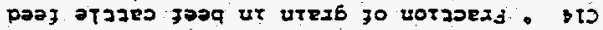

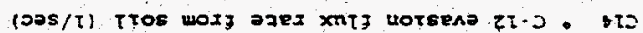

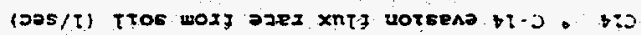

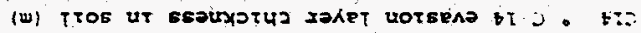

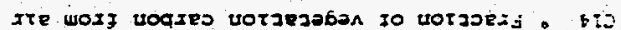
tros woxf voqxes vorsezoban zo votzoezs bis (6/5) Tros poseutueauos ut vorjeIzusouos zI-J : bIS

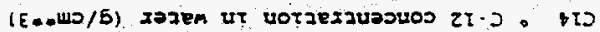

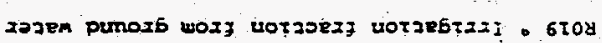

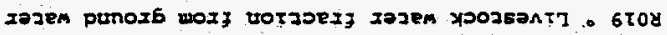

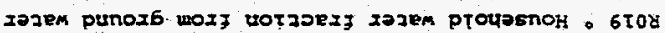

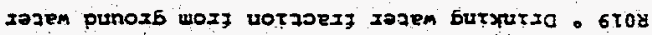

(w) 52002 jo yado0 0.6708

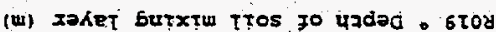

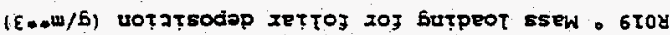

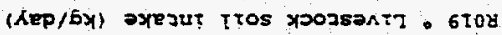

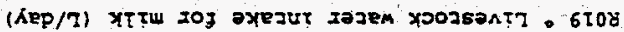

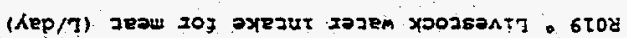

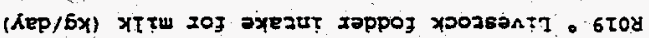

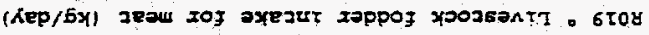

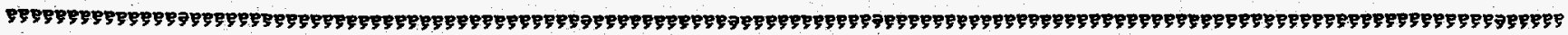

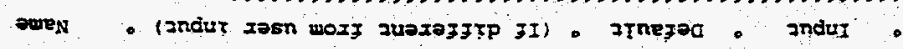

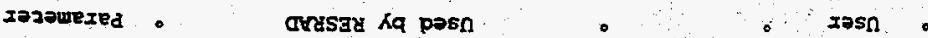

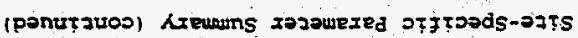

IRd C AHSOIEI : : : :

ET :BeC IT:ZT $L 6 / 90 / \varepsilon 0$

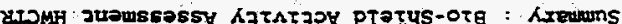

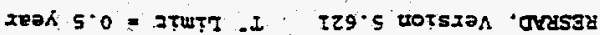


RESRAD, Version $5.623 \quad T^{-}$Limit $=0.5$ year

Sumary, : Bio-Shield Activity Assessment HACtR
03/06/97 12:11 Page 14

File: A: $\backslash$ BIOSHDD.DAT

Site-Specific Parameter Sumnary (continued)

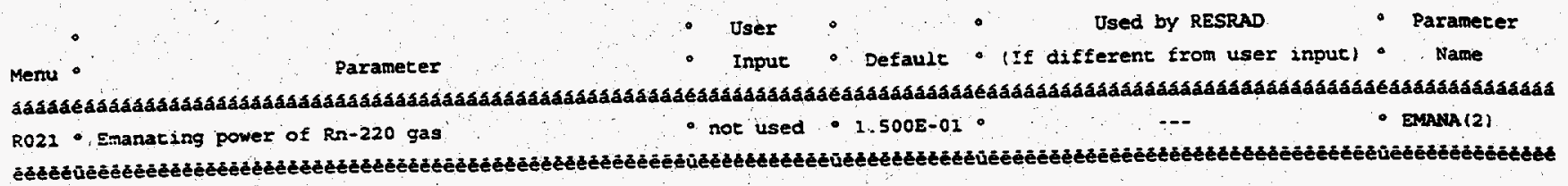

Summary of Pathway selections

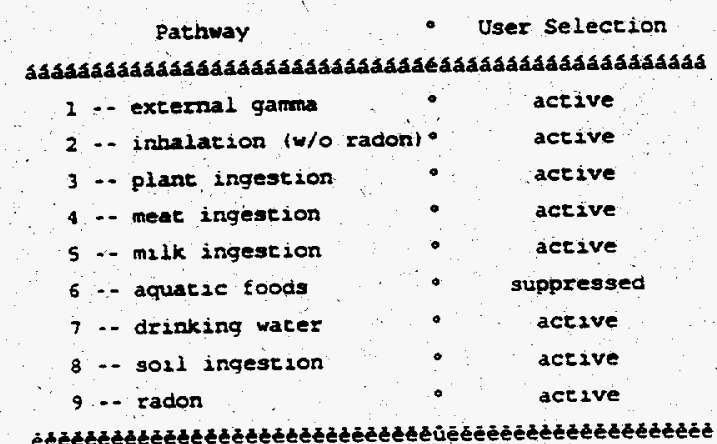




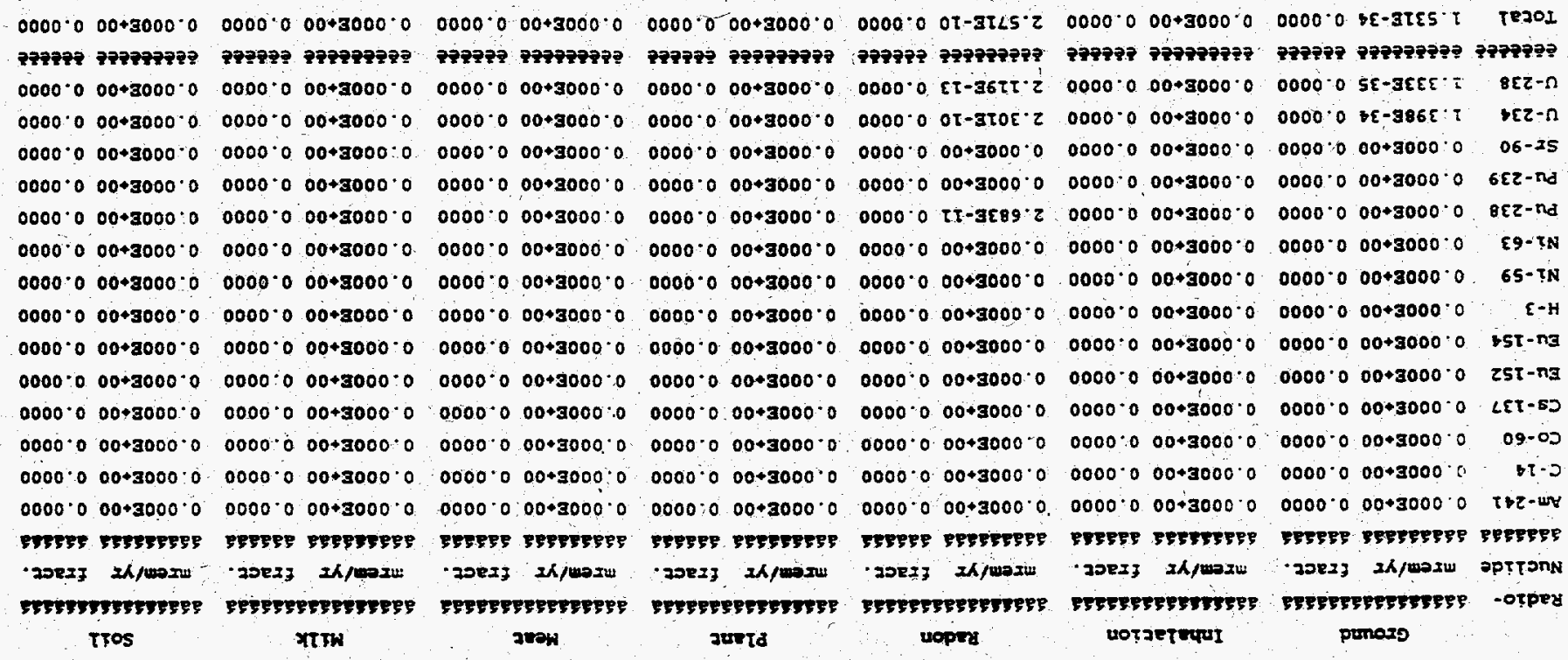

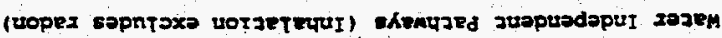

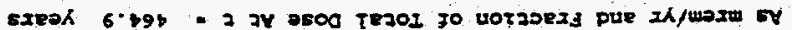

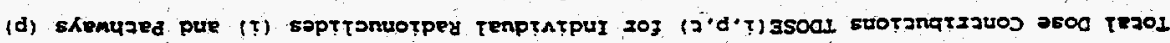

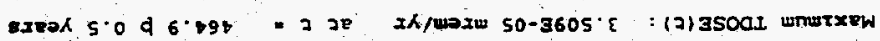

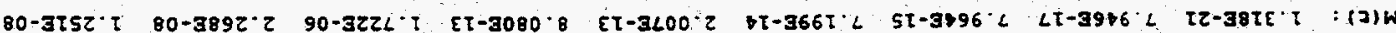

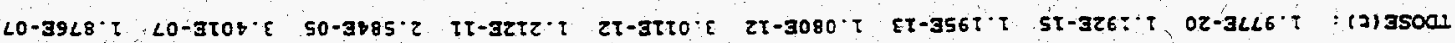

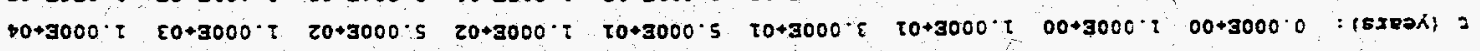

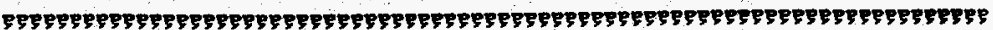

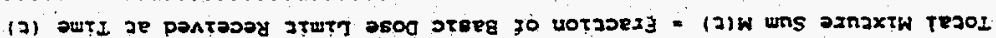

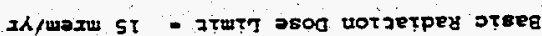

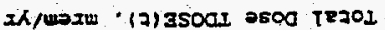

\begin{tabular}{|c|c|}
\hline $0-309 L \cdot c$ & $8 \varepsilon z-1$ \\
\hline $90-3029 \cdot \varepsilon$ & $\Delta \varepsilon z-\Omega$ \\
\hline To-309E' & $06-25$ \\
\hline$=0-3055^{-} \tau$ & $6 \varepsilon z-$ ind \\
\hline$\varepsilon 0-30 \$ 6 \cdot T$ & BEz-nd \\
\hline $00+3029: \varepsilon$ & $\varepsilon g-t N$ \\
\hline $20-30 \varepsilon 5$ & $6 S-\pi N$ \\
\hline $20+30<8^{\circ} \mathrm{T}$ & $\varepsilon-H$ \\
\hline $20+3086 \cdot \varepsilon$ & bt-ng \\
\hline$\varepsilon 0+306 L ; T$ & $2 S T-\pi g$ \\
\hline $20-3052 \div 1$ & LET-SD \\
\hline $80+3056^{\circ} 9$ & $09-00$ \\
\hline $80-3065^{\circ} 9$ & $t \tau-2$ \\
\hline$=0-\varepsilon 0 \varepsilon \tau \cdot \varepsilon$ & $\tau t$ \\
\hline
\end{tabular}

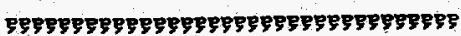

6/tod - suotaexzuosuos tros teṛțu

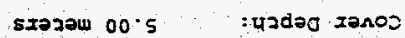

sxazaw $00^{\circ} \varepsilon$ :ssauxpty

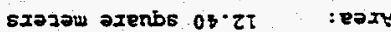

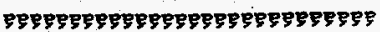

suotstawţ әtoz pazeuçweavos
I*O- CHHSOIE) : : :

St obed $\tau \tau: Z t \quad \angle 6 / 90 / \varepsilon 0$

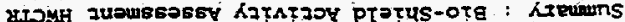

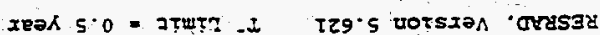




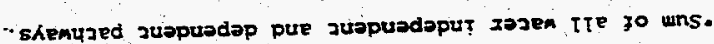

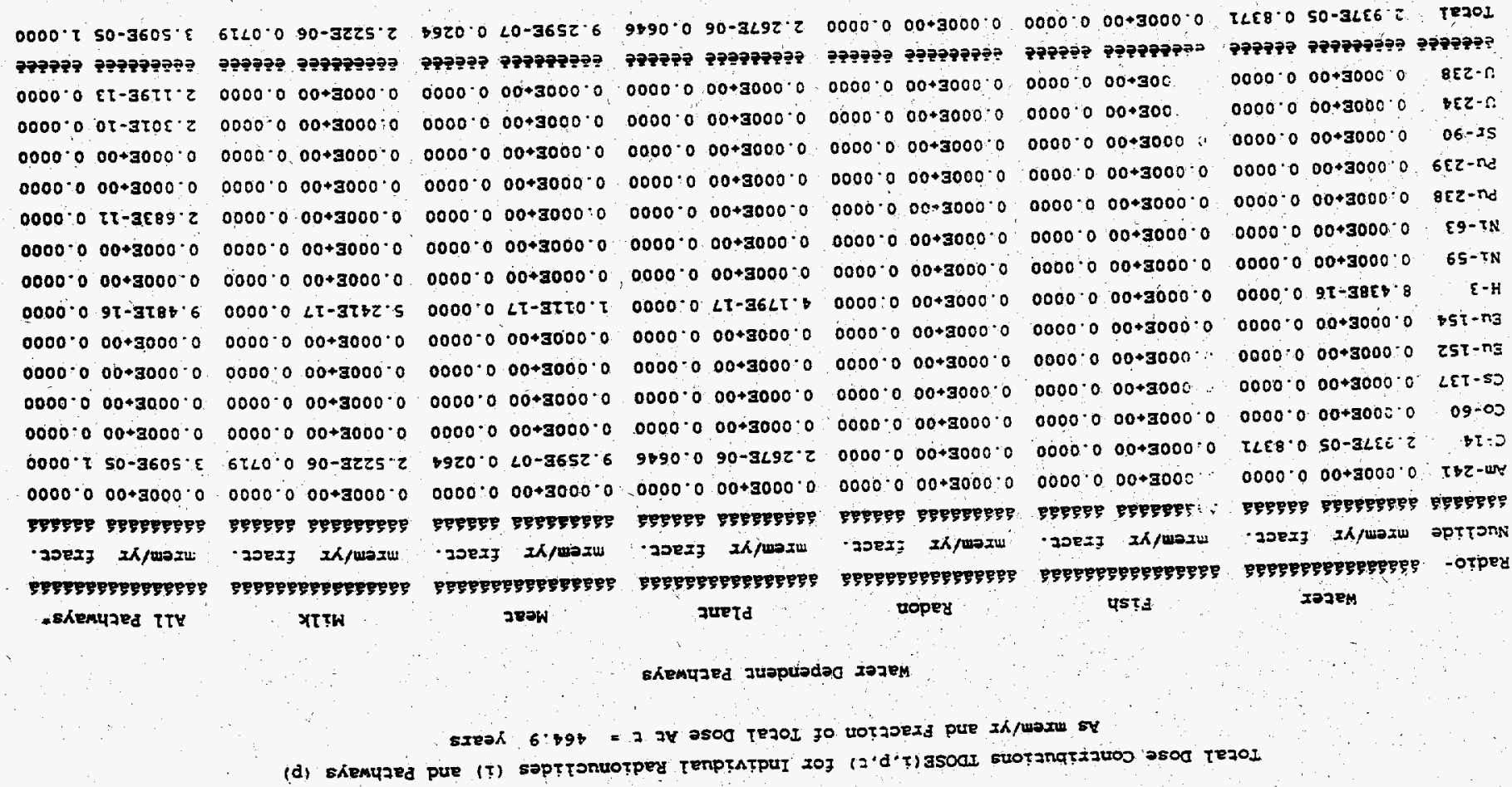

IYO बTHSOTE : : :

$9 \tau$ obed $\tau \tau: \tau T \quad \angle 6 / 90 / \varepsilon 0$

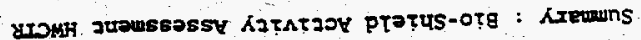

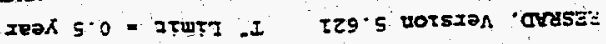


RESRAD, Version 5.621 T Limit $=0.5$ year

Sumary : Bio-Shield Activicy Assessment HWCTR
$03 / 06 / 97$ 12:11 Page: 17

File: A: IBIOSHID.DAT

Total Dose Contributions IDOSE (i,p, f) for Individual Radionuclides (i) and Pathways (p) As mrem/yr and Fraction of rotal Dose At $t=0.000 E+00$ years

Water Independent pathways (Inhalation excludes radon)

Ground

Radio- aasasaáakasaasa

Nuclice mrem/yr fract.

\section{dáasá sasadaasa aasaas}

Am-241 $0.000 E+00 \quad 0.0000$

C-14 $\quad 0.000 E+00 \quad 0.0000$

Co-60 1.973E-20 0.9980

Cs-137 4.5998-32 0.0000

Eu-152 3.613E-23 0.0018

Eu-154 $\quad 3.611 \mathrm{E}-24 \quad 0.0002$

$\mathrm{H}-3 \quad 0.000 \mathrm{E}+0 \mathrm{O} \quad 0.0000$

$\mathrm{N}_{2}-59 \quad 0.0008+00 \quad 0.0000$

$\mathrm{N}_{2}-63,0.000 \mathrm{E}+0 \mathrm{O} \quad 0.0000$

Pu-238 $0.000 E+00 \quad 0.0000$

PU-239 $0.000 E+00 \quad 0.0000$

$s r-90 \quad 0.000 E+00 \quad 0.0000$

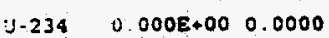

$\checkmark .2389 .784 E-36 \quad 0.0000$

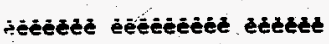

Total $1.9778-20 \quad 1.0000$
Inhalacion akáakadáakaasá mren/yr fract. áasaaada áasaá $0.000 E+00 \cdot 0.0000$ $0.000 E+00 \quad 0.0000$

$0.000 E+00.0 .0000$

$0.0000+00 \quad 0.0000$

$0.000 E+00 \quad 0.0000$

$0.0008+00 \quad 0.0000$

$0.000 E+00 \quad 0.0000$

$0.000 E+00 \cdot 0.0000$

$0.0008+00 \quad 0.0000$

$0.000 E+00 \quad 0.0000$

$0.000 E+00 \quad 0.0000$

$0.0008+00 \quad 0.0000$

$0.0008+00 \quad 0.0000$

0: $000 \mathrm{E}+00 \quad 0.0000$

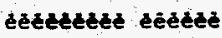

$0.000 E+00 \quad 0.0000$
Radon

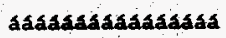

mrem/yr fract.

aadadasaa akakáa

$0.000 E+00 \quad 0.0000$

$0.000 E+00 \quad 0.0000$

$0.000 E+00 \quad 0.0000$

$0.000 E+00 \quad 0.0000$

$0.000 E+00 \quad 0.0000$

$0.000 E+00 \quad 0.0000$

$0.000 E+00 \quad 0.0000$

$0.0008+00 \quad 0.0000$

$0.000 E+00 \quad 0.0000$

$0.000 E+00 \quad 0.0000$

$0.000 E+00.0 .0000$

$0.0005+00 \quad 0.0000$

$0.0008+00 \quad 0.0000$

$0.000 E+00 \quad 0.0000$

èètelede éécted

$0.000 E+00 \quad 0.0000$
Plant

dáasadakadasadá

mren/yr fract.

sasalasá áasá

$0.000 E+00 \quad 0.0000$

$0.000 E+00 \quad 0.0000$

$0.0008+00 \quad 0.0000$

$0.0008+00 \quad 0.0000$

$0.000 E+00 \quad 0.0000$

$0.000 E+00 \quad 0.0000$

$0.000 E+00 \quad 0.0000$

$0.000 E+00 \quad 0.0000$

$0.000 E+00 \quad 0.0000$

$0.000 E+00 \quad 0.0000$

$0.000 E+00,0: 0000$

$0.000 E+00 \quad 0.0000$

$0.000 E+00 \quad 0.0000$

$0.000 E+00 \quad 0.0000$

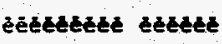

$0.000 E+00 \quad 0.0000$
Meat

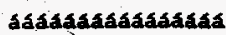
mren/yr Eract. áasasaáa dáasa $0.000 E+00$ ó.0000 $0.0008+00 \quad 0.0000$ $0.000 E+00 \quad 0.0000$ $0.000 E+00 \quad 0.0000$ $0.000 E+00 \quad 0.0000$ $0.0008+00 \quad 0.0000$ $0.000 E+00 \cdot 0.0000$ $0.000 E+00 \quad 0.0000$ $0.0008+00 \quad 0.0000$ $0.000 E+00 \quad 0.0000$ $0.000 E+00 \quad 0.0000$ $0.000 E+00 \quad 0.0000$ $0.000 E+00 \quad 0.0000$ $0.000 E+00 \quad 0.0000$

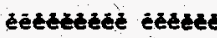
$0.000 E+00 \quad 0.0000$
Milk aasaakaakasasaá mrem/yr frace. aasadasá áajaa $0.000 E+00 \quad 0.0000$ $0.0008+00 \quad 0.0000$ $0.0008+00 \quad 0.0000$ $0.000 E+00 \quad 0.0000$ $0.000 E+00 \quad 0.0000$ $0.0008+00 \quad 0.0000$ $0.0005+00 \quad 0.0000$ $0.0008+00 \quad 0.0000$ $0.0008+00 \quad 0.0000$ $0.000 E+00 \quad 0.0000$ $0.000 E+00 \quad 0.0000$ $0.0008+000.0000$ $0.000 E+00 \quad 0.0000$ $0.0008+00 \quad 0.0000$

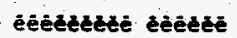
$0.0005+00 \quad 0.0000$
Soil

\section{aasadasasasaatas} mrem/yr fract. áasasaáá asasas $0.000 E+00 \quad 0.0000$ $0.000 E+00 \quad 0.0000$ $0.000 E+00 \quad 0.0000$ $0.000 E+00 \quad 0.0000$ $0.000 E+00 \quad 0.0000$ $0.0008+00 \quad 0.0000$ $0.0008+00 \quad 0.0000$ $0.000 \mathrm{E}+000.0000$ $0.000 E+00 \quad 0.0000$ $0.000 E+00 \quad 0.0000$ $0.0008+00 \quad 0.0000$ $0.000 E+00 \quad 0.0000$ $0.0008+00 \quad 0.0000$ $0.000 E+00 \quad 0.0000$

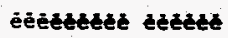
$0.000 E+00 \quad 0.0000$

Total Dose Contributions TDOSE (i,p,e) for Individual Radionuclides (i) and Pathways (p) As mrem/yr and fraction of Tocal Dose Ac $e=0.000 E+00$ years

wacer Dependent Pathways

\begin{tabular}{|c|c|c|c|c|c|c|c|c|c|c|c|c|c|c|}
\hline \multirow{2}{*}{$\begin{array}{l}\text { Radio- } \\
\text { Nuclide } \\
\text { aasasa }\end{array}$} & & \multicolumn{2}{|c|}{$\begin{array}{l}\text { Fian } \\
\text { aderastasasasad }\end{array}$} & \multicolumn{2}{|c|}{$\begin{array}{c}\text { Radon } \\
\text { dasuratadsasasa }\end{array}$} & \multicolumn{2}{|c|}{$\begin{array}{l}\text { Plane } \\
\text { adschasasadusas }\end{array}$} & \multicolumn{2}{|c|}{$\begin{array}{c}\text { Ment } \\
\text { adsamagsastasa }\end{array}$} & \multicolumn{2}{|c|}{$\begin{array}{l}\text { Malk } \\
\text { aasadasasasadad }\end{array}$} & \multicolumn{2}{|c|}{ 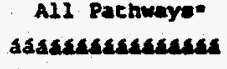 } \\
\hline & $\begin{array}{l}\text { mren/yt } \\
\text { dasasasat }\end{array}$ & $\begin{array}{l}\text { frace. } \\
\text { astids }\end{array}$ & $\begin{array}{l}\text { mren/yr } \\
\text { dasasast }\end{array}$ & $\begin{array}{l}\text { fract: } \\
\text { aasasa }\end{array}$ & $\begin{array}{l}\text { mrem/yr } \\
\text { adsisasas }\end{array}$ & $\begin{array}{l}\text { Eract. } \\
\text { asasas }\end{array}$ & $\begin{array}{l}\text { mreap/yr } \\
\text { sadasasa }\end{array}$ & $\begin{array}{l}\text { fract. } \\
\text { adacia }\end{array}$ & $\begin{array}{l}\text { mrea/yr } \\
\text { datususad }\end{array}$ & $\begin{array}{l}\text { fract. } \\
\text { dassas }\end{array}$ & mrem/yr & $\begin{array}{l}\text { fract. } \\
\text { asasas }\end{array}$ & $\begin{array}{l}\text { mrew/yr } \\
\text { asasucas }\end{array}$ & $\begin{array}{l}\text { frace. } \\
\text { asside. }\end{array}$ \\
\hline & 00 & 0.0000 & $E+00$ & 0.0000 & $.000 E+00$ & 0.0000 & $0.000 E+00$ & 0.0000 & $.000 E+00$ & 0.0000 & $.000 E+00$ & jo & $.000 E+00$ & 0 \\
\hline-14 & 0 & 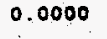 & $+\infty$ & 0.0000 & 00. & 0 & +00 & 0. & 0 & 0. & 00 & 10 & $.000 E+00$ & 10 \\
\hline ) & so & 0 & . & 0 & 8 & 0 & +00 & 0.0 & +00 & 0 & 10 & & 0 & 30 \\
\hline$C s-137$ & 00 & 0 & . & 0 & $c$ & C & .00 & 0. & & & 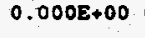 & & 2 & \\
\hline 2 & $.0008+00$ & 0.0 & $.0008+00$ & 00 & . & 0 & 00 & 0 & o: & 0 & 0 & & 3 & \\
\hline $1-254$ & 0 & . & 10 & 0 & 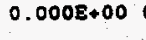 & 200 & $8+00$ & 0. & $\$ 00$ & 0. & $.000 \mathrm{O}+00$ & 0 & $.611 E-24$ & 02 \\
\hline $\mathrm{H}-3$ & 0. & ( & : & & i & c & 0 & 0 & 0 & & 10 & & po & 100 \\
\hline$i-59$ & 0.000 & c & +00 & & & & & & & & & & & \\
\hline$i-63$ & $0.000 \mathrm{E}+00$ & 30 & 80 & 0 & 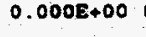 & & 0 & & & & 0 & & 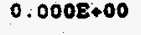 & \\
\hline $2-238$ & $8+00$ & 0.0000 & .00 & 0. & $0.000 \mathrm{E}+00$ & 0 & 0. & 0 & 0 & 0 & $\$ 00$ & 0 & 0 & \\
\hline 39 & 00 & 0.0000 & $.000 E+00$ & 0.0000 & $.000 E+00$ & 0.0 & 0.000 & 0.0000 & $0.0008+00$ & 0.0000 & $0.000 \mathrm{~B}+00$ & 0. & $.0008+00$ & 200 \\
\hline$x-20$ & 00 & 00 & $O E+00$ & 0.0000 & $.000 E+00$ & 0.0000 & $0.0008+00$ & 0.0000 & $0.000 \Sigma+00$ & 0.0000 & $0.0008+00$ & 0.0000 & $0.000 E+00$ & 0.0000 \\
\hline 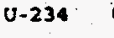 & 0 & i & 00 & 00 . & $008+00$ & 0.0000 & $000 E+00$ & 0.0000 & $0.0008+00$ & 200 & $00 \mathrm{~B}+00$ & 100 & $0,05+00$ & 0.0000 \\
\hline 238 & 0 & 0 & 10 & 0 & .00 & 0 & 00 & 00 & $\mathbf{E}+00$ & 0 & 00 & 0. & 36 & 0.0000 \\
\hline & seceses & e른 & 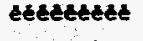 & ed & \pm & et & 8 & 2 & t. & 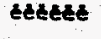 & at & 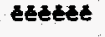 & $=$ & tet \\
\hline & $0.000 E+00$ & 0.0000 & $0.000 E+00$ & $0.0000^{\circ}$ & $0.000 E+00$ & 0.0000 & $0.0005+00$ & 0.0000 & $0.0008+00$ & 0.0000 & $0.000 E+00$ & 0.0000 & $1.977 \mathrm{E}-20$ & 1.0000 \\
\hline
\end{tabular}

- Sun of all wacer independent and dependent pathways. 
RESRAD, Version S.621:T Limit $=0.5$ year

Sumuary : Bio-Shield Activity Assessment HACTR
$03 / 06 / 97 \quad 32: 11$ Page 18 File: A: IBTOSHLD.DAT

Total Dose Coneributions IDOSE (i,p,t) for Individual Radionuclides (i) and Pathways (p) As mrem/yz and Fraction of Total Dose At $t=1.000 E+00$ years

Wacer Independent Pathways (Inhalation excludes radon)

Ground

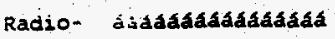

Nuclide mrem/yr sract.

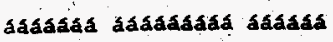

Am-242 $0.000 E+00 \quad 0.0000$

C-24 $\quad 0.000 \mathrm{E}+00 \quad 0.0000$

Co-60 1.729E-20 0.0000

Cs-137 4.499E-32 0.c: 00

Eu-152 $3 .+33 E-230.0000$

Eu-254 $3.342 E-240.0000$

$\mathrm{H}-3 \quad 0.000 \mathrm{E}+00 \quad 0.0000$

Ni-59 $\quad 0.000 \mathrm{E}+00 \quad 0.0000$

$N_{2}-63 \quad 3.000 E+00 \quad 0.0000$

Pu-2: $0.000 E+00 \quad 0.0000$

PU-239 $0.000 E+00 \quad 0.0000$

SE-90 $2,000 E+00 \quad 0.0000$

U.234 $0.000 E+00 \quad 0.0000$

uj-238 $\div 790 \mathrm{E}-360.0000$

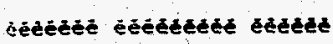

roeal $\quad 2.732 E-20 \quad 0.0000$
Inizalacion

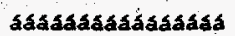

mrem/yr fract.

adasajasa ááasa

$0.0008+00.0 .0000$

$0.000 E+00 \quad 0.0000$

$0.000 E+00 \quad 0.0000$

$0.000 E+00 \quad 0.0000$

$0.0005+00 \quad 0.0000$

$0.000 E+00 \quad 0.0000$

$0.000 E+00 \quad 0.0000$

$0.0008+00 \quad 0.0000$

$0.0008+00 \quad 0.0000$

$0.000 E+000.0000$

$0.0008+00 \quad 0.0000$

$0.000 E+00 \quad 0.0000$

$0.0008+00 \quad 0.0000$

$0.000 E+00 \quad 0.0000$

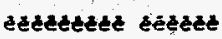

$0.000 E+00 \quad 0.0000$
Radon

daááááááaááá

\section{rrem/yr fract.}

aáakakáa aasaáa

$0.000 E+00 \quad 0.0000$

$0.000 E+00 \quad 0.0000$

$0.0008+00 \quad 0.0000$

$0.000 \mathrm{E}+00 \quad 0.0000$

$0.000 E+00 \quad 0.0000$

$0.000 E+00 \quad 0.0000$

$0.000 E+00 \quad 0.0000$

$0.000 E+00 \quad 0.0000$

$0.000 E+00 \quad 0.0000$

$6.0228-190.0005$

$0.000 E+00 \quad 0.0000$

$0.000 E+00 \quad 0.0000$

1. :915-25 0.9995

$2 .+24 E-210.0000$

èceteret tertet

$1.292 E-25 \quad 2.0000$
Plant

áááasasasaáaáa nren/yx fract áaaasaas aasasa

$0.0008+00 \quad 0.0000$

$0.0008+00 \quad 0.0000$

$0.000 E+00 \quad 0.0000$

$0.000 E+00 \quad 0.0000$

$0.000 E+000.0000$

$0.000 E+00 \quad 0.0000$

$0.0008+00 \quad 0.0000$

$0.0008+00 \quad 0.0000$

$0.000 E+00 \quad 0.0000$

$0.000 E+00 \quad 0.0000$

$0.000 E+00 \quad 0.0000$

$0.000 E+00 \quad 0.0000$

$0.000 E+00 \quad 0.0000$

$0.000 E+000.0000$

éejtatese exthet

$0.000 E+00 \quad 0.0000$

Total Dose Coneributions TDOSE(i.p.t) for Individual Radionuclides (i) and Pathrays (p) As mrem/yr and Fraction of Total Dose At $c=1.0008+00$ years

\begin{tabular}{|c|c|c|c|c|c|}
\hline \multirow{2}{*}{\multicolumn{2}{|c|}{$\begin{array}{c}\text { Meat } \\
\text { akadáakasaáaka }\end{array}$}} & \multicolumn{2}{|c|}{ Milk. } & \multicolumn{2}{|c|}{ Soil } \\
\hline & & 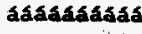 & áa & áá & Sas \\
\hline mrem/yx & fI & mirea/yx & $\mathbf{f}$ & mrem/yr & fract. \\
\hline & aAaAaA & adasasasa & asá & 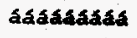 & sassax \\
\hline : & 0.0000 & $0.000 E+00$ & 0.0000 & $0.0008+00$ & 0.0000 \\
\hline 10 & 0.0000 & $0.000 \mathrm{~B}$ & 0.0000 & $0.000 E+00$ & 0.0000 \\
\hline 0 & 0. & $0.000 E+00$ & 0.0000 & $0.000 E+00$ & 0.0000 \\
\hline$E+00$ & 000 & $0.000 E+00$ & 0.0000 & $0.000 \mathrm{E}+00$ & 0.0000 \\
\hline$E+00$ & .0 .0000 & $0.000 \mathrm{E}+00$ & 0.0000 & $0.000 \varepsilon+00$ & 0.0000 \\
\hline$E+00$ & 0.0000 & $0.000 E+00$ & 0.0000 & .000 & 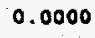 \\
\hline$E+C 0$ & 0.0000 & $0.000 E+00$ & 0.0000 & 0 & 0 \\
\hline $2+00$ & 0.0000 & $0.000 E+00$ & 0.0 & 0.00 & 0.000 \\
\hline $8+00$ & 0.0000 & $0.000 \mathrm{E}+00$ & 0 . & $0.000 E+00$ & 0.0000 \\
\hline$\$+00$ & 0.0000 & $0.000 E+00$ & 0. & $0.0008+00$ & 0.0000 \\
\hline$E+00$ & 0.0000 & $0.000 E+00$ & 0.00 & $0.000 \mathrm{E}+00$ & 0.0000 \\
\hline $0 E+00$ & 0.0000 & $0.000 E+00$ & 0.0000 & $0.000 \varepsilon+00$ & 0.0000 \\
\hline$D E+00$ & 0.0000 & $0.000 E+00$ & 0.0000 & $0.000 E+00$ & 0.0000 \\
\hline $08+00$ & 0.0000 & $0.000 E+00$ & 0.0000 & $0.000 \mathrm{E}+00$ & 0.0000 \\
\hline eteted & éécelt & Séétetedè & ejodet & èétedeted & cejedt \\
\hline $008+00$ & 0.0000 & $0.000 E+00$ & 0.0000 & $0.000 E+00$ & 0.00 \\
\hline
\end{tabular}

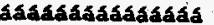

.

(1)

(1)

wacer Dependent Pachways

\begin{tabular}{|c|c|c|c|c|c|c|c|c|c|c|c|c|c|c|}
\hline \multirow{2}{*}{$\begin{array}{l}\text { Radio- } \\
\text { yelide } \\
\text { idasada }\end{array}$} & \multicolumn{2}{|c|}{$\begin{array}{l}\text { Wacer } \\
\text { sdasusasasadadada }\end{array}$} & \multicolumn{2}{|c|}{$\begin{array}{c}\text { Fish } \\
\text { sasusasastadadad }\end{array}$} & \multicolumn{2}{|c|}{$\begin{array}{c}\text { Radon } \\
\text { dadestasadasuassa }\end{array}$} & \multicolumn{2}{|c|}{$\begin{array}{c}\text { Plant } \\
\text { adsusangastiest }\end{array}$} & \multicolumn{2}{|c|}{$\begin{array}{c}\text { Meat } \\
\text { asamasasasasasa }\end{array}$} & \multicolumn{2}{|c|}{$\begin{array}{c}\text { Milk } \\
\text { adastadisadastasa }\end{array}$} & \multicolumn{2}{|c|}{ 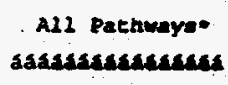 } \\
\hline & $\begin{array}{l}\text { mrem/yr } \\
\text { adasadaua }\end{array}$ & $\begin{array}{l}\text { frace. } \\
\text { aasasa }\end{array}$ & $\begin{array}{l}\text { mrewo/yr } \\
\text { adsusasas }\end{array}$ & $\begin{array}{l}\text { fract. } \\
\text { asassa }\end{array}$ & $\begin{array}{l}\text { rem/yr } \\
\text { susuasa }\end{array}$ & $\begin{array}{l}\text { fract. } \\
\text { asasas }\end{array}$ & $\begin{array}{l}\text { mrem/ye } \\
\text { adsusas }\end{array}$ & $\begin{array}{l}\text { frace. } \\
\text { aasas }\end{array}$ & $\begin{array}{l}\text { mrem/yr } \\
\text { sasalsasas }\end{array}$ & Erac & $\begin{array}{l}\text { gem/yx } \\
\text { Masasas }\end{array}$ & 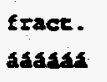 & wys & \\
\hline-7 & $.000 E+00$ & 0.0000 & $=00$ & 0.0 & & 0 & $.000 E+00$ & & $008+00$ & 0 & $.0008+00$ & & $.000 \mathrm{E}+00$ & 00 \\
\hline & 0.00 & & $5+00$ & $0 . c$ & $1008 \cdot 00$ & 0.0000 & $0.0008+00$ & 200 & $.000 E+00$ & 00 & $0,000 \mathrm{E}+00$ & 100 & $0.0008+00$ & 00 \\
\hline 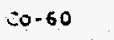 & so. & & $:+\infty$ & 0. & $: 00$ & 0.0000 & $E+00$ & 0. & $.000 E+00$ & 100 & $0.0008+00$ & 100 & $E-20$ & 10 \\
\hline & 00 . & & .00 & 0 & $+\infty 0$ & 00 & $E * 00$ & 100 & $.0008 * 00$ & 100 & $E+00$ & 000 & -32 & 10 \\
\hline 52 & .00 & & & & 00 & 20 & $8+00$ & 00 & $8+00$ & & & 100 & -23 & \\
\hline 154 & .00 & & & & .00 & 00 & $.0008+00$ & 100 & $0.000 E+00$ & 200 & $0.0008+00$ & 200 & & \\
\hline-3 & 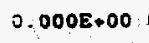 & & & & & . & $.0008 * 00$ & 200 & $0.0008+00$ & & $0.000 E+00$ & & $+\infty$ & \\
\hline & 00 & 0 & 0 & 0 & & & & & & & & & & \\
\hline .63 & .00 & 00 & a. & 00. & 10 & $\therefore$ & 00 & & & 0 & & & $0.0008 * 00$ & \\
\hline & 00 & 00 & $0005+00$ & 100 & 00 & 00 & +00 & 0 & $E \nrightarrow 00$ & 00 & $8+00$ & & -19 & Nos \\
\hline - & & 20 & ? & 000 & $.000 E+00$ & 0.0000 & $0.000 E+00$ & 000 & $0.000 E+00$ & 0.0 & $8+00$ & 100 & $0.0008+00$ & 000 \\
\hline 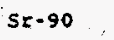 & & & 400 & 100 & .00 & 0.0000 & $.000 E+00$ & 0.0000 & $0.000 \mathrm{E}+00$ & 200 & $E+00$ & 0.0000 & $0.000 E+00$ & 000 \\
\hline-234 & & & $8+00$ & 0.0000 & $0.000 \mathrm{E}+00$ & 0.0000 & $0.000 E+00$ & 000 & $0.000 E+00$ & 0.0000 & $08+00$ & 0.0000 & $1.191 E-15$ & \\
\hline & & & $\varepsilon+00$ & 0.0 & $0.000 \mathrm{E}$ & 0.0000 & $0.000 E+00$ & 100 & 0.000 & 0.0 & $0 E+00$ & 0.0000 & $2.424 E-21$ & 0.0000 \\
\hline & & & eteet & es & èettestet & Secket & 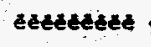 & & & & & & èēet & 6 \\
\hline 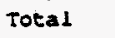 & $0.000 \mathrm{E} * 00$ & 0.0000 & $0.0008+00$ & & $0.000 \mathrm{E}+00$ & 0.0000 & $.0008+00$ & 0.0000 & $0.000 E+00$ & & $.000 E+00$ & & $1.2928-15$ & 1.0000 \\
\hline
\end{tabular}


$0000^{\circ} \tau$ ET-356T'T

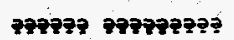
$0000 \cdot 0$ : $82-3 \angle 0+2$

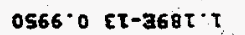
$0000 \cdot 00+3000 \cdot 0$ $0000.000+3000^{\circ} 0$ $6200 \div 09 \pi-3506 . \mathrm{s}$ $0000 \cdot 00+3000 \cdot 0$ $0000 \div 00+3000 \div 0$ $0000 \% 00+3000 \% 0$ $0000 \% 0+2-3659$. $\tau$ 0000 0 Ez-30LI $z$. $0000^{\circ} 0$ zE-3E69. $\varepsilon$ 0000.0 Iz-35sz's $0000 \div 00+3000: 0$ $0000 \cdot 00+3000 \cdot 0$

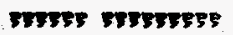
- zosez IX/mori browstreneses -exmayjea tTV
$0000.000+3000 \cdot 0$

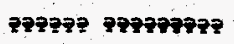
$0000000+3000: 0$ $0000.000+3000.0$ $0000 \cdot 000+3000.0$ $0000: 000+3000.0$ $0000 \cdot 000+3000 \cdot 0$ $0000 \cdot 000+3000.0$ $0000 \cdot 000+2000 \cdot 0$ $0000 \cdot 0 \quad 00+3000 \cdot 0$ $0000.000+2000.0$ $0000 \cdot 000+2000 \cdot 0$ $0000.000+3000.0$ $0000 \cdot 000+2000 \cdot 0$ $0000 \cdot 000+3000 \cdot 0$ $0000000+3000 \cdot 0$ eEsegs retrisese - כ5exI 2K/0oIw 55F9e5954) XTFW
$0000 \cdot 000+3000 \cdot 0$

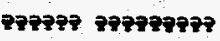
$0000 \% 00+3000 \%$ $0000 \cdot 0.00+2000 \cdot 0$ $0000000+9000.0$ $0000 \% 00+3000 \%$ $0000 \cdot 0.00+8000 \cdot 0$ $0000=00+8000 \cdot 0$ $0000000+9000=0$ $0000-00+2000 \% 0$ $0000 \div 00+3000: 0$ $0000 \cdot 0 \quad 00+3000 \% 0$ $0000.000+3000 \%$ $0000000+3000.0$ $0000 \cdot 00+3000 \cdot 0$ $0000 \div 00+3000 \cdot 0$

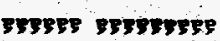
-208x; دर/ 535355535545355 2000
$0000 \% 00+3000 \%$

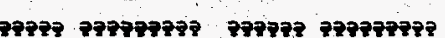
$0000 \cdot 00+3000 \cdot 0 \quad 0000 \cdot 0: 00+3000 \cdot 0$ $0000000+3000 \cdot 0000000+30000$ $0000.000+3000-0000 \% 00+3000 \%$ $0000-0,00+3000 \cdot 0 \quad 0000 \cdot 000+3000 \%$ $0000 \div 00+3000 \% 0000 \div 00+3000=0$ $0000000+3000.00000 \% 00+3000.0$ $0000 \% 00+3000 \% 0000 \% 00+3000 \% 0$ $0000 \% 00+3000.00000 .000+3000.0$ $0000 \% 00+3000 \% 0000.000+3000.0$ $0000 \% 00+3000 \cdot 00000 \cdot 000+3000=0$ $0000 \% 00+3000 \% 0000 \div 00+3000 \cdot 0$ $0000.000+3000.0 \quad 0000 \cdot 0 \quad 00+3000 \cdot 0$ $0000.000+3000 \% 00000000+3000 \%$ $0000-00+3000 \div 0000000+3000 \% 0$

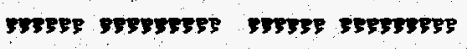

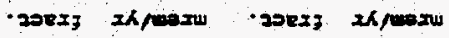
resserseserserse 2tret voper
$0000 \cdot 000+30000$

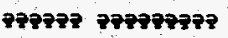
$0000 \div 0009000.0$ $0000.000+3000 \cdot 0$ $0000 \cdot 00+3000 \%$ $0000-000+3000=0$ $0000.000+3000.0$ $0000 \% 00+3000.0$ $0000 \times 0.00+3000 \cdot 0$ $0000: 000+3000 \% 0$ $0000 \div 00+3000 \%$ $0000 \cdot 00+3000 \%$ $0000.00+3000 \%$ $0000 \cdot 00+3000.0$ $0000.000+3000.0$ $0000: 000+30000$

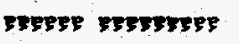
- zoras zk/moxi. 4อTd

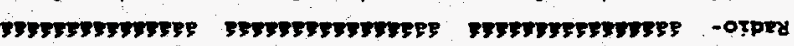

$0000 \% 00+3000 \%$ tejos

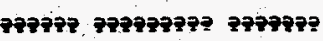
$0000000+9000 \cdot 0$ BEz$0000.00+3000 \%$ H $0000 \cdot 000+3000 \div 0$ 06-25 $0000 \cdot 0 \quad 00+3000 \cdot 0 \quad 6 \varepsilon z-n d$ $0000 \% 00+3000: 0$ 8Ez-nd $0000^{-0} 00+9000^{-0}$ Eg-th $0000000+3000$ 0 $65-5 N$ $0000.000+3000 \% \quad E-H$ $0000 \cdot 0.00+3000: 0$ \$5T-nב $0000 \% 00+3000^{\circ} 0 \quad 25 \pi-\pi$ $0000.000+30000$ LET-50 $0000 \% 00+3000 \% 09-00$ $0000 \cdot 000+3000 \cdot 0 \quad \square[-5$ $0000.00+3000.0$ toz-w

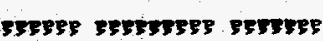
-20ex 3 2X/woxw optTonk sesen

exenuzed zuopubded I0JEM

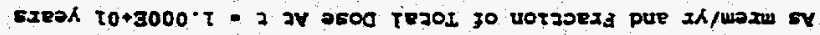

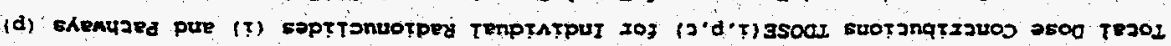

$0000 \cdot 000+3000 \cdot 0$

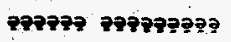
$0000: 00+3000 \cdot 0$ $0000 \cdot 0 \cdot 00+3000: 0$ $0000000+3000.0$ $0000 \% 00+3000 \%$ $0000.000+3000.0$ $0000.00+3000.0$ $0000 \cdot 000+3000 \%$ $0000 \cdot 0.00+3000 \%$ $0000000+3000 \%$ $0000 \div 000+3000.0$ $0000-00+3000 \cdot 0$ $0000.000+3000.0$ $0000-000+3000.0$ $0000 \cdot 000+3000=0$ resere pepespeps -20exz 2A/mark 9eperpsegeregeps. tros
$0000 \cdot 000+30000$

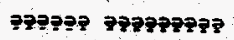
$0000 \cdot 000+3000.0$ $0000000+3000.0$ $0000.00+3000 \%$ $0000.000+3000.0$ $0000 \% 00+30000$ $0000 \cdot 000+3000.0$ $0000 \cdot 000+3000: 0$ $0000 \cdot 000+2000.0$ $0000 \cdot 0.00+8000.0$ $0000 \cdot 00+3000 \cdot 0$ $0000 \cdot 000+3000 \cdot 0$ $0000=00+3000=0$ $0000 \cdot 000+2000.0$ $0000.000+2000.0$

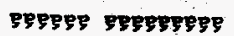
20eבI xK/w02w

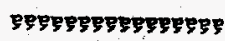
xt Tw

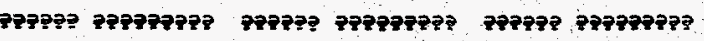
$0000000+3000 \div 0000 \% 00+3000 \cdot 00000 \% 0$ 8 $-3.000: z$ $0000.000+3000.0 \quad 0000 \% 00+3000 \% 0 \quad 0566.0 \varepsilon \tau-368 \tau T$ $0000 \% 00+3000 \cdot 0 \quad 0000 \div 00+3000 \% 0000 \% 00+3000 \%$ $0000 \cdot 000+3000 \cdot 0 \quad 0000 \cdot 000+3000 \cdot 00000-000+3000 \cdot 0$ $0000 \% 00+3000 \cdot 0 \quad 0000 \% 00+3000 \cdot 06400 \% 9 \tau-3506.5$ $0000 \cdot 000+3000 \cdot 0 \quad 0000 \cdot 0 \quad 00+3000 \cdot 0 \quad 0000 \% 00+3000 \cdot 0$ $0000 \cdot 0 \quad 00+3000 \cdot 0 \quad 0000 \cdot 0 \quad 00+3000 \div 0000 \cdot 0 \quad 00+3000 \cdot 0$ $0000-000+3000-00000-000+3000-00000-000+3000.0$ $0000 \% 00+3000 \cdot 0 \quad 0000 \% 00+3000 \cdot 0 \quad 0000 \div 000+3000 \cdot 0$ $0000 \% 00+3000 \% 0000 \% 00+3000 \% 0000 \div 000+3000 \% 0$ $0000: 0 \quad 00+3000 \% 0000 \% 00+3000-0 \quad 0000 \div 000+3000=0$ $0000000+3000 \% 00000000+3000 \% 0000 \cdot 000+3000 \%$ $0000000+3000 \cdot 0 \quad 0000 \% 00+3000 \% 0000 \% 00+3000 \cdot 0$ $0000000+3000-00000-00+300000000-000+3000=0$

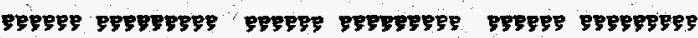
- joes; $x$ K/worw

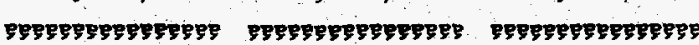
วnew zureta

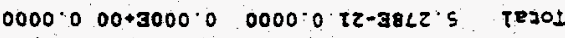

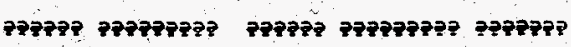
$0000 \cdot 00+3000 \div 000009 \varepsilon-36+6$ 8 $8 E z-n$ $0000000+300000000 \cdot 000+3000 \cdot 0$ bez-n $0000000+3000 \% 0000 \% 00+3000 \%$ 06-35 $0000000+3000 \% 0000000+3000 \cdot 0$ 6EZ-nd $0000 \cdot 000+3000 \% 0000 \% 00+3000 \%$ BEz-nd $0000 \% 00+3000 \cdot 0 \quad 0000 \cdot 000+3000 \%$ E9-7N $0000 \cdot 000+3000 \cdot 0 \quad 0000 \div 000+3000 \cdot 0 \quad 6.5 \mathrm{NN}$ $0000 \% 00+3000 \cdot 00000000+30000 \quad \varepsilon+H$ $0000 \% 00+3000 \% 0000 \% 0 z-3659 \cdot$ I b5t-n3

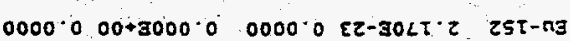
$0000000+3000.0 \quad 0000.0$ ZE-3E69.E LET-SJ $0000 \% 00+3000 \% 0000 \%$ Iz-35sz 5 09-00 $0000000+3000 \% 0000+00+3000 \% \quad 3 \tau-5$

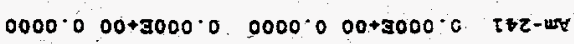

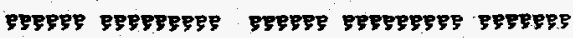

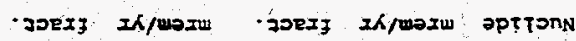

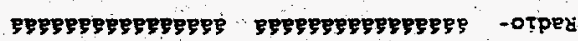

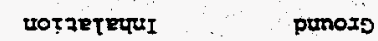

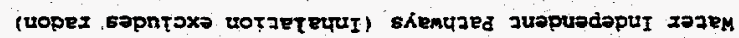

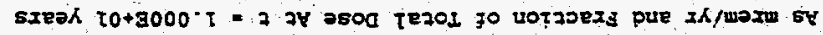

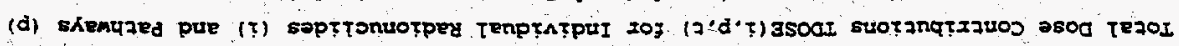

$$
\begin{aligned}
& \text { IHO ansorel: } \\
& \text { 6T Dsed TT:ZT L6/90/عO }
\end{aligned}
$$

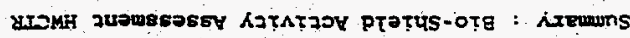

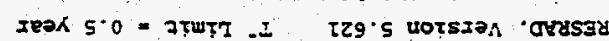


RESRAD, Version 5.621 T Limit $=0.5$ year

Sumary : Bio-Shield Activity Assessment hwCTR
03/06/97 12:11. Page 20

Eile: A: \BIOSHWD.DAT

Totsi Dose Contributions IDOSE(i,p.t) for Individual Radionuclides (i) and Pachways (p) As mrem/yr and Fraction of Total Dose At $t=3.000 E+0 ?$ years

Water Independent Pathways (Inhalation excludes radon)

\begin{tabular}{|c|c|c|c|c|c|c|c|c|c|c|c|c|c|c|}
\hline \multirow[b]{2}{*}{$\begin{array}{l}\text { Radio- } \\
\text { Nuclide } \\
\text { aásadas }\end{array}$} & \multicolumn{2}{|c|}{$\begin{array}{c}\text { Ground } \\
\text { adastakakakakasa }\end{array}$} & \multicolumn{2}{|c|}{$\begin{array}{c}\text { Iribalation } \\
\text { áasasaasaááasaa }\end{array}$} & \multicolumn{2}{|c|}{$\begin{array}{c}\text { Radon } \\
\text { áááááasaáááá }\end{array}$} & \multicolumn{2}{|c|}{ 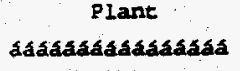 } & \multicolumn{2}{|c|}{$\begin{array}{c}\text { Meat } \\
\text { asaasaááááááá }\end{array}$} & \multicolumn{2}{|c|}{ Milk } & \multicolumn{2}{|c|}{$\begin{array}{c}\text { Soil } \\
\text { áasasasasasasas }\end{array}$} \\
\hline & $\begin{array}{l}\text { adádidadá } \\
\text { mrem/yx }\end{array}$ & $\begin{array}{l}\text { fract. } \\
\text { aááas }\end{array}$ & $\begin{array}{l}\text { mrem/yr } \\
\text { áakadada }\end{array}$ & fract. & mrem/yr & $f$ & lys & fra & ys & $\cdot$ & Yas & 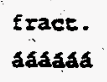 & yas & \\
\hline-241 & ? & 0 & 00 & 0.0 & 00 & 0.0000 & 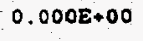 & & & & . & . & 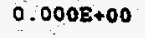 & 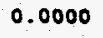 \\
\hline 4 & o & 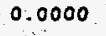 & 0. & 00 & 00 & 0. & 400 & 10 & 00 & & 00 & 100 & 7 & 7 \\
\hline .60 & 2 & 10. & 0 & Con & 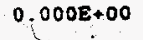 & 0 & 00 & 0 & 00 & & 00 & 0 & 0 & \\
\hline$\$ 237$ & 32 & so & & & . & c & 0 & 0 & 00 & & +00 & 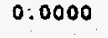 & 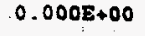 & \\
\hline-152 & 8.8 & 20 & & & & & 0 & & 20 & & 00 & 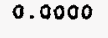 & & \\
\hline $1-154$ & $3: 5$ & 10 & & & 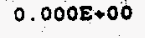 & & $\mathbf{0}$ & & 0 & & 10 & & & \\
\hline$\rightarrow$ & 0.0 & 0.0 & . & & & & 0 & & & & o & & & \\
\hline$->9$ & . & 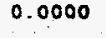 & 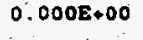 & & $\because$ & & & & & & & & & \\
\hline-63 & 0.00 & 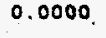 & ? & ( & . & & & & & & & & & \\
\hline $1-238$ & $0.0008+00$ & 0.0 & $+\infty$ & 0 & 4 & & & & & & & & & \\
\hline $2-239$ & $0.0008+00$ & 0.0000 & $.000 E+00$ & 0.0000 & 0.00 & 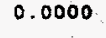 & ? & & $2+00$ & & 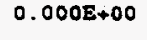 & & & \\
\hline$\because \quad \ln$ & $0.0008+00$ & 0.0000 & $.0005+00$ & 0.0000 & 0.00 & 0. & 10 & & 00 & & 00 & 0 & 00 & s. \\
\hline & $0.000 E+00$ & $0: 0000$ & $+\infty$ & 000 & -12 & 630 & 0 & & +00 & & $+\infty$ & 000 & .00 & 0. \\
\hline$\therefore$ & $9: 98$ & & $.000 \varepsilon+00$ & 00 & $6.460 E-17$ & 0.0001 & 0 & ( & $+\infty$ & 100 & $8+00$ & 000 & $2+00$ & ?ou \\
\hline r & Ext & & te & & $e 3$ & ee & cet & e & seed & es & ee & & teet & \\
\hline & -22 . & & +00 & 10 & -12 & 1. & $0.0008+00$ & 0. & $000 \mathrm{E}+00$ & 0.0000 & +00. & 00 & 00 & \\
\hline
\end{tabular}

Total Dose Coneribueions IDOSE (i,p,e) for Iridividual Radionuclides (i) dnd Pathways (p) As mrem/yr and Fraction of Total Dose At $\varepsilon=3.000 E+01$ years

Water Dependent Pathways

wacer

Radio- sasmassasasasa Nuclide nzew/yr fract.

sanasa dasasasa asasas

Aw-24: $: .000 E+00 \quad 0.0000$

$C-24 \quad 0.0008+00 \quad 0.0000$

Co-60 $0.000 E+00 \quad 0.0000$

Cs-23? $0.000 E+00 \quad 0.0000$

Eu-152 $0.000 E+00 \quad 0.0000$

su-154 $\quad 0.0008+00 \quad 0.0000$

$\mathrm{H}-3 \quad 0.000 \mathrm{E}+00 \quad 0.0000$

Ni-59 $0.0008+00 \quad 0.0000$

Ni-63 0.0008+00 0.0000

Pu-238 $0.000 E+00 \quad 0.0000$

pu-239 $0.000 \mathrm{E}+00 \quad 0.0000$

$S r-90 \quad 0.0008+00 \quad 0.0000$

$u-234 \quad 0.000 E+00 \quad 0.0000$

U-238 $\quad 0.0008+00 \quad 0.0000$

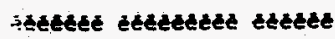

Toeal $0.0008+00 \quad 0.0000$
Fish

Rish Radon mren/yr Erace.

\section{asalnasis adasad}

$0.0008+00 \quad 0.0000$

$0.0008+00 \quad 0.0000$

$0.0000 \quad 0.0008+00 \quad 0.0000$

0.0008000 .00000 .00080000 .0000

$0.0008+000.000$

$0 . ; 000 E+00 \quad c .0000$

$0.000 E+00.0 .0000$

$0.000 E+00 \quad 0.0000$

$0.0008+00 \quad 0.0000$

$0.0008+000.0000$

$0.0008 * 00 \quad 0.0000$

$0.000 E+00 \quad 0.0000$

$0.000 E+000.0000$

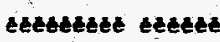

$0.000 E+00 \quad 0.0000$
Plant

adadusasassasasa

mrew/yr Erace.

\section{dascasas sacuas}

$0.0008+00.0 .0000$

$0.000 E \rightarrow 00 \quad 0.0000$

$0.000 E+00 \quad 0.0000$

$0.0008+00 \quad 0.0000$

$0.000 E+00 \quad 0.0000$

$0.0008+00 \quad 0.0000$

$0.0008+00 \quad 0.0000$

$0.000 E+00 \quad 0.0000$

$0.000 E+00 \cdot 0.0000$

$0.0008+00 \quad 0.0000$

$0.000 E+00 \quad 0.0000$

$0.0008+00 \quad 0.0000$

$0.000 \mathrm{E}+00 \quad 0.0000$

$0.000 E+00.0 .0000$

jecteteter cetet

$0.0008+00 \quad 0.0000$
Ment

dassuasadassanas mrem/yr frace.

dastusass sasss

$0.000 E+00 \quad 0.0000$

$0.0008+00 \quad 0.0000$

$0.0005+00 \quad 0.0000$

$0.0008+00 \quad 0.0000$

$0: 000 E+00 \quad 0.0000$

$0.000 E+00 \quad 0.0000$

$0.000 E+00 \quad 0.0000$

$0.0005+00 \quad 0.0000$

$0.000 E+00 \quad 0.0000$

$0.000 E+00 \quad 0.0000$

$0.000 E+00 \quad 0.0000$

$0.000 E+00 \cdot 0.0000$

$0.0008+00 \quad 0.0000$

$0.0008+00 \quad 0.0000$

destekted ected

$0.000 E+00 \quad 0.0000$
Milk adscasalasasadad mrem/yr fract.

\section{adsusasaa aasasa}

$0.000 E+00 \quad 0.0000$

$0.000 E+00 \quad 0.0000$

$0.000 E+00 \quad 0.0000$

$0.0008+00 \quad 0.0000$

$0.000 E+00 \quad 0.0000$

$0.0005+00 \quad 0.0000$

$0.0008+00 \quad 0.0000$

$0.0008+000.0000$

$0.000 E+00 \quad 0: 0000$

$0.000 E+00 \quad 0.0000$

$0.000 E+00 \quad 0.0000$

$0.000 E+00 \quad 0.0000$

$0.000 E+00 \quad 0.0000$

$0.0008+00 \quad 0.0000$

stectestet entert

$0.0008+00 \quad 0.0000$
All Pachwaye dancsuascaseses mrew/ys fract. assarsas senes $0.0008+00 \quad 0.0000$ $0.000 E+00 \quad 0.0000$ $3.727 E-22 \quad 0.0000$ $2.382 E-32 \quad 0.0000$ ?.826E-24 0.0000 3.5018-25 0.0000 $0.0008+00 \quad 0.0000$ $0.0008+00 \cdot 0.0000$ $0.0008+00 \quad 0.0000$ $1.528 E-14 \quad 0.0241$ $0.0008+00 \quad 0.0000$ $0.0008+00 \quad 0.0000$ $1.065 E-12 \quad 0.9858$ $6.4608-17 \quad 0.0002$ èctatedes colut 1. $0808-121.0000$

- Sum of all wacex independent and dependent pathways. 
RESRAD, Version $5.621 \quad T$ Limit $=0.5$ year

Sumary : Bio-Shield Accivity Assessmenc iwCTR
03/06/97 12:12 Page 21

File: A: SBrOSHTD.DAT

Total Dose Contributions IDOSE(i.p. i) for Individual Radionuclides (i) and Pathways (p) As $m r e m / y$ and Fraction of Total Dose At $t=5.000 E+01$ years

Water Independent pathways (Inhalation excludes radon)

\section{Ground}

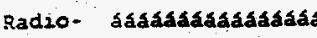
Nuclide mrem/yr Eract. adáasá áasaasasa asasada $A m-241 \quad 0.000 E+00 \quad 0.0000$ c-14 $\quad 0.000 E+00 \quad 0.0000$ Co-60 $\quad 2.643 E-23 \quad 0.0000$ Cs-137 1.536E-32 0.0000 Eu-152 2.823E-24 0.0000 Eu-154 T.390E-26 0.0000 $\mathrm{H}-3 \quad 0.000 \mathrm{E}+00 \quad 0.0000$ Ni-59 $0.0008+00 \quad 0.0000$ Ni $-63: 0.0008+00 \quad 0.0000$ Pu-238 $\quad 0.000 \mathrm{E}+00 \quad 0.0000$ Pu-239: $0: 000 E+00 \quad 0.0000$ Sr-90 $\quad 0.000 E+00 \quad 0.0000$ U.234 $0.000 E+00 \cdot 0.0000$ $J-238 \quad 1 \quad 012 E-35 \quad 0.0000$

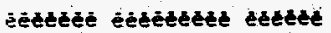
Total $=933 \mathrm{E}-230.0000$
Inhalation aasasadaasadaaáa mren/ye fract. adasaadaa aasaá

$0.000 E+00 \quad 0.0000$ $0.000 E+00 \quad 0.0000$ $0.000 E+00 \quad 0.0000$ $0.000 E+00 \quad 0.0000$ $0.0008+00 \quad 0.0000$ $0.0008+00 \quad 0.0000$ $0.000 E+00 \quad 0.0000$ $0.000 E+000.0000$ $0.000 E+00 \quad 0.0000$ $0.000 E+00 \quad 0.0000$ $0.000 E+00 \quad 0.0000$ $0.000 E+00 \quad 0.0000$ $0.000 E+00 \quad 0.0000$ $0.000 E+00 \quad 0.0000$

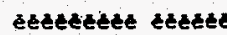
$0.000 E+00 \quad 0.0000$
Radon aáasasaáasaadá mrem/yx fract. afasasaka adaasa $0.000 E+00 \quad 0.0000$ $0.0008+00 \quad 0.0000$ $0.0008+00 \quad 0.0000$ $0.0008+00 \quad 0.0000$ $0.000 E+00 \quad 0.0000$ $0.0008+00 \quad 0.0000$ $0.000 \mathrm{E}+00 \quad 0.0000$ $0.000 E+00 \quad 0.0000$ $0.000 E+00 \quad 0.0000$ $6.782 E-240.0225$ $0.000 E+00 \quad 0.0000$ $0.000 E+00 \quad 0.0000$ $2.943 E-12 \quad 0.9774$ $2.974 E-16 \quad 0.0001$

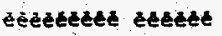
3.0215-12 1.0000
Plane áasaakaaasaaaá rrem/yr frack. sáassasa aasasa $0.000 E+00 \quad 0.0000$ $0.000 E+00 \quad 0.0000$ $0.000 E+000.0000$ $0.000 E+00 \quad 0.0000$ $0.000 E+000.0000$ $0.000 E+00 \quad 0.0000$ $0.0008+00 \quad 0.0000$ $0.000 E+00 \quad 0.0000$ $0.000 E+00 \quad 0.0000$ $0.0008+00 \cdot 0.0000$ $0.000 E+00 \cdot 0.0000$ $0.000 E+00 \quad 0.0000$ $0.000 E+000.0000$ $0.000 E+00 \quad 0.0000$ fécteteter ctutest $0.0008000 \quad 0.0000$
Meac adaสałaakáałaá mrem/yr fract. assasaaáa áasasa $0.000 E+00 \quad 0.0000$ $0.000 E+00 \quad 0.0000$ $0.000 E+00 \quad 0.0000$ $0.000 E+00 \quad 0.0000$ $0.000 E+00 \quad 0.0000$ $0.000 E+00 \quad 0.0000$ $0.000 E+00 \quad 0.0000$ $0.000 E+00 \quad 0.0000$ $0.0005+00 \quad 0.0000$ $0.000 E+00 \quad 0.0000$ $0.000 E+000.0000$ $0.000 E+00 \quad 0.0000$ $0.000 E+00 \quad 0.0000$ $0.000 E+00 \quad 0.0000$

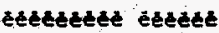
$0.0008+00 \quad 0.0000$
Milk

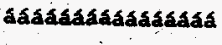
mrem/yr fract. dáasaaáá asaasá $0.000 E+00 \quad 0.0000$ $0.0008+00 \quad 0.0000$ $0.0005+00 \quad 0.0000$ $0.000 E+00 \quad 0.0000$ $0.000 E+00 \quad 0.0000$ $0.000 E+00 \quad 0.0000$ $0.000 E+00 \quad 0.0000$ $0.0008+00 \quad 0.0000$ $0.000 E+00 \quad 0.0000$ $0.000 E+00 \quad 0.0000$ $0.000 E+00 \quad 0.0000$ $0.000 E+00 \quad 0.0000$ $0.000 \mathrm{E}+000.0000$ $0.000 E+00 \quad 0.0000$ ejeztedict ejectes $0.0008+000.0000$ soil

gakasasadadasasa mrem/yr fract. cáasakasa kastas $0.0008+00 \quad 0.0000$ $0.0008+00 \quad 0.0000$ $0.0005+00 \quad 0.0000$ $0.0008+00 \quad 0.0000$ $0.0008+00 \quad 0.0000$ $0.0005+00 \quad 0.0000$ $0.0008+00 \quad 0.0000$ $0.0005+00 \quad 0.0000$ $0.000 \mathrm{E}+000.0000$ $0.0008+00 \quad 0.0000$ $0.000 E+00 \quad 0.0000$ $0.000 E+00 \quad 0.0000$ $0.0002+00 \quad 0.0000$ $0.000 E+00 \quad 0.0000$ exezdeted etctid $0.000 E+00 \quad 0.0000$

Total Dose Contributions fDose(i.p.e) for Individual Radionuelides (i) and Pachways (p) As mrem/yz and Fraction of Toeal Dose Ae $t \cdot 5.000 E+01$ years

Water Dependent Pathways

\begin{tabular}{|c|c|c|c|c|c|c|c|c|c|c|c|c|c|c|}
\hline \multirow{2}{*}{ iclide } & \multicolumn{2}{|c|}{$\begin{array}{c}\text { Woeer } \\
\text { dasassasasasusa }\end{array}$} & \multicolumn{2}{|c|}{ 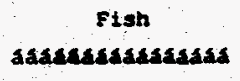 } & \multicolumn{2}{|c|}{$\begin{array}{c}\text { Radon } \\
\text { adasuasadasasasa }\end{array}$} & \multicolumn{2}{|c|}{ 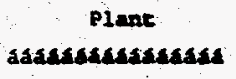 } & \multicolumn{2}{|c|}{$\begin{array}{l}\text { Mene } \\
\text { adasmasasadasada }\end{array}$} & \multicolumn{2}{|c|}{ Milk } & \multicolumn{2}{|c|}{ 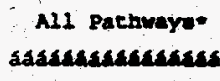 } \\
\hline & & & $\begin{array}{l}\text { Yr } \\
\text { atas }\end{array}$ & & /ys & & tyx. & & asas & & $m x$ & $\begin{array}{l}\text { fra } \\
\text { aAt }\end{array}$ & sas & \\
\hline & 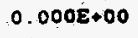 & & 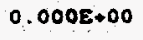 & 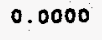 & 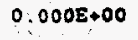 & & 00 & & & & & & & \\
\hline & . & & & & 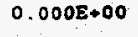 & & 10 & & & & & & & \\
\hline & 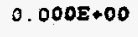 & & & & ? & & 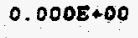 & & & & & & & \\
\hline & 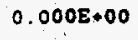 & & & & & & & & & & & & & \\
\hline & .00 & & & & 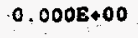 & & 0. & & & & & & & \\
\hline & 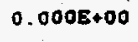 & & & & & & & & & & 00 & & 6 & \\
\hline & & & & & & & & & & & $0.000 E+00$ & & & \\
\hline & : & & & 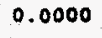 & 0 & 0 & ? & & $.0008+00$ & & & & & \\
\hline & .00 & & & & & & 0.00 & & & & $3+00$ & & & \\
\hline & .00 & & . & & & & & & & & & & & \\
\hline & .00 & & $+\infty 0$ & & +00 & & $?$ & & 00 & & $+\infty$ & & $+\infty 0$ & \\
\hline & 00 & & $1008+00$ & & $0.0008+00$ & 0 & $0.000 E+00$ & . & $0.0008+00$ & & +00 & & $\bullet 00$ & \\
\hline & 0 & & +00 & 00 & $0.000 \mathrm{E}+00$ & & 0.0001 & & 00 & 0 & 10 & & & \\
\hline & & & $E \div 00$ & oo & $0.0008+00$ & 0.0000 & $0.0005+00$ & & & & 10 & & & . \\
\hline & & & & & te & & & & & & & & & \\
\hline & 100 & 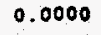 & 00 & & $E+00$ & 00. & $8+00$ & 00 & & & 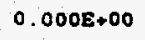 & & $.0112-12$ & \\
\hline
\end{tabular}

- Sum of all water independent and dependent pathways 
$0000 \cdot \tau \tau t-3 z t z \cdot \tau$

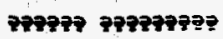
$2000 \cdot 0$ 5T-376E' 2 E656.0 TT-3E9T' $x$ $0000 \cdot 000+3000 \% 0$ $0000.000+3000.0$ 5070.0 ET-3106. $0000 \cdot 000+3000 \%$ $0000 \cdot 0.00+3000 \cdot 0$ $0000 \cdot 000+3000 \cdot 0$ 0000.0 cz-3zts : 1 0000.0 sz-390z'z $0000 \cdot 000+3000 \cdot 0$ $0000 \cdot 0.92-3085 \cdot \varepsilon$ $0000 \cdot 000+3000 \cdot 0$ $0000: 000+3000 \cdot 0$ surses serwespe -20023 xh/602w nowversserves -akemujed ITY
$0000 \cdot 000+3000 \cdot 0$ $0000 \% 00+3000.0$ $0000 \div 0.00+3000 \cdot 0$ $0000.0 \quad 00+3000 * 0$ $0000 \cdot 000+2000.0$ $0000 \% 00+a 000 \%$ $0000.000+3000.0$ $0000: 000+2000 \cdot 0$ $0000.000+3000.0$ $0000.000+3000 \%$ $0000.000+3000.0$ $0,000 \cdot 0.00+3000: 0$ $0000000+3000.0$ $0000 \cdot 000+3000 \%$ $0000.000+3000.0$

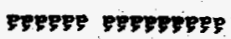
-20ex IX/moxw

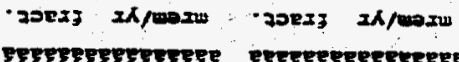
ytกพ JWow $0000 \cdot 0-00+3000 \cdot 0$
$0000 \cdot 0$ 00+3000.0 $0000 \cdot 000+3000 \cdot 0$ $0000 \cdot 000+3000 \cdot 0$ $0000 \cdot 00+3000 \cdot 0$ $0000 \cdot 000+3000.0$ $0000 \cdot 000+3000 \cdot 0 \quad 0000 \cdot 0 \quad 00+3000.0$ $0000 \% 00+3000: 0,0000: 0 \quad 00+3000 \div 0$ $0000 \cdot 000+3000.0 .0000 \cdot 0.00+3000 \% 0$ $0000 \% 00+3000 \% 0000 \% 00+3000 \%$ $0000 \% 00+3000 \% 0000 \cdot 000+3000 \% 0$ $0000 \cdot 0 \quad 00+3000 \cdot 00000 \% 00+3000 \% 0$ $0000000+300000000000+30000$

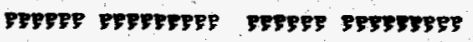

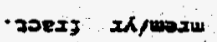
BFrsestersuses วखeTd
$0000.000+3000.0$

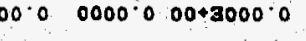
$0000 \% 00+3000: 0$ $0000 \cdot 00+3000 \%$ $0000 \cdot 00+3000 \cdot 0$ $0000 \cdot 000+3000 \cdot 0$ $0000000+3000 \cdot 0$ $0000000+3000 \cdot 0$ $0000 \cdot 00+3000=0$ $0000 \div 000+3000 \cdot 0$ $0000 \% 00+3000.0$ $0000 \% 00+3000 \%$ $000000+30000$ $0000.000+3000.0$ $0000 \% 00+3000 \cdot 0$ $0000000+3000 \%$ psepge perentes -20ex; IK/moxw esestreproterest uopred

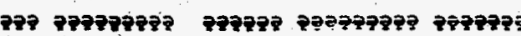
$0000000+300000000 \% 00-3000 \%$ 8Ez-n $0000.000+3000 \cdot 00000.000+3000.0 \quad \square z-0$ $0000 \cdot 0 \quad 00+3000 \cdot 0 \quad 0000 \cdot 0 \quad 00+3000 \cdot 0 \quad 06-35$ $0000 \cdot 000+3000 \% 0000 \cdot 000+3000$ o $6 \varepsilon z-n d$ $0000 \% 00+3000 \% 0000.000+3000.0$ eEz-nd $0000 \cdot 0 \quad 00+3000 \cdot 0$ 0000.0 00+3000\%0 E9-7N $0000 \cdot 000+3000 \cdot 0 \quad 0000 \cdot 0 \quad 00+3000: 0 \quad 65-T_{N}$ $0000 \cdot 000+3000.0 \quad 0000 \cdot 000+3000 \%$ e E-H $0000 \cdot 000+3000 \% 0000 \div 00+3000 \%$ bst-n3 $0000 \cdot 00+3000 \% 0 \quad 0000 \div 000+3000 \div 0$ zst-na $0000.000+3000 \% \quad 0000 \cdot 0 \quad 00+3000 \%$ LET-6 $0000.000 \cdot 3000 \% 0000 \cdot 000+3000 \% 09-02$ $0000000+3000 \% 0000000+3000 \%$ t 0 $0000000+300000000000+3000.0$ t? $0-0$

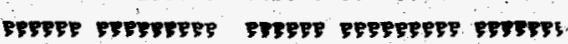
soex $2 \Lambda /$ max

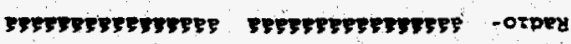

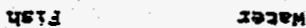

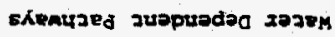

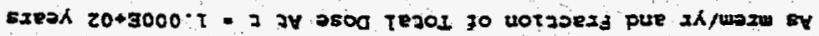

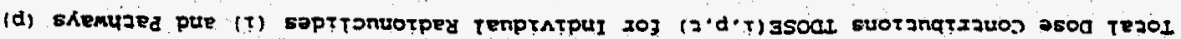

$0000 \cdot 000+3000 \cdot 0$

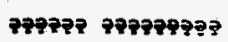
$0000 \cdot 0 \quad 00+3000 \cdot 0$ $0000.000+3000 \cdot 0$ $0000.000+3000.0$ $0000 \cdot 000+3000 \cdot 0$ $0000.00+3000.0$ $0000 \cdot 00+3000 \cdot 0$ $0000.000 \times 3000.0$ $0000 \cdot 00+3000 \cdot 0$ $0000.000+3000 \% 0$ $0000 \cdot 000+3000 \cdot 0$ $0000.000+3000.0$ $0000-0.00+3000.0$ $0000 \cdot 0.00+3000.0$ $0000 \% 00+3000 \%$

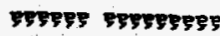

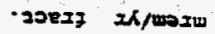

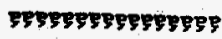
T ţos
$0000 \% 00+3000 \%$

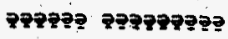
$0000 \cdot 0 \quad 00+3000: 0$ $0000.000+3000.0$ $0000.00+3000.0$ $0000.00+3000.0$ $0000.000+9000 \%$ $0000.00+3000 \cdot 0$ $0000 \div 00+3000 \div 0$ $0000 * 00+3000 \% 0$ $0000 \cdot 00+3000 \%$ $0000 \cdot 0 \cdot 00+3000 \times 0$ $0000-00+3000-0$ $0000 \cdot 00+3000 \cdot 0$ $0000 \% 00+9000.0$ $0000.000+3000.0$ eseses pepsespes 7OEבI 2X/100xw

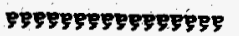
*T?

\begin{abstract}
$0000 \cdot 00+3000 \% 0$

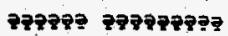
$0000 \% 00+3000 \%$ $0,000 \cdot 000+2000: 0$ $0000.00+3000 \%$ $0000 \% 00+3000.0$ $0000: 000+3000: 0$ $0000 \% 00+3000 \%$ $0000 \% 00+3000 \%$ $0000 \cdot 000+3000 \%$ $0000 \% 00+3000 \%$ $0000 \div 000+3000 \%$ $0000 \cdot 000 \div 3000 \%$ $0000 \cdot 0 \cdot 00+9000 \cdot 0$ $0000 \cdot 000+3000 \cdot 0$ $0000.000+3000.0$ 595959 595959959 208xz $\quad$ XX/waxi:

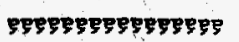
7ean
$0000 \cdot 000+3000.00000 . t$ tr-3ztz.t

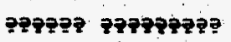
$0000000+3000 \%$ $0000 \cdot 0 \quad 00+3000 \cdot 0$ $0000 \cdot 0 \quad 00+3000.0$ $0000: 000+30000$ $0000000+3000.0$ $0000 \cdot 000+3000 \%$ 0000.0003000 .0 $0000 \% 00+3000.0$ $0000.00+3000.0$ $0000.000+3000.0$ $0000.000+3000.0$ $0000 \cdot 00+3000.0$ $0000.000+3000.0$ $0000 \cdot 00+3000 \%$

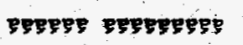
-208IJ IX/worw Ppgeppespepsepse

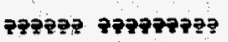
$2000: 0$ ST-JBBE-z

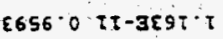
$0000000+3000 \%$ $0000 \cdot 00+3000 \cdot 0$ 5050.0 Ez-as06 $0000.000+3000 \%$ $0000.000+3000 \%$ $0000 \cdot 00+3000 \cdot 0$ $0000000+3000 \%$ $0000000+30000$ $0000.000+30: .0$ $0000-000+3000.0$ $0000000+9000 \%$ $0000 \% 00+3000 \%$ 5epges pegespegs

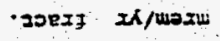

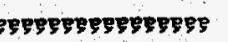
uoper
$0000.000+2000.0$ 0000.0 25-35L5:z T6z0广

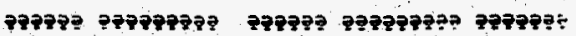
$0000 \% 00 \div 3000 \% 0000 \%$ sE-396c: $8 \varepsilon z+\delta$ $0000 \% 00+3000 \% 0000 \%$ gE-3EE6 b bE-n $0000 \% 00+3000 \% 0: 0000.000+3000 \% 0$ 06-25 $0000000+3000 \cdot 0$ 0000:0 00+3000.0 6E2-nd $0000 \% 00+3000 \% 0 \quad 0000 \times 00+3000 \%$ bez-nd

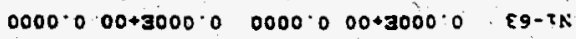
$0000 \% 00+3000 \% 0000 \% 00+3000 \%$ 65-TN $0000 \% 00+3000 \% 0000 \% 00+3000 \%$ E-H $0000 \% 00+3000 \cdot 0 \quad 0000 \%$ Lz-3zTL I b5T-na $0000 \cdot 0 \quad 00+3000 \cdot 0 \quad 0000.0$ 5z-390z z z5t-na. $0000 \cdot 000+3000 \cdot 0 \quad 0000 \cdot 0 \quad 00+3000.0$ LET-5D $0000 \cdot 0 \quad 00+3000 \cdot 0 \quad 0000 \cdot 0$ 9z-3055. $0000-000+3000 \cdot 0$ 0000:0.00+3000.0 का-3 $0000000+300000000000+3000 \%$ tњz-ury

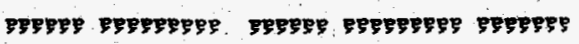

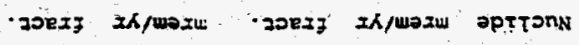

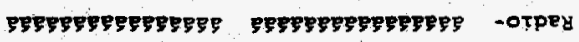
UOTAETEYUI punass

(copex səprtoxa uotzeterur) skemyjed juapuodopar sozen

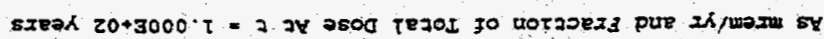

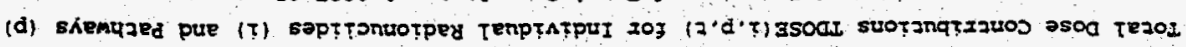

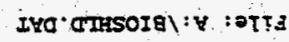

zZ DEEd IT: $2 T$ L6/90/EO

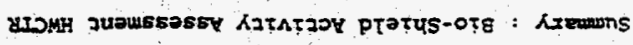

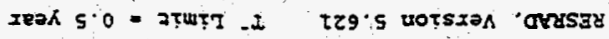


RESRAD, Version 5.621 T Limit -0.5 year

Sumary : Bio-Shield Accivicy Assessmene twiCTR
$03 / 06 / 97 \quad 12: 11$ Page 23

File: A: \BIOSHW.DAT

Total Dose Contributions TDOSE (i,p,t) for Individual Radionuclides (i) and Pathways (p) As mrem/yr and Fraction of Total Dose At $t=5.000 E+02$ years

Wacer Independent Pathways (Inhalation excludes radon)

\begin{tabular}{|c|c|c|c|c|c|c|c|c|c|c|c|c|c|c|}
\hline \multirow{2}{*}{ ludio- } & Groun & & $I$ & n. & \multicolumn{2}{|c|}{$\begin{array}{c}\text { Radon : } \\
\text { aasasaasasasaaaa }\end{array}$} & \multicolumn{2}{|c|}{ Plane } & \multicolumn{2}{|c|}{$\begin{array}{l}\text { Meac } \\
\text { aasasasaasasadá }\end{array}$} & \multicolumn{2}{|c|}{ Milk } & \multicolumn{2}{|c|}{$\begin{array}{c}\text { Soil } \\
\text { aasadasadadassas }\end{array}$} \\
\hline & & & as & & $y x$ & & & & & & & & $\mathrm{~s}$ & \\
\hline 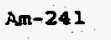 & & & & & & & 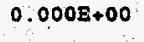 & & +00 & & & & & \\
\hline 4 & . & & & & 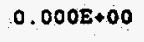 & & & & & & & & & \\
\hline 0 & 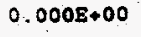 & & & & & & & & & & & & & \\
\hline 137 & & & & & & & & & & & & & & \\
\hline & & & & & & & +00 & & & & & & & \\
\hline & & & & & & & & & & & & & & \\
\hline & & & & & & & & & & & & & & \\
\hline & & & & & & & & & & & & & & \\
\hline & & & & & & & & & & & & & & \\
\hline & & & & & & & & & & & & & & \\
\hline & & & & & 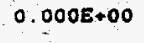 & 00 & 0 & & & & & & & \\
\hline & & & & & & & & & & & & & & \\
\hline & & & & & & & & & & & & & & \\
\hline & & & & & & & 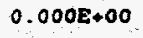 & & & & & & & \\
\hline & & & & & & & & & & & & & & \\
\hline & & & .00 & & -10 & 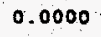 & 00 & 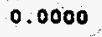 & 00 & & & & & \\
\hline
\end{tabular}

Total Dose Concribueions rDose (i,p,t) for Individual Radionuclides (i) and Pathways (p) As mrem/yr and Fraction of Total Dose At $t=5.0008+02$ years

wacer Dependene Pachways

\begin{tabular}{|c|c|c|c|c|c|c|c|c|c|c|c|c|c|c|}
\hline & & & & & then & 24 & ate & Warat & 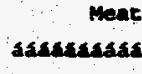 & & & & - & \\
\hline 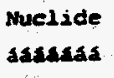 & $\begin{array}{l}y x \\
\text { axa }\end{array}$ & $\begin{array}{l}\text { Eraet. } \\
\text { sasas }\end{array}$ & $\begin{array}{l}\text { mrem/yx } \\
\text { adancasa }\end{array}$ & $\begin{array}{l}\text { fract. } \\
\text { asarax }\end{array}$ & $\begin{array}{l}\text { mrem/yr } \\
\text { asasasass }\end{array}$ & $\begin{array}{l}\text { Erace. } \\
\text { asalst }\end{array}$ & $\begin{array}{c}\text { mrem/yr } \\
\text { sascascas }\end{array}$ & $\begin{array}{l}\text { Erace } \\
\text { asadst }\end{array}$ & $\begin{array}{l}\text { mrena/yr } \\
\text { astrassass }\end{array}$ & . & $\begin{array}{l}\text { mrem/yx } \\
\text { arassass }\end{array}$ & . & $=/ y x$ & \\
\hline & 0 & 10 & & & j & 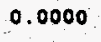 & 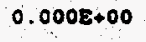 & s & & & & & & \\
\hline & 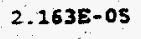 & & D & 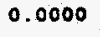 & & & & & & & 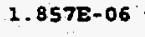 & & & \\
\hline & 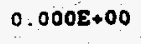 & & . & 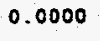 & 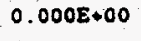 & & 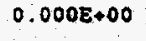 & & & & 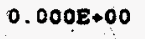 & & & \\
\hline & . & & & & & & & & & & & & & \\
\hline & & & & & & & & & & & & & & \\
\hline & 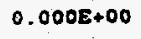 & & 0 & 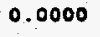 & & & & & & & & & & \\
\hline & & & $\mathbf{0}$ & 0. & & & & & 9. & & 18 & & & \\
\hline & & & .00 & 0 & D & & 400 & & & & 00 & & & \\
\hline 3 & & & 00 & & & & +00 & & & & & & & \\
\hline & & & & & & & & & & & & & & \\
\hline & & & & & & & & & & & & & & \\
\hline & 0 & & & & & & : & & & & & & & \\
\hline & 0 & & & & & & & & & & 9 & & & \\
\hline & & & & & 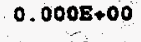 & & & & 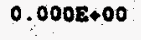 & & & & & \\
\hline & & & & & & & & & & & & & & \\
\hline & .05 & 0.8371 & $.000 \mathrm{E}+00$ & 0,0000 & $0.000 E+00$ & 0.0000 & $1.6708-06$ & 0.0646 & $.8285-07$ & 0.0264 & $1.857 E-06$ & 0.0719 & $2.584 E-05$ & 2.0000 \\
\hline
\end{tabular}

- Sum of all water independent and dependene pachways. 


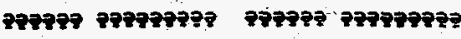

$0000 \cdot 02 \tau-30 \tau 9 \cdot 2$ 0000.0 $00+3000.0$

$8200 \cdot 0$ ot-zr8E' 6

$0000.000+3000 \cdot 0$

0000.00049000 .0

$1000 \cdot 0$ ot-aszb:t

$0000 \cdot 000+3000.0$

$0000 \cdot 000+3000 \cdot 0$

$0000 \cdot 000+3000 \cdot 0$

$0000 \% 0043000 \div 0$

$0000 \cdot 00+3000 \cdot 0$

$0000 \cdot 0 \quad 00+3000 \cdot 0$

$0000 \% 00+3000.0$

$8966^{\circ} 0$ LO-3T6E. $\varepsilon$

$0000.000+3000.0$

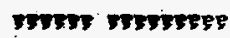

:200x; 2h/nosw

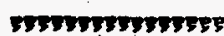

- exvarard TTY
0000.0000000 $0000.000 \cdot 3000^{\circ} 0$ $0000 \% 00+9000 \%$ $0000 \cdot 000+3000.0$ $0000 \cdot 000+3000 \cdot 0$ $0000 \cdot 000+2000.0$ $000+3000.0000 .00+3000.0$ $000+3000=0$. 0000.00048000 .0 0000 $000+3000.0$ $0000 \cdot 000+3000 \cdot 0 \quad 0000 \cdot 0.00+3000 \cdot 0$ $9 \tau \angle 0.0$ 80-3LEF $Z$ E920.0 60-38\$6. $0000 \% 00+3000.0$ 0000\%0 00+3000.0

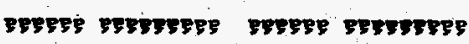
200aj 2 K/maxw

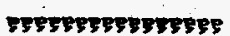
xTin 208x3 $2 x /$ mani pepsestrestrites دvew
$1990.080-376 \mathrm{~T} \cdot z$

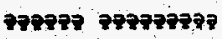
$0000 \cdot 00+3000.0$ $0000 \cdot 0.00+3000 \cdot 0$ $0000.00+3000.0$ $0000.000+3000.0$ $0000 \% 00+3000.0$ $0000.00+3000 \%$ $0000 \% 00+2000 \%$ $0000=00+3000 \%$ $0000: 000+30000$ $0000.00+3000 \cdot 0$ $0000=00+3000 \%$ $0000 \cdot 00+3000 \%$ พ90.0 80-3t6t? 0000 : $00+3 \dot{0} 00$ o nwe pswerese - popaz 2h/maris meresstrestese aveta

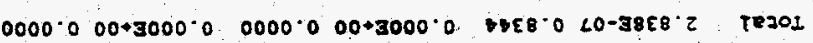

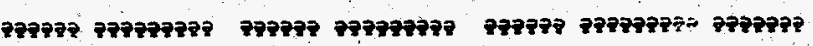
$0000 \cdot 0.00+3000 \% 0000 \% 00+3000 \cdot 0 \quad 0000 \cdot 000+3000 \cdots \quad$ Bez-n

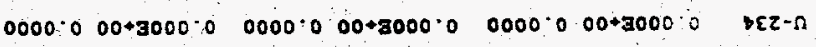
$0000000+3000 \% 0000000+3000 \cdot 0 \quad 0000 \cdot 000+3000 \% 06-x 5$ $0000.000+3000 \div 00000 \% 00+3000.0 \quad 0000.000+3000 \% 0$ Gez-ne

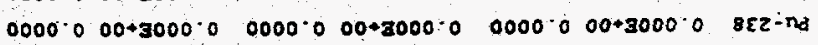
$0000 \% 00+3000 \cdot 0 \quad 0000 \% 00+3000 \cdot 0 \quad 0000.000+3000 \% 0 \quad \varepsilon 9-? \mathrm{~N}$

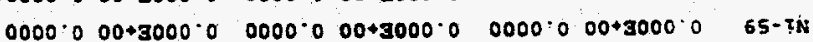
$0000.000+3000 \cdot 0 \quad 0000.000+3000 \cdot 0 \quad 0000 \% 00+3000 \%$ E E $0000 \% 00+3000 \% 0000 \% 00+3000 \cdot 0$ 0000.0 00+3000\% BSt-ng $0000: 000+3000 \cdot 0 \quad 0000 \cdot 000+3000 \cdot 0 \quad 0000 \cdot 0 \quad 00+3000 \cdot 0 \quad 257-n 3$ $0000 \% 00+3000 \% 0000 \% 00+3000.00000 \% 00+3000 \%$ LET-SO $0000 \cdot 0.00+3000 \% 0000000+300000000 \cdot 0,00+3000 \cdot 0$ 09-05

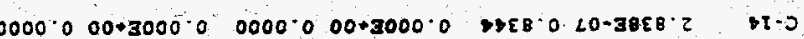

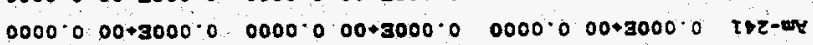

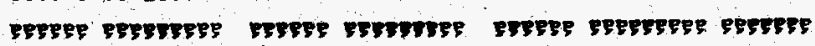

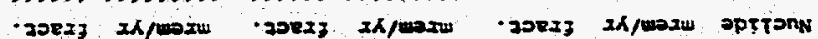

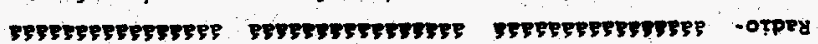
topre uots soskn

\section{oxenuzed zuepuedod zozRM

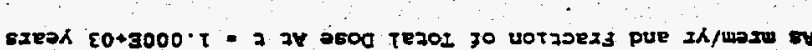

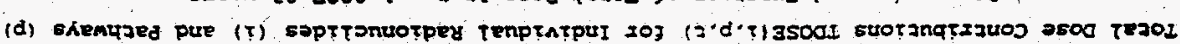

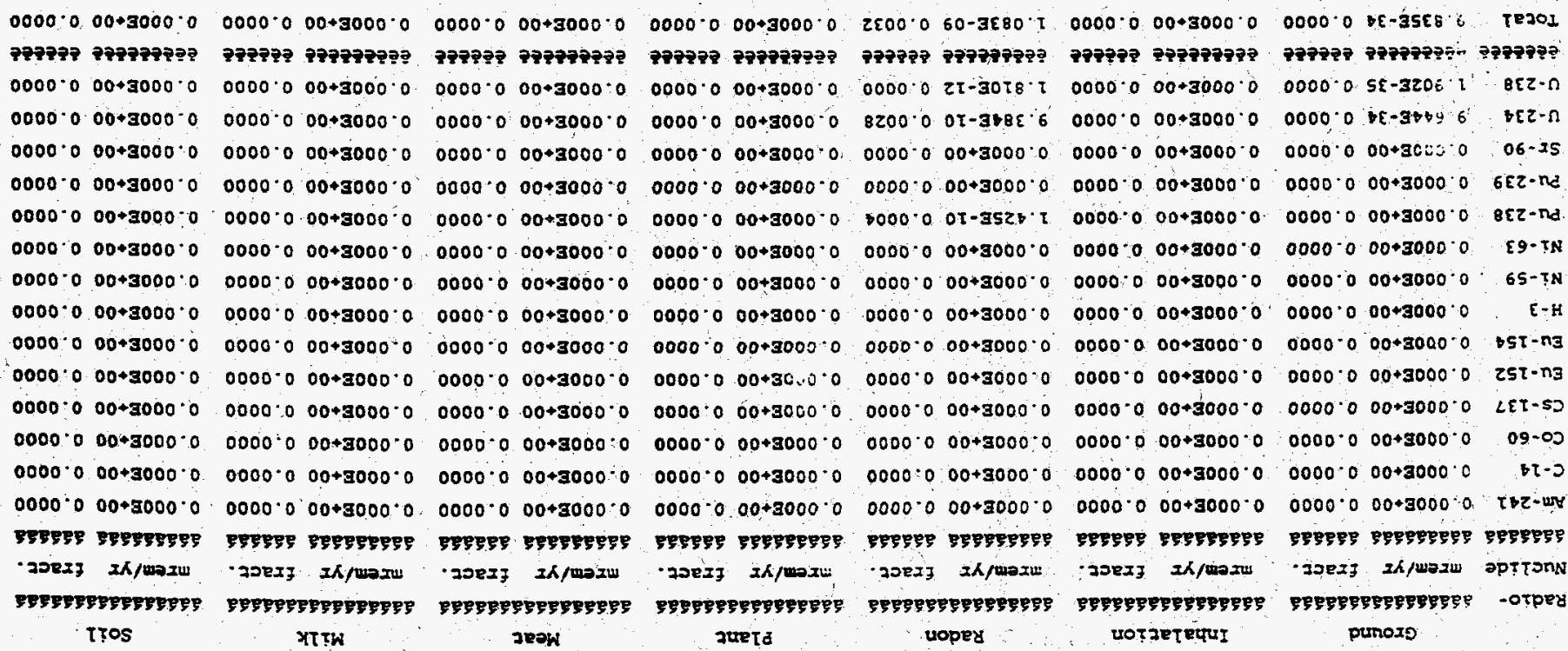

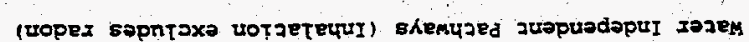

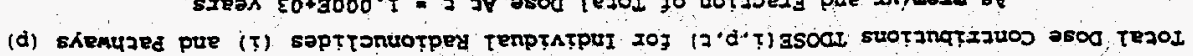

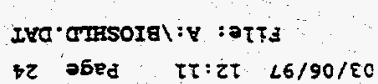


RESRAD, Vergion 5.621 Timit $* 0.5$ year Summary : Bio-Shield Activiey Assessment HWCTR
$03 / 06 / 9712: 11$ Page 25 File: A: \BIOSHLD.DAT

Total Dose Contributions TDOSE (i,p,e) for Individual Radionucildes (i) and Pathways (p) As mrem/yr and Fraction of Total Dose At $t=1.000 E+04$ years

Water Independent Pachways (Inhalation excludes racion)

\begin{tabular}{|c|c|c|c|c|c|c|c|c|c|c|c|c|c|c|}
\hline \multirow[b]{2}{*}{$\begin{array}{l}\text { Lejide } \\
\text { sááá }\end{array}$} & \multicolumn{2}{|c|}{ Ground } & \multicolumn{2}{|c|}{ Inhalation } & \multicolumn{2}{|c|}{ 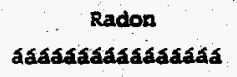 } & \multicolumn{2}{|c|}{ 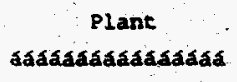 } & \multicolumn{2}{|c|}{ 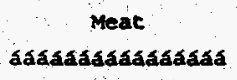 } & \multicolumn{2}{|c|}{ Milk } & \multicolumn{2}{|c|}{$\begin{array}{c}\text { Soil } \\
\text { Gasasasáasadá }\end{array}$} \\
\hline & 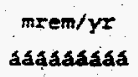 & strats & (yz & 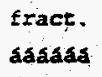 & as & & $\begin{array}{l}\text { mre } \\
\text { dáad }\end{array}$ & 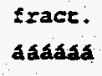 & ateo & s & $\mathbf{r}$ & $\begin{array}{l}\text { frace. } \\
\text { áasas }\end{array}$ & $m=$ & \\
\hline $1-241$ & 00 & 0000 & & & .000 & c & $1+00$ & ( & •00 & 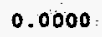 & +00 & 0000 & & \\
\hline & & & & & & & & & & & & & $.000 \mathrm{E}+00$ & 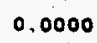 \\
\hline & & & & & & & & & & & $+\infty$ & & 20 & \\
\hline-1 & & & & & & & & & & & & & & \\
\hline- & , & & & & & & & & & & & & $+\infty 0$ & \\
\hline$=$ & 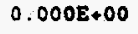 & & & & & & & & & & +00 & & & \\
\hline & & & & & & & & & & & & & 年 & \\
\hline & & & & & & & & & & & & & & \\
\hline & & & & & & & & & & & & & & \\
\hline & & & & & & & & & & & & & & \\
\hline & & & & & & & & & & & $+00^{\circ}$ & & & \\
\hline & & & & & & & & & & & & & & \\
\hline & & & & & & & & & & & & & & \\
\hline & & & & & & & & & & & & & & \\
\hline & & & & & & & & & & & & & 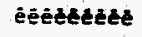 & \\
\hline & $1.806 E-28$ & & 0 & & $.5568-08$ & & $.000 E+00$ & & ת & & a & & & \\
\hline
\end{tabular}

Total Dose Concributions TpOSE $(i, p, t)$ for Individual Radionuclides (i) anc Pachways (p) As $m r e m / y$ and Fraction of Total Dose Ae $\varepsilon=1.000 E+04$ years

Wher Dependent Pathways

\begin{tabular}{|c|c|c|c|c|c|c|c|c|c|c|c|c|c|c|}
\hline \multirow{2}{*}{$\begin{array}{l}\text { Radio- } \\
\text { Nuclide } \\
\text { dasasaa }\end{array}$} & \multicolumn{2}{|c|}{$\begin{array}{c}\text { water } \\
\text { asarususurass }\end{array}$} & \multicolumn{2}{|c|}{$\begin{array}{l}\text { Finh } \\
\text { admusudasadusa }\end{array}$} & \multicolumn{2}{|c|}{ 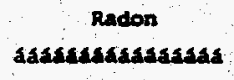 } & \multicolumn{2}{|c|}{$\begin{array}{c}\text { Plent } \\
\text { sadigadastasad }\end{array}$} & \multicolumn{2}{|c|}{ 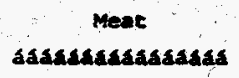 } & \multicolumn{2}{|c|}{$\begin{array}{c}\text { Milk } \\
\text { aasadsatadasasa }\end{array}$} & \multicolumn{2}{|c|}{$\begin{array}{l}\text { All Pathweye } \\
\text { sadisasusususe }\end{array}$} \\
\hline & & & & & $\begin{array}{l}\text { yrx } \\
\text { actad }\end{array}$ & & $\begin{array}{l}\text { mren/ye } \\
\text { sasmadada }\end{array}$ & t. & $x$ & : & istas & & ast & \\
\hline & S44E-10 & 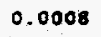 & $0000+00$ & 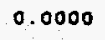 & $.0008+00$ & 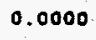 & 1 & & 3 & 0 & $2.762 \mathrm{E}-12$ & & . & \\
\hline & 0 & & 000 & 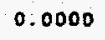 & $.000 \mathrm{E}+00$ & & & 0 & so. & & $0.000 E+00$ & & 0 & \\
\hline $0-6$ & 0.00 & & 0 & & 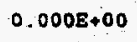 & & 0 & & 0 & & $O E+O O$ & & .00 & \\
\hline 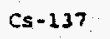 & 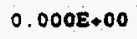 & & 0 & & 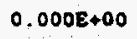 & & & & & & 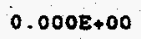 & & $2 E+00$ & \\
\hline 7 & & & 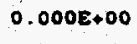 & & 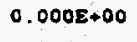 & & & & & & $E+00$ & 00 & $: 000 E+00$ & . \\
\hline & & & & & & & & & & 0. & $8+00$ & 0 & 00 & 0.0000 \\
\hline 3. & & & 0 & & & & 20 & & 000 & 0 & 00 & & & \\
\hline- & 0 & & & & & & 10 & 0 & 0 & 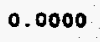 & $a$ & & & \\
\hline- & 0 & & 10 & & 6 & & & & & & 00 & & 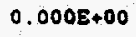 & \\
\hline 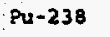 & & & & & & & & & & & -20 & & & \\
\hline$u=23$ & 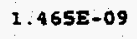 & & & & & & & & & & 12 & & 9 & \\
\hline$=$ & D & & 0 & . & 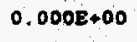 & & 10 & & 0 & D. & 10. & & o. & \\
\hline-27 & 8 & & ? & & 21 & & & & & & 0 & 0.0049 & -08 & \\
\hline & 08 & 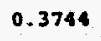 & 00 & & 2 & & 8. & & & 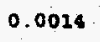 & 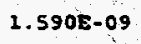 & 5 & $E-68$ & 0 \\
\hline & & & & & & & & & $=$ & e & Ee & 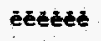 & zetere & et \\
\hline & $1.283 E-07$ & 0.6 & $0.000 \mathrm{E}+00$ & 0 & $.278 E-11$ & 0.0003 & $2 E-08$ & 0.1 & $5.518 E-10$ & 0.0029 & $2.7105-09$ & 0.0144 & $1.876 E-07$ & 2.0000 \\
\hline
\end{tabular}

- Sur of all water independent and dependene pachways. 
RESRAD, Version $5.621 \quad T^{-}$Limit $=0.5$ year Sumary : Bio-Shield Activity Assessment HWCTR
$03 / 06 / 97 \quad 12: 13$ Page 26 File: A: IBIOSHD.DAT

Dose/Source Ratios Summed Over AlI Pathways

Parent and Progeny Princtpal Radionuclide Contributions Indicated

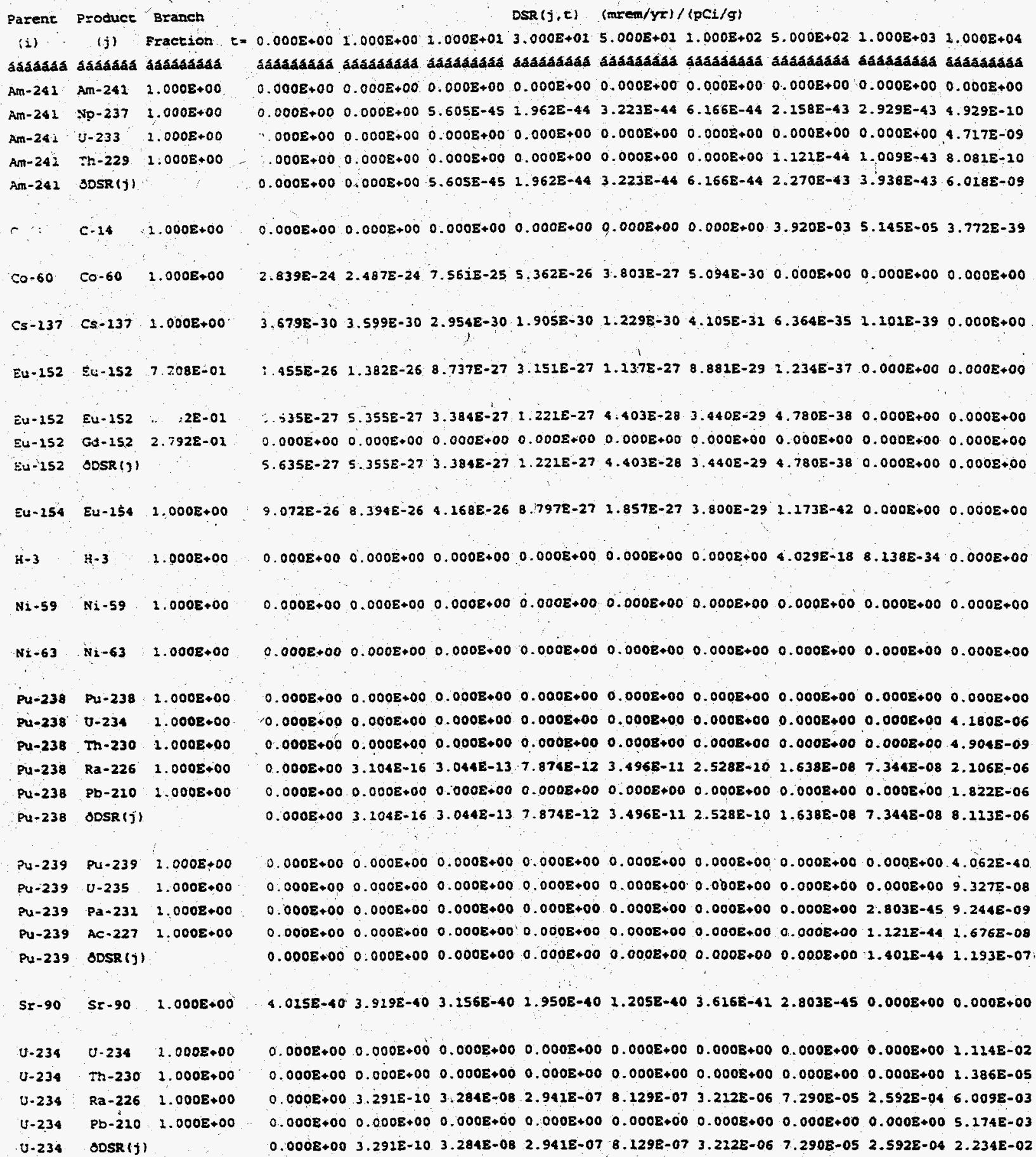


RESRAD, Version $5.621 T$ Limit $=0.5$ year

Sumary : Bio-Shield Activity Assesament :HCTR
$03 / 06 / 97 \quad 12: 11$ Page 27

File: A: IBIOSHID.DAT

Dose/Source Ratios Surmed Over Al1 Pathways

Parent and Progeny Principal Radionuclide Contributions Indicated

parent Produce Branch

(i) (j) Fraceion

$U-238 \quad U-238 \quad 1.000 E+00$

U-238 U-234 $1.0008+00$

U-238 Th-230 $1.000 E+00$

$\begin{array}{llll}\mathrm{U}-238 & \mathrm{Ra}-226 & 1.000 \mathrm{E}+00\end{array}$

U-238 Pb-210 1.000E+00

U-238 ODSR(j)

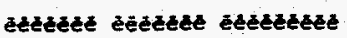

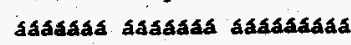

DSR (j.t) (arem/yr)/(PCi/g)

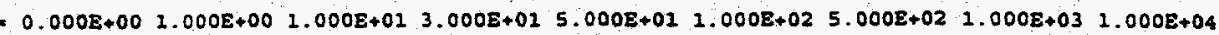

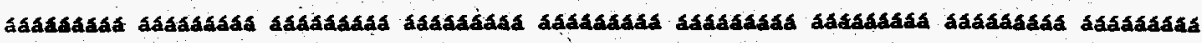
1.261E-30 1.262E-30 1.269E-30 $1.286 E-30 \quad 1.303 E-30 \quad 1.347 E-30 \quad 1.758 E-30 \quad 2.451 E-30 \quad 1.089 E-02$ $\begin{array}{lllllllll}0.000 E+00 & 0.000 E+00 & 0.000 E+00 & 0.000 E+00 & 0.000 E+00 & 0.000 E+00 & 0.000 E+00 & 0.000 E+00 & 3.203 E-04\end{array}$ $0.000 E+00 \quad 0.0008+00 \quad 0.000 E+00 \quad 0.000 E+00 \quad 0.000 E+00 \quad 0.000 E+00 \quad 0.000 E+00 \quad 0.000 E+00 \quad 3.279 E-07$ $0.000 E+00 \quad 3.123 E-16 \quad 3.10 .2 E-13 \quad 8.325 E-12 \quad 3.832 E-12 \quad 3.021 E-10 \quad 3.363 E-08 \quad 2.332 E-07 \quad 3.469 E-05$ $0.000 E+00 \quad 0.000 E+00 \quad 0.000 E+00 \quad 0.000 E+00 \quad 0.0008+00 \quad 0.000 E+00 \quad 0.000 E+00 \quad 0.000 E+00 \quad 2.147 E-04$ $\begin{array}{lllllllll}1.261 E-30 & 3.123 E-16 & 3.102 E-13 & 8.325 E-12 & 3.832 E-11 & 3.021 E-10 & 3.363 E-08 & 2.332 E-07 & 1.146 E-02\end{array}$

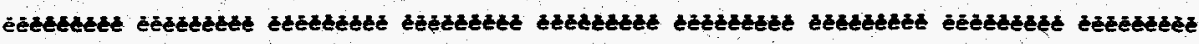
Granch Fraction is the cumulative factor for the jeth principal radionuclide daugher: CUMBRf(j) = BRF (1)-BRF(2) ... BRF(j). The DSR, includes concributions from associated (half-life 0.5 yr) daugheers.

Single Radionuelide Soil Guidelines $G(i . t)$ in $\mathrm{pci} / \mathrm{g}$ Bavic Radiation Dose Limit = $15 \mathrm{mrem} / \mathrm{Yr}$

\section{Nuclide}

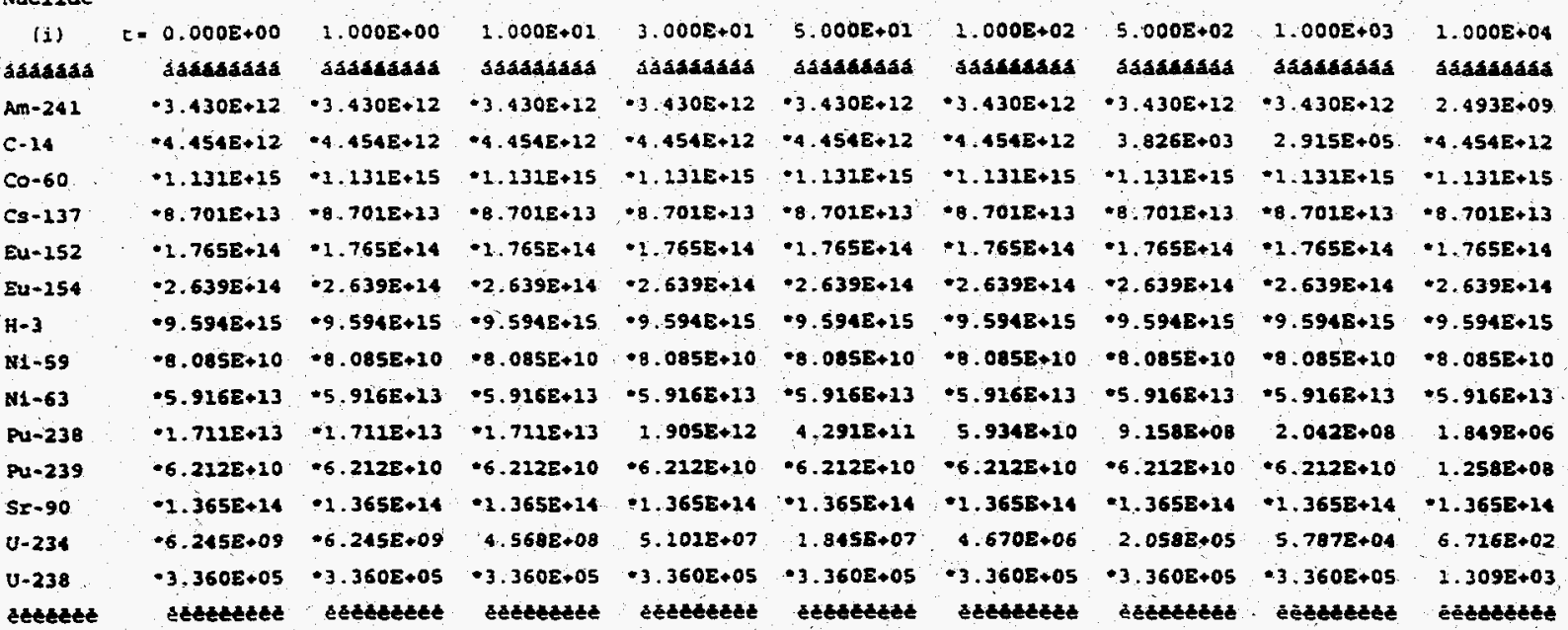


RESRAD, Version $5.621 \quad T$ Limit $=0.5$ year Sumbery : Bio-Shield Activity Assesisment HWCIR
$0.3 / 06 / 97 \quad 12: 12$ Page 28

File: A: lBtostido.dAT

Summed Dose/Source Ratios DSR (i,c) in (mrem/rt)/(pCi/g)

and Single Radionuclide Soil Guidelines $G(i, t)$ in $\mathrm{pCi} / \mathrm{g}$

at emin - rime of minimum single radionuclide soil guideline

and as cmax $=$ cime of maximum total dose $=464.9$ b 0.5 years

\begin{tabular}{|c|c|c|c|c|c|c|}
\hline $\begin{array}{c}\text { Nuclide } \\
\text { (i) }\end{array}$ & $\begin{array}{c}\text { Initial } \\
\mathrm{pci} / \mathrm{g}\end{array}$ & $\begin{array}{c}\text { tmin } \\
\text { (years) }\end{array}$ & $\operatorname{DsR}(i$, imin) & $\begin{array}{l}G(i, \text { emin }) \\
\quad(p C i / g)\end{array}$ & $\operatorname{DSR}(i, \operatorname{tmax})$ & $\begin{array}{c}G(i, \operatorname{tmax}) \\
(p C i / g)\end{array}$ \\
\hline áasaá & åáaลaลá & 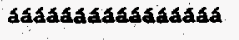 & 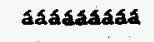 & aáasabaá & ąáasááa & aakasaáạ \\
\hline$A m-241$ & $3.230 \mathrm{E}-02$ & $1489 \mathrm{Dl}$ & $2.389 \mathrm{E}-04$ & $6.2798+04$ & $0.000 \mathrm{E}+00$ & $.3 .430 \mathrm{E}+12$ \\
\hline-14 & $6.590 E-03$ & 465.3 p 0.5 & $5.3058-03$ & $2.828 E+03$ & $5.324 E-03$ & $2.8178+03$ \\
\hline Co-60 & $6.9508+03$ & $0.000 E+00$ & $2.839 \mathrm{E}-24$ & $-1.1315+15$ & $0.000 \mathrm{E}+00$ & $+1.131 E+15$ \\
\hline$C s-137$ & $2.2505-02$ & $0.0008 \div 00$ & $3.679 E-30$ & $-8.701 E+13$ & $0.0008+00$ & $.8 .701 E+13$ \\
\hline Eu-152 & $1.7908+03$ & $0.000 E+00$ & $2.018 E-26$ & $\because 2.765 E+14$ & $0.0008+00$ & $-1.765 E+14$ \\
\hline $5 u-154$ & $3.980 E+02$ & $0.000 E+00$ & $9: 072 E-26$ & $-2.639 \mathrm{E}+14$ & $0.000 \mathrm{E}+00$ & $-2.639 \mathrm{E}+14$ \\
\hline $\mathrm{H}-3$ & $1.8708+02$ & $258.2 p 0.3$ & $1.541 E-10$ & $9.737 \mathrm{E}+10$ & $5.070 E-17$ & $-9.594 E+15$ \\
\hline $\mathrm{Ni}=59$ & $4,530 \mathrm{E}-02$ & $0.0000<0.0000$ & $0.000 \mathrm{E}+00$ & $-0.085 E+10$ & $0,0008+00$ & $-8.085 E+20$ \\
\hline$N i-63$ & $3.620 \mathrm{E}+00$ & 0.0000 o 0.0000 & $0.000 E+00$ & $-5.916 \mathrm{E}+13$ & $0.000 \mathrm{E}+00$ & $=5.9168+13$ \\
\hline Pu-238 & $1.9408-03$ & 7558 P $B$ & $2.6305-05$ & $9.201 E+05$ & $1.383 E-08$ & $1.085 E+09$ \\
\hline Pu-239: & $1.550 \mathrm{E}-02$ & $1.0008+04$ & $1.293 E-07$ & $1.258 E+08$ & $0.0008+00$ & $-6.2128+10$ \\
\hline$S x-90$ & $3.3608-01$ & 2219 p 2 & $2.230 E-23$ & $+365 E+14$ & $0.000 E+00$ & $-1.3658+14$ \\
\hline $0-234$ & $3.620 \mathrm{E}-06$ & $7282 \mathrm{p} 7$ & $5.125 E-02$ & $2.927 E+02$ & $6.357 E-05$ & $2.3608+05$ \\
\hline$U-238$ & $7.760 E-06$ & $7282 \mathrm{P} 7$ & $4.654 E-02$ & $3.223 \mathrm{E}+02$ & $2.7318-08$ & $-3.3608+05$ \\
\hline atefet & ieféctedst & éetextetejectectè & éttetsete & esteteres & écectedse & esteceste \\
\hline
\end{tabular}


RESRAD, Version 5.621 T Limic $=0.5$ year Sumary : Bio-Shield Activity Asseasment HWCTR
03/06/97 12:11 Page 29

File: A: \BIOSER.DAT

Individual Nuclide Doge Surmed Over All Pachways

parent Nuclide and Branen Fraction Indicated

Nuclide parent BRF(i)

(j) : (i)

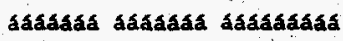

$A n-241, A m-241 \quad 1.000 E+00$

$\mathrm{Np}-237$ Am-24I $1.000 \mathrm{E}+00$

$\mathrm{U}+233 \quad \mathrm{Am}-242 \quad 1.0008+00$

$T h-229 \quad A m=241 \quad 1.000 E+00$

$c-14 \quad c-14 \quad 1.000 E+00$

$60-60 \quad 00-60 \quad 1.0008+00$

$C s-137 \quad 5-237 \quad 1.000 E+00$

Eu-152 Eu-152 7.208E-02

Eu-152 Eu-152 2.792E-01

Eu-152 ODOSE $(j)$ :

Gd-152 Eu-152 2.792E-02

Eu-154 Eu-154 $1.000 E+00$

$H-3 \quad H-3 \quad 1.0008+00$

Ni-59 Ni-59 $1.000 E+00$

$N i-63 \quad N i-63 \quad 1.000 E+00$

$P u-238$ Pu-238 $1.000 E+00$

$0.234 \quad P U-238 \quad 1.000 E+00$

U-234 U-234 i. $\quad 0008+00$

U.234 U-238 $\quad 2,0008+00$

U.234 SDOSE $(j)$ :

In-230 Pu-238 $2.000 E+00$

$\begin{array}{llll}7 \mathrm{~m}-230 & 0-231 & 2.0008+00\end{array}$

Th-230 J-238 $\quad 1.0008+00$

$m-230$ ODOSE (j) :

$R a-226 \quad P L-238 \quad 1.0008+00$

Ra-226 U.234 $\quad 1.000 \varepsilon+00$

Ra-226 U-23B 2.000E+00

Ra-226 ODOSE (j):

Pb-210 Pu-238 $1.000 E+00$ Pb-210 U-234 2.000E*00 Pb-210 U-238 $2.0008+00$ PD-210 ODOSE (j):
Doss $(j, t), m z e m / Y^{2}$

$E=0.000 E+00 \quad 1.000 E+00 \quad 1.000 E+01 \quad 3.000 E+01 \quad 5.0008+01 \quad 1.000 E+02 \quad 5.000 E+02 \quad 1.0008+03 \quad 1.000 E+04$

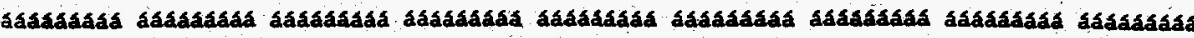
$\begin{array}{llllllll}0.000 E+00 & 0.000 E+00 & 0.000 E+00 & 0.000 E+00 & 0.000 E+00 & 0.000 E+00 & 0.000 E+00 & 0.000 E+00 \quad 0.000 E+00\end{array}$

$0.000 E+00 \quad 0.000 E+00 \quad 0.000 E+00 \quad 0.000 E+00 \quad 0.000 E+00 \quad 0.000 E+00 \quad 0.000 E+00 \quad 0.000 E+00 \quad 1.592 E-11$

$0.000 E+00 \quad 0.000 E+00 \quad 0.000 E+00 \quad 0.000 E+00 \quad 0.000 E+00 \quad 0.000 E+00 \quad 0.000 E+00 \quad 0.000 E+00 \quad 1.524 E-10$

$0.000 E+00 \quad 0.000 E+00 \quad 0.000 E+00 \quad 0.000 E+00 \quad 0.000 E+00 \quad 0.000 E+00 \quad 0.000 E+00 \quad 0.000 E+00 \quad 2.610 E-11$

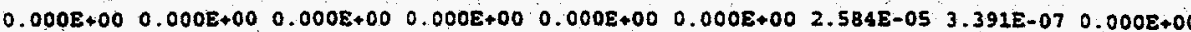

$1.973 E-20 \quad 1.729 E-20 \quad 5.255 E-21 \quad 3.727 E-22 \quad 2.643 E-23 \quad 3.540 E-26 \quad 0.000 E+00 \quad 0.000 E+00 \quad 0.000 E+00$

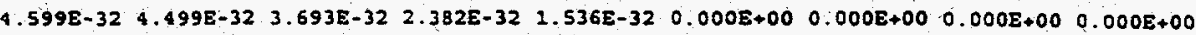

$2.604 E-23 \quad 2.474 E-23 \quad 1.564 E-23 \quad 5.641 E-24 \quad 2.035 E-24 \quad 1.590 E-25 \quad 0.000 E+00 \quad 0.000 E+00 \quad 0.000 E+00$

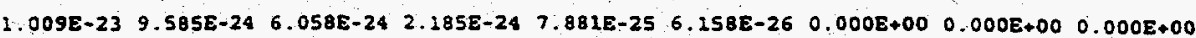

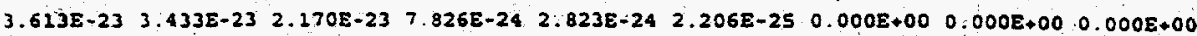

$\begin{array}{llllllllll}0.000 E+00 & 0.000 E+00 & 0.000 E+00 & 0.000 E+00 & 0.000 E+00 & 0.000 E+00 & 0.000 E+00 & 0.000 E+00 & 0.000 E+00\end{array}$

$3.611 E-24 \quad 3.341 E-24 \quad 1.659 E-24 \quad 3.501 E-25 \quad 7.390 E-26 \quad 1.512 E-27,0.000 E+00 \quad 0.000 E+00 \quad 0.000 E+00$

$0.000 E+00 \quad 0.000 E+00 \quad 0.000 E+00 \quad 0.000 E+00 \quad 0.000 E+00 \quad 0.000 E+00 \quad 7.534 E-17 \quad 0.000 E+00 \quad 0.000 E+00$

$0.000 E+00 \quad 0.000 \Sigma+00 \quad 0.000 \varepsilon+00 \cdot 0.000 \varepsilon+00 \quad 0.000 \Sigma+00 \quad 0.000 E+00 \quad 0.000 E+00 \quad 0.000 E+00 \quad 0.000 \varepsilon+00$

$\begin{array}{lllllllll}0.000 E+00 & 0.000 E+00 & 0.000 E+00 & 0.000 E+00 & 0.000 E+00 & 0.000 E+00 & 0.000 E+00 & 0.000 E+00 & 0.000 E+00\end{array}$

$\begin{array}{llllllll}0.000 E+00 & 0.000 E+00 & 0.000 E+00 & 0.000 E+00 & 0.000 E+00 & 0.0008+00 \quad 0.000 E+00 & 0.000 E+00 & 0.000 E+00\end{array}$

$\begin{array}{lllllllll}0.000 E+00 & 0.000 E+00 & 0.000 E+00 & 0.000 E+00 & 0.000 E+00 & 0.000 E+00 & 0.000 E+00 & 0.000 E+00 & 8.09 E-09\end{array}$ $\begin{array}{lllllllll}0.000 E+00 & 0.000 E+00 & 0.000 E+00 & 0.000 E+00 & 0.000 E+00 & 0.000 E+00 & 0.000 E+00 & 0.000 E+00 & 4.033 E-08\end{array}$ $\begin{array}{llllllll}0.000 E+00 & 0.000 E+00 & 0.000 E+00 & 0.000 E+00 & 0.000 E+00 & 0.000 E+00 \quad 0.000 E+00 \quad 0.000 E+00 \quad 2.486 E-09\end{array}$ $0.000 E+00 \quad 0.000 E+00 \quad 0.000 E+00 \quad 0.000 E+00 \quad 0.000 E+00 \quad 0.000 E+00 \quad 0.000 E+00 \quad 0.000 E+00 \quad 5.092 E-08$

$0.000 E+00 \quad 0.000 E+00 \quad 0.000 E+00 \quad 0.000 E+00 \quad 0.000 E+00 \quad 0.000 E+00 \quad 0.000 E+00 \quad 0.000 E+00 \quad 9.514 E-12$ $\begin{array}{llllllllllll}0.000 E+00 & 0.0008+00 & 0.000 E+00 & 0.000 E+00 & 0.000 E+00 & 0.000 E+00 & 0.000 E+00 & 0.000 E+00 & 5.016 E-11\end{array}$ $\begin{array}{llllllllll}0.000 E+00 & 0.000 E+00 & 0.000 E+00 & 0.000 E+00 & 0.000 E+00 & 0.000 E+00 & 0.000 E+00 & 0.000 E+00 \quad 2.544 E-12\end{array}$

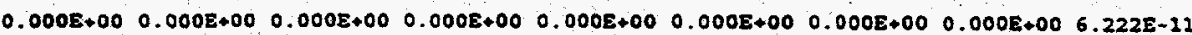

$0.000 E+00 \quad 6.021 E-19 \quad 5.905 E-16 \quad 2.528 E-14 \quad 6.782 E-14 \quad 4.904 E-13 \quad 3.278 E-12 \quad 1.425 E-10 \quad 4.085 E-09$ $0.000 E+00 \quad 1.191 E-15 \quad 1.289 E-13 \quad 1.065 E-12 \quad 2.943 E-12 \quad 2.163 E-11 \quad 2.639 E-10 \quad 9.384 E-10 \quad 2.175 E-08$ $0.0008+00 \quad 2.424 E-21 \quad 2.407 E-18 \quad 6.4608-17 \quad 2.974 E-16 \quad 2.344 E-15 \quad 2.610 E-13 \quad 2.810 E-12 \quad 2.692 E-10$ $0.000 E+00$ 1. $192 E-15$ 1. $195 E-13$ L.080E-12 3.011E-12 1.212E-11 2.959E-10 3.083E-09 2.61.2E-08

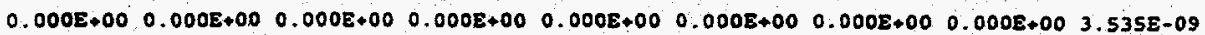

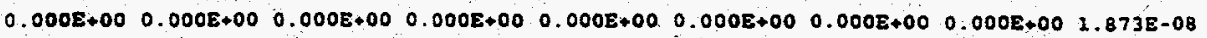
$\begin{array}{lllllllllll}0.000 E+00 & 0.000 E+00 & 0.000 E+00 & 0.000 E+00 & 0.000 \Sigma+00 & 0.000 E+00 & 0.000 E+00 & 0.0008+00 & 1.666 E-09\end{array}$

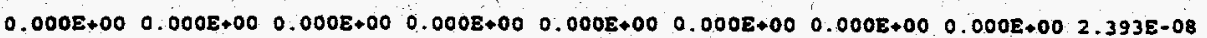


RESRAD, Version $5.623 \quad T^{*}$ Limic $=0.5$ year Sumary : Bio-Shield Activity Assessment HACTR
$03 / 06 / 97 \quad 12: 12$ zage 30

File: A: \BIOSHD.DAT

Individual Nuclide Dose Sumed over All Pathways

Parent Nuclide and Branch Fraetion Indicated

Nuclide Parent BRF(i)

$$
\text { (j) (i) }
$$

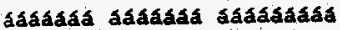

Pu-239 Pu-239 $1.000 E+00$

J-235 Pu-239. 1.000E+00

Pa-231 Pu-239 $1,000 E+00$

Ac-227 Pu-239 2.000E+00

$5 x-90 \quad 5 x-90 \quad 1.000 E+00$

$U-238 \cdot \quad-238 \quad 1.000 E+00$

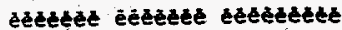
BRF (2) is the branch fraceion of the parene nuclide.
DOSE (j, t), mrem/yr

$E=0.000 E+001.000 E+001.000 E+01 \quad 3.000 E+01 \quad 5.000 E+01 \quad 1.000 E+02 \quad 5.000 E+02 \quad 1.000 E+03 \quad 1.000 E+04$

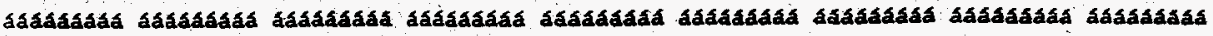
$0.000 E+00 \quad 0.000 E+00 \quad 0.000 E+00 \quad 0.000 E+00 \quad 0.000 E+00 \quad 0.000 E+00 \quad 0.000 E+00 \quad 0.000 E+00 \quad 0.000 E+00$ $0.000 E+00,0.000 E+00 \quad 0.000 E+00 \quad 0.000 E+00 \quad 0.000 \Sigma+00 \quad 0.000 E+00 \quad 0.000 E+00 \quad 0.000 E+00 \quad 2.446 E-09$

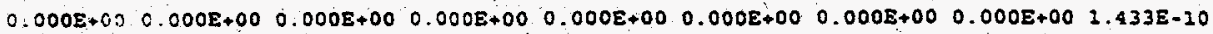
$0.000 E, 0.000 E+00 \quad 0.000 E+00 \quad 0.000 E+00 \quad 0.000 E+00 \quad 0.000 E+00 \quad 0.000 E+00 \quad 0.000 E+00 \quad 2.598 E-10$ $0.000 E+00 \quad 0.000 E+00 \quad 0.000 E+00 \quad 0.000 E+00 \quad 0.000 E+00 \quad 0.0008+00 \quad 0.0008+00 \quad 0.000 E+00 \quad 0.000 E+00$

$9.784 E-36 \quad 9.790 E-36 \quad 9.849 E-36 \quad 9.981 E-36 \quad 1.021 E-35 \quad 1.046 E-35 \quad 1.364 E-35 \quad 1.902 E-35 \quad 8.453 E-08$

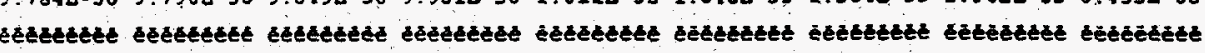


RESRAD, Version $5.621 \quad T$ Limit $=0.5$ year Sumary : Bio-shield Activity Assessment HACTR
03/06/97:12:11 Page $3 i$ File: A: \BTOSHD.DAT

Individual Nuclide Soil Concencration parene Nuclide and Branch Fraction Indicated

Muclide Parent ERF(i)

(j) (i)

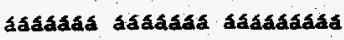

$\begin{array}{lll}A m-241 & \mathrm{~A} m-241 & 2.000 \mathrm{E}+00\end{array}$

$N p-237 \quad 2 m-241,1.000 E+00$

$\mathrm{U}-233 \quad \mathrm{Am}-242 \quad 2.0003+00$

Th-229 Am-242 2.000E+00

$c-14=-14 \quad 1.0008+00$

$\operatorname{co-60} \quad 00-60 \quad 2.000 E+00$

Cs-137 Cs-137 1.000E+00

Eu-152 Eu-152 7.208E-01

Eu-152 Eu-152 2.792E-0I

Eu-152 OS(j)

Gd-152 Eu-152:2.792E-01

$E u-154$ Eu-154 $1.000 E+00$

$\mathrm{H}-3$ H-3 $\quad 1,000 \mathrm{E}+00$

$N i-59 \quad \mathrm{Ni}-59 \quad 1.000 \mathrm{E}+00$

$N i-63 \quad N i-63 \quad 1.000 E+00$

Pu-238 Pu-23B $\quad 3,000 E+00$

U-234 2u-238 $1.0008+00$

U-234 U-234 I. $\quad 000 E+00$

$\mathrm{U}-234 \quad \mathrm{U}-238 \quad 2.000 \mathrm{E}+00$

u.234 OS(j):

$T h-230$ Pu-238 $1.0008+00$

$\mathrm{Th}-230 \mathrm{U}-234 \quad 1.000 \mathrm{E}+00$

Th-230 U-238 $1,000 E+00$

$m-230$ os $(j)=$

Ra-226 Pu-238 1.000E+00

Ra-226 U-234 $1.0008+00$

RA-226 U-238: $1.000 E+00$

Ra-226 As $(j)$ :

Pb-21O Pu-238 1.000E+00

Pb-210 U-234 2.000E+00

Pb-210 U-238 1.000E+00

$\mathrm{Pb}-210$ os $(j)$ :

$$
\text { s(j.t). pci/g }
$$

$E=0.000 E+00 \quad 1.000 E+00 \quad 1.000 E+01 \quad 3.000 E+01 \quad 5.000 E+01 \quad 1.000 E+02 \quad 5.000 E+021.000 E+03 \quad 1.000 E+04$

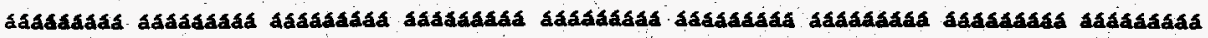
3.230E-02 3:225E-02 3.178E-02 3.077E-02 2.9B0E-02 2.749E-02 1.442E-02 6.434E-03 3.176E-09.

$0.000 E+00 \quad 2.043 E-08 \quad 1.029 E-07 \quad 2.903 E-07$ 4.594E-07 $8.077 E-07 \quad 1.482 E-06 \quad 9.074 E-07 \quad 5.197 E-13$

$0.000 E+00 \quad 2.283 E-14 \quad 2.244 E-12 \quad 1.944 E-11 \quad 5.200 E-11 \quad 1.893 E-10 \quad 2.308 E-09 \quad 4.0728-096.840 E-11$ $0: 000 E+007.290 E-19 \quad 7.097 E-16 \quad 1.861 E-14 \quad 8.372 E-14 \quad 6.234 E-13 \quad 4.531 E-11 \quad 1.971 E-10 \cdot 6.803 E-10$ $6.590 E-03 \quad 6.533 E-03 \quad 6.039 E-03 \quad 5.072 E-03 \quad 4.2608-03 \quad 2.754 E-03 \quad 8.394 E-05 \quad 1.069 E-06 \quad 8.328 E-41$ $6.950 E+03 \quad 6.083 E+03 \quad 1.832 E+03 \quad 1.273 E+02 \quad 8.849 E+00 \quad 1.127 E-02 \quad 7.778 E-26 \quad 0.000 E+00 \quad 0.000 E+00$

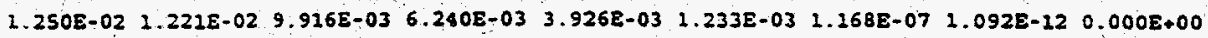

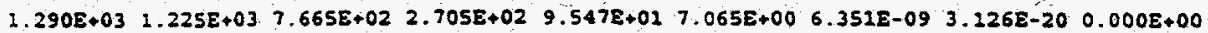

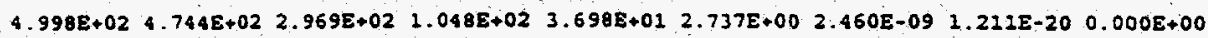
$1.790 E+031.699 E+03 \quad 1.063 E+03 \quad 3.753 E+02 \quad 1.325 E+02 \quad 9 . B 01 E+00$ 8.B21E-09 4.337E-20 0.000E+00 $0.000 E+00$ 3.225E-12 2.500E-12 4.865E-11 5.697E-11 6.110E-12 6.065E-11 5.968E-11 4.470E-11 3. $980 E+01 \quad 3.678 E+02 \quad 1.809 E+01 \quad 3.738 E+00 \quad 7.724 E-01 \quad 1.499 E-02 \quad 3.017 E-16 \quad 2.286 E-33 \quad 0.000 E+00$ $1.870 E+01 \quad 2.340 E+01 \cdot 9.083 E+00 \quad 2.243 E+00 \quad 5.055 E-01 \quad 1.366 E-02 \quad 3.895 E-15 \quad 8.114 E-310.000 E+00$ 4.530E-02 4.530E-02 4.527E-02 4.522E-02 4.527E-02 4.505E-02 4.406E-02 4.286E-02 2.601E-02 $3.6208+00 \quad 3.5948+00 \quad 3.366 E+00 \quad 2.9218+00 \quad 2.517 E+00 \quad 1.750 E+00 \quad 9.568 E-02 \quad 2.529 E-03 \quad 1.001 E-31$

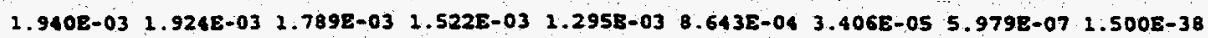
$0.0008+00 \quad 5.476 E-095.269 E-0 B \quad 2.453 E-07 \quad 2.230 E-07 \quad 3.661 E-07$ 5.459E-07 4.286E-07 3.664E-09 3.620E-06 3.618E-06 3.601E-06 3.563E-06 3.525E-06 3.433E-06 2.778E-06 2.133E-06 $1.822 E-08$ $0.000 E+00 \quad 2.199 E-11 \quad 2.288 E-10 \quad 6.496 E-10 \quad 1.071 E-09 \quad 2.087 E-09$ 8.449E-09 $1.298 E-08 \quad 1.123 E-09$ 3.620E-06 3.624E-06 3.654E-06 3.709E-06 3.750E-06 3.802E-06 3.333E-06:2.574E-06. 2.301E-08

$0.000 E+002.468 E-14 \quad 2.4065-12 \quad 2.047 E-11 \quad 5.382 E-11 \quad 2.887 E-10 \quad 2.076 E-09 \quad 4.247 E-09 \quad 1.019 E-08$

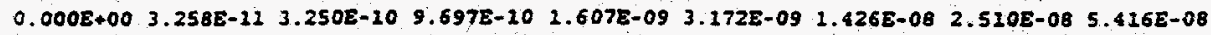
$0.000 E+00 \quad 9.898 E-17 \quad 9.867 E-15$ B.817E-14 2.432E-13 9.555E-13 2.075E-11 6.982E-11 $6.241 E-10$

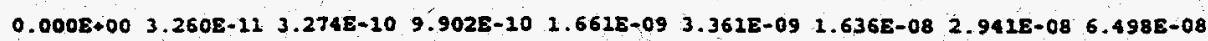

$0.000 E+00 \quad 3.567 E-18 \quad 3.495 E-15 \quad 9.024 E-14 \quad 3.999 E-13 \quad 2.878 E-12 \quad 1.797 E-10 \quad 7.694 E-10-9.283 E-09$ $0.000 E+00 \quad 7.056 E-15 \quad 7.035 E-13 \quad 6.289 \mathrm{E}-12 \quad 1.735 \mathrm{E}-11 \quad 6.824 \mathrm{E}-11$ 1.493E-09 $5.067 \mathrm{E}-09 \quad 4.943 \mathrm{E}-08$ $0.000 E+00 \quad 1.436 E-20 \quad 1.424 E-17 \quad 3.816 E-16 \quad 2.753 E-15 \quad 1.376 E-14 \quad 1.476 E-12 \quad 9.774 E-22 \quad 5.353 E-10$ $0.000 E+00 \quad 7.060 E-15 \quad 7.070 E-13 \quad 6.379 E-12 \quad 1.775 E-11 \quad 7.1148-11 \quad 1.674 E-09 \quad 5.846 E-09 \quad 5.925 E-08$

$0.000 E+00 \quad 2.756 E-20 \quad 2.565 E-16 \quad 1.783 E-14 \quad 1.191 E-13 \quad 1.371 E-12 \quad 1.539 E-10 \quad 7.157 E-10 \quad 9.183 E-09$ $0.000 E+00 \quad 7.254 E-17 \quad 6.756 E-14 \quad 2.572 E-12$ 6.353E-12 $3.783 E-11 \quad 1.313 E-09 \quad 4.750 E-09 \quad 4.890 E-08$ $0.000 E+00 \quad 1.402 E-22 \quad 1.042 E-18 \quad 7.467 E-17 \quad 5.144 E-26 \quad 6.402 E-15 \quad 1.227 E-12$ 8.900E-12 5.290E-10 $0.000 E+007.257 E-17 \quad 6.782 E-14 \quad 1.590 E-12 \quad 6.472 E-12 \quad 3.921 E-11 \quad 1.468 E-09$ 5.475E-09 5.861E-08 
RESRAD, Version 5.621 TH Limie $=0,5$ year Sumary : Bio-Shield Activity Assessment HWCTR
03/06/97 $12: 11$ Page 32

File: A: \BIOSHTD.DAT

Individual Nuclide soil concentration

Parent Nuclide and Branch Fraction Indicated

\begin{abstract}
vuclịde Parenc BRF(i)
(j)

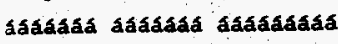

Pu-239. Pu-239 $1.000 E+00$

$0-235 \quad 3-2391.0008+00$

$P a-232$ Pu-239 $1.000 E+00$

Ac-227 Pu-239, $1.000 E+00$

$S I-90 \quad S=-90 \quad 1.000 E+00$

$3-238, \quad 0-238 \quad 2.000 E+00$

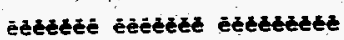
BRF (i) is the branch fraction of the parent nuclide.

$$
s(j, t) . p c i / g
$$

$\tau=0.000 E+00 \quad 1.000 E+00 \quad 1.000 E+01.3 .000 E+02 \quad 5.000 E+01 \quad 1.000 E+02 \quad 5.000 E+02 \quad 1.000 E+03 \quad 1.000 E+04$

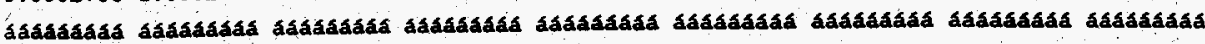

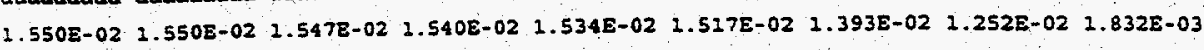
$0.000 E+00 \quad 1.526 E-12 \quad 1.521 E-10 \quad 4.529 E-10 \quad 7.493 E-10 \quad 1.471 E-09 \quad 6.350 E-09 \quad 1.059 E-08 \quad 5.516 E-09$ $0.000 E+00 \quad 1.615 E-16 \quad 1.611 E-14 \quad 1.442 E-13 \quad 3.984 E-13 \quad 1.573 E-12 \quad 3.541 E-111-245 E-10 \quad 1.737 E-09$ $0.000 E+00 \quad 1.700 E-18 \quad 2.582 E-15 \quad 3.680 E-14 \quad 1.486 E-13 \quad 8.853 E-23 \quad 3.142 E-12 \quad 1.277 E-10 \quad 1.734 E-09$ $3.360 E-01 \quad 3.275 E-01 \quad 2.600 E-01 \quad 1.558 E-01 \quad 9.330 E-02 \quad 2.591 E-02 \quad 9.157 E-07 \quad 2.496 E-12 \quad 0.000 E+00$ 7.760E-06 7.756E-06 7.719E-06 7.638E-06 J.558E-06 7.362E-06 5.964E-06 4 $584 E-06 \quad 4.019 E-08$

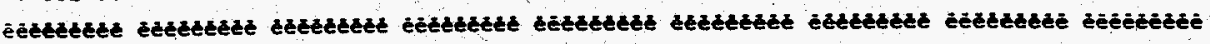


RESIDUAL RÁDIOACTIVITY GUIDELINES FOR THE HWCTR

\title{
Appendix E
}

RESRAD Summary Report

\section{Contaminated Concrete and}

\author{
Embedded Piping
}



RESRAD, Version 5.621 $T^{*}$ Limit $=0.5$ year $\quad 03 / 07 / 97 \quad 08.54$ Page 1

Summary : Radioactivity associated surface concaminated concrece and drainlines File : A: YRUBBLE.DAT

Table of Contents

aasasaááááááá

Part I: Mixture Sums and Single Radionuelide Guidelines

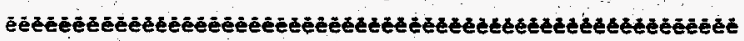

Dose Conversion Factor land Related Parameter Summary ... 2

Site-Specific paramecer, Sumary .................. 6

Sumary of pathway selections $\ldots \ldots \ldots \ldots \ldots \ldots \ldots \ldots \ldots$ i3

Contaminated zone and Total Dose Summary ........... 14

Total Dose Components

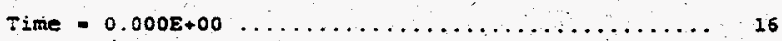

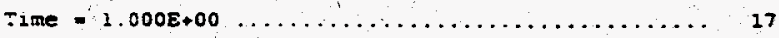

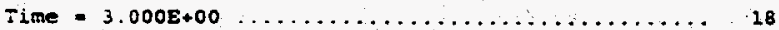

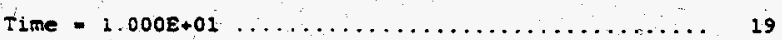

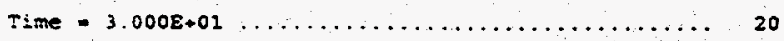

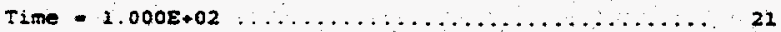

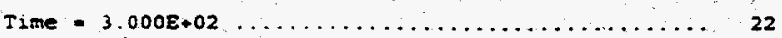

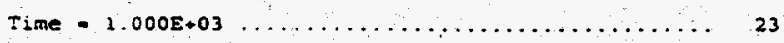

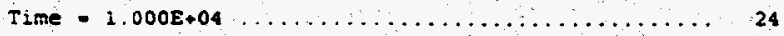

Dose/Source Ratios sumened over All pathways ........... is

Single Radronuclide Soil Guidelınes $\ldots \ldots \ldots \ldots \ldots \ldots \ldots \ldots 26$

Dose Per Nuclide Sumned over All Pathways ........... 28

Solt Concencration Per Nuclide ................... 30 
STSRAD, Versic: $521, T^{-}$Limit $=0.5$ year $03 / 07 / 97$ 08:54 page 2 Sumary : Radioaccivicy associated surface contaminaced concrete and drainlines File A: ARUaze.Dat

Dose Conversion Factor (and Related) Paramecer Sumary File: DOSFAC.BIN

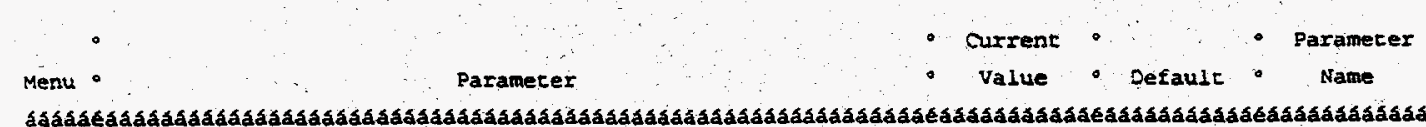

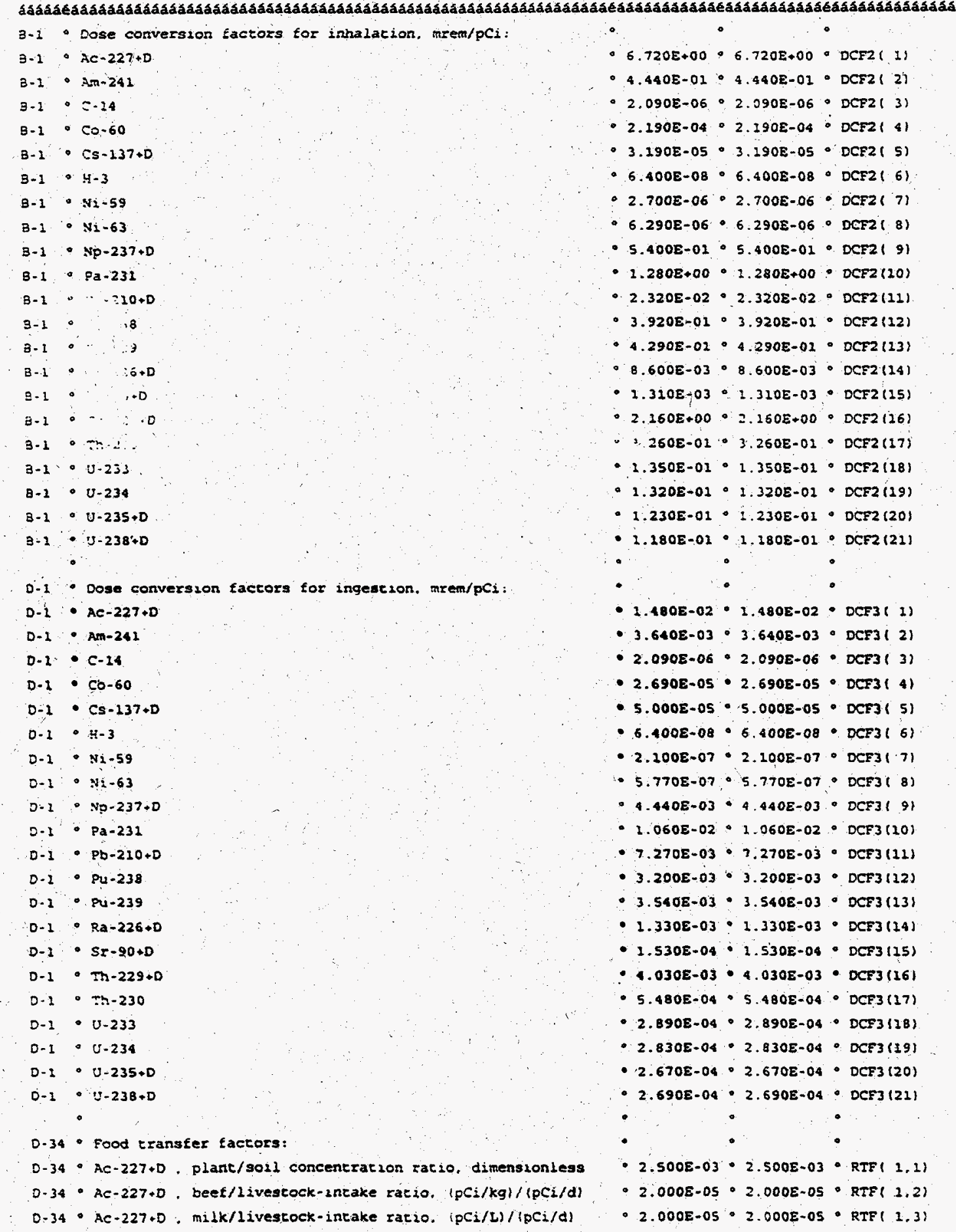


RESRAD, version 5.622 TLimit $=0.5$ year $03 / 07 / 97$ 08:54 Page 3

Sumary : Radioactivity associated surface concaminated concrete and drainlines

File : A: YRUBBLE.DAT

Dose Conversion Factor (and Related) Parameter Sumnary (continued)

File: DOSFAC. BIN

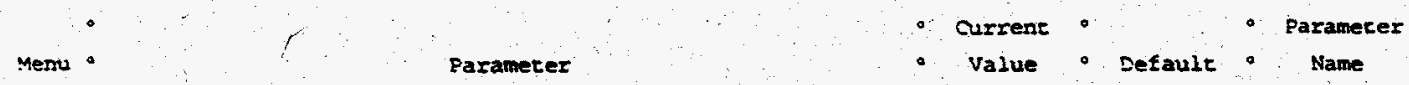

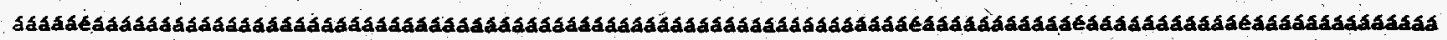

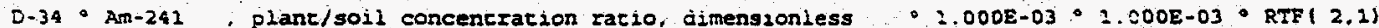

2-34. Am-24I, beef/livescock-incake Iatio, (pCi/kg)/(pCi/d).

D-34 Am-241 milk/livegtock-incake ratio, $(p C i / L) /(p C i / d)$

$0 \cdot 34 \cdot$

D-34 - C-14 plant/soil concentracion ratio, dimensionless

D-34 - c-14. beef/livestock-intake xatio. (pCi/kg)/(pCi/d)

$0-34 \cdot c-14$ milk/livestock-incake racio, $(p C i / L) /(p c i / d)$

$0-34 \cdot$

D-34 Co-60 plant/soil concencration racso. dimensionless

$0.34 \cdot c 0-60$. beef/livescock-intake racio. $(p c j / k g) /(p c i / d)$

D-34 Co-60 milk/livescock-intake ratio. $(\mathrm{pCi} / \mathrm{L}) /(\mathrm{pCi} / \mathrm{d})$

D-34:

D-34 Cs-137+D . plane/soil conceneration racio, dimensionless

D-34 Cs-137+D. beef/livestock-intake ratso. $(\mathrm{pCi} / \mathrm{kg}) /(\mathrm{pCl} / \mathrm{d})$

2-34. Cs-137.D. mi ik/livestock-intake ratzo. $(\mathrm{pCi} / \mathrm{L}) /\left(\mathrm{p} \mathrm{C}_{2} / \mathrm{d}\right)$

$=34$.

$=34 \cdot \mathrm{H}=3$

$=.34 \cdot H \cdot 3$

$2.34 \cdot \mathrm{H}-3$

0.34 .

$0-34: N_{1}-59$

$\mathrm{D}-34 \cdot \mathrm{Ni}-59$

D.34-Ni.59

0.34 .

D-34.Ni-63

$\mathrm{D}-34 \cdot \mathrm{Ni}-63$

mik/livestock-intake rat $20,\left(p C^{2} / L\right) /\left(p C_{2} / d\right)$

plant/soll concentration racio. dimensioniess beef/livestock- 2ntake racso. $\left(p c_{j} / \mathrm{kg}\right) /\left(p C_{2} / d\right)$ - m2lk/livestock-intake rat 20 , (pCi/L)/(pCs/d)

D-34. Ni-63

- plane/soil concencracion rat 10, dimensionless beef/Livestock-incake rat $10 . \quad(p C 2 / \mathrm{kg}) /\left(p C_{1} / d\right)$

milk/livestock-intake rat $10 .(p c i / L) /(p c i / d)$

$0-34$.

plant/soil concentration ratio, dimensionless beef/livescock-2ntake racio, (pci/kg)/(pCi/d) milk/liveatock-incake racso. $(p C i / t) /(p \mathrm{ci} / d)$

D-34 - Np-237+D . plane/soil concentration raclo. dimensioniess D-34 Np-237+D, beef/livestock-2ncake ratio, (pCi/kg)/(pCi/d) D-34 - Np-237+D milk/livescock-incake rar1o, $(p C i / L) /(p C i / d)$

$D-34$.

$0.34 \div \mathrm{Pa}-231$

$=-34 \cdot \mathrm{Pa}-231$

plant/soil concentration ratso. dimenszoriess beef/1ivestock-1ntake raclo, $(p \mathrm{Ci} / \mathrm{kg}) /(\mathrm{pCi} / \mathrm{d})$ j-34. Pa-231 milk/livegtock-2ncake, rat 20. (pCi/L)/(pCi/d)

$0-34 \cdots$

$0-34$ Pb-210+D. plant/soil concentration rat20, dimensionless 0-34 - pb-210+D, beef/livestock-intake rat10, (pCi/kg//(pCi/d) $0-34$ - pb-210+D milk/iivestock-intake rat10, (pci/L)/(pCi/d) 2.34 .

$0.34 \cdot P u-238$

$0-34 \cdot P u-238$

plant/soil concentration zat 20 . dimensionless

$0-34 \cdot P u-238$ beef/livestock-1ntake rat2o. (pCi/kg)/(pCi/d) $0-34$. milk/livestock-intake racio, (pCi/L)/(pCj/d)

$0.34 \cdot P u=239$

plant/soil concentration ratio. dimensionless

$0-34 \cdot$ Pu-239

plane/soll concentration rat 10 . dimensioniess

$0.34 \cdot P 1-239$

milk/livestock-1ntake rac $10 .(p C i / L) /(p C i / d)$

. 5.000E-05.5.000E-05 RTF(2.2)

2.000E-06*2.000E-06* RTF1 2.3)

- $5.500 E+00 \cdot 5.500 E+00 \cdot \operatorname{RTE}\{3,1\}$

- 3..1008-02 $\cdot 3.1008-02 \cdot \operatorname{RTF}(3,2)$

- 1.200E-02 $: 1.2008-02 \cdot R T E(3.3)$

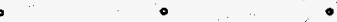

$0.000 E-02 \cdot 8.000 E-02 \cdot$ RTF $(4,1)$

- 2.000E-02 $2.000 E-02-\operatorname{RTF}(4.2)$

-2.000E-03 $\cdot 2.000 E-03 \bullet \operatorname{RTF}(4.3)$

-.$\quad 0$

$\because 4.000 E-02 \cdot 4.000 E-02 \cdot \operatorname{RTF}(5,1)$

- 3.000E-02 3.000E-02 - RTF( 5,2)

- 0.000E-03 8.000E-03 RTF( 5.3$)$

-

- . B00E+00 4. BOOE+00 RTFI 6.11

- 1.200E-02 $\cdot 2.200 E-02 \cdot \operatorname{RTF}(6.2)$

- 1.000E-02 1,000E-02 - RTF (6,3)

-.$\quad 1.0000^{0}$.

0 5.000E-02 5.000E-02 - RTF ( T,1)

- 5.000E-03 $: 5.000 E-03 \cdot \operatorname{RTE}(7,2)$

2.000E-02 $2.0008-02 \cdot \operatorname{RTF}(7.3)$

- 5.000E-02 $\cdot 5.000 E-02 \cdot \operatorname{RTF}(a, 1)$

- 5.0008-03 $\cdot 5.0008-03 \cdot \operatorname{RTF}(8.2)$

2.000E-02 2.000E-02 - RTF( 8.3)

2.000E-02 -2.0008-02 RTFt 9.1

- $1.0008-03 \cdot 1.000$ E-03 RTF(9.2)

- 5.000E-06 5.000E-06 RTF 9.31

$\therefore \quad \cdots \cdots$

- 1.000E-02:I. C00E-02 $\operatorname{RTF}(20,1)$

$-5.0008-03 \cdot 5.0008-03 \cdot \operatorname{RTF}(10.2)$

- 5.0008-06 \$.000E-06 RTF $(10.3)$

- $\quad 0 \quad 0$

- 1.000E-02 $1.000 \mathrm{E}-02 \cdot \operatorname{RTT}(11,1)$

- 8,0008-04 8,0008-04 - RTE(11,2)

-3.0008-04-3.0008-04-RTF(11.3)

- $\quad \cdots \quad$ C

- :.0008-03 1.0008-03 RTF $(12,1)$

-1.000E-04:1.000E-04 RTE(12.2)

- 1.000E-06 2.000E-06 RTE(22,3)

$-10005-03 \cdot 10005-03 \bullet$ OTE 123,1

$-1.0008-04 \cdot 1.000 E-04 \cdot \operatorname{RTE}(13,2)$

-1.000E-06 1.000E-06 $\cdot \operatorname{RTF}(13,3)$

$3-34 \cdot$ 
RESRAD, version $5.621 \quad$ Limit $=0.5$ year $03 / 07 / 97$ 08:54 2age 4 Summary : Radioactivity associaced surface contamsnated concrete and drainlines File : A: \RUBBLE.DAT

Dose Conversion Factor (and Related) Parameter Sumary (continued) File: DOSFAC.BIX

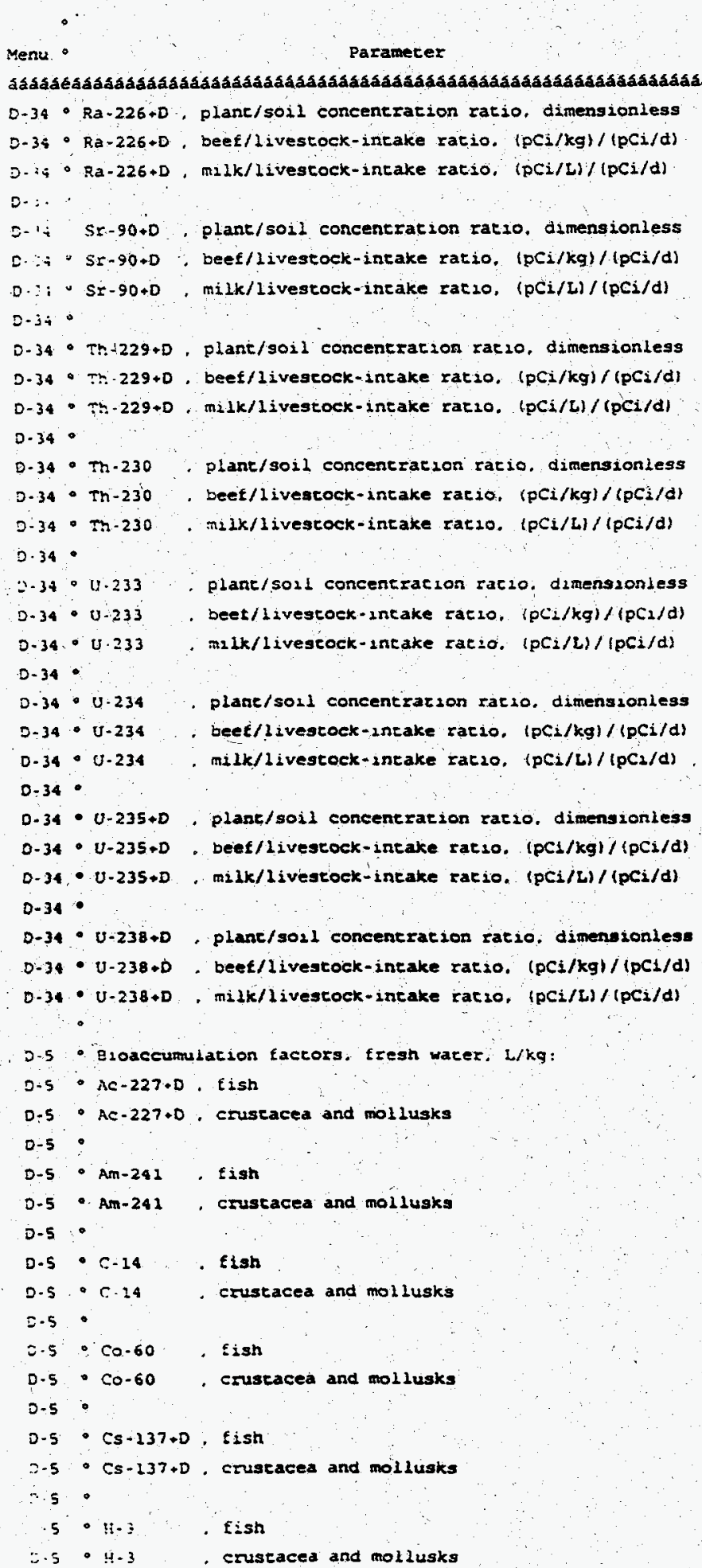

- Current - Palue - Pefaule - Namecer

5

$0-34-R a-226+D$, planc/soil concentration ratio, dimensionless

- 4.000E-02 $\cdot 4.0008-02 \cdot \operatorname{RTF}(14,1)$

- 1.000E-03:1.200E-03 RTF(14.2)

$\therefore 1,000 E-03 \cdot 1,000 E-03 \bullet R T E(14.3)$

- $\quad$.

- 3.000E-01 3.000E-01 RTE\{15.1\}

-8.000E-03 - 8.000E-03 - RTF $(15,2)$

-2.000E-03 2.000E-03 RTF (25.3)

- 1.000E-03 $\cdot 2.000 E-03 \cdot \operatorname{RTF}(16.1)$

$\because 1.0008-04 \cdot 1.0008-04 \cdot \operatorname{RTF}(16.2)$

- 5.000E-06:5.000E-06 RTF(16.3)

- $\quad \vdots \quad a$

- 1.000E-03 $1.000 E-03 \cdot \operatorname{RTF}(17,1)$

- 1.000E-04 2.000E-04 RTF(17.2)

- S.000E-06 5.000E-06 RTF (27.3)

- $\therefore \quad \therefore \quad 0$

$-2.500 E-03 \cdot 2.500 E-03 \cdot \operatorname{RTF}(28.1)$

$-3.400 E-04 \cdot 3.400 E-04 \cdot \operatorname{RTE}(18,2)$

$\therefore 6.000 E-04 \cdot 6.000 E-04 \cdot \operatorname{RTF}(28,3)$

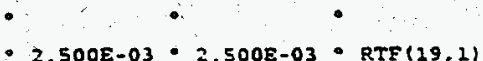

- 3.400E-04-3.400E-04 $\operatorname{RTF}(19.2)$

6.000E-04 6.000E-04 RTF(19.3)

- $\quad \therefore, \quad-\ldots$

-2.500E-03 $2.500 E-03 \cdot \operatorname{RTF}(20.1)$

3.400E-04 3.4008-04 RTF (20.2)

- $6.0008-04 \cdot 6.0008-04 \cdot \operatorname{RTF}(20,3)$

- $2.5008-03 \cdot 2,500 E-03 \cdot R T F(21.1)$

-3.400E-04 -3.400E-04 RTF $(21,2)$

- $6.000 E-04 \cdot 6.000 E-04 \cdot R T F(21.3)$

- $\quad$ a

- 1.500E+01 1.500E+01 - agogaci 1.1)

- 1.000E+03 - 1.000e+03 - grofacl 1.2)

- 1 -

- 3.000E+02 - 3.000E+02 - BrofaCi 2,11

- $1.0008+03 \cdot 2.0008+03 \cdot$ BIOFAC $(2,2)$.

- 1 -

- 5.000E+04 5.000E+04 - BIOEAC( 3.1$)$

$0.100 E+03 \cdot 9.1008+03 \cdot$ BIOFAC $(3.2)$

- $\quad \therefore \quad$ C

- 3.000E+02 $-3.0002+02:$ BIOFAC $(4,1)$

- $2.0008+02 \cdot 2.000 E+02 \cdot \operatorname{BIOFAC}(4.2)$

- $\quad . \quad-$

- 2.000E+03 $\cdot 2.0008+03 \cdot \operatorname{BIOFAC}(5.1)$

$\because 1.000 E+02 \cdot 1.0008+02 \cdot \operatorname{Btosac}(5.2)$

- C C

- $1.000 E+00 \cdot 1.000 E+00 \cdot$ BIOFAC $(6,1)$

- 1.000E+00 1.000E+00 BIOFAC( 6.2$)$ 
RESRAD. Version 5.621. $I^{-}$imie $=0.5$ year $03 / 07 / 97$ 08:54 Page 5

Sumary : Radioactivity associated surface contaminated conerete and drainilines

File : A: IRUBBLE.DAT

Dose Conversion Factor (and Related) Parameter Sumary (contimued)

File: DOSFAC.BIN

Menu - Parameter $\quad \therefore$ Current $\bullet$ Parameter

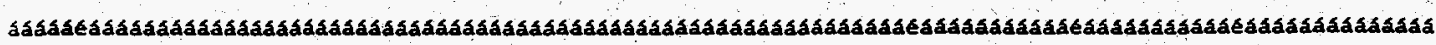

D.5 $\cdot \mathrm{Ni-59}$, Eish

D-5. Ni-59. Cristacea and mollusks

$0-5 \cdot$

$0.5 \cdot \mathrm{N}_{2}-63 \cdots$. fish

$\mathrm{D}-5 \circ \mathrm{N}-63$, crustacea and mollusiks

D-5

$D-5 \cdot N o-237+D ; f i s h$

$D-5 \cdot N p-237+D$, cruscacea and mollusks

$D .5$.

$D-5 \cdot P a-231$ Eish

$0-5$ - $9-231$, crustacea and mollusks

$D-5$.

D-5 Pb-210+p. fish

$0+5$ - $P b+210+D$. Crugtacea and mollusks

$0-5:$

$0-5 \cdot M-238$ : $\quad$ ish

0.5 Pu-238 . Cxustacea and molluskg

0.5 .

D-5 Pu-239, fish

$0-5$ Pu-239 Crustaces and mollusks

$0-5 \cdot$

$0.5-R a-226+D, f i s h$

$0-5$ - Ra-226+D. crustacea and mollusks

0.5 .

D-5 $5 x-90+0$, Eish

D-5 - SE-90+D. Crustinced and mollusks

D-S .

D-5 In-229+D fish

D-5 In-229+D cruacecea and mollusks

$0-5$.

D-5 Th-230 Eish

D.5 Th-230, chistacea and mollusks

$0-5$.

D.5 U.233 fish

$0.5 \cdot 0.233$ erustaces and mollusks

$0-5:$

$0-5 \cdot U-234 \quad$ E1sh

D.5 - U-234 ersatacea and mollusks

$0-5$.

$0-5 \cdot U-235+D$. Eish

$0-5 \cdot U-235+D$ crustacea and mollusks

$D-5$.

$0.5 \cdot U-238+0 \%$ figh

0.5 U-238+D, erustacea and mollusks
- 1.000E+02 $\cdot 1.000 E+02 \cdot$ eIOFAC 7,11

- $1.000 E+02 \cdot 1.000 E+02 \cdot \operatorname{BIOFAC}(7.2)$

- $\therefore$

- 1.000E+02 $1.0008+02$ BIOFACl 8.11

- $1.000 E+02 \cdot 1.000 E+02 \cdot \operatorname{aIOEAC}(8,2)$

$\therefore \quad 0$.

- 3.000E+01 3.000E+01 - BrofAc( 9.1)

- 4.000E+02 - 4.000E+02 - BIOFAC 9,21

- $\quad$. 0

-1.000E+02 $1.000 E+01 \cdot \operatorname{grOFAC}(20.2)$

- $1.1008+02 \cdot 1.1008+02 \cdot \operatorname{BLOFAC}(10.2)$

- a c a

- $3.000 E+02 \cdot 3.0008+02 \cdot$ BIOFAC $(11.1)$

- 1.000E+02 $1.000 E+02 \cdot \operatorname{BIOFAC}(21,2)$

-

- 3.000E+01-3.0008+01- ETOFAC (12.1)

-1.000E+02 1.000E+02. BIOFAC (12.2)

- 3.000E+01 -3.000E+01 BIOFAC (13.2)

- $1.000 E+02 \cdot 2.000 E+02 \cdot$ BIOFAC $(13.2)$

- 5.000E+01 5.000E+01 BIOFAC(14.1)

- $2.500 E+02 \cdot 2.500 E+02 \cdot$ BIOEAC (14.2)

-6.000E+01 6. 000E+01 BIOFAC (15,1)

-1.0008+02 $\cdot 2.0008+02 \cdot$ BIOFAC $(15.2)$

- $1.0008+02 \cdot 2.0008+02 \cdot \operatorname{BIOFAC}(16,1)$

$-5.0008+02 \cdot 5.0008+02 \cdot$ BIOFAC $(16.2)$

- 1.000E+02 $-1.000 E+02 \cdot \operatorname{arOFAC}(17.1)$

-5.000E+02 -5.000E+02 BIOFAC (27,2)

- 1.0008+01 - 1.0008+02 - arOfac(18.1)

-6.000E+01 $6.000 \mathrm{E}+01 \cdot \operatorname{BrOFAC}(18.2)$

-

- 1.000E+01 - 1.000E+01 - BroFAC $(19,1)$

-6.000E+01 $6.0008+02 \cdot \operatorname{srOFAC}(29.2)$

-

- $1.0008+01 \cdot 1.0005+01 \cdot \operatorname{BtOFac}(20,1)$

-6.000E+01 6.000E+01 $\operatorname{BIOFAC}(20,2)$

-

- $1.0008+01 \cdot 1.0008+01 \cdot \operatorname{BIOFAC}(21,1)$

- 6.000E+01 $6.0008+01 \cdot \operatorname{BIOFAC}(21,2)$

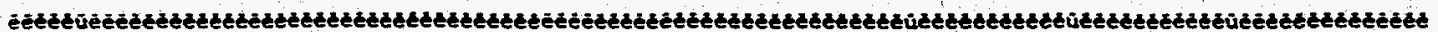


ESRAD, Version 5.521 T Limit $=0.5$ year 03/07/97 08:54 Page 6

junmary : Radioactivity associated surface concaminated concrece and drainlines

File : A: \RUABLE.DAI

Site-Specific Paramerer Sumnary

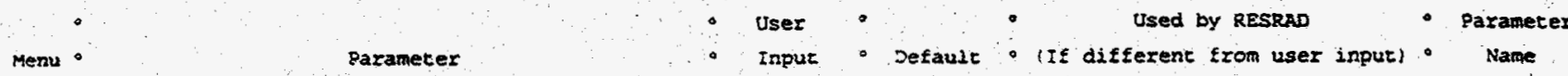

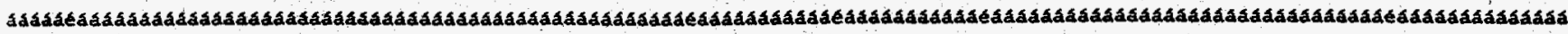

\$011 - Area of contaminated zone $\left(m^{* * 2}\right)$

2022 - Thickness of concaminated zone im

2012 - Lengen parallel to aquifer flow (mi

ROI1 - Sasle radiacion dose limit (mrem/yr)

ro12 - Time since placemenc of marerial (yr)

R012. Times for calculations (yr)

F011 - Iimes for calculations (yr)

R012. Tintes tor calculations $(y x)$

Ro11 - Times for calculations (yr)

Ro11. Iimes for calculations (yx)

R022 - Times tor calculations (yz)

R011 - Times for calculations (yr)

Ro11 - Times for calculations (yr)

R012 - Tames for calcularzons (yz)

Ro12 - Injelal pxincipal radionuclide $(p C i / g \mid$

3012 - Initial principal radionuclide ( $\left.p C_{1} / g\right)$

r012 : sirlal prancipal fadionuch 2 de $\left(p c_{2} / q\right)$

Ro12 - Initial principal radionuclide $(\mathrm{pC} / \mathrm{g})$

2022 - Inicsal principal zadionuclide (pci/g):

R022 - Inleial principal radionuclide (pCj/g):

Ro12 - Isicial principal radiomelide (pCi/g):

R012 - Inirial primeipal radionuclide (pCi/g\};

R022 - Instial principal radionuclide $(\mathrm{pci} / \mathrm{g})$;

R012 - Initial principal radionuclide $(\mathrm{pCi} / \mathrm{g})$ :

R012 - Initial principal radionvelide (pci/gl:

Ró2 - Initial principal radionuclide (pci/g).

Ro12 - Initial pxincipal radionuclide (pCl/g):

R012 - Concentration in groundwacer

R012 - Concentration in groundwacer

R012 - Concentration in groundwacer

R012 - Concentration in groundwater

R012 Concentration in groundwater

Rol2 - Conceneration in groundwacex

Ro12 - Concentration in groundwacer

RO12 - Concentration in groundwater

8012 Concencration in groundwater

R012 - Concentration in groundwater

Ro12 - Conceneration in groundwacer

8022 - Concentracion in groundwacer

2012 - Concentracion in grountwacer

(pcL/to) :

(pcs/L)

(pCL/L)

(pci/l)

(pci/t)

(pci/L)

(pCi/L):

(pCi/L)

(pcis/L) :

(pCl/L)

(pci/L)

$(p c i / L)$

(pei/t):

Ro13 - Covex deprh (m)

2023 - Ders $25 y$ of cover material ( $/$ / cmm-3)

R013, - ( $\because 2 x$ depen erosion sace $(\mathrm{m} / \mathrm{yr})$

Ros3 : Sersicy of concaminated zone $(5 / \mathrm{cm} * 3$

R013 - Contaminaced zone erosion race $(\mathrm{m} / \mathrm{yr})$

r013. Contaminated zone cotal porosaty

$20: 3$ - Contaminated zone effeceive porosity

- 3.150E+02-2.000E+04.

- $5.000 E+00 \cdot 2.0008+00 \cdot$

- 2.100E+02:2.000E+02

$-1.5008+01 \cdot 3.0000+01$.

$0.000 E+00 \cdot 0.000 E+00$.

- $1.000 E+00 \cdot 1.0008+00$.

-3.000E+00:3.000E+00.

$=1.000 E+01 \cdot 1.000 E+02$.

$3.000 E+01 \cdot 3.000 E+01$.

$1,000 E+02 \div 2.000 E+02 \circ$

$-3.000 E+02 \cdot 3.0008+02$.

$\therefore 1.0008+03 \cdot 1.0008+03 \cdot$

- $1.000 E+00-0.000 E+00$.

- not used $0.000 E+00$.

-

$A m-242 \therefore 2.0708-05 \div 0.000 E+00 \circ$

c.14 - 2.200E-01 -0.000E+00.

$60.60 * 1770 E-02 * 0,000 E+00$.

$0-137 \cdot 1.5208+00 \cdot 0.0008+00$

$4-3 \quad \because 4.4902-01 \cdot 0.0008+00$

$N i=59 \quad 2.8908-05 \cdot 0.000 E+00$ a

$\mathrm{N}_{2}-63 \div 2.3208-03 \div 0.0008+00$.

Pu-238 $: 270 \mathrm{E}-06 \cdot 0.000 \mathrm{E}+00$.

Pu-239 $-1.8008-04 \cdot 0.0008+00 \cdot$

sx-90 $\because 2.0908-01 \cdot 0.0008+00$.

$v-234 \cdot 200 E-03 \cdot 0.000 E+00$.

U-235 $\cdot 2.780 \mathrm{E}-04 \cdot 0.000 \mathrm{E}+00$

G-238 $\quad 2.7108-02-0.000 E+00$

am-242 not used $0.000 E+00$

C-14 not used $0.0008+00 \cdot$

Co-60 noe used $0.0008+00$

Cs-137. not used $-0.000 E+00 \%$

H-3 not used $\div 0.000 E+00$

$N_{2}-59$ not used $0.000 E+00$

Ni-63 a not used $0,000 E+00$.

pi-23B - nor used $0.000 E+00$.

Pu-239 not used $0.000 E+00$.

$5 r-90$ at used $0,000 E+00$.

U-234 not used $0.000 E+00$.

U-235 not used $0.0008+00$.

- not used - $0.000 \mathrm{E}+00$ -

$\therefore 1.3008+01 \div 0.0008 * 00$.

$1.4408+00 \cdot 1.5008+00 \cdot$

-9.000E-05 - 2.000E-03.

- $2.400 E+00 \cdot 1.500 E+00$.

- $0.000 E+00 \cdot 1,000 E-03$

- $4.3008-02 \cdot 4.000 E-02$.

- 3.300E-02 - 2.000E-02.

7013 - Concaninated zorie hydraulic conductivicy (m/yr)

- $1.520 z+00 \cdot 1.000 E+01$.

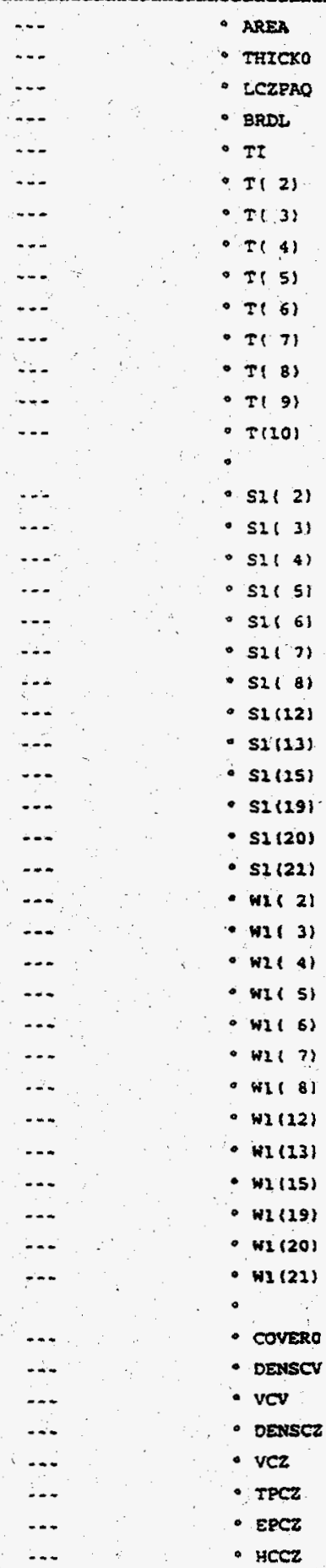


RESRAD, Version $5.621 \quad T^{*}$ Limit $=0.5$ year $03 / 07 / 9708: 54$ Fage 7

Sumary : Radioactivity associated surface concaminated concrece and crainlines

File, A: ARUBBLE.DAT

Site-Specific Paramecer Sumary (continued)

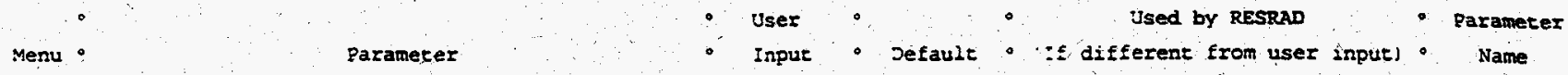

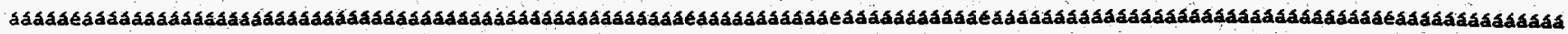

$R O 13$ - Concaminated zone b parameter

Ro13 - Fumzdicy in asr $(\mathrm{g} / \mathrm{cm} * * 3)$

R013 - Evapotranspiracion coefficient

Ro13 - Precipleation (m/yr)

R013 - Inzigacion (m/yr)

R013 - Irxigacion mode

Ro13 - Runotf coefficient

R013 - watershed area for nearby siream or pond (m* $* 2$ )

R013 - AccuracY for water/s011 compucations

-

R014 Densicy of sacurated zone $1 \mathrm{~g} / \mathrm{cm} * * 31$

R014 : Sacuraced zone cocal porossey

R014 - Saturaced zone effective porosity

R014 - Saturated zone hydraulic conductiviey $(\mathrm{m} / \mathrm{yr})$

R014. Saturated zone hydraulic gradzent

R024 Sacuraced zone b parameter

R014 - Water cable drop race $(\mathrm{m} / \mathrm{yr})$

2014 Walt pump incake depen (m below wacer rables

R014 Model: Nondispersion (ND) or Mass-Balance (MB)

Ro14 Well pumping-rate $(\mathrm{m} *-3 / y r)$

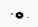

R015 - Number of unsaturated zone strata

R015 - Unsat, zone 1. thickness $(\mathrm{m})$

Ro15 - Unsat. zone 1 , soll densicy $(\mathrm{g} / \mathrm{cm} \cdots 3)$

Ro15 Unsac. zone 1. tocal porosity.

Ro15 - Ungat zone 1 , effective porosity.

Ro1s - Unsat. zone 1, soil-apecific b parameter

R015 - Unsac: zone 1, hydraulic conductiviey $(\mathrm{m} / \mathrm{y}=)$

$\because$

R016 Digeribution coefficients for Am-241

R026 - Contaminated zone $(\mathrm{cm} * 3 / \mathrm{g})$

R016. Unsaturaced zone $1(\mathrm{~cm} *-3 / \mathrm{g})$.

R016 - Saturated zone $(\mathrm{cm}-0.3 / \mathrm{g})$

2016 : :each race (/yr)

Ro16 Solubiltey constant

R016 - Distribution coefficients for $\mathrm{C}-14$

RO16 - Concaminaced zone $(\mathrm{cm}+0) / \mathrm{g})$

R016 - Unsaturated zone 1 (cm*3/g)

Roi6 - Sacuraced zone $(\mathrm{cm} * 3 / \mathrm{g})$

Ro16 - Leach rate $(/ \mathrm{Yr})$

R016. Solubility constane

R016 Distrabution coefficienes for Co- 60

R016 - Contaminaced zone $(\mathrm{cm} *-3 / \mathrm{g})$

Ro16 Unsaturated zone 1 ( $\mathrm{cm}=3 / \mathrm{g})$

R016 - Saturated zone $(\mathrm{cm}=3 / \mathrm{g})$

Rd16 - veach race $(/ Y T)$

R016 Solubslity constant
- $5.3008+00 \cdot 5.300 E+00$.

$\because 1.1008+01$ - $8.0008+00$.

-9.100E-01 5.000E-01.

- $1.200 \mathrm{E}+00 \cdot 2.000 \mathrm{E}+00$

-7.600E-01 2.000E-01?

- overhead - overhead -

- 4.000E-01 2.000E-01.

- $1.0008+06 \cdot 2.0008+06$.

-1.000E-03 :.000E-03 -

$\therefore \quad \therefore \quad \therefore$

- $1.4408+00 \cdot 1.500 E+00$

- 4.300E-01 4.000E-01.

-3.3008-02 2.000E-02

$\because 5.4608+02 \bigcirc \div .000 E+02$.

- 3.500E-03 2.000E-02

- 5.300E+00 5. $300 E+00$.

- $0.0008+00 \cdot 2.0008-03$.

- 5.100E+00? O.000E+01。

- ND ND $2500 E+02 \cdot 2500 E+02 \circ$

$2.500 E+02 \cdot 2.500 E+02 \circ$

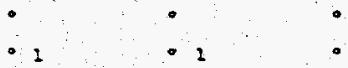

$1.550 E+03 \cdot 4.0008+00$

- $1.440 E+00 \cdot 1.500 E+00$.

- 4.300E-02 4.000E-01.

$: 3.3008-01 \cdot 2.0008-01$.

- $5.3008+00 \bullet$. $3.3002+00$.

$1.520 E+00 \cdot 1.000 E+02$

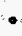

- $1.900 E+03 \cdot 2.000 E+01$

-1.900E+03 2.000E+01.

- $1.900 E+03 \cdot 2.000 E+01$.

- $0.000 \varepsilon+00 \div 0.000 \varepsilon+00$.

- $0.000 E+00 \cdot 0.000 E+00$

.

$\therefore$

$2.000 E+00 \cdot 0.000 E+00$.

- 2.000E+00 $0.0008+00$.

- 2.000E+00 $\cdot 0.000 E+00$.

$-0.000 E+00 \div 0.000 E+00$.

- $0.000 E+00 \cdot 0.0008+00$.

.

-

- $1.000 E+01 \div 1.000 E+03$.

- $1.000 E+01 \div 1.0008+03$.

- 1.000E+01 2.000E+03.

- $0.000 E+00 \cdot 0.0008+00$.

- $0.000 E+00 \cdot 0.000 E+00$.

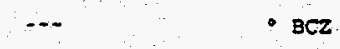

.- $\quad$ HUMID

-. - EVAPTR

-. $\quad$ C PRECIR

-. $\quad$ O RI

-.. $\quad$ IOITCh

$\cdots \quad \because \quad$ RUNOFF

… D WAREA

$\therefore$ EPS

- D DENSAO

-. $\quad$ - TPSz

$\therefore$ - EPSZ

-. $\quad$ Hesz

-- $\quad$ HorT

.. $\quad \therefore$ Bsz

-. VWT

$\cdots \quad \therefore \quad$ DWIBWT

-.

$\ldots \quad \cdots$

$\cdots \quad \cdots \quad 0$

$\because \quad \div$ NS

.. $\quad$ H(I)

-. - DEnsuz (2)

-- $\quad \therefore \quad \bullet \operatorname{TPUz}(1)$

$\ldots \quad \because$ EPUZ (1)

C. . auz(2)

-.. $\quad$ HCOz(1)

$\therefore \therefore \cdots$

$\ldots \quad$ Danuce $(2)$

-. ocrucur 2.1

... V DCUuCs ( 2$)$

5.842E-06 ALEACH( 2)

not used $\quad$ SOLUBK( 2$)$

- Darucer 31

- Darucosi 3.1 .

- Dorucs (3)

- aleacal 31

- soluexi 31

not used

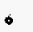

$\ldots \quad$ Davuces 4 )

$\cdots$

$1.094 E-03$

- bavucs ( 4 )

noc used

- AlEach (4)

- soluex ( 4$)$ 
RESRAD, Version $5.621 \quad T$ Limie $=0.5$ year $03 / 07 / 97$ 08:54 Fage 8 Sumnary : Radioactivicy associated surface contaminated concrete and drainlines File : A: \RUBBLE:DAT

Site-Specifis Paramecer Summary (continued)

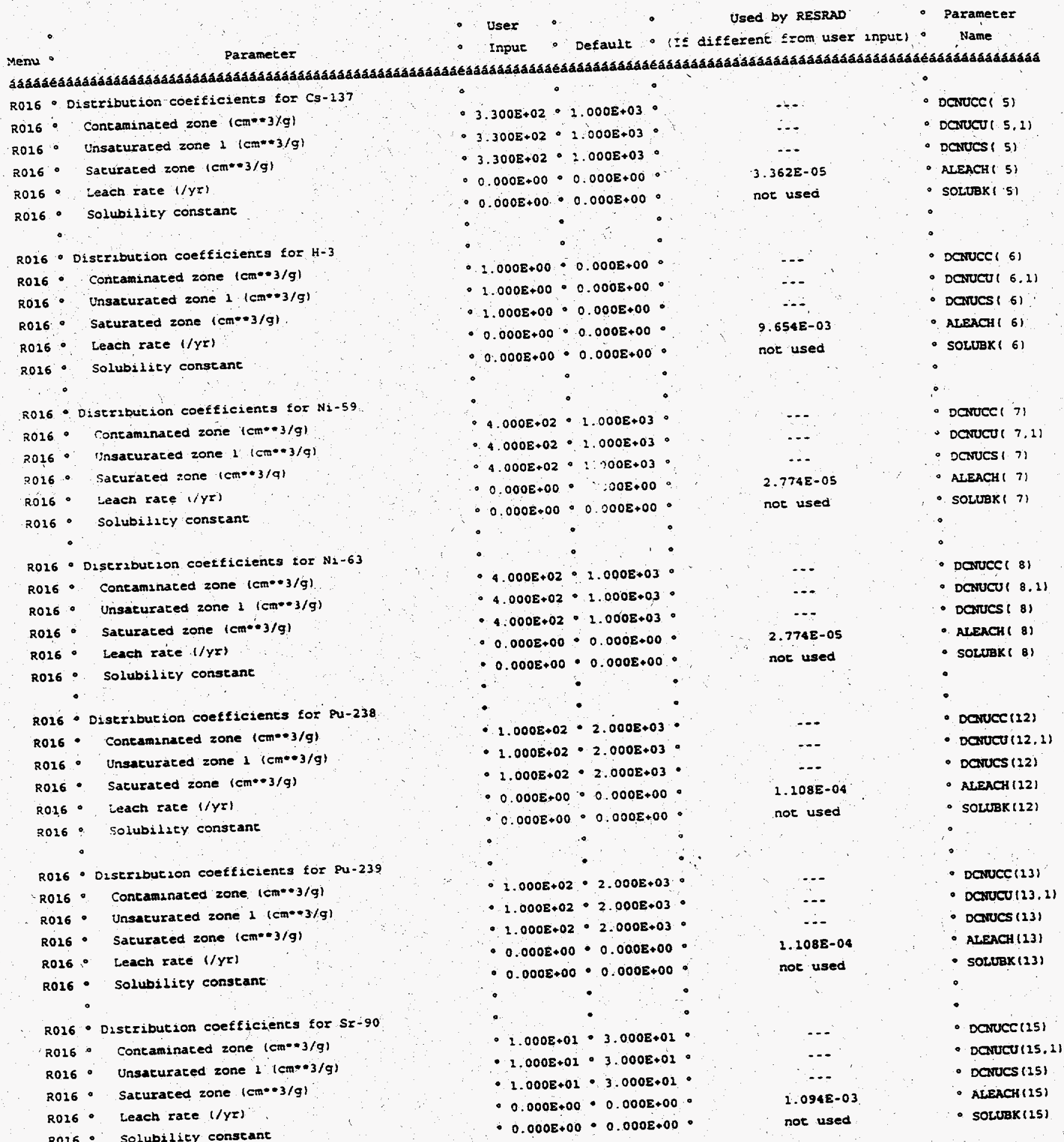


FISRAD. Version 5.621 T' Limie $=0.5$ year $03 / 07 / 97$ 08:54 page 9

Summary : Radioactivity associated surface contaminated conerete and drainlines

File $\quad \therefore$ A:IRUBBIE.DAT

Site-Specific Paramecer Summary (continued)

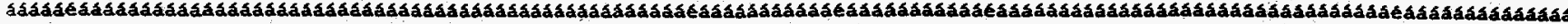

2026 - Distribution coefficients for U-234

3016 - Contaminated zone $(\mathrm{cm} * * 3 / g)$

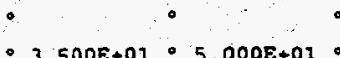

- $3.5008+01 \cdot 5: 000 E+01$.

- $3.500 E+01 \cdot 5.000 E+01$.

- $0.000 E+00 \cdot 0.000 E+00$

$0.000 E+00 \div 0.000 E+00$

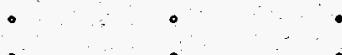

$3.5005+01 \cdot 5.0005+01$.

- $3.5008+01 \cdot 5.000 E+01 \cdots$

- $3.500 E+02 \cdot 5.0008+01 \cdot$

- $0.000 \mathrm{E}+00 \cdot 0.000 \mathrm{E}+00$.

- $0.000 E+00 \cdot 0.0008+00$.

3.500E+01 $5.000 E+01$

- 3.500E+01 5.000E+01

- 3.500E+01 $-5.000 E+01$.

- $0.000 E+00 \cdot 0.000 E+00$ ?

- $0.000 E+00 \cdot 0.000 E+00$

- 4.000z+02 *2.000z+01.

-4.000E+02 $\cdot 2.000 E+01$

- $4000 E+02$ - 2.000E+02

$0.000 E+00-0.000 E+00$

- $0.0008+00 \cdot 0.0008+00$

50000000000000

- $5.0008+00-1.0005+00$.

- $5.000 E+00-2.000 E+00$

- $0.000 E+00 \cdot 0.000 E+00$

- $0.000 E+00 \cdot 0.000 E+00$

- $5.5008+02 \cdot 5.0008+01$

-5.500E+02 -5.000E+01.

- $5.5008+02 \cdot 5.000 E+02$

- $0.0008+00 \cdot 0.0008+00$.

$0.000 E+00 \cdot 0.000 E+00$

- 5.500E+01 $\cdot 1.0008+02$

- 5.500E+02 - 1.000E+02

- $5.500 E+01 \cdot 1.0008+02$.

- $0.000 E+00 \div 0.000 E+00$

- $0.000 E+00 \cdot 0.000 E+00$.
-

- Donucc (29)

- Dovucu(19,1)

- Dorrocs (29)

$\cdots$

- Alexach (19)

- soLUdK (19)

not used

- Daruec (20)

$-\infty$

$\ldots$

$3.158 E-04$

not used

- derues (20)

- aleacilzo

- soLUak $(20)$

- DCNuCC(21)

- $\operatorname{DanuCU}(21,1)$

- DCIUCs (21)

- Aleachi (21)

- soludaxi22)

not used

- davucer 13

- parucur 1.1

- DCrucs ( 1)

- arsacar 11

- sounaki 11

noc used

- DCNucei 91

- donueur 9,1)

- davucs 91

- areacar 91

- soluBKI 91

nor used

- ocruce (10)

- Dovucu(10.1)

- Danucs (20)

- AZEACH(20)

- soltrexizo

noe used

- danuccial

- Darucu $\{21.1\}$

- Danucs(i2)

- ALEACH (11)

- solvex(11)

$2.013 E-04$

not used

- Leach race (/yr)
2016 - Solubilicy conscant 
RESRAD, Version $5.621 \quad \tau$ Limit $=0.5$ year $: 3 / 07 / 9708: 54$ Page 20 Sumaiy : Radioactivzty associaced surface consaminated concrete and drainilinea File : i: IRUBBLE.DAT Site-Specific pazameter Sumary (continued)

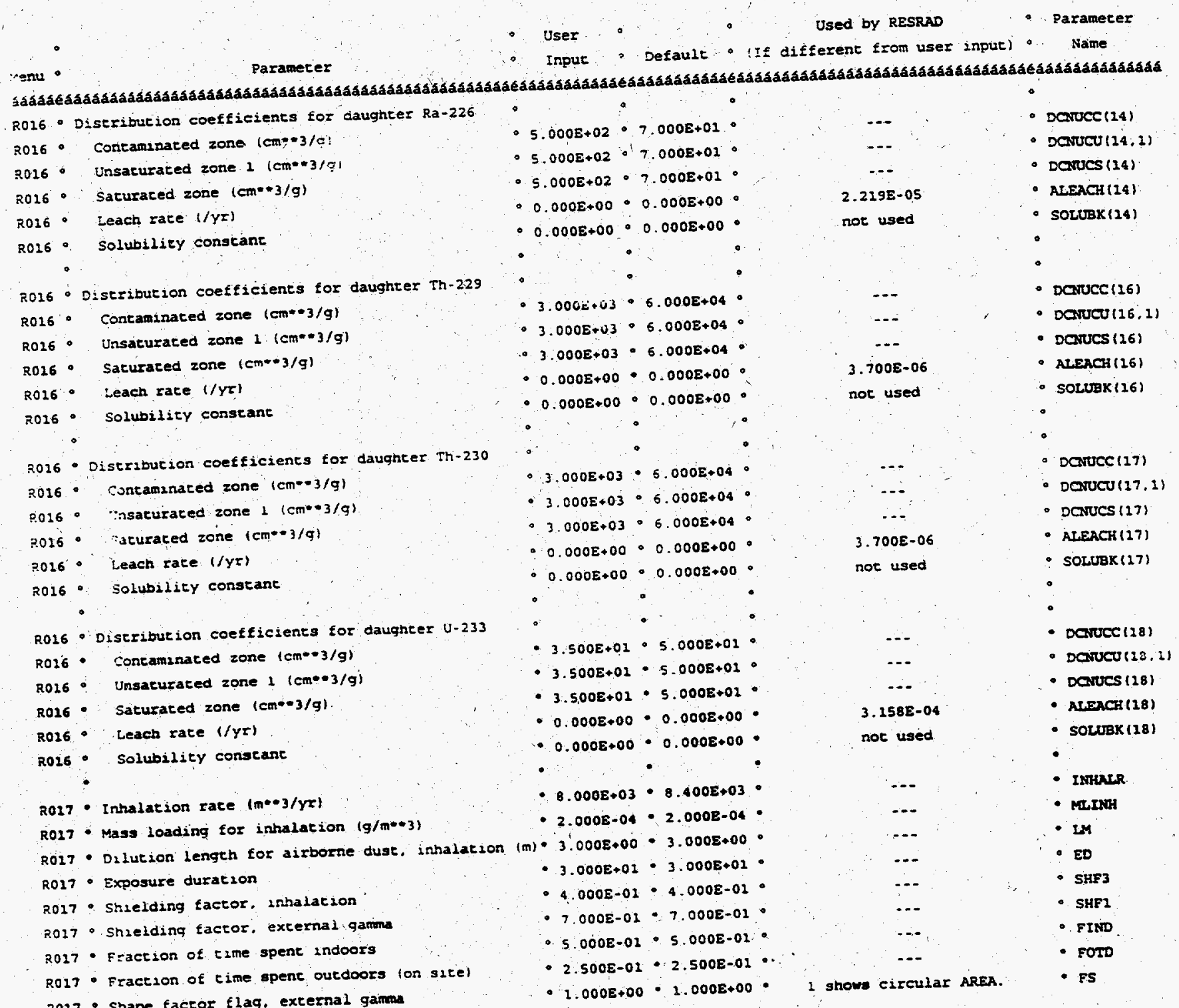




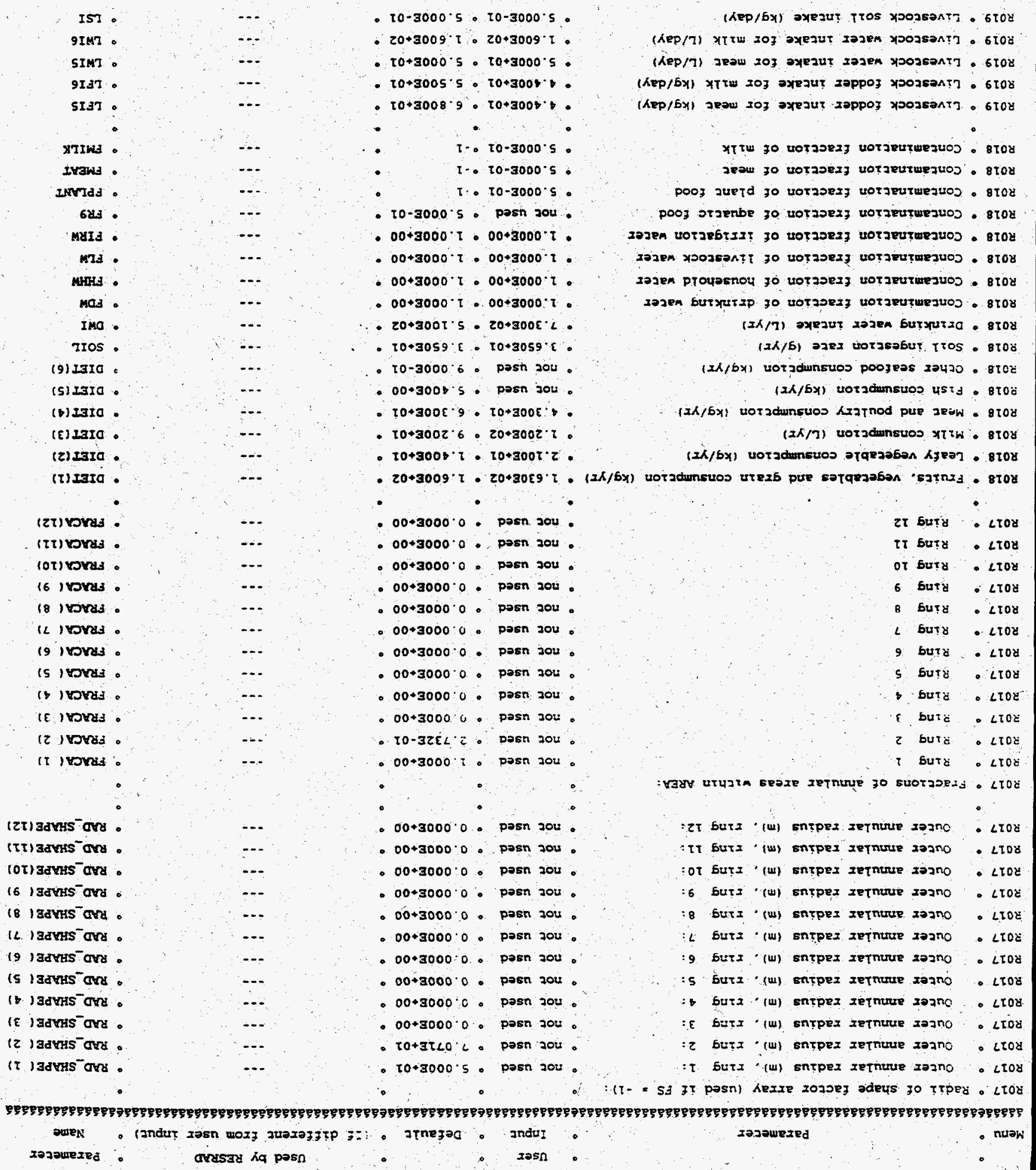

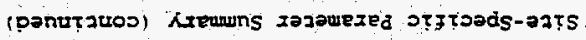


RESRAD, version $5.621 \quad T^{*}$ Limit $=0.5$ year 03/07/97 08:54 Page 12 Sumary : Radioactivity associaced surface contaminated concrete and drainline File : A: \RUBBES.DAT Site-Specific Paramecer Sumary (concinued)

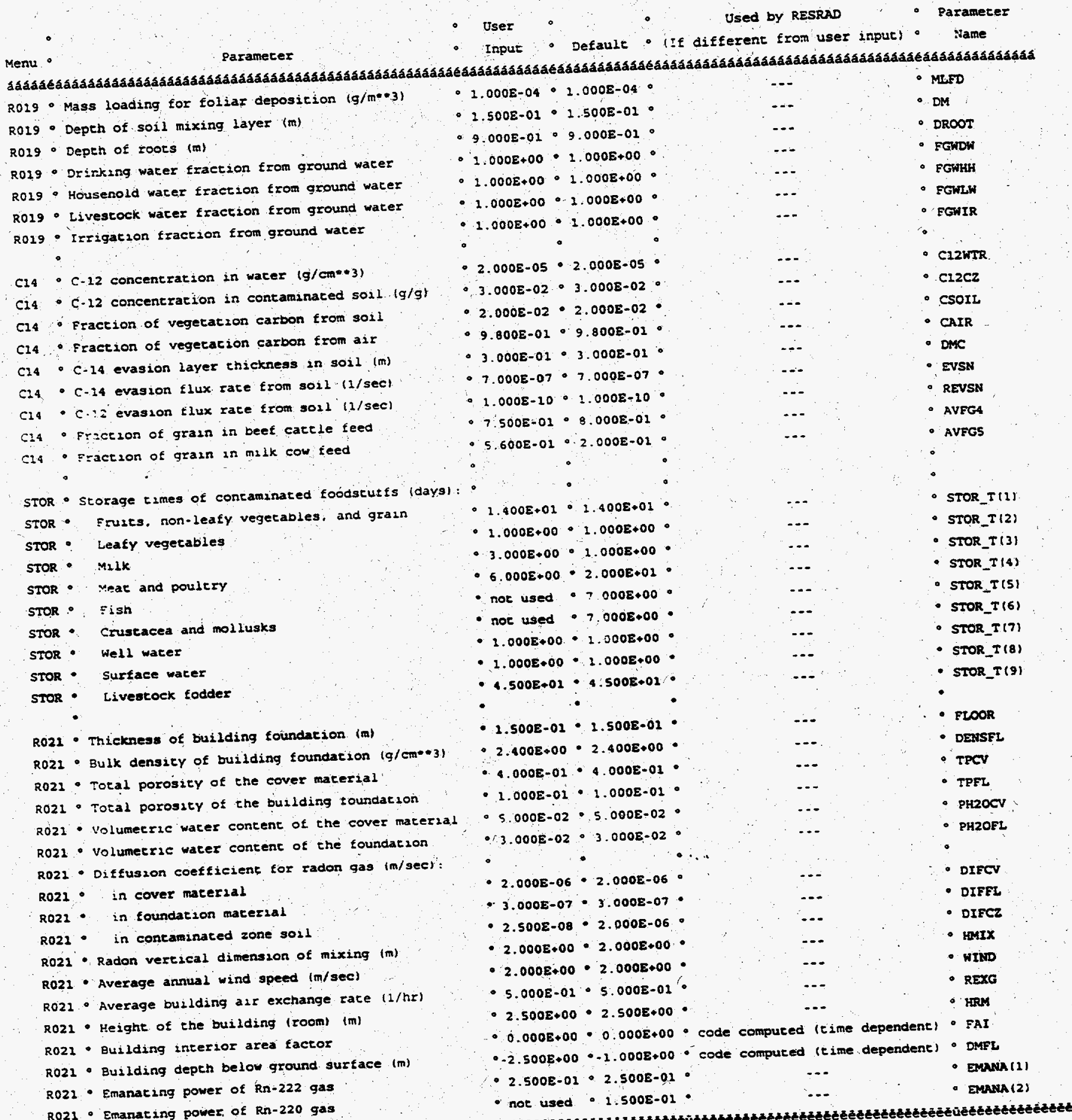

R023 - Emanating poiver of Rin-220 gas

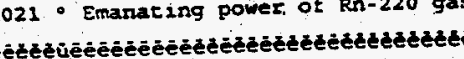




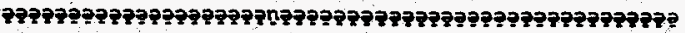

\begin{tabular}{|c|c|c|}
\hline DATבDe & - & 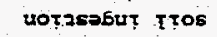 \\
\hline DתтุכDE & 。 & xəzem GuţนTIP \\
\hline seardins & 。 & spooj otrenbe \\
\hline องţวDe & $\circ$ & 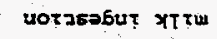 \\
\hline DAŢ=OP & - & UoT3805ut levw \\
\hline จATุ๋อ & 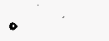 & torzsabu? دuejd \\
\hline DATzop & - (uopez & O/M \} uotzetequT \\
\hline קATTOEE & 0 & eunes feurosxo \\
\hline
\end{tabular}

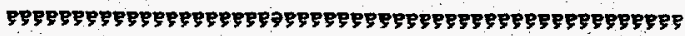

uotzoetas zasn

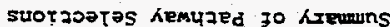


RESRAD, Version $5.621 \quad T$ Limit $=0.5$ year $03 / 07 / 97$ 08:54 Page 14 Sumary : Radioactivicy associaced surface contaminated concrete and drainlines File : A: IRUBBLE.DAT

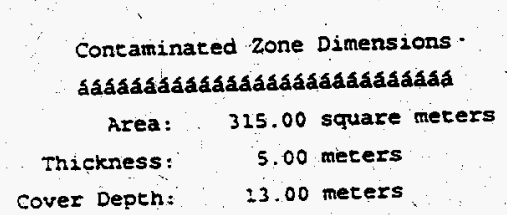

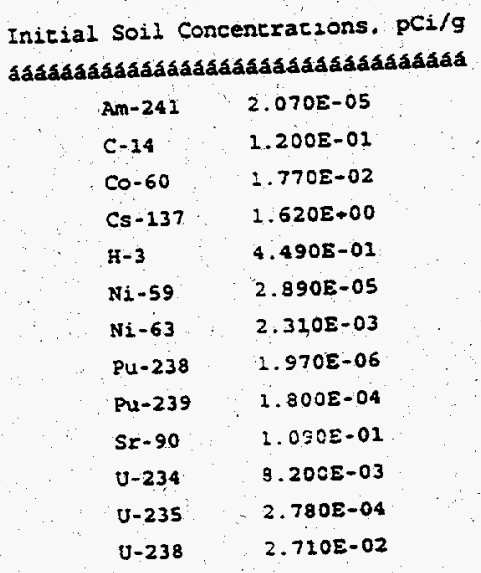

Total Dose TDOSE(c), mrem/yr

Basic Radiation Dose Limic - $15 \mathrm{mrem} / \mathrm{Yr}$

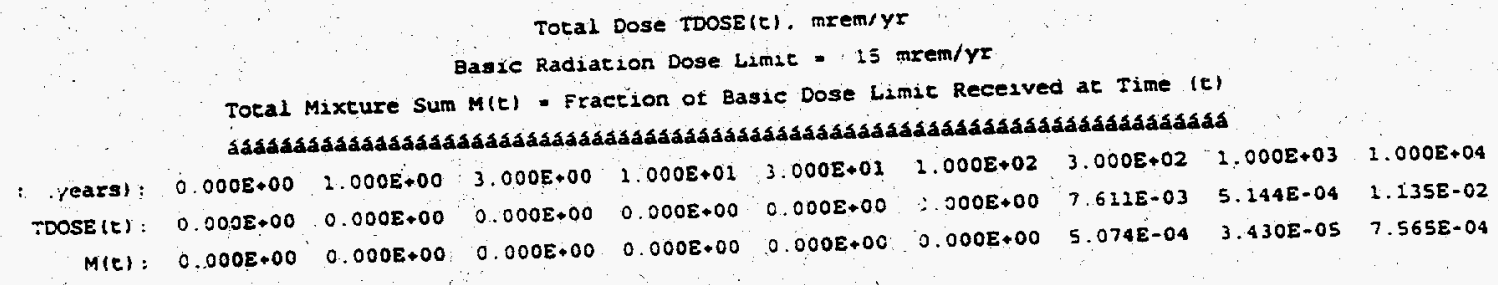

Maximum TDOSE(E): $4.020 E-02 \mathrm{mrem} / \mathrm{yr}$ at $=\quad 4965$ p 5 years .

Total Dose Concributions TDOSE (i,p,t) for Indzvidual Radionuclides $(i)$ and Pattways (p) As mrem/yr and Fraction of Total Dose Ae $==4965$ years

water Independent Pathways (Inhalation excludes radon)

\begin{tabular}{|c|c|c|c|c|c|c|c|c|c|c|c|c|c|c|}
\hline$A m-241$ & $0.0005+00$ & 0.0000 & $0.000 E+00$ & 0.0000 & $0.000 E+00$ & 0.0000 & $0.000 E+00$ & & $0.0008+00$ & c & 00 & 0.0 & 00 & 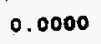 \\
\hline & $E+000$ & 0.0000 & $0.000 E+00$ & 0.0000 & $0.000 E+00$ & 0.0000 & $0.000 E+00$ & 0. & $0,0008+00$ & 0.0000 & 0 & 0 & .00 & 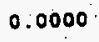 \\
\hline 00 & $0.000 \varepsilon+00$ & 0.0000 & $0.000 \mathrm{E}+00$ & 0.0000 & $0.000 E+00$ & 0. & $0.000 E \div 00$ & 0. & $0 E+00$ & 0.0000 & $0.000 E+00$ & c & $000+000$ & \\
\hline 13 & $0.0008+00$ & 0.0000 & $0.0008+00$ & 0.0000 & $0.000 E+00$ & 0.0000 & $0.000 E+0$ & 000 & $0 \mathrm{E}+0,0$ & 0 & $0.000 \mathrm{E}+00$ & 0 & $18+000$ & 000 \\
\hline . & $0.000 E+00$ & 0.0 & $0.000 E+00$ & 0.0000 & 0.0 & 0.0000 & $0.0000+00$ & 0 & $0.000 E+00$ & 200 & $28+00$ & 0 & $2+000$ & 100 \\
\hline & $0.000 E+00$ & 0.0000 & $8+00$ & 0.0000 & $0.000 E+00$ & 0.0 & $0.0008+00$ & 0.000 & $000 E+00$ & 0.0000 & 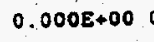 & 100 & 000 & 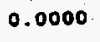 \\
\hline & $.000 E+000$ & 0.0000 & 0.0 & 000 & $0.000 \varepsilon+00$ & 0.000 & $0 E+00$ & 0.0000 & $D E+00$ & 000 & 0.0001 & & $0 E+00$ & 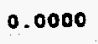 \\
\hline .238 & $E+00$ & 0.0000 & $0.0008+00$ & 0. & $0.000 \mathrm{E}+00$ & 0.0000 & 00 & 0.0000 & $0.0008+00$ & 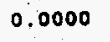 & $0.000 E+00$ & & $.000 E+00$ & 0 \\
\hline 239 & $0.000 E+00$ & 00 & $0.000 E+00$ & 0.0 & $E+00$ & 0.0000 & .00 & 0.0000 & $0.000 \mathrm{E}+00$ & 0 & $0.000 \mathrm{E}+00$ & 0. & $E+00$ & 200 \\
\hline-90 & $0.000 E+00$ & 0. & $0.000 \mathrm{E}+00$ & 0.0000 & $E+00$ & 0.0000 & $0.000 \varepsilon+00$ & 00 & $0.000 E+00$ & 0 & $00 \mathrm{E}+00$ & 0.0000 & . & 1000 \\
\hline 3 & $.00:$ & 10. & $0.000 \mathrm{E}+00$ & 0.0000 & $0.000 E+00$ & 0.0000 & $0.000 \mathrm{E}+00$ & 0. & $0.000 E+00$ & 0.000 & 0 & 000 & $8+00$ & is \\
\hline .235 & 00 & 0.0000 & .000 & 0.0000 & $0.0000+00$ & 0.0000 & $0.000 E+00$ & 0. & $0.000 E+00$ & 0.0000 & $10 E+00$ & c & $E+00$ & 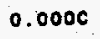 \\
\hline 238 & $0.000 \mathrm{E}+00$ & 0.0000 & $0.000 \mathrm{E}+00$ & 0.0000 & $0.000 E \div 00$ & 0.000 & $0.000 E+00$ & 0. & $0.000 E+00$ & 200 & $E+00$ & $\mathbf{0}$ & OOE +00 & 0.0000 \\
\hline 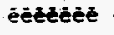 & e & 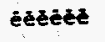 & éeztetetet & Eet & bet & ééèces & te & trat & éexterest & 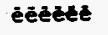 & 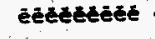 & 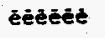 & beet & 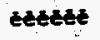 \\
\hline ta & $0.000 E+00$ & 0.0000 & $0.000 \mathrm{E}+00$ & 0.0000 & $0.000 \mathrm{E}+00$ & 0.0000 & $.0008+00$ & 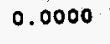 & $0.000 E+00$ & 0. & 00 & & 00 & \\
\hline
\end{tabular}




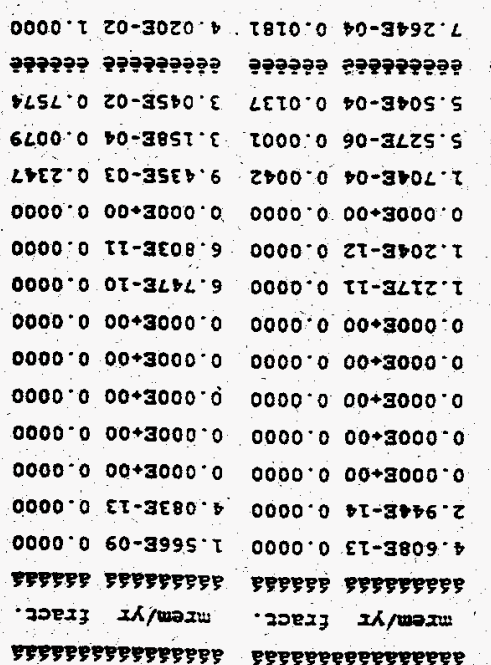

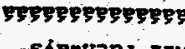

หTพ
$8200 \cdot 0 \quad 0-38 z: \cdot \mathrm{t}$

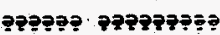
TzO0 $0 \cdot 50-3055 \cdot 8$ $0000-090-38 \tau t \cdot t$ $1000^{\circ} 0$ 50-3059.2 $0000 \cdot 000+3000 \cdot 0$ $0000 \cdot 0$ EL-3L6: 2 $0000^{\circ} 0$ zt-3506. $0000-00+3000 \cdot 0$ $0000=00+3000 \div 0$ $0000 \cdot 000+300=0$ $0000 \cdot 00+3002 \cdot 0$ $0000 \cdot 000+30000$ $0000 \cdot 0$ b $T-367 \div \cdot \mathrm{T}$

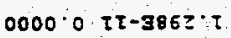
eperge gesersess

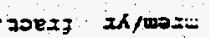

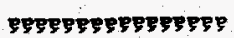
Jew
$2 \angle 8 T \cdot 00-3245 \cdot C$

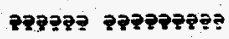
TZZT. 0 EO-3ETL. 5200.0 50-3L26.5 $0 \rightarrow+0^{\circ} 0$ 50-30LL"T $0000000+3000.0$ $0000^{\circ} 0$ IT-3LLZ T $0000 \%$ or-395z $t$ $0000.000+3000 \cdot 0$ $0000000+3000 \cdot 0$ $0000 \div 00+3000 \% 0$ $0000 \cdot 0.00+3000 \%$ $0000-000+3000.0$ $0000-0, \tau-30<8 \cdot 8$ $0020 \circ 0$ ot-3\$86. epsepe pepsespes - Joex $\mathrm{x} \alpha /$ maxw इеsеg zuetd
$0000 \cdot 0$ 80-3LEE L $2000 \cdot 000+3000.0$

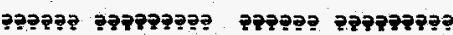
$0000: 0 \quad 00-3668 \cdot 2$ $0000 \cdot 000+3000 \cdot 0$ $0000 \%$ 80-3eE? $0000 \div 00+3000=0$ $0000 \% 00+3000.0$ $0000 \cdot 0.5 T-38 \pi L$ $0000 \times 00+3000 \cdot 0$ $0000 \cdot 00+3000 \cdot 0$ $0000000+3000 \cdot 0$ $0000: 000+3000: 0$ $0000 \cdot 000+3000 \%$ $0000000+3000-0$ $0000 \cdot 00+3000 \cdot 0$

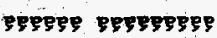
JDEII IX InOXW eppepep p̈pereger
ST6L:0 zo-3tвT't Tezo:

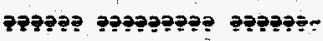
$5665^{\circ} 0$ 20-30Tt $z$ 8Ez-ก 2900.0 b0-3005. SEZ.: $858 T^{\circ} 0$ E0-3L95 $L$ DEZ. $0000.000+3000$ 0 06-25 $0000 \cdot 0$ tt-358E's 6Ez-n 0000.0 0z-30b5.s gez-nd 0000\% 00+3000\% E E-TN $0000-0 \quad 00+3000 \cdot 0 \quad$ 62-Tn $0000.000+3000 \%$ $0000 \cdot 000+3000 \cdot 0$ LET-SD $0000 \cdot 0.00+3000 \% 09-00$ $0000 \cdot 0[\tau-3 \angle S L=2 T-5$ $0000 \%$ 60-365z I Ibz-ur

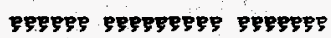
-20exy $2 \kappa /$ waxu aptronk

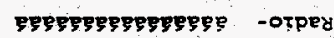
uoped पsţ

Shemujed zuspuadad sesem

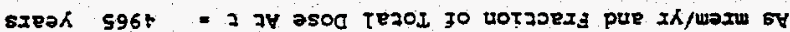

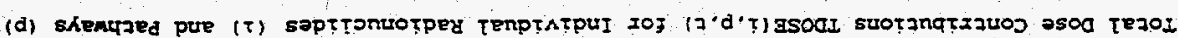

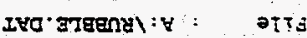

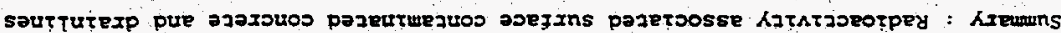

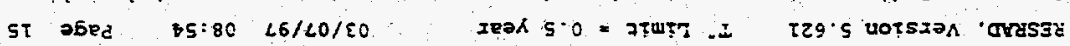


RESRAD, Version $5.621 \quad T$. Limit $=0.5$ year $03 / 07 / 97$ 08:54 Page 16 Suraary : Radioactivity associated surface concaminated concrete and drainines File : A: \RUBBLE.DAT

Total Dose Coneributions TDose(i,p, f) for Individual Radionuclides (i) and Pathways (p) As mrem/yr and Fraction of Total Dose At $t=0.000 \mathrm{E}+00$ years

Water Independens Pathways (Irhalation excludes radon

\begin{tabular}{|c|c|c|c|c|c|c|c|c|c|c|c|c|c|c|}
\hline \multirow{2}{*}{$\begin{array}{l}\text { Radio- } \\
\text { Nuelide }\end{array}$} & \multicolumn{2}{|c|}{ Ground } & \multicolumn{2}{|c|}{ Inhalacion } & & & & \multicolumn{2}{|l|}{ Meat } & \multicolumn{2}{|l|}{ Milk } & \multicolumn{2}{|l|}{ Soil } \\
\hline & $r$ & & $\boldsymbol{r}$ & & mren/yr & & & & & & & & & et. \\
\hline ałấá & aasa & ast & aá & $\mathbf{a a}$ & saa & fasa & sat & & & & aasa & & ááa & ásasta \\
\hline$m-242$ & $0.000 E+00$ & 0.0000 & $0.000 E+00$ & 0.0000 & $.0008+00$ & 0.0000 & $0.000 E+00$ & 0.0000 & $0.0008+00$ & 0.0000 & $0.0008+00$ & 0.0000 & $.000 \mathrm{E}+00$ & 100 \\
\hline-14 & $0.000 E+00$ & 0.0000 & $000 E+00$ & 0.0 & $.0008+00$ & 0.0000 & $.0008+00$ & 200 & $.000 \mathrm{E}+00$ & 0.0000 & $0.000 E+00$ & 0.0000 & +00 & 100 \\
\hline $2-60$ & 0.0 & 10 & .00 & 00 & +00 & 1000 & $8+00$ & 000. & $+\infty 0$ & 000 & 0. & & 0 & 10 \\
\hline 37 & 0.0 & 00 & Do & 00 & 10 & 000 & .00 & 0 & roo & ra & .00 & & & 100 \\
\hline $1-3$ & $0: 0$ & 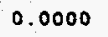 & 00 & 00 & 0 & 0 & 0 & 0 & +00 & 20 & 0 & 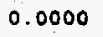 & $+\infty$ & 0000 \\
\hline $1 i-59$ & $0.000 \mathrm{E}+00$ & 00 & $.000 E+00$ & 0.0000 & 00 & 000 & .00 & 00 & +00 & 200 & +00 & 100 & $08+00$ & 000 \\
\hline $\mathrm{Ni}-63$ & $0.0008+00$ & 0.0000 & $.000 E+00$ & 0.0000 & $0.0005+00$ & 0.0000 & $08+00$ & 0.0000 & $0.000 E+00$ & 000 & $E+00$ & 0.0000 & $8+00$ & \\
\hline 38 & $0.000 \varepsilon+00$ & 0.0 & $.000 E$ & 00 & 00 & 300 & 0 & 100 & +00 & 0 & $E+00$ & & 0 & \\
\hline 9 & $E \div 00$ & $x$ & .000 & ( & . & 0 & $E+\infty$ & 0 & 0 & C & & 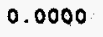 & $E+00$ & 0.0000 \\
\hline 990 & $0.0008+00$ & 00. & 0.000 & 0.0000 & 100 & 1000 & $:+0$ & 0.0000 & $E+\infty 0$ & 100 & $8+00$ & 00 & $0.000 \varepsilon+00$ & 0. \\
\hline-234 & $0.000 E+00$ & 0.0000 & $0.000 E+00$ & $0: 0000$ & $.000 \varepsilon+00$ & 0.0000 & $0.000 \mathrm{E}+00$ & 0.0000 & $0.000 E+00$ & 0.0000 & $0.000 E+00$ & 0.0000 & $0: 000 E+00$ & 0.0000 \\
\hline 235 & $0.000 E+00$ & 0.0000 & $0.000 E \times 00$ & 0.0000 & $.0008+00$ & 0.0000 & $10+00$ & 100 & $O E+00$ & 00 & $000 E+00$ & 0 & 00 & tha \\
\hline 38 & $a:$ & po & 20 & 100 & 00 & 00 & 00 & $a$ & 0 & 0 & 00 & 0 & oo. & ( \\
\hline . & $\bar{e}$ & 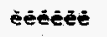 & & 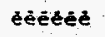 & è & $\dot{s}$ & 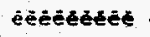 & $\dot{e}$ & e & 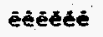 & e & e & tè & ( \\
\hline oeal & $0.000 E+00$ & 0.0000 & $0.0008 \div 00$ & 0.0000 & $0.000 \varepsilon+00$ & 0.0000 & $0.000 E+00$ & 0.0000 & $0.000 E+00$ & 0.0000 & $0.000 E+00$ & 0.0000 & $0.000 E+00$ & 0.0000 \\
\hline
\end{tabular}

Total Dose Concributions TDOSE(i.p., f) for Individual Radionuclides (1) and Pathways (p) As mrem/yr and Eraction of Total Dose At $t=0.000 E+00$ years

Wacer Dependenc Pachways

\begin{tabular}{|c|c|c|c|c|c|c|c|c|c|c|c|c|c|c|}
\hline \multirow{2}{*}{$\begin{array}{l}\text { Radio- } \\
\text { Muclide }\end{array}$} & \multicolumn{2}{|c|}{$\begin{array}{c}\text { Water } \\
\text { asastasadasasá }\end{array}$} & \multicolumn{2}{|c|}{$\begin{array}{c}\text { Fish } \\
\text { sasasuadaadaadaa }\end{array}$} & \multicolumn{2}{|c|}{$\begin{array}{c}\text { Radon } \\
\text { sadssadadadasasa }\end{array}$} & \multicolumn{2}{|c|}{$\begin{array}{l}\text { Plant } \\
\text { a }\end{array}$} & \multicolumn{2}{|c|}{$\begin{array}{l}\text { Meat } \\
\text { asasasadasasadas }\end{array}$} & \multicolumn{2}{|c|}{$\begin{array}{c}\text { Milk } \\
\text { sadagasaasasaas }\end{array}$} & \multicolumn{2}{|c|}{$\begin{array}{l}\text { All Pathwayo" } \\
\text { adasasasasuse }\end{array}$} \\
\hline & & & & & isa & & as & • & a & 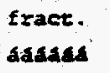 & 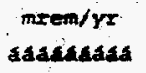 & & as & \\
\hline & & & & & & 3000 & 00 & & +00 & & & & & \\
\hline & t & & 0 & & 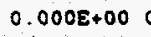 & & & & 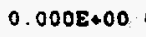 & & & & & \\
\hline & 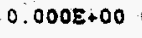 & & & & & & & & & & & & & \\
\hline & & & & & & & & & & & & & & \\
\hline & & & & & & & & & & & & & & \\
\hline & & & & & & & & & & & & & & \\
\hline & & & & & & & & & & & & & & \\
\hline & & & & & & & & & & & & & & \\
\hline & & & & & & & & & & & & & & \\
\hline & & & & & & & & & & & & & & \\
\hline & & & & & & & 0 & & 0 & & & & & \\
\hline & 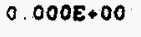 & & a. & & 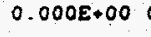 & & & & & & & & & \\
\hline & & & & & & & & & & & & & & \\
\hline & & & & & & & 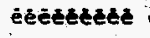 & & 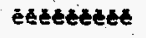 & & & & & \\
\hline . & $0.000 \mathrm{E}+00$ & 0.000 & $.000 E+00$ & 0.0000. & $0.000 E+00$ & $0: 0000$ & $.0005+00$ & 0.0000 & $.000 E+00$ & 000 & $0.000 \mathrm{E}+00$ & 0.0000 & $0.0008+00$ & 0.000 \\
\hline
\end{tabular}

- Sum of all water independent and dependeric pathways. 
RESRAD. Version 5.623 T Limit $=0.5$ year 03/07/97 08:54 Fage 17

Sumary : Radioactivicy associated surface concaminated concrece and drainlines

File : A: IRUBBLE.DAT

Total Dose Contributions TDOSE(i.p.e) for Individual Radionuclides (i) and Pathways (p)

Ground

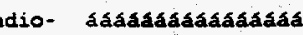

Nuclide mrem/yr fract.

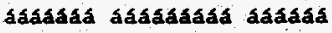

Am-241 $\quad 0.000 E+00 \quad 0.0000$

C.14. $\quad 0.0008+000.0000$

Co-60. $0.000 \mathrm{E}+00 \quad 0.0000$

Cs-137 $0.000 E+00 \quad 0.0000$

H-3 $\quad 0.000 E+00 \quad 0.0000$

Ni-59 $0.0005+00 \quad 0.0000$

$N_{i}-63 ; \quad 0.000 E+00 \quad 0.0000$

Pu-238 $0.000 E+00 \quad 0.0000$

PU-239 $0.000 E+00 \quad 0.0000$

$S r-90 \quad 0.000 E+00 \quad 0.0000$

U.234 $0.000 E+00 \quad 0.0000$

$0.235 \quad 0.000 E+00 \quad 0.0000$

U.238 O 000E+00 0.0000

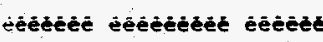

Total O $000 E+000.0000$
As mrem/yx and Eraction of Total Dose At $c=1.000 E+00$ years

Water Independent Pathways (Inhalation excludes radon)
Total Dose Contrlbutiong IDOSE(i,p.t) for Individual Radionuclides (i) and Pathways (p) As mrem/yz and Fraction of Total Dose At $t=1.000 E+00$ years

water Dependene Pathways

\section{water}

Radio- adachasasadasas

Nuclide mrea/yr fract.

\section{assasas assasasa sasasa}

Am-241 $0.0008+00 \quad 0.0000$

C.14 $0.000 E+00 \quad 0.0000$

Co-60 $0.000 E+00 \quad 0.0000$

$c s-137 \quad 0.0008+00 \quad 0.0000$

H-3 $\quad 0.000 E+00 \quad 0.0000$

Ni-59 $\quad 0.000 \mathrm{E}+00 \quad 0.0000$

$\mathrm{Ni}-63 \quad 0.000 \mathrm{E}+00 \quad 0.0000$

Pu-238 $0.000 E+000.0000$

Pui-239 $0.000 E+00 \quad 0.0000$

SE-90 $\quad 0.000 E+00 \quad 0.0000$

U-234 $\quad 0.0008+00 \quad 0.0000$

U-235 $0.000 E+00 \quad 0.0000$

$\begin{array}{lllll}U-238 & 0.000 E+00 & 0.0000\end{array}$

ejecté ejedtedete dected

Total $0.000 E+00 \quad 0.0000$
Inhalacion

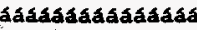

rem/yr fract

$0.000 \varepsilon+00 \quad 0.0000$

$0.0005+00 \quad 0.0000$

$0.000 \Sigma+00 \quad 0.0000$

$000 E+0.0 \quad 0.0000$

$0.0000+00$ o.0000

$0.0008+00 \quad 0.0000$

$0.000 E+00 \quad 0.0000$

$0.000 E+00 \quad 0.0000$

$000 E+00 \quad 0.000$
Radón

aáasaááááááá

mrem/yr fract.

dakáa áasáa

0.0000

$0008+00 \quad 0.0000$

$0.0008+00 \quad 0.0000$

$0.000 E+00 \quad 0.0000$

$0.000 E+00.0 .0000$

$0 E+00 \quad 0.0000$

$000 E+00 \quad 0.000$

000.0000

$000 E+00 \quad 0.000$

$0.0000+000.0000$
Plane

áasaáakaaasá

rem/yr fract.

aadaáa aáaá

0005

$0.0008+00 \quad 0.0000$

$000 E+00$

$000 \mathrm{E}+00 \quad 0.0000$
Meat mrem/yx Eract.

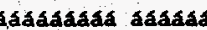
$0.0008+00 \quad 0.0000$ $0.0008+00 \quad 0.0000$ $0.0008+00 \quad 0.0000$ $0.0008+00 \quad 0.0000$ $0.000 E+00 \quad 0.0000$ $0.000 E+00 \quad 0.0000$ $0.000 E+00 \quad 0.0000$ $0.000 E+00 \quad 0.0000$ $0.000 E+00 \quad 0.0000$ $0.000 E+00 \quad 0.0000$ $0.000 E+00 \quad 0.0000$ $0.000 E+00 \quad 0.0000$

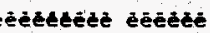
$0.000 E+00.0 .0000$
Milk

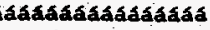
mrem/yr Exact. sadasaaka cakaka $0.000 \mathrm{E}+00 \cdot 0.0000$ $0.000 E+00 \quad 0.0000$ $0.000 E+00-0.0000$ $0.000 E+00 \quad 0.0000$ $0.000 E+00 \quad 0.0000$ $0.000 E+00 \quad 0.0000$ $0.000 E+00 \quad 0.0000$ $0.000 E+00 \quad 0: 0000$ $0.000 E+00 \quad 0.0000$ $0.0008+00 \quad 0.0000^{\circ}$ $0.000 E+00 \quad 0.0000$ $0.000 E+00 \quad 0.0000$ $0.000 E+00 \quad 0.0000$

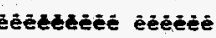
$0.000 E+00 \quad 0.0000$

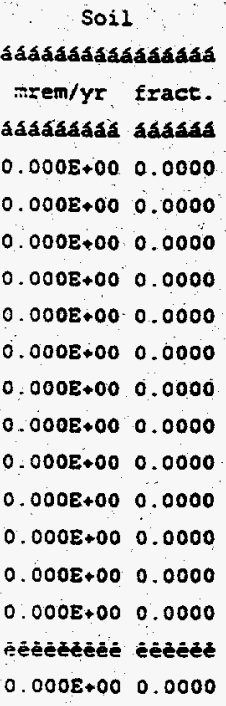

- Sum of all water independent and dependent pachways. 
RESRAD. Version $5.621 \quad T$ Limit $=0.5$ year $\quad 03 / 07 / 9708: 54 \quad$ Fage 18

Summary : Radioactivity associaced surface contaminated concrete and draininines

File : A: \RUBELE.DAT

Total Dose Contributions tDoSE $(i, p, t)$ for Individual Radionuclides (i) and Pathways (p)

As mrem/yr and Eraction of Total Dose Ac $t=3.000 \mathrm{E}+00$ years

water Isdependent pathways (Irhalation exciudes radon)

\begin{tabular}{|c|c|c|c|c|c|c|c|c|c|c|c|c|c|c|}
\hline \multirow{3}{*}{ adso- } & \multicolumn{2}{|c|}{ Ground. } & \multicolumn{2}{|c|}{ Inhalacion } & \multicolumn{2}{|c|}{ Radon } & \multicolumn{2}{|c|}{ Plant } & \multicolumn{2}{|l|}{ Meat } & \multicolumn{2}{|l|}{$I \pi$} & \multicolumn{2}{|l|}{ So } \\
\hline & & & $\begin{array}{c}\text { madabaaaa } \\
\text { mrem/yr }\end{array}$ & $\mathrm{abc}$ & a. & & & & & & & & & \\
\hline & 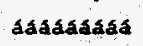 & & a & & & & & & & & & & & \\
\hline $1-241$ & 00 & & & & & & & & 0 & & & & 0 & \\
\hline .34 & 0 & & & & & & & & & & & & & \\
\hline & 0 & & & & & & & & & & & & & \\
\hline & 0 & & o. & & & & & & & & & & & \\
\hline & 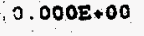 & & Do & & & & & & & & & & & \\
\hline & 0.0 & & .00 & & & & & & & & & & & \\
\hline & 00 & 00 & $\bullet 00$ & & & & & & & & & & & \\
\hline 38 & .00 & pa & .00 & & 30 & & 0 & & & & & & & \\
\hline 39 & 0.0 & 00. & 0. & & 00 & & & & & & & & & \\
\hline 90 & 00 & & & & & & & & & & & Ong & & \\
\hline 4 & 0 & & & & & & & & & & & & & \\
\hline & 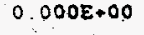 & & & & & & & & & & +00 & & & \\
\hline & & & 0 & & 0 & 0 & & & & & 00 & & 10 & \\
\hline & & $\ddot{e}$ & et & 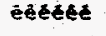 & 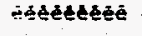 & $\dot{\theta}$ & $E$ & & 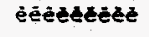 & & & & & \\
\hline & $0.000 E+00$ & 200 & $.000 E+00$ & 0.0000 & . & 100 & .00 & 0.0000 & +00 & & & & . & \\
\hline
\end{tabular}

Total Dose Concributions TDOSE(i.p.tel for Individual Radionuclides (i) and Pathways (p)

As mrem/yr and Fraction of Total Dose At $C=3.000 E+00$ years

Water Dependent Pachways

\begin{tabular}{|c|c|c|c|c|c|c|c|c|c|c|c|c|c|c|}
\hline \multirow{2}{*}{$\begin{array}{l}\text { Radio- } \\
\text { vuelide } \\
\text { assasa }\end{array}$} & & & \multicolumn{2}{|c|}{$\begin{array}{c}\text { Fish } \\
\text { daditadasassisa }\end{array}$} & \multicolumn{2}{|c|}{$\begin{array}{r}\text { Radon } \\
\text { asasuasasasasuas }\end{array}$} & \multicolumn{2}{|c|}{ 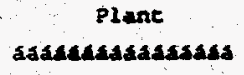 } & \multicolumn{2}{|c|}{$\begin{array}{c}\text { Meat } \\
\text { adasurasasasas }\end{array}$} & \multicolumn{2}{|c|}{$\begin{array}{c}\text { Milk } \\
\text { adseasadasasasa }\end{array}$} & \multicolumn{2}{|c|}{ 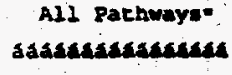 } \\
\hline & & & rá & & ada & & as & & & & $x$ & & as & \\
\hline-241 & 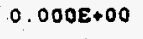 & & 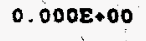 & 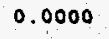 & 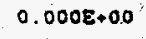 & 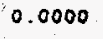 & 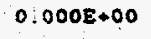 & & 0 & & +00 & & & \\
\hline & 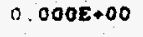 & & . & & & & & & & & , & & & \\
\hline 0.60 & 0 & & 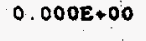 & & & & & & & & . & & & \\
\hline & & & & & & & & & & & & & & \\
\hline & & & & & & & & & & & & & & \\
\hline & & & & & & & & & & & 100 & & & \\
\hline & & & o. & & & & & & & & +00 & & & \\
\hline & & & 0 & & & & & & & & .00 & & & \\
\hline & & & 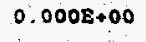 & & & & & & & & 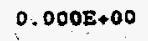 & & & \\
\hline & & & & & & & & & & & 0 & & & \\
\hline & & & & & & & & & & & .00 & & & 000 \\
\hline 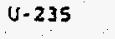 & & & & & & & & & +00 & & 00 & & 00 & 000 \\
\hline & & & & & & & & & 0. & & , & & & \\
\hline & & & & & & & & & & & & & & \\
\hline & & & & & & & & & & & (2) & & encis & 0.0000 \\
\hline
\end{tabular}

- Sum of all water 1 ndependent and dependent pathways. 
$0000000+3000 \cdot 0000 \% 00+3000 \% 0$

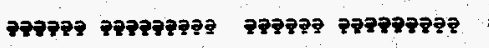
$0000 \div 00+3000 \% 0000 \% 00+3000 \div 0$ $0000 \cdot 000+300000000: 000+3000 \% 0$ $0000 \cdot 000+3000 \% 0000: 000+3000 \%$ $0000 \cdot 000+3000.00000 \% 00+3000 \%$ $0000.000+3000 \% 0000 \% 00+3000 \cdot 0$ $0000.000+3000 \% 0000.000+3000.0$ $0000 \% 00+3000 \% 0000.000+3000.0$ $0000 \cdot 000+3000 \% 0000 \% 00+3000: 0$ $0000 \cdot 000+3000 \% 0000000+3000 \%$ $0000.000+300000000.000+3000 \% 0$ $0000.000+3000 \div 00000.000+3000 \%$ $0000 \cdot 000+3000 \cdot 0 \quad 0000 \times 00+3000 \cdot 0$ $0000.000+3000.0 \quad 0000.000+3000.0$

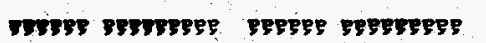

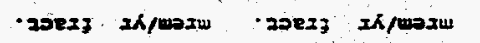

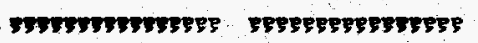
- sxeayaed tTY
$0000000+30000$ ㄱำรจำ ㄱำรำㄱำ $0000 \% 000+3000 \cdot 0$ $0000 \cdot 000+3000 \cdot 0$ $0000 \div 00+3000: 0$ $0000 \cdot 000+3000: 0$ $0000.000+3000.0$ $0000.000+3000 \%$ $0000.00+3000 \% 0$ $0000: 000+3000: 0$ $0000: 000+3000: 0$ $0000 \cdot 00+3000.0$ $0000: 000+3000 \cdot 0$ $0000.00+3000 \%$ $0000 \cdot 0 \cdot 00+3000 \cdot 0$

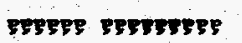
- בoex IN/moxi

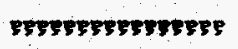
Jeaw
$0000 \cdot 000+3000: 0$

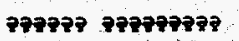
$0000 \% 00+3000 \%$ $0000000+3000: 0$ $0000 \% 00+3000.0$ $0000 \cdot 000+9000 \cdot 0$ $0000 \cdot 000+3000 \cdot 0$ $0000 \cdot 00+30000$ $0000 \cdot 00+3000 \%$ $0000 \cdot 000+3000: 0$ $0000 \% 00+3000 \%$ $0000000+3000.0$ $0000.000+3000.0$ $0000 \% 00+3000.0$ $0000 \cdot 000+3000 \%$ potere stsheseps -20ex IK/WOa BwrFerstricese gureta
$0000000+3000: 0$

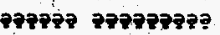
$0000 \cdot 00+3000 \% 0$ $0000: 0 \quad 00+3000 \%$ $0000000+3000 \%$ $0000 \cdot 0 \quad 00+3000 \cdot 0$ $0000 \cdot 00+3000 \cdot 0$ $0000 \% 00+3000 \% 0$ $0000.00+5000.0$ $0000 \cdot 00+3000.0$ $0000 \cdot 00+3000.0$ $0000.00+3000.0$ $0000 \% 00+30000$ $0000 \cdot 00+3000.0^{\circ}$ $0000.000+3000.0$ Tresese Essisteps -200x3 2a/pusw

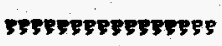
uopry
$0000000+30000$

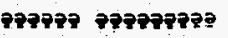
$0000 \% 00+3000 \% 0$ $0000: 0: 00+3000.0$ $0000.00+3000 \%$ $0000 \cdot 00+3000 \cdot 0$ $0000.00+3000.0$ $0000000+30000$ $0000000+3000 \%$ $0000: 000+3000 \cdot 0$

$0000: 000+3000 \%$ $0000000+3000: 0$ $0000 \div 00+3000: 0$ $0000: 00+3000 \div 0$ $0000.000+3000 \%$

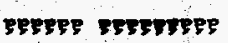

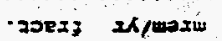
eeseresserstrepes 4อ?
$0000000+3000$ Traol

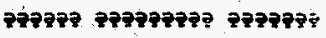
$0000 \cdot 000+3000 \%$ вعz-n $0000 \cdot 000+3000 \cdot 0$, sez-n $0000-000+3000: 0$ bez-n $0000.000+3000 \cdot 0$ 06-25 $0000 \cdot 000+3000 \cdot 0$ 6हz-nd $0000.000+3000.0$ bez-nd $0000: 000+3000 \cdot 0 \quad$ Eg- th $0000 \cdot 000+3000 \%$ 65-3N $0000.000+3000 \%$ E-H $0000 \% 00+3000 \%$ LET-SD $0000 \cdot 000+3000.0 \quad 09-05$ $0000 \cdot 00+3000.0$ OT $0000000+3000.0$ Trz-uy

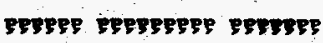
- zoez 3 akpuaw optrions segstesegresteps -otpey roares

8Rеm4วед zuopuadiog zәzем

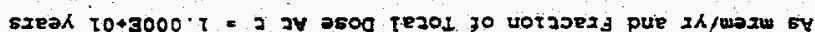

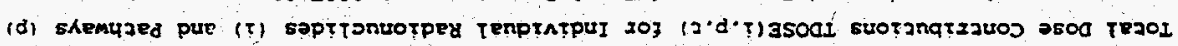

$0000.00+3000 \cdot 0$

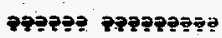
$0000 \cdot 00+3000 \cdot 0$ $0000 \cdot 000 \div 3000 \cdot 0$ $0000 \cdot 000+3000: 0$ $0000000+3000 \cdot 0$ $0000 \div 00+3000 \cdot 0$ $0000 \cdot 000+9000^{\circ} 0$ $0000 \div 00+3000 \cdot 0$ $0000 \cdot 00+3000 \cdot 0$ $0000.000+3000.0$ $0000: 00+3000 \%$ $0000 \cdot 000+3000.0$ $0000 \cdot 000+3000-0$ $0000 \% 00+3000 \% 0$ 595959 pepsespes asedz xk/woxw FEsepegespepepg tTos
$0000 \div 00-3000 \cdot 0$

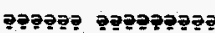
$0000000+3000.0$ $000+3000 \div 0000.000+3000 \% 0$ $0000.000+3000.0 \quad 0000 \% 00+3000.0$ $0000 \cdot 0.00+3000: 0 \quad 0000 \% 00+3000 \cdot 0$ $0000 \cdot 0 \quad 00+3000 \cdot 0 \quad 0000.00+3000 \div 0$ $0000 \% 00+3000 \cdot 00000 \% 00+3000 \%$ $0000.00+3000 \% 0000: 000+3000 \div 0$ $0000=00+3000=0 \quad 0000 \cdot 000+3000 \div 0$ $0000: 000+3000 \cdot 0 \quad 0000: 000+3000 \cdot 0$ $0000 \div 00+3000 \cdot 0 \quad 0000: 000+3000 \cdot 0$ $0000 \cdot 000+3000 \cdot 0 \quad 0000 \cdot 000+3000 \cdot 0$ $0000-0 \quad 00+3000 \cdot 0 \quad 0000 \cdot 0 \quad 00+3000.0$ $0000 \cdot 000+3000 \cdot 0 \quad 0000 \cdot 0 \quad 00+3002 \cdot 0$

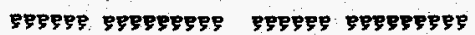

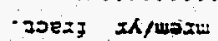
בכexj $2 \alpha / m=2$

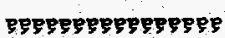
วеow
$0000000+300000000000+3000 \%$

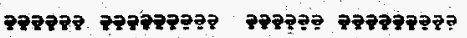
$0000000+300000000-00+3000: 0$ $0000 \% 00+3000 \% 0000: 000+3000 \%$ $0000.00+3000 \% 0000.00+3000 \%$ $0000 \cdot 00+3000 \% 0000 \cdot 0.00+3000 \%$ $0000 \cdot 00+3000 \cdot 0 \quad 0000 \cdot 000+3000 \%$ $0000 \times 00+3000 \% 0000 \cdot 00+3000 \%$ $0000 \% 00+3000 \cdot 00000 \% 00+3000 \%$ $0000 \cdot 000+3000: 0 \quad 0000 \cdot 0 \quad 00+3000 \cdot 0$ $0000000+3000 \div 00000 \cdot 000+3000 \% 0$ $0000000+300000000 \% 00+3000 \cdot 0$ $0000000+3000 \cdot 0000000+3000.0$ $0000 \div 0 \quad 00+3000 \times 0 \quad 0000 \div 000+3000 \cdot 0$ $0000000+3000 \% 0000 \cdot 000+3000 \cdot 0$

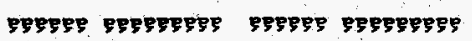

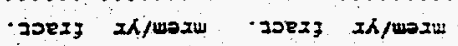

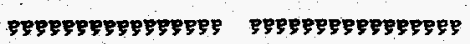
Juetd uoped
$0000 \cdot 000+3000 \cdot 0$

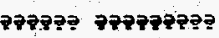
$0000.00+3000^{\circ} 0$ $0000 \div 00+3000 \cdot 0$ $0000 \% 00+3000: 0$ $0000 \div 00+3000: 0$ $0000 \cdot 00+3000 \%$ $0000000+3000 \%$ $0000 \% 00+3000 \div 0$ $0000 \% 00+3000 \%$ $0000 \cdot 000+3000 \%$ $0000-00+3000.0$ $0000.000+3000.0$ $0000=000+3000 \cdot 0$ $0000 \cdot 00+2000 \cdot 0$

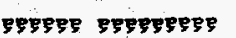
-20EIF IK/WO2U pepesestregseges vorjeteruI
0000.0003000 .0 TEJ0j

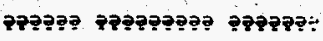
$0000000+3000 \%$ BEZ. $0000 \% 00+3000 \% \quad 5 \varepsilon z-n$ $0000.000+3000.0$ bez-s $0000 \% 00 \div 3000 \%$ 06-35 $0000 \cdot 0 \quad 00+3000 \cdot 0$ 6rz-nd $0000 \cdot 000+3000 \% 0$ bez-nd $0000: 000+3000 \div 0$ E9-TN $0000 \cdot 0.00+3000 \cdot 0$ 65-TK $0000 \cdot 0.00+3000 \% \quad \varepsilon-H$ $0000 \cdot 0 \quad 00+3000 \cdot 0 \quad$ LET-5 $0000 \cdot 000+9000: 0 \quad 09-00$ $0000^{\circ} 00+3000.0$ \& 0 $0000000+3000: 0$ isz-uz

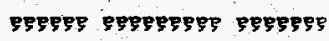

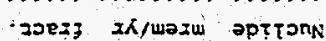

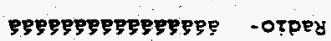
punoxs

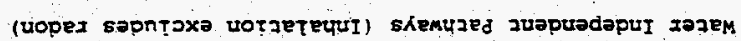

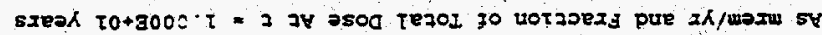

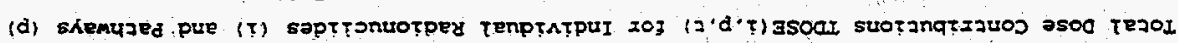

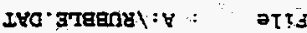

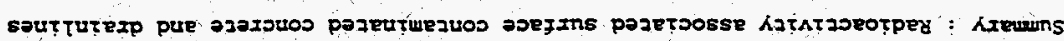

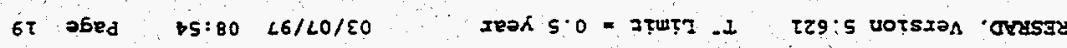


- Shemuzed juopuadop pue zuspuedoput rojem tTe jo wnS.

$0000 \div 000+3000 \div 0$

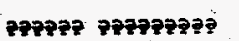
$0000 \cdot 000+3000 \cdot 0$ $0,000 \cdot 000+3000 \cdot 0$ $0000 \cdot 00+3000.0$ $0000.000+2000.0$ $0000 \div 00+3000: 0$ $0000 \cdot 000+3000 \cdot 0$ $0000 \cdot 00+3000 \cdot 0$ $0000.000 \div 3000 \%$ $0000 \% 00+3000.0$ $0000.000+3000.0$ $0000 \cdot 000+3000.0$ $0000 \% 00+3000 \%$ $0000.00 \times 3000 \%$ HTES stressegs. -20exz ak/worw verserenteres - shomuara tTy
$0000 \% 000+3000.0$ 국ำ 779797972 $0000 \cdot 000+2000 \cdot 0$ $0000 \cdot 00+3000=0$ $0000 \div 000+3000 \% 0$ $0000.000+3000.0$ $0000.000+3000 \cdot 0$ $0000 \cdot 00+3000.0$ $0000 \cdot 00+3000 \cdot 0$ $0000 \cdot 0 \quad 00+3000 \cdot 0$ $0000.000+3000.0$ $0000: 0 \quad 00+3000 \cdot 0$ $0000: 000+3000 \cdot 0$ $0000.000+3000.0$ $0000-0 \quad 00+3000=0$

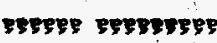
- 70ex Ix/maxw.

Pexpepeepretrep xTรW
$0000 \cdot 00043000 \cdot 0$

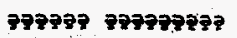
$0000 \cdot 000 \div 9000 \div 0$ $0000 \cdot 00+3000 \% 0$ $0000 \% 00+3000 \%$ $0000 \cdot 0 \quad 00+3000.0$ $0000: 000+3000 \%$ $0000.00+3000.0$ $0000000+3000.0$ $0000 \% 0003000: 0$ $0000 \% 0043000: 0$ $0000.000+3000^{\circ} 0$ $0000000+3000: 0$ $0000 \times 00+3000 \% 0$ $0000 \% 0043000 \%$ essese estrestes -7sexz 2k/wozu poperfeperterpe วอพ
$000000+3000 \div 0 \quad 0000: 000+3000 \% 0000 \div 000+3000 \% 00000000+3000 \%$ TE20

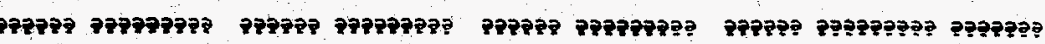

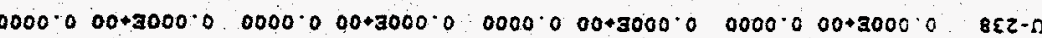
$0000.0,00+3000.00000 \cdot 0.00+3000 \cdot 0 \quad 0000 \cdot 000+300000000.000+30:$ sहz-n $0000 \% 00+3000 \% 0000 \% 00+3000 \% 0000 \% 00+3000 \% 0000.000+30 \%$ bez-n $0000 \cdot 000+3000 \% 0000 \% 00+3000 \% 0000 \% 00+3000 \% 0000 \cdot 000+30000$ 06-35

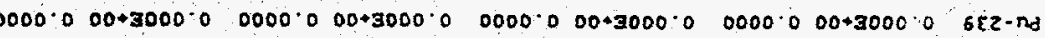
$0000 \% 00+3000 \% 0000 \% 00+3000.0$ 0000.0 00+3000.0 $0000.000+3000.0 \quad 8\left[z-n_{\mathrm{d}}\right.$

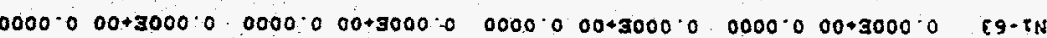
$0000 \% 00+300000000.000+3000 \% 0000000+3000 \% 0000 \cdot 000+30000 \quad 65-T N$ $0000.000+3000 \% 0000000+3000 \div 00000000+300000000000+3000$ \& 0.4 $0000.000+3000.00000 .000+3000.0 \quad 0000 \% 00+3000 \cdot 0$ 0000.0 00+3ct o LET-s) $0000.000+3000.00000 \% 00+3000 \% 0000 \% 00+3000 \% 0000 \% 00+3000 \% 09.00$ $0000000+300000000.000+300000000 \% 00+3000 \% 0000 \% 00+3000 \%$ b1.s $0000000+3000.00000 \% 00+3000 \% 0000 \% 00+3000 \% 0000000+30000$ itz-un

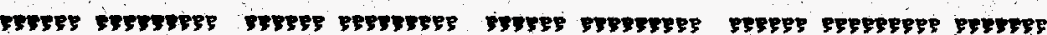

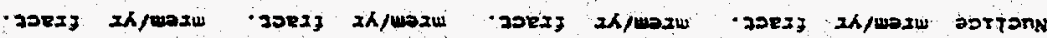

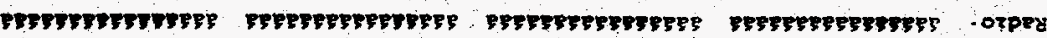
zuetd uopey ucts sezen

8xemysed suopusdoo sepen

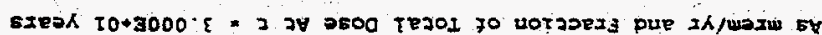

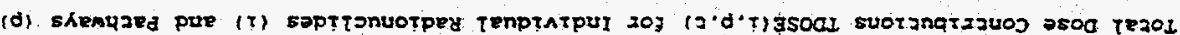

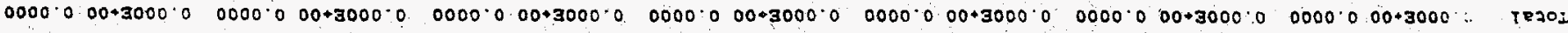

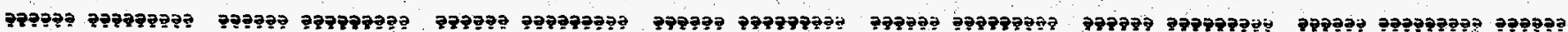

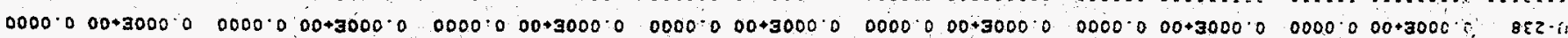

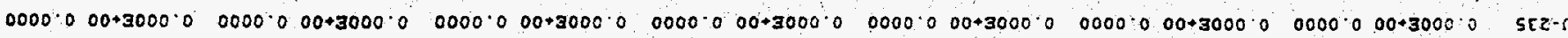

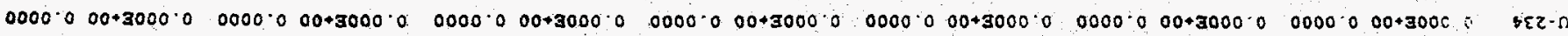

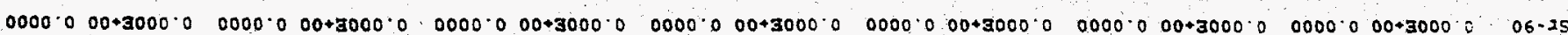

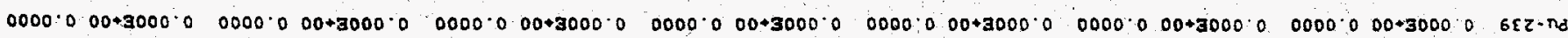

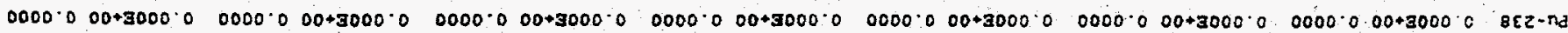

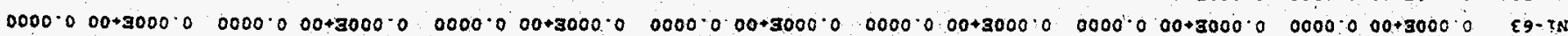

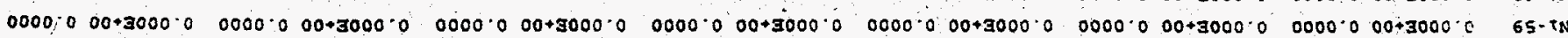
$0000 \% 00+3000 \% 0000 \% 00+3000 \% 00000 \% 00+3000 \% 0000 \% 00+3000 \% 0000 \% 00+3000 \% 0000 \% 00+3000 \% 0000 \% 00+3000 \% \quad 0 \quad 0-4$

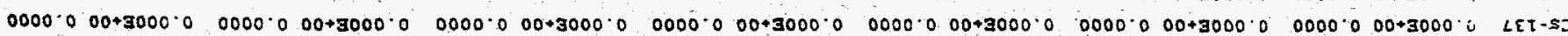

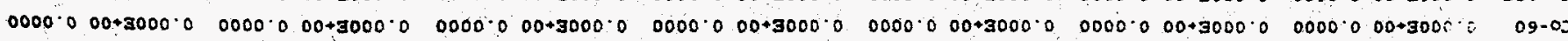

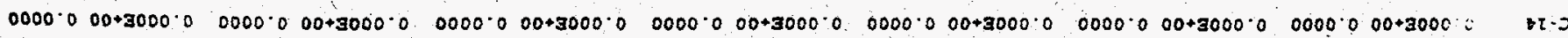

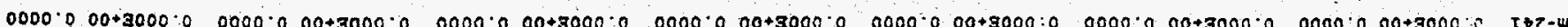

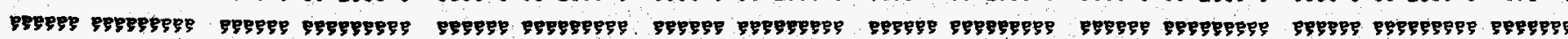

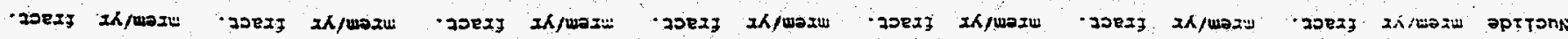

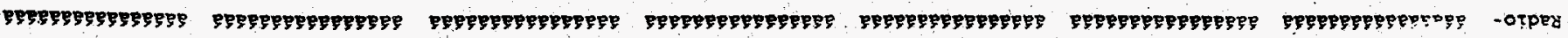
Tros

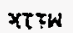
วeow มีe uopey uotวeT EuTI pumox:
\end{abstract}

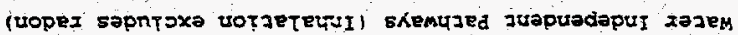

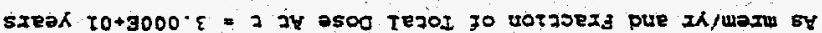

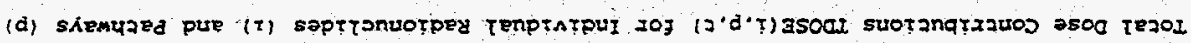


$0000 \cdot 00+3000 \cdot 0 \quad 0000 \cdot 0 \quad 00+3000 \cdot 0$

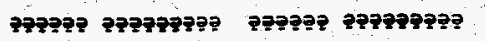
$0000 \cdot 000+3000 \cdot 0 \quad 0000 \cdot 000+3000 \cdot 0$ $0000 \cdot 000+3000 \cdot 0 \quad 0000 \div 00+3000 \% 0$ $0000 \cdot 0 \quad 00+3000 \cdot 0 \quad 0000 \div 00+3000 \cdot 0$ $0000 \cdot 0 \quad 00+3000 \cdot 0 \quad 0000 \cdot 0 \quad 00+3000 \cdot 0$ $0000 \cdot 00+3000 \cdot 00000 \cdot 000+3000 \cdot 0$ $0000 \div 00+3000 \cdot 0 \div 0000 \div 00+3000 \cdot 0$ $0000 \cdot 000+3000 \div 00000: 0 \quad 00+3000 \cdot 0$ $0000 \cdot 000+3000 \cdot 0 \quad 0000 \cdot 000+3000 \cdot 0$ $00000.00+3000 \% 0000 \cdot 00+3000 \%$ $0000 \% 00+3000 \% 0000 \div 00+3000 \%$ $0000 \div 00+3000 \cdot 0000 \div 00+3000 \% 0$ $0000 \cdot 00+3000 \% 0000 \cdot 000+3000: 0$ $0000 \cdot 000+3000.0 \quad 0000 \cdot 000+30000$

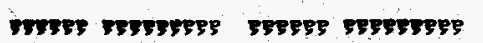

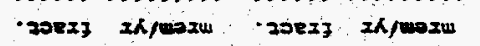

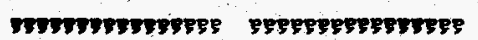
- exentraea tro xt ţผ
$0000 \cdot 000+30000$

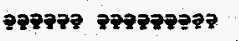
$0000 \cdot 000+3000 \cdot 0$ $0000.000+3000.0$ $0000-00+3000=0$ $0000 \div 00+3000 \div 0$ $0000 \cdot 000+3000 \%$ $0000 \cdot 00+2000 \%$ $0000-000+3000 \cdot 0$ $0000 \% 00+3000 \% 0$ $0000.000+3000.0$ $0000-000+3000=0$ $0000000+3000^{\circ} 0$ $0000.000+3000 \%$ $0000 \% 00+3000 \%$ vesepe perserses 20ez3 xh/wozit GFrensersenterse Jกอพ
$0000 \cdot 00+30000$

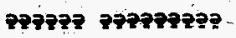
$0000 \cdot 000+3000.0$ $0000: 000+3000 \div 0$ $0000 \cdot 00+3000.0$ $0000 \cdot 00+3000: 0$ $0000 \cdot 000+3000 \% 0$ $0000.000+3000.0$ $0000 \cdot 000+3000 \cdot 0$ $0000 \cdot 0$ 00+8000.0 $0000 \cdot 00+3000 \%$ $0000 \cdot 00+3000.0$ $0000 \cdot 00+3000 \cdot 0$ $0000 \cdot 000+3000-0$ $0000 \cdot 000+3000 \% 0$ mutes shstrses 20RxJ IK/unin whossterentese 7urta
$0000000+3000 \%$

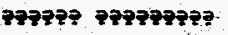
$0000: 00+3000 \cdot 0$ $0000.000+3000 \%$ $0000.000+2000 \% 0$ $0000: 0 \quad 0043000 \cdot 0$ $0000: 000+3000 \%$ $0000^{\circ} 000+3000^{\circ} 0$ $0000 \cdot 00+3000.0$ $0000 \cdot 000+3000 \% 0$ $0000 \cdot 000+3000 \cdot 0$ $0000 \% 00+3000 \div 0$ $0000: 00+3000.0$ $0000 \div 00+3000 \div 0$ $0000 \cdot 000+3000 \cdot 0$

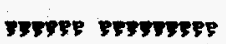
- zoex $x$ K/mera. HEW noper
$0000 \cdot 000+3000 \cdot 00000 \cdot 000+3000 \cdot 0$ TE301

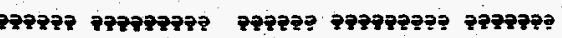
$0000 \cdot 000+3000 \% 0000 \cdot 000+3000 \cdot 0$ BEz-n $0000 \% 00+3000: 00000.000+3000 \cdot 0$ sez-n $0000 \cdot 000+3000=0 \quad 0000 \cdot 000+3000=0$ tez-n $0000.000+3000 \% 00000.0 \quad 00+3000 \% 0$ 06-xs $0000 \cdot 0 \quad 00+3000 \cdot 0 \quad 0000 \cdot 0 \quad 00+3000 \cdot 0 \quad 6 \varepsilon z-n_{d}$ $0000000+3000 \% 0000.000+3000 \cdot 0$ Bez-na $0000 \% 00+3000=0$ 0000:0 00+3000 0 Eg-TK $0000 \cdot 000+3000 \div 00000 \div 000+3000 \% \quad 65-\frac{1 N}{N}$ $0000 \div 000+3000 \cdot 0 \quad 0000 \cdot 000+3000.0 \quad$ E-H $0000 \% 00+3000 \% 0000.000+3000 \cdot 0$ LET-EO $0000.000+3000.0 \quad 0000.000+3000.0 \quad 09-05$ $0000.000+3000 \cdot 0000.000+3000.0 \quad 75-5$ $0000.000+3000 \% 0000 \% 00+3000 \%$ isz-4\%

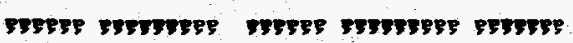

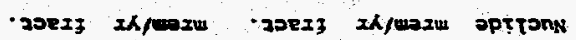

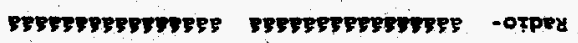
4s?a xeasy

EkBnuzed Juopuedod xojen

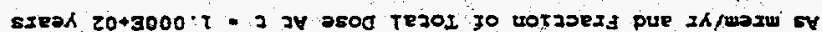

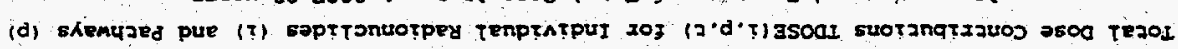

$0000 \cdot 000+3000 \cdot 0$

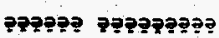
$0000: 00+3000: 0$ $0000 \cdot 00+3000.0$ $0000000+3000 \%$ $0000 \cdot 000+3000 \cdot 0$ $0000 \cdot 000+3000 \cdot 0$ $0000000+3000.0$ $0000 \cdot 00+3000.0$ $0000000+3000 \%$ $0000 \cdot 00+3000.0$ $0000 \% 00+3000 \%$ $0000 \cdot 000+3000 \%$ $0000 \cdot 00+3000 \cdot 0$ $0000 \% 0.00+3000 \% 0$. 5eseres pespespes - 20exj - 2X/waxw

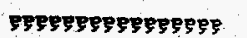
โṬos
$0000=0 \quad 00+30000$ ำวซำ 쿨ำำำ $0000 \cdot 000+3000 \cdot 0$ 000000000000000000 $0000.000+3000 \% \quad 0000 \% 00+3000 \%$ $0000 \% 00+3000 \% 0000 \% 00+3000 \%$ $0000 \% 00+3000 \cdot 0 \quad 0000 \% 00+3000 \%$ $0000 \cdot 000+3000 \cdot 0 \quad 0000 \cdot 000+3000 \cdot 0$ $0000 \div 00+3000 \cdot 0000 \% 00+3000 \div 0$ $0000 \cdot 000+3000=00000 \% 00+3000 \% 0$ $0000 \% 00+3000 \cdot 0 \quad 0000 \cdot 000+3000 \cdot 0$. $0000000+3000 \% 00000 \% 00+3000=0$ $0000 \cdot 000+3000 \cdot 0 \quad 0000-000+3000: 0$ $0000 \cdot 000+3000 \% 0000-000+3000.0$ $0000 \cdot 00+3000 \cdot 0,0000 \cdot 000+3000 \cdot 0$

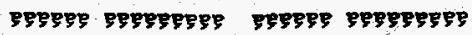
- zoexz $2 x /$ waxu - DOEIJ xK/WOIW

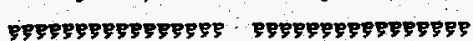
2อaw
$0000 \cdot 0 \cdot 00+3000 \cdot 0$

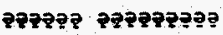
$0000 \% 00+3000.0$ $0000 \cdot 000+3000 \cdot 0$ $0000 \cdot 000+3000 \%$ $0000 * 000+3000 * 0$ $0000 \cdot 000+3000 \cdot 0$ $0000 \cdot 000+3000: 0$ $0000 \cdot 000+3000 \%$ $0000: 000+3000 \cdot 0$ $0000 \cdot 000+3000 \%$ $0000.000+3000 \%$ $0000 \div 00+3000 \%$ $0000: 00+3000.0$ $0000 \cdot 0.00+3000-0$

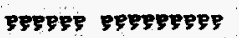
- 7oex; Ih/uszu pespepsegeseses
zuetd
$0000 \% 00+3000 \%$

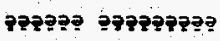
$0000000 \div 3000 \%$ $0000.000+3000.0$ $0000 \% 00+3000 \%$ $0000 \% 00+3000=0$ $0000 \cdot 00+3000^{\circ} 0$ $0000 \cdot 00+3000 \%$ $0000 \cdot 00+3000=0$ $0000 \cdot 00+3000=0$ $0000 \cdot 0.00+3000: 0$ $0000 \cdot 000+3000-0$ $0000 \cdot 000+3000 \cdot 0$ $0000 \div 00+3000-0$ $0000 \cdot 00+3000+0$

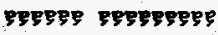
$208 x 7 \quad 2 \Lambda /$ wasx. epsepespegserges topey
$0000.00+3000.0$

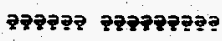
$0000.00+3000.0$ $0000 \% 00+3000.0$ $0000 \cdot 000+30000$ $0000 \% 00+3000=0$ $0000 \cdot 000+3000 \cdot 0$ 0000 0 00+3000.0. $0000 \cdot 00+3000.0$ $0000 \cdot 000+3000.0$ $0000: 000+3000.0$ $0000.000+3000.0$ $0000.000+3000^{\circ} 0$ $0000.00+3000.0$ $0000 \% 00+3000 \% 0$

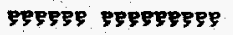
- zoezz IN/Lesw

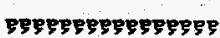
norjeT EYLI

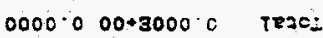

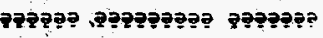
$00000.00+3000 \% 0$ 8दz$0000 \cdot 0 \quad 00+3000 \%$ sez-r. $0000 \cdot 000+3000 \%$ ।हZ-r $0000 \cdot 000+3000.0$ 06-25 $0000 \% 000+3000.0$ 6Ez-nd $0000 \cdot 0$ 00+3000:0 oEz-nd $0000-00+3000 \cdot 0$ E9-1n $0000 \cdot 0 \quad 00+3000 \cdot 0$ 65-TK $0000.000+3000 \% 0$ \&-4 $0000 \cdot 0 \quad 00+3000 \cdot 0$ LET-5z $0000 \cdot 000+3000 \cdot 0$. 09-cs $0000 \cdot 000+3000 \div 0: \quad 5 T-2$ $0000000+3000=0$ t\$z-ur

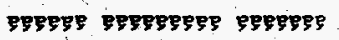
- joexi $x \Lambda /$ was oprtonk

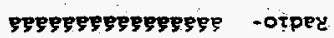
punais

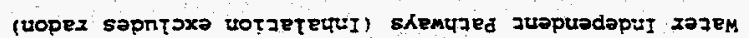

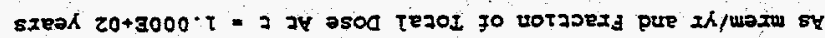

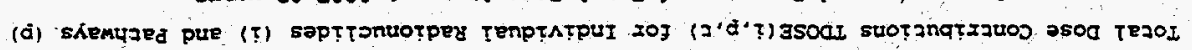

LYG $\operatorname{argand~:~}$

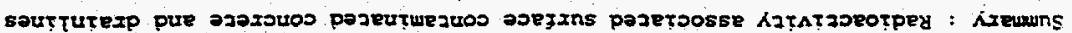

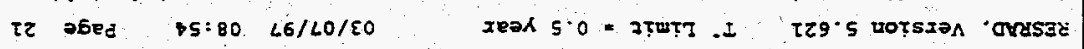




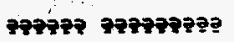

$0000 \% 00+3000 \% 0$

$0000 \cdot 000+3000 \cdot 0$

$0000 \cdot 000+3000 \cdot 0$

$0000.000+3000 \% 0$

$0000 \cdot 000+3000 \div 0$.

$0000 \cdot 000+3000 \cdot 0$

$0000 \div 00+3000 \% 0$

$0000^{\circ} 00+3000^{\circ} 0$

0000.0 rt-39xL.

$0000.00+3000.0$.

$0000 \cdot 000+3000 \% 0$

$0000 \cdot r$ ro-artg.L

$0000.00+3000.0$

rine Thuses

-2oexz $2 x$ moxus

rophrshenteps

- exemuped TTY

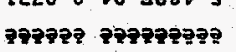
$0000 \cdot 000+3000 \%$ $0000.000+3000.0$ $0000 \% 00+3000 \%$ $0000.00+2000.0$ $0000 \% 00+3000.0$ $0000 \cdot 000+3000.0$ $0000.000+3000.0$ $0000.000+2000.0$ $0000 \div 0$ 2I-3109:2 $0000 \% 00+3000 \%$ $0000 \cdot 0 \cdot 00+3000 \cdot 0$ IZLO.0 ro-368:5 $0000.000+2000.0$

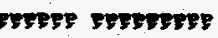
-30253 $2 x /$ woxw

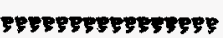
xT TW 국ำ 구구국ำ $0000: 0 \quad 0.0+8000 \div 0$ $0000 \cdot 0.00+3000 \cdot 0$ $0000.00+3000: 0$

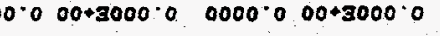
$0000.000+3000.0 \quad 0000 \% 00+3000.0$ $0000: 0.00+3000 \cdot 0 \quad 0000 \div 0.00+3000 \%$ $0000 \cdot 0 \quad 00+3000 \% 0000 \cdot 000+3000 \% 0$ $0000.000+3000 \% 0000.000+3000 \% 0$ $0000: 000+3000 \cdot 0 \quad 0000 \cdot 0$ 0043000 0 0000.0 <T-3820.5 $0000-0 \mathrm{zZ-3080:z}$ $0000 \div 00+3000 \% 0000: 000+3000=0$ $0000 \cdot 000+3000 \cdot 0 \quad 0000 \cdot 0: 00 \div 3000 \cdot 0$ 55E0.0 *0-3TOL' $z$ zLTZO EO-3ESg: $0000-000+3000-0000000+3000.0$

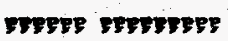
- 2005z ixpmoxur

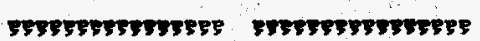
anaw

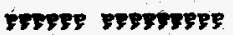
- poezj zipuaru. aretd

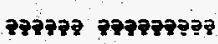
$0000 \div 000+3000 \cdot 0$ $0000 \cdot 000+3000 \cdot 0$ $0000 \cdot 00+3000 \cdot 0$ $0000 \cdot 000+3000.0$ $0000.000+3000 \cdot 0$ $0000.000+3000 \% 0$. $0000 \cdot 000+3000 \cdot 0$ $0000 \cdot 00+3000 \cdot 0$. $0000: 000+3000 \cdot 0$ $0000 \cdot 00+3000 \%$ $0000 \cdot 0.00+3000 \cdot 0$ $0000.000+3000.0$ $0000 \div 0.0+3000 \cdot 0$ TFEPSE EFETSESE 20ex; 21 rearu creverenterseres. voper
25L9.0 E0-38ET'S TEZOI

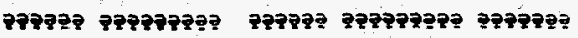
$0000 \cdot 000+3000 \cdot 0 \quad 0000 \cdot 000+3000 \cdot 0 \quad 8 \varepsilon z \cdot n$ $0000000+300000000000+3000 \%$ sez-n $0000000+3000 \% 0000 \% 00+3000 \%$ VEz-n $0000 \div 00+3000 \div 0 \quad 0000 \div 000+3000 \div 0$ 06-3S $0000000+3000 \cdot 0 \quad 0000 \cdot 000+3000 \div 0$ 6Ez-nd $0000 \% 00+3000 \% 0000000+3000.0$ 8Ez-nd $0000.0003000 .00000 .000 \times 3000 \%$ E $\quad 0-t N$ $0000 \cdot 000+3000: 0 \quad 0000.000+30000 \quad 65-1 \mathrm{~N}$ $0000.000+3000000000 t r-3 \angle 620 E-H$ $0000000+3000 \% 0000 \% 00+3000$ O LET-sइ $0000 \cdot 0 \quad 00+3000 \cdot 0 \quad 0000 \cdot 0 \quad 00+3000 \cdot 0 \cdots 09-03$ $0000 \% 00+3000.0 \quad 25 \angle 9.0$ E0-38ET's BT.5 $0000000+300000000000+3000 \%$ toz-w

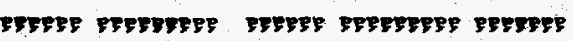

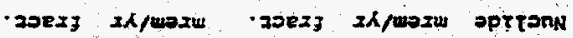

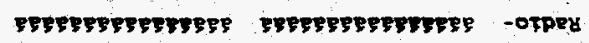
บร? 3 دO2EM

skemuzed suspusdod sosen

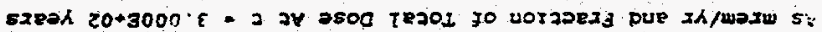

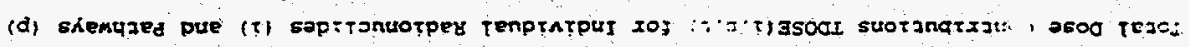

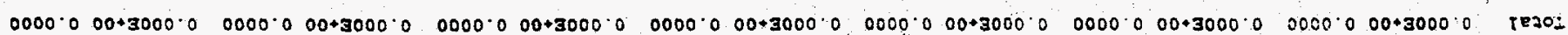

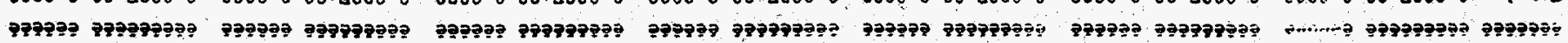

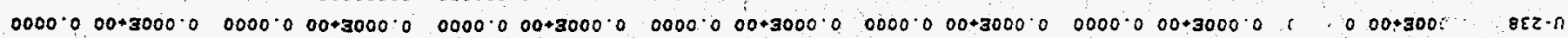

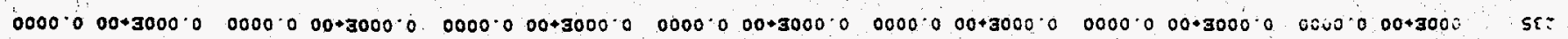

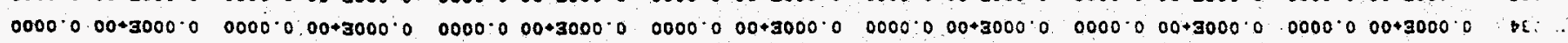

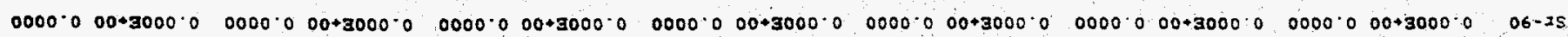

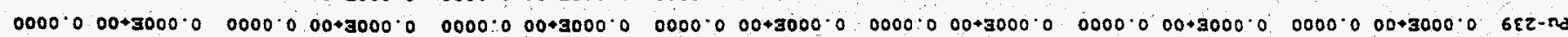

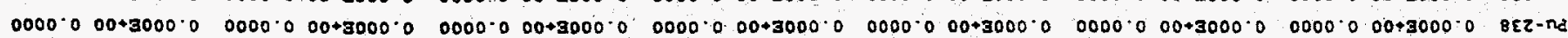

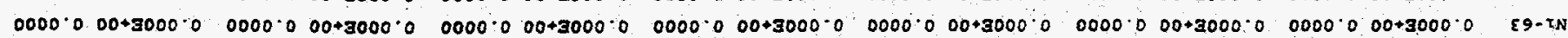

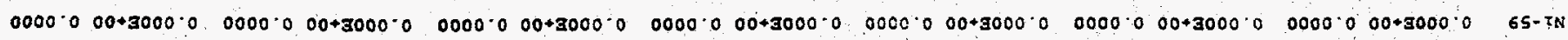

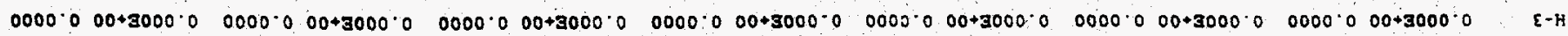

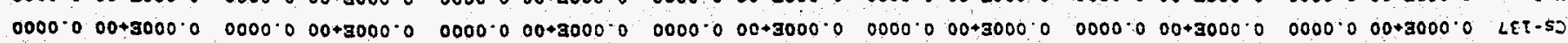

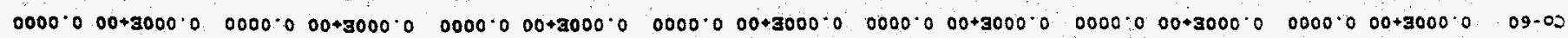

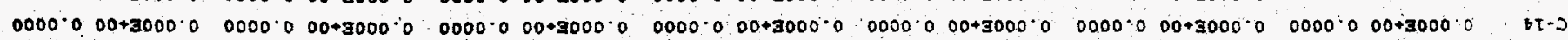

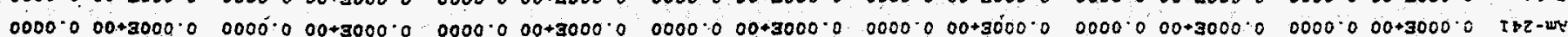

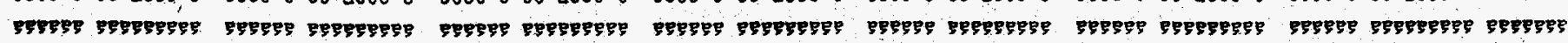
- Joex IK/Wox -

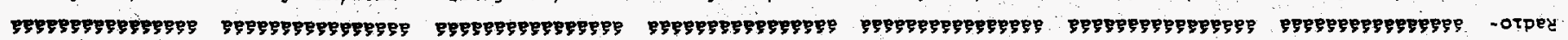
tros หโT seak zueta uopey uotaetruit purnois
\end{abstract}


$0000 \cdot T \cdot 0-306 t 5$

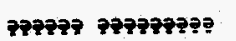
$0000 \cdot 0.00+3000 \cdot 0$ $0000 \cdot 000+3000 \cdot 0$ $0000 \cdot 0 \cdot 00+3000 \%$ $0000-000+3000 \div 0$ $0000-0003000 \cdot 0$ $0000 \cdot 000+3000 \cdot 0$ $0000 \div 00+3000: 0$ $0000 \div 00+3000 \%$ $0000 \cdot 00+3000 \cdot 0$ $0000 \% 00+3000.0$ L666.0 D0-3EDT'S TZLOOO 50-360L:C $8000 \cdot 0$ LO-356t't $0000 \%$ Tt-3EBZ. $z$

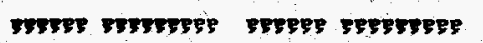
2oext IA/mons moveswerrepes - Exenuasa tTr
זรLO.0 SO-360L E $0000.000+3000.0$ $0000 \cdot 0 \cdot 00+3000 \cdot 0$ $0000 \% 00+3000 \% 0$ $000 \div 00+3000 \% 0$ $0000.000 \cdot 3000.0$ $0000.0 \quad 00+3000.0$ $0000 \% 00 \div 3000 \div 0$ $0000 \cdot 000+3000 \cdot 0$ epese sestroses
zorif IK/woxw

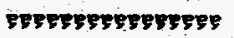
ทีTK $0000 \% 00+3000: 0$
2560.0 50-39.2.

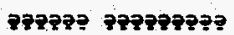
$0000 \cdot 00+3000.0$ $0000 \cdot 00+3000: 0$ $0000.00+3000.0$ $0000.00+3000.0$ $0000 \cdot 000+3000 \cdot 0$ $0000.000 \times 3000.0$ $0000 \% 00+3000.0$ $0000.000+3000: 0$ $0000: 000+3000 \cdot 0$ $0000 \% 00+3000.0$ $0000.000+3000 \%$ $5580^{\circ} 0$ 50-3528 $\tau$ $0000^{\circ} 0.60-3 L 8 z \cdot \tau$ pepere crestreps

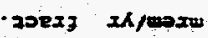
EFPSEFFETSTSERS בNow

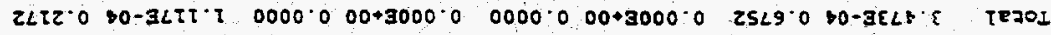

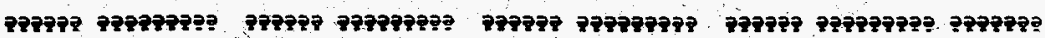

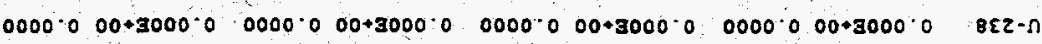
$0000 \cdot 000+3000 \div 0000000+3000 \% 0000000+3000 \% 0000 \% 00+30000$ sez-r $0000 \% 00+3000 \cdot 0 \quad 0000 \div 000+3000: 0 \quad 0000 \% 00+3000 \% 0000 \% 00+3000 \%$ B $0 z-\Omega$ $0000 \cdot 0 \quad 00+3000 \cdot 0 \quad 0000 \cdot 0 \quad 00+3000 \cdot 0 \quad 0000 \cdot 0 \quad 00+3000 \cdot 00000 \cdot 000+3000 \cdot 0$ 06-2s $0000 \% 00+3000 \% 0 \quad 0000 \% 00+3000 \% 0000 \% 00+3000 \% 0000 \% 00+3000 \%$ GEZ-n $0000.000+3900 \% 0 \quad 0000: 000+3000 \% 00000000+3000 \% 0000 \% 00+3000 \%$ BEZ-nd $0000 \% 00+3000 \% 0000 \% 00+3000 \% 0000 \cdot 000+3000 \% 0000 \% 00+3000 \%$ Eg-TN

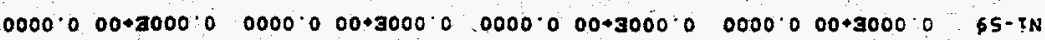
$0000 \% 00+3000 \% 00000.00+3000 \% 0000000+3000 \% 0000 \% 00+3000 \%$ \& $0000 \% 00+3000 \% 0000 \% 00+3000 \% \quad 0000 \% 00+3000 \%$ 0000 0 00+3000\% LET-5: $0000000+300000000 \% 00+3000 \div 00000000+3000 \% 0000 \% 000+3000 \% 09-02$

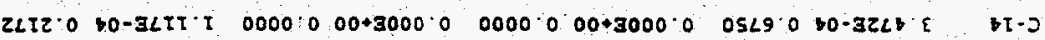
T000.0 B0-3058.z 0000\% 00+3000\% $0000 \% 0003000 \%$ 2000.0 L0-3L6I'I Ibz-w

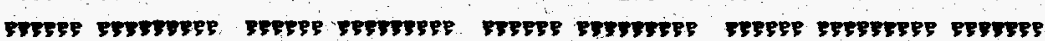

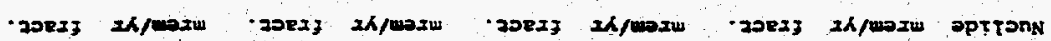

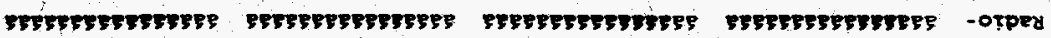
zurTd uoped ucta xasen

skenuzed zuspuriad zajen

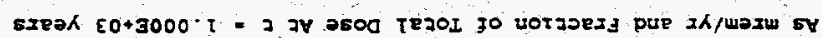

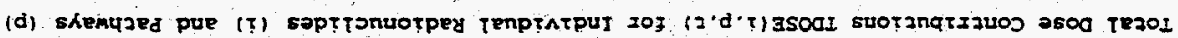

$0000 \cdot 00+3000 \cdot 0$

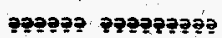
$0000 \cdot 0 \cdot 00+3000 \cdot 0$ $0000 \cdot 00+3000 \cdot 0$ $0000.000+3000 \%$ $0000 \div 00+3000=0$ $0000.000+3000.0$ $0000.000+2000.0$ $0000 \cdot 000+9000 \%$ $0000 \% 00+3000 \div 0$ $0000.000+3000.0$ $0000: 0 \quad 00+3000 \%$ $0000.000+3000 \%$ $0000: 0-00+3000 \div 0$ $0000 \cdot 000+3000 \%$

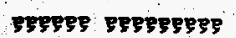
-2SEXI, IK/WOIU

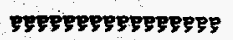
Tros
$0000000+3000.0$

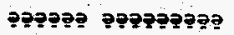
$0000 \cdot 000450000$ $0000 \% 00+3000 \%$ $0000.000+3000.0$ $0000 \cdot 000+3000-0$ $0000=00+3000=0$ $0000 \% 00+3000 \% 0$ $0000 \cdot 000+3000=0$ $0000 \% 00+3000 \%$ $0000 \% 00+3000=0$ $0000.000+3000 \cdot 0$ $0000: 000+3000 \% 0$ $0000.000+3000.0$ $0000: 000+3000 \% 0$

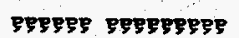
-joexf 2א/wexw

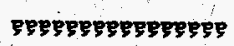
xโ Țแ
$0000 \cdot 00+3000.0$

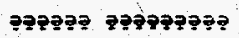
$0000.000+3000.0$ $0000-000+3000: 0$ $0000=00+3000: 0$ $0000 \cdot 00+3000 \cdot 0$. $0000^{\circ} 00+3000.0$ $0000 \% 00+3000.0$ $0000 \cdot 000+3000: 0$ $0000 \cdot 000+3000^{\circ} 0$ $0000 \% 00+3000 \%$ $0000 \cdot 000+3000 \cdot 0$ $0000 \cdot 000+3000 \cdot 0$ $0000 \cdot 000+3000=0$ $0000.000+3000.0$

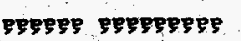

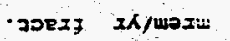

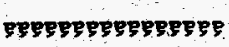
วeaw

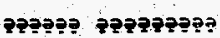
$0000 \div 00 * 3000 \% 0$. $0000 \cdot 000+3000 \cdot 0$ $0000 \div 000+3000 \% 00$ $0000: 000+3000.0$ $0000 \cdot 00+3000 \cdot 0$ $0000 \div 00+3000: 0$ $0000 \cdot 00+3000 \%$ $000+3000 \div 0000 \div 00+3000.0$ $0000000+300000000000+3000 \%$ $0000000+3000 \% 0000000+30000$ $0000 \cdot 00+3000 \% 0000 \cdot 000+3000 \cdot 0$ $0000000+300000000 \cdot 000+3000=0$ $0000000+3000 \% 0000000+3000 \% 0$

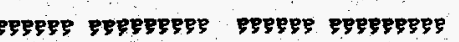
20exz xk/wazw

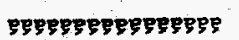
zreta

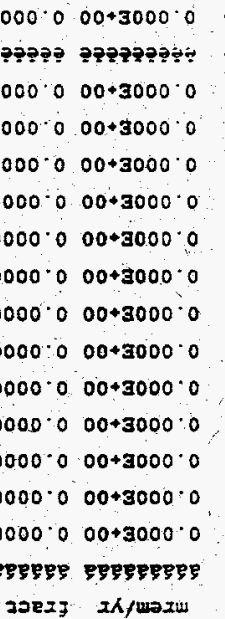

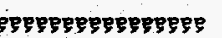
ropey
$0000 \cdot 00 \cdot 3000 \cdot 0$

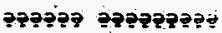
$0000000+30000$ $0000 \cdot 00+3000 \cdot 0$ $0000 \cdot 00+3000.0$ $0000 \cdot 0 \quad 00+3000 \cdot 0$ $0000 \cdot 000+3000 \cdot 0$ $0000 \div 00+3000 \%$ $0000 \cdot 000+3000 \cdot 0$ $0000 \cdot 00+3000.0$ $000000+3000 \%$ $0000 \cdot 000+3000 \%$ $0000 \cdot 00+3000 \cdot 0$ $0000 \% 00+3000 \%$ $0000 \% 0.00+3000: 0$ pepses peppegpes. zoeż $2 \mathrm{~K}$ /wazw

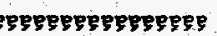
NOTJETEYUT
$0000 \% 00+3000.0$ TEJ0:

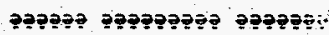
$0000000+30000$ esz-t: $0000000+30000$ sहz-? $0000 \% 00+3000 \%$ bez-r $0000 \% 0.00+3000 \cdot 0$ 06-Is $0000 \cdot 000+3000.0$ 6Ez-nz $0000: 000+3000 \%$ Bez-nd $0000 \cdot 0 \quad 00+3000 \cdot 0$ Eg-TN $0000 \cdot 0 \quad 00+3000 \%$ 65-TN $0000-000+3000 \cdot C \quad \varepsilon-f i$ $0000 \cdot 0$ 00+3000.0 LEI-SD $0000 \cdot 00+3000 \cdot 0 \quad 09-02$ $0000 \cdot 000+3000 \cdot 0$ t $0000.000+3000.0$ t\$2-4r

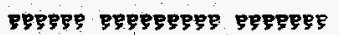
zoeI IN/waxu aptTonN

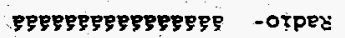
punoro

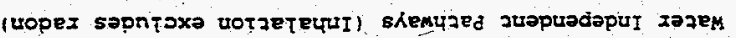

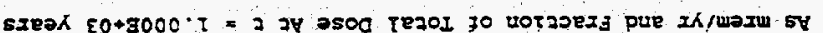

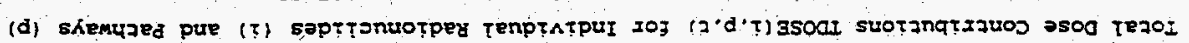

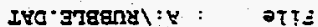

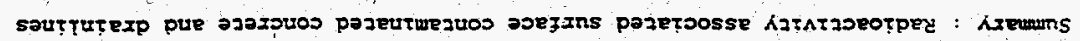

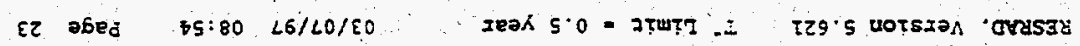


RESRAD, Vezsion $5.621 \cdot T$ Eimic $=0.5$ year $03 / 07 / 97$ 08:54 Fage 24

Sumary : Radjoactivity associaced surface contaminated concrete and drainlines File : M YRUBBLE.DAT

Tocal Dose contribueions toose (i,p,c) for Individual Radionuclides (i) and Pathways (p) As mrem $y x$ and Fraction of Tocal Dose Ac $=3.000 E+04$ years

Water Independent Pathways (Inhalation excludes radon)

Ground

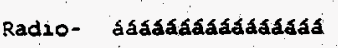
Nuclide mrem/yr fract.

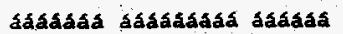

Am-241 $0.000 E+00 \quad 0.0000$

C. 14

Co-60 $0.000 E+00 \quad 0.0000$

$6 s-137 \quad 0.000 E+00 \quad 0.0000$

$\mathrm{H}-3 \quad 0.000 \mathrm{E}+0 \mathrm{OO} 0.0000$

$\mathrm{N}_{1}-59 \quad 0.000 \mathrm{E}+00 \quad 0.0000$

Ní63 $0.000 \mathrm{E}-000.0000$

Pu-238 $0.0008+00 \quad 0.0000$

Pu-239 $0.0008+00 \quad 0.0000$

$\$ 5.90 \quad 0.0008+00 \quad 0.0000$

U-234 $0.000 E+00 \quad 0.0000$

J-235 $0.000 E+00 \quad 0.0000$

$U .238,0.000 E+000.0000$

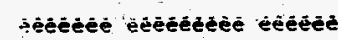

Total $: 000 E-00 \% 0000$
Inhalation sásasasáasáasáạa mren/yr tract. áasakaá aakasa $0.000 E+00 \quad 0.0000$ $0.000 E+00 \quad 0.0000$ $\begin{array}{ll}0.000 E+00 & 0.0000 \\ 0.000 E+00 & 0.0000\end{array}$ $0.0008+00 \quad 0.0000$ $0.0008+00 \quad 0.0000$ $0.000 E+00 \quad 0.0000$ $0.0008+00 \quad 0.0000$ $0.000 E+00 \quad 0.0000$ $0.000 E+00 \quad 0.0000$ $0.000 E+00 \quad 0.0000$ $0.000 E+00 \quad 0.0000$ $0.000 \varepsilon+00 \quad 0.0000$

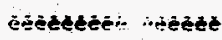
$0.000 E+06 \quad 0000$
Radon.

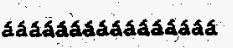
mrem/yx fract.

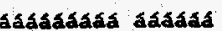

$0.000 E+00 \quad 0.0000$

$0.000 E+00 \quad 0.0000$

$0.000 E+00 \quad 0.0000$

$0.000 \mathrm{E}+00 \quad 0.0000$

$0.0008+00 \quad 0.0000$

$0.0008+00 \quad 0.0000$

$0.0002+00.0 .0000$

$0.0008+00 \quad 0.0000$

$0.000 E+00 \quad 0.0000$

$0.000 E+00 \quad 0.0000$

$0.000 E+00 \quad 0.0000$

$0.000 E+00 \quad 0.0000$

$0.000 E+00 \quad 0.0000$

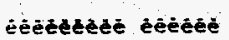

$0.000 E+00$ O.0000
Plant aáasááááááá mrem/yr fract. áadaasaá saakas $0.000 \mathrm{E}+00 \quad 0.0000$ $0.000 E+00 \quad 0.0000$ $0.000 E+00 \quad 0.0000$ $0.000 \mathrm{E}+00 \quad 0.0000$ $0.000 z+00 \quad 0.0000$ $0.000 E+00 \quad 0.0000$ $0.000 E+00 \quad 0.0000$ $0.000 E+00 \quad 0.0000 \quad 0.000 E+00 \quad 0.0000$ $\begin{array}{llllllll}0.0005+00 & 0.0000 & 0.0002+00 & 0.0000\end{array}$ $0.000 E+00 \quad 0.0000 \quad 0.000 E+00 \quad 0.0000$ $0.000 E+00 \quad 0.0000$ $0.000 \varepsilon+00 \quad 0.0000$ èécezté éécezz $0.000 E+00 \quad 0.0000$
Neat

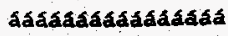
mrem/yr frace.

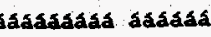
$0.000 E+00 \quad 0.0000$ $0.000 E+00 \cdot 0.0000$ $0.0008+00 \quad 0.0000$ $0.000 E+00 \quad 0.0000$ $0.0008+00 \quad 0.0000$ $0.000 E+00 \quad 0.0000$ $\begin{array}{cc}.0008+00 & 0.0000 \\ 0.0008+00 & 0.0000\end{array}$ $0.000 E+00 \quad 0.0000$ $0.000 E+00 \quad 0.0000$

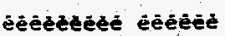
$0.000 E+00 \quad 0.0000$
Milk aakasaakáakaáá mrem/yr fract. áakakas akaká $0.000 E+00 \quad 0.0000$ $0.000 E+00 \quad 0.0000$ $0.000 \mathrm{E}+00 \quad 0.0000$ $0.0008+00 \quad 0.0000$ $0.000 E+00 \quad 0.0000$ $0.000 E+000.0000$ $0.000 E+00 \quad 0.0000$ $0.000 E+00 \quad 0.0000$ $0.000 E+00 \quad 0.0000$ $0.0008+00 \quad 0.0000$ $0.000 E+00 \quad 0.0000$ $0.000 E+00 \quad 0.0000$ $0.000 E+00.0 .0000$

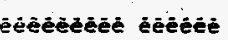
$0.0005+00 \quad 0,0000$
Soil

aakasakakakasasa mrem/yr fract. acáasasa sakasa $0.000 E+00 \quad 0.0000$ $0.000 E+00 \quad 0.0000$ $0.000 E+00 \quad 0.0000$ $0.000 E+00 \quad 0.0000$ $0.000 E+00 \quad 0.0000$ $0.000 E+00 \quad 0.0000$ $0.000 E+00 \quad 0.0000$ $0.0005+00 \quad 0.0000$ $0.000 E+00 \quad 0.0000$ $0.000 E+00 \quad 0.0000$ $0.000 E+00 \quad 0.0000$ $0.000 E+00 \quad 0.0000$ $0.000 E+00 \quad 0.0000$

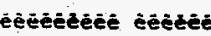
$0.000 E+00 \quad 0.0000$

Total Dose Contributzons TDOSE(i.p.t) for Individual Radionuclidés (i) and Pathways (p) As mrem/yr and Fraction of Total Dose At $t=1.000 E+04$ years

wacer Dependent Pachways

\begin{tabular}{|c|c|c|c|c|c|c|c|c|c|c|c|c|c|c|}
\hline \multirow{2}{*}{$\begin{array}{l}\text { Radio- } \\
\text { Nuclide } \\
\text { aserada }\end{array}$} & \multicolumn{2}{|c|}{ Wacer } & & & & \multicolumn{2}{|c|}{ Ment } & $\begin{array}{l}\text { Milk } \\
\text { atasa }\end{array}$ & as & $\begin{array}{l}\text { All Pach } \\
\text { gasasasal }\end{array}$ & $\begin{array}{l}\text { hwaya: } \\
\text { casesed }\end{array}$ \\
\hline & & & $\begin{array}{l}\text { mrem/yx } \\
\text { sasisass }\end{array}$ & $\begin{array}{l}\text { fract. } \\
\text { sasats }\end{array}$ & $\begin{array}{c}\text { mrem/yr } \\
\text { assasasas }\end{array}$ & $\begin{array}{l}\text { Eract: } \\
\text { asasat }\end{array}$ & $\begin{array}{l}\text { mrea/yr } \\
\text { sasuisust }\end{array}$ & $\begin{array}{l}\text { frace. } \\
\text { sasast }\end{array}$ & $\begin{array}{l}\text { /yr } \\
\text { anda }\end{array}$ & $\begin{array}{l}\text { fract. } \\
\text { aasisa }\end{array}$ & $\begin{array}{l}y \mathbf{x} \\
\operatorname{asa}\end{array}$ & ar: & $\begin{array}{l}\text { aryz } \\
\text { atase }\end{array}$ & \\
\hline 241 & $E-12$ & 0.0000 & $.000 E+00$ & 0.0000 & $100 \mathrm{E}+00$ & 0.00 & $2 E-13$ & 0.0 & $3 E-14$ & 00. & 4 & & 2 & D. \\
\hline 4 & $7.698 \mathrm{E}-25$ & 00 & 0 & 000 & 00 & 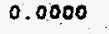 & -25 & 0 & .26 & 0.0000 & 26 & 0 & $E-24$ & 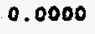 \\
\hline 60 & a. & 0 & $E+00$ & 0 & $E+\infty$ & ( & $.000 E+00$ & 0.0000 & o & 0.0 & 0.0 & 0 & i & \\
\hline 7 & $0.000 \mathrm{E} \cdot 00$ & 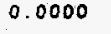 & OOOFAO & r & $8+00$ & 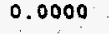 & $E+00$ & & 00 & 0. & +00 & 0 & 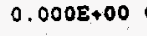 & \\
\hline & .00 & & & & & & & & 0 & 0 & $+\infty$ & 20 & $2 E+00$ & 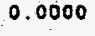 \\
\hline-59 & 0.00 & Do & 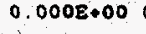 & 0.0 & $\infty 0$ & & . & & $0.05+00$ & & $2+00$ & 00 & $00 E+00$ & 0 \\
\hline & 0.000 & & 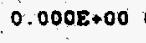 & . & 0 & & 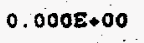 & & & & 0 & & $0 \varepsilon+00$ & c \\
\hline 238 & 3.18 & & 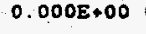 & & & & . & & & & & & $7 E-20$ & 0.000 \\
\hline 239 & -10 & & 0. & & 00 & & 10. & 0 & 2 & & & & $E-09$ & 000 \\
\hline 90 & $0.000 \mathrm{E}+00$ & & 0 & & 00 & & 00 & & & & & & 00 & 000 \\
\hline 34 & 3.692 & 53 & 0 & & 5 & & & & 5 & 0 & 5 & & 03 & 104 \\
\hline & 04 & 4 & 00 & & 0 & 0 & 5 & 6 & 6 & 0 & 26 & & E-04 & .0169 \\
\hline & 03 & & $.000 E+00$ & 0.0 & $E-07$ & 0.0 & $E-03$ & 0. & -05 & 7 & 34 & 2 & $98 E-03$ & .5726 \\
\hline & & & eet & $\mathbf{t}$ & cet & $e 8$ & tee & es & see & 4 & aeㄴ & $\varepsilon$ & et & 4 \\
\hline tral & 3 & & $000 E+00$ & 0.0000 & $397 E-06$ & 0.0006 & $18-03$ & 0.1878 & $.456 E-05$ & 0.0039 & L. $773 E-04$ & 0.0156 & .02 & 300 \\
\hline
\end{tabular}


RESRAD. Version 5.621 T Limit $=0.5$ year $03 / 07 / 97$ 08:54 Page 25 Sumary : Radioactivity associated surface contaminated concrete and drainilines File : A: IRUBBLE.DAT

Dose/Source Ratios Summed Over All Pathways

Parent and Progeny principal Radionuclide Contributions Indicated

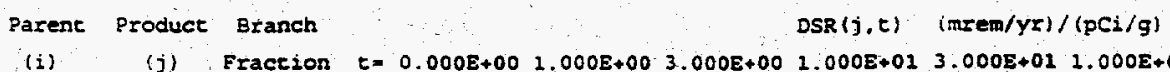

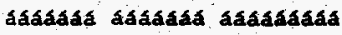
$A m-241: A m-241 \quad 1.000 E+00$ An-241. Np-237 $1.000 E+00$ Am-241 $\quad 0.233 \quad 2.0 .00 E+00$ An-241 Th-229 1.000B 400 $A n-241$ ODSR (j)

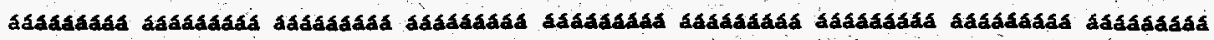

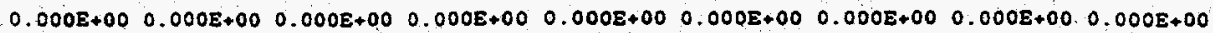
$\begin{array}{lllllllllll}0.000 E+00 & 0.000 E+00 & 0.000 E+00 & 0.000 E+00 & 0.000 E+00 & 0.000 E+00 & 0.000 E+00 & 7.223 E-03 & 2.516 E-08\end{array}$ $\begin{array}{llllllll}0.000 E+00 & 0.000 E+00 & 0.000 E+00 & 0.000 E+00 & 0.000 E+00 & 0.000 E+00 \quad 0.000 E+00 \quad 6.543 E-08 & 1.389 E-07\end{array}$ $\begin{array}{llllllllllll}0.000 E+00 & 0.000 E+00 & 0.000 E+00 & 0.000 E+00 & 0.000 E+00 & 0.000 E+00 & 0.000 E+00 & 1.316 E-10 & 3.217 E-08\end{array}$ $0.000 E+00 \quad 0.000 E+00 \quad 0.000 E+00 \quad 0.000 E+00 \quad 0.000 E+00 \quad 0.000 E+00 \quad 0.000 E+00 \quad 7.223 E-03 \quad 1.952 E-07$

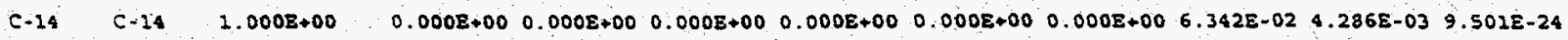

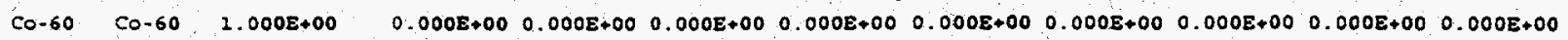

Cs-137. Cs $-137 \quad 1.000 E \bullet 00$

$0,000 E+00 \quad 0.000 E+00 \quad 0.000 E+00 \quad 0.000 E+00 \quad 0.000 E+00 \quad 0.000 E+00 \quad 0.000 E+00 \quad 0.000 E+00 \quad 0.000 E+00$

H-3 H-3 $\quad 1.000 E+00 \quad 0.000 E+00 \quad 0.000 E+00 \quad 0.000 E+00 \quad 0.000 E+00 \quad 0.000 E+00 \quad 0.000 E+001.050 E-10 \quad 1.057 E-30 \quad 0.000 E+00$

$N_{2}-59 \quad N_{2}-59 \quad 1.000 E+00 \quad 0.000 E+00 \quad 0.000 E+00 \quad 0.000 E+00 \quad 0.000 E+00 \quad 0.000 E+00 \quad 0.000 E+00 \quad 0.000 E+00 \quad 0.000 E+000.000 E+00$

$N_{2}-63 \quad N_{2}-63 \quad 1.000 \varepsilon+00 \quad 0.000 \varepsilon+00 \quad 0.000 \varepsilon+00 \quad 0.000 \varepsilon+00 \quad 0.000 E+00 \quad 0.000 E+00 \quad 0.000 \varepsilon+00 \quad 0.000 E+00 \quad 0.000 \varepsilon+00 \quad 0.000 \varepsilon+00$

PU-238 PU-238 1.000E+00 $0.000 E+00 \quad 0.000 E+00 \quad 0.000 E+00 \quad 0.000 E+00 \quad 0.000 E+00 \quad 0.000 E+00 \quad 0.000 E+00 \quad 0.000 E+00 \quad 0.000 E+00$ Pu-238 U.234:1.000E+00

Pu-238 Th-230 $1.0008+00$.

Pu-238 Ra-226 $1.000 E+00$

Pu-238. Pb-210 1.000E+00

$P(1-238$ ODSR (j)

Pu-239 Pu-239 $1.000 E+00$

Pus-239, J-235 $1.000 \mathrm{E}+00$

Pu-239 Pa-231 $1.000 \mathrm{E}+00$

Pu-239 AC-22? $2.000 E+00$

Pu-239 ODSR (j)

$5 z-90 \quad 5=-90 \quad 1.000 E+00$

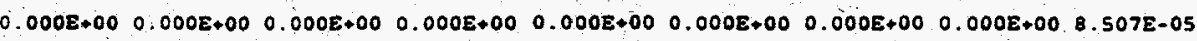
$0.000 E+00 \quad 0.000 E+00 \quad 0.000 E+00 \quad 0.000 E+00 \quad 0.000 E+00 \quad 0.000 E+00 \quad 0.000 E+00 \quad 0.000 E+00 \quad 2.198 E-07$

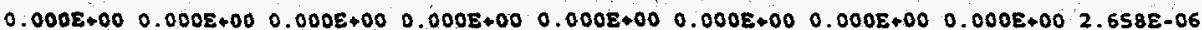
$0.000 E+00 \quad 0.000 E+00 \quad 0.000 E+00 \quad 0.000 E+00 \quad 0.000 E+00 \quad 0.000 E+00 \quad 0.000 E+00 \quad 0.000 E+00 \quad 1.160 E-04$ $0.000 E+00 \quad 0.000 E+00 \quad 0.000 E+00 \quad 0.000 E+00 \quad 0.000 E+00 \quad 0.000 E+00 \quad 0.000 E+000.000 E+00 \quad 2.039 E-04$

$\begin{array}{lllllllll}0.000 E+00 & 0.000 E+00 & 0.000 E+00 & 0.000 E+00 & 0.000 E+00 & 0.000 E+00 & 0.000 E+00 & 0.0008+00 & 0.000 E+00\end{array}$

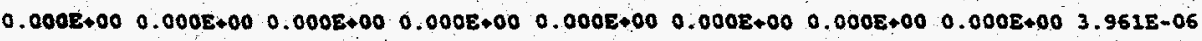

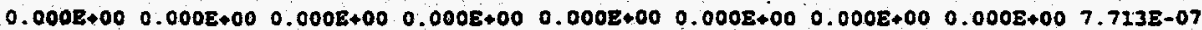

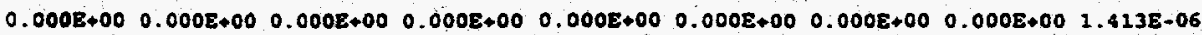
$0.000 E+00 \quad 0.000 E+00 \quad 0.000 E+00 \quad 0.000 E+00 \quad 0.000 E+00 \quad 0.000 E+00 \quad 0.000 E+00 \quad 0.000 E+00 \quad 6.145 E-06$

$0.000 \varepsilon+00 \quad 0.000 \varepsilon+00 \quad 0.000 \varepsilon+00 \quad 0.000 E+00 \quad 0.000 \varepsilon+00 \quad 0.000 \varepsilon+00 \quad 0.000 \varepsilon+00 \quad 0.000 \varepsilon+00 \quad 0.000 \varepsilon+00$

J-234 U-234 $\quad 2.000 E+00$

U.234 Th-230 $1.000 \mathrm{~T}+00$

U.234 Ra-226 $1.000 E+00$

U-234 Pb-210 I.000E+00

U-234 ODSR $(j)$

$u-235 \quad U-235 \quad 1.000 E+00$
0.235

U-235 Pa-231 $1.0008 * 00$

U-235 AC-227 $\quad 1.0008+00$

U-235 ODSR(j)

$\begin{array}{lllllllll}0.000 E+00 & 0.000 E+00 & 0.000 E+00 & 0.000 E+00 & 0.000 E+00 & 0.000 E+00 & 0.000 E+00 & 0.000 E+00 & 2.308 E-01\end{array}$

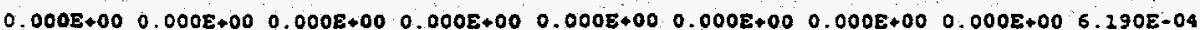

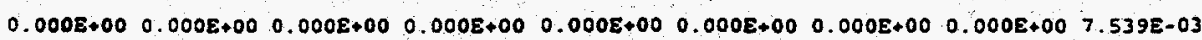
$0.000 E+00 \quad 0.000 E+00 \quad 0.000 E+00 \quad 0.000 E+00 \quad 0.000 E+00 \quad 0.000 E+00 \quad 0.000 E+00 \quad 0.000 E+00 \quad 3.290 E-01$ $0.000 E+00 \quad 0.000 E+00 \quad 0.000 E+00 \quad 0.000 E+00 \quad 0.000 E+00 \quad 0.000 E+00 \quad 0.0008+00 \quad 0.000 E+00 \quad 5.680 E-01$

$\begin{array}{llllllllll}0.000 E+00 & 0.000 E+00 & 0.000 E+00 & 0.000 E+00 & 0.000 E+00 & 0.000 E+00 & 0.000 E+00 & 0.000 E+00 & 2.240 E-02\end{array}$ $\begin{array}{ccccccccc}0.000 E+00 & 0.000 E+00 & 0.0008+00 & 0.000 E+00 & 0.000 E+00 & 0.000 E+00 & 0.0008+00 & 0.000 E+00 & 1.645 E-01\end{array}$ $0.000 E+00 \quad 0.000 E+00 \quad 0.000 E+00 \quad 0.000 E+00 \quad 0.000 E+00 \quad 0.000 E+00 \quad 0.0008+000.000 E+00 \quad 3.029 E-01$ $0.000 E+00 \quad 0.000 E+00 \quad 0.000 E+00 \quad 0.000 E+00 \quad 0.000 E+00 \quad 0.000 E+00 \quad 0.000 E+00 \quad 0.000 E+00 \quad 6.904 E-01$
0 
RESRAD, Version 5.621 I Limit $=0.5$ year $33 / 07 / 97$ 08.54 page 26

Sumary : Radioactivity associated surface concammated concrete and drainilines

File $\quad A$ IRUBBLE.DAT

Dose/Source Ratios Summed Over All Pachways

parent and Progeny Principal Radionuclide Contriburions Indicated

parent Produce Branch

$\operatorname{LSR}(j, t) \quad(\mathrm{mrem} / \mathrm{yr}) /(\mathrm{pci} / \mathrm{g})$

(i) (j) Fraction

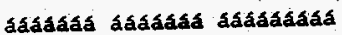

U-238 U-23a $\quad 1.000 \mathrm{E}+00$

U.238 U-234 $\quad 2.000 E+00$

U-238 $=2-230 \cdot 1.000 E-$

$\mathrm{J}-238$ Ra-226 $1.000 \mathrm{E}$.

U.238 Pb-210 1.000E+a:

U-238: CDSR (3)

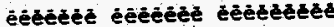

2.000E+01 3.000E+02 $2.000 E+02 \quad 3.000 E+02 \quad 1.000 E+03 \quad 1.000 E+04$

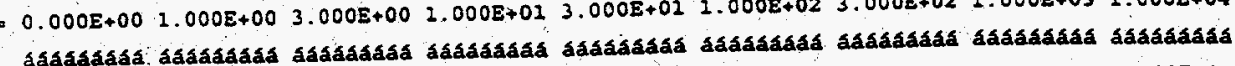

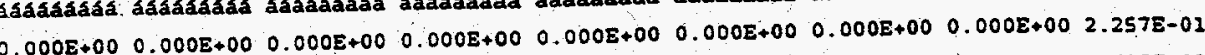

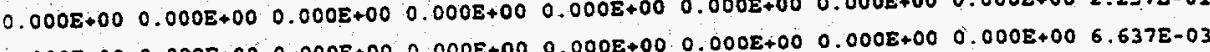
$0000+0000+000.0008+000.0008+001,178 E-05$ $J .000 E+00 \quad 0.000 E+00 \quad 0.000 E+00 \quad 0.000 E+00 \quad 0.000 E+00 \quad 0.000 E+00 \quad 0$ $0.000 E+00.000 E+00 \quad 0.000 E+00 \quad 0.000 E+00 \quad 0.000 E+00 \quad 0.000 E+00 \quad 0.000 E+00 \quad 0.000 E+00 \quad 1.670 E-04$

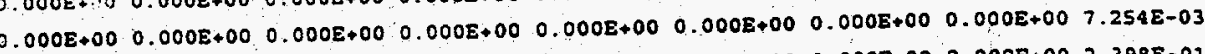

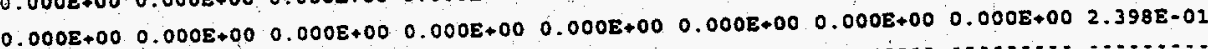

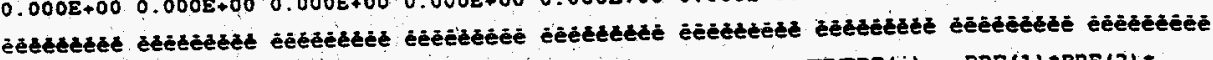
Branch Fraction is the cumulative factor for the $j$ ' th principal radionuclide
The DSR includes contributions from associated (half-life $\mu 0.5$ yr) daughters.

Single Radionuclide Soil Guidelines $G(i, t)$ in $\mathrm{pCi} / \mathrm{g}$ Basic Radiacion Dose imit = 15 mrem/yx

\begin{tabular}{|c|c|c|c|c|c|c|c|c|c|}
\hline ááá & Iadadáá & jabaakada & áasaajá & çsaadaaá & suááááá & asaạakaaa & adaasaasáa & cáabaaja & adáasaaáa \\
\hline-241 & $\because 430 E+12$ & $-3.430 E+12$ & $-3.430 E+12$ & $-3: 430 E+12$ & $-3.430 E+12$ & $-3.430 E+12$ & $-3.430 E+12$ & $2.077 E+03$ & $7.684 E+07$ \\
\hline$C-14$ & $-4.454 \varepsilon+12$ & $-4.454 E+12$ & $-4.454 E+12$ & $.4 .454 E+12$ & $4.454 E+12$ & $-4.454 E+12$ & $2.365 E+02$ & $3.5008+03$ & $-4.454 E+12$ \\
\hline Co-60 & $-1.131 E+15$ & $-1.131 E+15$ & $-1.131 E+15$ & $-2,232 E+15$ & $-1+132 E+15$ & $-1.131 E+15$ & $-1.233 E+25$ & $-1,131 E+15$ & $=1.131 E+15$ \\
\hline$(5-137$ & $-8.7028+13$ & $-8.7025+23$ & $-8.701 E+13$ & $-8.7028+13$ & $-8.702 E+13$ & $-8.701 E+13$ & $-8.702 \mathrm{E}+1.3$ & $-8.701 E+13$ & $-8.701 E+13$ \\
\hline$H-3$ & $-9.594 E+15$ & $-9.594 E+15$ & $-9.594 E+15$ & $-9.594 E+15$ & $-9.594 E+15$ & $\cdot 9.594 E+15$ & $1.428 E+1 I$ & $.9 .594 E+15$ & $\because 9.594 E+15$ \\
\hline$N_{i}-59$ & $.085 E+10$ & $-8.085 E+10$ & $-8.085 \mathrm{E}$ & $-8.085 E+10$ & $-8.085 E+10$ & -8.085 & $-8.085 E+10$ & $-8.085 E+10$ & $-8.085 E+20$ \\
\hline$N 2-63$ & $-5.9168+13$ & $+5.916 \mathrm{E}+13$ & $-5.926 E+13$ & $16 E+13$ & $-5.916 E+13$ & $-5.916 E+13$ & $\cdot 5.916 \mathrm{E}+13$ & $+5.916 E+13$ & $E+13$ \\
\hline Pu-238 & $1.7112+13$ & $12.711 E+13$ & $-1.712 \mathrm{E}+23$ & $-1.7228+23$ & $-1.72128+23$ & $-1.712 E+13$ & $712 E+13$ & 1.. $721 E+13$ & 7.3551 \\
\hline Pu-239 & $* 6.212 E+10$ & $-6.212 E+10$ & $-6.212 E+10$ & .6 .21 & $-6,212 \mathrm{E}+10$ & $-6.212 \mathrm{E}+10$ & $25+10$ & $-6.212 E+20^{\prime}$ & $2.441 E+06$ \\
\hline $5 r-90$ & $1.365 E+14$ & $-1.365 E+14$ & $-1.3658+14$ & $=2,365 E+24$ & $-1.365 E+14$ & $=1.365 \mathrm{E}+14$ & $65 E+14$ & $1.365 E+14$ & $+1.365 E+14$ \\
\hline$u-2$ & $.245 E+09$ & $-6.245 E+09$ & $-6.245 E+09$ & $-6.245 E+09$ & $-6.245 E+09$ & $-6.245 E+09$ & $-6.245 E+09$ & $-6.245 E+09$ & $2.641 E+01$ \\
\hline$u-235$ & $2.160 E+06$ & $2.160 E+06$ & $=.1608+06$ & $-2.1608+06$ & $-2.2608+06$ & $-2.160 E+06$ & $-2.160 E+06$ & $=2.160 \bar{E}+06$ & $2.173 E+01$ \\
\hline 238 & $-3.360 z+05$ & $605+05$ & $-3: 3608+05$ & $-3.360 \mathrm{E}+05$ & $3.3605+05$ & $3.3608+05$ & $-3.360 E+05$ & $-3.3608+05$ & $6.256 \mathrm{E} * 0$ \\
\hline & teted & édectetet & exteteted & ècestakde & éfected & esetweted & etetereted & ederetede & ēètetęetd \\
\hline
\end{tabular}

$$
\text { - Ae specific activity limic }
$$


RESRAD, Version 5.621 T Limit $=0.5$ year $\quad 03 / 07 / 97$ 08:54 Page 27 Sumary : Radioactivity associated surface concaminated concrete and drainilines

File : A: \RUBBLE.DAT

Summed Dose/Source Ratios DSR $(i, t)$ in $(m x e m / y r) /(p C i / g)$

and Single Radionuclide Soil Guidelines $\mathrm{G}(\mathrm{i}, \mathrm{t})$ in $\mathrm{pCi} / \mathrm{g}$

at tmin - time of minimum single radionuclide soil guideline

and at emax $=$ time of maximum cotal dose $=4965$ p 5 years

\begin{tabular}{|c|c|c|c|c|c|c|}
\hline $\begin{array}{l}\text { Nuclide } \\
\text { (i) }\end{array}$ & $\begin{array}{l}\text { Initial } \\
\mathrm{pCi} / \mathrm{g}\end{array}$ & $\begin{array}{l}\text { cmin } \\
\text { (years) }\end{array}$ & $\operatorname{DSR}(i, \operatorname{cmin})$ & $\begin{array}{l}G(i, t m i n) \\
(p C i / g)\end{array}$ & DSR 12 & $\begin{array}{c}G(2, t \max ) \\
(p C i / g)\end{array}$ \\
\hline aáaลâá & aasasaááa & 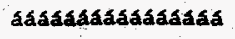 & aáasasaá & asadasaad & 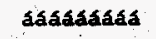 & 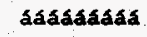 \\
\hline$A m-241$ & $2.0708-05$ & $=1243$ p 1 & $8,397 E-03$ & $1.786 \varepsilon+03$ & $7.564 \mathrm{E}-05$ & $1.983 \mathrm{E}+05$ \\
\hline$c-14$ & $1.200 \mathrm{E}-01$ & 327.5 p 0.3 & $579 E-01$ & $9.500 E+01$ & $3 E-12$ & 4.4 \\
\hline $\operatorname{co-} 60$ & $1.770 E-02$. & 0.0000 p 0.0000 & $0.000 E+00$ & $-1.1312+25$ & $0.000 E+00$ & $-1.131 E+15$ \\
\hline $\operatorname{cs}-137$ & $1.620 \mathrm{E}+00$ & 0.0000 p 0.0000 & $0.000 \mathrm{E}+00$ & $-8.701 E+13$ & $0.0008+00$ & $-8: 701 E+13$ \\
\hline $4-3$ & 4.490 & 176.5 p 0.2 & $3.5498-07$ & $4.227 E+07$ & $0.0008+00$ & $-9.594 E+15$ \\
\hline $\mathrm{Ni}-59$ & 2.8 & 0.0000 p 0.0000 & $0.000 E+00$ & $-8.085 E+10$ & $0.000 \mathrm{E}+00$ & $-8.085 E+10$ \\
\hline Ni -63 & 2.3 & $0.0000 p 0.0000$ & io & $8+13$ & OOOE+00: & $-5: 926 \mathrm{E}+13$ \\
\hline Pu-238 & $1.9708-06$ & 5156 P 5 & $3.9445-04$ & $3.804 E+04$ & $3.425 E-04$ & .04 \\
\hline Pu-239 & $1.800 E-04$ & $1.000 E+04$ & $6: 145 E-06$ & $2.442 E+06$ & $3.779 E-07$ & $3.969 E+07$ \\
\hline Sr-90 & $1.0908-01$ & 1360 b 1 & $7.460 \mathrm{E}-15$ & $-1.3658+14$ & $0.000 \mathrm{E}+00$ & $-1.365 E+14$ \\
\hline $0-234$ & 6. 200E-03 & 4964 b 5 & $1.151 E+00$ & $1.303 E+01$ & $1,151 E+00$ & $1.304 \mathrm{E}+01$ \\
\hline$U-235$ & $2.780 E-04$ & 4964 ? & $1.236 \mathrm{E}+00$ & $1.320 E+01$ & $2.136 E+00$ & 1. $320 E+01$ \\
\hline$v-238$ & $2.710 E-02$ & 4962 P 5 & $1.125 E+00$ & i. $334 E+01$ & $1.1238+00$ & $1.335 E+01$ \\
\hline ėętedé & éezatejét. & 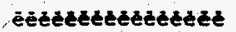 & éextedaxy & échetces. & extedtete & ééèlteted \\
\hline
\end{tabular}

- Ac speczfic accivity limit 
RESRAD. Version 5.627. I LimiL $=0.5$ year $\quad 03 / 07 / 9708: 54$ Page 28 Sumary : Radioactivicy associated surface concaninaced concrece and draznlines File $\quad$ A :IRUBBLE.DAT

Individual Nuclide Dose Sumed Over All Pathways

parent Nuclide and Branch Fraction indicaced

\begin{tabular}{|c|c|c|}
\hline Nuclide & Parent & $\operatorname{ERF}(i)$ \\
\hline (j) & $\therefore 1$ & \\
\hline ááááa & adá & adaabaáa \\
\hline$A m-241$ & $\because \quad 41$ & $1.000 \mathrm{E}+00$ \\
\hline$N D-237$ & $\sin -241$ & $1.000 \mathrm{E}+00$ \\
\hline $0-233$ & An-241 & $1.000 \mathrm{E}+00$ \\
\hline Th-229 & $2 m-241$ & $1.0008+00$ \\
\hline$c-14$ & $c-14$ & $1.000 E+00$ \\
\hline $\operatorname{co-} 60$ & $C 0-60$ & $1.000 E+00$ \\
\hline cs -137 & $\operatorname{cs}-137$ & $1.000 E+00$ \\
\hline $\mathrm{H}-3$ & $: a-3$ & $1.000 \varepsilon+00$ \\
\hline$N_{1}=59$ & $\therefore:-59$ & $1000 E \cdot 00$ \\
\hline$N_{2} \rightarrow 3$ & $\therefore 2-63$ & $1.000 \varepsilon+00$ \\
\hline Pu-2: = & $P u-238$ & $1.000 E+00$ \\
\hline$U .234$ & $\therefore 4-238$ & 1. $.000 \varepsilon+00$ \\
\hline$U-234$ & $U-234$ & $1.0008+00$ \\
\hline$u-234$ & $\mathrm{U}-\mathbf{2 3 8}$ & $1.0008+00$ \\
\hline 0.234 & ODOSE (j) & j1: \\
\hline$\because$ & . & \\
\hline $\operatorname{Th}-230$ & Fu -238 & $1.000 \mathrm{E}+00$ \\
\hline $\operatorname{Th}-230$ & $0-234$ & $1.000 E+00$ \\
\hline$m-230$ & $0-238$ & $1.000 E+00$ \\
\hline$T h=230$ & ODOSE $(j)$ & j!: \\
\hline Ra-226 & $P u-238$ & $1.000 E+00$ \\
\hline$R a-226$ & $\because 234$ & $1.000 E+00$ \\
\hline $\operatorname{Ra}-226$ & $y-=38$ & $1.000 E+00$ \\
\hline $8 a \times 226$ & ODOSE $(j)$ & j): \\
\hline$P b-210$ & $P u-23 a$ & $2.0008+00$ \\
\hline$P b-210$ & 0.234 & $1.000 E+0.0$ \\
\hline$P b-210$ & $0-238$ & $1.0008 \cdot 00$ \\
\hline $\mathrm{Pb}-210$ & CDOSE $(3)$ & 3: \\
\hline$P 4=239$. & $P r-239$ & $1.000 E+00$ \\
\hline$U-235$ & $\mathrm{Pu}-239$ & $1.000 E+00$ \\
\hline$U .235$ & 0.235 & $1.000 E+00$ \\
\hline U-235. & DDOSE (j) & \\
\hline
\end{tabular}

DOSE (j.t). mrem/yr

$\tau=0.000 E+00 \quad 1.00 .0 E+00 \quad 3.000 E+00 \quad 1.000 E+02 \quad 3.000 E+02 \quad 1.000 E+02 \quad 3.000 E+02 \quad 1.000 E+03 \quad 1.000 E+04$

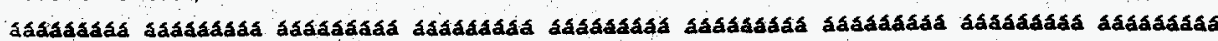
$\begin{array}{lllllllll}0.000 E+00 & 0.000 E+00 & 0.000 E+00 & 0.000 E+00 & 0.000 E+00 & 0.000 E+00 & 0.000 E+00 \quad 0.000 E+00 \quad 0.000 E+00\end{array}$ $0.000 E+00 \quad 0.000 E+00 \quad 0.000 E+00 \quad 0.000 E+00 \quad 0.000 E+00 \quad 0.000 E+00 \quad 0.0008+00 \quad 1.495 E-07 \quad 5.208 E-13$ $\begin{array}{lllllllll}0.000 E+00 & 0.000 E+00 & 0.000 E+00 & 0.000 E+00 & 0.000 E+00 & 0.000 E+00 & 0.000 E+00 \quad 1.354 E-12 & 2.875 E-12\end{array}$ $0.000 E+00 \quad 0.000 E+00 \quad 0.000 E+00 \quad 0.000 E+00 \quad 0.000 E+00 \quad 0.000 E+00 \quad 0.000 E+00 \quad 2.723 E-15 \quad 6.452 E-13$ $0.000 E+00 \quad 0.000 \varepsilon+00 \quad 0.000 \Sigma+00 \quad 0.000 E+00 \quad 0.000 E+00 \quad 0.000 E+00 \quad 7.612 E-03 \quad 5.143 E-04 \quad 1.140 E-24$ $0.000 E+00 \quad 0.000 E+00 \quad 0.000 E+00 \quad 0.000 E+00 \quad 0.000 E+00 \quad 0.000 E+00 \quad 0.000 E+00 \quad 0.000 E+00 \quad 0.000 E+00$ $0.000 E+00 \quad 0.000 E+00 \quad 0.000 E+00 \quad 0.000 E+00 \quad 0.000 \varepsilon+00 \quad 0.000 E+00 \quad 0.000 E+00 \quad 0.000 E+00 \quad 0.000 E+00$ $0.000 E+00 \quad 0.000 E+00 \quad 0.000 E+00 \quad 0.000 E+00 \quad 0.000 E+00 \quad 0.000 E+00 \quad 4.716 E-12 \quad 0.000 E+00 \quad 0.000 E+00$ $\therefore .000 E+00 \quad 0.000 E+00 \quad 0,000 \varepsilon+00 \quad 0.000 E+00 \quad 0.000 E+00 \quad 0.000 E+00 \quad 0.000 E+00 \quad 0.000 E+00 \quad 0.000 E+00$ $0.000 E+00 \quad 0.000 E+00 \quad 0.000 E+00 \quad 0.000 E+00 \quad 0.000 E+00 \quad 0.000 E+00 \quad 0.000 E+00 \quad 0.000 E+00 \quad 0.000 E+00$

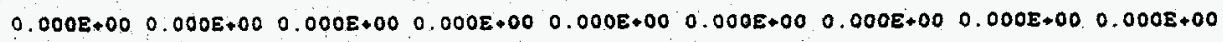
$0.000 E+00 \quad 0.000 E+00 \quad 0.000 E+00 \quad 0.000 E+00 \quad 0.000 E+00 \quad 0.000 E+00 \quad 0.000 E+00 \quad 0.000 E+00 \quad 1.676 E-10$ $0.000 E+00 \quad 0.000 E+00 \quad 0.000 E+00 \quad 0.000 E+00 \quad 0.000 E+00 \quad 0.000 E+00 \quad 0.000 E+00 \quad 0.000 E+00 \quad 2.893 E-03$ $0.000 E+00 \quad 0.000 E+00 \quad 0.000 E+00 \quad 0.000 E+00 \quad 0.000 E+00 \quad 0.000 E+00 \quad 0.000 E+00 \quad 0.000 E+00 \quad 1.799 E-04$ $0.000 E+00 \quad 0.000 E+00 \quad 0.000 E+00 \quad 0.000 E+00 \quad 0,000 E+00 \quad 0.000 E+00 \quad 0.000 E+00 \quad 0.000 E+00 \quad 2.073 E-03$

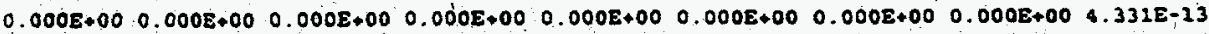
$0.000 E+00 \quad 0.000 E+00 \quad 0.000 E+00 \quad 0.000 E+00 \quad 0.000 E+00 \quad 0.000 E+00 \quad 0.000 E+00 \quad 0.000 E+00 \quad 5.076 E-06$ $0.000 E+00 \quad 0.000 E+00 \quad 0.000 E+00 \quad 0.000 E+00 \quad 0.000 E+00 \quad 0.000 E+00 \quad 0.000 E+00 \quad 0.000 E+00 \quad 3.293 E-07$ $0.000 E+00 \quad 0.000 E+00 \quad 0.000 E+00 \quad 0.000 E+00 \quad 0.000 E+00 \quad 0.000 E+00 \quad 0.000 E+00 \quad 0.000 E+00 \quad 5.395 E-06$

$0.000 E+00 \quad 0.000 \varepsilon+00 \quad 0.000 E+00 \quad 0.000 E+00 \quad 0.000 E+00,0.000 E+00 \quad 0.0008+00 \quad 0.000 E+00 \quad 5.236 E-12$ $0.000 E+00 \quad 0.000 E+00,0.000 E+00 \quad 0.000 E+00 \quad 0.000 E+00 \quad 0.000 E+00 \quad 0.000 E+00 \quad 0.000 E+00 \quad 6.282 E-05$ $0.000 E+00 \quad 0.000 E+00 \quad 0.000 E+00 \quad 0.000 E+00 \quad 0.000 E+00 \quad 0.000 E+00 \quad 0.000 E+00 \quad 0.000 E+00 \quad 4.525 E-06$ $0.000 E+00 \quad 0.000 E+00 \quad 0.0008+00 \quad 0.000 E+00 \quad 0.000 E+00 \quad 0.000 E+00 \quad 0.000 E+00 \quad 0.000 E+00 \quad 6.635 E-05$

$0.000 E+00 \quad 0.000 E+00 \quad 0.000 E+00 \quad 0.000 E+00 \quad 0.000 E+00 \quad 0.000 E+00 \quad 0.000 E+00 \quad 0.000 E+00 \quad 2.285 E-10$ $0.000 E+00 \quad 0.000 E+00 \quad 0.000 E+00 \quad 0.000 E+00 \quad 0.000 E+00 \quad 0.000 E+00 \quad 0.0008+00 \quad 0.000 E+00 \quad 2.698 E-03$ $\begin{array}{lllllllll}0.000 E+00 & 0.000 E+00 & 0.000 E+00 & 0.000 E+00 & 0.000 E+00 & 0.000 E+00 & 0.000 E+00 & 0.000 E+00 & 3.966 E-04\end{array}$ $0.000 E+00 \quad 0.000 E+00 \quad 0.000 E+00 \quad 0.000 E+00 \quad 0.000 E+00 \quad 0.000 E+00 \quad 0.000 E+00 \quad 0.000 E+00 \quad 2.895 E-03$

$0.000 E+00 \quad 0.000 E+00 \quad 0.000 E+00 \quad 0.000 E+00 \quad 0.000 E+00 \quad 0.000 E+00 \quad 0.000 E+00 \quad 0.000 E+00 \quad 0.000 E+00$

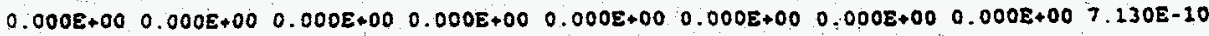
$\begin{array}{lllllllll}0.000 E+00 & 0.000 E+00 & 0.000 E+00 & 0.000 E+00 & 0.000 E+00 & 0.000 E+00 & 0.000 E+00 & 0.000 E+00 \quad 6.228 E-05\end{array}$

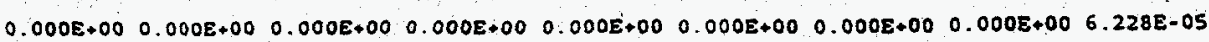




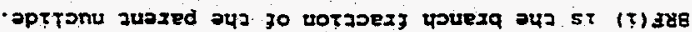

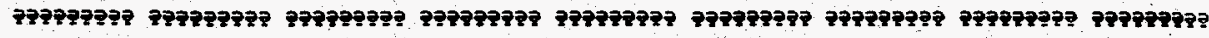
$\varepsilon 0-3<T T=00+3000 \cdot 000+3000 \cdot 000+3000 \cdot 0 \quad 00+3000 \cdot 000+3000 \div 000+3000-0,00+3000 \cdot 000+3000 \cdot 0$ $00+3000 \div 00+3000 \% 00+3000 \div 000+3000 \div 000+3000 \% 000+3000 \div 00+3000 \cdot 000+3000 \cdot 000+3000-0$ $50-3 \varepsilon 6 \varepsilon \cdot 800+3000 \cdot 0 \quad 00+3000 \div 000+3000 \div 000+3000 \div 000+3000 \cdot 000+3000 \cdot 000+3000 \cdot 000+3000 \cdot 0$

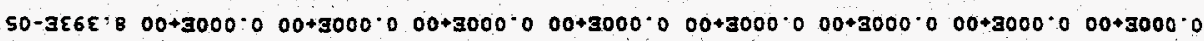
$0 z-3 \varepsilon \$ 5 \cdot z 00+3000 \cdot 0 \quad 00+3000 \cdot 0 \quad 00+3000 \div 0 \quad 00+3000 \div 0 \quad 00+3000 \cdot 000+3000 \div 0 \quad 00+3000 \div 000+3000-0$

SO-3ELS $00+3000 \div 00+3000 \div 000+3000 \times 000+3000 \cdot 0 \quad 00+3000 \div 000+3000 \% 00+3000 \% 00+3000 \div 0$ SO-3ELS $00+3000 \cdot 0 \quad 00+3000 \cdot 0 \quad 00+3000 \cdot 0 \quad 00+3000 \cdot 0 \quad 00+3000 \cdot 0 \quad 00+3000 \cdot 000+3000 \cdot 0 \quad 00+3000 \cdot 0$ $0 \tau-388 \varepsilon \cdot 00+3000 \cdot 0 \quad 00+3000 \cdot 0 \quad 00+3000 \cdot 0 \quad 00+3000 \cdot 0 \quad 00+3000 \cdot 0 \quad 00+3000 \cdot 000+3000 \cdot 0 \quad 00+3000 \cdot 0$

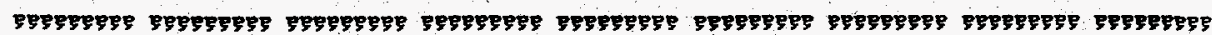
$30+3000^{\circ} \tau \varepsilon 0+3000 \cdot \tau 0+3000^{\circ} \varepsilon z 0+3000 \cdot \tau \tau 0+3000^{\circ} \varepsilon \tau 0+3000 \cdot \tau 00+3000 \cdot \varepsilon 00+3000 \cdot \tau 00+3000 \cdot 0=2$

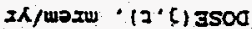

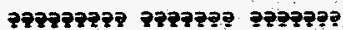
$00+3000-T$ BEZ- $\Omega$ BEZ-O $00+3000 \cdot 1 \quad 06-25 \quad 06-25$

$=(5)$ ssoco tzz-oy $00+3000 \cdot T \quad 5 \varepsilon z-n \quad L z Z-2 Y$ $00+3000 \cdot$ I $6 \varepsilon z-$ nd $\quad 2 z z-58$

: (c) ssogo tहz-Ed $00+3000 \cdot I$ sez-n zEz-6z

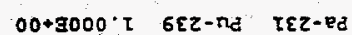

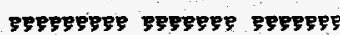
(?) (द)

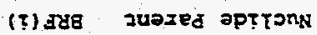

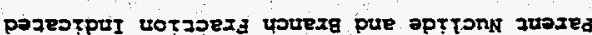

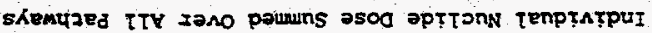

$$
\begin{aligned}
& 62 \text { abed } 55: 80 \quad \text { L6/LO/عO }
\end{aligned}
$$

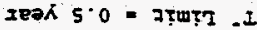


RESRAD, version $5.621 \quad T^{-}$imit $=0.5$ year $03 / 07 / 9708: 54$ Eage 30 Sumary : Radioactivity assoclated surface contaminated concrete and drainines File A: $\quad$ RUBELE.DAT

Individual Nuclide soil Concentration Parent Nuclide and Branch Fract 20 . Indicated

Nuclide Parent ERF(i)

(j) (i)

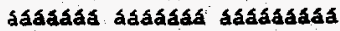

Am-241 Am-241, $1.000 \mathrm{E}+00$

$N p-237 \quad A m-241 \quad 1.0008+00$

ij-233 An-242, $1,0008+00$

$\operatorname{Th}-229$ Am-242 $1.000 E+00$

$C-14 \quad C .14 \quad 1.200 E+00$

Co-60 Co-60 1.000E+00

Cs-137, Cs-137 $1.0008+00$

$\mathrm{H}=3$ ii-3 $\quad 000 \mathrm{E}+00$

$N_{1}-59+N_{2}-59 \cdot 1.000 E+00$

$N i-63 \quad N_{1}-63: 1.0008+00$

$P_{4}=238 \quad P u-238 \cdot 1,0008+00$

U-234 Pu-238 $1.000 E+00$

U-234 U-234 $1.000 E+00$

U-234 U-238 $1.000 E+00$

$U-234$ os $(j)$

$\mathrm{m}-230$ Pu-238 2.000E+00

$\mathrm{m}-230 \quad \mathrm{v}-234 \quad 1.000 \mathrm{E}+00$

Th-230 U-233 1.000E +00

$\operatorname{Th}-230$ os $(j)$ :

Ra-226. Pu-238 $1.000 E+00$

$R a-226$ U-234 $\quad 1.000 E+00$

$R a-226 \quad U-238 \quad 1.000 E+00$

$R a-226 \Delta S(j)$

Pb-210 Pu-238 $1.000 E * 00$

Pb-210 U-234 1.000E+00

$\mathrm{Pb}-210 \quad 0-238 \quad 1.000 \mathrm{E}+00$

pb-210 os $(j)$ :

Pu-239 Pu-239 $1.000 E+00$

U-235 $\quad P u-239 \quad 1.000 E+00$

U-235 U-235 $\quad 1.0008+00$

U.23s $O S(j)$ $s\{j, t), \mathrm{pCi} / \mathrm{g}$

$=0.0008+001.000 E+00 \quad 3.000 E+00 \quad 2.000 E+01 \quad 3.0008+01 \quad 1.000 E+02 \quad 3.000 E+021.000 E+03 \quad 1.000 E+04$

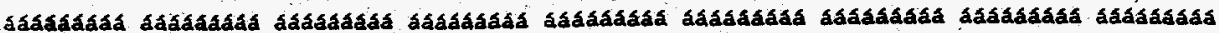
2.070E-05 2.067E-05 2.050E-05 2.037E-05 1.972E-05 1.762E-05 1.277E-05 4.139E-06 2.126E-12

$0.000 E+00 \quad 6.692 E-12 \quad 2.000 E-11 \quad 6.580 E-21 \quad 2.901 E-10 \quad 5.555 E-10 \quad 1.145 E-09 \quad 1.033 E-09$ 1.250E-15 $0.000 E+00 \quad 2.464 E-17 \quad 1.314 E-16 \quad 2.446 E-15 \quad 2.267 E-14 \quad 1.280 E-13 \quad 8.829 E-13 \quad 4.056 E-12 \quad 5.041 E-13$ $0.000 E+00 \quad 4.620 E-22 \quad 1.242 E-20 \quad 4.567 E-19 \quad 1.208 E-17 \quad 4.160 E-16 \quad 9.165 E-15 \quad 2.744 E-13 \quad 1.289 E-12$ 1.200E-01 1.194E-01 1.181E-01 2.138E-01 1.024E-0. 7.074E-02 2.459E-02 6.085E-04 1.349E-24 $1.770 E-02 \quad 1.550 E-02 \quad 1.189 E-02+4.700 E-03 \cdot 3.314 E-04 \quad 3.086 E-08 \quad 9.382 E-20 \quad 0.0008+00 \quad 0.000 E+00$ $1.620 E+00 \quad 1.583 E+00 \quad 1.521 E+00 \quad 1.285 E+00$ B. $092 E-01 \quad 1.602 E-01 \quad 1.566 E-03 \quad 1.447 E-10 \quad 0.000 E+00$ $4.490 E-01 \quad 4.204 E-02 \quad 3.686 E-012.326 E-01 \quad 6.241 E-02 \quad 6.244 E-04 \quad 1.20 B E-09 \quad 1.215 E-29 \quad 0.000 E+00$ $2.390 E-05 \quad 2.490 E-052.890 E-052: 389 E-05 \quad 2.887 E-05 \quad 2.879 E-05$ 2.858E-05 2.785E-05 $1.997 E-05$ $2.310 E-03 \quad 2.293 E-03 \quad 2.2608-03 \quad 2.148 E-03 \quad 3.859 E-03 \quad 1.119 E-03 \quad 2.626 E-04 \quad 1.644 E-06 \quad 7.689 E-35$ $1.970 E-06 \quad 1.954 E-06 \quad 1.923 E-06 \quad 2.828 E-06$ 1.549E-06 B.842E-07 1.781E-07 6.537E-10 3.190E-41

$0.000 E+00 \quad 5.562 E-12 \quad 2.655 E-11 \quad 5.358 E-11 \quad 1.482 E-10 \quad 3.774 E-10-5.942 E-10 \quad 5.277 E-10 \quad 3.000 E-11$ 8. 200E-03 B.197E-03 B. $192 E-03$ B.174E-03 8.122E-03 7.943E-03 J.452E-03 5.963E-03 3.389E-04 $0.000 E+00 \quad 7.6908-08 \quad 2.303 E-07 \quad 7.658 E-07 \quad 2.283 E-06 \quad 7.443 E-06 \quad 2.096 E-05 \quad 5.594 E-05 \quad 3.220 E-05$ 8.200E-03 B.197E-03 \&.192E-03 8.175E-03 9.124E-03 7.950E-03 7.473E-03 6.019E-03 3.711E-04

$0.000 E+00 \quad 2.507 E-17 \quad 2.244 E-16 \quad 2.445 E-15 \quad 2.0 B 4 E-14 \quad 1.935 E-13 \quad 2.126 E-12 \quad 4.753 E-12 \quad 1.721 E-11$ 0.000E+00 7.380E-08 2.213E-07 7.369E-07 2.203E-06 7.261E-06 2.108E-05 6.279E-05 2.025E-04 $\begin{array}{llllllll}0.000 E+00 ~ 3.457 E-13 & 3.210 E-12 & 3.451 E-11 & 3.092 E-10 & 3.384 E-09 & 2.928 E-08 & 2.794 E-07 & 5.304 E-06\end{array}$ 0.000 E $007.380 E-08 \quad 2.213 E-07 \quad 7.370 E-07 \quad 2.204 E-06 \quad 7.264 E-06 \quad 2.111 E-05 \quad 6.307 E-05 \quad 2.078 E-04$

$0.000 E+00 \quad 3.522 E-21 \quad 9.737 E-20 \quad 3.551 E-18 \quad 9.294 E-17 \quad 2.945 E-15 \quad 5.538 E-14 \quad 8.421 E-13 \quad 1.541 E-11$ $0.0008-00 \quad 1.598 E-11 \quad 1.438 E-10$ 1.595E-09 1.428E-08 1.558E-07 1.333E-06 1.235E-05 1.819E-04 $0.000 E+00$ S.013E-17 1.347E-15 4.980E-14 1.337E-12 4.868E-11 3.242E-09 3.801E-08 4.250E-06 $0.000 E+00 \quad 1.598 E-12 \quad 1.438 E-10 \quad 1.595 E-09$ 1.428E-08 $\quad$ L.558E-07 $1.332 E-06 \quad 1.239 E-05 \quad 1.862 E-04$

$0.0008+00 \quad 2.797 E-23 \quad 2.231 E-21 \quad 2.607 E-19 \quad 2.815 E-27 \quad 1.404 E-15 \quad 4.243 E-14 \quad 7.838 E-13 \quad 1.530 E-12$ $0.000 E+00 \quad 1.643 E-13 \quad 4.367 E-12$ 1.532E-10 3.572E-09 8.642E-08 1.077E-06 1.160E-05 1.806E-04 $0.000 E+00 \quad 2.277 E-19 \quad 3.063 E-17 \quad 3.642 E-15 \quad 2.616 E-13 \quad 2.261 E-11 \quad 9.201 E-10 \quad 3.464 E-08$ 4.211E-06 $0.000 E+00 \quad 1.643 E-13 \quad 4.367 E-32 \quad 1.532 E-10 \quad 3.571 E-09$ 8.645E-08 1.078E-06 $1.164 E-05 \quad 1.848 E-04$

1.800E-04 1.600E-04 2.799E-04 2.797E-04 1.792E-04 1.775E-04 1.726E-04 1.565E-04 4.455E-05

$0.000 E+001.772 E-13 \quad 5.315 E-13 \quad 1.769 E-12 \quad 5.282 E-12 \quad 1.733 E-11 \quad 4.968 E-11 \quad 1.414 E-10 \quad 2.063 E-10$ $\begin{array}{lllllllllll}2.780 E-04 & 2.779 E-04 & 2.777 E-04 & 2.771 E-04 & 2.754 E-04 & 2.6948-04 & 2.529 E-04 & 2.027 E-04 & 1.182 E-05\end{array}$ 2.780E-04 2.779E-04 2.777E-04 2.771E-04 2.754E-04 2.694E-04 2.529E-04 2.027E-04 1.182E-05 
optTonu zused sta jo vo

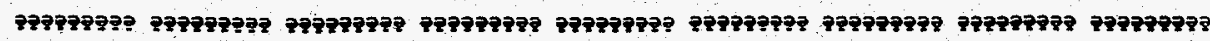

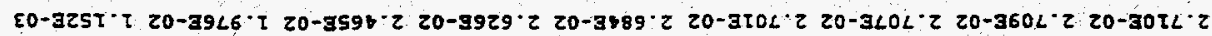

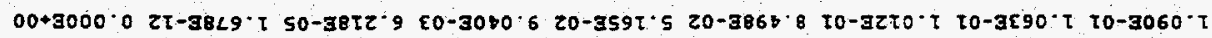

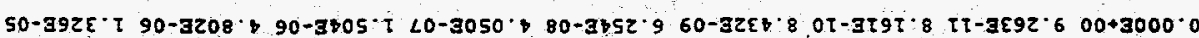

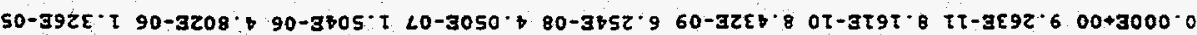

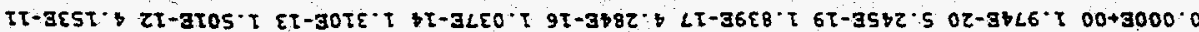

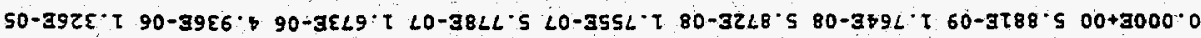

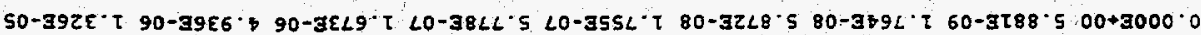

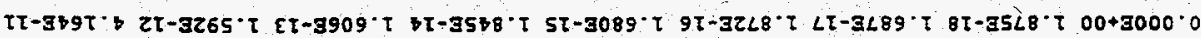

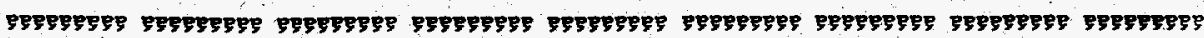

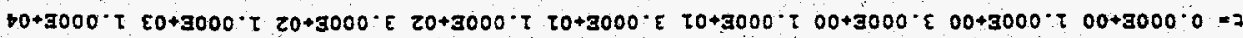

$$
\text { S/Tod } \cdot(2 \cdot 5) S
$$

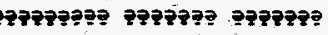 $00+3000 \cdot \tau \quad 8 \varepsilon z-\Omega \quad 8 \varepsilon z-\Omega$ $00+3000^{\circ}$ I $\quad 06-x S \quad 06-x S$

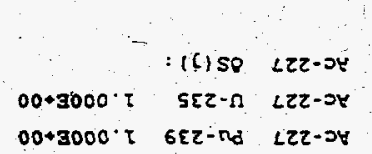 \\ (5) 58 tहZ-e

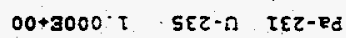 $00+3000^{\circ}$ T 6EZ-nd tez-eo

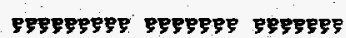

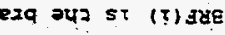

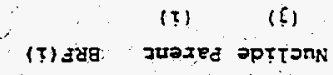

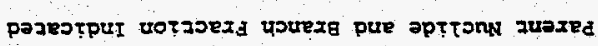
uorzerzuastos tros apt tork TEnpthtpuI 



\section{Appendix F}

\section{RESRAD Summary Report}

Radioactive Equipment,

Contaminated Concrete and

\section{Embedded Piping}




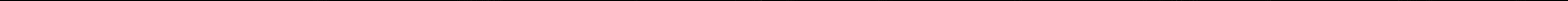




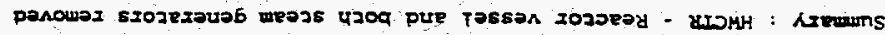


RESRAD, Version 5.621 H Limie = 0.5 year $04 / 16 / 9713: 17$ Page 2

Sumany : HWCRR - Reactor vessel and both steam generacors removed

File : A:VTOT_INV.DAT

Dose Conversion Factor (and Relaced) Parameter Summary

File: DOSFAC. BIN

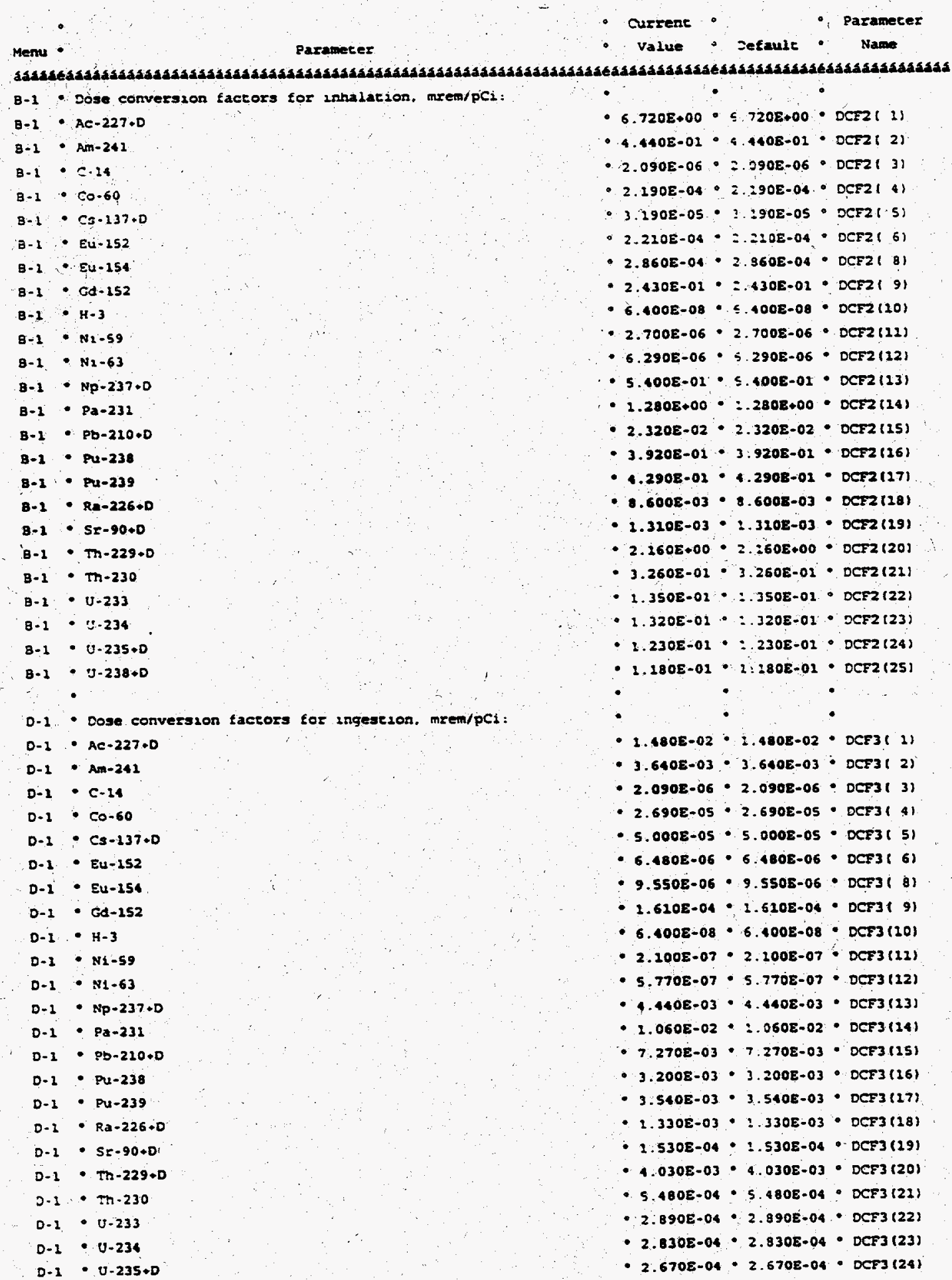


RESRAD. Version 5.621 T Limit = 0.5 year P $04 / 16 / 9713: 17$ Page 3

Summary : HWCTR - Reaccor vessel and both sceam generacors removed

File : A:lTOT_INV.DAT

Dose Conversion factor (and Related) Paramecer Summary (conesnued) File: DOSFAC.BIA

Menu - Parametex Daramecer

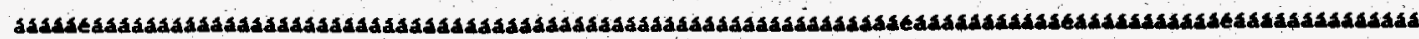

$D-1 \cdot 0-238 \cdot 0$

2.6908-04 $2.6908-04 \cdot$ DCF3 (25)

$0-34$ - Food eranster faccors:

D-34 AC-227+D plane/sosl concentration rac10, dimensionless

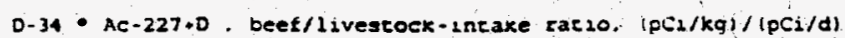

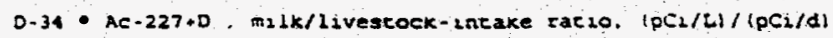

$0.34 \cdot$

0.34 - Am-241 planc/sozl concencracion racso. dimenasoniess

D-34 Am-241 : beet/livestock-incake rac10, $\left(\mathrm{pCl}_{1} / \mathrm{kg}\right) /\left(\mathrm{pCl}_{1} / \mathrm{d}\right)$

D-34 - Am-241 ?mlik/livestock-1neake rat20. (pCi/h)/(pCi/d)

$0.34 \cdot$

$0.34 \cdot c .14$

$0.34 \cdot c-14$

D-34:C-14

D-34:

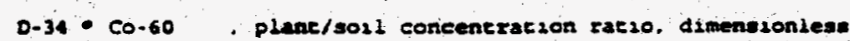

D-34 - Co-60 a bet/livestock-1ncake ratso, $(\mathrm{pCl} / \mathrm{kg}) /(\mathrm{pCl} / \mathrm{d})$

D-34 c0-60 . milk/livestock-2ntake racio. (pCL/L)/(pcl/d)

D-34.

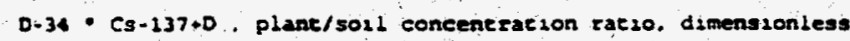

D-34 $-\mathrm{Cs}-137+0$, beef/livescock-2ncake ratio, $(\mathrm{pCi} / \mathrm{kg}) /(\mathrm{pCi} / \mathrm{d})$

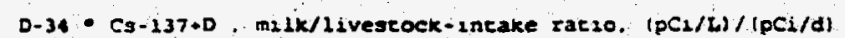

D.34 -

D-34 - Eu-152 flanc/soll contentracion ratio, dimensionless

D-36 - Eu-1S2 setk/livestock-1ncake ratio. $(p C i / k g) /(p C 1 / d)$

D-34: Eu-152 , m1lk/livestock-2ncake rat1o. (pCi/L)/(pCi/d)

$0-34:$

D-34 Eu-154 planc/sozl concentracion racio. dimenesonleas

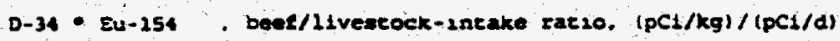

D-34 Eu-154 milk/Livescock-incake racioe (pCl/L)/(pC)/d)

$0-34 \cdot$

0-30 - Gd-152 . plant/sozl concentracion raczo. dimensionless:

D-34: Gd-152 beet/livestock-incake ratso. (pCl/kg)/(pCi/d)

D-34 cd-152 m2lk/livestock-2ntake ratso. (pCi/L)/(pCi/d)

D-34

D-34 - H-3 planc/soll concencration zatso. dimensionless

D-34 $-\mathrm{H}-3$

$0-34 \cdot k-3$

$0-34 \cdot$

D-34 - Ni-59 - plane/soll concencration zakso, dimensionless

D-34 Ni.59 beef/liveatock-intake rat1o. $(\mathrm{pCi} / \mathrm{kg}) /(\mathrm{pCi} / \mathrm{d})$

D-34 - Ni.59 amilk/liveatock-incake rat20. (pCi/L)/(pCi/d)

$0-34 \cdot$

D-34 - Ni-63 planc/soll concencracion rat 20 . dimenosoniess

D-34 - $\mathrm{N}_{1-63}$, beef/livescock-2ntake ratio, $(\mathrm{pCl} / \mathrm{kg}) /(\mathrm{pCi} / \mathrm{d})$

D-34 - Ni-63 : milk/liveseock-2ntake rat10. $(\mathrm{pCi} / \mathrm{L}) /(\mathrm{pCl} / \mathrm{d})$

0.34 .

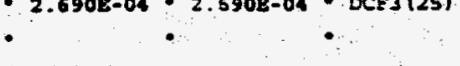

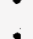

$02.5008-03 \cdot 2.5008-03 \cdot \operatorname{RTE}(2.1)$

$\therefore$ 2.0008-05 2.2008-05 RTF( 1.2$)$

$=2.000$-05 $=.000$-05 RTE( 1.31

- $2.0008-050.0000050$

- $\quad 0008-03 \cdot: 2008-03 \cdot \operatorname{RTF}(2.1)$

- 5.0008-05 - 5.0008-05 - RTF( 2.2)

2.000E-06 2.0008-06 : RTF(2.3)

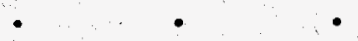

- 5.5008+00 - 5.5008+00 - RTF( 3.1)

$-3.1008-02 \cdot 3.2008-02 \cdot \operatorname{RTPl} 3.2\}$

$-2.2008-02 \cdot 2.2008-02 \cdot \operatorname{RTF}(3.3)$

- $\bullet$.

- 8.0008-02 - 8.0008-02 - RTP( 4.1)

-2.0008-02 2.0008-02 - RTF( 4.2)

- 2.0008-03 $2.0008-03 \cdot \operatorname{RTF}(4.3)$

- $\because \quad \bullet \quad 2$

- 1.0008-02 4.0008-02 - RTFI 5.11

- 3.000E-02 3.0008-02 - RTF( 5.2)

6.0008-03 -9.0008-03 - RTFI 5.31

$=2.5005 .03 \cdot 2.5005-03 \cdot$ RTF( 6.1$)$

- 2.0008-03 2.0008-03 - RTFI 6.21

2.0008-05 : 2.0008-05 - RTF( 6,3$)$

- $\quad$.

- 2.5008-03 $\cdot 2.5008-03 \cdot \operatorname{RTF}(8.2)$

- 2.0008-03 $2.0008-03 \cdot$ RTF( 8.2)

-2.0005-05 2.0008-05 - RTPI 8.3)

-2.5008-03 - 2.5008-03 - RTFI 9.11

-2.0008-03 - 2.0008-03 - RTF( 9,2)

2.0008-05 $\bullet 2.0008-05 \cdot \operatorname{RTE}(9.3)$

$\cdot$

- $1.8008+00 \cdot 4.800 E+00 \cdot \operatorname{RTF}(20,1)$

$1.2008 .02 \cdot 1.2008-02 \cdot \operatorname{RTF} 120.21$

- $1.0005-02 \cdot 2.000 \mathrm{~B}-02 \cdot \operatorname{RTF}(30.3)$

- $\quad$.

- 5.0008-02 5.000E-02 RTF(12.1)

5.0005-03 - 5.000E-03 RTF (11.2)

$-2.000 \mathrm{~B}-02 \cdot 2.000 \mathrm{E}-02 \cdot \operatorname{RTE}(11.3)$

5:000E-02 5.000E-02 RTF (12.1)

$05.0008-03 \cdot 5.0008-03 \cdot \operatorname{RTF}(12.2)$

- 2.000E-02 $\cdot 2.000 E-02 \cdot R T F(22.3)$

- $2.000{ }^{2} 02$. $2.0008-02$

$2.000 E-02 \cdot 2.000 E-02 \cdot \operatorname{RTF}(13.1)$

- 1.0008-03 - 1.0008-03 - RTF (13.2)

D-34 - Np-237+O : beef/livescock-2ntake racio, $(p \mathrm{Ci} / \mathrm{kg}) /(\mathrm{pCi} / \mathrm{d}$

D-34 - Np-237+0. mlik/2ivestock-incake racio. (pCi/L)/(pCi/d) 
RESRAD. Version 5.621 T- Limic - 0.5 year o 04/16/97 13:17 page 4

Summer : HiwCTR - Reaccor vessel and both steam generacors removed

File: A:ITOT_INV.DAT

Dose Conversion Factor land Relaced Parameter Summary leonesnued

File: DOSFAC.BIN

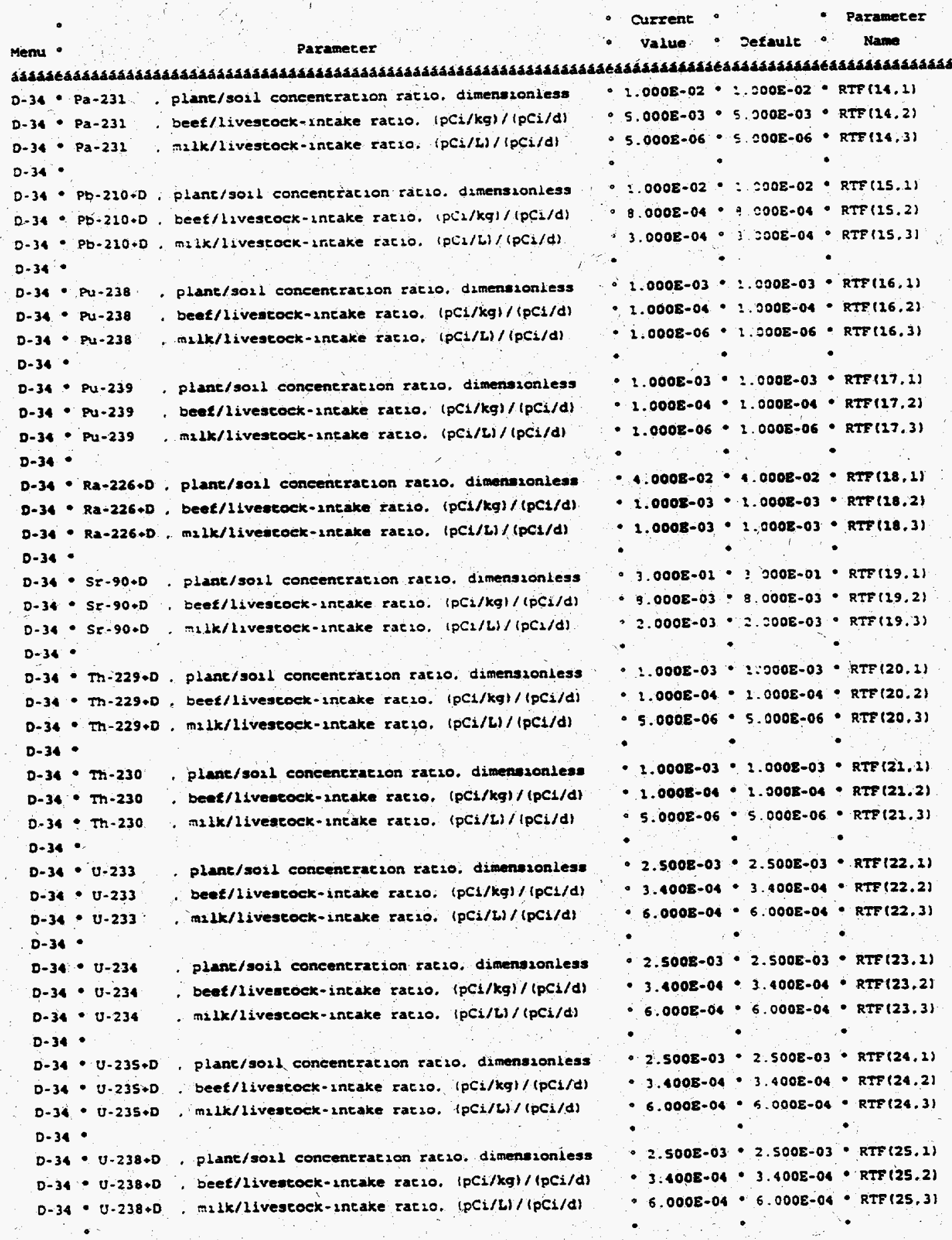


RESRAD. Version 5.621 T: Limit - 0.5 year $04 / 16 / 9713: 17$ Page 5

Sumary : HwCTR - Reactor vessel and boch scean generacoss removed

File : A:ITOT_rWV.DAT

Dose Conversion factor (and Relaced Parameter Summery Iconcamued, File: DOSFAC.BIN

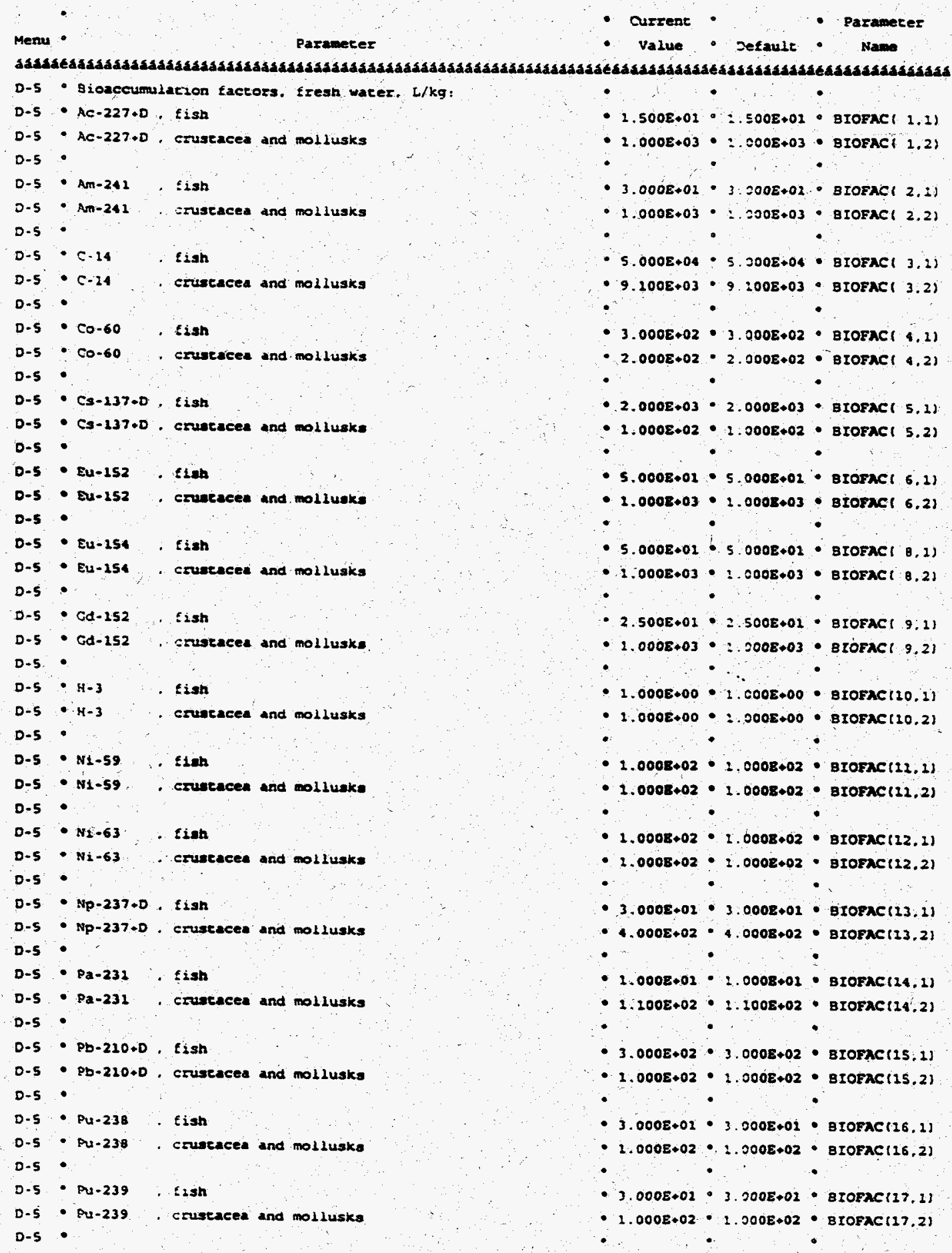


RESRAD; Version 5.621 T Limit = 0.5 year $\quad 04 / 26 / 97 \quad 13: 17$ Page 6

Sumary : HWCTR - Resctor vessel and both steam generacors removed

File : A:lTOT_INY.DAT

Dose Conversion Factor (and Relaced) Paramecer Summer (conc2nued)

File: DOSFAC. $\mathrm{EIN}$

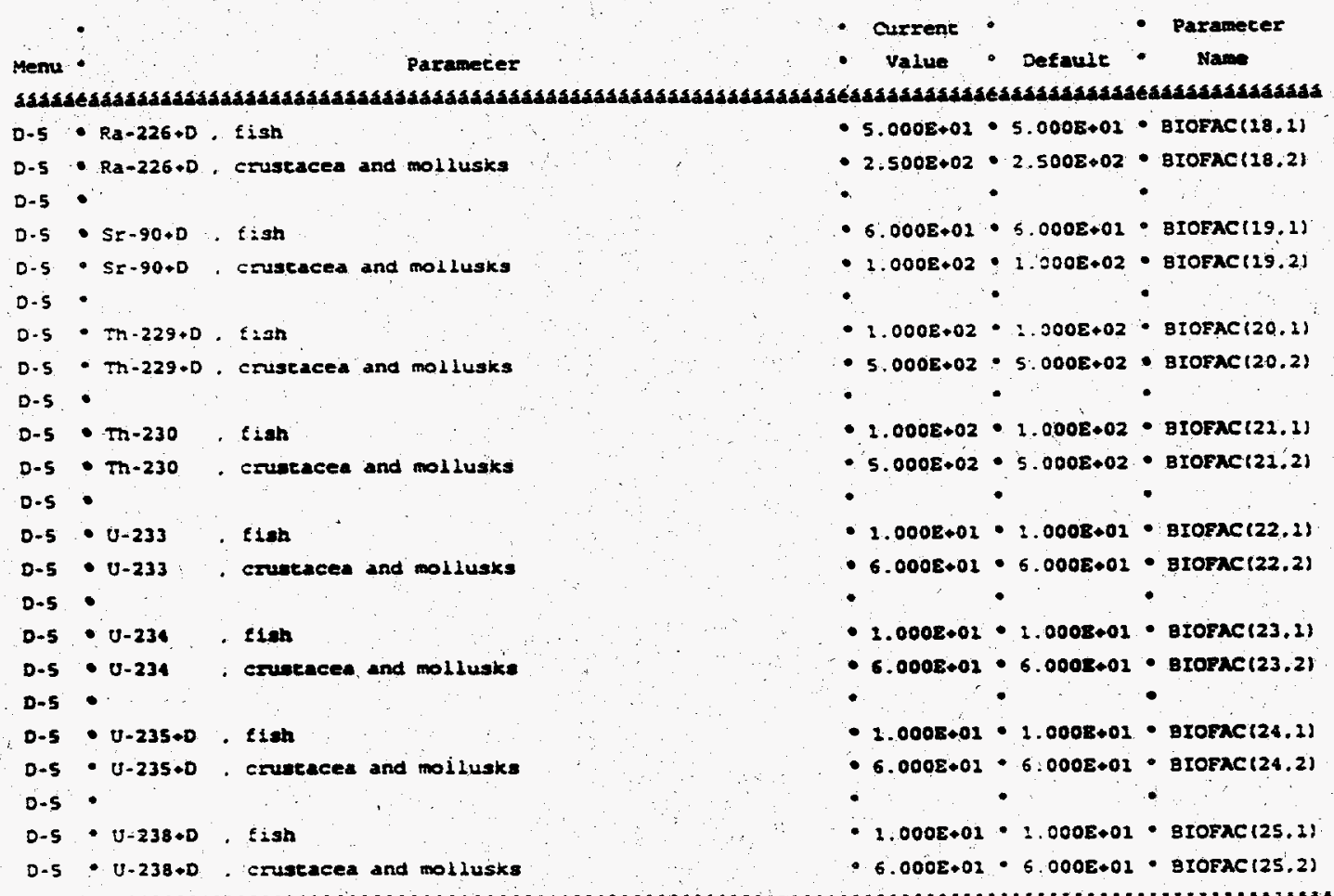

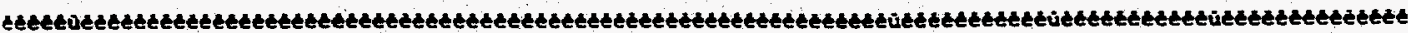


RESRAD, Version 5.621 Limic - 0.5 year $04 / 16 / 9713: 17$ jage,

Sumary : HWCIR - Reaceor vessel and both steam generacors removed

File : A: ITOT_INV.DAT

Site-Specific Paramecer sumery

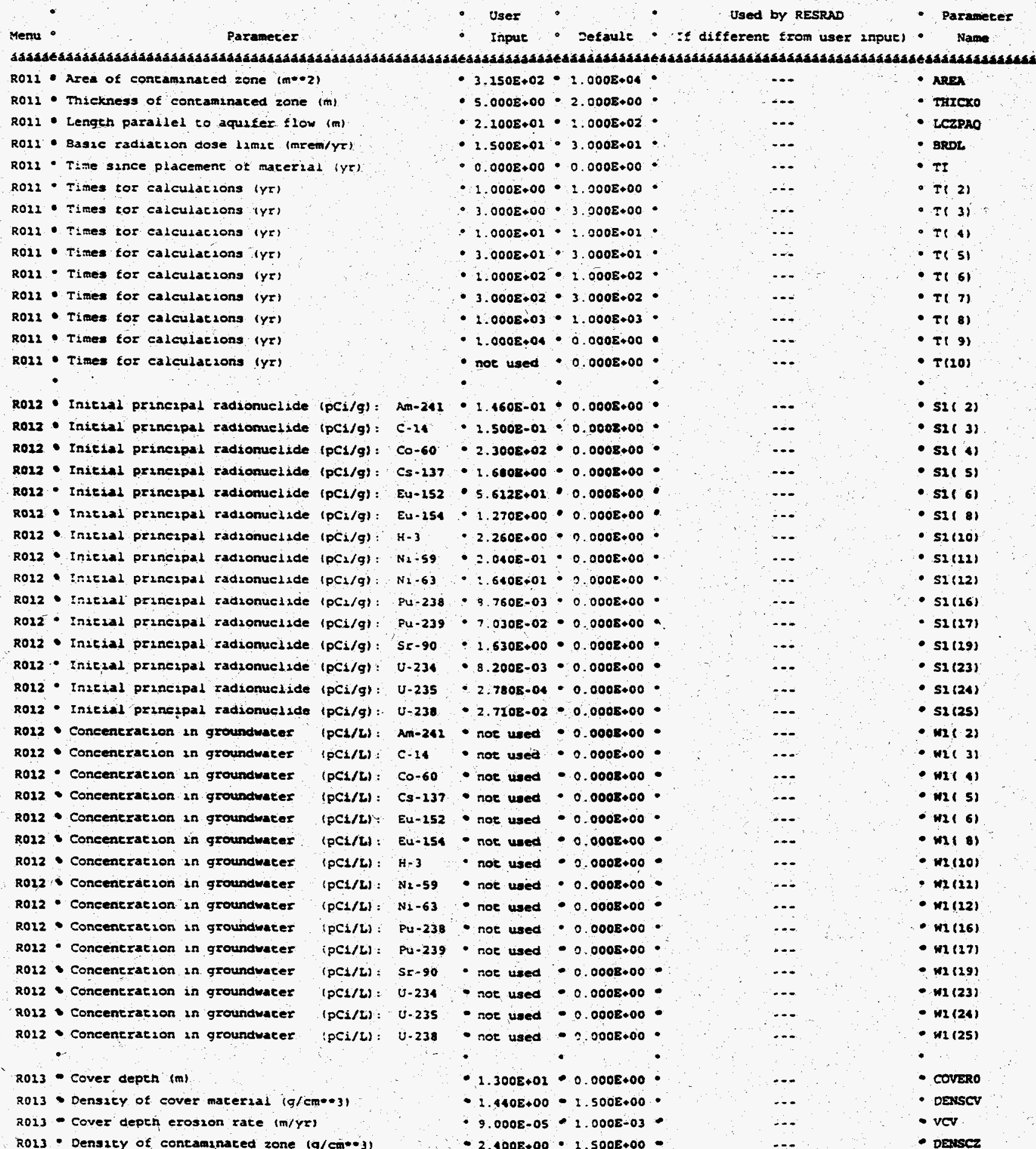


RESRAD. Versson $5.622 \quad I^{*}$ Limic = 0.5 year $\quad 04 / 16 / 97 \quad 13: 17$ page : 8 Sumary : HWCTR - Reactor vessel and boch sceam generators removed File : A:ITOT_INV.OAT

Site-Specsfic Parameter Summary (continued)

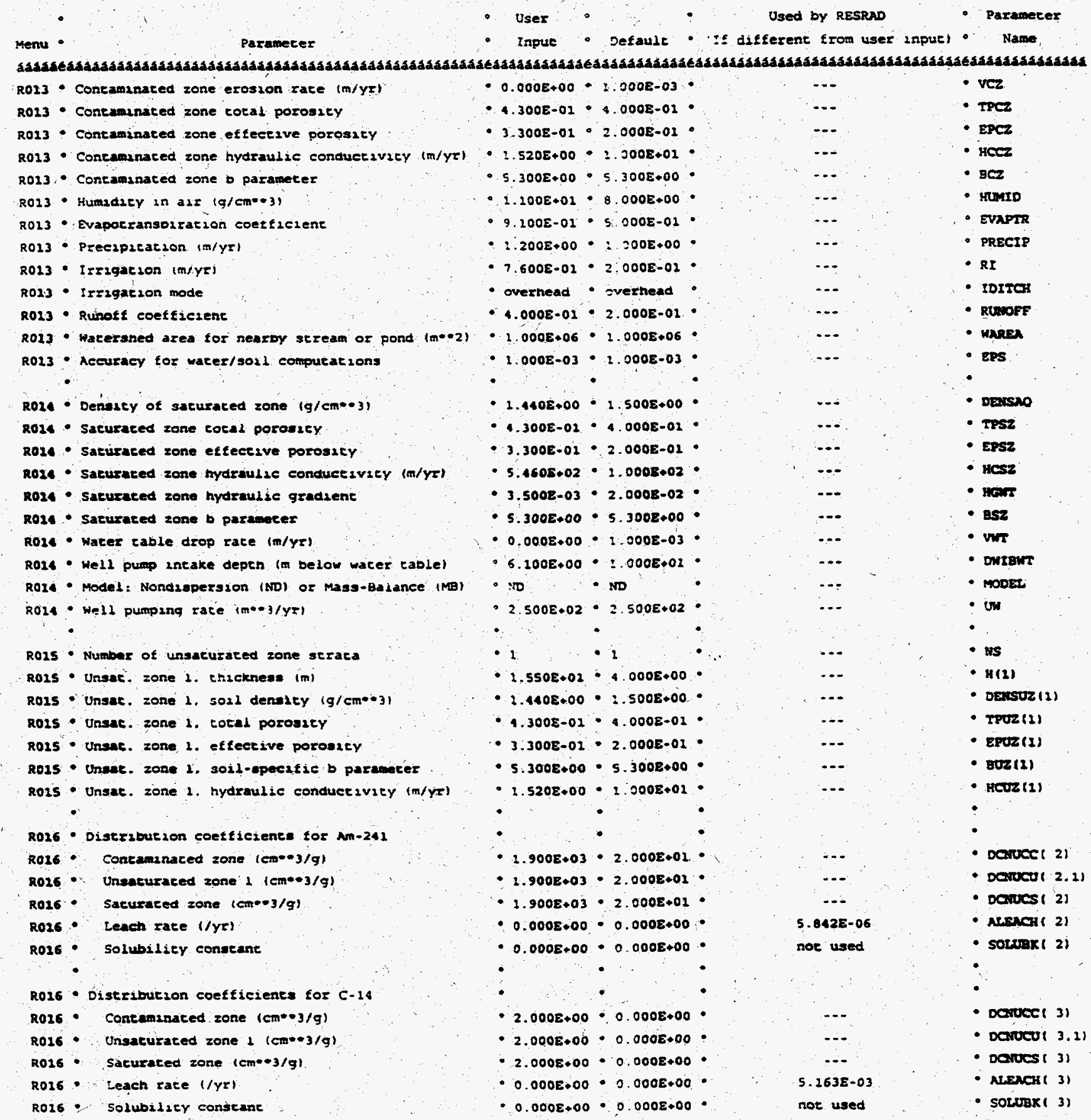




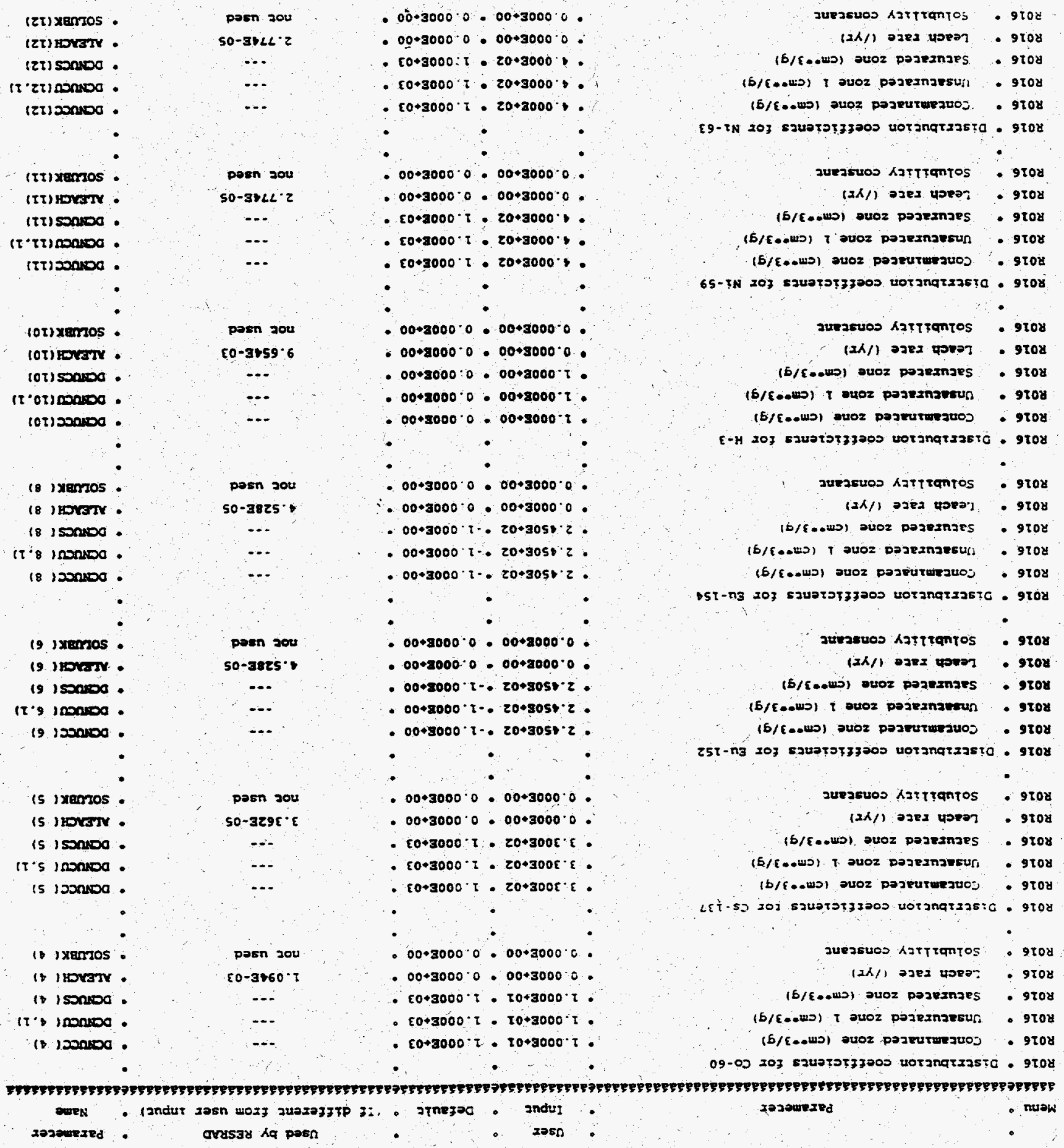

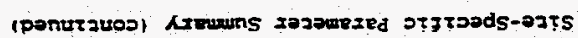

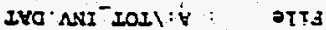

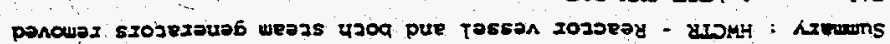

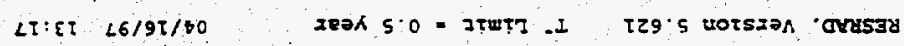


RESRAD. Version $5.623 \quad \tau$ Limit $=0.5$ year $04 / 16 / 97 \quad 13: 27$ Page 10

Summary : HWCTR - Reactor vessel and both steam generators renoved

File A:ITOT_INV.DAT

Sice-Specific Parameter Sumary (continued

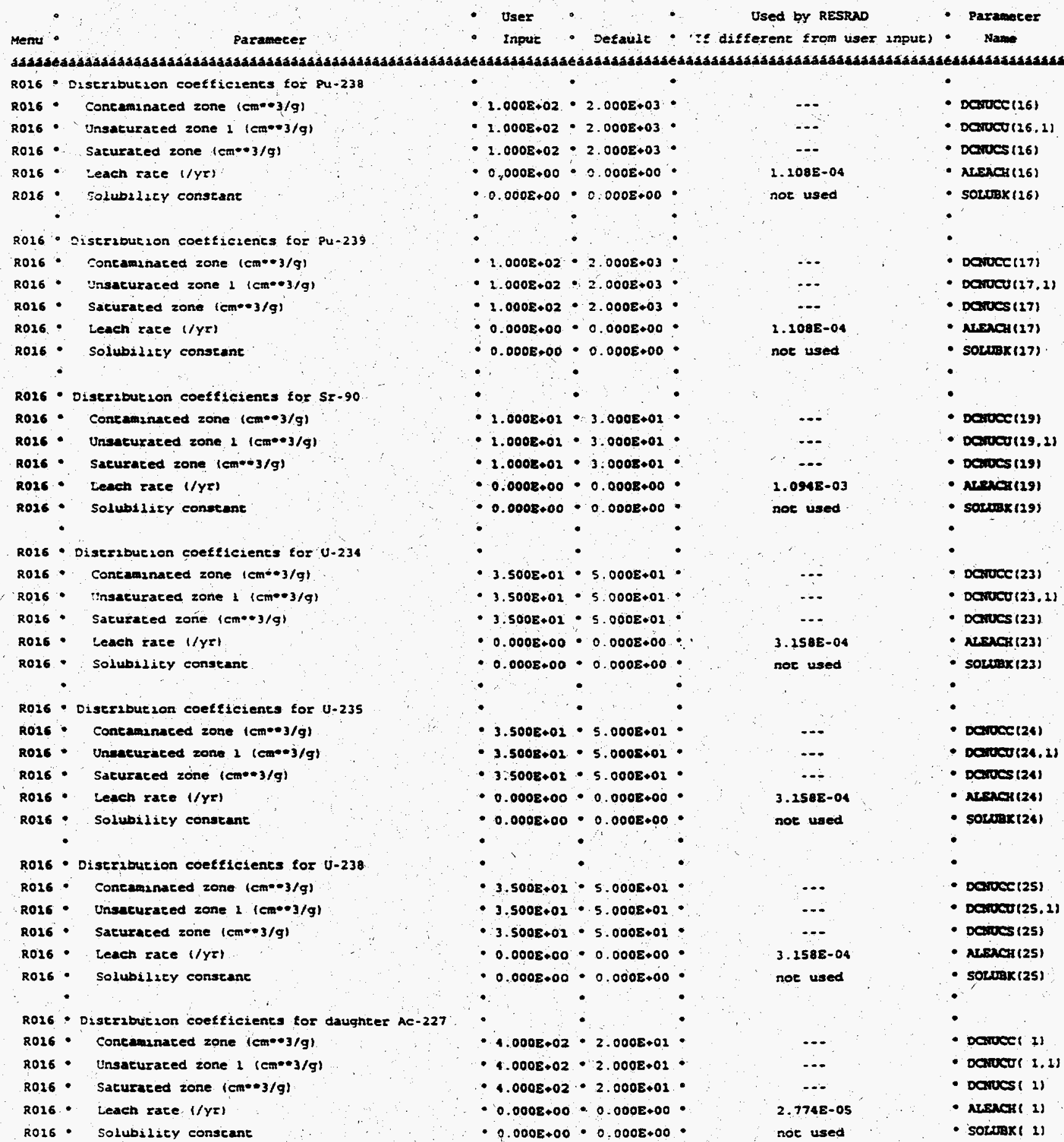


RESRAD Version 5.621 I Limit $=0.5$ year gean generacors renoved

\section{$04 / 16 / 97 \quad 13: 17$ Page 11}

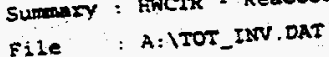

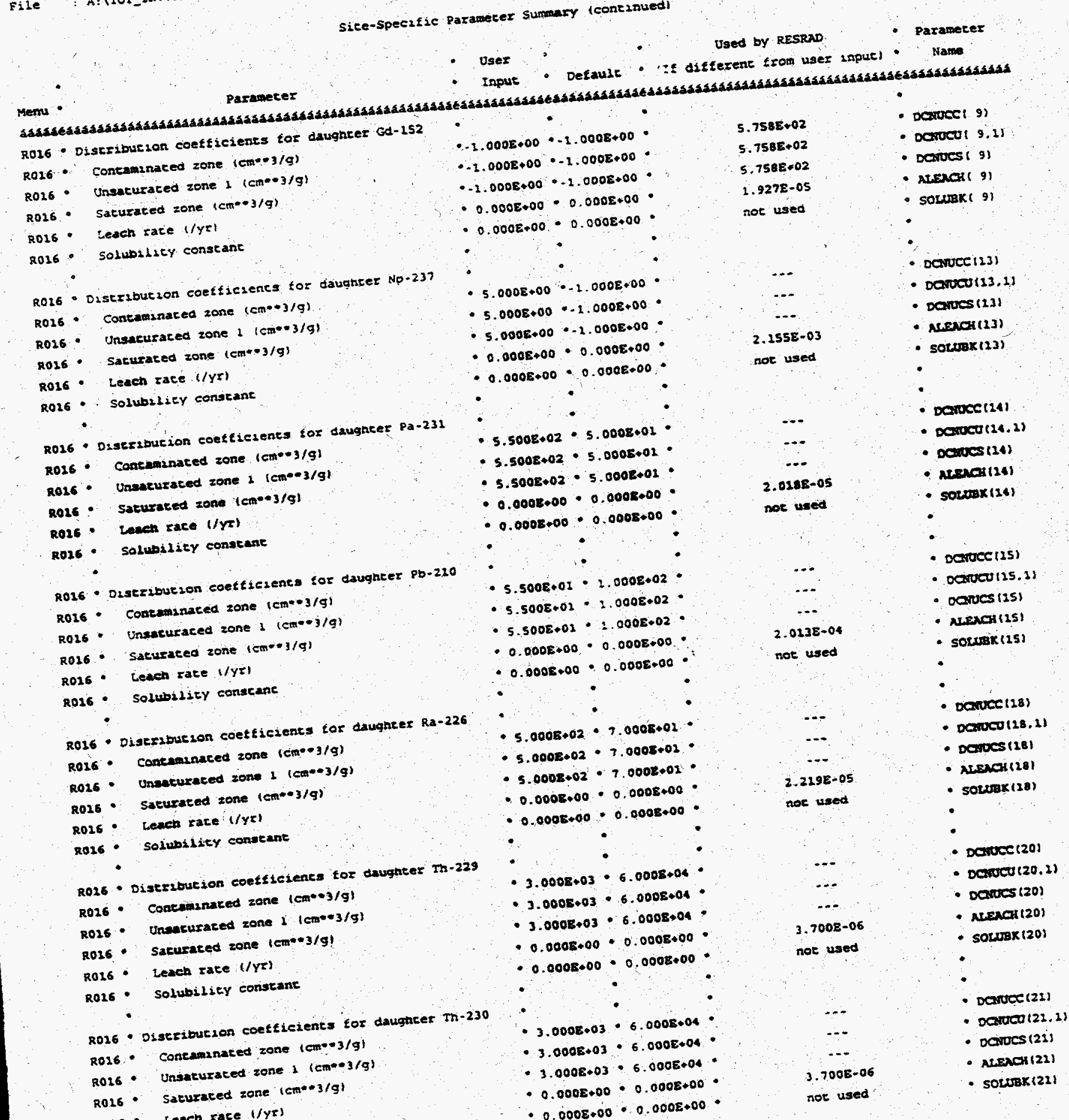


RESRAP. Version 5.621 T Limie 0.5 year $04 / 16 / 97 \quad 13: 17$ Page 12

Sumary : HWCTR - Reactor vessel and boch stean generacors renoved

File : A:ITOT_INV.DAT

Site-Specific Parameter Summary (continued

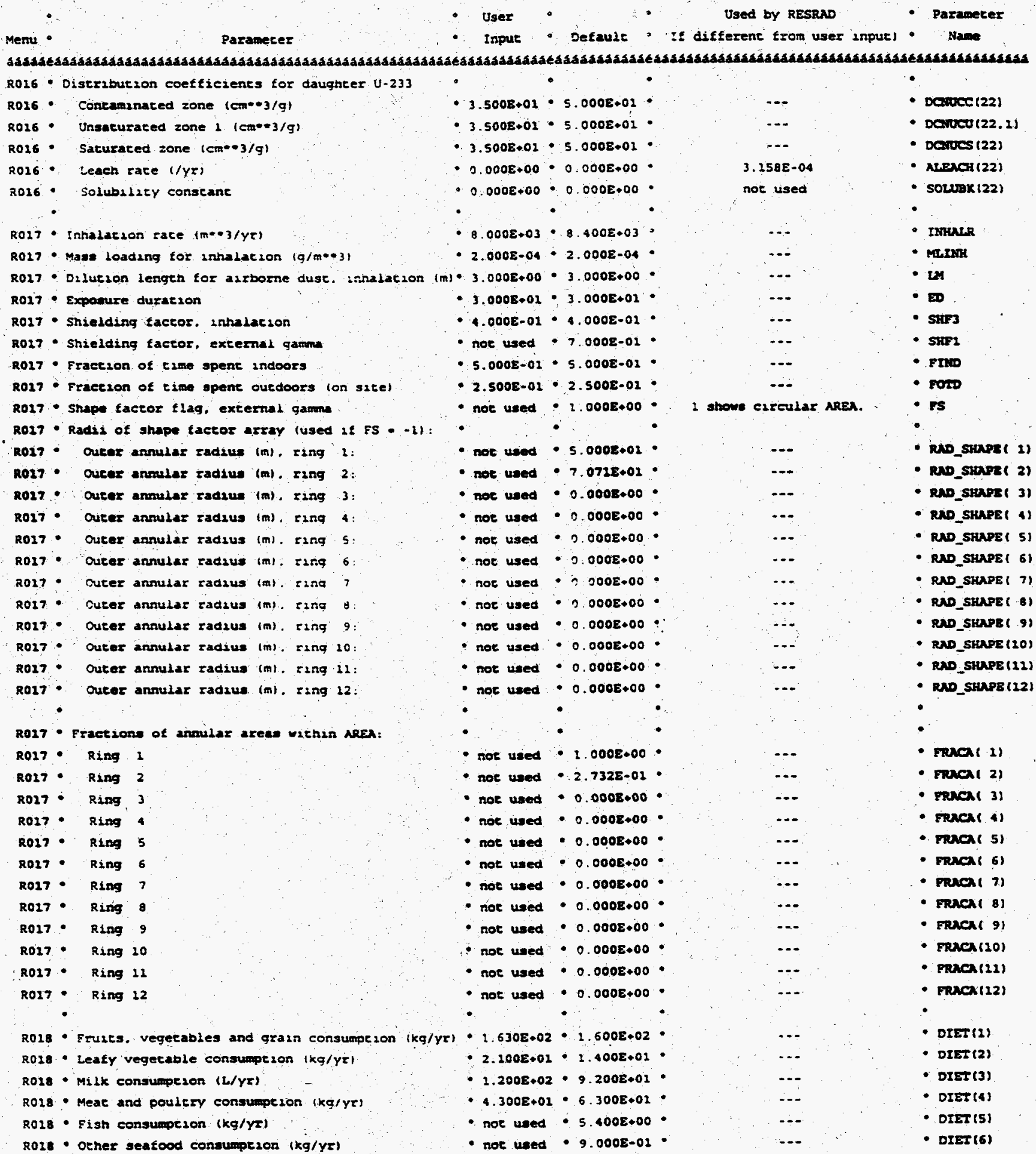


RESRAD, Version $5.621 \quad T$ Limit $=0.5$ year $04 / 16 / 97 \quad 13: 17$ page 13

Sumary : HWCTR - Reaceor vessel and both sceam generacors removed

File : A:ITOT_INS.DAT

Site-Specific Parameter Suminy (continued)

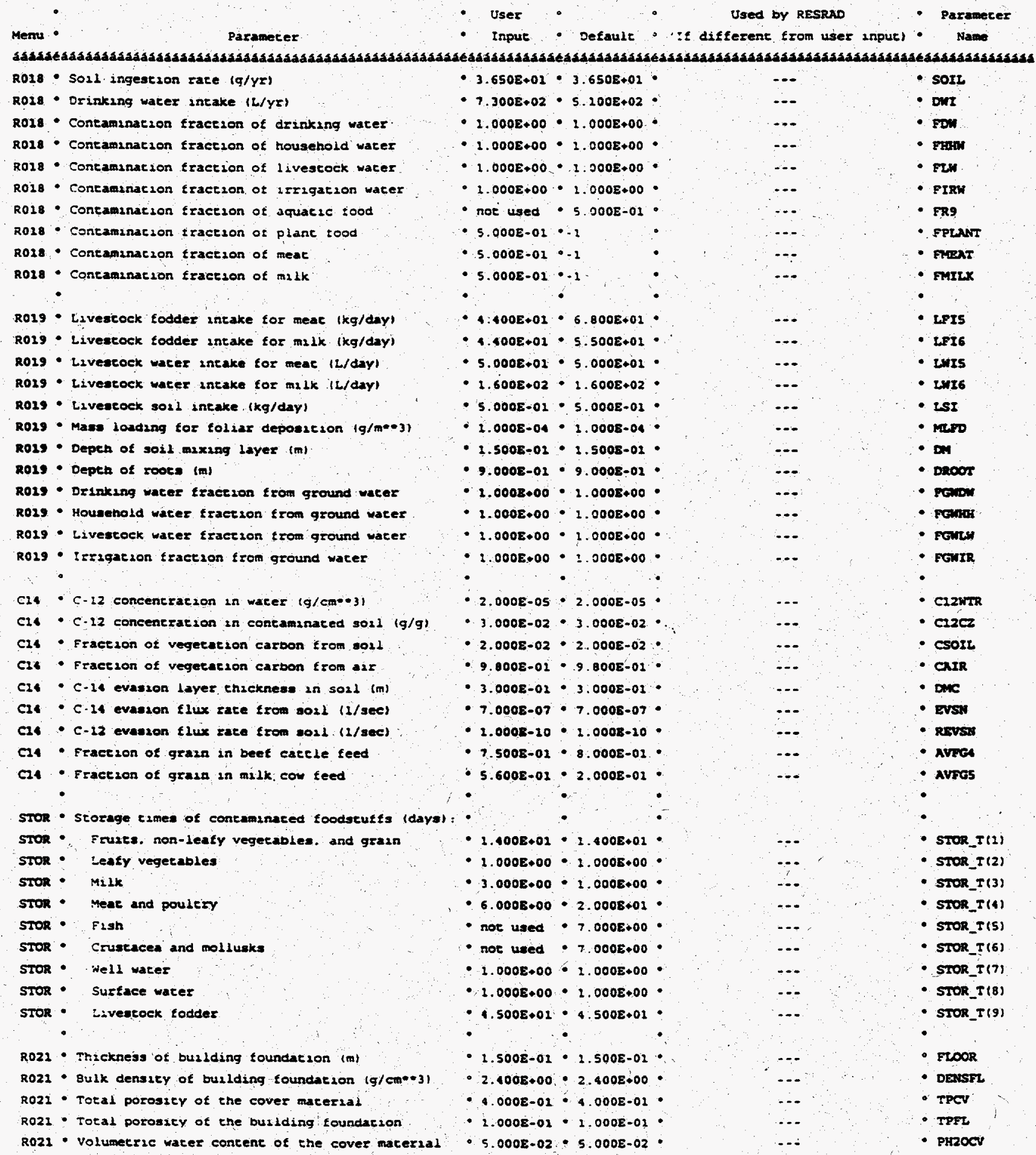


RESRAD. Versyon 5.621 T Lima 0.5 year $04 / 16 / 9713: 37$ Page 14 Summary : hicTR - Reaccor vegsel and boch steam generacors removed File $\quad \therefore$ ATOT_INV.DAT

Site-Specific Pazameter Summary (conesnued)

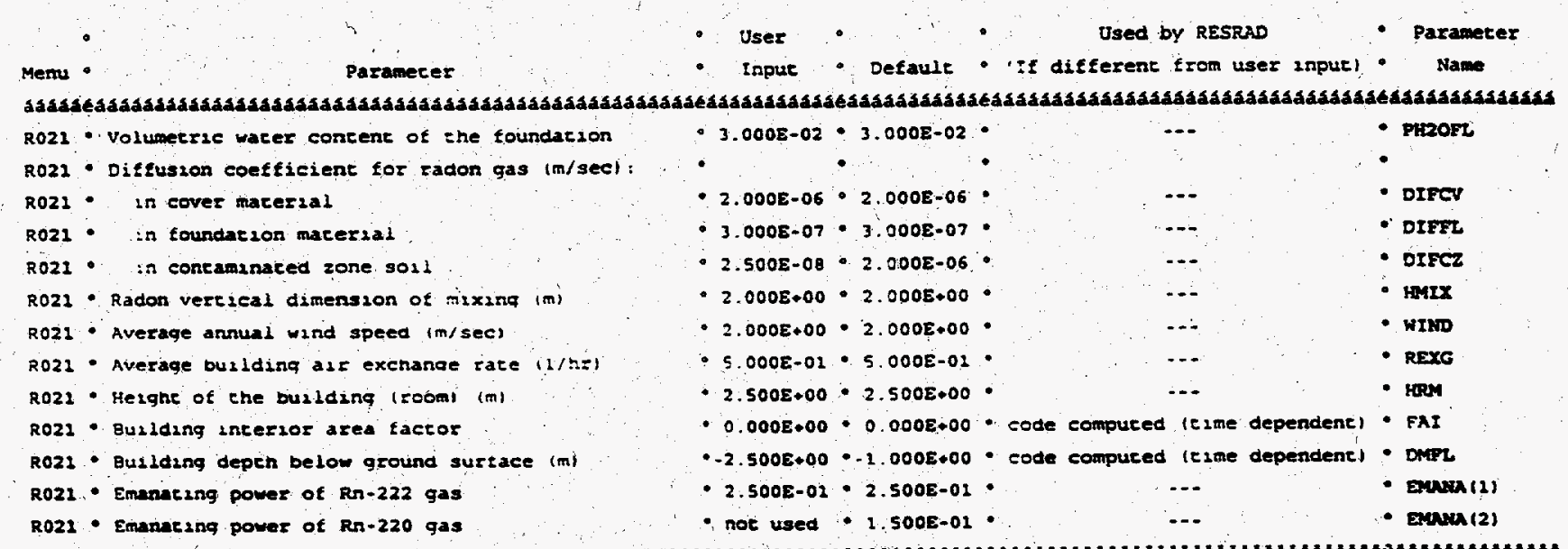

Sumary of Pachway Selections

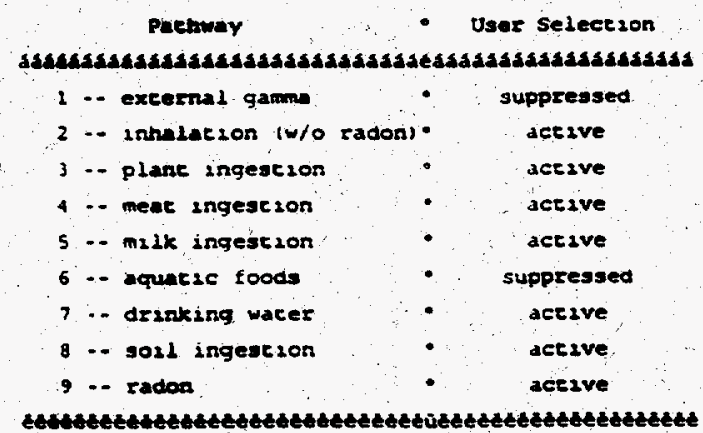


$0000.000+3000 \cdot 0 \quad 0000 \cdot 000+3000.0$

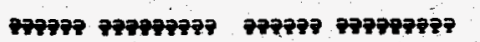
$0000.000+3000 \% 0000.000+3000.0$ $0000 \cdot 000+3000 \cdot 0$ $0000 \cdot 0.00+3000 \cdot 0$ $0000 \cdot 000+3000 \cdot 0$ $0000 \cdot 000+3000 \cdot 0$ $0000 \cdot 000 \times 3000 \cdot 0$ $0000.000+20000.0$ $0000.000+3000 \cdot 0000$ $0000.000+2000 \%$ $0000.000+3000 \cdot 0$ $0000 \cdot 000+3000: 0$ $0000.000+2000 \cdot 0$ $0000.000 \div 3000 \% 0$ $0000 \cdot 000+2000 \div 0$ $0000 \cdot 000+3000.0$ mist istures vesex 2hpoxil

wronsmenterses tros
$0000 \cdot 000.3000 \cdot 0$

$0000000+3000 \%$

$0000 \cdot 000+3000 \div 0$

$0000000+3000.0$

$0000=000+3000 \% 0$

$0000 \cdot 0.00+3000 \cdot 0$

$0000-00+3000 \%$

$0000 \% 00+3000 \%$

$0000 \div 000+3000 \%$

$0000 \cdot 00+2000 \cdot 0$

$0000000+3000.0$

$0000 \% 00+3000=0$

$0000 \% 00+9000.0$

nowes rreneses

- 30ez 7 xh/moxin

HeEpereventse XTTh
$0000 \div 00+2000: 0$
$0000.000+3000.0$

$0000 \cdot 000+3000 \cdot 0$

$00+3000.0$

$0000.000+3000.0$

$0000 \cdot 000+3000 \%$

$0000.00+3000.0$

$0000 \cdot 00+3000.0$

$0000.000+3000.0$

$0000.000+3000.0$

$0000 \cdot 00+3000.0$

$0000 \cdot 000+3000 \cdot 0$

$0000.00+3000.0$

$0000 \cdot 000+3000.0$

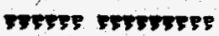

- zoezf 2X/mazu

Hestretresterse
$0000 \cdot 000+3000 \%$

$0000 \div 00+3000 \times 0$ 777797 797927\%7\%

$0000 \cdot 000 \cdot 3000.0$

$0000 \div 00+3000 \cdot 0$

$0000 \% 00+3000.0$

$0000 \cdot 0$ 00+a000 0

$0000.000+3000 \%$

$0000=00+3000=0$

$0000 \div 000+3000 \div 0$

$0000 \cdot 0.00+3000 \cdot 0$

$0000 \div 00+3000 \div 0$

$0000.0003000 \% 0$

$0000 \div 00+3000 \div 0$

0000 00+2000:0

$0000 \cdot 000-3000.0$

meses smenter

-20es 2र/

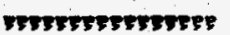

$0000 \div 000 \div 3000 \div 00000 \div 000+3000 \div 0000 \div 00+3000 \div 0$ TF20I

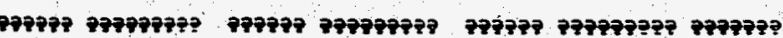
$0000000+3000 \% 00000: 000+3000 \cdot 0$ 0000.0 00+3000.0 sez-n $0000 \% 00+3000 \% 0000 \% 00+3000 \cdot 00000 \cdot 000+3000 \cdot 0 \quad 58 z-n$ $0000 \cdot 000+3000 \cdot 00000 \cdot 000+3000 \% 0000 \% 00+3000 \cdot 0$. tez-n $0000 \% 00+3000 \cdot 00000.000+3000 \cdot 0 \quad 0000 \cdot 000+3000 \cdot 0$ 06-35 $0000: 000+300000000 \cdot 000+3000 \% 0000000+3000 \cdot 0$ 6rz-nd $0000000+300000000000+3000 \% 0000 \% 00+3000-0$ oez nd $0000 \div 000+3000 \% 0000 \% 00+3000 \% 0 \quad 0000 \div 000+3000 \%$ E9-TH $0000 \cdot 000+3000 \% 0000 \cdot 000+3000.00000000+3000 \%$ 65-18 $0000: 000+3000: 00000 \cdot 000+3000 \div 00000: 000+3000 \%$ \& -1 $0000000+3000 \% 0000 \% 0043000: 0 \quad 0000.000+3000 \%$ ust-n3 $0000 \cdot 000+3000 \cdot 0 \quad 0000 \cdot 0 \quad 00+3000 \cdot 0 \quad 0000 \% 00+3000 \cdot 0$ zst-ns $0000.000+3000 \% 0000-000+3000 \% 0000 \% 00+3000 \%$ LET-ED $0000.000+3000 \% 0000.000+3000.00000 \% 00+3000 \%$ 09-02 $0000=00+9000.00000 .000+3000.00000 \% 00+3000 \%$ ut-s $0000000+3000 \cdot 0 \quad 0000000+3000 \cdot 0$ 0000.0 00+3000.0 Tre-w

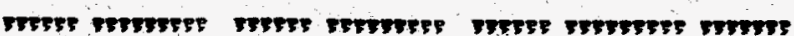

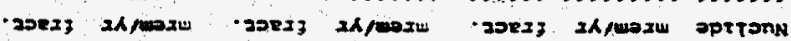

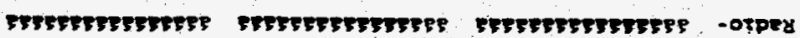
vorjeTtur $\quad$ pemoso

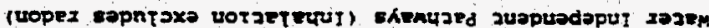

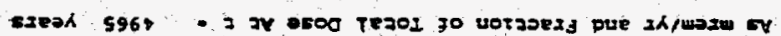

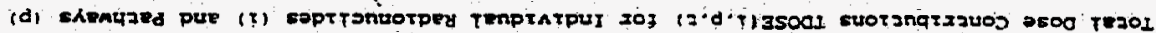

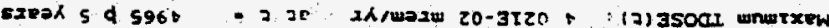

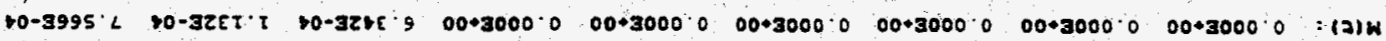

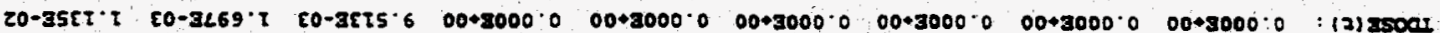

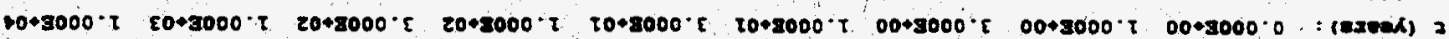

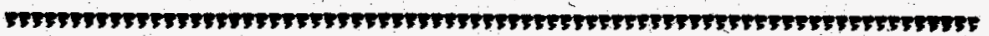

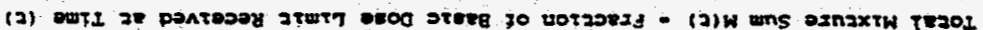

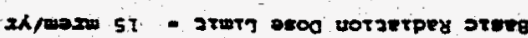

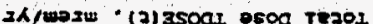

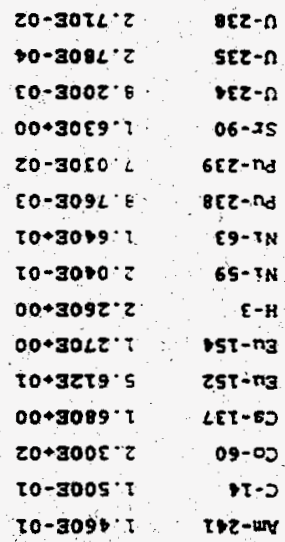

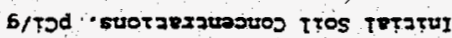

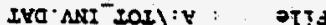

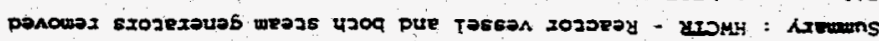

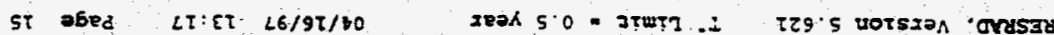

sxozaw

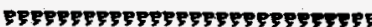

strotsuow? otuoz poseutweatos 
- shemuzed zuspusdop pup juspuedoput zojem tTe jo uns.

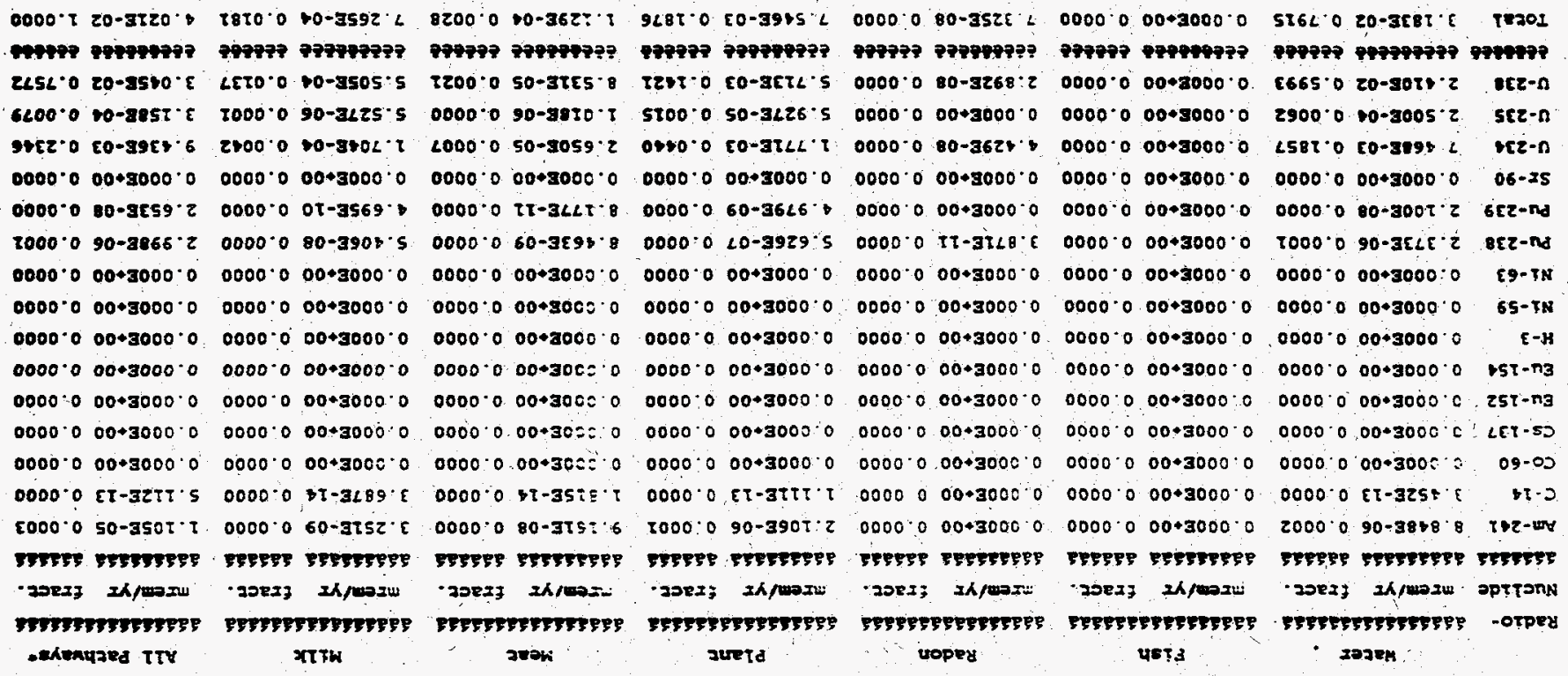

axemused zurpuradog sojen

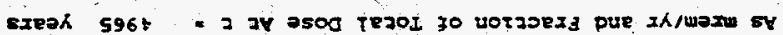

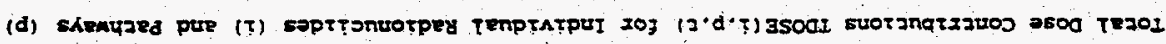

IYOANT IOII:Y : गTT3

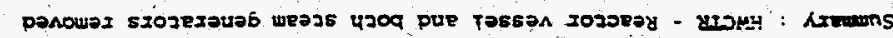

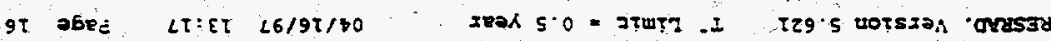


RESRAD, Version S.621 T Limit $=0.5$ year $04 / 16 / 97$ 13:17 Page 17

Summary : HWCIR - Reactor vessel and boch sceam generacors removed

File :A:ITOT_INV:DAT

Total Dose Coneribueions TDOSE(i,p, f) for Individual Radionuclides (i) and Pachways (p)

As mrem/yx and Fraction of Total Dose Ac $t=0.0008+00$ years

water Independene partways (Inhalation excludes radon!

\begin{tabular}{|c|c|c|c|c|c|c|c|c|c|c|c|c|c|c|}
\hline \multirow{2}{*}{$\begin{array}{l}\text { Radio- } \\
\text { Nuclide } \\
\text { dastasa }\end{array}$} & \multicolumn{2}{|c|}{$\begin{array}{c}\text { Ground } \\
\text { adastatasiatsasas }\end{array}$} & \multicolumn{2}{|c|}{$\begin{array}{c}\text { Inhalation } \\
\text { adasdissisassadad }\end{array}$} & \multicolumn{2}{|c|}{$\begin{array}{c}\text { Radon } \\
\text { sasastasassastak }\end{array}$} & \multicolumn{2}{|c|}{ 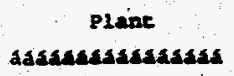 } & \multicolumn{2}{|c|}{ Ment } & \multicolumn{2}{|c|}{$\begin{array}{l}\text { Milk } \\
\text { dasmanasasadias }\end{array}$} & \multicolumn{2}{|c|}{ 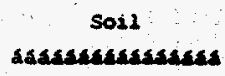 } \\
\hline & & $\begin{array}{l}\text { frace. } \\
\text { adass }\end{array}$ & $\begin{array}{l}\text { mrem/ra } \\
\text { asasasada }\end{array}$ & $\begin{array}{l}\text { Erace: } \\
\text { asasad }\end{array}$ & $\begin{array}{l}\operatorname{mrem} / y x \\
\text { adsatisas }\end{array}$ & $\begin{array}{l}\text { frace. } \\
\text { asisas }\end{array}$ & $\begin{array}{l}\text { mrem/yr } \\
\text { assassass }\end{array}$ & $\begin{array}{l}\text { trace. } \\
\text { datast }\end{array}$ & $\begin{array}{l}\text { meen/yr } \\
\text { dasdusas }\end{array}$ & $\begin{array}{l}\text { Erace. } \\
\text { aasas }\end{array}$ & $\begin{array}{c}\text { mrew/yr } \\
\text { adasasats }\end{array}$ & $\begin{array}{l}\text { Erace. } \\
\text { sastis }\end{array}$ & $\begin{array}{l}\text { mrem/yr } \\
\text { adsususas }\end{array}$ & $\begin{array}{l}\text { Erace. } \\
\text { sessis }\end{array}$ \\
\hline$=-24$ & 0 & $c$ & 0 & 0 & 0 & & 0 & & 0 & & $.000 E+00$ & & 0 & \\
\hline-14 & 00 & & 0 & 0 & 0 & & 10 & & 0 & & 0 & & & \\
\hline - & 10 & & .00 & 10 & $+\infty$ & & .00 & & $2+00$ & & $2+00$ & & $0.000 E+00$ & \\
\hline 7 & 10 & 0 & $8=00$ & 0.0 & $0.000 E+00$ & & $8+\infty$ & 0 . & $0 .=008+00$ & & $0.0008+00$ & & $0,0008+00$ & \\
\hline 52 & 00 & 0. & 00 & 00 & $=00$ & 0. & $0.000 z+00$ & & $E+00$ & & $8+00$ & & $0.0008+00$ & t \\
\hline 54 & 00 & 0.0 & .00 & 100 & $+\infty$ & 0 & $.0008+00$ & 0. & $0.0008+00$ & & $-\infty 0$ & 0 & $0.0008+00$ & 0.000 \\
\hline-3 & 00 & 0.0 & 100 & 00 & $+\infty$ & & 000 & 0. & $0.000 \Sigma+00$ & & $\$+\infty$ & & $0.0008+00$ & 0.0000 \\
\hline $1-5$ & $E+\infty$ & 0.0 & $=00$ & $\infty 0$ & $=+\infty$ & 0 & $.000 E+00$ & 0. & $0.0008+00$ & 0.0 & $0.0008+00$ & 0.0 & $0.000 \mathrm{E}+00$ & 0.0000 \\
\hline 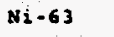 & $0.0008+00$ & 0.0000 & $000 E+00$ & 100 & $5+00$ & 0 & .00 & 0 & .00 & & $0.000 \mathrm{E}+00$ & & $0.0008+00$ & 0.000 \\
\hline 36 & $0.000 E \bullet 00$ & 0.0000 & $0008+00$ & 100 & .00 & 0 & .00 & 0 & .00 & o & $3+00$ & $\mathbf{0}$ & $8+00$ & 0.000 \\
\hline 39 & $.000 E+00$ & 0.0 & 10 & $n$ & 00 & c & . & 0 & 10 & 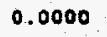 & 400 & 0 . & 00 & . \\
\hline--00 & $0.0008+00$ & 0.0000 & 0 & 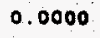 & .00 & 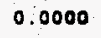 & 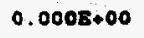 & c & 0 & & .00 & 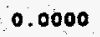 & 0 & \\
\hline$=2$ & $0.0008+00$ & t & $08 * 00$ & & .00 & & & & & & 100 & & & \\
\hline & & & & & & & 8.00 & & & & .00 & & 400 & \\
\hline & & & $1008 * 00$ & & & & $8+00$ & & $18 * 00$ & & $0.0008+00$ & & $+\infty$ & \\
\hline & & & & & & 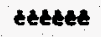 & obet & & & & $=6$ & & 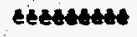 & \\
\hline otant & 00 & so & 00 & 0000 & $8+00$ & 0000 & $05: 00$, & 00 & 0008.00 & 0.0000 & $.000 E+00$ & 0.0000 & $0.0008 \times 00$ & 0.0 \\
\hline
\end{tabular}

Total Dose Conerzbuesons TDOSE(b.p.e) for Iadividual Radionuclides (2) and pachways. (p)

As mremiyr and fraceson of Jotal Dose Ac $E \cdot 0.000 \mathrm{E}+00$ years

water pependent pacmays

water

Radio- assusussusasss

Muclide mrewirg fract.

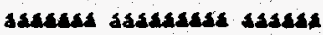

Nan-241 $0.0008 \cdot 00 \quad 0.0000$

C-14: $0.0008+00 \quad 0.0000$

Co-60 $0.0008+00 \quad 0.0000$

Cs-137 $0.0008+00 \quad 0.0000$

Eu-2S2 $0.000 E+00 \quad 0.0000$

Eu-254 $0.0008+00 \quad 0.0000$

H-3 $\quad 0.0008+00 \quad 0.0000$

Ni.59 $0.000 E+00 \quad 0.0000$

Ni.63:0.000E+00 0.0000

Pu-238 $0.0008 \times 00 \quad 0.0000$

Pu-239, $0.0008+00 \quad 0.0000$

SE+90 $0.000 E+00 \quad 0.0000$

U-234 $\quad 0.0008+00 \quad 0.0000$

$\begin{array}{lll}0.235 & 0.0008+00 \quad 0.0000\end{array}$

$\begin{array}{llll}0.238 & 0.000 E+00 \quad 0.0000\end{array}$

éettete éejejetet tetek

Fish

\section{adscusassassasss}

mrem/yr tract.

\section{dascusasususasa}

dascas dadus dastasss dsasus

$\begin{array}{lllll}0.000 E+00 & 0.0000 \quad 0.000 z+00 & 0.0000\end{array}$

$0.0008 \cdot 00 \quad 0.0000$

$0.0008+00 \quad 0.0000$

$\begin{array}{llll}0.0000 & 0.000 \varepsilon+00 \quad 0.0000\end{array}$

$0.0008 .00 \quad 0.0000$

$0.0008+000.0000$

$0.000 \varepsilon+00 \quad 0.0000$

$0.000 \varepsilon+00 \quad 0.0000$

0.0008 .000 .0000

0.0008 .000 .0000

$0.0008+00 \quad 0.0000$

$0.000 E+00 \quad 0.0000$

$0.0008+00 \quad 0.0000$

$0.0000+00 \quad 0.0000$

ééttete tetete

$0.000 E+00 \quad 0.0000$
Radon

mreanyr fraet.

Plase

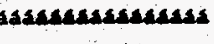

mronlyr trace.

\section{dasusess sasses}

$0.0008+00 \quad 0.0000$

$0.0008+00 \quad 0.0000$

$0.0002+000.0000$

$0.0008+00 \quad 0.0000$

$0.000 E+000.0000$

$0.0008+00 \quad 0.0000$

$0.0005+00 \quad 0.0000$

$0.0008+00 \quad 0.0000$

$0.0008+00 \quad 0.0000$

$0.0008+00 \quad 0.0000$

$0.0008+00 \quad 0.0000$

$0.0008+00 \quad 0.0000$

$0.000 E+00 \quad 0.0000$

$0.0008+00 \quad 0.0000$

$0.000 E=00 \quad 0.0000$

$0.000 E+00 \quad 0.0000$

diblente bettet

$0.0008+000.0000$

etchbbute detete

$0.000 E+00 \quad 0.0000$
Meat

\section{dasucuansasassse}

mxen/yr fraet.

\section{dasasessa sassas}

$0.000 E+00 \quad 0.0000$

$0.0008+00 \quad 0.0000$

$0.0008+00 \quad 0.0000$

$0.0008+00 \quad 0.0000$

$0.0008+00 \quad 0.0000$

$0.0008+00 \quad 0.0000$

$0.0008+00 \quad 0.0000$

$0.0008+00 \quad 0.0000$

$0.0008+00 \quad 0.0000$

$0.0008+00 \quad 0.0000$

$0.0008+00 \quad 0.0000$

$0.0008+00 \quad 0.0000$

$0.000 E+00 \quad 0.0000$

$0.0008+00 \quad 0.0000$

$0.0008+00 \quad 0.0000$

écituted tettet

$0.0008+00 \quad 0.0000$
MIIx

dasususassassas

mrea/yr Eract.

\section{adsacksas sasssa}

$0.0008+00 \quad 0.0000$

$0.0008+00 \quad 0.0000$

$0.0008+00 \quad 0.0000$

$0.000 E+00 \quad 0.0000$

$0.000 E+00 \quad 0.0000$

$0.0008+00 \quad 0.0000$

$0.0002+000.0000$

$0.0008+00 \quad 0.0000$

$0.0008 .00 \quad 0.0000$

$0.000 E+00 \quad 0.0000$

$0.0008+00 \quad 0.0000$

$0.0008+00 \quad 0.0000$

$0.000 E+00 \cdot 0.0000$

$0.000 E+00 \quad 0.0000$

$0.000 E+00 \quad 0.0000$

etstetes etetes

$0.0008+00 \quad 0.0000$
All pachuaye* sasulusanassasa mreadyr Erace. sasasase caseas $0.0008+00 \quad 0.0000$ $0.0008+00 \quad 0.00000$ $0.0008+00 \quad 0.0000$ $0.000 E+000.0000$ $0.0008+00 \quad 0.0000$ $0.0008+00 \quad 0.0000$ $0.0008+00 \quad 0.0000$ $0.0008+00 \quad 0.0000$ $0.0008+00 \quad 0.0000$ $0.0008+00.0 .0000$ $0.0008+00 \quad 0.0000$ $0.000 \varepsilon+00 \quad 0.0000$ $0.000 E+00 \quad 0.0000$ $0.0008+00 \quad 0.0000$ $0.0008+00 \quad 0.0000$ extetted tetied $0.000 E+00 \quad 0.0000$

- Sum of all wacer independene and dependent pachways. 


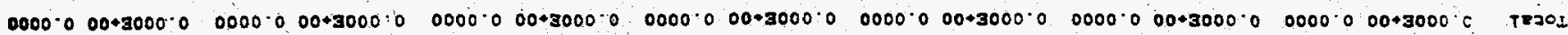

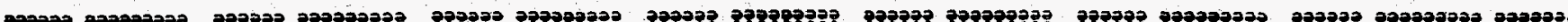

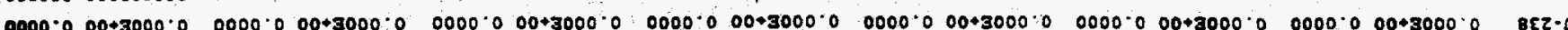

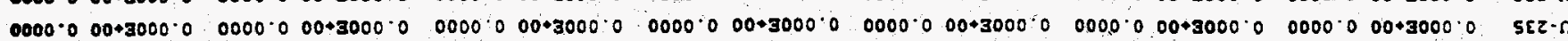

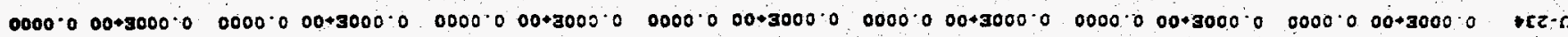

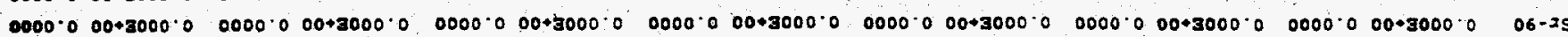

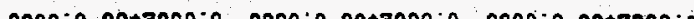

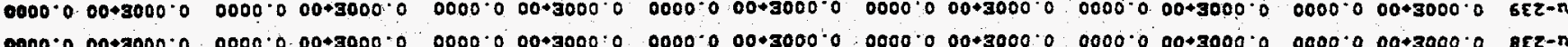

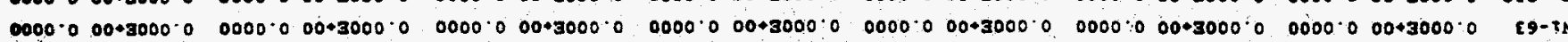
$0000.000 \cdot 3000.00000 \cdot 0.00+3000 \cdot 0$ $0000.000+2000.0 \quad 0000.000+3000.0$ $0000.00049000 \% 0000.000+3000.0$ 0000 -0 00+8000 0 0000;0 00+3000 0 $0000.00+3000.00000 \% 00+3000.0$ $0000.000+3000 \cdot 0 \quad 0000.000+3000.0$ $0000 \cdot 000+8000 \cdot 0$ 0000:0 00+3000.0

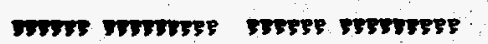
-2oszz xk/merw misinswerseg exisaysed tTY. -205a3 2 / 5erstrestrostege xTtw $0000=00+3000 \div 0 \quad 0000 \% 00+3000.00000 \% 00+3000 \% 0$ $0000 \cdot 000+3000=0000 \cdot 00+3000=00000 \cdot 00+3000 \%$ $0000.000+3000 \times 0 \quad 0000 \cdot 000+3000 \cdot 0 \quad 0000 \cdot 000+3000.0$ $0000 \cdot 000+3000 \cdot 0$ $0000000+3000.00000: 000+3000.00000-000+3000.0$ $0000 \% 00+3000 \% 0000 \% 00+3000 \% 0000 \cdot 000+30000$ $0000 \cdot 000+3000 \cdot 0$ $0000.000+9000 \% 0000 \cdot 000 \times 3000 \cdot 0 \quad 0000 \cdot 0 \quad 00+3000.0$ perees pestrises 20exz $2 x /$ maxi.

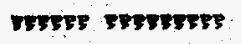

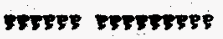
$0000 \div 00+3000 \% 0000 \cdot 000+3000 \cdot 0$ $0000 \cdot 000+3000 \cdot 0000 \% 00+3000 \cdot 0$ $0000.000+3000 \cdot 0 \quad 0000 \cdot 0 \quad 00+3000.0 \quad 15 t-n 3$ $0000 \% 00+3000.0 \quad 0000: 0 \quad 00+3000 \cdot 0$ z5t-ng $0000.000+3000 \cdot 0$ 0000.0 00+3000.0 LET-50 $0000000+3000 \% 0000 \div 00+3000 \% 0$ 09-05 $0000 \% 00+3000 \% 0000 \div 000 \times 3000 \cdot 0$. it-5 $0000 \div 000+8000 \% 0$ 0000.0 00+3000.0 Tiz-w

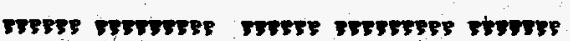

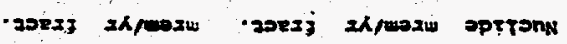

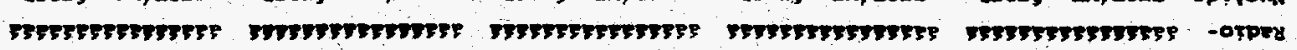
200w oureta uoperd บe? 3 searm

elenused suspuedoo seaven

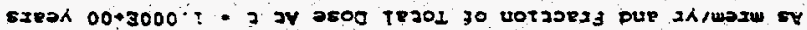

,

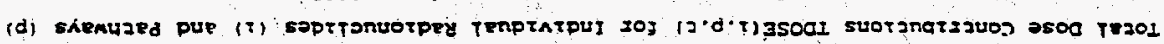

$0000.000+2000 \% 0000 \% 00+3000 \% 0$

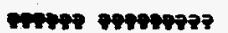
$0000.00045000 \% 0$ $0000 \cdot 000+8000 \cdot 0$ $0000 \cdot 000+9000: 0$ $0000.00+2000^{\circ} 0$ $0000^{-0} 00+2000^{\circ} 0$ $0000.000+3000 \% 0$ $0000.000+3000^{\circ} 0$ $0000 \cdot 0003000.0$ $0000.000+3000 \cdot 0$ $0000.00+3000.0$ $0000 \cdot 0.00+3000.0$ $0000.000+3000.0$ $0000.000+3000.0$ $0000.00+3000^{\circ} 0$ $0000^{\circ} 000+3000^{\circ} 0$

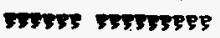
-25exj 2x/maxu mistrstsersegs tros - 708x3 2K/w024

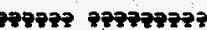
$0000.000+2000.0$ $0000.000+3000 \%$ $0000 \cdot 000+3000 \cdot 0$ $0000 \% 00+9000 \%$ $0000 \cdot 000+3000.0$ $0000.000+3000.0$ $0000.000+3000 \%$ $0000.000+9000.0$ $0000.000+3000.0$ $0000.00+3000.0$ $0000 \cdot 000+3000 \cdot 0$ $0000 \cdot 00+3000.0$ 0000.000 .3000 .0 $0000.000+3000.0$ $0000-0$ 00+3000-0

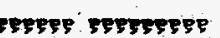
xTกต
$0000 \cdot 000+30000$

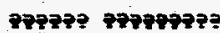
0000.0 00+3000.0 $0000.00+3000 \%$ $0000 \times 00+3000 * 0$ $0000.000+3000.0$ $0000.000+3000^{\circ} 0$ $0000 \% 00+3000 \cdot 0$ 0000 0043000\% $0000.0043000 \%$ $0000.000+300=0$ $0000: 0 \quad 00+300=0 \quad 0000 \cdot 0 \quad 00+3000 \cdot 0 \quad 0000 \cdot 0 \quad 00+3000 \%$ $0000.0 \quad 00+300=0 \quad 0000 \cdot 0.00+3000.0 \quad 0000 \cdot 000+3000.0$ $0000.000+300=:=0000: 0.00+3000 \cdot 0 \quad 0000 \% 000+3000 \%$ $0000 \cdot 0 \quad 00+300=0 \quad 0000 \cdot 000+3000 \div 0 \quad 0000 \div 00+3000=0$ $0,000 \cdot 0$ 00+30c: 0 0000:0 00+3000\% 0000.0 00+3000:0. $0000 \cdot 0,00+300=0.0000 .000+3000 \% 0000.000+3000 \%$

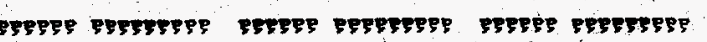

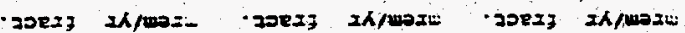

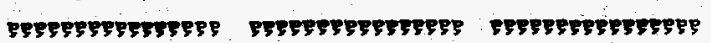
วEN

$0000000+300000000 \times 00+3000 \times 0$

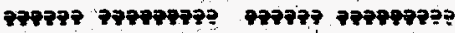

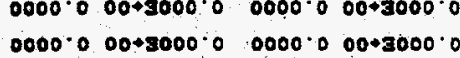
$0000.000 \times 3000: 0 \quad 0000.000 \times 3000.0$ $0000.0 \quad 00 \times 3000 \cdot 0 \quad 0000 \cdot 000+3000.0$ $0000000+3000 \cdot 0 \quad 0000 \% 00+3000 \%$ $0000 \div 00+3000 \cdot 0.0000 \cdot 0.00+300010$ $0000: 000+3000 \% 0000 \cdot 000+3000 \%$ $0000-000+3000.0 \quad 0000.000+3000.0$ $0000 \cdot 000+3000 \cdot 0$ $0000 \% 00+3000 \% 0000 \% 00+3000 \%$-5t-nz $0000.000+3000.0 \quad 0000.000+3000.0$ z5t-ns $0000: 0.00+3000 \% 0000 \% 00+3000 \%$ LET-ED $0000 \cdot 00+3000 \% 0000 \cdot 00+3000009-02$ $0000.00+3000 \% 0000.000 \times 3000.0$ ot. $0000 \% 00+3000 \% 0000.00+3000: 0$ tre-ur

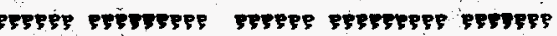

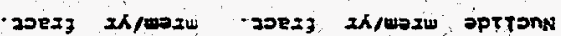

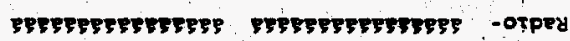
vot כetwert : punoxs

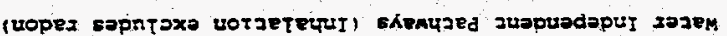

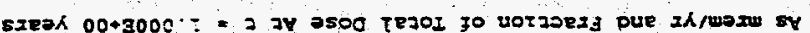

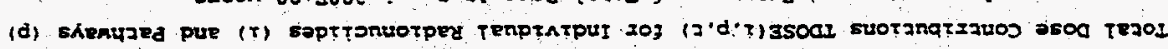

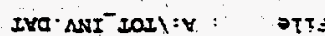

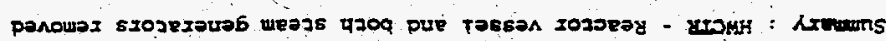

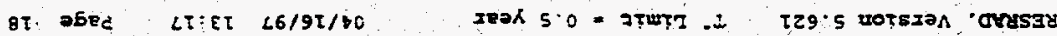


$0000.000+3000.00000 \cdot 000+3000.0$

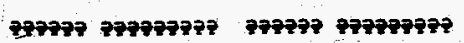
$0000.000+3000 \cdot 0 \quad 0000.000+3000.0$ $0000 \cdot 0.00+3000 \cdot 0 \quad 0000 \cdot 000+3000 \cdot 0$ $0000 \cdot 000+3000 \cdot 0 \quad 0000 \cdot 0.00+3000 \cdot 0$ $0000 \% 00+3000 \% 0000 \cdot 000+3000 \%$ $0000 \cdot 0.00+3000 \cdot 0 \quad 0000 \cdot 0 \cdot 00+3000 \cdot 0$ $0,000 \div 00+3000 \cdot 0 \quad 0000 \div 000+3000 \% 0$ $0000 \% 00+3000 \% 0000.000+3000 \% 0$ $0000 \div 000+3000 \cdot 0 \quad 0000 \div 00+3000.0$ $0000 \cdot 000+3000 \cdot 0 \quad 0000-000+3000 \cdot 0$ $0000 \cdot 00+3000 \% 0000 \% 00+2000 \% 0$ $0000 \cdot 000+3000.0 \quad 0000 \div 000+3000 \cdot 0$ $0000 \% 00+3000.0 \quad 0000.000+8000 \%$ $0000 \div 000+3000 \% 0000 \% 00+9000 \%$ $0000.000+2000.0 \quad 0000.000+2000.0$ $0000: 000+3000.00000000+3000.0$

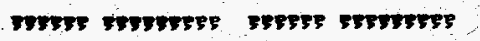

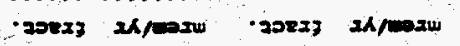

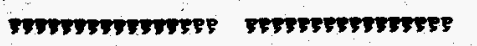
- exentraed TTV หกเพ
$0000 \div 00+3000 \cdot 0$

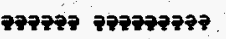
$0000.000+3000.0$ $0000 \cdot 000+3000: 0$ $0000 \cdot 000+3000.0$ $0000 \cdot 000+2000.0$ $0000: 000+9000 \%$ $0000-000+3000$ $0000 \cdot 000+3000 \cdot 0$ $0000: 00+3000 \div 0$ $0000: 000+2000.0$ $0000^{\circ} 000+8000.0$ $0000.000+8000^{\circ} 0$ $0000.00+2000.0$ $0000.000+2000.0$ $0000.000+8000.0$ $0000.000 \cdot 3000 \%$

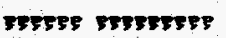
asez 2 a/oris. srrpstriscurves anow
$0000 \cdot 00+3000 \cdot 0$

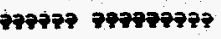
$0000: 00+3000 \div 0$ $0000.00+3000 \%$ $0000 \cdot 0 \quad 00+3000: 0$ $0000 \div 000+3000: 0$ $0000.00+3000.0$ $0000 \cdot 00+3000.0$ $0000 \% 00+3000 \% 0$ $0000.000+3000.0$ $0000 \cdot 0003000 \% 0$ $0000-000+2000=0$ $0000.00+3000 \%$ $0000.000+9000 \% 0$ $0000.00+3000.0$ $0000.000+2000 \cdot 0$ $0000000+2000=0$ trrese restress -2oexs skpmext meverstruste aterd
$0000 \cdot 000+3000 \% 0$

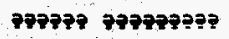
$0000.00 \div 3000 \%$ $0000000+30000$ $0000000+3000 \%$ $0000 \div 000+3000 \div 0$ $0000 \% 00 \times 3000 \%$ $0000 \cdot 00+30000$ $0000 \div 00+3000 \cdot 0$ $0000.000+30000$ $0000-000+3000.0$ $0000.0009000 \%$ $0000.00+3000 \%$ $0000 \cdot 00+3000.0$ $0000.00+3000.0$ $0000000+3000.0$ $0000000+3000$ : Trsere sereseres. -20exz xA/ewis. stereterstreses. uopry
$0000 \div 0 \cdot 00+3000 \cdot 0$

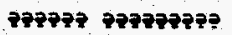
$0000 \div 00 \times 3000 \%$ $0000.000 \times 3000 \%$ $0000000+3000 \%$ $0000.00+3000.0$ $0000000+30000$ $0000000+30000$ $0000.000 \times 3000 \% 0$ $0000 \cdot 00+3000 \%$ $0000 \% 00+3000 \% 0$ 0000 0 004apoo 0 0000 0 00+3000.0 $0000.000+2000.0$ 0000 0 0098000.0 $0000.000 \times 2000.0$ $0000^{\circ} 0002000.0$ crefes ereverses -25eis 2x/mosu creverserswepe पธतु
$0000 \cdot 000+3000 \cdot 0 \quad$ TE20I

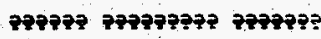
0000 0 00+3000 0 eEz-n $0000 \cdot 000+3000 \%$ sez-n $0000 \% 00+3000 \%$ vez-n $0000 \div 00+3000 \% 06-25$ $0000-000+3000 \cdot 0$ 6ez-nd $0000^{\circ} 000+2000^{\circ} 0$ gez-nd $0000 \cdot 0.00+3000: 0$ E9-TN $0000 \cdot 000+3000 \cdot 0$ 65-7N $0000.00+3000$ O E-H $0000 \cdot 0$ 004a000.0 STt-ng 0000 0 00+a000.0 zst-ng $0000 \cdot 000+3000^{\circ} 0$ LET-85 0000.00043000 .0 09-02 $0000.000+3000 \cdot 0$ ו $0000 \cdot 0.004 a 000.0$ tre-we

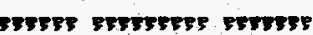
-2oesz IA/mozw optTon

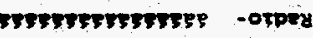
xazen

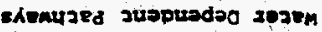

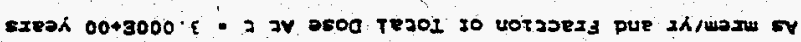

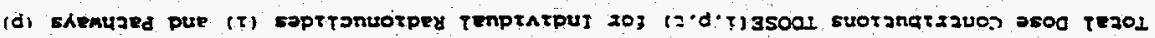

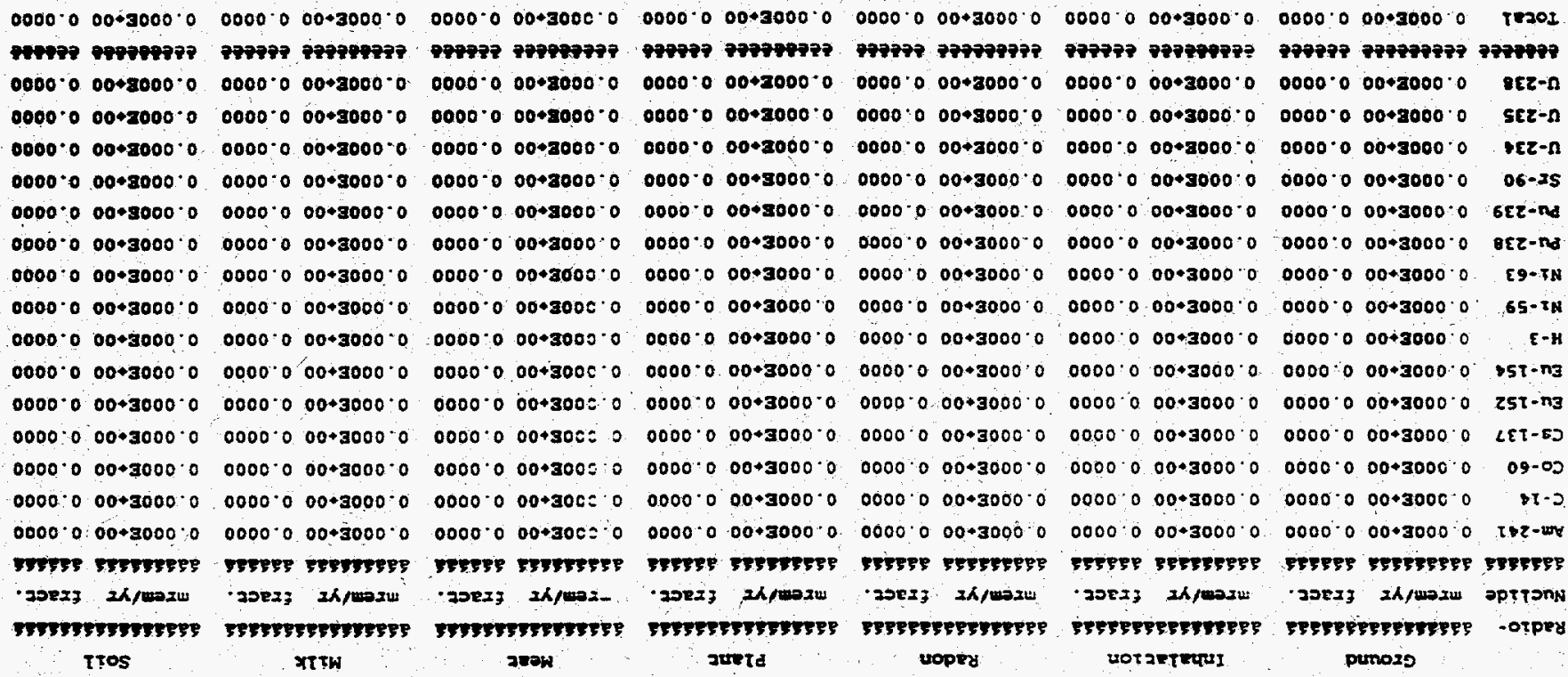

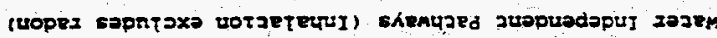

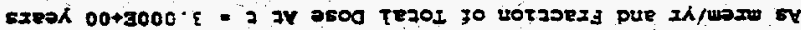

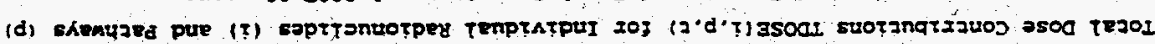

IRO MUI

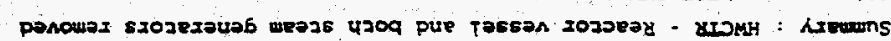

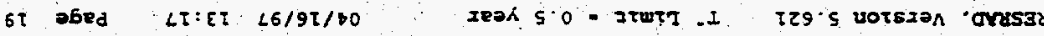


RESRAD, Version $5.621 \quad T^{\circ}$ Limit $=0.5$ year $04 / 16 / 97 \quad 13: 17$ Fage 20

Summiny : HWCrR - Reactor vessei and both scean generacors irenoved

File : A:ITOT_INV.DAT

Total Dose Contributions TDOSE(i,p.c) for Individual Radionuelides (i) and pachways (p) As mrem/yr and Fraction of Total Dose Ac $t=2.000+01$ years

Water Independent Pathways (Inhalation excizdes radon

\begin{tabular}{|c|c|c|c|c|c|c|c|c|c|c|c|c|c|c|}
\hline \multirow{2}{*}{$\begin{array}{l}\text { Radio- } \\
\text { Nuelide } \\
\text { adsuas }\end{array}$} & & \multicolumn{2}{|c|}{ Inhalacion } & & & Maskisa & \multicolumn{2}{|l|}{ Neec } & & \multicolumn{2}{|l|}{ Soll } \\
\hline & & Erac & $\begin{array}{c}\text { mrea/yr } \\
\text { adadstsas }\end{array}$ & $\begin{array}{l}\text { zraec. } \\
\text { asaaas }\end{array}$ & $\begin{array}{l}\text { mrem/yr } \\
\text { asaakasa }\end{array}$ & $\begin{array}{l}\text { frace. } \\
\text { saAasa }\end{array}$ & $\begin{array}{l}\text { nrem/yr } \\
\text { adaspasas }\end{array}$ & $\begin{array}{l}\text { fraec. } \\
\text { datasa }\end{array}$ & $\begin{array}{c}\text {-tem/yr } \\
\text { dadsasada }\end{array}$ & $\begin{array}{l}\text { fract. } \\
\text { asasaa }\end{array}$ & $\begin{array}{c}\text { mrem/yr } \\
\text { adasarsas }\end{array}$ & $\begin{array}{l}\text { frace. } \\
\text { asasas }\end{array}$ & $\begin{array}{c}\text { mrem/yr } \\
\text { asascasas }\end{array}$ & $\begin{array}{l}\text { Erace. } \\
\text { sasase }\end{array}$ \\
\hline$a-242$ & $0.000 \varepsilon+00$ & 0.0000 & $.0008=00$ & 0.0000 & $E * 00$ & 0.0000 & 0 & 0.0000 & $=208$ & 0.0 & 0 & 0 & $E+0.0$ & 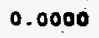 \\
\hline 4 & c. $000 \mathrm{E}+00$ & 200 & .00 & bo. & $.000 E+00$ & 0 & 10 & 00 & 0. & 0. & 0 & & 10 & 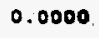 \\
\hline.-60 & $0.0005+00$ & 000 & .00 & jo & $E+00$ & 1000 & 10 & 1000 & $=$ & 0 & 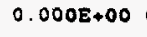 & & 0 & 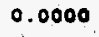 \\
\hline 37 & $.0008+00$ & 0.0000 & $0 \varepsilon+00$ & $0 .$. & $.0008+00$ & 000 & 0 & 0 & $\approx$ & $?$ & 10 & & & \\
\hline 52 & $.0008+00$ & 0.0000 & 8.00 & 0. & 0 & 000 & 10 & 0 & 10 & 0 & $+\infty$ & & 0 & 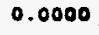 \\
\hline $2-254$ & $.0005+00$ & 0.0000 & .00 & 0.0000 & .00 & 0.0000 & .00 & 0 & $8+00$ & 0 & 20 & & 10 & 0 \\
\hline-3 & $0.000 E+00$ & 0.0000 & $8+00$ & 0.0000 & +00 & 0.0000 & $0.0008+00$ & 0. & $8+00$ & & 00 & & 10 & 0 \\
\hline $1-59$ & $0.000 z+00$ & 0.0000 & $0.0008+00$ & 0.0000 & $8+00$ & 0.0000 & $E+00$ & 0. & $0.2008+00$ & 0 & $E+00$ & 0 & 20 & 0 \\
\hline $1-63$ & $0.0005+00$ & 0.0000 & $.000 E+00$ & 0.0000 & $0008 \cdot 00$ & 000 & 0.00 & 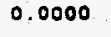 & 00 & 0 & 0.0 & 8 & .00 & 0 \\
\hline $12-238$ & $0.0008+00$ & 0.0000 & $.0008 * 00$ & 0.0000 & $.000 E+00$ & 0.0000 & $0.000 E+00$ & 0 & $=008+00$ & 0 & $8+00$ & 00 & 00 & 0 \\
\hline $1-239$ & $0.000 \Sigma+00$ & 0.0000 & $0.000 \varepsilon+00$ & 0.0000 & 0.0008 .00 & 0.0000 & $0.000 E+00$ & 0. & $0.0008+00$ & 100 & $0.0008+00$ & 00 & $2+00$ & 0. \\
\hline$r-90$ & $0.000 E+00$ & 0.0000 & $0.0008+00$ & 0.0000 & $0.0008 \times 00$ & 0.0000 & $0.000 E+00$ & 0.0000 & $0.000 E+00$ & 0.0000 & $0.0008+00$ & 0.0000 & 0.0005000 & 0.0000 \\
\hline-234 & $0.0005+00$ & 0.0000 & $.000 \varepsilon \bullet 00$ & 0.0000 & $0.000 E+00$ & 0.0000 & $0.0008+00$ & 0.0000 & $0.3008+00$ & 0.0000 & $0.000 \mathrm{E}+00$ & 0.0000 & $0.0008+00$ & 0.0000 \\
\hline-235 & $0.0008+00$ & 0.0000 & $.0008 \cdot 00$ & 0.0000 & $0.000 E+00$ & 0.0000 & $0.0008+00$ & 0.0000 & $0.0008 \bullet 00$ & 0.0000 & $0.00018+00$ & 0.0000 & $0.000 \mathrm{~B}+00$ & 0.0000 \\
\hline-239 & $0.0008 * 00$ & 0.0 & $.0005 * 00$ & 0.0000 & $108+00$ & 0.0000 & $0.0008+00$ & 0 & $28 \bullet 00$ & 00 & $0.000 E+00$ & 0.0000 & $0.0008+00$ & 0.0000 \\
\hline & etectedet & ede & Let & ejectes & et & हe & scetes & $=$ & exeet & e & exceteres & ee & tetebetes & 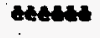 \\
\hline eal & $0: 000 E+00$ & & $0.000 E \cdot 00$ & 0.0000 & $0.0008 \cdot 00$ & 0.0000 & $0.0008 \div 00$ & 0.0000 & $200 E+00$ & 0.0000 & $0.000 E \bullet 00$ & 10 & $000 E+00$ & \\
\hline
\end{tabular}

Total Dose Contrzbutzons TDOSE (2,p, fi for Individual Radionvelides (2) and Pathways (p) As mrem/yr and Fraetion of Toeal Dose Ac $c: 000 E+01$ years

Wacer Dependent Pathways

\begin{tabular}{|c|c|c|c|c|c|c|c|c|c|c|c|c|c|c|}
\hline \multirow{2}{*}{$\begin{array}{l}\text { Radio- } \\
\text { thelite } \\
\text { adistat }\end{array}$} & \multicolumn{2}{|c|}{ 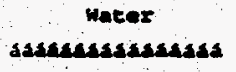 } & \multicolumn{2}{|c|}{ 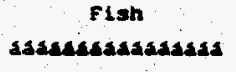 } & \multicolumn{2}{|c|}{ 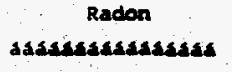 } & \multicolumn{2}{|c|}{ 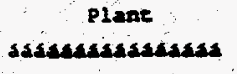 } & \multicolumn{2}{|c|}{$\begin{array}{c}\text { Ment } \\
\text { disuranasadasas }\end{array}$} & \multicolumn{2}{|c|}{ 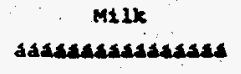 } & \multicolumn{2}{|c|}{ 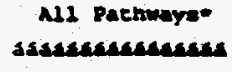 } \\
\hline & & & $\begin{array}{l}\text { yr } \\
\text { asa }\end{array}$ & & $x$ & & rai & & as & & 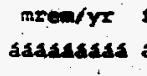 & & a & \\
\hline & & & $8+00$ & & 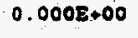 & & & & & & 00 & & & \\
\hline & 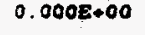 & & 0 & & .00 & & ? & & 0 & & 00 & & & \\
\hline & & & 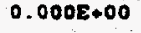 & & 10 & & 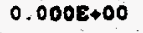 & & $\bullet 00$ & & 0 & & & \\
\hline & & & & & & & & & & & & & & \\
\hline & & & & & & & & & & & & & & \\
\hline & & & & & & & & & & & & & & \\
\hline & & & & & & & & & & & & & & \\
\hline & & & & & & & & & & & & & & \\
\hline & & & & & & & & & & & & & & \\
\hline & & & & & & & & & & & & & & \\
\hline & & & & & & & & & & & & & & \\
\hline & & & & & 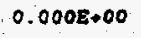 & & & & & & & & & \\
\hline & & & o & & 0 & & & & & & & & & \\
\hline & & & 0 & & & & & & & & 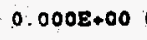 & & & \\
\hline & & . & 0 & & 0 & & 0 & & 0 & & 00 & & & \\
\hline & & & & & & & & & & & & & & \\
\hline Scat & $E+00$ & 00 & $E \bullet 00$ & 100 & $0.000 \mathrm{E} * 00$ & ov & $E+\infty$ & 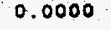 & $0.000 \mathrm{E}+00:$ & 00 & $0.0008+00$ & 0 & $0.000 z+00$ & . \\
\hline
\end{tabular}


$0000 \cdot 000+3000 \cdot 0 \quad 0000 \cdot 000+3000 \div 0$

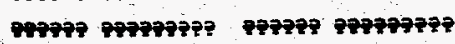
$0000.000+2000: 0 \quad 0000 \div 000+3000.0$ $0000 \cdot 000+2000 \cdot 0 \quad 0000-000+3000 \cdot 0$ $0000 \cdot 000+3000 \% 0000 \div 00+3000=0$ $0000.000+3000: 0 \quad 0000 \cdot 000+3000 \cdot 0$ $0000.000 \times 3000.00000 .000+3000-0$ $0000 \div 00+3000 \% 0000 \div 000+3000 \div 0$ $0000 \cdot 000+3000 \cdot 0 \quad 0000 \div 00+3000 \cdot 0$ $0000 \div 00+8000: 0$ 0000 $000 \times 3000 \div 0$ $0000 \cdot 000 \cdot 2000 \times 00000000+3000 \% 0$ $0000-000+9000 \div 0000 \div 000+3000 \cdot 0$ $0000 \cdot 000+2000 \% 0000 \cdot 00+2000 \% 0$ $0000 \cdot 000+3000 \cdot 0000000+3000 \cdot 0$ $0000 \cdot 0$ 00+8000\% $0000 \cdot 0.00+3000 \%$ $0000.000+2000 \% 0000 \% 00+2000 \%$ $0000000+2000 \% 0000 \% 00+3000 \%$

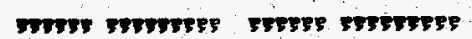

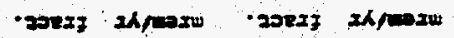

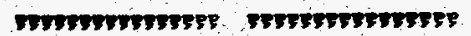
exexaysed tTY xtris.
$0000: 000+3000 \cdot 0$

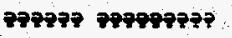
$0000 \cdot 000+2000 \cdot 0$ $0000 \cdot 000+2000 \cdot 0$ $0000.00+3000.0$ $0000.000+2000 \cdot 0$ $0000.000+8000.0$ 0000.000420000 $0000 \cdot 000+3000 \cdot 0$ $0000000+2000 \cdot 0$ $0000.000+2000 \%$ $0000 \cdot 000+2000 \cdot 0$ $0000 \% 00+2000: 0$ $0000 \% 00+2000 \%$ $0000.000+8000.0$ $0000 \cdot 0.00+2000^{\circ} \circ$ $0000.000+2000^{\circ} 0$

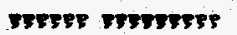
-aoexz zh/ment Frsentrensese 20ew
$0000 \% 000+3000 \%$

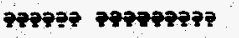
$0000 \div 000 \times 3000 \div 0$ $0000 \cdot 000+3000 \% 0$ $0000.000+3000.0$ $0000 \cdot 000+3000 \cdot 0$ $0000 \cdot 000+3000 \cdot 00000 \cdot 0$ 00+3000.0 $0000.000+300000000000+3000=0$ $0000.000+3000 \% 0000.000+3000 \% 0$ $0000 \% 00+3000 \% 0000 \% 00 \times 3000 \%$ $0000 \% 00+3000 \% 0000 \div 000+3000 \%$ $0000 \% 00+3000 \% 0000: 000+3000 \% 0$ $0000 \% 00+3000 \% 0000 \cdot 000+3000.0$ $0000 \% 00+3000 \% 0000 \div 00+3000 \%$ $0000.000+3000.00000 .000+2000.0$ $0000.00042000 .0 \quad 0000.000+2000.0$ $0000.000+2000.00000 \times 00+2000.0$

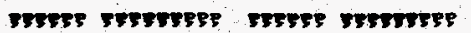
- 200xz xh/ueriw meststisumes asezz anpers Triversursestep uopry
$0000 \cdot 000+3000 \cdot 0000000+3000 \%$ TE20I

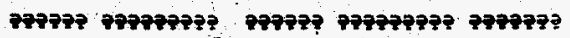
$0000.000+5000.00000 \cdot 000+30000$ ecz- 0 $0000000+300000000 \cdot 000+30000$ sez-n $0000: 000+300000000.000+30000$ bez-n 0000:0 00+3000 $0 \quad 0000 \cdot 000+3000 \cdot 0$ 06-xs $0000 \% 00+3000 \cdot 0$ 0000.0 00+3000.0 6rz-na $0000000+3000.00000 \cdot 000+3000 \%$ 8Ez-nd $0000 \% 00+3000 \% 0000 \% 00+3000 \%$ E9-5M $0000000+3000 \% 0000.00+3000 \%$ o 65-7f $0000 \% 00 \times 3000 \% 0000 \% 00 \times 3000$ C E-H $0000.000+3000: 0 \quad 0000.000+3000.0$ ist-nz $0000.000+3000.0 \quad 0000.000+3000.0 \quad z s \tau-n 3$ $0000: 000+3000 \cdot 0$ 0000:0 00+3000:0 LET-s0 $0000.00+3000 \% 0 \quad 0000.000+2000 \%$ os.0J $0000 \% 00+2000 \% 0 \quad 0000000+2000 \% 0$ > $\tau-5$ $0000.000+3000 \cdot 0 \quad 0000 \cdot 000+3000.0$ IVz-m

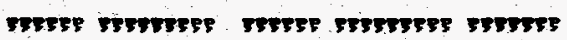

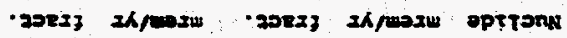

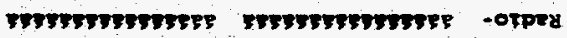
पะts

enenuzed zuepuedeo josen

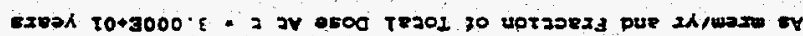

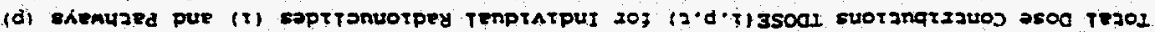

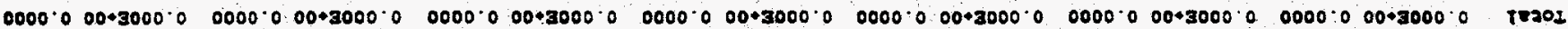

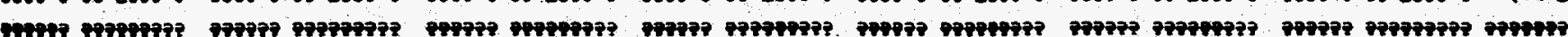

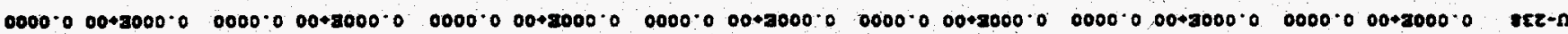

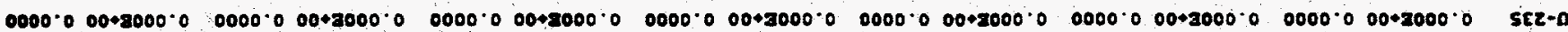

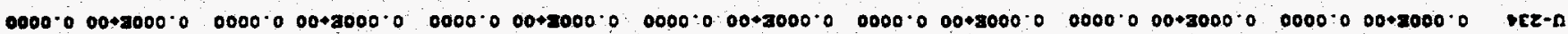

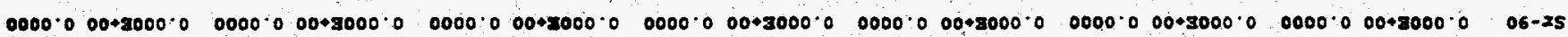

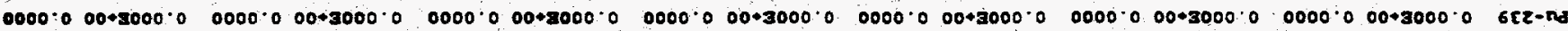

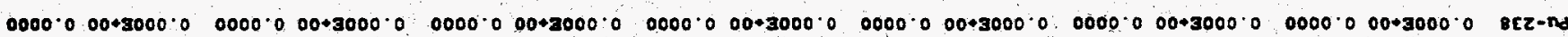

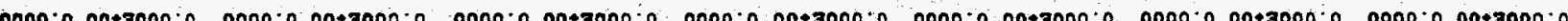

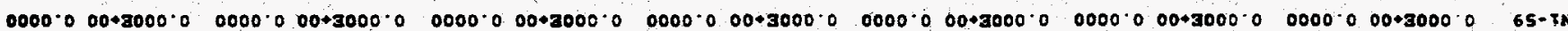

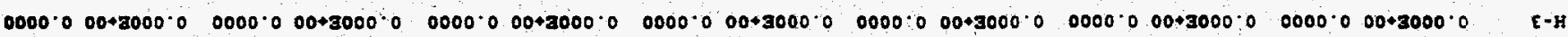

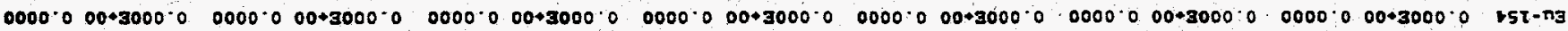

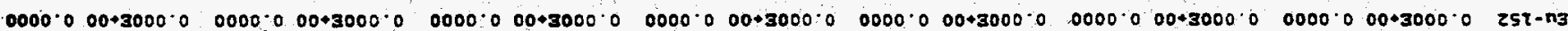

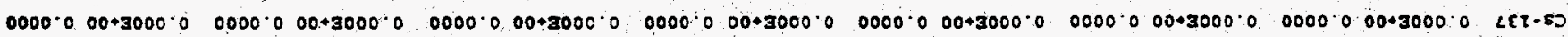

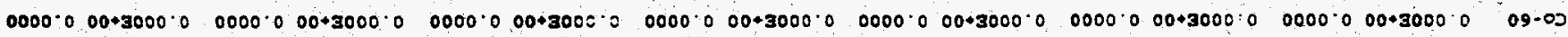

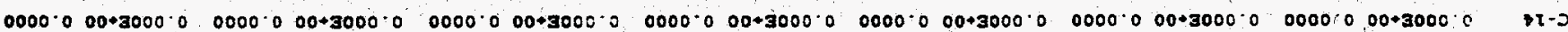

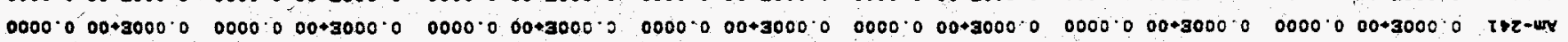

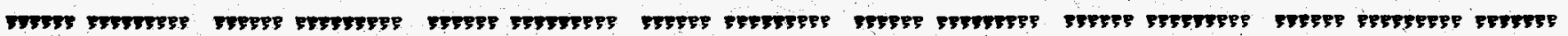

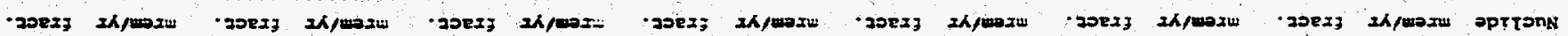

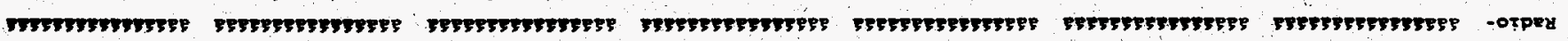

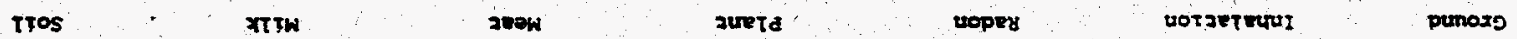

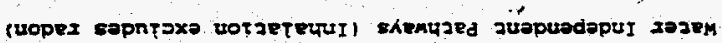

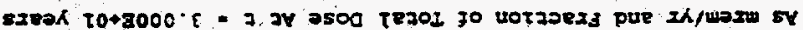

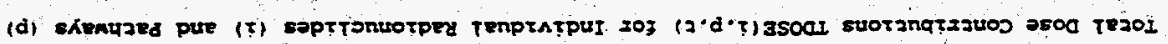

IXO ANITIOIl:Y : :TT]

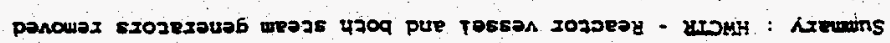

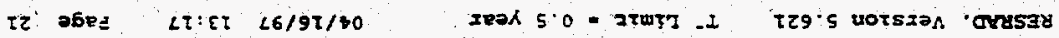


REsRap. Version 5.622 T. Limic $=0.5$ yeaz $04 / 16 / 97 \quad 13: 17$ Page. 22

Sumniry : HWCTR - Reaccor vessel and boch scean generacors removed

File : A:ITOT INV.DAT

Total Dose Contr2butions Toose(i.p.t) for Individual Radionuclides (i) and Pachways (p)

As mrem/yr and Fraction of Total Dose At $c=1.000 E+02$ years

water Independent Pachways inhalation exciudes radon

\begin{abstract}
Ground
\end{abstract}
Radio- áasusadasassas

Nuclide mrem/yr frace.

asasass dadastada saada

Am-241 $0.000 E 000 \quad 0.0000$

$0.14 \quad 0.0008 .000 .0000$

C0-60 $0.2008+00 \quad 0.0000$

Cs-137, $0.0008+00 \quad 0.0000$

Eu-is2 $0.0008+00 \quad 0.0000$

Eu-154 $0.000 E+00 \quad 0.0000$

$H-3 \quad 0.0008+00 \quad 0.0000$

Ni-59 $0.000 \mathrm{E}+00 \quad 0.0000$

$\begin{array}{llll}N i-63 \quad 0.000 E .00 & 0.0000\end{array}$

$\begin{array}{lll}P & -238 \quad 0.000 E \cdot 00 \quad 0.0000\end{array}$

Pu-239 $0.0008+00 \quad 0.0000$

Sx-90 $\quad 0.0008+00 \quad 0.0000$

U-234 $\quad 0.0008 .00 \quad 0.0000$

u-235 $0.0008+00 \quad 0.0000$

U-230 $\quad 0.0008+00 \quad 0.0000$

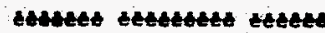

Toen $0.0008+00 \quad 0.0000$
Inhalacion

sasatsasasasasa

mrem/yr fract.

\section{jasassada sadasa}

$0.000 E+00 \quad 0.0000$

$0.000 \varepsilon+00 \quad 0.0000$

$0.000 E+00 \quad 0.0000$

$0.000 E+00000,00$

$0.000 \varepsilon+00 \cdot 0.0000$

$0.006=000.0000$

$0.0008+00 \quad 0.0000$

$0.000 E+00 \quad 0.0000$

$0.0008+00 \quad 0.0000$

$0.0008 .00 \quad 0.0000$

$0.000 E+000.0000$

$0.000 E+000.0000$

$0.0008+00 \quad 0.0000$

$0.0008+00 \quad 0.0000$

$0.0005+00 \quad 0.0000$

eschetect cuthas

$0.0005+00 \quad 0.0000$
Radon

adasusatadatias

mrem/yr erace.

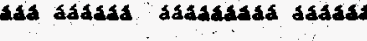

$0.0008+00 \quad 0.0000 ; 0.0008+00 \quad 0.0000$

$0.0000 \quad 0.0008+00 \quad 0.0000$

$0.000 \varepsilon+00 \quad 0.0000 \quad 0.000 \varepsilon+00 \quad 0.0000$

$0.0008+00 \quad 0.0000 \quad 0.0008+00 \quad 0.0000$

$0.000 E+00 \quad 0.0000 \quad 0.0005+00 \quad 0.0000$

$0.000 E+00 \quad 0.0000 \quad 0.0008+00 \quad 0.0000$

$0.0008+00 \quad 0.0000 \quad 0.0008 .00 \quad 0.0000$

$0.0008+00 \quad 0.0000 \quad 0.0008+00 \quad 0.0000$

$0.0008+00 \quad 0.0000 \quad 0.0008+00 \quad 0.0000$

$0.0008+00 \quad 0.0000 \quad 0.0005+00 \quad 0.0000$

$\begin{array}{llllll}0.0008+00 & 0.0000 & 0.0008+00 & 0.0000\end{array}$

$0.0008 \times 00 \quad 0.0000 \quad 0.0008+00 \quad 0.0000$

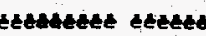

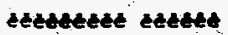

$0.000 E+00 \quad 0.0000 \quad 0.000 E+00 \quad 0.0000$
Meac

adasussasasassa

-rem/yr trace.

sassissaa sasas

$0.000 E 000 \quad 0.0000$

$0.0008+00 \quad 0.0000$

$0.0008+000.0000$

$0.0008+00 \quad 0.0000$

$0.0008+00 \quad 0.0000$

$0.0008 .00 \quad 0.0000$

$0.0008+00 \quad 0.0000$

$0.0008+00 \cdot 0.0000$

$0.0008+00 \quad 0.0000$

$0.0008+00 \quad 0.0000$

$0.0008+00 \quad 0.0000$

$0.0008+00 \quad 0.0000$

$0.0008+00 \quad 0.0000$

$0.0008+00 \quad 0.0000$

$0.0005+000.0000$

cstertetet estede

$0.000 \mathrm{E}+000.0000$
Mi2k

\section{adsugasadasadas}

mrem/yr fraet.

aasusasas asasas

$0.0008+00 \quad 0.0000$

$0.0008+00 \quad 0.0000$

$0.0008+00 \quad 0.0000$

$0.0008+00 \quad 0.0000$

$0.000 \varepsilon+00 \quad 0.0000$

$0.0008+00 \quad 0.0000$

$0.0008+00 \quad 0.0000$

$0.0008+00 \quad 0.0000$

$0.0005+00 \quad 0.0000$

$0.0008+00 \quad 0.0000$

$0.0008+00 \quad 0.0000$

$0.0008+00 \quad 0.0000$

$0.0008+00 \quad 0.000$

$0.0008 .00 \quad 0.0000$

$0.0008+00 \quad 0.0008$

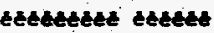

$0.0000+000.0000$
Soil

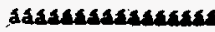

mrem/yr frace. atasasass stasu

$0.0008+00 \quad 0.0000$

$0.0008+00 \quad 0.0000$

$0.000 E+000.0000$

$0.0008+00 \quad 0.0000$

$0.0008+00 \quad 0.0000$

$0.000=000 \quad 0.0000$

$0.0008+00 \quad 0.0000$

$0.0008 .00 \quad 0.0000$

$0.0008+000.0000$

$0.0008 .00 \quad 0.0000$

$0.0008+00 \quad 0.0000$

$0.0008+00 \quad 0.0000$

$0.0008+00 \quad 0.0000$

$0.000 z+00 \quad 0.0000$

$0.0008+00 \quad 0.0000$

zecensere sesur

$0.000 E+00 \quad 0.0000$

Total Dose Coneribuezons TDOSE (i.p.e) for Indiviqual Radsonuelzdes ( 1 ) and Pathways (p) As mrem/yr and fraction of Total Dose Ae $c \cdot 1.0008+02$ years

Wácer Dependent Pachwaye

Hater

Radio- damesasadasad

Suclide mreiv/yr trace.

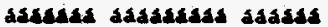

$\operatorname{sm} 2410.0008+00 \quad 0.0000$

c. $24: 0.0008+00 \quad 0.0000$

co-60 $0.0008+00 \quad 0.0000$

Cs-137 $\quad 0.000 E+00 \quad 0.0000$

$\begin{array}{llll}\text { Eu-252 } & 0.0008+00 \quad 0.0000\end{array}$

Eu-154 $0.0008+00 \quad 0.0000$

H-3

Ni-59

$0.0008+00 \quad 0.0000$

$\begin{array}{lll}N i-63 & 0.000 E+00 & 0.0000\end{array}$

Pu-238 $0.0008+00 \quad 0.0000$

Pu-239 $0.0008+00 \quad 0.0000$

SF-90 $\quad 0.000 E+00 \quad 0.0000$

$0.234 \quad 0.0008+00 \quad 0.0000$

U-235 $0.0008+00: 0.0000$

U.238 $0.000 E+00 \quad 0.0000$

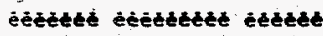

Total $0.0008+00 \quad 0.0000$
Finh

adsumsandasuas

mroin/yr trace.

sadusad dasids

$0.0008+00 \quad 0.0000$

$0.0008+00 \quad 0.0000$

$0.0008+00 \quad 0.0000$

$0.0008+00 \quad 0.0000$

$0.0008+00 \quad 0.0000$

$0.0005+00 \cdot 0.0000$

$0.000 E+00 \quad 0.0000$

$0.000 E+00 \quad 0.0000$

$0.000 E+00 \quad 0.0000$

0.00050000 .0000

$0.0002+00 \quad 0.0000$

$0.0008+00 \quad 0.0000$

$0.000 E+00 \quad 0.0000$

$0.000 E+00 \quad 0.0000$

$0.0008+00 \quad 0.0000$

dejètest téètth

$0.000 E+00 \quad 0.0000$
Radion

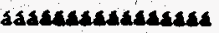

mrew/yr fract.

dadsucisa sasasa

$0.0005+00 \quad 0.0000$

$0.0008 \div 00 \quad 0.0000$

$0.0008+00 \quad 0.0000$

$0.0008+000.0000$

$0.0000+00 \quad 0.0000$

$0.0008+00 \quad 0.0000$

$0.0008+00 \quad 0.0000$

$0.000 E+00 \quad 0.0000$

$0.0008+00 \quad 0.0000$

$0.000 E+00 \quad 0.0000$

$0.0008+00 \quad 0.0000$

$0.0008+00 \quad 0.0000$

$0.000 E+00 \quad 0.0000$

$0.0008+00 \quad 0.0000$

$0.000 E+00 \quad 0.0000$

ejectetyet etetet

$0.000 E+00 \quad 0.0000$
Plane

dassagasastages

mrem/yr frace:

adassasa sasua

$0.000 \mathrm{E}+00 \quad 0.0000$

$0.0008+00 \quad 0.0000$

$0.0008+00 \quad 0.0000$

$0.0008+000.0000$

$0.0008+00 \quad 0.0000$

$0.000 E+00 \quad 0.0000$

$0.0008+00 \quad 0.0000$

$0.0008+00 \quad 0.0000$

$0.000 E+00 \quad 0.0000$

$0.0008+000.0000$

$0.000 E+00 \quad 0.0000$

$0.000 E+00 \quad 0.0000$

$0.0008+00 \% 0.0000$

$0.000 E+00 \quad 0.0000$

$0.000 \mathrm{E}+00 \quad 0.0000$

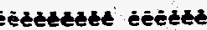

$0.0008+000.0000$
Mene

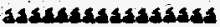

mrea/yt eract.

sasusasta sasala

$0.0008+00 \quad 0.0000$

$0.0005+00 \quad 0.0000$

$0.000 E+00 \quad 0.0000$

$0.0008+00 \quad 0.0000$

$0.0008+00 \quad 0.0000$

$0.0008+00 \quad 0.0000$

$0.000 E+00 \quad 0.0000$

$0.0008+00 \quad 0.0000$

$0.0008+00 \quad 0.0000$

$0.0008+00 \quad 0.0000$

$0.0008+00 \quad 0.0000$

$0.000 E+00 \quad 0.0000$

$0.000 E+00 \quad 0.0000$

$0.000 E+00 \quad 0.0000$

$0.000 \mathrm{E}+00 \quad 0.0000$

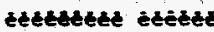

$0.0000+00 \quad 0.0000$
Milk

adsusuastansas

mrew/ye Exace.

adasudasa dasala

$0.0008+00 \quad 0.0000$

$0.0008+00 \quad 0.0000$

$0.000 E+00 \quad 0.0000$

$0.000 E+00 \quad 0.0000$

$0.0008+00 \quad 0.0000$

$0.0008+00 \cdot 0.0000$

$0.0008+00 \quad 0.0000$

$0.000 E+00 \quad 0.0000$

$0.000 E+00 \quad 0.0000$

$0.0008+00 \quad 0.0000$

$0.0008+000.0000$

$0.0008+00 \quad 0.0000$

$0.000 E+00 \quad 0.0000$

$0.0008+00 \quad 0.0000$

$0.000 \varepsilon+00 \quad 0.0000$

éettecest éectet

$0.0008+00 \quad 0.0000$

Al] Paemway

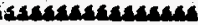

mrea/re sract.

dasumsas disus

$0.0008+00 \quad 0.0000$

$0.0008+00 \quad 0.0000$

$0.0008+00 \quad 0.0000$ 


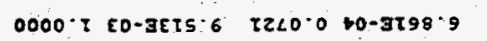

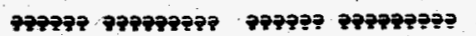
$0000 \cdot 00+2000 \% 0000 \cdot 0.00+3000 \cdot 0$ $0000 \div 00+3000 \cdot 0 \quad 0000 \div 00+3000 \%$ $0000 \cdot 00+3000 \cdot 0 \quad 0000 \cdot 000+3000 \cdot 0$ $0000.000+3000 \% 0000.000+3000.0$ $0000 \cdot 000+3000.0,0000 \% 00+3000 \% 0$ $0000 \div 00+3000 \cdot 0-0000.000+3000 \% 0$ $0000 \div 0 \quad 00+3000 \div 0000 \% 0 \quad 00+3000.0$ $0000.000+3000.00000 \% 00+3000 \%$

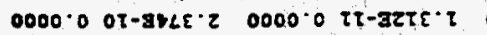
$0000.000+3000 \cdot 0 \quad 0000 \cdot 000+3000.0$ $0000 \div 0 \quad 00+3000 \cdot 0 \quad 0000 \div 0 \quad 00+3000.0$ $0000 \% 00+3000 \% 0 \quad 0000=00+3000 \% 0$ $0000 \div 000+3000.0 \quad 0000 \cdot 000+3000 \%$ 0000 'T co-acts: 6 $0000: 0$ 00+3000 0 rwest renceres -20es3 2א/mezw

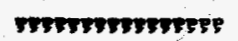
exenurea TTY
I2<0.0 $>0-3 \pi 98.9$ $0000 \cdot 000+3000 \cdot 0$ 5respe Prestepes -20Exz 2h/waxu

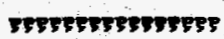
xา เพื $\therefore$.

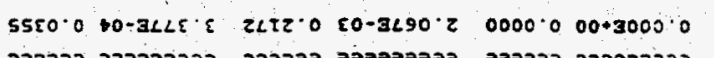

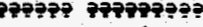
$0000.000+3000.0$ $0000: 00+3000 \cdot 0$ $0000 \cdot 000+3000 \cdot 0$ $0000 \cdot 000+3000 \div 0000 \div 0003000 \%$ $0000: 000+3000 \cdot 0 \quad 0000 \cdot 000+3000 \cdot 0$ $0000 \% 00+3000 \% 0000 \% 00+3000 \% 0000000+3000 \%$ 0000.0 zI-3tEs. $20000 \cdot 0$ IT-3Lr0. $0000 \% 00+3000 \%$ $0000 \% 00+9000 \% 0000000+3000-0 \quad 0000 \cdot 000+3000 \%$ $0000000+3000 \% 0000 \% 00+3000 \% 0000 \% 00+3000 \%$ $0000 \% 00+3000.00000 .000+2000.0 \quad 0000.000+3000.0$ $0000 \% 00+3000 \% 0000 \% 00+3000 \% 0000 \% 00+30000$ S5E0:0 to-3LLE:E zLTZ.0 C0-3L90.Z 0000-0 00+3000:0 $0000.000 \times 9000 \% 0000 \% 00 \times 9000 \% 0000 \% 00+3000 \%$

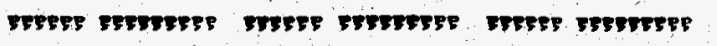

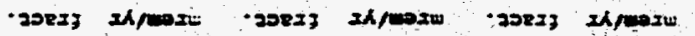

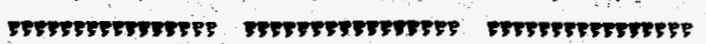
20\%W atretd uepry

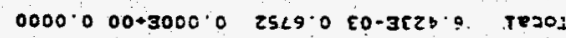

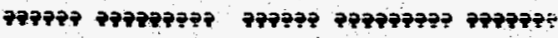
$0000 \% 00+3000 \% 0000 \cdot 00+3000 \%$ ocz-n $0000 \% 00+3000 \% 0000 \cdot 000+3000 \cdot 0 \quad 5 \varepsilon z-\Omega$ $0000000 \cdot 300000000000+30000 \quad b z=\Omega$ $0000 \div 000+3000 \% 0000 \div 00+3000 \% 0 \quad 0 \overline{-35}$ $0000 \% 00 \times 3000 \% 0000.000+3000.0 \quad 6 \varepsilon z-$ nd $0000000+3000 \cdot 00000.000+3000 \circ 0$ 8Ez-nd $0000 \cdot 000+3000 \cdot 0 \quad 0000.000+30000$ Eg-\$N $0000 \% 00+3000 \% \quad 0000000+3000 \% \quad 65-7 \mathrm{~N}$

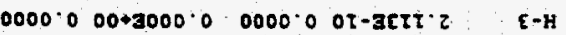
$0000.000+3000.0 \quad 0000.000+3000 \cdot 0$ sst-ns $0000 \% 00 \times 3000: 0 \quad 0000 \cdot 0 \quad 00+3000 \cdot 0$ zst-na $0000 \% 00+3000 \% 0$ 0000-0 00+3000\% LET-83 $0000.000+3000.0 \quad 0000 \cdot 0.00+3000 \% 00000$ $0000.000+3000 \% 0$ 2SE9.0 E0-3E2\%.9 lt-3 0000.0 00+3000.0 0000.0 00+3000.0 toz-ur

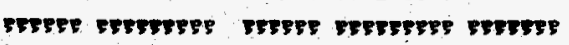

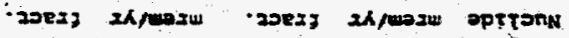

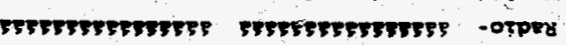
uets $\quad$ Ieam

ExEayzed zuepusdoa sezem

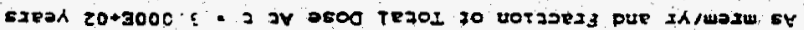

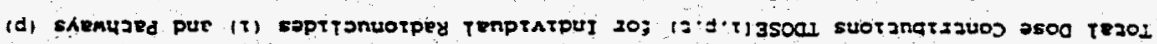

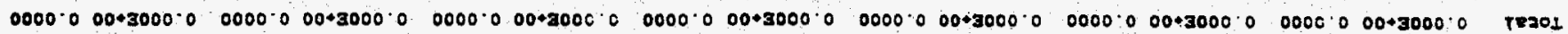

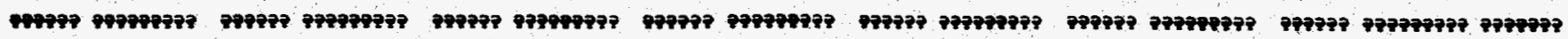

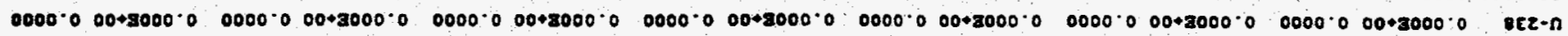

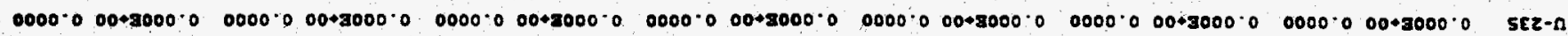

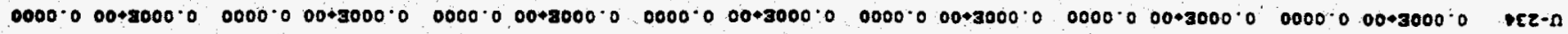

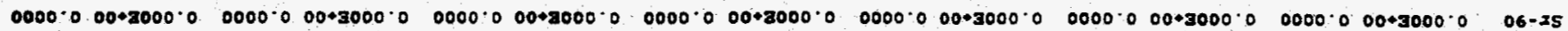

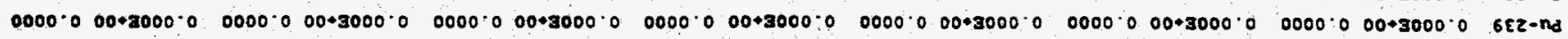

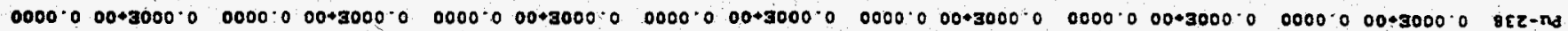

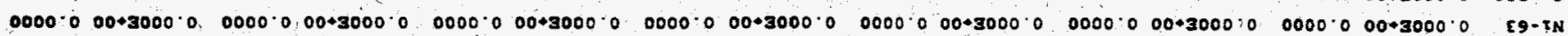

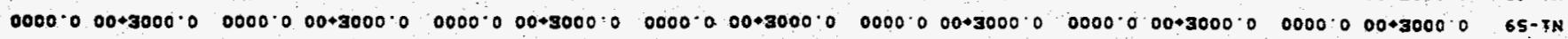

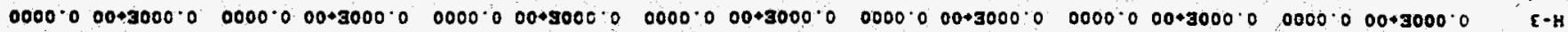

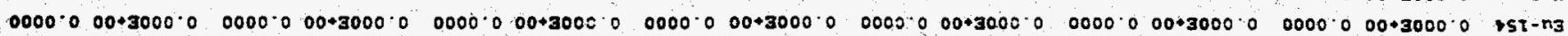

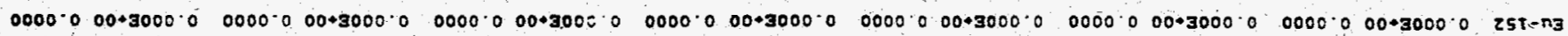

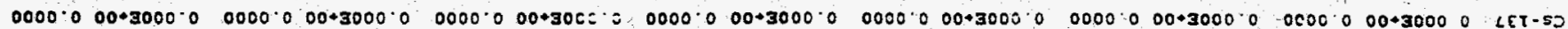

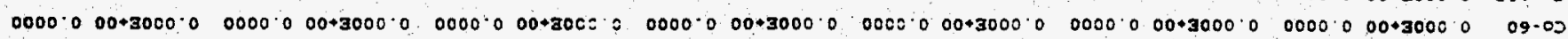

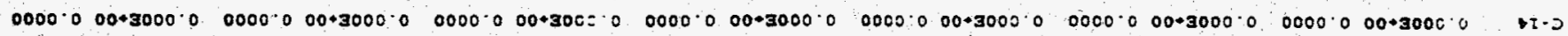

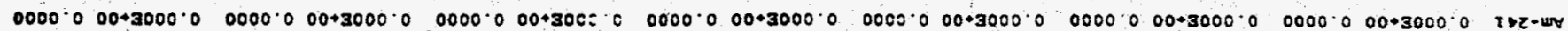

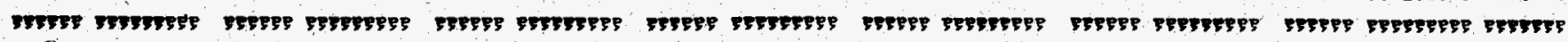

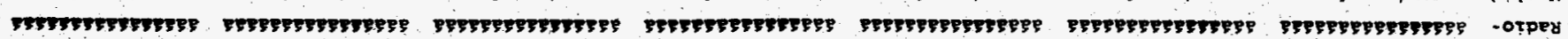
tros หт Th 200w zuretd noper. นoT JPTE्यuI punoxis
\end{abstract}

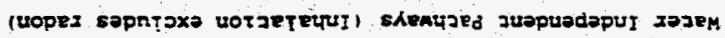

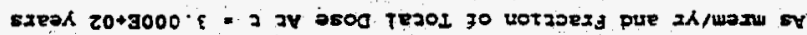

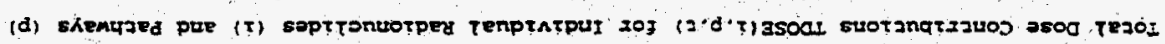


RESRAD. Version $5.621 \quad T$ Limiz = 0.5 year $\quad 04 / 16 / 9713: 17$ page 24

Sumary : HATR - Reaccor vessel and both sceam generacors removed

File : A:ITOT_INV.DAT

Total Dose Contrabutzons TDOSE (i.p.e) for Individual Radionuclides (i) and Ratmays (p) As mrem/yr and Fraction of TOtal Dose AC $C=: .2008+03$ years

Water. Independent Pachways (Inhalation exciludes radon)

\begin{tabular}{|c|c|c|c|c|c|c|c|c|c|c|c|c|c|c|}
\hline \multirow{2}{*}{$\begin{array}{l}\text { Radio- } \\
\text { Nuclide } \\
\text { adatias }\end{array}$} & \multicolumn{2}{|c|}{$\begin{array}{l}\text { Ground } \\
\text { asaluastasastata }\end{array}$} & \multicolumn{2}{|c|}{$\begin{array}{c}\text { Intulation } \\
\text { sadastadasadsta }\end{array}$} & \multicolumn{2}{|c|}{ Radon } & \multicolumn{2}{|c|}{$\begin{array}{c}\text { Plane } \\
\text { dassasadasadssask }\end{array}$} & \multicolumn{2}{|c|}{$\begin{array}{c}\text { Ment } \\
\text { dasasassasasasass }\end{array}$} & \multicolumn{2}{|c|}{ Mask } & \multicolumn{2}{|c|}{ 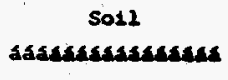 } \\
\hline & $\begin{array}{l}\text { mrem/yr } \\
\text { adsadsasa }\end{array}$ & $\begin{array}{l}\text { frace. } \\
\text { sadast }\end{array}$ & $\begin{array}{l}\text { mreen/yr } \\
\text { adasusasa }\end{array}$ & $\begin{array}{l}\text { fract. } \\
\text { asdad }\end{array}$ & $\begin{array}{c}\text { mren/yr } \\
\text { adadasasa }\end{array}$ & $\begin{array}{l}\text { fract. } \\
\text { asadad }\end{array}$ & $\begin{array}{l}\text { mrem/yr } \\
\text { sassasada }\end{array}$ & $\begin{array}{l}\text { fract. } \\
\text { dasas }\end{array}$ & $\begin{array}{l}-s e n / y x \\
\text { adsustaks }\end{array}$ & $\begin{array}{l}\text { frace. } \\
\text { agáaAa }\end{array}$ & $\begin{array}{l}\text { mrem/yr } \\
\text { adasastat }\end{array}$ & $\begin{array}{l}\text { sract. } \\
\text { adase }\end{array}$ & $\begin{array}{l}\text { mren/yr } \\
\text { asarasers }\end{array}$ & $\begin{array}{l}\text { Erace. } \\
\text { atsus }\end{array}$ \\
\hline 41. & $E+00$ & 0.0000 & $.000 E+00$ & 0.0000 & $.000 E+00$ & 0.0000 & $000 E+00$ & 0.0000 & $=00 E+00$ & 0.0000 & $0.0008+00$ & 0.0000 & $.000 E+00$ & 0.0000 \\
\hline 4 & 0.0 & 0.0000 & $00 E+00$ & 0.0000 & $000 E+00$ & 0.0000 & $.000 E+00$ & 0.00 & $\therefore .=008+00$ & 0.0000 & $0.0008+00$ & 0.0000 & $.0005+00$ & 10 \\
\hline 0 & 0 & 0. & 0 & 0 & 0 & 0 & .00 & 0 & $0=0$ & 0.0000 & $0.000 E+00$ & 0 & 00 & \\
\hline 337 & 00 & 0.0 & 0 & po & 0 & 0 & 0 & 2 & 10 & 0 & 0.0 & D & 00 & 0 \\
\hline 52 & +00 & 10 & a & 0 & 0 & 0 & 0. & & 0 & . & 0 & & & \\
\hline 54. & $0.000 E+00$ & oo & o & & r & & 0 & & 0 & & 0 & & & $\bullet$ \\
\hline 3 & $0.0005+00$ & 0.0 & $1008 \bullet 00$ & 0. & 0 & 0 & 0 & 0 & 0. & 0 & 0. & 0 & 0 & - \\
\hline $1-59$ & $0.000 E+00$ & 0.0000 & .00 & 0. & 00 & 0 & 10 & ? & 0. & 0 & 0. & 0. & 20 & $\bullet$ \\
\hline$i-63$ & $0.000 \varepsilon+00$ & 0.0000 & 00 & 0 & $+\infty$ & 0 . & 400 & ? & $c:$ & 0 & 0. & 0. & 00 & 90 \\
\hline $4-238$ & $0.000 E+00$ & 0.0 & -00 & 0.0 & 0.00 & 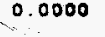 & $0: 000 E+00$ & 0 & 0.00 & . & 0.000 & 0. & 400 & 100 \\
\hline $4-239$ & $0.000 E+00$ & 0.0000 & $.0008+00$ & 0.0000 & $.000 E+00$ & 0.0000 & $0.0008 \cdot 00$ & 0. & $0.2008+00$ & 0.0000 & $0.0008 \bullet 00$ & 0.0000 & $\bullet 00$ & 100 \\
\hline$=-90$ & $0.000 E+00$ & 0.0000 & $.000 E * 00$ & 0.0000 & $: 0008+00$ & 0.0000 & 108.00 & 0. & $0.0008+00$ & 0.0000 & $0.000 E+00$ & 0.0000 & $0.0008+00$ & 000 \\
\hline-234 & $0.0008+00$ & 0.0000 & $.000 E+00$ & 0.0000 & $.000 E+00$ & 0.0000 & $008+00$ & 10 & $0.000 E+00$ & 0.0000 & $005+00$ & 00 & $08+00$ & 0 \\
\hline 35 & $2+00$ & 20 & 008 & 00 & 00 & 0 & $D E \bullet 00$ & o & 00 & $?$ & $+\infty$ & & .00 & 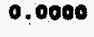 \\
\hline & ०० & 20 & 0 & a & 0 & & 20 & . & 0 & 0 & 0 & $\bullet$ & 00 & 000 \\
\hline & & & & & & & 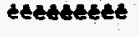 & & e & & 4 & e & $\mathbf{e s}$ & egens \\
\hline 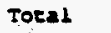 & $0.000 E+00$ & 0.0000 & .00 & 00 & .00 & 00 & .00 & 0 & 0.0 & $\mathrm{c}$ & $+\infty 0$ & 10 & $8 \div 00$ & 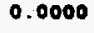 \\
\hline
\end{tabular}

Total Dose Coneriout zons TDOSEli.p.e, for Individual Radionuclides (1) and Pachways (p) As mrem/ye and Fraction of Total Dose Ac $C .: 000 E+03$ years

Water Dependenc Pachweys

waeer

Fion

Radio- dassustadsatas muclide mrem/yr trace.

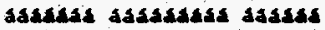

Am-242 0.446E-04 0.4976

C.14 $+.340 E-040.2557$

C0.60 $\quad 0.000 E+00 \quad 0.0000$

C9-237 $0.0008+00 \quad 0.0000$

su-152 $0.0008 * 00 \quad 0.0000$

Eu-154 $0.0008+00 \quad 0.0000$

H -3

Ni-59 $0.000 \mathrm{E}+00 \quad 0.0000$

$N i-63 \quad 0.000 E+00 \quad 0.0000$

Pu-238 $0.0008 .00 \quad 0.0000$

Pu-239 $0.0008+00 \quad 0.0000$

$S=-90 \quad 0.000 E+00 \quad 0.0000$

U-234 $0.000 E * 00 \quad 0.0000$

$U-235 \quad 0.000 E+00 \quad 0.0000$

U-238 $\quad 0.000 E+00 \quad 0.0000$

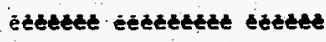

rocal ' $L=798-03 \cdot 0.7533$

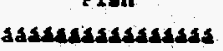
mrem/yr frace. adsastas sadist

$0.000 \mathrm{e}+00 \quad 0.0000$

$0.000 E \div 00 \quad 0.0000$

$0.000 E+00 \quad 0.0000$

$0.0008+00 \quad 0.0000$

$0.000 E+90 \quad 0.0000$

$0.0002+00 \quad 0.0000$

$0.0008+00 \quad 0.0000$

$0.0008 .00 \quad 0.0000$

$0.000 E+00 \quad 0.0000$

$0.000 E+00 \quad 0.0000$

$0.0008+000.0000$

$0.0008+00 \quad 0.0000$

$0.000 E+00 \quad 0.0000$

$0.000 E+00 \quad 0.0000$

$0.000 E+00 \quad 0.0000$

éctecter eteest

$0.000 E+00 \quad 0.0000$
Radon

\section{sassustassisast}

mren/yt sract.

assalsads sasass

$0.000 E+00 \quad 0.0000$

$0.0008+00 \quad 0.0000$

$0.0008+00 \quad 0.0000$

$0.0008+00 \quad 0.0000$

$0.0008+00 \quad 0.0000$

$0.0008+00 \quad 0.0000$

$0.000 E+00 \quad 0.0000$

$0.0008 \times 00 \quad 0.0000$

$0.000 E+00 \quad 0.0000$

$0.000 E+00 \quad 0.0000$

$0.000 E+00 \quad 0.0000$

$0.0000+000.0000$

$0.000 E+00 \quad 0.0000$

$0.000 E+00 \quad 0.0000$

$0.0008+00 \quad 0.0000$

exterthe extet

$0.000 \varepsilon+00 \quad 0.0000$
Plane

\section{dasulasasusats} tarea/yr tract.

\section{dascesss dases}

$2.0208-04 \quad 0.2184$

2. 396 E-04 0.0823

$0.0005+000.0000$

$0.0008+00 \quad 0.0000$

$0.0008+00 \cdot 0.0000$

$0.0008000 \quad 0.0000$

$0.000 E+00 \quad 0.0000$

$0.000 E+00 \quad 0.0000$

$0.0008+00 \quad 0.0000$

$0.0008 .00 \quad 0.0000$

$0.0008+00: 0.0000$

$0.0005000 \quad 0.0000$

$0.000 E+00 \quad 0.0000$

$0.0008+00 \quad 0.0000$

$0.000 E+00 \quad 0.0000$

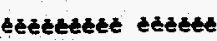

$3.4078-040.2007$
Meat

adsivisasalasates -rew/rr tract.

\section{dascissa dadata}

$\begin{array}{lll}0.792 E-06 & 0.0052\end{array}$

$2.2825-05 \quad 0.0134$

$0.0008+00 \quad 0.0000$

$0.000 \mathrm{0}+00 \quad 0.0000$

$0.0008+00 \quad 0.0000$

$0.0008+00 \quad 0.0000$

0.0008 .000 .0000

$0.000 E+00 \quad 0.0000$

$0.0008+00 \quad 0.0000$

$0.000 E+00.0 .0000$

$0.0008+000.0000$

$0.0008+00 \quad 0.0000$

$0.0008+00 \quad 0.0000$

$0.000 E+00 \quad 0.0000$

$0.0008+00 \quad 0.0000$

cejteter jested

$3 .: 618-05 \quad 0.0186$
Milx

asascasassastase mrea/yr fract. dassusuas asaras

1.610E-07 0.0001 $4.6368-05 \quad 0.0273$

$0.0005+00 \quad 0.0000$

$0.0008+00 \quad 0.0000$

$0.0008+00 \quad 0.0000$

$0.0008 .00 \quad 0.0000$

$0.0008+00 \quad 0.0000$

$0.000 E+00 \quad 0.0000$

$0.0008+00 \quad 0.0000$

$0.0008+00 \quad 0.0000$

$0.000 E+00 \quad 0.0000$

$0.0008 .00 \quad 0.0000$

$0.000 E+00 \quad 0.0000$

$0.000 E+00 \quad 0.0000$

$0.0008+00 \quad 0.0000$

etelected exter

$4.652 E-05 \quad 0.0274$
Al1 Rathweye

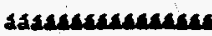
mreidyr trace. asascasas sasea

$1.0558-03 \quad 0.6213$

$6.4298-04 \quad 0.3714$

$0.0008 * 00 \quad 0.0000$

$0.0008+00 \quad 0.0000$

$0.0008+00 \quad 0.0000$

$0.0008+00 \quad 0.0000$

$0.0008+00 \quad 0.0000$

$0.0008+00 \quad 0.0000$

$0.000 E+00 \quad 0.0000$

$0.000 \mathrm{E}+00 \quad 0.0000$

$0.0008+00 \quad 0.0000$

$0.0008+00 \quad 0.0000$

$0.0002+00 \quad 0.0000$

$0.0008+00 \quad 0.0000$ $0.0008+000.0000$ chreseces cesed $3.6978-031.0000$

-Sum of all wacer sndependent and dependent pachmays. 
RESRAD. Version 5.621 Limit -0.5 year $04 / 16 / 97 \quad 13: 17$ Page 25

Sumary : HWCTR - Reactor vessed and both stean generacors renoved

File : A: ITOT_INV.DAT

Total Dose Contributions rDOSE(i,p.e) for Individual Radionuclides (i) and Pathways (p) As mrem/yr and Fraction of Total Dose At $t=1.0008+04$ years

Water Independent Pathways IInhalation excludes radonl

\begin{tabular}{|c|c|c|c|c|c|c|c|c|c|c|c|c|c|c|}
\hline \multirow{2}{*}{$\begin{array}{l}\text { Radio } \\
\text { Nucl de } \\
\text { assasas }\end{array}$} & \multicolumn{2}{|c|}{$\begin{array}{l}\text { Ground } \\
\text { adassusasasadasa }\end{array}$} & \multicolumn{2}{|c|}{$\begin{array}{l}\text { Intulation } \\
\text { dasarassassassas }\end{array}$} & \multicolumn{2}{|c|}{$\begin{array}{c}\text { Radon } \\
\text { asassisusassassisa }\end{array}$} & \multicolumn{2}{|c|}{$\begin{array}{l}\text { Plane } \\
\text { adasidsasastassad }\end{array}$} & \multicolumn{2}{|c|}{$\begin{array}{c}\text { Mone } \\
\text { dastassasadasadx }\end{array}$} & \multicolumn{2}{|c|}{$\begin{array}{c}\text { Milk } \\
\text { dassisusasasdaka }\end{array}$} & \multicolumn{2}{|c|}{$\begin{array}{c}\text { soit } \\
\text { sasursasususases }\end{array}$} \\
\hline & $\begin{array}{c}\text { mrem/yr } \\
\text { sadasasas }\end{array}$ & fra & $\begin{array}{l}\text { mrem/yr } \\
\text { sadustass }\end{array}$ & $\begin{array}{l}\text { Erace. } \\
\text { dasasa }\end{array}$ & $\begin{array}{l}\text { mreap/yr } \\
\text { asastasas }\end{array}$ & $\begin{array}{l}\text { fract: } \\
\text { sadsa }\end{array}$ & $\begin{array}{l}\text { mrew/yr } \\
\text { dasasassa }\end{array}$ & $\begin{array}{l}\text { sract } \\
\text { adasas }\end{array}$ & $\begin{array}{l}\text { nrem/yr } \\
\text { dastatast }\end{array}$ & & $\begin{array}{l}\text { mrem/yr } \\
\text { adsasssas }\end{array}$ & & $\begin{array}{l}\text { a/ys } \\
\text { ans }\end{array}$ & \\
\hline$m-241$ & $0.000 E+00$ & $0: 0000$ & $.000 E=00$ & 0.0000 & $0.000 \mathrm{E}=00$ & 0.0000 & $0.000 \mathrm{E} \cdot 00$ & 0.0000 & $2008-00$ & 00 & $0: 000 E+00$ & 0 & $E+\infty 0$ & $\theta^{-1}+2$ \\
\hline-14 & $0.000 E \cdot 00$ & 0.0000 & $.000 E+00$ & 0.0000 & $.000 E+00$ & 0.0000 & $.000 E+00$ & 0.0000 & $0008+00$ & & HOE +00 & 100 & $08+00$ & \\
\hline $00-60$ & 00 & 0.0 & $.000 E+00$ & 0.0 & $0 E+00$ & 000 & $.0008 \cdot 00$ & 000 & .00 & & $8+00$ & 100 & $D E+00$ & (5) \\
\hline$s-237$ & bo & $0:$ & $\rightarrow \infty$ & 10 & .00 & 100 & .000 & 000 & .00 & & $E+\infty$ & & $0 E+00$ & 0.0000 \\
\hline $4-152$ & 10 & 30 & o & 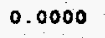 & 00 & 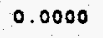 & $0.000 E+00$ & 1000 & +00 & & $8+00$ & & $0.000 E \div 00$ & \\
\hline $2-154$ & 0 & 00 & .00 & & & & & & $0.00008+00$ & & & & $0.0008+00$ & \\
\hline-3 & & 100 & $0.000 E+00$ & & & & & & & & & & & \\
\hline & & & & 0 & $0.0008+00$ & & & & & & & & & 0.0000 \\
\hline- & & & $0.000 \mathrm{E}+00$ & 100 & $0.000 E+00$ & 0.0000 & $0.0008+00$ & ? & $0.0008+00$ & 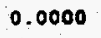 & & 0.0000 & $8+00$ & 0.0000 \\
\hline $0<+30$ & $0.000 E+00$ & 100 & 0.0008 .00 & 0.0 & $0.000 \mathrm{E}+00$ & 0.0000 & $0.0008+00$ & 0.0000 & $0.000 \mathrm{E}+00$ & 000 & $0.0008+00$ & 0.0000 & $0.0008+00$ & 0.0000 \\
\hline $4-239$ & $0.000 E+00$ & 100 & $0.0008+00$ & 0.0000 & $0.000 \mathrm{E}+00$ & 0.0000 & $0.0008+00$ & 0.0000 & $0.0002+00$ & 000 & $0.0008+00$ & 0.0000 & $0.0008+00$ & 0.0000 \\
\hline 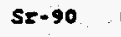 & $0.0008=00$ & 0.0000 & $0.0008+00$ & 0.0000 & $0.000 E+00$ & 0.0000 & $0.0008+00$ & 0.0000 & 0.0002000 & 0.0000 & $0.0008+00$ & 0.0000 & $0.0008+00$ & 0.0000 \\
\hline-234 & $0.0008+00$ & 0.0000 & $0.0008+00$ & 0.0000 & $0.0008+00$ & 0.0000 & $0.0008+00^{\circ}$ & 0.0000 & $0.0002+00$ & & $0.0008+00$ & 0.0000 & $0.0008+00$ & 0.0 \\
\hline 20 & $0.0008+00$ & 0.0000 & 0.0008 .00 & 0.0000 & $0.0008+00$ & 0.0000 & $0.0008+00$ & 0.0000 & $0.0002+00$ & & $0.0008+00$ & 0.0000 & $0.0008+00$ & 0000 \\
\hline 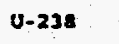 & 0.000 & 0.0 & $0.000 E+00$ & 0.00 & $0.0008+00$ & $0: 0000$ & $0.0008+00$ & 0.0 & $0.0002+00$ & & so & 0.0 & $28+00$ & \\
\hline eorete & 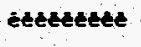 & 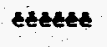 & 논 & est & ee & eed & et & eet & te & 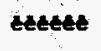 & s. & & neted & \\
\hline eat & .00 & 20 & $0008 * 00$ & 0.0000 & $.000 E+00$ & 0.0000 & $.000 E+00^{\circ}$ & 0.0000 & $.0008 * 00$ & .0000 & $.000 E+00$ & 0.0000 & $.000 \mathrm{E}+0$ & .000 \\
\hline
\end{tabular}

Total Dose Coneribuesons TDOSE (i.p.t) for Individual Radionuelides ( $)$ and Paenways (p) As mrem/yr and fraceson of Total Dose Ae $=2.000 E+04$ years

Water Dependene Pacmays

wicer

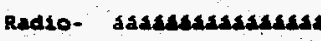

Muelide mrem/yr fract.

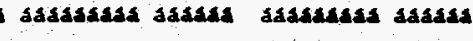

C-I4 $\quad 9.622 E-25 \quad 0.0000 \quad 0.0002+00 \quad 0.0000 \quad 0.0005+00 \quad 0.0000$

Co-60 $0.0008+00 \quad 0.0000 \quad 0.0008+00 \quad 0.0000 \quad 0.000 E+00 \quad 0.0000$

$\begin{array}{cccccccc}C s-237 & 0.0008+00 & 0.0000 & 0.0008+00 & 0.0000 & 0.000 E+00 & 0.0000\end{array}$

Eu-152 $0.0008+00 \quad 0.0000 \quad 0.000 E+00 \quad 0.0000 \quad 0.0008+00 \quad 0.0000$

Eu-154 $0.000 E+00 \quad 0.0000 \quad 0.0006 \$ 00 \quad 0.0000 \quad 0.0008+00 \quad 0.0000$

H-3 $\quad 0.0008+00 \quad 0.0000 \quad 0.0008+00 \quad 0.0000 \quad 0.0008+00 \quad 0.0000$

$\begin{array}{lllllllllll}\mathrm{Ni}-59 & 0.0008+00 & 0.0000 & 0.0008+00 & 0.0000 & 0.0008,00 & 0.0000\end{array}$

$\mathrm{Ni}-63 \quad 0.000 \mathrm{E}+00 \quad 0.0000 \quad 0.000 \mathrm{0}+00 \quad 0.0000 \quad 0.0008+00 \quad 0.0000$

Pu-238 $1.416 E-06 \quad 0.0001 \quad 0.000 E+00 \quad 0.0000 \quad 2.242 E-09 \quad 0.0000$

$\begin{array}{llllllll}P \text { P }-239 & 3.426 E-07 & 0.0000 & 0.000 E+00 & 0.0000 & 0.000 E+00 & 0.0000\end{array}$

Sr-90 $0.0008+00 \quad 0.0000 \quad 0.0008+00 \quad 0.0000 \quad 0.0008+00 \quad 0.0000$

$\begin{array}{lllllllll}0-234 \quad 392 E-03 & 0.3253 & 0.000 E+00 & 0.0000 & 5.952 E-06 & 0.0005\end{array}$

$\begin{array}{lllllll}U-235 & 1.525 E-04 & 0.0134 & 0.0008+00 & 0.0000 & 0.0008+00 & 0.0000\end{array}$

$\begin{array}{llllllll}U-238 & 5.143 E-03 & 0.4532 & 0.0008+00 & 0.0000 \quad & .3568-07 & 0.0000\end{array}$

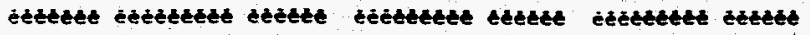

$\begin{array}{lllllllll}\text { Total } & 9908-03 & 0.7921 & 0.0008+00 & 0.0000 & 6.3908-06 & 0.0006\end{array}$
Plane

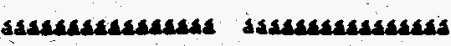
mreo/yr Erace. aasuscas asasa

$5.3768-090.0000$

$3.0965-250.0000$

$0.0008+00 \quad 0.0000$

$0.0008+00 \div 0.0000$

$0.000 E+00 \quad 0.0000$

$0.0008+00 \quad 0.0000$

$0.000 z+00 \quad 0.0000$

$0.0008+00 \quad 0.0000$

$0.0008+00 \quad 0.0000$

3.359E-07 0.0000

8.125E-08 0.0000

$0.000 E+00 \quad 0.0000$

$0.758 E-040.0772$

$3.617 E-05 \quad 0.0032$

$1.219 E-030.1074$

dentevets elctet

2.132E-03 0.1878 $\therefore$

- Sum of all water andependent and dependent pachwaya. 
REsRaD. Version S.6921 T* Limit - 0.5 year $04 / 16 / 97 \quad 13: 17$ Page 26 Sumary : HWCTR - Reaccor ressel and boch steam generacors removed File A: ITOT_INV.DAT

Dose/Source Racios Sumed over All Pathways

Parent and Progeny primeipal Radionuelide Concributions indicaced

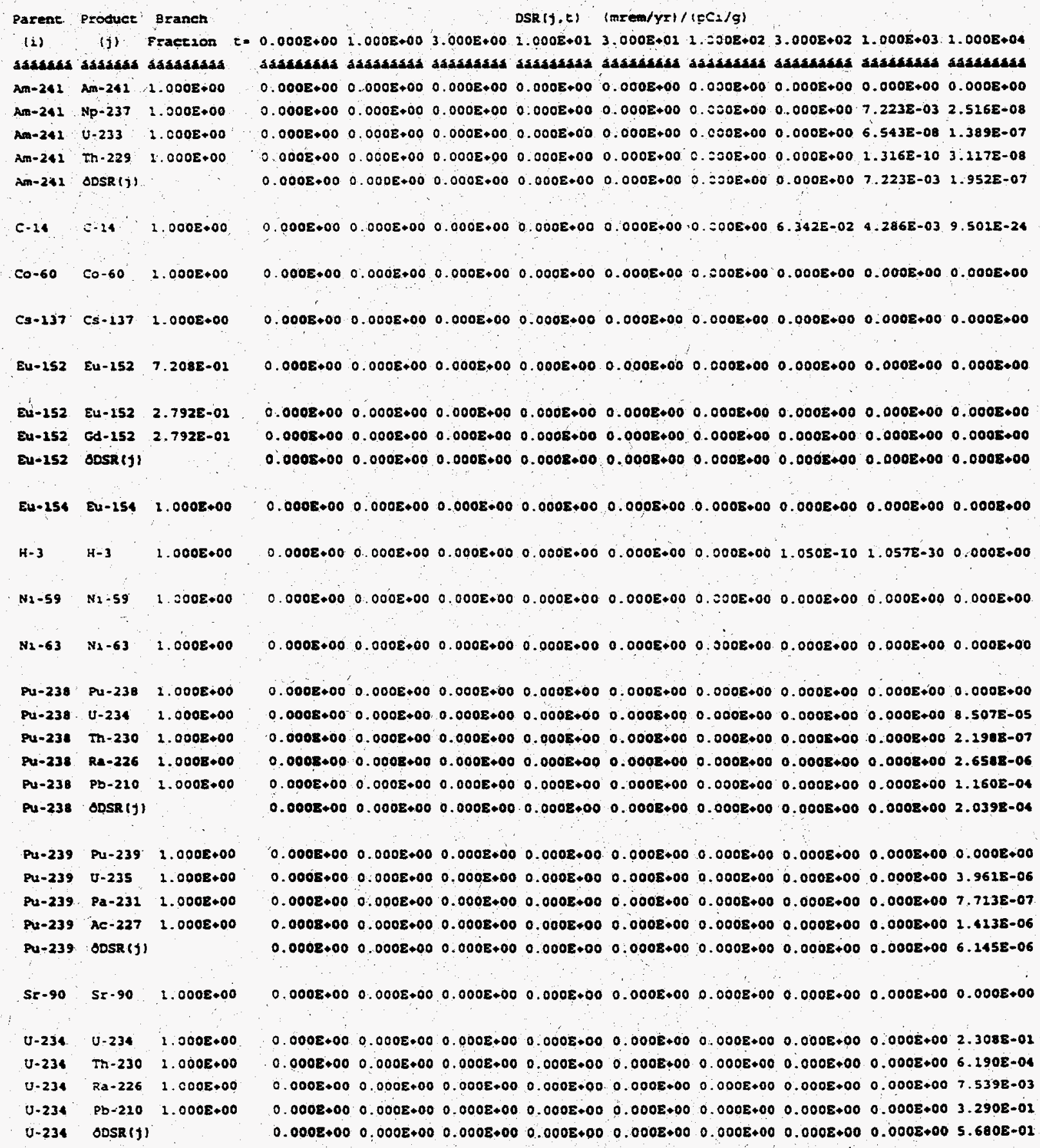


RESRAD, Version S.621 I Jimit $=0.5$ year $\quad 04 / 26 / 97 \quad 13: 17$ Page 27

Suminy : HWCTR - Reactor vessel and both steam generacors removed

File $\quad \because$ A:ITOT_Iav. DAT

Dose/Source Ratios Sumed Over All Pathwaya

Parent and' Progeny Principal Radionuclide Coneributions Indieated

Pareint Produce Branch

DSR (g,t) (mrem/Yr)/(pCi/g)

(i) (j) Fraction $t=0.0008+001.0008+00 \quad 3.0008+00 \quad 1.0008+02 \quad 3.0008+02 \quad 1.0008+02 \quad 3.000 E+021.0008+03 \quad 1.000 E+04$

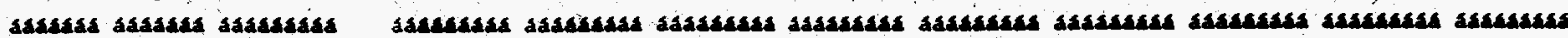
U-23S U-235 I.000E+00 $0.000 E+00 \quad 0.000 E+00 \quad 0.000 E+00 \quad 0.000 E+00 \quad 0.000 E+00 \quad 0.0008+00 \quad 0.000 E+00 \quad 0.000 E+00 \quad 2.240 E-01$

U-235 Pa-231 1.000E+00 $\quad 0.000 E+00 \quad 0.000 E+00 \quad 0.000 E+00 \quad 0.000 E+00 \quad 0.000 E+00 \quad 0.000 E+00 \quad 0.000 E+00 \quad 0.000 E+001.645 E-01$

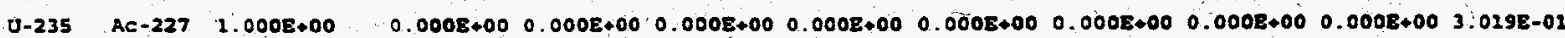
U-235 ODSR(f) $\quad 0.000 E+00 \quad 0.000 E+00 \quad 0.000 E+00 \quad 0.000 E+00 \quad 0.000 E+00 \quad 0.000 E+00 \quad 0.000 E+00 \quad 0.000 E+00 \quad 6.904 E-01$

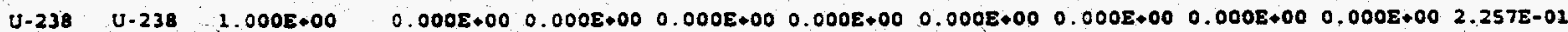
U.238 U-234 1.000E+00 $0.000 E+00 \quad 0.000 E+00 \quad 0.000 E+00 \quad 0.000 E+00 \quad 0.0008+00 \quad 0.0008+00 \quad 0.000 E+00 \quad 0.000 E+00 \quad 6.637 E-03$

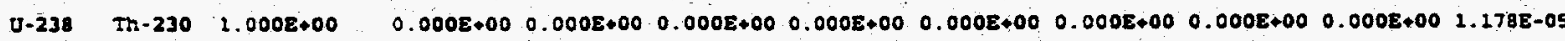

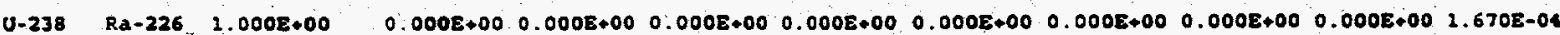
U-231 Pb-210 $1.000 E+00 \quad 0.000 E+00 \quad 0.0008+00 \quad 0.000 E+00 \quad 0.000 E+00 \quad 0.000 E+00 \quad 0.000 E+00 \quad 0.000 E+00 \quad 0.000 E+00 \quad 7.254 E-03$ U-238 ODSR(j) $\quad 0.0008+00 \quad 0.000 E+00 \quad 0.000 E+00 \quad 0.000 E+00 \quad 0.0008+00 \quad 0.0008+00 \quad 0.0008+00 \quad 0.000 E+00 \quad 2.398 E-02$

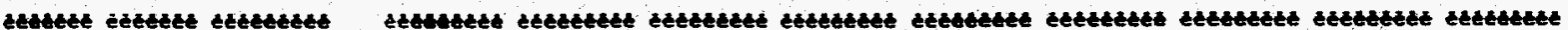

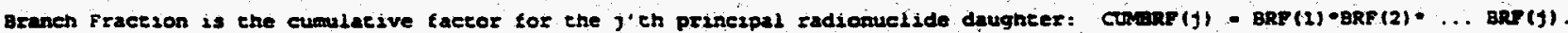
The DSR includes contribution from essocsaced (half-life 10.5 yr) daugheers.

SLogle Radtonuclide soil cildelined G(1.e) in pCi/g Banic Radiation Dose limit - is mom/yr

\begin{tabular}{|c|c|c|c|c|c|c|c|c|c|}
\hline (i) & $t=0.0008+00$ & $1.000 E 000$ & $3.0008+00$ & $1.000 E+01$ & $3.0008+01$ & $1.0005+02$ & $3.0008+02$ & $1.0008+03$ & $1.000 E+04$ \\
\hline iscasa & ascenses & dasengasa & sasursas & adsususe & dasurst & dasusans & asasesesa & aascesas & sadsuases \\
\hline$m=242$ & $-3.430 E+12$ & $-3.4308+22$ & $-3430 E+22$ & $+3: 430 E+12$ & $-3.430 E+12$ & $-3.4305+12$ & $-3.4308+12$ & $2.0778 * 03$ & $7.6046+07$ \\
\hline-16 & $-4.454 E+12$ & $94.4545+22$ & $-4.454 E+12$ & $* 4.454 E+12$ & $\because 4.454 E+12$ & $-4.454 E+12$ & $2.3658+02$ & $3.5002 * 03$ & $\because 4: 454 E+12$ \\
\hline $60-60$ & $+1.2318+25$ & $-1.1318+15$ & $-1.131 E+15$ & $=2.132 E+25$ & $-1.1328+15$ & $-1.1318+15$. & $\because 1.2318+15$ & $-2.1315+25$ & $-2.132 E+15$ \\
\hline $3 \cdot 237$ & $8+13$ & $\cdot 8.701 E+13$ & $1 E+13$ & $\cdot 0.702 E+13$ & $-8.7028+13$ & $-8.7018+13$ & $-8,7018+13$ & $-8.7018+13$ & $-8.701 E+13$ \\
\hline $4=152$ & $5 E+24$ & $5 E+24$ & $\because 1$ & $-2.765 E+14$ & 1.7655 & 24 & $3658+14$ & $.7655+14$ & $\because 1.765 \mathrm{E}+14$ \\
\hline$x+154$ & $-2.6392+14$ & $=2.6395+14$ & -2.6398 & -2.6 & -2 & 46 & .24 & $8+14$ & $398+14$ \\
\hline $4-3$ & $\bullet 9.5948+25$ & $\cdot 9.5945+15$ & $* 9.5948+15$ & $\cdot 9.594 \mathrm{~B}+15$ & $-9.5948+15$ & $-9.5948+15$ & $1.6208+21$ & 25 & $\cdot 9$ \\
\hline $11-59$ & $-8.085 E+20$ & $-0.0858+10$ & -8.0258+10 & $-0.0458+10$ & $\because 0: 0058+10$ & $\bullet .0058+10$ & $-0.0458+20$ & $-0.0058+20$ & $\bullet 0.0058$ \\
\hline $91-63$ & $-5.926 E+23$ & $-5.9168+13$ & $-5.916 E+13$ & $-5.916 E+13$ & $05.916 E+13$ & $-5.9168+13$ & $-5.9268+13$ & $75.9168+13$ & .5. \\
\hline$P u=238$ & $-1.7225+13$ & $1.7128+13$ & $-2.7218+13$ & $-1.711 E+13$ & $-2,7125+13$ & $-2.7128+13$ & $-1.711 E+13$ & $-1.7118+13$ & 7.35 \\
\hline$P u-239$ & $+6.222 E+10$ & $-6.2128+10$ & $-6.2128+10$ & $-6.212 E+10$ & $-6.2128+10$ & $.6 .2225+10$ & $-6.2228+20$ & $06.222 E+10$ & 2.44 \\
\hline $5 x-90$ & $-1.365 E+14$ & $\cdot 1.3658+14$ & $\cdot 1.365 E+14$ & $-1.3655+24$ & $-1.365 E+14$ & $\cdot 2.365 E+14$ & $\cdot 1.3658+24$ & $-1.3658+14$ & $-2.365 E+24$ \\
\hline $0-234$. & -6.2458 .09 & $-6.2458+09$ & $\bullet 6.245 E+09$ & $\cdot 6.2455 \cdot 09$ & $-6.245 E+09$ & $.6 .2458+09$ & $-6.2458+09$ & $\bullet 6.245 B+09$ & 2.64 \\
\hline 235 & $2.2608 * 06$ & $-2.1608+06$ & $-2.160 E+06$ & $-2.2605 \bullet 06$ & $-2.160 E+06$ & $-2.1608 \bullet 06$ & $-2: 1608+06$ & $\bullet 2.160 \mathrm{E}+06$ & $2.273 E+01$ \\
\hline $0-238$ & 05 & $3608 \bullet 05$ & $50 E+05$ & 05 & $-3.360 \mathrm{E}+05$ & $3608+05$ & os & .3 .360 & $6.2568+01$ \\
\hline set & eew & stucted & sebcestes & sentete & écruede & enseated & cetrestas & zetreseed & sestedetet \\
\hline
\end{tabular}

- At specific activity limit 
RESRAD, Version $5.621 \quad T$ Limit $=0.5$ year $04 / 16 / 97 \quad 13: 17$ Page 28 Sumany : HWCHR - Reactor vessel and both sceam generators removed

File : A:ITOT_INY.DAT

Sumed Dose/SOurce Ratios OSR (i,t) in (nrem/yr)/(pCi/g)

and single Radionucizde soil Guidelines G(i.e) in pci/g

at tmin - time of manimum sangle radionuclide soil guideline

and af tmax - ime of maximum total dose - 4965 o 5 years

\begin{tabular}{|c|c|c|c|c|c|c|}
\hline (i) & $\mathrm{pCl} / \mathrm{g}$ & $\begin{array}{c}\text { tmin } \\
\text { (yenrs) }\end{array}$ & & $\begin{array}{l}\text { (i. emin) } \\
(\mathrm{pcc} / \mathrm{g})\end{array}$ & & inceses \\
\hline & adsastada & Marasada & da & & & \\
\hline & -01 & 31 & 03 & 23 & & 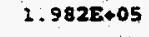 \\
\hline & .01 & 317.5 p 0.3 & 12 & 01 & 12 & +12 \\
\hline & .02 & $0.0000 p 0.0$ & 00 & $\cdot 1$ & .00 & -1 \\
\hline 137 & $00^{-}$ & $200 \% 0$ & .00 & -8.7 & $2+00$ & $\bullet 8$. \\
\hline 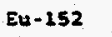 & 02 & 0.0000000000 & +00 & -1 & .00 & -1 \\
\hline$\therefore$ & 00 & $0.0000 \mathrm{p} 0$. & .00 & $\cdot 2$. & +00 & -2 \\
\hline & $\therefore ?$ & 176.5 b 0.2 & & 1.07 & $2+00$ & $\bullet 9$ \\
\hline & -02 & $0.0000 * 0.00$ & & & & -8 \\
\hline & & $0.0000 p 0.0000$ & & & & $* 5$ \\
\hline & & $5156 \times 5$ & & & & \\
\hline & & $D O E+04$ & & & & \\
\hline & 1.6 & $1360 \mathrm{Pl}$ & & & & -1 \\
\hline & -03 & $4964 \mathrm{ps}$ & & & & $8<0$ \\
\hline & 04 & 4 p 5 & & & & 2. \\
\hline & & p 5 & & & & \\
\hline & crestes & 8 & exter & haser & seter 10 & 론론 \\
\hline
\end{tabular}

- he speestic acesvity linite 
RESRAD. Version $5.621 \quad T$ Limit $=0.5$ year $04 / 16 / 9713: 17$ page 29

Summiry : HWCIR - Reactor vessei and both steam generacors removed

File : A:ITOT_INV.DAT

Individual Nuclide Dose Summed Over All Pathways

Parent Nuclide and Branch Fraction Indicaced

\begin{tabular}{|c|c|c|c|c|c|c|c|c|c|c|c|}
\hline Nuclide. & Parene & $\operatorname{BRF}(i)$ & & & & DOSE $\{?$ & $(j, t)$, mrem & a/yx & & & \\
\hline (j) & (i) & & $t=0.000 \Sigma+00$ & $2.0008+00$ & $3.0008+00$ & 3.000E +01 & $3.0008 * 01$ & 1. $200 \mathrm{E}+02$ & $3.0008+02$ & $1.0008+03$ & $1.000 E+04$ \\
\hline sanksas & SAasasa & asasanas & adsasura & adasusas & asadassiak & atassasas & asastias & astastast & adtisistas & atastatas & astassase \\
\hline $\operatorname{Mn}-241$ & $A m-241$ & $1.000 E+00$ & $0.000 E+00$ & $0.0008+00$ & $0.000 E+00$ & $0.0008+00$ & $0.0008+00$ & $0.000 E+00$ & $0.000 E+00$ & $0.0002+00$ & $0.0008+00$ \\
\hline $1 p-237$ & $A n-241$ & $1.0008 \times 00$ & $0.000 E+00$ & $0.000 E+00$ & $0.000 E+00$. & $0.0008+00$ & $0.000 E+00$ & $0.0008+00$ & $0.0008+00$ & $1.055 E-03$ & $3.673 E-09$ \\
\hline $0-233$ & $A m-241$ & $1.000 E+00$ & $0.000 \mathrm{E}+00$ & $0.000 E+00$ & $0.000 E+00$ & $0.0008+00$ & $0.0008+00$ & $0.000 E+00$ & $0.000 \mathrm{E}+00$ & $9.552 E-09$ & $2.028 E-08$ \\
\hline$\pi n-229$ & $\operatorname{Am}-241$ & $1.0008+00$ & $0.000 E \cdot 00$ & $0.000 E+00$ & $0.000 E+00$ & $0.000 E+00$ & $0.000 E+00$ & $0.000 E+00$ & $0.000 E+00$ & 1. $922 E-21$ & $\$ 5508-09$ \\
\hline$c-24$ & $c \cdot 14$ & $1.000 E+00$ & $0.000 \mathrm{E}+00$ & $0.000 \mathrm{E}+00$ & $0.000 \mathrm{E}+00$ & $0.000 E+00$ & $0.0008+00$ & $0.0008+00$ & $9.513 E-03$ & $6.429 E .04$ & $2.425 E-24$ \\
\hline Co-60 & $50-60$ & $2.000 E+00$ & $0.000 E * 00$ & $0.000 E \cdot 00$ & $0.0008 \times 00$ & $0.000 E+00$ & $0.0008 * 00$ & 0.0008 .00 & $0.0005+00$ & $0.000 E+00$ & $0.0008+00$ \\
\hline$c=-137$ & $69-137$ & $1.0008 * 00$ & $0.000 E+00$ & $0.000 E+00$ & $0.000 \mathrm{E}+00$ & $0.000 E+00$ & $0.000 \mathrm{E}+00$ & $0.0008+00$ & $0.000 \mathrm{E}+00$ & $0.0008+00$ & $0.000 E+00$ \\
\hline Dis-152 & $8 u-152$ & $7.2088-02$ & & & & & & & & 0 0anson & \\
\hline $52-152$ & $2 u-152$ & $2.7928-02$ & $0.0008+00$ & $0.000 E 000$ & $0.000 \mathrm{z}+00$ & $0.0008+00$ & $0.0008+00$ & $0.0008+00$ & $0.0008+00$ & $0.0008+00$ & $0.0008+00$ \\
\hline bu-152 & $800 \operatorname{se}(j)$ & & $0.0008+00$ & $0.000 E+00$ & $0.000 \mathrm{E}+00$ & 0.0008 .00 & $0.0008+00$ & $0.0008+00$ & $0.0008 \div 00$ & $0.0008+00$ & $0.0008+00$ \\
\hline $\operatorname{cod}-152$ & Eu-252 & $2.7925-01$ & $0.0005+00$ & $0.000 E+00$ & $0.0008+00$ & 0.0008 .00 & $0.0008+00$ & $0.0008+00$ & $0.0008+00$ & $0.0005+00$ & $0.0008+00$ \\
\hline$E u-254$ & $8 u-154$ & $2.000 \mathrm{~B}+00$ & $0.000 \mathrm{E}-00$ & $0.000 E+00$ & $0.000 E-00$ & $0.0008+00$ & $0.0008+00$ & $0.000 \mathrm{E} \bullet 00$ & $0.000 E+00$ & $0.000 E+00$ & $0.0008 * 00$ \\
\hline $\mathrm{H}-3$ & $\mathrm{H}-3$ & $1.0008+00$ & 0.0008 .00 & $0.000 \mathrm{E}+00$ & $0.000 E+00$ & $0.0008+00$ & $0.000 E+00$ & $0.000 E+00$ & $2.374 E-10$ & $0.000 E+00$ & $0.000 E+00$ \\
\hline N1-59 & $N i-59$ & $1.0008+00$ & $0.0008+00$ & $0.0008+00$ & $0.000 \varepsilon+00$ & $0.0005+00$ & $0.000 \Sigma+00$ & $0.000 \mathrm{E}+00$ & $0.000 \mathrm{E}+00$ & $0.000 \varepsilon+00$ & $0.0008+00$ \\
\hline$M 1-63$ & $N i-63$ & $1.000 \mathrm{~B} \cdot 00$ & $0.0008+00$ & $0.000 \mathrm{E} \times 00$ & $0.000 E+00$ & $0.000 E, 00$ & $0.000 E+00$ & $0.000 \mathrm{E}+00$ & $0.000 \mathrm{E}+00$ & $0.000 E+00$ & $0.000 \mathrm{E}+00$ \\
\hline Pu-238 & $P u-238$ & $1.0008+00$ & $0.0008 \times 00$ & $0.0008+00$ & $0.0008+00$ & $0.0008 \times 00$ & $0.000 E+00$ & $0: 000 B+00$ & $0.000 E+00$ & $0.0008+00$ & $0.000 E+00$ \\
\hline $0-234$ & & & $0.000 E+00$ & $0.000 E+00$ & 0.0008 .00 & 000080 & 0 onoricon & & & & \\
\hline $\mathbf{0 - 2 3 4}$ & $v-234$ & $1.0008+00$ & $0.000 \mathrm{E}+00$ & $0.0008+00$ & $0.0008 \times 00$ & $0.0008+00$ & $0.0008 \div 00$ & $0.0008+00$ & $0.000 \Sigma+00$ & $0.0005 \times 00$ & $1.8938-03$ \\
\hline $0-234$ & 0.238 & $1.0008=00$ & $.000 E+00$ & $0.000 z+00$ & $0.000 E+00$ & $0.0008+00$ & $0.000 E+00$ & $0.0008+00$ & $0.000 z+00$ & $0.0008+00$ & $1.7998-04$ \\
\hline$u-234$ & ocose (y) & $1:$ & $0.0008+00$ & $0.000 E+00$ & $0.0008+00$ & $0.000 \mathrm{E}+00$ & $0.0008+00$ & 0.0008400 & $0.0008+00$ & $0.000 E+00$ & $2.073 E-03$ \\
\hline$m-230$ & $P u-238$ & $2.0008+00$ & $0.000 \mathrm{R}+00$ & $0.0008+00$ & $0.0008+00$ & $0.000 E+00$ & $0.000 E+00$ & $0.000 \mathrm{~B}+00$ & $0.0008+00$ & $0.000 \mathrm{E}+00$ & $1.926 \mathrm{E}-09$ \\
\hline$m-230$ & $v-234$ & $1.0005 * 00$ & $0.0008-00$ & $0.000 E+00$ & 0.0005000 & $0.000 \varepsilon+00$ & $0.000 \mathrm{~B}+00$ & $0.0008+00$ & $0.000 E+00$ & $0.0008+00$ & $5.0768-06$ \\
\hline$m-230$ & U-238. & $1.0008 \times 00$ & $0.0008<00$ & $0.000 E+00$ & $0,000 E * 00$ & $0.000 \mathrm{E}+00$ & $0.0008+00$ & $0.0008+00$ & $0.0008+00$ & $0.0008+00$ & $3.1938-07$ \\
\hline $\operatorname{mn-230}$ & $O D O S E(j)$ & & $0.0008+00$ & $0.000 E+00$ & $0.000 E=00$ & $0.000 \mathrm{E} \cdot 00$ & $0.0008 \times 00$ & $0.0008+00$ & $0.0008 \cdot 00$ & $0.000 E+00$ & $5.397 E-06$ \\
\hline$R=-226$ & Pus -238 & $1.000 E+00$ & $0.0008+00$ & $0.000 \mathrm{E}+00$ & $0.000 E+00$ & $0.000 E+00$ & $0.000 \bar{E}+00$ & $0.000 E \times 00$ & $0.000 E * 00$ & $0.0008+00$ & $2.328 E-08$ \\
\hline$R=-226$ & $u-234$ & 1.0000 .00 & $0.000 \varepsilon+00$ & $0.000 E+00$ & $0.0008+00$ & $0.000 \mathrm{E}+00$ & $0.0008+00$ & $0.0008+00$ & $0.000 E+00^{\circ}$ & $0.000 E \div 00$ & $6.2828-05$ \\
\hline Ra-226 & $0-238$ & $1.0005 \div 00$ & $0.000 E+00$ & $0.000 E+00$ & $0.000 E+00$ & $0.0008-00$ & $0.000 E+00$ & $0.0008+00$ & $0.0008+00$ & $0.000 \mathrm{E}+00$ & $4.525 z-06$ \\
\hline$R a-226$ & $\Delta \cos E(j)$ & & $0.0008 \cdot 00$ & $0.000 \varepsilon .00$ & $0.000 E+00$ & $0.000 E+00$ & $0.0008+00$ & $0.000 E+00$ & $0.000 E+00$ & $0.000 \mathrm{E}+00$ & $6.637 E-05$ \\
\hline $\mathrm{Pb}-210$ & $P U-238$ & 1. $0008+00$ & $0.0008+00$ & 0.0008 .00 & $0.0005+00$ & $0.0008+00$ & 0. & $0.0008+00$ & $0: 0008+00$ & $0.0008+00$ & $1.0168-06$ \\
\hline$P b-210$ & $U=234$ & $1.0008+00$ & $0.0008+00$ & $0.000 E+00$ & $0.000 \mathrm{E}+00$ & $0.0008+00$ & $0.000 E+00$ & $0.0008+00$ & $0.000 E+00$ & 00 & $2: 698 \varepsilon-03$ \\
\hline$P b-210$ & $0-236$ & $1.000 E+00$ & $0.0008+00$ & $0.000 E+00$ & $0.0008+00$ & $0.0008+00$ & $0.000 z+00$ & $0.000 \Sigma+00$ & $0.000 E+00$ & $0.000 E+00$ & $1.966 E-04$ \\
\hline$P b-210$ & ODOSE (j) & & $0.0005 \cdot 00$ & $0.000 E+00$ & $0.000 E+00$ & $0.000 E+00$ & $0.0008+00$ & $0.000 E+00$ & $0.000 E+00$ & $0.000 \Sigma+00$ & $2.896 \mathrm{E}-03$ \\
\hline
\end{tabular}


REsrad, Version $5.621 \quad T$ Limit $=0.5$ year $04 / 16 / 97$ 13:17 Page 30 Sumary : HWCTR - Reactor vessel and both sceam generacors removed File : A:ITOT_INV.DAT

Individual Nuclide Dose Sumped Over All Pathvays

Parene Nuclide and Branch Fraetion Indicated




REsRaD, Version $5.622 \quad T^{-}$Liait $=0.5$ year $04 / 16 / 97$ 13:17 Page 31

Sumery : HWCTR - Reactor ressel and both steam generacors Ienoved

File : A:ITOT_INV.DAT

Individual Nuclide Soil Concencracion

Parent Nuclide and Branch Fraceion Indieaced

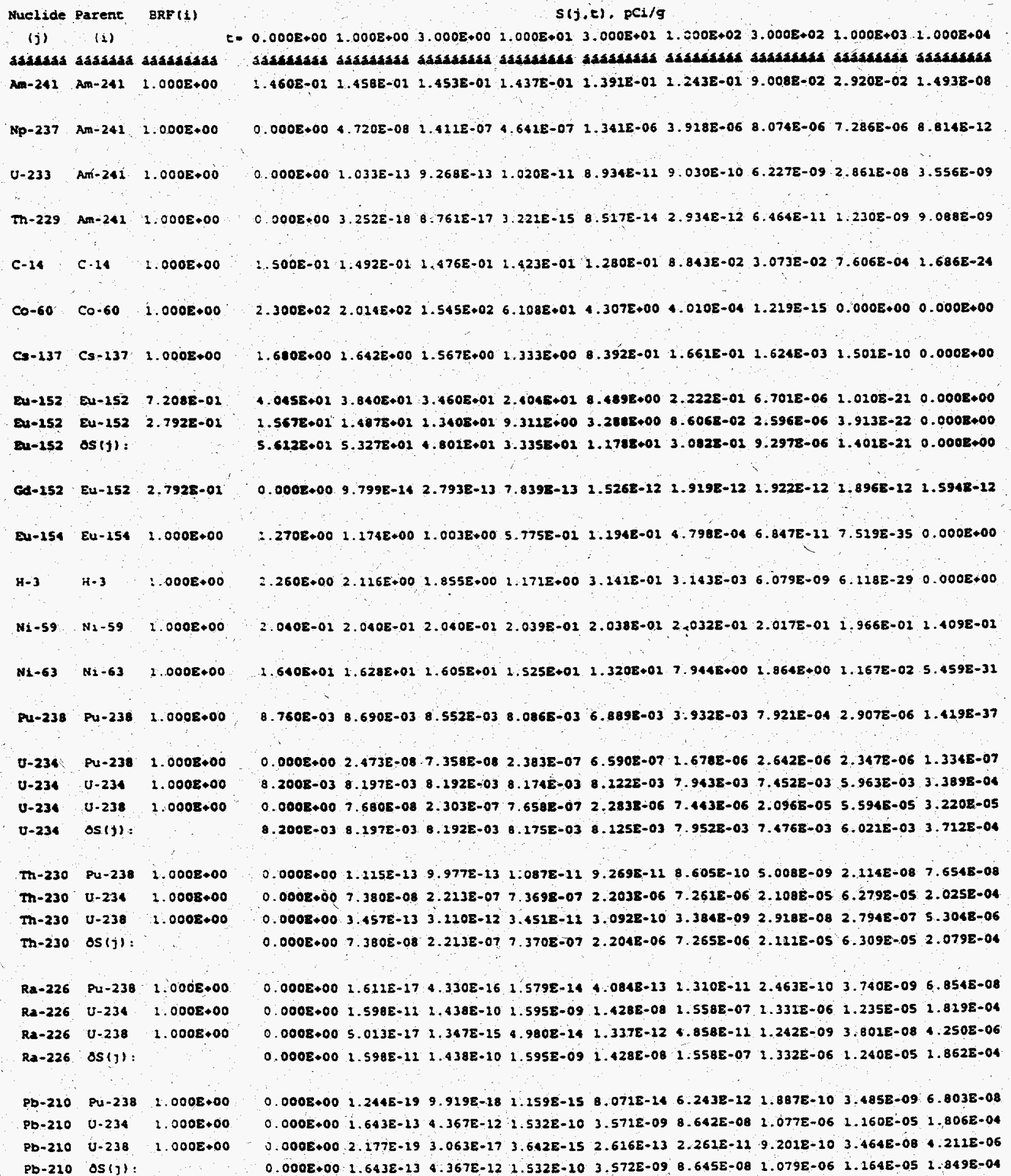


RESRAD. Version $5.621 \quad T^{-}$Limit $=0.5$ year $\quad 04 / 16 / 97 \quad 13: 17$ Page 32

SUmary : HWCTR - Reaccor vessel and boch steam generators removed

File : A: ITOT_INW.DAT

Individual Nuclide Soll Coneencracion

parent Nuclide and Eranch fraction Indicated

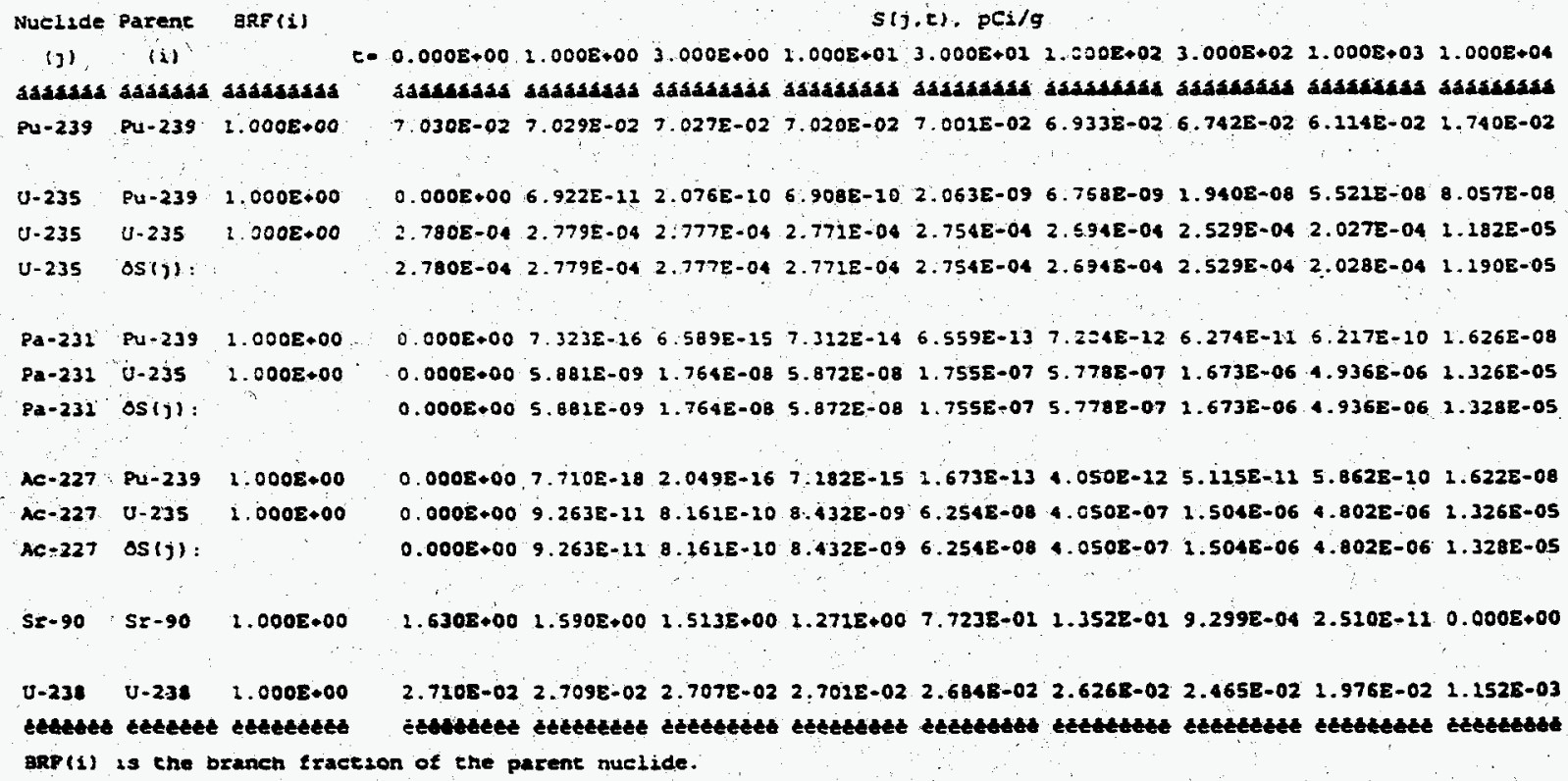


RĖSIDUAL RADIOACTIVITY GUIDELINES FOR THE HWCTR

\title{
Appendix G
}

\author{
RESRAD-BUILD
}

\section{Summary Report}



SSRAD-3UILD Program Outpue, Version 2.10 03/06/97 13:41 Page: $0-0: 1$ ** : Building on the HWCTR site

File : FWCTRE.DAT

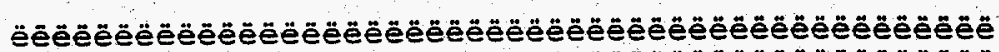

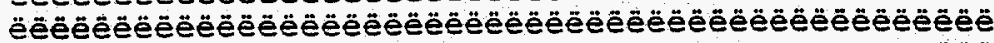
ëëè

ëēè

ëè RESRAD-BUILD Table of Contents ëëe

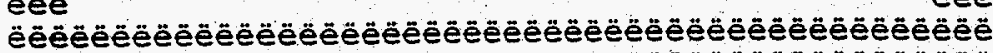

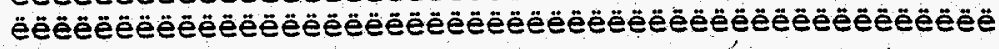

Inpue Parameters............... $0-1$

For Each Time (I) : $\ldots \ldots \ldots \ldots \ldots \ldots \ldots$

Time specific Parameters............. I-I

Receptor-Source Dose Summary......... I-2

Dose by Pathway Detail............... I-3

Dose by Nuclide Detail............. I-4

Full Summary...................F -1 
* RESRAD-BUILD Program Output, Version 2.10:03/06/97 13:41 Page:0-1: Title: Building on the HWCTR site

Input File : HWCTRE.DAT

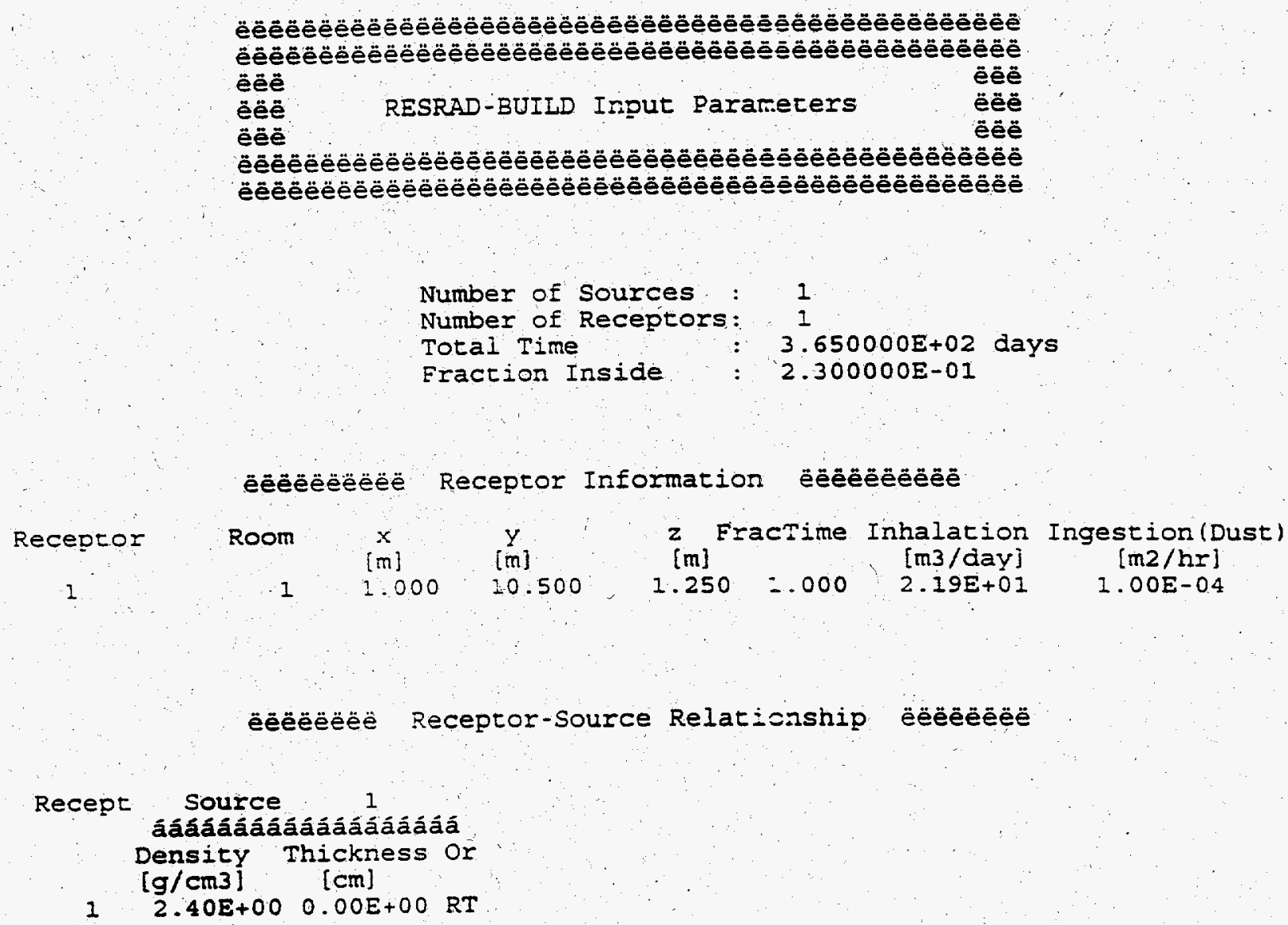

$\cdots$

$\therefore$

ëëëëëëë Receptor-Source Relat icnship ëëëëëë 
SRAD-BUILD Program Output, Version 2.10 03/06/97 13:41 Page: $0-2: 2$ ** : Building on the HWCTR site

File : HWCTRE.DAT

\section{ëëëëëëë Building Information ëẹëëëëe}

Building Air Exchange Rate: $8.00 \mathrm{E}-0 \mathrm{l}$ 1/hr

ieight [m]

Area [m2]

$13: 2.500$

rea 36.000

H2: $\quad 2.500$

rea $\quad 36.000$

HI : 2.500

rea 53.300
Air Exchanges [m3/hr]

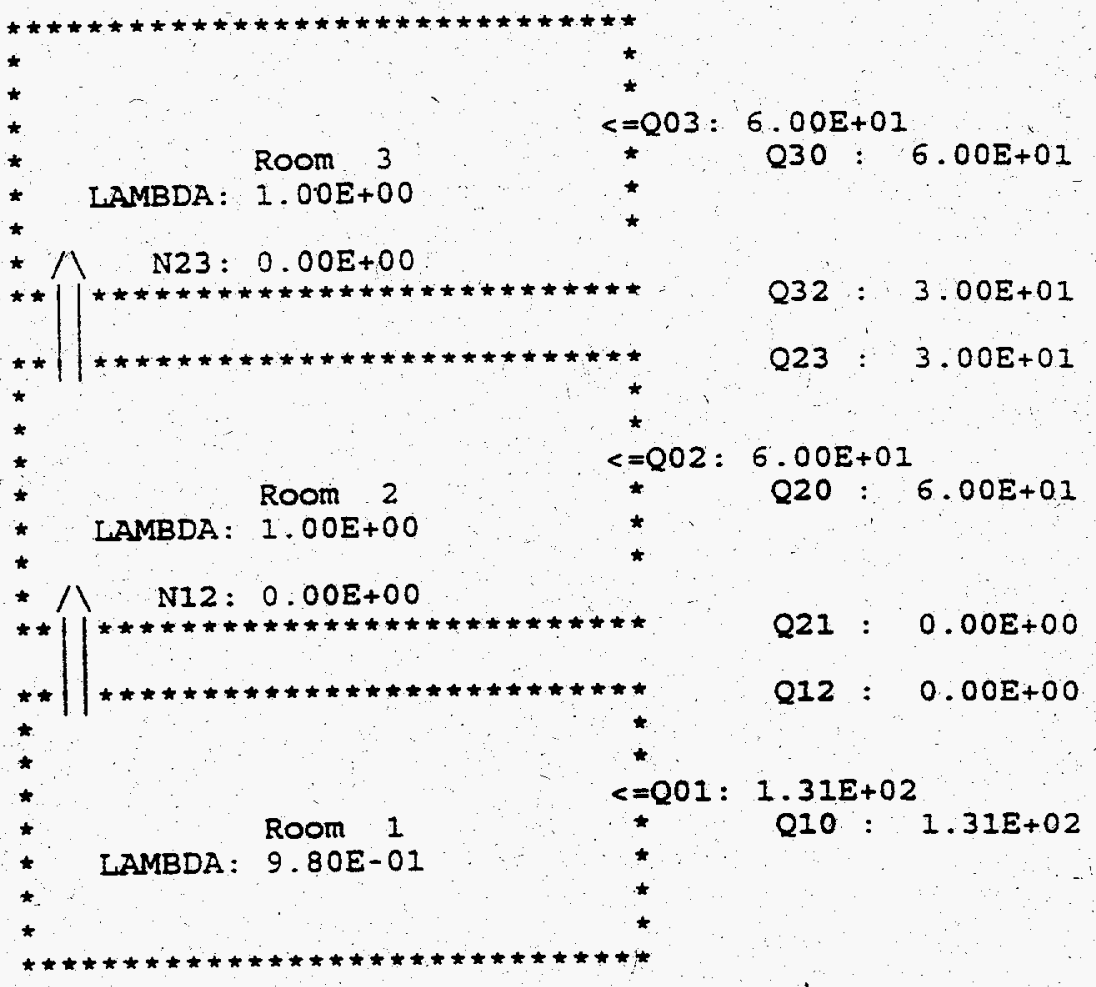

Deposition velocity: $1.00 \mathrm{E}-02[\mathrm{~m} / \mathrm{s}]$ Resuspension Rate: $5.00 \mathrm{E}-07[1 / \mathrm{s}]$ 
** RESRAD-BUILD Program Output, Version $2.10 \quad 03 / 06 / 97$ 13:41 Page: $0-3:$ : $^{\prime} 4$ ** Titie : Building on the HWCTR site

Input File : HWCTRE.DAT

ëeёëëëë source Information ëëëè̈ëë

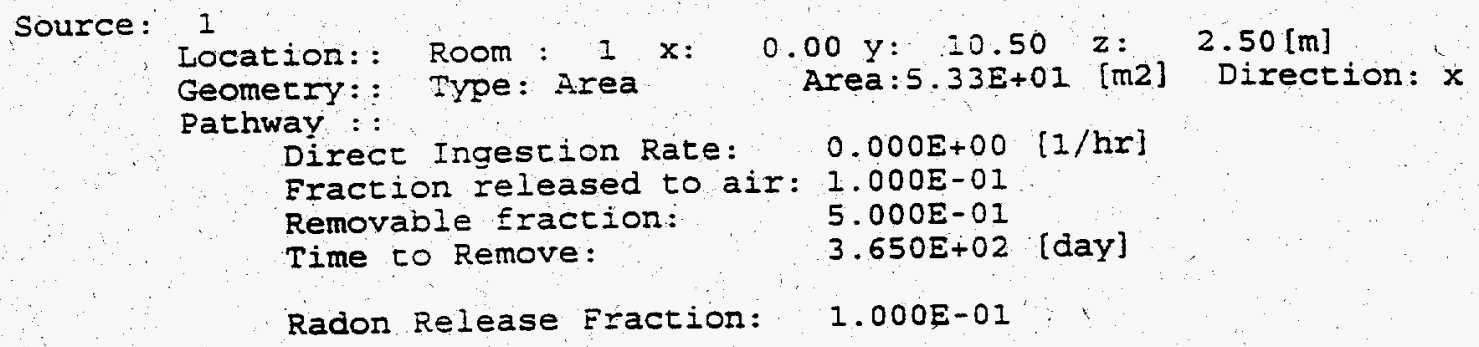

Contamination: :

Nuclide Concentration

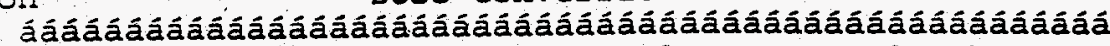
Ingestion Inhalation External (Surface)

$[\mathrm{pci} / \mathrm{m} 2]$ [mrem/pci] [mrem/pCi] [mrem/yr] (pci/m2)]

$A M-241$
$P U-239$
$N P-237$
$U-238$
$U-235$
$U-234$
$U-233$
$P A-231$
$T H-230$
$T H-229$
$A C-227$
$R A-226$
$P B-210$
$C S-137$
$S R-90$
$C O-60$
$C-14$
$H-3$

$6.630 E+03$

$7.490 E+03$

$0.000 E+00$

$2.870 E+04$

$0.000 E+00$

$0.000 E+00$

$0.000 E+00$

$0.000 E+00$

$0.000 E+00$

$0.000 E+00$

$0.000 E+00$

$0.000 E+00$

$0.000 E+00$

$2.680 E+06$

2.210E+06

7. $140 E+05$

$5.170 \mathrm{E}+08$

1. $830 \mathrm{E}+10$

$4.500 E-03$

4. 30OE-03

3. $900 \mathrm{E}-03$

2. 500E-04

2. 500E-04

$2.600 \mathrm{E}-04$

2.700E-04

1. $100 \mathrm{E}-02$

5. 300E-04

4. 30OE-03

1. 50OE-02

I. $100 \mathrm{E}-03$

$6.700 E-03$

5. $000 E-05$

1. $400 \mathrm{E}-0.4$

2. $600 E-05$

2. $100 \mathrm{E}-06$

6. $300 E-08$

5.200E-0I

5. 1OOE-0I

4.900E-OI

1. 200E-01

1. 200E-01

1. 300E-0I

1. $300 E-01$

1. $300 \mathrm{E}+00$

3.200E-0I

2. $000 E+00$

6. $700 E+00$

7. $900 \mathrm{E}-03$

2. 100E-02

3. 200E-05

1. $300 \mathrm{E}-03$

1. $500 \mathrm{E}-04$

2. $100 E-06$

$6.300 E-08$

3. $220 \mathrm{E}-06$

4. 290E-08

2. $620 \mathrm{E}-05$

3. $530 E-06$

1. 950 E-05

8. $750 E-08$

8. $380 E-08$

4. $760 E-06$

8. 780E-08

3. $680 \mathrm{E}-05$

4. 53OE-05

1. $940 E-04$

4. $140 E-07$

$6.490 E-05$

6. 560E-07

2. 750 E- 04

1. 880 E- 09

$0.000 E+00$
External Submersion

(Volume)

[mrem/yr/ [mrem/yr] $(\mathrm{pCi} / \mathrm{m} 3)](\mathrm{pCi} / \mathrm{m} 3)]$

$2.740 E-08.9 .570 E-05$

$1.850 E-10 \quad 4.960 E-07$

$6.880 E-07$ I.210E-03

9.510E-08 1.600E-04

$4.740 E-07 \quad 9.030 E-04$

$2.520 E-10 \quad 8.930 E-07$

$8.750 E-10 \quad 1.910 E-06$

$1.190 E-07 \quad 2.010 E-04$

$7.570 \mathrm{E}-10 \quad 2.040 \mathrm{E}-06$

$9.870 E-07 \quad 1.720 E-03$

$1.260 \mathrm{E}-06 \quad 2.160 \mathrm{E}-03$

7.000 E-06 1.040 E-02

$3.820 \mathrm{E}-09$ 1.430E-05

2.140E-06 3.190E-03

1.54OE-08 2.310E-05

1. $020 \mathrm{E}-05$ 1.470E-02

8.420E-12 2.620E-08

$0.000 E+00 \quad 3.870 E-08$ 
RESRAD-BUILD Program Output, Version 2.10 03/06/97 13:41 Page: 1- 1 : le : Building on the HWCTR site
it File : HWCTRE.DAT
Evaluation Time:
0.000000
years

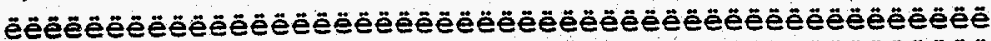

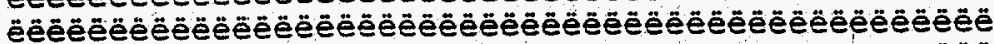
ëëe Assessment for Time: $Z$ ëëẻ ëëe $\quad$ Time $=0.00 E+00 \mathrm{yr}$ ẽëé

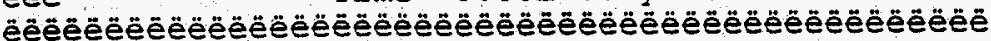

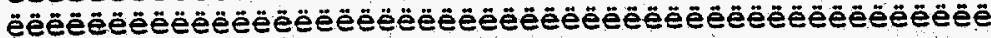

ëëëēëëè source Information ëëëëëëë

urce : 1

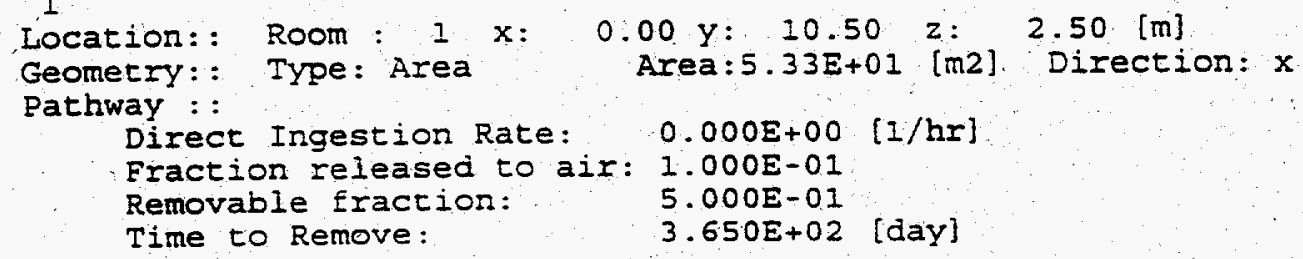

Contamination: : Nuclide

$A M-241$
$P U-239$
$N P-237$
$U-238$
$U-235$
$U-234$
$U-233$
$P A-231$
$T H-230$
$T H-229$
$A C-227$
$R A-226$
$P B-210$
$C S-137$
$S R-90$
$C O-60$
$C-14$
$H-3$

Concentration

[pCi/m2]

$6.630 \mathrm{E}+03$

$7.490 E+03$

$0.000 E+00$

$2.870 E+04$

$0.000 E+00$

$0.000 \mathrm{E}+00$

$0.000 E+00$

$0.000 E+00$

$0.000 E+00$

$0.000 E+00$

$0.000 E+00$

$0.000 E+00$

$0.000 E+00$

2. $680 \mathrm{E}+06$

2. $210 E+06$

7. $140 E+05$

5. $170 E+08$

1. $830 \mathrm{E}+10$ 
** RESRAD-BUILD Program Output, Version 2.10 $03 / 06 / 97$ 13:41 Page: 1- 2 : $6 \star \star$ Title : Building on the HWCTR site Input File : HWCTRE.DAT

\author{
Evaluation Time:
}

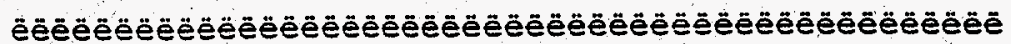

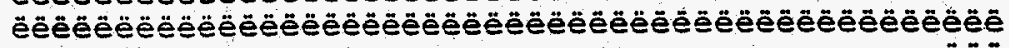
ëëè ëëë ëëè RESRAD-BUILD Dose Tables èể ëëë

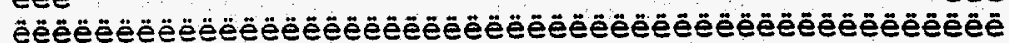

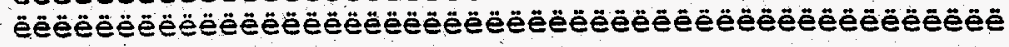

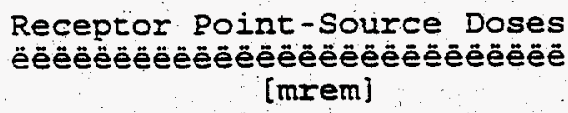

$\begin{array}{lll} & \text { Source } & \text { Total } \\ \text { Receptor I } & 1.2 E+02 & 1.2 E+02 \\ \text { Total } & 1.2 E+02 & 1.2 E+02\end{array}$


RESRAD-BUILD Program Output, Version 2.10 03/06/97 13:41 Page: 1- 3 :

le : Building on the HWCTR site

ut File : HWCTRE.DAT Evaluation Time: 0.000000 years

Pathway Detail of Doses

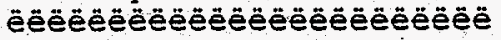
[mrem]

rce: 1

Receptor 1

otal

External

$2.57 E+01$

$2.57 E+0 I$
Inhalation

$6.80 E+01$

$1.15 E+00$

1. $15 E+00$

$9.91 E-03$

$9.91 E-03$

$6.80 \mathrm{E}+0 \mathrm{I}$

Radon

Ingestion

$3.42 E-32 \quad 2.53 E+01$

$3.42 E-32 \quad 2.53 E+01$ 
* RESRAD-BUIID Program Output, Version 2.10 03/06/97 13:41 Page: I- 4: Title : Building on the HWCTR site Input File : FWCTRE. DAT

\section{Evaluation Time:}

0.000000

\section{Nuclice Detail of Doses

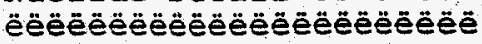 [mrem]}

\begin{tabular}{|c|c|c|}
\hline Nuclide & Receptor & Total \\
\hline $\begin{array}{l}A M-24 I \\
A M-241\end{array}$ & $1.50 E+01$ & $1.50 E+01$ \\
\hline $\begin{array}{l}P U-239 \\
P U-239\end{array}$ & $1.67 \mathrm{E}+01$ & $1.67 E+01$ \\
\hline $\begin{array}{r}U-238 \\
U-238 \\
C S-137\end{array}$ & $1.48 E+01$ & $1.48 \mathrm{E}+01$ \\
\hline $\begin{array}{l}C S-137 \\
S R-90\end{array}$ & $1.46 \mathrm{E}+01$ & $1.46 \mathrm{E}+01$ \\
\hline $\begin{array}{c}S R-90 \\
C 0-60\end{array}$ & $1.50 \mathrm{E}+01$ & $1.50 E+01$ \\
\hline $\begin{array}{l}C O-60 \\
C-14\end{array}$ & $1.43 E+01$ & $I .43 E+0 I$ \\
\hline $\begin{array}{l}\mathrm{C}-14 \\
\mathrm{H}-3\end{array}$ & $1.48 E+01$ & $1.48 E+01$ \\
\hline$H-3$ & $1.49 E+01$ & $1.49 E+01$ \\
\hline
\end{tabular}


KESRAD-BUIID Program Output, Version 2.10 03/06/97 13:41 Page: 2- I :

le : Building on the HWCTR site

ht File: EWCTRE.DAT Evaluation Time: 1.00000 years

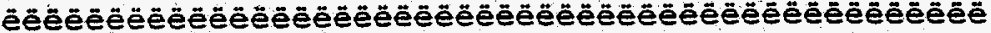

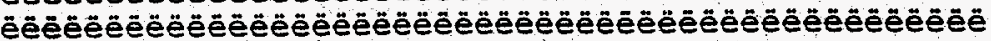
ëēé Assessment for Time: 2 ëë ëë : Time $=1.00 E+00 \mathrm{yr}$ ëë

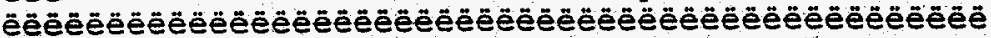

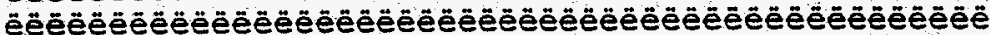

ëēëëëëëe Source Information ëëēëëëë

urce: 1 Location:: Room : $1 \mathrm{x}: 0.00 \mathrm{y}: 10.50 \mathrm{z}: 2.50[\mathrm{~m}]$ Geometry: : Type: Area Area:5.33E+01 [m2] Direction: $x$ Pathway : :

Direct Ingestion Rate:

$0.000 E+00$

$[1 / \mathrm{hr}]$

Fraction released to air: 1.000E-01

Removable fraction: 5.000E-01

Time to Remove:

$3.650 E+02$ [day]

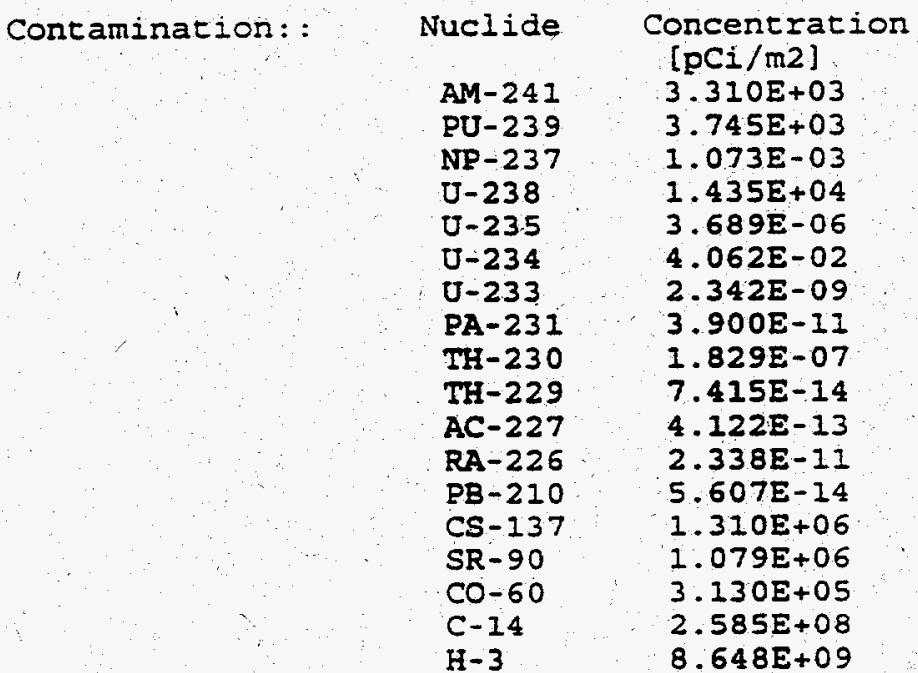


* * RESRAD-BUIID Program Output, Version 2.1003/06/97 13:41 Page:2-2: $10 * *$ Title; Builaing on the HWCTR site Input File : HWCTRE:DAT Evaluation Time:

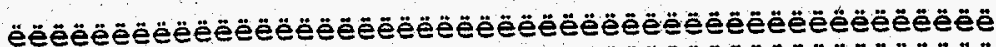

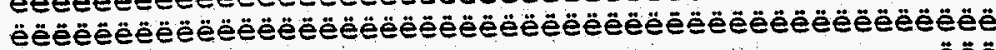
ëêe ëè ëëë ëè ëèe

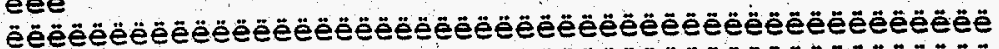

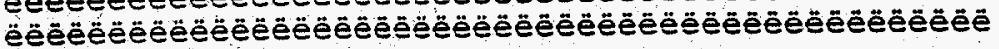

Receptor Point-Source Doses

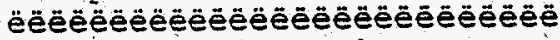

[mrem]

$\begin{array}{lll} & \text { Source } & \text { Total } \\ \text { Receptor I } & 1.2 E+01 & 1.2 E+01 \\ \text { Total } & 1.2 E+01 & 1.2 E+0 I\end{array}$


RESRAD-BUIID Program Output, Version 2.10 03/06/97 13:41 Page: 2-3: $11 * *$ e : Building on the HWCTR site

it File : HWCTRE.DAT

Evaluation Time:

1.00000

years

Pathway Detail of Doses

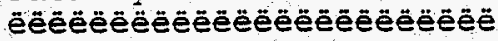
[mrem]

ce : 1

Receptor

1

btal

External

Deposition Immersion

$0.00 E+00$

$0.00 E+00$

1. $19 E+01$

$0.00 E+00$

$0.00 E+00$

Inhalation $0.00 E+00$

Radon

2.74E-17

$0.00 E+00$

2. $74 E-17$
Ingestion

$0.00 E+00$

$0.00 E+00$ 
** RESRAD-BUILD Program Output, Version 2.10 03/06/97 $13: 41$ Page: 2- 4 : Title : Building on the HWCTR site

Input File : HWCTRE.DAT

Evaluation Time:

1.00000

years

Nuclide Detail of Doses

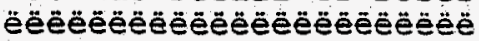

[mrem]

Source: I

Nuclide Receptor Total

$A M-24 I$

AM-241 1.03E-03 1.03E-03

NP-237 2.24E-09 2.24E-09

$U-233 \quad 2.30 E-17,2.30 E-17$

TH-229 2.13E-19 2.13E-19

PU -239

PU-239 2.93E-05 2.93E-05

U-235 5.90E-12 5.90E-12

PA-23I 1.54E-17 I.54E-I7

AC-227 1.44E-18 1.44E-18

$\mathrm{U}-238$

U-238 $\quad 3.97 E-03 \quad 3.97 E-03$

U-234 4.92E-10 4.92E-10

TH-230 I.93E-I5 $1.93 E-15$

$R A-226 \quad 3.38 E-16 \quad 3.38 E-16$

PB-210 2.57E-21 2.57E-2I

CS-137

CS-137 $\quad 6.07 E+00 \quad 6.07 E+00$

$S R-90$

SR -90

CO-60

$C 0-60 \quad 5.76 E+00 \quad 5.76 E+00$

$\mathrm{C}-14$

C-I4 0.00E+00 0.00E+00

$\mathrm{H}-3$

H-3

$0.00 E+00 \quad 0.00 E+00$ 
RESRAD-BUILD Program Output, Version 2.10 03/06/97 $13: 41$ Page: $3-1$ :

le : Building on the HWCTR site

it File : HWCTRE.DAT Evaluation Time: 10.0000 years

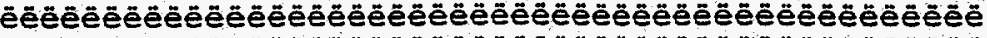

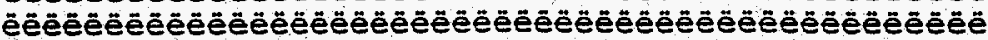

ëë Assessment for Time: 3 ëëë

ëe Time $=1.00 E+01$ yr ëëë

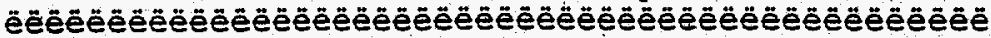

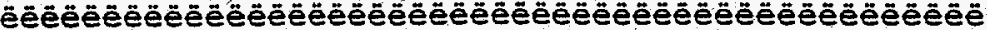

êëëëëëëè Source Information ëēëëëëë

urce : I

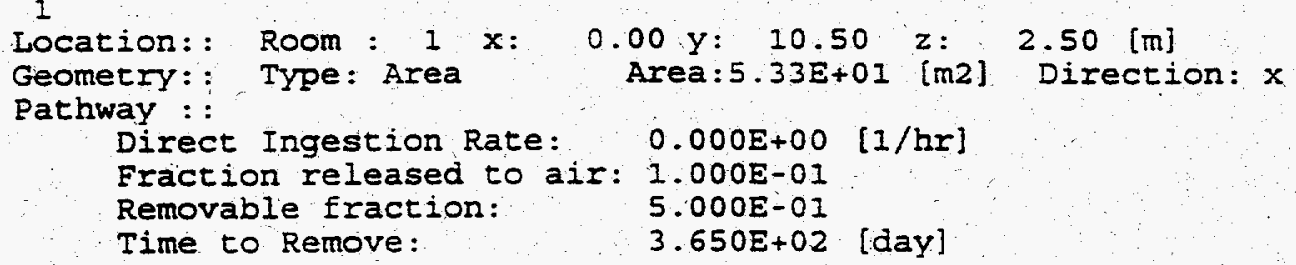

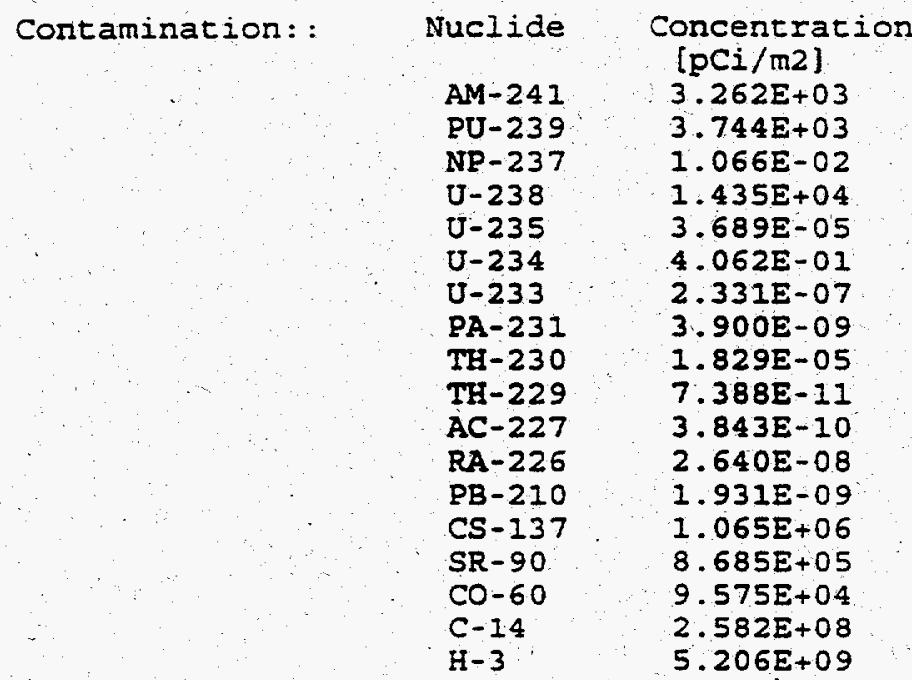


* RESRAD-BUILD Program Output, Version 2.10 03/06/97 13:41 Page: 3-2:

Title : Building on the HWCTR site

Input File : HWCTRE.DAT

Evaluation Tine:

10.0000

years

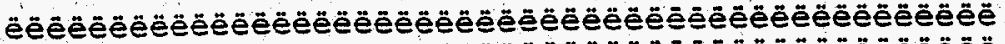

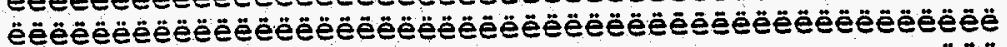

ëëe ëè

Ëë RESRAD-BUILD Dose Tables ëëë

ëë ëèè

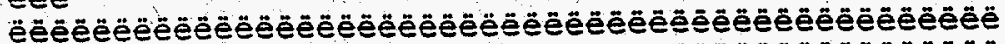

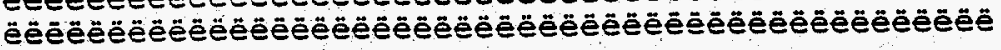

Receptor Point-Source Doses

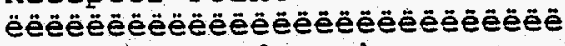

[mrem]

$\begin{array}{lll} & \text { Source } & \text { Total } \\ & 1 & \\ \text { Receptor } 1 & 6.7 E+00 & 6.7 E+00 \\ \text { Total } & 6.7 E+00 & 6.7 E+00\end{array}$


* RESRAD-BUILD Program Output, Version 2.10 03/06/97 13:41 Page:3-3: $15 \star \star$ itle : Building on the HWCTR site npit File : HWCTRE.DAT Evaluation Time: 10.0000 years

Pathway Detail of Doses

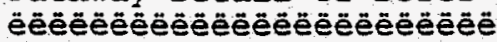
[mrem]

ource: I

Receptor Total
External

$6.74 E+00$

$6.74 E+00$
Deposition Immersión

$0.00 E+00$

$0.00 \mathrm{E}+00$

$0.00 E+00$

$0.00 E+00$
Inhalation
$0.00 \mathrm{E}+00$

Radon

3. $10 E-14$

$0.00 E+00$

$3.10 \mathrm{E}-14$

Ingestion

$0.00 E+00$

$0.00 E+00$ 
* RESRAD-BUIID Program Output, Version $2.1003 / 06 / 97$ 13:41 Page: 3 - 4 :

Title: Building on the HWCTR site

Input File : HWCTRE.DAT

Evaluation Time:

10.0000

years

Nuclide Detail of Doses

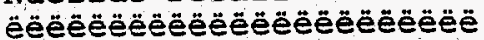

[mrem]

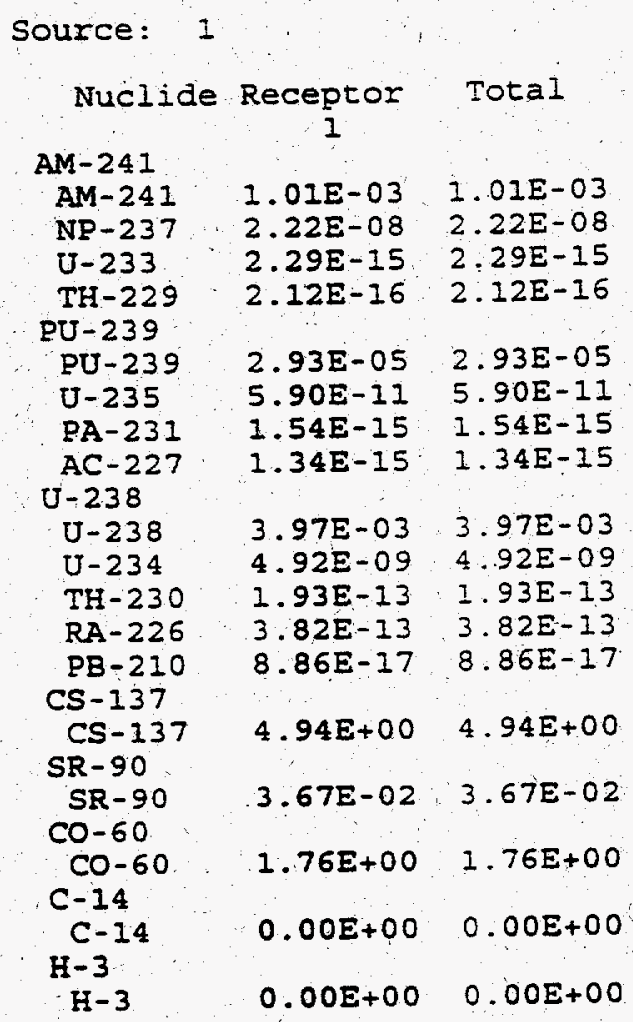


** RESRAD-BUIID Program Output, Version 2.10 03/06/97 13:41 Page: 4- $1:$

Title : Building on the HWCTR site

Input File : HWCTRE.DAT

Evaluation Time:

30.0000

years

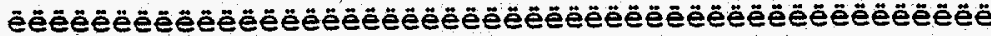

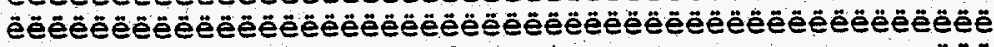
ëëë Assessment for Time: 4 ëëe

ëëè Time $=3.00 E+01 \mathrm{yr}$ ëëĕ

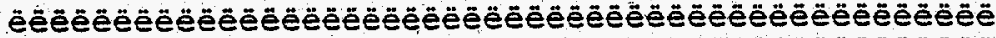

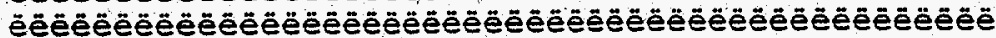

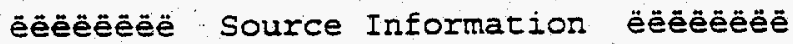

Source: 1

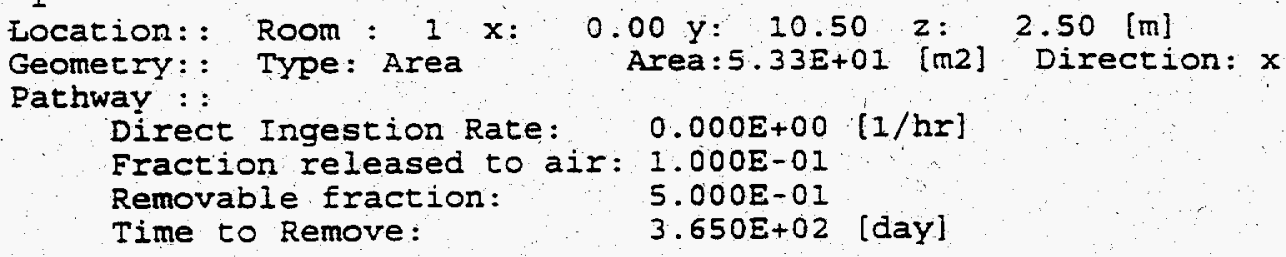

\begin{tabular}{|c|c|c|}
\hline $\begin{array}{r}\text { Contamination: : } \\
\qquad\end{array}$ & $\begin{array}{l}\text { Nuclide } \\
\text { AM-241 } \\
\text { PU-239 } \\
\text { NP-237 } \\
U-238 \\
U-235 \\
U-234 \\
U-233 \\
P A-231 \\
T H-230 \\
T H-229 \\
A C-227 \\
R A-226 \\
P B-210 \\
C S-137 \\
S R-90 \\
C O-60 \\
C-14 \\
H-3\end{array}$ & $\begin{array}{l}\text { Concentration } \\
\text { [pCi/m2] } \\
3.159 E+03 \\
3.742 E+03 \\
3.147 E-02 \\
1.435 E+04 \\
1.106 E-04 \\
1.219 E+00 \\
2.075 E-06 \\
3.509 E-08 \\
1.646 E-04 \\
1.978 E-09 \\
8.970 E-09 \\
7.113 E-07 \\
1.393 E-07 \\
6.728 E+05 \\
5.366 E+05 \\
6.888 E+03 \\
2.576 E+08 \\
1.686 E+09\end{array}$ \\
\hline
\end{tabular}


* RESRAD-BUILD Program Output, Version 2.10 03/06/97 13:41 Page: 4- 2 :

Title : Building on the HWCTR site

Input Eile : HWCTRE.DAT

Evaluation Time:

30.0000

years

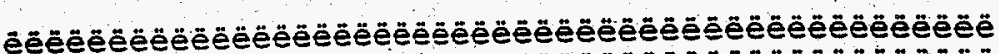

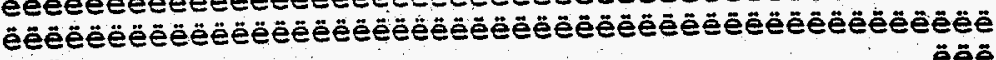
èè

êể

ëèé

RESRAD-BUIID DOSE Tables

ēēe

ëèe

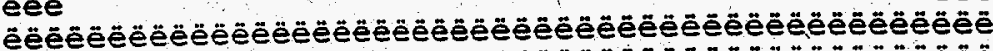

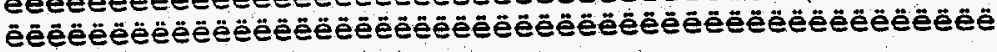

Receptor Point-Source Doses

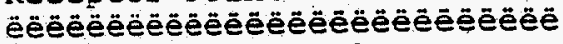
[mrem]

Source Total

1

Receptor 1

3. $3 E+00 \quad 3.3 E+00$

Total.

3. $3 E+00 \quad 3.3 E+00$ 
* RESRAD-BUILD Program Output, Version 2.10 03/06/97 13:41 Page: 4- 3 :

itle : Building on the HWCTR site

nput File : HWCTRE.DAT

Evaluation Time:

30.0000

years

Pathway Detail of Doses

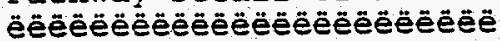

[mrem]

Source: 1 Receptor 1

Tota1
External

$3.27 E+00$

$3.27 \mathrm{E}+00$
Deposition Immersion

$0.00 E+00$

$0.00 E+00$
$0.00 E+00$

$0.00 \mathrm{E}+00$
Inhalation

$0.00 E+00$

$0.00 E+00$
Radon

8. 35E-13

$8.35 E-13$
Ingestion

$0.00 E+00$

$0.00 E+00$ 
** RESRAD-BUIID Erogram Output, Version 2.10 03/06/97 13:4I Page: $4-4$ : Title : Building on the HWCTR site [mrem]

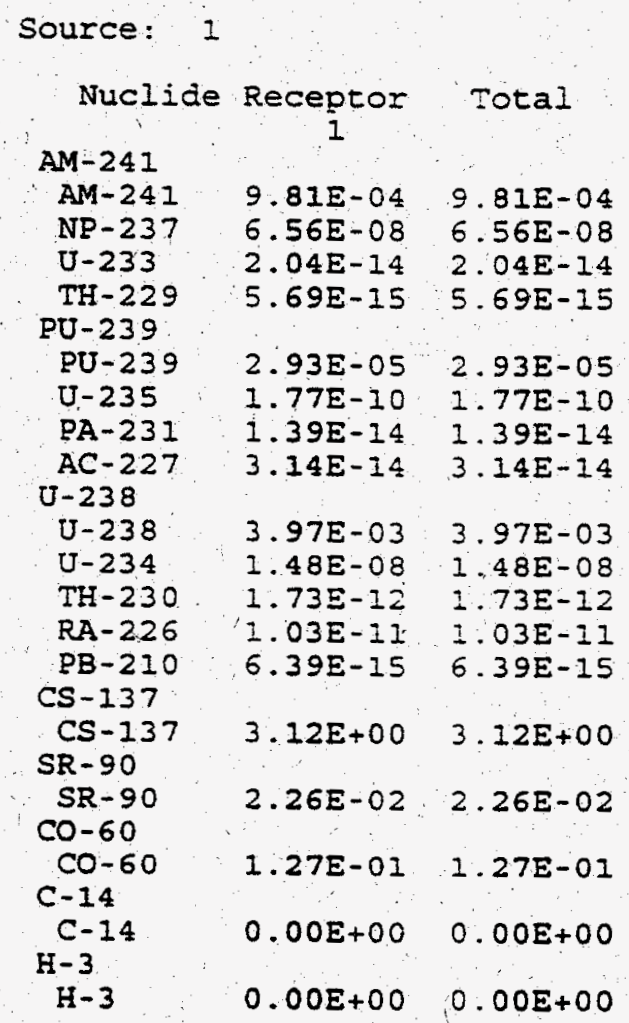

